\title{
WAVE POWERED DESALINATION
}

by

Alan J. Crerar

B.Sc. (Edinburgh)

Thesis presented for the Degree of Doctor of Philosophy University of Edinburgh

November, 1989

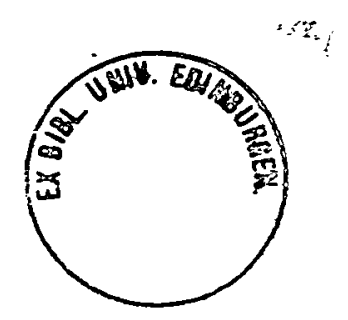


To my family and to Anne. 
"Learn to think."

W.G. Crerar, Architect.

iii 
The work described in this thesis is the original work of the author except where specific reference is made to other sources. It has not been submitted, in whole or in part, for any degree at any other University 
UST OF FIGURES

ACKNOWLEDGEMENT

ABSTRACT

xiii

1 INTRODUCTION

1.1 OVERVIEW

1.1.1 Desalination

1.1.2 The Edinburgh Duck Wave Absorber

1.2 WAVE POWERED DESALINATION

1.2.1 Process Description

1.2.2 Auxiliary Equipment

1.3 PROJECT OVERVIEW

1.3.1 Research Programme

1.3.2 Scope of Thesis

2 UTERATURE REVIEW AND THEORY

2.1 DESALINATION

2.1.1 Overview

2.1.2 The MSF Process

2.1.3 Other Distillation Methods

2.1.4 Other Desalination Processes

2.1.5 Desalination Using Renewable Energy

2.1.6 Trends in Desalination

2.2 OCEAN WAVE ENERGY

2.2.1 Introduction

2.2.2 Linear Wave Theory

2.2.3 Wave-Device Interactions

2.2.4 The Wave Energy Resource

3 MOdELING AND SIMULATION OF THE DESALINATION PROCESS 68

3.1 INTRODUCTION

\subsection{THE FIRST MODEL}


3.2.4 Implementation of the First Model 77

$\begin{array}{ll}\text { 3.3 THE SECOND MODEL } & 78\end{array}$

$\begin{array}{ll}\text { 3.3.1 Objectives } & 78\end{array}$

3.3.2 The Model Basis $\quad 79$

3.3.3 Derivation of the Equations $\quad 79$

3.3.4 Implementation of the Second Model 92

3.4 SIMULATION OF EXPERIMENTAL APPARATUS 94

3.4.1 The First Model as a Design Tool 94

3.4.2 Modifications to the Second Model 95

4 DESIGN AND DEVELOPMENT OF THE EXPERIMENTAL FACILTY 101

4.1 INTRODUCTION 102

4.1.1 The Experimental Approach 102

4.1.2 Use of an Analogue Working Fluid 104

4.2 DETAILED DESIGN OF COMPONENTS 105

4.2.1 Design of Condenser/Evaporator Unit 105

4.2.2 Design of Liquid Cooler and Trim Heater 107

4.2.3 Design of the Compressor 109

$\begin{array}{ll}4.3 \text { INSTRUMENTATION AND CONTROL } & 114\end{array}$

4.3.1 Introduction $\quad 114$

4.3.2 Piston Position Control . 115

$\begin{array}{ll}\text { 4.3.3 Data Logging and Process Control } & 121\end{array}$

$\begin{array}{ll}5 \text { RESULTS AND ANALYSIS } & 163\end{array}$

5.1 RESULTS FROM THE FIRST MODEL 164

5.1.1 Transient Performance Predictions 164

5.1.2 Performance Predictions $\quad 165$

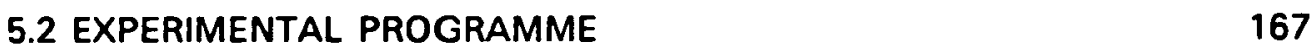

$\begin{array}{ll}\text { 5.2.1 Procedure } & 167\end{array}$

5.2.2 Analysis of Experimental Data $\quad 170$

5.3 RESULTS FROM THE SECOND MODEL 173

5.3.1 Transient Performance Predictions 173

$\begin{array}{ll}\text { 5.3.2 Performance Predictions } & 174\end{array}$

$\begin{array}{ll}5.4 \text { SUMMARY } & 180\end{array}$

6 CONCLUSIONS AND RECOMMENDATIONS $\quad 225$

7 APPENDICES $\quad 229$ 
I.I Alkaline Scales

I.II Calcium Sulphate Scaling

I.III Methods of Scale Control

234

II ENHANCED HEAT TRANSFER IN FALUNG FILMS

236

II.I Condensation

236

II.II Evaporation

238

III PROPOSALS FOR FUTURE WORK

III.I Addition of Compliance to the Compressor

III.II Verification of the Fluid Piston Equation

III.III New Model for the Non-Return Valves

III.IV New Model of the Heat Recovery Exchanger

IV PROGRAM FLOWSHEETS AND LSTINGS

IV.I First Model

IV.II Second Model 
1.2 Development of Edinburgh Duck: "Tadpole"

1.3 Development of Edinburgh Duck: the Duck

1.4 Development of Edinburgh Duck VC System

1.5 Detail of Edinburgh Duck VC System

1.6 Cross Section through Spiral Plate

1.7 Inertial Loop Pump

2.1 Schematic of Simple Still

2.3 Schematic of MSF Plant

2.6 Temperature Profile Through MSF Plant with Inifite Number of Stages

2.8 Total Cost vs. Number of Stages for MSF and MEB Plants

2.9 Schematic of VTE Plant

2.10 Cross Section Through Double Fluted Tube 62

2.11 Schematic of Simple VC Unit 62

2.12 Simple RO Plant 63

$2.12 \quad$ Principle of Electrodialysis $\quad 63$

$\begin{array}{lll}2.13 & \text { Principle of Electrodialysis } & 64\end{array}$

$\begin{array}{lll}2.14 & \text { Global Rainfall Patterns } & 64 \\ 2.15 & 65\end{array}$

$\begin{array}{lll}2.15 & \text { Progressive Water Wave } & 65 \\ 2.16 & \text { Circulation Beneath Progressive Waves } & 65\end{array}$

$\begin{array}{lll}2.17 & \text { Momentum Transfer in Water Waves } & 66 \\ 2.18 & \text { Simply Shaped Body in a Wave Field } & 66\end{array}$

$\begin{array}{lll}2.18 & \text { Simply Shaped Body in a Wave Field } & 66 \\ 2.19 & \text { Global Wave Climates }\end{array}$

3.1 2 Chamber/4 Chamber Basis of the First Model 97

3.2 Definition of $\delta V$ for the First Model 97 
3.3 Fluid Piston as a U-Tube

3.4 Fluid Piston as a Solid Semi-Cylinder

3.5 4 Chamber Basis of the Second Model

3.6 Fractional Opening vs. $\Delta p$ for the Non-Return Valves $\quad 99$

3.7 Definition of $\delta V$ for the Second Model

3.8 Heat Recovery Exchanger Model

4.1 Early Design of Compressor

4.2 Compressor with Compliance Feedback Loop

Sketch of Still with Liquid and Vapour Lines

Distributor Components Simple Flowsheet for MFI Communication Improved Flowsheet for MFI Communication Flowsheet for MFI Control and 
4.28 Flowsheet for Control Parameter

$$
\text { Setting Program }
$$

4.29 Interface Circuit for BBC Microcomputer 156

4.30 Flowsheet for Menu System 157

4.31 Multiplexer Circuit Diagram 158

4.32 Solenoid Valve Relay Wiring Diagram 159

$4.33 \quad$ Tachometer i.c. Circuit Diagram 160

4.34 Opto-Isolator i.c. Circuit Diagram 161

4.35 Thermocouple Probe Assemblies 162

4.36 Level Measurement Boxes 162

5.1 Plot of $\Delta p$ vs. $\theta$ from equation (5.1) 182

$5.2 \quad$ Plot of $\Delta p$ vs. $\theta$ from the First Model 182

5.3 Plots of $\Delta p$ vs. $t$ for Time Dependency 183

Testing; First Model 183

$\begin{array}{llr}5.6 & \text { Output vs. Area; First Model } & 185 \\ 5.7 & \text { Output vs. T } & 185\end{array}$

$\begin{array}{llr}5.7 & \text { Output vs. T } T_{\text {feed }} ; \text { First Model } & 185 \\ 5.8-.24 & \text { Plots of } \Delta p \text { vs. } \theta ; & 186-194\end{array}$

Experimental and Predicted for Various

Amplitudes and Periods

$5.25-.41$ Plots of $\Delta p_{\text {sim }}$ vs. $\Delta p_{\text {exp }}$

for Various Amplitudes and Periods

5.42 Gradient, Intercept and Period from

Figures (5.25) to (5.41) vs. Period

5.43 Gradient, Intercept and Period from

Figures (5.25) to (5.41) vs. Amplitude

5.44 - .60 Plots of $p$ vs. $\theta$;

Experimental and Predicted

for Various Amplitudes and Periods

5.61 Transient Behaviour Prediction from

the Second Model

5.62 Output vs. Period and Amplitude from the Second Model 
5.63 SEC vs. Period and Amplitude from the Second Model 216

5.64 $\begin{array}{ll}\alpha \text { vs. Period } & 217\end{array}$

5.65 a vs. Amplitude

5.66 Output vs. Period without the Fluid Piston

5.67 Output vs. Area from the Second Model

$5.78 \Delta p$ vs. $\theta$ for Various Values of $\Delta p_{o}$

7.2 Scaling Thresholds of $\mathrm{CaSO}_{4}$

$$
\text { Crystalline Forms after [53] }
$$

7.6 Block Diagram for Pressure Compliance Control System

7.7 Simplified Circuit Diagram for Pressure Compliance System 


\section{ACKNOWLEDEMENT}

The author would like to express his gratitude to the following people who have contributed to the completion of this research project:

1. Dr. C.L. Pritchard for his supervision throughout the project.

2. The workshop staff at the Department of Chemical Engineering, Edinburgh University, for the construction of the experimental apparatus.

3. The members of the Edinburgh Wave Power Group for their help, advice and loan of equipment.

4. Mr. R.E. Low, Mr. A.T. Doig and Dr. B.H. Cooke for discussion and encouragement. 


\section{ABSTRACT}

Desalination of brackish or seawaters may be used to provide fresh water in arid areas. The cost of energy required for conventional desalination processes has, however, limited their adoption to certain specific geographical locations. Much work has been carried out on the use of renewable resources (particularly solar power) as possible energy sources for desalination. This study was concerned with the investigation of the use of the "Edinburgh Duck" ocean wave energy absorption device as the prime mover in a novel vapour compression distillation system; a process originally proposed by Professor S. H. Salter of Edinburgh University.

Two mathematical models of the unsteady-state heat and mass transfer processes taking place in the process under simulated sea conditions were developed and subsequently implemented in computer programs. The principles upon which the final model was based were experimentally tested through the operation of a small-scale analogue plant using the refrigerant $\mathrm{R}-11, \mathrm{CCl}_{3} \mathrm{~F}$, as the working fluid. Comparisons between the predicted performance of the equipment (from a mathematical simulation of the pilot plant based on the same fundamental equations used to describe the full-scale system) and the actual behaviour of the plant suggested that the model could provide indications of the characteristics of the proposed process with reasonable confidence. The mathematical model was then used to investigate the effects of various process parameters upon the system.

From the results of this study, it would seem that the proposed process would be capable of meeting initial expectations, but that further work would be required in specific areas before moving on to any more detailed design work. 
CHAPTER 1

INTRODUCTION 


\subsection{OVERVEW}

\subsubsection{Desalination}

The desalination industry is geared to the production of very large amounts of a product for sale at a low price. Like many such processes, this implies that a small change in the running costs or output of a given plant can make a large difference to the economics of the process. As the fundamentals of desalination are quite straightforward (i.e. the separation of a volatile solvent from a solution containing a non-volatile solute) so it becomes clear that the running costs involved in a process must be, to a first approximation at least, energy costs and capital amortization charges. At the moment, the only countries for which large scale desalination is feasible are those with access to large fossil fuel deposits, ie. the countries of the Arabian peninsula, with the USA and the USSR running poor second and third places respectively. Much of the research in desalination, therefore, has been driven by the need to minimise energy usage in the face of rising fuel costs. Considerable advances have been made in this field, but it should be recognised that, eventually, alternative sources of energy to oil or gas must be exploited. One solution may be to use nuclear power; this has been thoroughly investigated and is probably a realistic proposition for states with the necessary technical expertise and financial resources. For most developing countries (in particular) nuclear desalination cannot be considered as a practical option; many of these countries, however, urgently require increased fresh water supplies and thus represent a large potential desalination market. Consequently, it is important to study the means by which the available renewable energy resources may be utilised to provide fresh water. A review of the research interest in this field is included in chapter 2, section 2.1.5.

Ocean wave energy is briefly discussed below and in more detail in chapter 2 , section 2.2.; an important point is, however, that wave energy is most easily and efficiently converted to mechanical energy. If one wishes to use wave energy for desalination, therefore, it would seem to be logical to look first at desalination processes which can directly use mechanical work. There are three such processes currently in use: reverse osmosis, freezing and mechanical vapour compression desalination. Each of these processes is described in detail in chapter 2, sections 2.1.3 and 2.1.4.; but it is possible here 
to evaluate each for its suitability in a wave powered system, at least approximately. Reverse osmosis (RO) is a membrane process; crudely, it may be thought of as pushing water molecules under pressure through a semi-permeable membrane, impervious to salt, to produce a fresh water stream and a concentrated brine stream. A compact and energy efficient process, RO has been increasing in popularity over the past decade or so with the development of improved membranes. Freezing processes rely upon the difference in the melting points of pure and salt water ice. The actual process flowsheets can appear complex, but basically a refrigeration cycle using a direct contact refrigerant (often butane) is the proposed method. Plant complexity and numerous technical difficulties have lessened the appeal of freezing processes and no large scale commercial plants have been successfully operated. Mechanical vapour compression distillation (VC) is a well established process, both in desalination and in the processing industries in general. In desalination plants (one of its simplest applications) the water vapour from evaporating brine is compressed (to raise its enthalpy and temperature) and then condensed in a heat exchanger against the evaporating brine. As all the latent heat of the vapour is "recycled" this process can offer energy efficiencies on a par with RO and many plants have been commercially operated, although usually on a small scale.

The various advantages and disadvantages of each of these processes are discussed in detail in chapter 2, sections 2.1.3 and 2.1.4 in terms of their use in more-or-less "conventional" desalination applications. Their use in a wave powered context may place different requirements upon them and so it is important to consider what these requirements might be. Firstly, the power input to the process will vary with time in a random manner and so the process must be capable of flexibility of operation. Secondly, it is likely that the plant may be extremely inaccessible and, therefore, maintenance and/or servicing should be kept to a minimum.. Thirdly, and finally, it is desirable to minimise the number of energy conversion steps from the wave to fresh water so that the overall energy requirement of the process can be kept as low as possible; in general a device which could operate in a wave climate of only 10-20kW. $\mathrm{m}^{-1}$ would find many suitable locations (see chapter 2, section 2.2.4). All of these tenets suggest that the chosen process should be as "simple" as practical; for example, the number of moving parts or parts that need to be carefully machined should be taken into account in any evaluation of the 
process. This tends to mitigate against a freezing process: such processes tend to be inflexible, complex and to carry a fairly heavy service burden. The differences between the remaining two, $R O$ and VC, are not so marked. RO involves mass transfer across a boundary driven by a pressure difference; VC involves heat (and therefore mass) transfer across a boundary driven by a temperature (and therefore pressure) difference; RO requires a pump, VC a compressor. In fact, both processes have been suggested for use in wave powered systems: RO by Pleass, 1983, [52], and VC by Salter, 1983, [60] (the basis of this project). Work has also been carried out into the suitability of RO for wind powered systems, which exhibit some of the same characteristics of wave powered plants (variability of supply, lack of external power for auxiliary units, etc); see chapter 2, section 2.1 .5 for details. Any comparative analysis must take into account the specific wave energy absorption device under consideration; in this case VC has been proposed as the most suitable method, as explained in sections 1.1 .2 and 1.2.

\subsubsection{The Edinburgh Duck Wave Absorber}

Interest in renewable energy resources in general rose during the 1970's as a result of the energy crisis arising from the Yom Kippur war. In the United Kingdom wave power received the most attention, as this country is perhaps one of the best sited in the world in terms of this resource (see chapter 2 , section 2.2.4). After an initial screening and evaluation exercise, seven absorber devices were chosen to be the recipients of significant amounts of research funding under the auspices of the Department of Energy. A review of the UK government's involvement in the wave power research programme may be found in Davies et al, 1983, [12]. One of the most promising of these devices was the Edinburgh, or Salter's (after Professor Stephen Salter, the original proponent of the device), "duck". It is upon the use of this device that this project is based.

The development of the duck concept started from a simple backless flap as shown in figure (1.1). This device consisted of a float or raft attached to an axis by trailing arms running back from it in the direction of the wave travel. As the waves passed, the rise and fall of the float could be converted into useful work at the axis of rotation. This idea developed into a device known as the "tadpole" shown in figure (1.2). The arrangement was similar to the 
backless flap, but with a much greater volume concentrated around the axis of rotation. Instead of a float riding over the waves, the waves exerted a force on the forward part of the device causing it to nod about the axis in a manner similar to the earlier flap. Further experimentation evolved this shape into the "duck" as shown in figure (1.3). The duck is cam shaped in cross section and is moored, as before, with its long axis normal to the direction of travel of the waves. In calm water it would be around $60-80 \%$ submerged, with the point of the cam facing the oncoming wave. A wave reaching the duck would, once again, cause it to nod about the long axis; it was found that this shape and mode of motion allowed ducks to operate very efficiently, capturing in excess of $90 \%$ of the available energy in a random sea. From this point, research into ducks went on in great detail; specifically with a government design brief for a 2GW electricity generating station to be sited off the Scottish Hebrides in mind. One of the major problems facing the workers in this field was the problem of the conversion of the captured wave energy into electrical power for supply to the national grid network. The scheme proposed by the Edinburgh group involved the generation of high pressure hydraulic fluid by ring cam pumps mounted in gyroscopically stabilised cannisters about which the ducks would move (for a full description of the proposed system and of the duck design work in general, see Salter, 1985, [60]). The hydraulic fluid would then be used in a turbine-generator set. The technology involved in this method was undoubtably complex and some of it completely untried; the group was careful to stress, however, that the system was being designed for the future by which time it should have been possible to prove the concepts involved. Nevertheless, as energy prices stabilised, so the official UK interest in wave power weakened to the point where support of the Edinburgh wave power project finally ceased in $1986^{\prime}$.

\footnotetext{
'Note: recent policy has shifted slightly in favour of wave power with the limited funding of a small, experimental station to be built on the island of Islay off the west coast of Scotland, see Whittaker, 1987. [77].
} 


\subsection{WAVE POWERED DESALINATION}

\subsubsection{Process Description}

The desalination method chosen, VC distillation, is based on the use of a compressor. In accordance with the guidelines outlined in section 1.1.1, it was necessary to find a means of running a compressor from wave power, using a duck, with as few intermediate energy conversion stages as possible. A method evolved as follows (see figure (1,A)). A VC duck would have a hollow cylindrical core (figure $(1,4) a)$ half-filled with water. As the duck nodded, this water would stay approximately still, ecting as an inertial reference. Figure (1.4)b shows a barrier bisecting the vapour space above the fluid piston (as the body of water came to be known) and running the full length of the duck. Now if the duck were to nod in an anti-clockwise direction, so the vapour in the right-hand (forward) space would be expanded, while that in the left-hand (aft) space would be compressed. As the wave passed, the motion of the duck would be reversed and so too would the compression action. This, then, would form the basis of a very large, slow-moving, double-acting, reciprocating compressor. No additional energy conversions have been included; no moving parts would be needed; no machined components have been included: a process based on such a device would seem to meet the requirements laid down above.

For the distillation part of the system, a falling film evaporator/condenser unit would be located in the central barrier, as in figure (1.4)c, and connected to the vapour spaces by means of four non-return valves, as shown in figure (1.5). Falling film units can be very compact as well as offering (under the right operating conditions) very high heat transfer coefficients (see appendix II). The use of a novel type of heat transfer surface material was envisaged for the process. Consisting of a copper mesh sandwiched between two layers of resin plastic, this material has been proven to be very resistant to the formation of crystalline scales; normally a major problem for heat transfer surfaces in desalination duties (see appendix 1). Although the mesh plastic has a lower thermal conductivity than conventional metallic surfaces, the removal of the problems associated with scaling and thus the requirement for extensive chemical pre-treatment of the feedwater, was felt to compensate more than adequately (see Roach and Holtz, [57], and the United Wire Group Report, [74]). 
Preliminary calculations suggested that initial designs should be orientated towards the development of a device capable of producing $1000 \mathrm{~m}^{3} \cdot \mathrm{day}^{-1}$ of fresh water in wave climates of $10-20 \mathrm{~kW} . \mathrm{m}^{-1}$ (very moderate energy densities, see chapter 2, section 2.2.4). As a first estimate, a duck $20 \mathrm{~m}$ long by $6 \mathrm{~m}$ in diameter with a heat transfer surface area of $4000 \mathrm{~m}^{2}$ was suggested. Although the system would appear to be conceptually novel, nonetheless it would be mechanically simple: the only moving parts would be the non-return valves. The construction of the duck would not be complex, being cast formed from foam insulation concrete with steel stiffening and outer steel cladding.

Hydraulic power take off systems had already been developed for electricity generating ducks; clearly, such systems could also be applied to the development of a wave powered RO process. This method would, however, have been marginally more complex and expensive than that described above. The life of the membranes (and the cost of their replacement), which can be adversely affected by operation under variable conditions, and the cost and complexities of the necessary feed pre-treatment systems also had to be taken into account. This is not to suggest that wave powered VC would be better than using RO under all circumstances; either system could prove to be the best suited in any given situation.

\subsubsection{Auxiliary Equipment}

In the original paper by Salter (Salter, 1985, [59]) a number of devices were proposed as auxiliary machinery necessary for a practical desalination plant. All desalination equipment requires plant items additional to those forming the basis of the process: pumps, heat recovery exchangers, control systems, etc. are all common additions. Under normal circumstances (ie. in land based installations) such additions rarely pose new problems. On a wave powered system, however, with no available external power supplies, their design may require rather more care.

\section{Feed Preheating}

It will be desirable to operate the vC process at pressures above atmospheric in order to facilitate the removal of non-condensable gases from the condenser and to reduce the specific volume of the water vapour handled. This, of course, implies that the product water and brine blowdown would leave the duck at above $100^{\circ} \mathrm{C}$. For a practical process, therefore, some means 
of product+blowdown/feed heat recovery system will need to be included. The method suggested is to use a spiral plate heat exchanger as shown in cross section in figure (1.6). Very large areas can be incorporated in such exchangers in a compact form; once again, mesh plastic would be the preferred material of construction (see Salter, 1985, [59], for further details).

\section{Device/Shore Pumping}

Three designs of pump were proposed: inertial loop pumps; ring cam pumps, and reciprocating pumps acting off the variation in tension in the mooring lines. Ring cam pumps had already received a great deal of attention in connection with the research carried out on electricity generating ducks. The design of reciprocating pumps to act of the mooring line tension would be fairly straightforward, save for the problems of total immersion in seawater and the need to shed load during extreme conditions. Inertial loop pumps are a rather more novel idea; all the pump would consist of would be a helical coil of pipe with non-return valves at either end, as shown in figure (1.7). As the helix accelerates about the axis shown (with the nodding of the duck), so the inertia of the water inside it would create a head across the coil. The head available would be proportional to the radius of the coil, the length of pipe in the coil, the density of the fluid and the angular acceleration of the coil. Approximate calculations suggest that a few quite small pumps of this nature could provide all the pumping duties required by the process (see Salter, 1983, [60]).

\subsection{PROJECT OVERVIEW}

It is intended that this section should describe, in brief, the reasoning behind the development of the project. Detailed explanations of particular decisions taken at any point, or of actions carried out, may be found (as appropriate) elsewhere in the text and the reader shall be referred to these points as necessary.

\subsubsection{Research Programme}

At the start of the project, the information pertaining to the proposed process was that which was contained in Professor Salter's paper; that is, a description similar to that above, in section 1.2. As the performance of ducks as wave energy absorbers had already been thoroughly investigated with reference to 
power generation, it was decided to first study the vapour compression system in isolation from the "wave powered" aspects of the project. The unsteady-state nature of the process meant that this study took the form of the construction of a numerical model of the process and of a computer program to solve the set of equations derived thereby. It was intended to use the model to provide two types of information: firstly, regarding the performance of the duck as a desalination plant (ie. how much water it could produce, how much energy it would require etc.); secondly, to determine the power take-off characteristics of the duck for possible future use in small-scale tank tests on model ducks (see chapter 2, section 2.2.3 and chapter 5, section 5.1.1). Most importantly, the results of the model suggested that realistic small-scale testing of desalination ducks would not be possible because of the scaling laws involved (see chapter 2, section 2.2 .3 and chapter 5, section 5.1.1). The model also confirmed the initial performance predictions for the duck which had been based on a steady-state process (see chapter 5 , section 5.1.2).

Given that the project could not proceed in a similar manner to the projects involving the development of power generating ducks, an alternative method was required. It was decided that the numerical model of the plant should be subject to experimental verification of some sort, but, obviously, a scaled-down replica of the entire system would have been neither practical nor cost-effective. A scheme was devised whereby the results from a pilot plant consisting of an unsteady-state VC process would be compared with the results from a model of the experimental facility based on the same principles as the model of the duck. In this way, while it would not be possible directly to verify the duck simulation data, it might be possible at least to check the validity of the equations used in the attainment of these data. Work proceeded, therefore, on the design, construction and commissioning of a pilot plant and simultaneously on a mathematical model of the rig.

During this phase of the project, it became clear that the original model of the duck was unsatisfactory in some respects (see chapter 3 , section 3.3.1). The structure of the computer program was such that the rectification of the faults identified would have been extremely tedious and ultimately ineffective and so it was decided to construct an entirely new and hopefully more accurate model of both duck and rig. This new model was completed at about the 
same time as the pilot plant was being commisioned. The data from the rig and from the new model were compared as proposed and found to match reasonably well (see chapter 5 , section 5.2.2). With the model of the duck thus validated, as far as possible, by this rather circuitous method, it was then used to study the predicted performance of the desalination plant.

\subsubsection{Scope of Thesis}

The work presented in this thesis is divided into four main sections:

1. Review of pertinent literature and theory (chapter 2).

2. Development of numerical models (chapter 3).

3. Development of physical apparatus (chapter 4).

4. Analysis of data from numerical and physical models (chapters 5 and 6).

As the project was related to both wave power and desalination, the first of these sections is further divided into two subsections; the first on desalination and the desalination industry and the second covering some fundamental aspects of ocean waves and wave energy as a resource.

The second section is also divided into two subsections; the first on the first numerical model and the second on the second numerical model. Of the two, the second subsection is by far the larger, reflecting the increased complexity of the second model. The subsection starts with consideration of why the construction of the second model was necessary and how the faults apparent in the first model were to be remedied. It then goes on to describe the mathematical basis of the model (including details of the various physical and transport property correlations used) and ends with a brief description of the structure of the computer program used to contain the model.

The third section begins with a consideration of the overall design brief for the experimental facility; the various constraints involved, and the consequent evolution of the form of the apparatus. The detailed design of the constituent parts is then described: the evaporator/condenser unit; the compressor, and 
the monitoring, control and data logging system.

The fourth section first presents the results from the first model and contains an analysis of these results in terms of transient behaviour and performance prediction; time dependency in the pressure force characteristic is noted and described. The second subsection deals with the experimental program and covers the range of experimental work carried out, the measurements made and a description of the experimental procedure. The third subsection deals with the comparison of rig and simulated transient data to provide an estimate of the accuracy of the second model. The rest of the section presents results from the second computer model and their analysis in terms of the performance of the duck as a desalination process. Finally, chapter 6 contains a summary of the conclusions of the work and presents some recommendations for future research. 


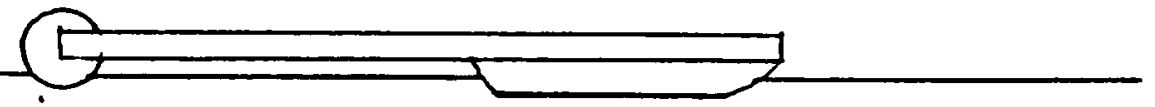

Figure (1.1) Development of the Duck: Backless Flap

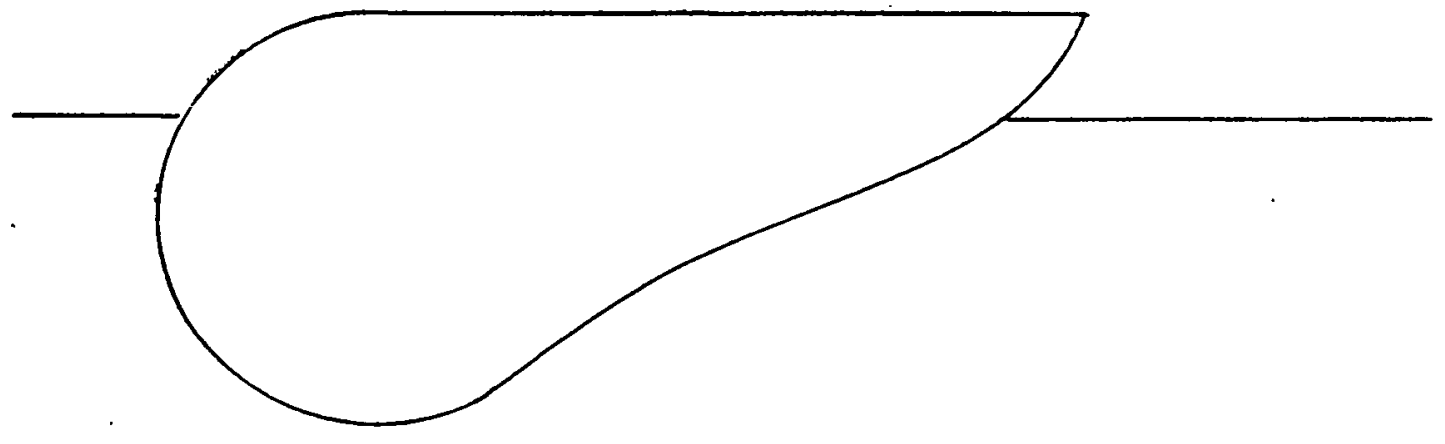

Figure (1.2) Development of the Duck: "Tadpole"

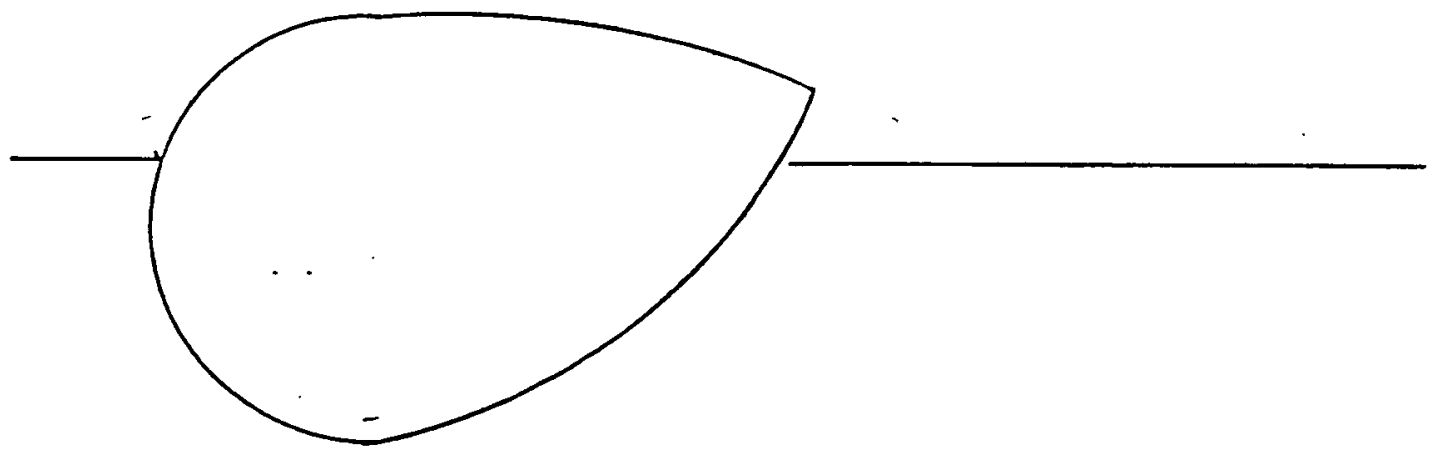

Figure (1.3) Development of the Duck: The Duck 


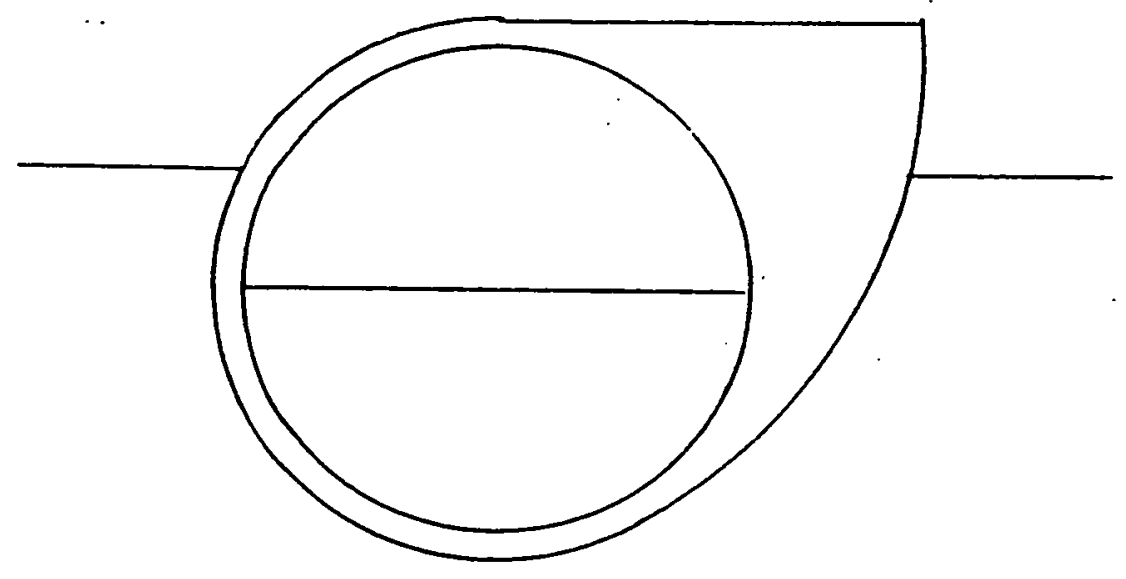

a.

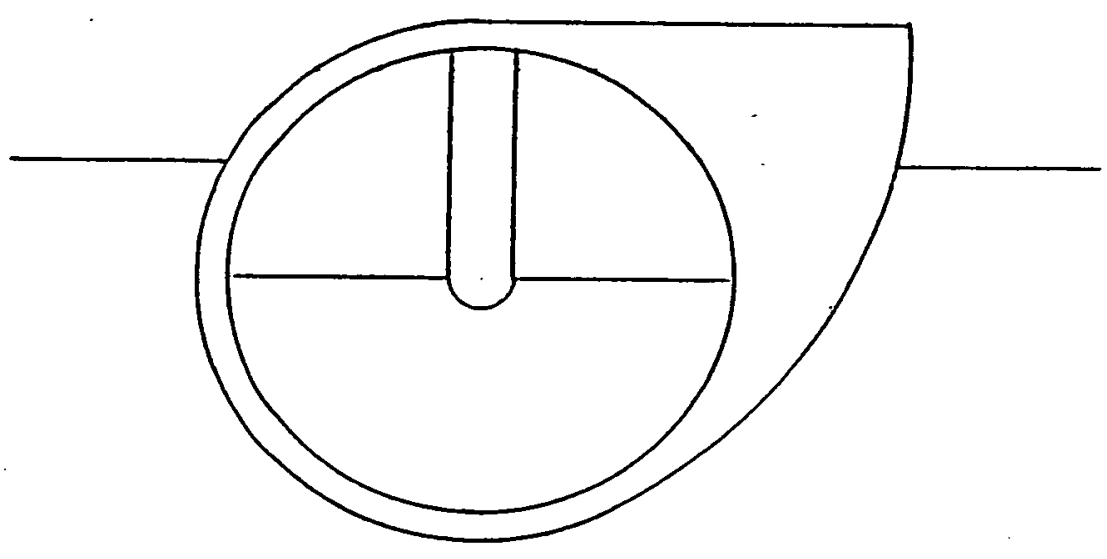

b.

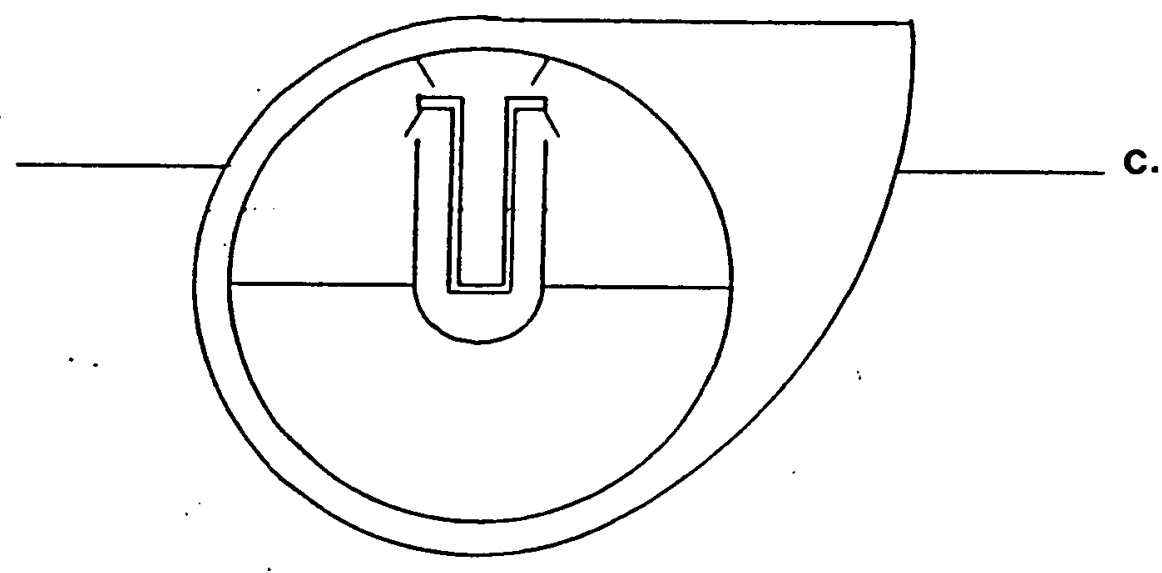

Figure(1.4) Development of Edinburgh Duck VC System 


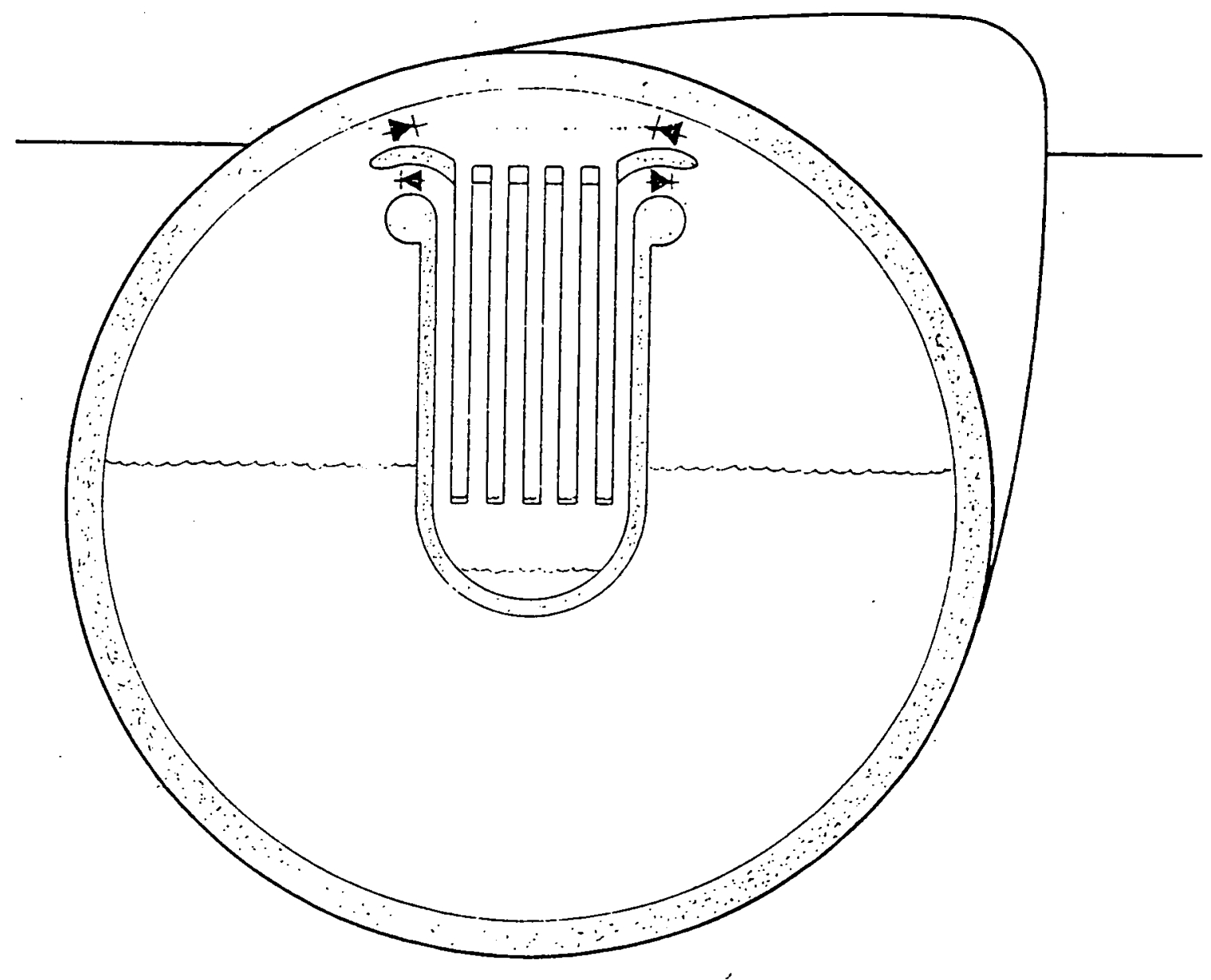

Figure (1.5) Detail of Duck VC System 


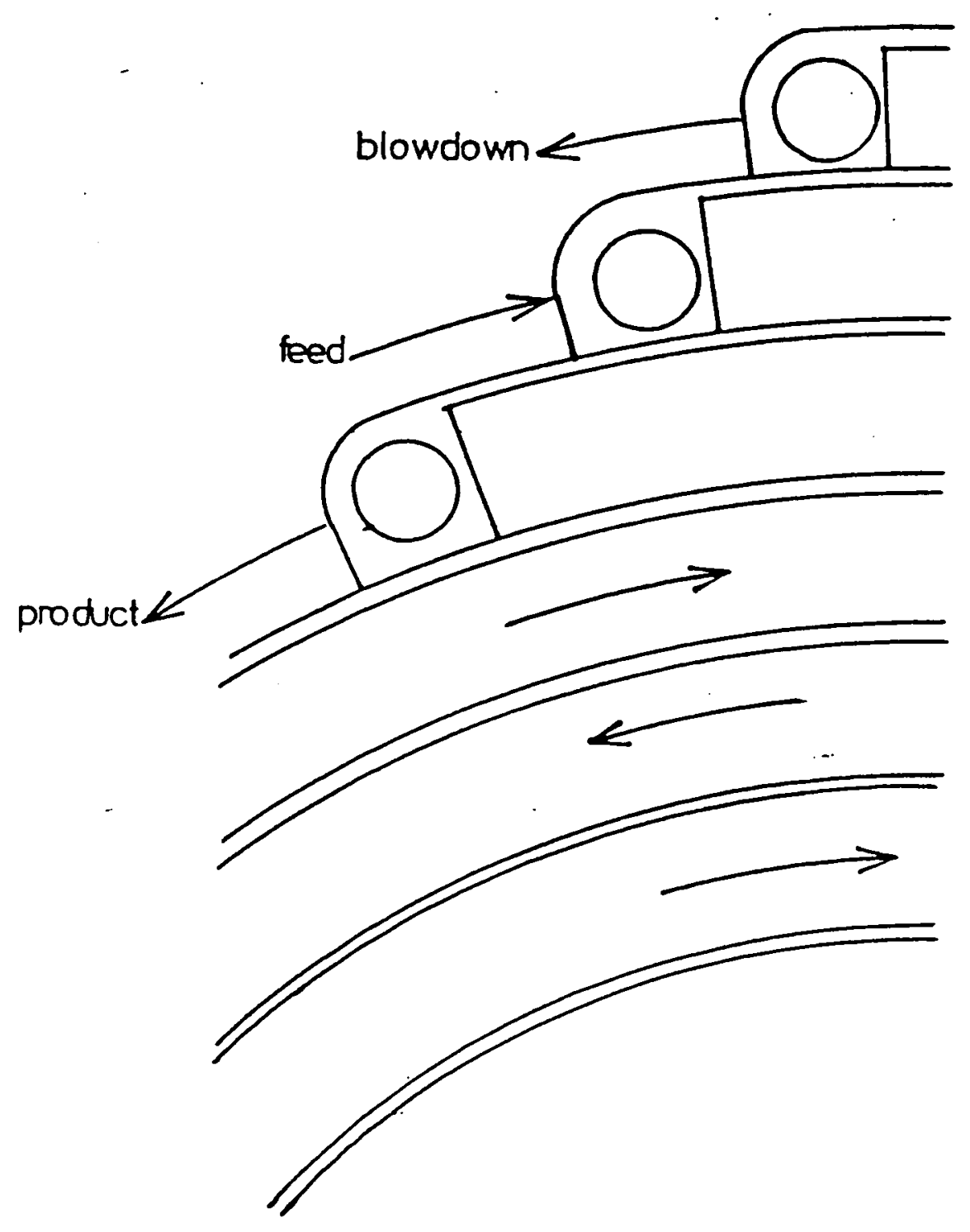

Figure (1.6) Cross-Section of Spiral Plate Heat Exchanger 


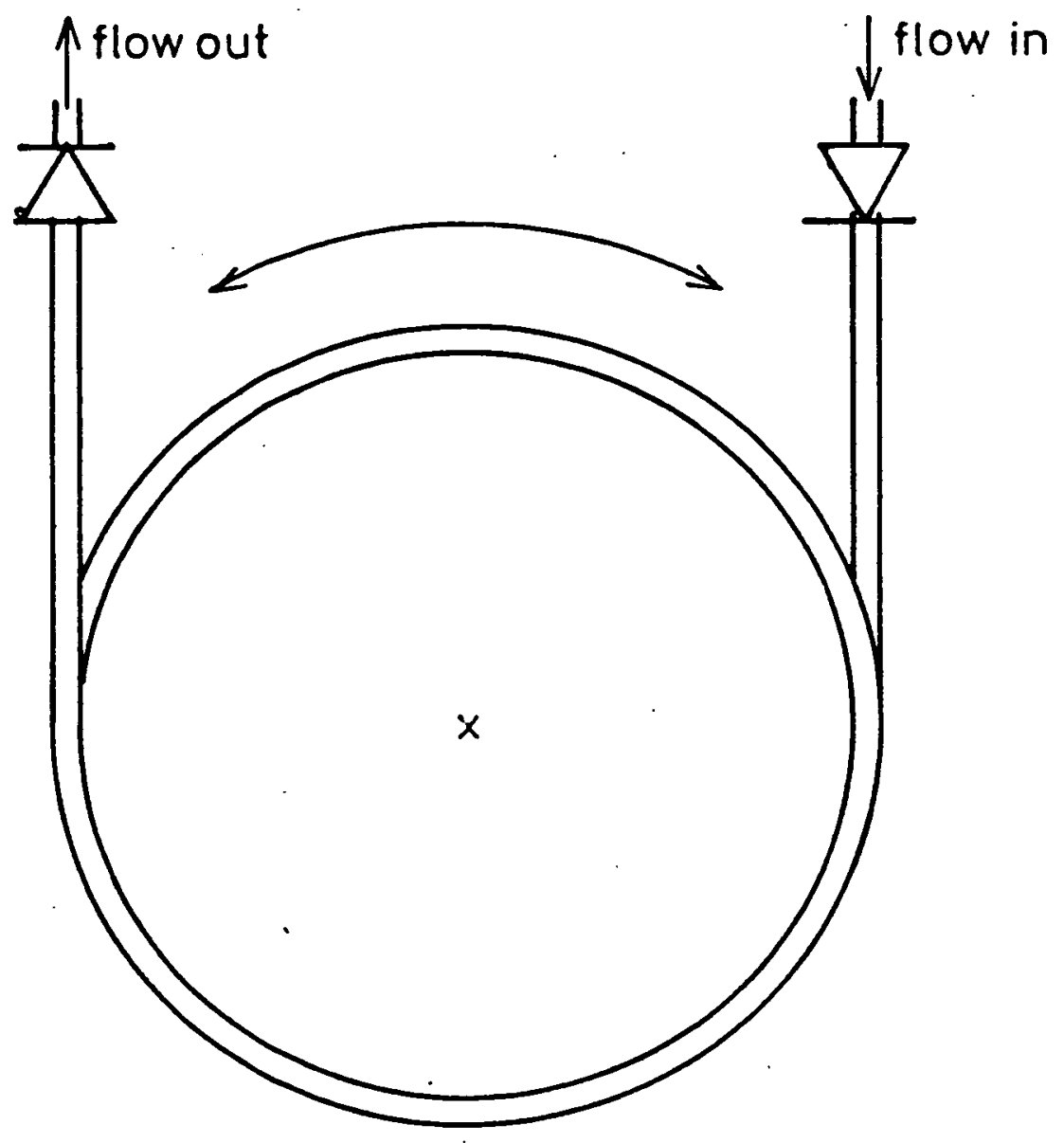

Figure (1.7) Inertial Loop Pump 
CHAPTER 2

UTERATURE REVIEW AND THEORY 


\subsection{DESALINATION}

\subsubsection{Overview}

The term "desalination", when used in this text, refers to the production of potable water from seawater. The processing of brackish waters, or of polluted waters, may be undertaken using techniques similar to those illustrated in this section and so shall not be dealt with separately for the sake of brevity. Seawater has an average salinity of around $35 \mathrm{~g} / \mathrm{kg}$ total dissolved solids (tds); the World Health Organisation (WHO) standard for potable water is $500 \mathrm{ppm}$ tds, or $0.5 \mathrm{~g} / \mathrm{kg}$ (see Porteous, 1975, [53]). It follows that, for a desalination plant to meet this internationally recognised standard, it must be capable of removing at least $98.6 \%$ (by weight) of the minerals dissolved in the feed brine. Fortunately, this does not present any fundamental problems, given that the feed water is a solution of essentially non-volatile components in a volatile solute. The most obvious means of effecting the separation would seem to be, therefore, distillation: it was simple stills, such as that shown diagrammatically in figure (2.1), that were first used to desalinate seawater to produce boiler make-up water on board steam ships (Hillier et al., 1952, [26]). In comparison to these early plants, modern desalination installations are incredibly complex; the motivation behind this increase in complexity has been, as always, the drive towards increased economy of operation.

The feed seawater to a desalination plant is usually cost free, aside from pumping costs (which are generally minimal); the only other costs are, therefore: interest on the capital used to construct the plant; the cost of the energy required to effect the separation; chemical pre- and post-treatment costs (see appendix I), and operational labour and maintenance costs. The last two items on this list may be reduced to an insignificant fraction of the product water price by careful design and operation of the plant and so the economic objective function for desalination equipment reduces to a balance between capital investment and ongoing energy consumption. Furthermore, as the supply of fresh water is often regarded as essential to the development of arid zones, the capital cost charges for desalination equipment may be reduced through the introduction of state grants, subsidies or low interest loans lone of the reasons for the difficulty in defining true costs for desalinated water). It has been suggested, albeit not entirely seriously, that 
desalination should be thought of not as a separation process, but rather as a process for the conversion of fuel oil into fresh water (Silver, in Porteous (Ed.), 1983, [54]). While this approach may not be particularly useful in the analysis of desalination plant design, it does serve to emphasise the fact that the cost of desalinated water is directly related to the cost of energy at a given location. Thus, modern desalination plants tend to include complex energy reclamation schemes which might not, in general, be justifiable on other industrial plants.

In order to fully understand the measures taken to improve desalination plant energy efficiency and the limitations of the methods used, it is useful to start from an analysis of the simplest plant which might be constructed, such as that shown in figure (2.1). A parameter often used in the analysis of desalination equipment is the "performance ratio", $r$ (also referred to as the "gained output ratio", GOR), which is defined as the mass of fresh water produced per equivalent unit of steam used in the process, ie. mass per unit energy in a dimensionless form (for a discussion of the use and abuse of this parameter, see Spiegler and Laird (Ed.s), 1980, [67]). Material and energy balances around the still shown in figure (2.1) show that:

$$
r=1 /\left(1+\left(m_{2} / m_{5}\right) \cdot\left(C_{p} / \lambda\right) \cdot(\delta+\Delta T)\right)
$$

where $\delta$ is the boiling point elevation of the brine at the blow-down concentration and $\Delta T$ is the minimum temperature approach in the recoverv heat exchanger. Thus it may be clearly seen that the theoretically attainable maximum value of $r$ is unity and that there are a number of thermodynamic irreversibilities which make the attainment of the ideal impossible. There are four means which might be used to improve the performance ratio for such a plant:

1. A reduction in the ratio $C_{p} / \lambda$.

2. A reduction in the boiling point elevation, $\delta$.

3. A reduction in the minimum temperature approach of the recovery exchanger.

4. A reduction in the ratio $m_{2} / m_{5}$. 
A reduction in the ratio of specific heat to latent heat could be achieved through the reduction of the temperature of operation, thereby increasing the volume of the vapour to be handled and thus the size of the equipment, and also resulting in a loss of availability of the primary steam, which is generally available at fairly high temperatures; this then cannot be considered as a practical or desirable option. A reduction in the boiling point elevation could be achieved through a reduction in the temperature of operation, which has already been discounted, and/or by a reduction in the concentration of the brine blow-down. A reduction in the salt concentration of the blow-down could only be achieved through an increase in the ratio $m_{2} / m_{5}$, which would tend to reduce the performance ratio by more than the amount which could be offset by a reduction in $\delta$.

A reduction in the minimum temperature approach in the recovery heat exchanger would be a more practical option and would involve increasing the area available for heat exchange and/or increasing the heat transfer coefficient, through the use of enhanced heat transfer surfaces, for example. Either of these options would incur additional capital costs which would need to be set against the reduction in energy costs attained. Once again, it would be possible to operate the still at a lower temperature and thus require less heat transfer surface area for the recovery of sensible heat, but one would then face the disadvantages detailed above.

A reduction in the ratio $m_{2} / m_{5}$ would be definitely feasible within certain limits; too small a value of this ratio would cause the concentration of salts in the blow-down brine to rise above that which is suitable for scale free operation. Seawater contains many dissolved minerals, some of which will form hard, crystalline scales of low thermal conductivity on heated surfaces in the process of distillation. The chemistry of scaling is discussed in some detail in appendix I, but it suffices to say here that, with current feed water treatments available, brine concentrations of around twice that of normal seawater, at temperatures up to around $120^{\circ} \mathrm{C}$, are the limits imposed by scaling considerations.

Thus, some of the most important factors in desalination plant design may be illustrated by a study of this very simple piece of equipment; the essential point is that energy economies can only be realised through increased capital 
expenditure; as one might intuitively expect.

The next stage in the historical development of desalination equipment was the introduction of multiple effect pool boiling evaporators (MEB's). Multiple effect evaporators use the vapour generated by one effect to produce more vapour in a second effect at a reduced pressure, which is in turn used in a third effect, and so on, as shown in figure (2.2). From an analysis similar to that for the single stage device, it may be seen that the maximum attainable performance ratio for an MEB is equal to $n$, the number of effects. However, as with the single stage device, thermodynamic irreversibilities are always present which reduce this maximum in practical equipment. The largest performance ratio that was economically achievable using an MEB was of the order of 5, for a plant with 6 effects (Porteous, 1975, [53]). The implementation of stage-wise operations was very important, however, as it led to the eventual development and introduction of the Multi-Stage Flash (MSF) system which was to lead, in turn, to the construction of large numbers of large scale desalination plants and a period of impressive growth in the industry.

\subsubsection{The MSF Process}

The design principles of MSF plants were developed simultaneously but separately by R.S. Silver (working for G\&J Weir Ltd., Glasgow) and A. Frankel (working for Richardsons, Westgarth \& Co. Ltd, Northumberland) towards the end of the 1950's, with the first commercial plants being built from around 1960 onwards. The process was developed from the Alberger flash process for producing salt from brine, itself in existence for some 30 years at that time (see for example, Kirk-Othmer, [33]). In MSF plants (see figure (2.3)) cool brine is preheated in stages by vapour condensing at successively higher pressures in the "recovery" section of the plant and finally by prime steam in the brine heater. The heated brine is then flashed down in pressure by stages, generating the vapour for the preheating duty. The condensate formed is also flashed down in the same manner, so that the total flashing flow is the same as the flow being heated. As a result the temperature rise and flashing range in all the heaters and flash chambers is approximately the same, thus minimising availability losses. A simple calculation may be used to show the relationship between the flashing temperature range and the fractional amount of distillate it is possible to produce: 


$$
\Psi=C_{p} \cdot\left(T_{\max }-T_{\min }\right) / \lambda
$$

Thus, with a maximum top temperature of (say) $120^{\circ} \mathrm{C}$, from scaling considerations, and a minimum of $45^{\circ} \mathrm{C}$, to allow for heat rejection to fresh feed, the fraction of the total flow that it would be possible to vaporise would be in the region of $12-13 \%$. For practical designs and in order to minimise the amount of feed pre-treatment, therefore, it is necessary for MSF plants to recirculate large amounts of brine through the cycle and this, then, requires that a heat rejection section (as shown) must be included in the flash train in which brine is flashed to reject heat to cooling water before being recirculated to the recovery section.

The similarities between an MSF plant and an MEB plant seem obvious, the differences perhaps less so and it is certainly not immediately obvious why MSF plants should have become so much more successful than MEB plants. The theoretical maximum value for an MSF plant is equal to $n$, the number of stages, as before. Unlike an MEB plant, however, an idealised MSF plant with no secondary losses and infinite heat transfer areas could not reach this ideal. The reason for this may be seen from the temperature profiles reproduced in figure (2.4): the log mean temperature difference between vapour and recirculating brine in a flash chamber and heater can never become zero, no matter how large is the heat transfer area, while it may for an MEB plant. Thus, if the same design principles are followed, MEB plants would actually be more thermodynamically efficient than MSF plants.

The advantages that MSF gained arose from differences in the construction of the two types of plant which allowed MSF plants to be built with a much higher number of stages, but with a much lower unit cost per stage. To understand why this should be important, it is useful to first look at the theoretical case of a plant with an infinite number of stages, such as that represented in figure (2.5), with corresponding temperature profiles shown in figure (2.6)). From this consideration it may be shown that, approximately:

$$
r=\left(T_{\max }-T_{\text {dis }}\right) /\left(T_{\max }-T_{\text {in }}\right)
$$

Thus $r$ is independent of the number of stages used. The specific area for heat transfer $\left(\mathrm{m}^{2} \mathrm{~s} . \mathrm{kg}^{-1}\right)$ in the recovery section is given by: 


$$
A_{s}=\lambda /\left\{U .\left(T_{\max }-T_{\text {in }}\right)\right\}
$$

Equations (2.3) and (2.4) may be combined to give:

$$
A_{s}=r \cdot \lambda /\left\{U \cdot\left(T_{\max }-T_{\text {dis }}\right)\right\}
$$

Thus, the designer is free to choose the performance ratio, as long as the number of stages is infinite, and $A_{s}$ will be fixed by the flashing range. Equation (2.5) is an identical relationship to that which may be derived for an MEB system, but for MSF $r$ may be chosen, whereas for an MEB system it is a function of the number of effects. It would be impossible, of course, to build a plant with an infinite number of stages, and the effect of using a finite number is to reduce the mean effective temperature difference for heat transfer $\left(T_{\max }-T_{\text {in }}\right)$. Eventually, it may be shown that, approximately:

$$
A_{s}=\left(\lambda / U \cdot\left(T_{\max }-T_{\text {dis }}\right)\right) \cdot n \cdot \log \{n /(n-r)\}
$$

Plots of $A_{s}$ versus $n$ for different values of $r$ are shown in figure (2.7), for a flash range of $75^{\circ} \mathrm{C}$.

The effect of this difference between MSF and MEB is shown qualitatively in figure (2.8); the optimum number of stages for an MSF plant will occur at much higher values of $n$ and $r$ than for MEB plants. The problem for the designers of MSF plants was then to reduce the unit cost per stage to a point where it could become competitive and without introducing too many avoidable thermodynamic losses into the system (Frankel, 1960, [20]). This was achieved through the construction of the plant as, essentially, rectangular metal boxes, with partitions to divide the internal space into stages and to add strength and rigidity to the structure. Orifices cut into the bottom of the dividing walls allowed the flashing brine to flow from one stage to the next, with the correct pressure drop for the flashing process to take place as planned. Thus the overall shell cost of the plant was reduced and interstage piping was kept to an absolute minimum. The brine pre-heating tubes could be mounted horizontally, running the length of the flash train (the long tube method), or vertically, that is, in a serpentine fashion through the plant, the exact method chosen depending very much on the specifications for individual plants. The distillate formed on the tubes would be collected on a tray mounted beneath them and carried from stage to stage to flash in the same 
manner as the brine. Generally, arrangements would be made to stop the carry-over of brine into the product stream, often consisting of a combination of baffles, splash plates, wire mesh demisters and the inclusion of extra disengagement volumes in the chambers. The reader will appreciate that, given the requirement to reach water purity of (at least) the WHO standard, even small amounts of brine/product contamination could seriously jeopardise the operation of these plants.

It is not proposed to discuss here the engineering design of MSF plants in detail. The reader is referred to the works of Frankel, [20], Porteous, [53], [54], Spiegler and Laird, [67], and the proceedings of any of the conferences on Fresh Water from the Sea and Desalination and Water Reuse for such information. Details of day-to-day operation of MSF (and other) desalination plants, and their economic performance etc., can be found in the trade journal, Desalination (published by Elsevier). It suffices to say here that the problems of scaling and feed pre-treatment; non-condensable ingress; corrosion; reduction of thermodynamic losses, and so on, occur in MSF plants just as they occurred in the simple still given as an example at the beginning of this section and that many ingenious means of reducing the problems have been found and put into operation.

\subsubsection{Other Distillation Methods}

MSF plants now account for the majority share of all the desalinated water produced; they are not the only method in use, however, for as local conditions vary, other types of plant have been constructed to suit these variations. Of the other types of plant in operation, only distillation methods shall be covered in this section; reverse osmosis, electrodialysis and freezing shall be covered in the next. There are two main distillation plant designs which fill the small gap left by MSF: long tube evaporation and vapour compression, of which long tube evaporation plants are by far the more common (see section 2.1.6).

\section{Long Tube Evaporation}

Long tube evaporation plants are a development of the MEB plants mentioned earlier. The basic difference between the two, aside from comparatively minor refinements, is the use of thin film condensation and evaporation, often on enhanced heat transfer surfaces, to reduce the area for heat transfer required 
to realise highly energy efficient operation. There are two types: Vertical Tube Evaporation (VTE) and Horizontal Tube Evaporation (HTE), and as their names suggest, they differ in terms of the orientation of the heat transfer surfaces. As the basic layout of both types is the same, the VTE plant will be considered in detail and the HTE plant referred to this.

A diagram of a VTE plant is shown in figure (2.9). The similarity with older MEB plants is apparent, with the difference that, in modern VTE plants, the distillate produced at each effect is flashed down between stages and the vapour evolved is used as part of the condensing stream in the next effect, in a similar manner to MSF plants. This step recovers some of the enthalpy of the distillate stream as it passes through the plant. An approximate analysis of this process may be carried out as for the single effect of the MSF plant above. For the first effect:

$$
m_{d 1}=m_{s 0}=m_{s 1}
$$

and

$$
m_{f 1}=m_{f 0}-m_{s 0}
$$

A heat balance on the flashing distillate gives:

$$
m_{d 1}^{\prime}=m_{d 1} \cdot C_{p} \cdot \Delta T / \lambda
$$

The term $C_{p} \Delta T / \lambda$, where $\Delta T$ is the difference in temperature between effects, is taken to be a constant for the process, so:

$$
m_{d n}^{\prime}=m_{d n} \cdot k
$$

Returning to the first effect, the total steam flow issuing from it is:

$$
m_{s 1}^{\prime}=m_{s 1}+k \cdot m_{d 1}=m_{d 1} \cdot(1+k)
$$

Now:

$$
m_{d 2}=m_{s 1}^{\prime}+m_{d 1}-k \cdot m_{d 1}=m_{d 1} \cdot(1+k)+m_{d 1} \cdot(1-k)
$$

So that: 


$$
m_{d 2}=2 \cdot m_{d 1}=2 \cdot m_{s 0}
$$

Eventually, it may be shown that:

$$
m_{d n}=n \cdot m_{s 0}
$$

The total amount of steam used by the plant must include the amount used in the final brine preheater:

$$
m_{s h}=\left(C_{p} / \lambda\right) \cdot m_{f 0} \cdot\left(\gamma_{t}+\delta\right)
$$

where $\gamma_{t}$ is the minimum heat transfer approach in the brine preheater and $\delta$ is the boiling point elevation in the first stage. Thus:

$$
m_{s}=\left(m_{d} / n\right)+\left(C_{p} / \lambda\right) \cdot m_{f 0} \cdot\left(\gamma_{t}+\delta\right)
$$

and so:

$$
r=n \cdot \lambda /\left(\lambda+n \cdot C_{p} \cdot \alpha \cdot\left(\gamma_{t}+\delta\right)\right)
$$

where:

$$
\alpha=m_{f 0} / m_{d}
$$

Thus $r$ is directly dependent upon the number of effects used. From a similar analysis it is also possible to find expressions for the total heat transfer area requirements for these plants (see Porteous, 1975, [53], for details):

$$
\left.A_{T} / m_{0}=n \cdot \lambda-\Delta T / U_{e} \cdot n \cdot(\Delta T-\alpha)+\left(1.5 n / U_{h}\right) \cdot \log \left[\left(\gamma_{t}+\Delta T\right) / \gamma_{t}\right)\right]
$$

Comparisons between VTE and MSF show that VTE plants require substantially less heat transfer area than MSF plants for a given performance ratio (see Howarth, 1976, [28], Kammal et al., 1980, [32]). They also have other advantages:

- The risk of scaling and corrosion and the effects of the bpe are minimised by treating the highest brine concentrations at the lowest temperatures.

- MSF plants need to heat the feed to the first stage above saturation and than flash it down. This inevitably leads to 
thermodynamic irreversibilities which are not present in VTE plants.

- MSF plants can, if not carefully designed, exhibit equilibration problems in the flash chambers. Such problems can markedly reduce the efficiency of the plant; this effect cannot occur in VTE plants where liquid and vapour streams are always at equilibrium with each other (for a discussion of equilibration problems in MSF plants, see Spiegler and Laird (Ed.s), 1983, [67]).

Furthermore, many VTE plants use enhanced heat transfer tubing to increase the heat transfer coefficients possible and thus reduce the heat exchanger area required. The most common method in practice is to use double fluted tubing, such as that shown in figure (2.10). On the condensate side, the liquid film is locally thinned on the crests of the flutes by surface tension gradients which draw liquid into the troughs (Webb, 1980, [76], reviews this and other methods of heat transfer enhancement). Despite the fact that little heat is transferred in the area taken up by the troughs, heat transfer coefficients may be increased by a factor of 3 or more on the condensate side. On the evaporation side, the rapid, turbulent flow in the troughs, combined with the effects of liquid splashing onto the surface of the crests, produces heat transfer coefficients of the order of 2 to 3 times those for plain tubes. More details of enhancement surfaces and the mechanisms of heat transfer involved may be found in appendix II. Enhancement of this kind could have only a very limited effect on the area requirements for MSF heat exchangers, as the overall coefficients in their case is limited by the brine side film coefficient; condensation coefficients generally being an order of magnitude greater than forced convection coefficients for aqueous media.

Horizontal tube evaporation, HTE, is exactly the same as VTE, save for the orientation of the heat exchange surfaces. Horizontal tubes can give higher heat transfer coefficients than vertical tubes, as their shorter liquid path length tends to produce smaller mean film thicknesses, and evaporation coefficients are at least compatible. The main problem with HTE is to ensure an even distribution of the evaporating feed on the outside of the tube bundles; also a 
design consideration for VTE, but rather more difficult to achieve for HTE. Poor film distribution can lead to local regions of high salt concentration and hence increased scaling and corrosion (see Porteous, (Ed.), 1983, [54]).

\section{Vapour Compression Distillation}

Vapour compression distillation should not, perhaps, be thought of as a single process, but rather as a technique which may be applied to any of the distillation schemes discussed above. To illustrate the principles of vapour compression, therefore, it is logical to begin with an analysis of the simplest type of operational plant, a direct equivalent of that shown in figure (2.1) (see figure (2.11)). The principle of operation is that the vapour produced by evaporation of brine is compressed so that its temperature and enthalpy are raised. The compressed steam is then condensed against the feed brine to produce the distillate and more vapour. Blow-down and distillate streams are cooled against the incoming feed stream in a three-way, liquid-liquid heat exchanger to recover as much sensible heat as possible. An auxiliary heater is generally included as shown, to raise the temperature of the feed stream to the operating temperature on start-up. The process can be highly energy efficient as may be seen, for all the latent heat of the product is "recycled", removing the need for a heat rejection system. Mass and energy balances around the system show, as before:

$$
r=\lambda \cdot(\gamma-1)) / R \cdot \gamma \cdot(\Delta T+\delta)
$$

where $\gamma$ is the polytropic compression index (see chapter 3 , section 3.2.3). Thus, even for a single effect, performance ratios greater than 10 may be obtained without much difficulty.

There are however problems associated with VC distillation:

1. The cost of the compressor can be considerable, so that most VC systems are only be economical in situations where energy costs are very high indeed, thus justifying a larger capital investment than normal.

2. It is important to consider the primary energy source for the compressor. Compressors are usually driven by electric motors and the conversion and transmission losses 
associated with electrical drives means that the apparent energy economy for VC systems must be reduced by a factor, normally taken as approximately 3, to present a true figure of primary energy use.

3. There is the problem of compressor specification for desalination duties. For maximum performance ratios the temperature difference across the evaporator/condenser unit should be as low as possible. Very low $\Delta T^{\prime}$ s are economically feasible if enhanced heat transfer falling film surfaces are used. Low $\Delta T$ 's, however, lead to very low pressure ratios across the compressor and is has proved difficult to manufacture reliable and efficient compressors to handle such duties. This problem has been compounded by the incentives to work at reduced temperatures, and hence pressures, in order to reduce the surface area required for sensible heat exchange. Operation at reduced pressure means that very large volumes of vapour have to be handled and it is the combination of low pressure ratios and large volumetric flowrates that cause the problem. Large fan type compressors can be used, but these can suffer from corrosion and erosion of the blades unless careful droplet disentrainment precautions are taken. Such precautions tend to increase the capital costs of the equipment and at the same time introduce irreversible pressure losses into the system, thus reducing its performance ratio.

Some of these problems may be reduced through the use of multi-effect VC systems. These plants are analagous to MSF and VTE plants, except that the vapour from the last effect is compressed and used in the brine heater, or first effect, as heating steam. The compressor in such plants must raise the pressure of the vapour from the last effect to above that in the first and so more efficient and reliable centrifugal machines can be used. The volumes of vapour handled by the compressor are also greatly decreased for a given rate of production, as the compressor only deals with the steam produced by the last stage, whereas distillate is produced by each stage. Some fairly large 
plants of this type have been built (See Le Mertret, 1976, [36] and Lucas, 1985, [40]) but only in areas where energy costs are high compared to capital costs. as the costs of the compressor and liquid-liquid exchangers are substantial. Aside from these applications, VC stills have been used in remote areas where the cost of imported energy may be very high. Often these units have been designed to be rugged and portable, or semi-portable (Loebel, 1967, [39]; Fisher, 1977, [19]; Hoffman, 1977, [27]) and to operate in inaccessible regions where there may be a lack of proper maintenance facilities. VC plants have, therefore, tended to have poor reputation for economy in the field (Senatore, 1980, [62]; Senatore, 1982, [63]; Tliemat, 1967, [71]). Even in these applications, however, it seems likely that VC plants may be overtaken by small reverse osmosis plants in the future (see below).

\subsubsection{Other Desalination Processes}

\section{Reverse Osmosis}

If two salt solutions of differing concentrations were contained on either side of a porous membrane, which allowed the passage of water molecules but not salt, then, under the processes of osmosis, water would tend to flow through the membrane in order to reduce the difference in salt concentration. The normal osmotic pressure difference of this system would be the pressure difference, $\Delta \pi$, across the membrane, necessary to just stop the flux of water molecules. Reverse osmosis occurs when the pressure difference across the membrane is raised above this "threshold" value, so that water flows from a more concentrated to a less concentrated solution; for example, from a brine stream to a stream of fresh water. As no change of phase takes place, reverse osmosis, RO, offers the possibility of a route to a very compact; energy efficient, and technically simple desalination process.

The normal osmotic pressure of a given solution may be found from:

$$
\Delta \pi=-\left(R \cdot T / v_{w}\right) \cdot \log \left(a_{w 1} / a_{w 2}\right)
$$

where $a_{w}$ is the solvent (water) activity and the subscripts refer to the upstream and downstream solutions: the osmotic pressure difference for normal seawater is in the region of 30bar. Expressions predicting the performance of membranes have been developed from two separate analyses. Firstly, the solubility and diffusion model, which, as the name suggests, 
considers solvent and solute diffusing through the membrane at different rates; this produces two fundamental relationships:

$$
N_{w}=P_{w} \cdot[(\Delta p-\Delta \pi) / I]
$$

where $N_{w}$ is the solvent flux, $P_{w}$ is the membrane permeability and $I$ is the membrane thickness; and:

$$
N_{s}=-D_{s m} \cdot d C_{m} / d x+k_{s} \cdot C_{s m} / C_{w m} \cdot N_{w}
$$

where $N_{s}$ is the solute flux; $D_{s m}$ the diffusion coefficient in the membrane and $C_{s m}$ and $C_{w m}$ the concentrations of solvent and solute in the membrane respectively. $k_{s}$ is an empirical coupling coefficient with a value between zero and unity. The second approach to the analysis of RO is to view the membrane as a filter cloth; this gives rise to the sieve-mechanism model:

$$
N_{w}=K_{w} \cdot\left[(\Delta p-\Delta \pi) / 1 \cdot \mu_{w}\right]
$$

where;

$$
K_{w}=\varepsilon \cdot r^{2} / 20
$$

where $\mu_{w}$ is the dynamic viscosity of the solvent, $r$ is the mean hydraulic radius of the membrane pores and $\varepsilon$ is the porosity of the membrane. The constant 20 in equation (2.25), is purely empirical and is included to take account of pore size distribution, pore path tortuosity and so on. Solute transport through the membrane is supposed to take place by convection with the solvent through pores of a sufficient diameter:

$$
N_{s}=\left[(1-\Phi) \cdot N_{w}\right] \cdot C_{s 1} / C_{w 1}
$$

RO membranes suffer from an effect known as "concentration polarisation", which is somewhat analagous to the effect of filter cloth blinding in solids processing operations. As solvent passes through the membrane, a thin layer of high concentration solution is left at its surface. Salts in this layer will tend to diffuse back into regions of lesser concentration, but more salts will be brought into the region by bulk solution transport and thus the local $\Delta \pi$ at the membrane surface will tend to be higher than the expected bulk mean $\Delta \pi$. Concentration polarisation always takes place in RO systems to some extent and results in the need to use higher pressure differences to achieve a given 
mass flux. In extreme cases it may, however, result in damage to the membrane and this may limit the maximum production rate for any particular membrane module (see Speigler and Laird (Ed.s), 1980, [67]).

The membranes themselves are clearly the most important component of any RO system. The specifications for membranes are based on three characteristics:

1. Membrane selectivity.

2. Permeation rate of water per unit pressure gradient.

3. Membrane durability.

Membrane selectivity is dependent on $\$$ in equation (2.26) and determines the number of stages that an RO plant will require to produce product of a given purity. Permeation rate determines the membrane area required per stage and the pressure difference across each membrane required to produce a given product rate (analagous to the heat transfer coefficient in thermal systems). All membranes have a finite lifetime during which their selectivity and/or their permeability will decrease; membrane durability is, therefore, a factor which must be taken into account when considering the design of any RO plant. In order to achieve the best mix of these three parameters many plants use two or three stages, often with different types of membrane in each stage. The pressure difference used in commercial RO plants depends upon the feedwater concentration, the membrane type and the economic production rate, but top pressures in the region of 50-60bar are common with membrane replacement every 2 to 4 years (see Matz, 1981, [42]; Kremen, 1979, [34]; Kammal, 1980, [32]).

The work requirements for an RO process may be calculated (approximately) from the steady flow energy equation for incompressible flow, so to a first approximation the performance ratio for the simple plant shown in figure (2.12) is: 


$$
r=\left(m_{3} / m_{1}\right) \cdot(\lambda \cdot \rho / \Delta p)
$$

for an ideal system. Typically, RO plants with some energy recovery measures (such as a back pressure turbine on the brine outflow) can achieve performance ratios of around 25 , that is $95 \mathrm{~kJ}_{\mathrm{kg}}{ }^{-1}$. This is approximately the same, or slightly less than, VC distillation and the plant required is simpler, cheaper and easier to maintain (although Ro plants do require rather more in the way of feed pre-treatment than distillation plants, it is not difficult to devise automatic control systems for such duties). Like VC, however, it must be appreciated that, if RO is to be compared to thermal processes, its primary energy consumption must be considered; that is, approximately three times the figure given. When this is taken into account, it is clear that while RO is most certainly a competitive process, it may be most suited to fairly small scale operations (see below) as it does not exhibit the large economies of scale associated with, say, MSF.

The principle reason that RO does not exhibit significant economies of scale stems from the construction of the membrane modules. The membranes themselves are usually made of either cellulose acetate or one of a number of aromatic polyamides and polyamide hydrazides (see Sourirajan, 1959, [66]). The dominant membranes in desalination at the moment are the Loeb-Sourirajan type asymmetric celluiose acetate membrane and the aromatic polyamide fibre types. The asymmetric membranes are made in the form of a composite structure with a thin (approximately $10 \mu \mathrm{m}$ ) dense layer supported by a thicker $(2-5 \mathrm{~mm})$ spongy substrate with little or no resistance to permeation. The pores in these membranes are conical with their diameters increasing through the thickness of the membrane; this helps to stop clogging of the pores and thus increases membrane life. The most popular module construction is the spiral wound type; two sheets of membrane separated by a matrix layer are sealed together along three edges, the fourth open edge is attached to a perforated outlet pipe. The membrane-matrix "sandwich" is rolled up over the pipe to form a spiral; this unit is then inserted into a pressure canister. Feedwater is pumped into the canister, fresh water flows through the membrane and is collected via the perforated pipe. Other types of membrane module are the plate-and-frame type, which is similar in construction to plate and frame filters, and the tubular type where the membranes are supported inside porous or perforated tubes. Hollow fibre 
modules consist of bundles of fibres with an outside diameter of 25 to $250 \mu \mathrm{m}$ and a wall thickness of 5 to $50 \mu \mathrm{m}$. Pressurised feedwater flows outside the bundles and the desalinated water is collected from the ends. The bundles are generally "potted" in resin to form the "tube plates" at either end of the cannister. The maximum transfer areas available in a given module type are therefore limited by the problems of fabrication. This implies that large scale RO plants must be made up of multiples of small scale plants, and, therefore, that their capital costs would tend to be approximately linear functions of throughput.

It must be stressed that membrane development is under a constant state of flux; it is, therefore, impossible to give anything more than a very general guide to the RO systems in use. Once again, the reader is referred to Desalination and recent conference proceedings, for more up-to-date information.

\section{Electrodialysis}

Electrodialysis (ED) is, like RO, a membrane process. The principle of operation is as follows: a compartment, or cell, is divided into subsections by a series of membranes; each membrane is ion selective, that is to say that one type of membrane will preferentially admit the flux of cations (eg. $\mathrm{Na}^{+}$), and the other will admit the flux of anions (eg. $\mathrm{Cl}^{-}$). When a DC voltage is applied across the cell, therefore, cations and anions tend to migrate towards their respective terminals, thus creating sections of alternately concentrated and desalinated water in the subsections, as shown in figure (2.13). In a practical ED system, hundreds of membranes would be used in each cell, separated by narrow (approximately $1 \mathrm{~mm}$ thick is common) spacers. ED has been used to treat both seawater and brackish feed streams; the largest plant to be operated produced around $15,000 \mathrm{~m}^{3} / \mathrm{day}$ of fresh water from a brackish feed, (see Speigler and Laird (Ed.s), 1980, [67]). On the whole, however, ED has not proved to be a popular method of desalination. The main stumbling block has been the development of suitably robust yet selective membranes to be able to produce water of an acceptable purity from seawater feed streams; corrosion problems and technical complexity have also served to lessen the appeal of the process. In recent years, the successful development of RO desalination technology has meant that the advantages associated with ED. physical compactness and low energy consumption, have become less important when set against its 
disadvantages.

\section{Freezing Processes}

When a salt solution is frozen, crystals of pure water ice are formed leaving behind a concentrated brine solution. It is this phenomenon which forms the basis of freeze distillation processes. The most commonly proposed method utilises butane as a direct contact refrigerant: pre-cooled seawater enters a freezing chamber where liquid butane is bubbled through it; as the butane evaporates the brine is further cooled and ice crystals are formed. The ice is separated from the brine in a separate vessel (usually, about one half of the brine is solidified), and then washed with a small portion, about $5 \%$, of the product stream. The butane vapour from the freezing vessel is compressed and used to melt the ice by direct contact condensation to form the product water; the liquid butane and fresh water are than decanted. Not all of the butane produced by the freezer is required for use in melting, the remaining portion is further compressed in a secondary compressor and condensed against seawater. The liquid butane streams are then fed back to the freezer. The product water and reject brine-washings streams are used to pre-cool the incoming seawater feed.

Freezing has the potential of being a compact and energy efficient desalination process (its similarities with VC distillation are obvious). On the debit side, however, is the use of two compressors and the required ice slurry handling equipment, all of which is expensive and relatively complex to operate and maintain. More fundamentally, perhaps, freezing processes suffer from the difficulties of predicting rates of crystal growth accurately, which can lead to incorrect sizing of the freezing vessel and/or difficulties in achieving the required product purity. Freezing processes continue to attract research interest, but have yet to be proven commercially (for a discussion of freezing processes, see Tliemat, in Spiegler and Laird (Ed.s), 1980, [67];,Brian, 1971, [4], and Margolis et al., 1971, [41]).

\section{Dual-Purpose Systems}

Dual-purpose plants are plants which are designed to produce both electrical power and fresh water. The motivation behind the development of the technique is as follows: thermal electricity generating stations need to reject heat from their turbine exhaust steam (typically this steam is exhausted at 
around $40^{\circ} \mathrm{C}$ and condensed against the sea, river or lake water, or the atmosphere in a cooling tower). It would, however, be possible to utilise this steam as the primary energy source for a distillation desalination plant, but only if it could be made available at a somewhat higher temperature. This would result in a decrease in the efficiency of the electricity generation process, but the overall efficiency of the combined desalination/generation process would be about the same. In this way, better use could be made of the fossil fuel or nuclear power sources, which tend to release energy well in excess of the $120^{\circ} \mathrm{C}$ that is the current top limit for desalination plants. Cogeneration of water and electricity can also be a sensible move towards the development of a modern economy, in which both electricity supplies and adequate supplies of fresh water are prerequisites.

The schemes proposed for the integration of desalination and electricity generating plants are myriad and largely depend upon local requirements and local economic factors and social conditions; the reader is referred to such texts as Porteous, 1975, [53]; Porteous (Ed.), 1983, [54], and Lof, in Spiegler and Laird (Ed.s), 1980, [67], for more information.

\subsubsection{Desalination Using Renewable Energy}

All the desalination processes described in the preceding sections required a heat or work input derived, at some stage, from either a fossil fuel, or, conceivably, a nuclear source. There are areas of the world, however, which urgently require supplies of fresh water but cannot afford to service this need using conventionally produced desalinated water: for example under-developed nations or small island sites. For such areas, the use of renewable energy sources can present desalination systems in an attractive light, provided of course, that these systems can be designed to produce fresh water at an economic cost. For workers in the renewable energy field, desalination may provide interesting avenues for the development and exploitation of early technologies. Fresh water, unlike electricity, for example, can be stored simply and cheaply, reducing the problem of short term variability which is common to almost all renewable energy sources.

The economics of renewable energy based desalination processes substantially differ from those utilising conventional sources. There are, of course, no costs directly associated with the ongoing energy consumption of the plant; the 
product water cost is, therefore, almost completely determined by the capital cost of the plant and the cost of its maintenance. In order to keep product costs low, many renewable energy based plants have been designed following low technology principles in order to require a minimum of skilled labour for erection and hopefully none at all for day-to-day maintenance: this is a valid approach and one which still predominates in this field. Another approach, however, which has attracted increasing interest in recent years, has been to use relatively more complex plants to produce more water per unit capital investment and to increase the size of the plant so as to decrease the effect of maintenance costs as a fraction of the selling price. These two approaches have tended to produce fundamentally different plant designs and operating methodologies, as may be illustrated in particular from a consideration of solar powered desalination.

\section{Solar Energy}

Solar energy was probably the first form of energy used for desalination; indeed, simple solar stills have been indentified amongst ancient Grecian remains. The advantages of solar energy are that, in arid areas, it is generally in abundant supply; its variations may be predicted with some degree of accuracy, and that it may be converted directly into heat which may be used in any form of distillation feasible. Its principal disadvantages are that, by comparison with wind or ocean waves for example, it has a fairly low energy density and that it varies diurnally and annually, as well as on a much shorter time scale.

Perhaps because solar desalination is such a mature technology, there are many different designs of solar stills in operation, each developed to take best advantage of the resource in a particular location. By far the most common type of plant is the greenhouse still; these rely on the greenhouse effect to raise the temperature of the feed brine to a useful temperature by solar insolation. Glass, and some plastics, transmit short wave radiation, allowing any absorbent surface under the glass to be heated, whilst reflecting the long wave radiation transmitted by the heated surface. Simple glass surfaces can thus be used to "trap" up to around $85 \%$ of incident radiation, depending on the angle of incidence of the radiation. Most greenhouse stills are simple basin-types: these consist of a shallow pool with a leak tight and radiation absorbent liner; a supporting structure, and a glass or transparent plastic 
cover. Such stills are often operated in a batch mode, whereby the basin is filled with sea water, which reaches a steady-state temperature of, normally, around $50-65^{\circ} \mathrm{C}$. As the glass surface is transparent to short wave radiation, it remains well below this temperature and so the water vapour condenses onto its lower surface and trickles down the incline of the glass to be collected in condensate troughs. When the brine in the still reaches approximately twice its normal concentration it is run off and replaced with fresh feed. This usually takes place at intervals of three to seven days, depending upon the depth of the still and the intensity of the insolation. The performance of these stills is mainly a function of the insolation and of the heat loss through the bottom of the basin; a production rate of around $3-5 \mathrm{~m}^{3} / \mathrm{m}^{2}$.day is the norm. The only maintenance required is the periodic replenishment of the brine pool; occasional cleaning of the glass surfaces; leak checking, and clearing the condensate channels of any blockages which might form. This type of plant has proved popular with small island communities, such as the Greek islands, because of its low maintenance and simple construction: the economical unit size of these stills is probably best suited to this type of operation.

The next stage in complexity in the construction of solar stills is the use of continuous brine feeds. This may be achieved either by simply trickling a thin film of brine over an inclined liner in a still otherwise identical to those described above, or by the use of an absorbent wick through which brine flows by capillarity. Trickling film stills can suffer from dry patch formation which results in a degradation of the performance of the unit; wick stills have proved to be rather more promising. Ouahe et al, 1987, [47], have reported a novel form of wick still in which a gauze wick is held at the front of a glass cuboid inclined towards the sun. Water evaporating from the brine flowing through the wick condenses at the shaded rear surface of the still. The principal use of such stills, which are very easy and cheap to construct, is envisaged to be for small units to be operated by individual families or homesteads in areas where a distributed supply of water from a central generating point would be infeasible.

An alternative approach to the utilisation of solar energy for desalination is to use the sun to heat the feed brine to the range of temperatures where it can then be treated in a conventional desalination plant (MSF, VTE etc). Such plants have the advantages of traditional desalination facilities: high 
performance ratios; centralised production for minimum erection and maintenance costs, and ease of control of the water supply to ensure an even distribution; but they are also technically complex and require to be constructed on a fairly large scale if they are to be operated economically. Essentially, such stills can be thought of as conventional desalination processes with a solar thermal plant "added-on" to provide the driving energy; the economic parameters affecting the design of the plant may be different, but the technical aspects are virtually identical. The solar plants, in order to achieve the higher brine temperatures required for the operation of the desalination processes, differ from the simple types described above and there are a number of different arrangements which may be adopted.

The least complex type of collector is the solar pond. A solar pond is a shallow body of water made up of two layers. The lower laver is the more saline, and thus the more dense, and therefore the effects of natural convection, which would tend to mix the layers, are suppresed. The upper layer acts as an insulating blanket over the lower, which absorbs solar radiation. As the thermal inertia of the ponds is large, comparatively short term variations in insolation, and to some extent diurnal variations, may be smoothed out, simplifying the design and operation of the desalination plant. Using this simple technique, temperatures of up to $85^{\circ} \mathrm{C}$ can be reached; the disadvantage is that, in order to stop mixing of the pond, the residence time of the brine must be kept high, and therefore the ponds tend to take up large areas of land (see Posnansky, 1987, [55]).

Solar ponds represent a very simple type of solar collector; more efficient types have been devised capable of producing higher water temperatures and utilising more of the available energy. The flat plate collector is similar in some ways to the basin stills described earlier; it consists of a flat front glass surface; a number of transparent insulating layers, and a rear absorbent layer, through which brine circulates. With this type of still, temperatures of up to $90^{\circ} \mathrm{C}$ can be achieved. Variations on the flat plate collector include the use of movable concentrators to track the motion of the sun and focus the radiation onto the collector in order to maintain maximum output. Evacuated tube collectors operate along the same lines as flat plate collectors: a glass tube with a radiation-absorbent, brine-carrying pipe running through it is partially evacuated to minimise convection losses from the apparatus. Often, the tubes 
are mounted at the focal line of parabolic reflectors, either stationary or tracking; water temperatures up to $200^{\circ} \mathrm{C}$ have been produced (for a discussion of the use of concentrating collectors in solar powered plants, see Delyannis, 1987, [13]). Further sophistication tends to be aimed at the production of water temperatures suitable for the production of process steam; this generally involves the use of complex concentrators, and is not applicable to desalination in particular.

A completely different approach to the utilisation of solar energy is to use photo-voltaic cells to produce electrical power, which in turn may be used to power an RO plant, for example. As the price of PV cells has tended to fall, so more of these plants have been built and operated, although generally on a small scale (Petersen et al., 1979, [50]; 1981, [51]). In terms of the overall design of the plant, there is little difference between these and other RO plants, except that complex operating modes may be employed to take account of the variations of intensity of insolation.

\section{Wind Power}

Several studies have been made of the possibilities for the utilisation of wind energy for desalination, of which the most fully documented in open literature is that of Petersen et al., 1979, [50]; 1981, [51]. Their papers described the work carried out on the design, installation and operation of a wind powered RO plant on a small Fresian island in the North Sea. In common with many wind powered schemes proposed, this plant used an aerogenerator to produce mechanical shaft work, which was converted to electrical power for use in the main RO pump. Direct mechanical coupling of a wind turbine to the seawater pump of an RO system has been proposed by Alward et al., 1973, [1], but no papers have been produced to support their studies with operational data.

The principal problem facing designers of wind powered plants of any nature is the matching of the wind turbine system to the load; this can be a particularly difficult problem to solve because of the extreme short term variability of the resource and because the power available from an airstream is proportional to the cube of the wind speed: 


$$
P=1 / 2 \cdot n \cdot A \cdot \rho \cdot V^{3}
$$

Thus, small fluctuations in wind speed can produce very large variations in the power output from the wind turbine and could lead to overload on the system. In large scale, complex wind turbines, such as those constructed for electricity generation, control of the generator is effected through control of the pitch of the blades. For the smaller, less advanced models envisaged for use in desalination duties, this would not be practical and so arrangements must be made for the control of the load on the generator. Thus, the fundamental design problem for wind powered RO plants is to find the best means of matching the generator load with the requirements of the RO system. In the test installation built, Petersen et al. used a system of batteries which could be charged at times of high wind availability (thus de-loading the RO system) and used to power the RO pump when the wind failed. Much of the work reported, therefore, concentrated on the complex control scheme operated to maximise the availability of this system; the work by Feron, 1985, [18] and Unione et al., 1985, [72] concentrated on the theoretical analysis of the supply/demand matching problem.

Wind energy technology is well developed at the scale considered for desalination applications, with many simple aerogenerators operating reliably in remote areas with minimal maintenance (in water pumping duties, for example). Undoubtably, technical problems, such as materials fatigue, can be overcome through the application of the knowledge gained through a study of these existing systems. Given that both RO and wind-powered systems are well understood, therefore, there would seem to be no reason why wind powered RO systems should not be put into operation.

\section{Geothermal Energy}

The use of geothermal energy for desalination has been investigated with reference to a particular project: the desalination of geothermal brines in the Imperial Valley, California, USA (Awerbuch and Fernelius, 1976, [3]). The proposal was that hot geothermal brines could be used to produce fresh water and/or electrical power, through the use of some kind of dual cycle plant. The techniques suggested for the production of product water were essentially modifications of existing processes: MSF and VTE. The additional difficulties associated with the processing of geothermal brines were found to be the 
high rate of evolution of non-condensable gases and the very high mineral content of the brines involved. In particular, the brines contained high concentrations of dissolved silicon compounds, capable of forming very hard crystalline scales, and sulphur compounds, which produced corrosive and toxic aqueous solutions and gases. The problems facing the designers of the plant described were, therefore, primarily related to the selection of suitable materials; the development of an effective pre-treatment process, and the specification of suitable disposal methods for the waste gases and concentrated brines produced. It is clear that the design of any geothermal plant must be very site-specific, depending as it does upon the exact composition of the brine to be treated and the temperatures at which it is available. It is perhaps unlikely, therefore, that geothermal desalination could ever command a significant share of the renewable energy desalination market mix.

\section{Ocean Wave Energy}

Aside from this project, and the papers associated with it (Salter, 1983, [60]; Crerar and Pritchard, 1986, [10]; Crerar et al., 1987, [11]), only one other researcher has published any work on this topic: C.M. Pleass, 1978, [52]. Pleass' system was based on the use of ingenious pumps, operated directly by wave following rafts (or simple heaving buoys), providing the high pressure water for RO modules. Much of the work describes the design of these reciprocating pumps, which had to be robust, cheap and maintenance free. Pleass used cast polymer constructions to remove the problem of corrosion and to reduce the unit cost of the components (the parts were moulded rather than being machined, therefore for large production runs, the cost associated with the moulds per unit would tend to zero and the cost of the unit would tend to the cost of the materials of construction). It has been reported elsewhere, that operating units producing around $500 \mathrm{gal} / \mathrm{day}$ have been installed as a pilot plant by Pleass for the government of Puerto Rico, but as yet no information has been published on this aspect of the work (see Hicks and Pleass, 1985, [25]). 


\subsubsection{Trends in Desalination}

Up until the late 1950's and early 1960's, when the first commercial MSF plants began to be put into commercial operation, the size of the desalination plants that could be economically constructed was limited by the existing technology to a maximum of around $2,500 \mathrm{~m}^{3} \cdot \mathrm{day}^{-1}$ (based on the MEB process). The development of MSF meant that much larger plants could be built, bringing the economies of scale and technical advantages of the process to bear. Between 1952 and 1960, the rate of growth in desalination capacity was an average of approximately $10^{4} \mathrm{~m}^{3} /$ day/year; from 1960 the new capacity of MSF plants alone grew at a rate of approximately $2.5 \times 10^{4} \mathrm{~m}^{3} /$ day/year (Liberti et al., 1985 , [37]). According to recent (1985) figures (from Liberti et al., 1985, [37]), world desalination capacity has grown to around $10^{7} \mathrm{~m}^{3} \cdot \mathrm{day}^{-1}$ and was continuing to grow exponentially at a rate of around $20 \%$ p.a. (not including a larger surge in capacity between 1979 and 1986 caused by a massive investment programme by the Saudi Arabian authorities, the underlying trend was for a mean growth rate of the above figure). Of this total, approximately $60-65 \%$ was provided by MSF plants, with RO processes taking the next largest share at around $23 \%$ of the market (other processes, such as VTE and VC, claim much smaller fractional market shares in terms of installed capacity, for, although many plants have been built, they do not exhibit economies of scale to the extent of MSF and are therefore built in smaller units). In geographical terms, $60 \%$ of desalination capacity is situated in the Middle East; $50 \%$ is installed in Saudi Arabia alone, the only country in the world without a single permanent river inside its borders. The countries of the Arabian Peninsula are, of course, extremely arid and are capable of supporting an expansion of such an energy intensive industry.

The rate at which installed capacity will continue to grow is very difficult to predict, as it must depend upon social, economic and demographic circumstances in the countries concerned, the possible introduction of new technologies (the development of RO made a radical impact on the market) and even the possibility of global climatic changes in the future. There is some evidence that manufacturers and suppliers of desalination plant and services have based their development plans on continued exponential growth well into the 1990's, but comparatively recently published work suggests that this may be unrealistic (Liberti et al., 1985, [37]; Stone and Rogers, 1985, [68]). 
Depending on the assumptions made, either a linear growth rate or stagnation of the market is predicted. Intuitively, this would seem to be reasonable; there seems to be general agreement among equipment manufacturers and contractors that the Middle Eastern market is close to saturation and since this is the most important marketplace for desalination plant, this must have far-reaching effects on the entire industry. The market saturation scenario, as proposed by Stone and Rogers, suggests that arid areas would cease to increase the provision of fresh water from desalinated sources and would tend to turn towards water reuse, conservation and improved management and distribution services, for economic reasons. Under these circumstances, there would still exist a substantial replacement market for existing desalination plant, as well as water treatment equipment; such as low specification RO plant.

There are two obvious fundamental problems with these predictions and they both stem from the geographically concentrated nature of the existing desalination plant market. The first problem is accounting for possible political changes in the Middle East. Were the distribution of plant capacity more evenly spread across the world, changes of policy in one area alone would not affect the global market to a great extent. With the current situation, however, the industry is fragile to the vagaries of this particular area. The second problem arises from the dependence of the predictions on data received from a small geographical area, which may mean that it is difficult or even impossible to predict the performance of the industry in areas outside of the Arabian Peninsula. It is clear, from figure (2.14), that there are many areas in the world which may be classed as arid and are, say, close enough to the sea for desalination to be considered as a means of fresh water production. Care must be taken, however, when attempting to draw immediate conclusions from such apparently self-evident data. Figure $(2.14)$ is based on the annual precipitation in the areas shown, yet this is fairly meaningless unless the population, land-use and industrial development at a given location is taken into account. This means that it is virtually impossible to determine whether a given area would benefit from water produced by desalination at a given cost, without a detailed study being carried out in the area in conjunction with the relevant local agencies. This has important implications for the development of new technologies for water production, especially those suited for use in as yet unexploited areas. For example, it may be possible to predict that the cost 
of desalinated water in the areas which already produce it in quantity, in 1990, will be of the order of, say, $\$ 2.00 / \mathrm{m}^{3}$; it is not possible to predict, however, what the effect of being able to produce water at $\$ 1.00 / \mathrm{m}^{3}$ would be at that time and at a particular location, or to define a cost at which desalination would become a feasible process for countries which cannot at the moment afford it, without specific local information. The United Nations (UN) organisation has produced world-wide surveys of desalination activity, including data for developing countries (the United Nations Organisation, 1965, [731; 1973, [75]) but even this is inadequate as neither the UN nor the countries themselves have the resources to take account of a new technology being introduced, and so their conclusions and predictions have necessarily been based on the use of conventional processes.

\subsection{OCEAN WAVE ENERGY}

\subsubsection{Introduction}

Water waves are a complex phenomenon and the analytical description of them and of their interactions have attracted the attention of applied mathematicians for many years. It would be unnecessary, and it is not proposed, to include a detailed review of such work in this text: a brief review of simple, linear wave theory will be provided, in order that its implications for the development of wave power absorption devices may be explained; followed by a brief discussion of estimates of wave power as a global resource.

\subsubsection{Linear Wave Theory}

(Note: for the purpose of the derivation of the theory below, water shall be assumed to be incompressible and inviscid and its motion, as described, irrotational and two dimensional).

There are two generic types of water water wave which may be covered by this treatment: progressive (or travelling) waves and standing waves. A progressive wave is illustrated in figure (2.15). The points where the liquid free surface crosses the still water line are known as the zero crossing points. In a progressive wave these points move in the indicated direction, like the troughs and crests of the waves, with a speed known as the phase speed, 
phase velocity or celerity, c. The zero crossing point of a standing wave does not move, but remains in one place, known as the node of the wave. Points one quarter of a wavelength to either side of a node become sequentially trough, still water, crest, still water and trough again; this part of a standing wave is called the loop. Observed wave phenomena are always a mixture, to some extent, of standing and progressive waves; far out in the open sea, for example, progressive waves are dominant, but when progressive waves are incident upon a solid body, the reflections set up cause interference which can lead to standing waves.

For an incompressible, inviscid fluid in two dimensions, the continuity equation gives:

$$
\partial u / \partial x+\partial v / \partial v=0
$$

and if the motion is irrotational:

$$
\partial v / \partial x-\partial u / \partial y=0
$$

A velocity potential, $\Phi$, may be defined in the usual manner, such that:

$$
\begin{aligned}
& u=\partial \Phi / \partial x \\
& v=\partial \Phi / \partial y
\end{aligned}
$$

to satisfy equation (2.30). Substitution into equation (2.29) gives rise to:

$$
\partial^{2} \Phi / \partial x^{2}+\partial^{2} \Phi / \partial y^{2}=0
$$

Equation (2.33) is Laplace's equation for $\Phi(x, y, t)$. This has a progressive wave solution of the form:

$$
\Phi=A \cdot \cosh \{k \cdot(y+h)\} \cdot \cos \{k \cdot(x-c \cdot t)\}
$$

where $A, k, h$ and $c$ are constants. From the equation for conservation of momentum comes the unsteady state Bernoulli equation: 


$$
(p / p)+\left(1 / 2 q^{2}\right)+(g \cdot v)+\partial \Phi / \partial t=0
$$

Thus, if $A, k, h$ and $c$ can be found, then the pressure at all points in the wave can be evaluated, and momentum flux through a given plane of reference can be found. The constant $k$ may be found from a consideration of the periodicity of the wave:

$$
c=L \pi
$$

where $L$ is the wavelength and $T$ is the period. Now, at times $t=0$ and $t=T$, the $\cos$ term in equation (2.34), $\cos (k x)$ and $\cos \{k(x+L)\}$, must be equal, which implies that:

$$
k=2 \cdot \pi / L
$$

$A, h$ and $c$ are found from a consideration of the three boundary considerations present:

1. No water particles can cross the sea bed

2. No water particles can cross the free surface

3. The pressure at the free surface is equal to zero (gauge)

Condition (1) gives:

$$
v=\partial \Phi /\left.\partial y\right|_{y} \text { at sea bed }
$$

Condition (2) gives:

$$
\partial \eta / \partial t=\partial \Phi /\left.\partial y\right|_{y=n} \simeq \partial \Phi /\left.\partial y\right|_{y=0}
$$

for waves of moderate steepness (see Shaw, 1982, [65]). Equation (2.35) gives, at the free surface where $p=0$, and if velocities are assumed to be small so that $q^{2}$ is negligible:

$$
\partial \Phi / \partial t+g \cdot n=\left.0\right|_{v=\eta \simeq 0}
$$

Partial differentiation and combination of equation (2.40) with equation (2.39) gives: 


$$
\partial^{2} \Phi / \partial t^{2}+g \cdot \partial \Phi / \partial y=\left.0\right|_{y=n}
$$

which is known as the surface boundary condition. Substituting for $\Phi$ from equation (2.34) into equation (2.41) gives:

$$
c^{2}=(g / k) \cdot \tanh (k \cdot h)
$$

Substitution for $\Phi$ into equation (2.40) gives:

$$
n=\operatorname{a.sin}\{k .(x-c . t)\}
$$

where;

$$
a=\text { amplitude }=-A /\{\operatorname{cosinh}(k h)\}
$$

The sea-bed condition, equation (2.38), is only satisfied if $h$ is the depth of the sea-bed below the still water line. Thus:

$$
\Phi=-a . c .(\cosh \{k \cdot(y+h)\} / \sinh \{k \cdot h\}) \cdot \cos (k \cdot\{x-c . t)\}
$$

For standing waves, the same fundamental equations and boundary conditions apply, but the phase velocity $=0$, and the local amplitude is periodic in time. Thus:

$$
\Phi=A \cdot \cosh \{k \cdot(y+h)\} \cdot \sin \{k \cdot x\} \cdot \sin \{\sigma \cdot t\}
$$

where:

$$
\sigma=2 \cdot \pi / T
$$

Thus:

$$
n=a \cdot \cos (\sigma \cdot t) \cdot \sin (k \cdot x)
$$

and

$$
\Phi=-(a \cdot \sigma / k) \cdot\{\cosh (k \cdot\{y+h\}) / \sinh (k \cdot x)\} \cdot \sin (k \cdot x) \cdot \sin (\sigma \cdot t)
$$

The velocity components, $u$ and $v$, for a fluid element at an arbitrary point $P$, can be obtained from the expression for $\phi$, equation (2.45), and so to a first approximation, the displacements $\alpha$ and $B$ can be found: 


$$
\left(\alpha^{2} / \cosh ^{2} k_{.}\left(y_{0}+h\right)\right)+\left(B^{2} / \sinh ^{2} k_{0}\left(y_{0}+h\right)\right)=a^{2} / \sinh ^{2} k \cdot h
$$

in the form of an equation for an ellipse. For deep water $h \rightarrow \infty$ and thus equation (2.50) becomes:

$$
\alpha^{2}+B^{2}=\alpha^{2} \cdot\left(\exp \left(k \cdot y_{0}\right)\right)^{2}
$$

the equation of a circle of radius $\alpha \cdot \exp \left(k, y_{0}\right)$. Since $y_{0}$ can never be greater than 0 , it follows that the radius of this circle is a maximum at the surface of the sea, and decays exponentially with depth. In more shallow water, the circle becomes progressively more ellipsoid, as shown in figure (2.16).

Following a similar procedure for standing waves gives:

$$
\begin{aligned}
& \alpha=a \cdot\left(\cosh \left\{k \cdot\left(y_{0}+h\right)\right\} / \sinh \{k \cdot h\}\right) \cdot \cos \left(k \cdot x_{0}\right) \cdot \cos (\sigma \cdot t) \\
& B=a \cdot(\sinh \{k \cdot(y+h)\} / \sinh \{k \cdot h\}) \cdot \sin \left(k \cdot x_{0}\right) \cdot \cos (\sigma \cdot t)
\end{aligned}
$$

Thus, the particle motions are horizontal beneath the nodes and vertical beneath the crests. Between the nodes and the crests the motion is angled to the horizontal by an angle $\theta$, given by:

$$
\theta=\tan ^{-1} \cdot\left\{\tanh \left(k \cdot\left\{y_{0}+h\right\}\right) \cdot \tan \left(k \cdot x_{0}\right)\right\}
$$

The pressure variation beneath a wave may be readily determined once the expression for $\Phi$ and hence $\partial \Phi / \partial t$ is known; for a progressive wave:

$$
\Delta p=2 \cdot \rho \cdot g \cdot a \cdot c^{2} \cdot k \cdot \cosh \{k \cdot(y+h)\} / \sin \{k \cdot h\}
$$

and for a standing wave:

$$
\Delta p=2 \cdot \rho \cdot g \cdot a \cdot\left(\cosh \left\{k \cdot\left(y_{0}+h\right)\right\} / \cosh \{k \cdot h\}\right) \cdot \sin \left\{k \cdot x_{0}\right\}
$$

For deep water, equations (2.55) and (2.56) become, respectively:

$$
\begin{aligned}
& \Delta p=2 \cdot \rho \cdot g \cdot a \cdot \exp (2 \cdot \pi \cdot y / L) \\
& \Delta p=2 \cdot \rho \cdot g \cdot \sigma \cdot \exp \left(2 \cdot \pi \cdot y_{0} / L\right) \cdot \sin \left(k \cdot x_{0}\right)
\end{aligned}
$$

Thus, the maximum pressure variation occurs at the surface and decays exponentially with depth. These particular results have a direct relevance to the design of wave energy absorption devices and their siting. 
An expression for the power available per unit width from a given wave may be derived as follows. Consider the movement, in time interval $\delta t$, of the fluid element $\delta x \delta y$ as shown in figure (2.17). $\delta x$ is chosen so that the whole element moves across the plane of reference, and therefore it has a velocity $u$, defined as:

$$
\delta x=u . \delta t
$$

Thus, the rate of kinetic energy transport by the fluid element must be:

$$
\delta P_{1}=\rho . \delta x . \delta y \cdot\left\{\left(u^{2}+v^{2}\right) / 2\right\} / \delta t
$$

The rate of potential energy transfer is:

$$
\delta P_{2}=\rho . g . \delta x \cdot \delta y \cdot y / \delta t
$$

and the displacement work due to pressure variations within the fluid body is:

$$
\delta P_{3}=p . \delta y . \delta x / \delta t
$$

From equation (2.59), and writing $\left(u^{2}+v^{2}\right)$ as $q^{2}$, then:

$$
\delta p / \delta y=p \cdot u \cdot\left(q^{2} / 2+g \cdot y+p / p\right)
$$

Thus, integrating:

$$
P=\int_{-h \cdot p \cdot u \cdot\left(q^{2} / 2+g \cdot y+p / p\right) d y}
$$

From equations (2.31) and (2.35), equation (2.64) may be expressed in another form:

$$
P=\int_{-h \cdot \rho \cdot(\partial \Phi / \partial x) \cdot(-\partial \Phi / \partial t) d y}^{\eta}
$$

From equation (2.65), an expression for the mean power in a wave, averaged over a wavelength or a period may be thus obtained:

$$
P=1 / 4 \cdot \rho \cdot a^{2} \cdot g \cdot c \cdot\{1+(2 \cdot k \cdot h / \sinh \{2 \cdot k \cdot h\})\}
$$

For deep water, as $n \rightarrow \infty$ : 


$$
P=0 \cdot a^{2} \cdot g^{2} \cdot T / 8 \cdot \pi
$$

This expression holds well for monochromatic waves, such as those which may be generated in experimental wave tanks, but requires modification for real seas. For standing waves, there is no nett transfer of energy in the $x$-direction, although there are instantaneous fluxes present due to the periodic flow reversals which take place in the quarter wavelengths on either side of the nodes.

\subsubsection{Wave-Device Interactions}

The motion of a body, such as a wave energy absorption device, under the influence of water waves and the forces the body will experience, are particularly difficult to theoretically predict. Many attempts have been made to furnish theoretical descriptions of such situations, with varying degrees of success; such analyses are, however, outwith the scope of this text and the reader is referred to the texts such as Crapper, 1935, [9]; and Lighthill, 1978, [38], for more details. What will be described here will be the essential factors governing the behaviour of bodies in wave fields, and how these factors arise.

Figure (2.18) shows a simply-shaped body in a wave field, being acted upon, and reacting to, an incident wave induced force. The applied force, $F_{w}$, from the wave, is periodic (for monochromatic waves), and may be expressed as:

$$
F_{w}=F \cdot \cos (w \cdot t)
$$

The reactive forces are the restoring, or spring, force, a damping force and an inertial force. The spring force in this case is proportional to the displacement of the body, that is, arising from the buoyancy of the device. The inertial term is proportional to the acceleration of the body and its moment of inertia in that mode of motion (in this example equivalent to the mass of the body), and the damping force is due to energy extraction, frictional drag and radiation (see below). The combination of these forces gives rise to the classical equation for forced, damped oscillation:

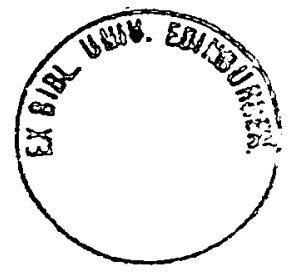




$$
\text { I. }\left(d^{2} y / d t^{2}\right)+D .(d y / d t)+S \cdot y=F \cdot \cos (w \cdot t)
$$

The parameters I, D, S and F are the key to the understanding of the motion of the body; unfortunately these are usually difficult to evaluate. The moment of inertia of the device, which affects $I$, is dependent upon the distribution of the mass of the device and on its axis of motion. The moment of inertia (I', say) can be, therefore, difficult to calculate for all but the simplest of device geometries, particularly if the device can move about more than one axis. $I^{\prime}$ is not, however, the only element of the inertial constant I; another factor is the "added mass" associated with the body, I $m$. The added mass term takes account of the mass of water which the device accelerates when it moves. Thus:

$$
I=I^{\prime}+I_{m}
$$

$I_{m}$ can be expressed theoretically for simple shapes; but for more complex devices, like ducks for example, it must be found experimentally. The spring term, $S$, is usually rather easier to calculate. This force is, in general, dependent on the buoyancy and gravity forces acting on the device and so can be found if the device geometry is well defined. The damping term, D, can be broken down into two parts, one dependent on the shape of the device and the other on the mechanism for power take-off used in the device. For example, damping could be applied by braking the device using friction pads, or by forcing fluid through a turbine. The device-dependent damping term depends upon fluid friction and upon radiation damping. Radiation damping is a measure of the amount of energy radiated by the device in creating waves of its own as it moves. Like the added mass, radiation damping terms are usually experimentally determined for complex devices. Thus, equation (2.69) may be expanded:

$$
\left.\left(I^{\prime}+I_{m}\right) \cdot\left(d^{2} y / d t^{2}\right)\right)+\left(D_{e}+D_{r}\right) \cdot(d y / d t)+S \cdot y=F \cdot \cos (w t)
$$

The choice of the parameters $I^{\prime}, D_{e}$ and $S$ affects the efficiency with which a given device can absorb wave energy for a given spectrum. For many designs, furthermore, motion is allowed in more than one mode and so equation (2.71) becomes a vectorial equation. For example, for optimal performance it has been found that ducks should be alfowed to move in three modes: nod (rotation), heave (vertical) and surge (horizontal) (see Salter, 1985, [59]). In each 
mode the three parameters have to be optimised, together with "cross-terms" (terms which take account of the ways in which, for example, motion in the nod mode can affect motion in the others and vice versa). It is clear, therefore, that the design of wave power absorption devices for high primary efficiency is a complex and subtle matter.

Equation (2.71) includes only terms in the frequency domain; that is, there are no time dependent terms. The inclusion of time dependence makes a theoretical description much more complex in its derivation and renders small scale modelling practically impossible. When small scale devices are tested (in wave tanks or inland waters, for example) they must be scaled in a dimensionally consistent manner if the results of the tests are to have any meaning. The relevant dimensionless quantities in this case are the Reynolds, Froude and Strouhal numbers:

$$
\begin{aligned}
& \text { Re }=\rho . u . L / \mu \\
& \mathrm{Fr}=u /(g . L)^{0.5} \\
& \text { St }=f . L / u=L / U . T
\end{aligned}
$$

In general, dynamical Reynolds consistency is impossible because of the requirement to work with a fluid of suitably scaled $\rho$ and $\mu$; small model tests, therefore, endeavour to use Froude and Strouhal numbers identical to the those of the full-scale system and include corrections to take account of the variations in the Reynolds' number. Thus, if the subscripts $m$ and $p$ represent the model and prototype devices respectively:

$$
\begin{aligned}
& u_{m} / J\left(g \cdot L_{m}\right)=u_{p} / V\left(g \cdot L_{p}\right) \\
& L_{m} / u_{m} \cdot T_{m}=L_{p} / u_{p} \cdot T_{p}
\end{aligned}
$$

Defining the scaling factor, s, such that:

$$
s=L_{m} / L_{p}
$$

then combining equations (2.75), (2.76) and (2.77) gives: 


$$
T_{m}=T_{p} \cdot \sqrt{s}
$$

So for a $1 / 100^{\text {th }}$ scale model, the wave period would be one-tenth that of a full-scale prototype. It can be seen, therefore, that time dependencies in the force characteristic of the device would be impossible to incorporate into small-scale tests.

\subsubsection{The Wave Energy Resource}

Ocean waves are generated by complex interactions between the wind and the surface of the sea. The exact mechanics of the process are not well understood, but it is estimated that the nett power input from wind to waves is normally in the region of $1 \mathrm{~W} \cdot \mathrm{m}^{-2}$. Given that the mean value for solar insolation is around $350 \mathrm{~W} \cdot \mathrm{m}^{-2}$, how, then, can wave power be seriously considered as an energy resource of a practical nature? The reason is that, once the energy has been transferred to the waves, it is dissipated only very slowly (in deep water) and hardly at all for waves of periods of nine seconds or greater. At the same time, waves travel vast distances, around 500 to 1000 $\mathrm{km}^{\mathrm{daay}}{ }^{-1}$ and thus the wave energy in a given area of ocean can be "harvested" along its boundaries, giving rise to energy densities of 40 to 50 kW. $\mathrm{m}^{-1}$ for chosen sites (see, for example, Shaw, 1982, [65]; Count (Ed.), 1980, [8]). Ocean waves are randomly distributed in both amplitude and period: the relation between the two is usually described using some sort of cross-correlation function. A radial density function may also be included to account for the directionality of the waves, which is influenced by the sea-bed topography and the prevailing wind direction. The most common representation of real sea spectra and that which is most often used in tank testing of small-scale devices, is the Pierson-Moskowitz (PM) spectra. The details and the difficulties associated with the use of this statistical expression of natural wave distributions are discussed in detail by Mollison, 1985, [43].

Global estimates of the availability of the ocean wave energy resource are scarce; the best estimates (Mollison, 1985, [43]; Shaw, 1982, [65]) available suggest that it may be around $1000 \mathrm{GW}$, (see figure (2.19)). Reliable prediction of the wave climate in a given area depends to some extent upon the scale of the project considered: for example, a large scale electricity generation project, which might require the installation of devices along several kilometres of wave front, would require information on the average wave climate over that 
distance, as well as on the incidence of extreme conditions (see Mollison, 1985, [43]; Salter, 1985, [59]). For a smaller scale project, however, perhaps for a single device, it has been shown (Budal et al, 1985, [6]) that it is more important to obtain detailed information on localised conditions around the likely siting areas. It is possible, for example, for coastal and/or sea-bed formations to focus surface waves into "hot-spots" where higher than average energy densities occur and it may be advantageous to site the device at such a location (the converse is, of course, also true: calm or sheltered areas with a lower than average wave climate may also exist). For either scale the prediction of the occurrence of extreme conditions often presents more problems than the estimation of average values. Cost factors to account for the likely survivability of a given device in extreme conditions and of the incidence of weather windows, during which maintenance and/or repair of the device can take place, must be included in the analysis of any proposed wave energy scheme (see Davies et al., 1985, [12]). The determination of these factors has proved to be a contentious issue in the debate over the future of wave energy as an exploitable resource. As the majority of the schemes put forward are more or less untried at full-scale, it has been difficult to produce suitable data from which reliable estimates of these factors may be made. 


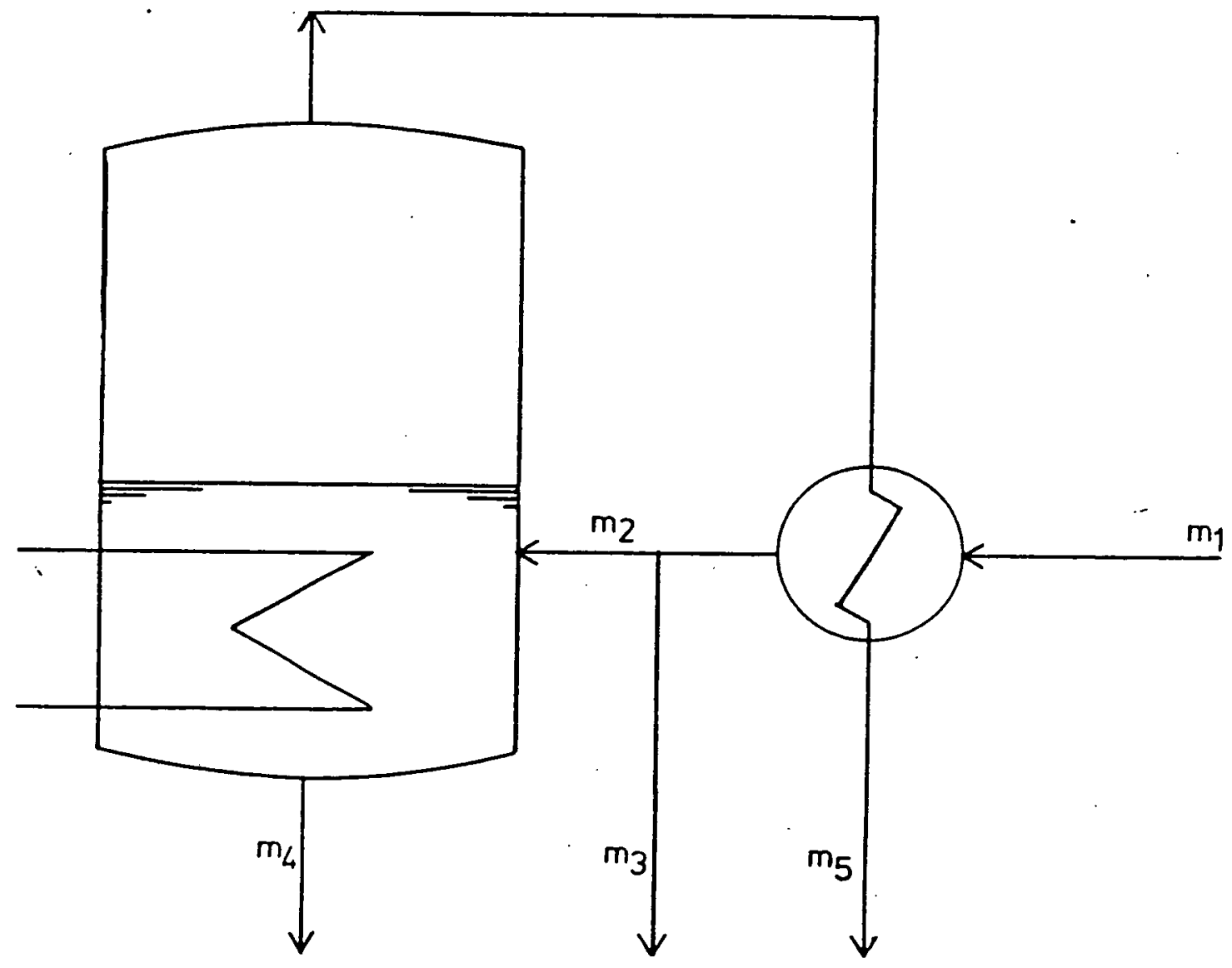

Figure (2.1) Schematic of Simple Still 


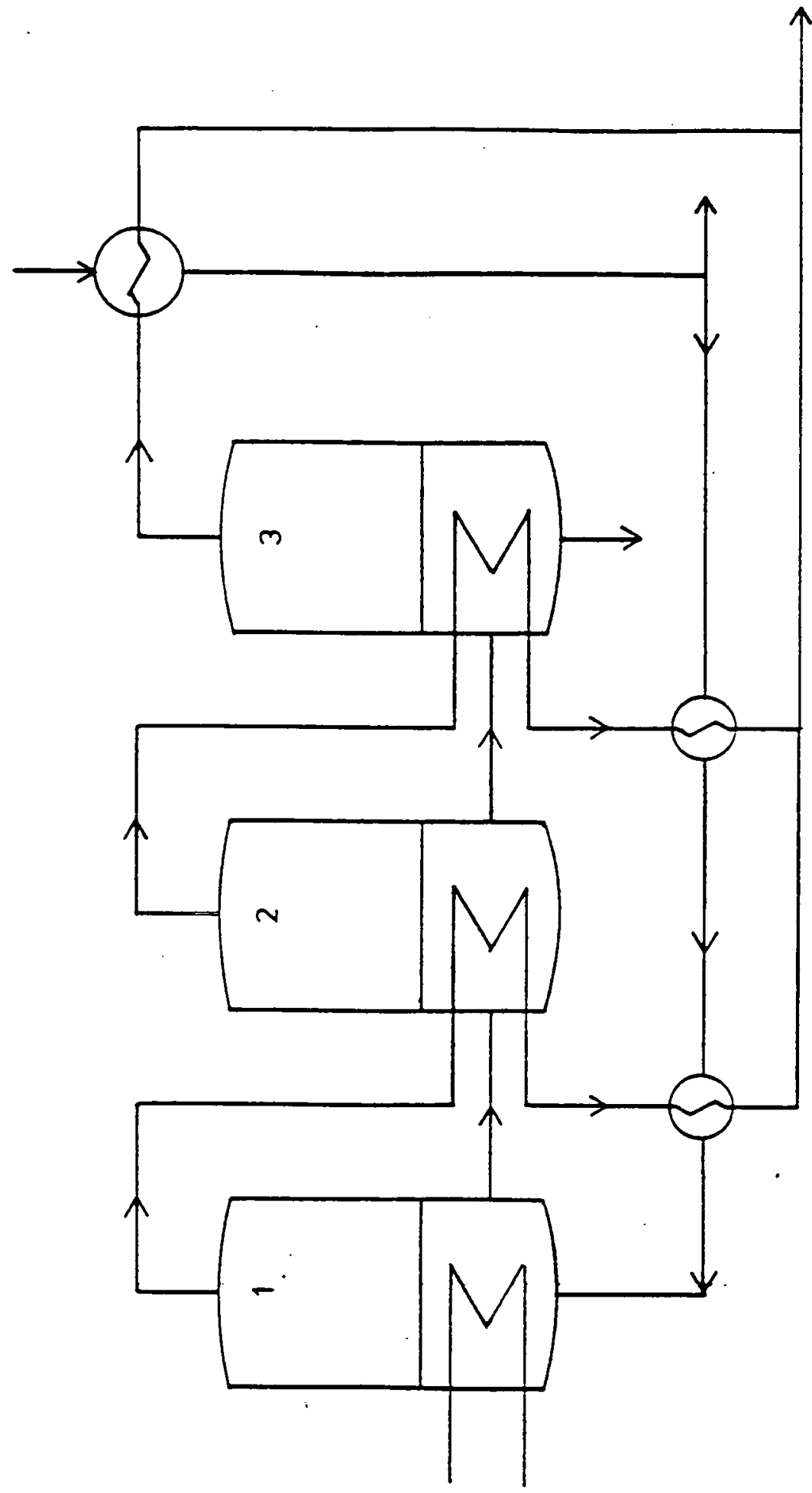

Figure (2.2) MEB Plant with 3 Stages 


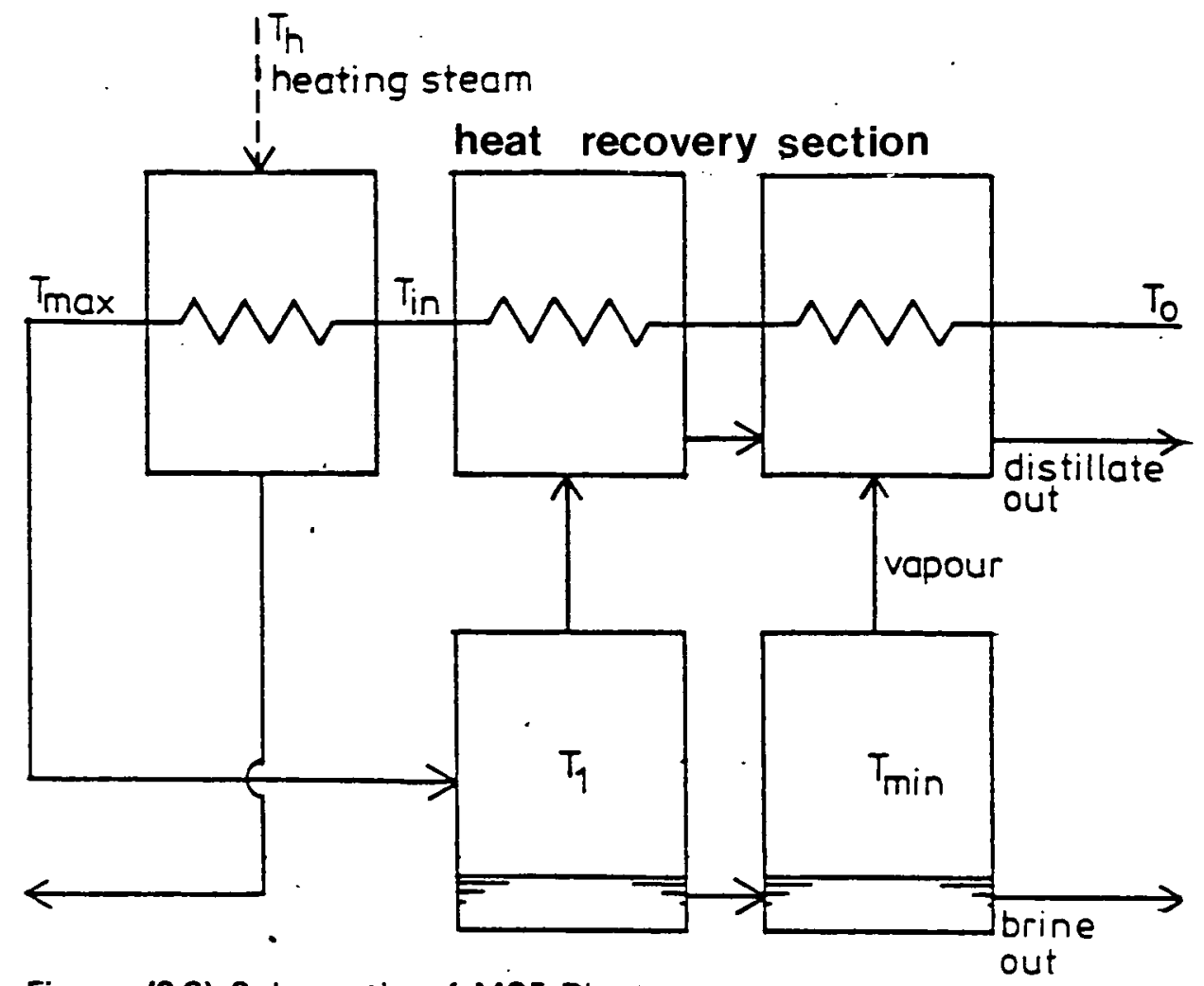

Figure (2.3) Schematic of MSF Plant

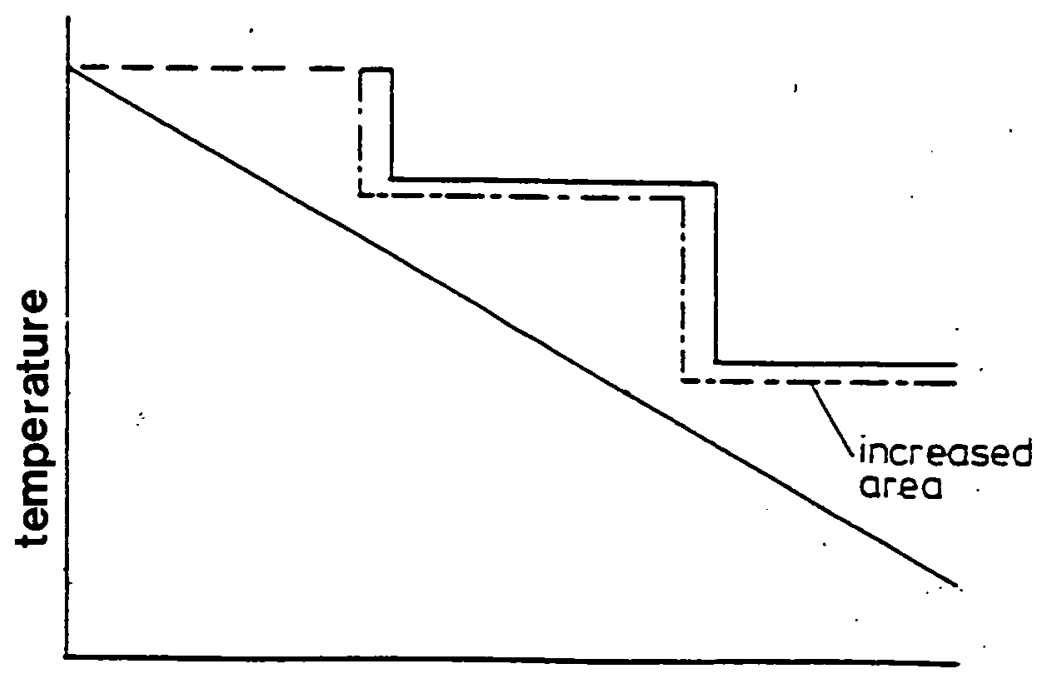

Figure (2.4) Temperature Profile Through MSF Plant 


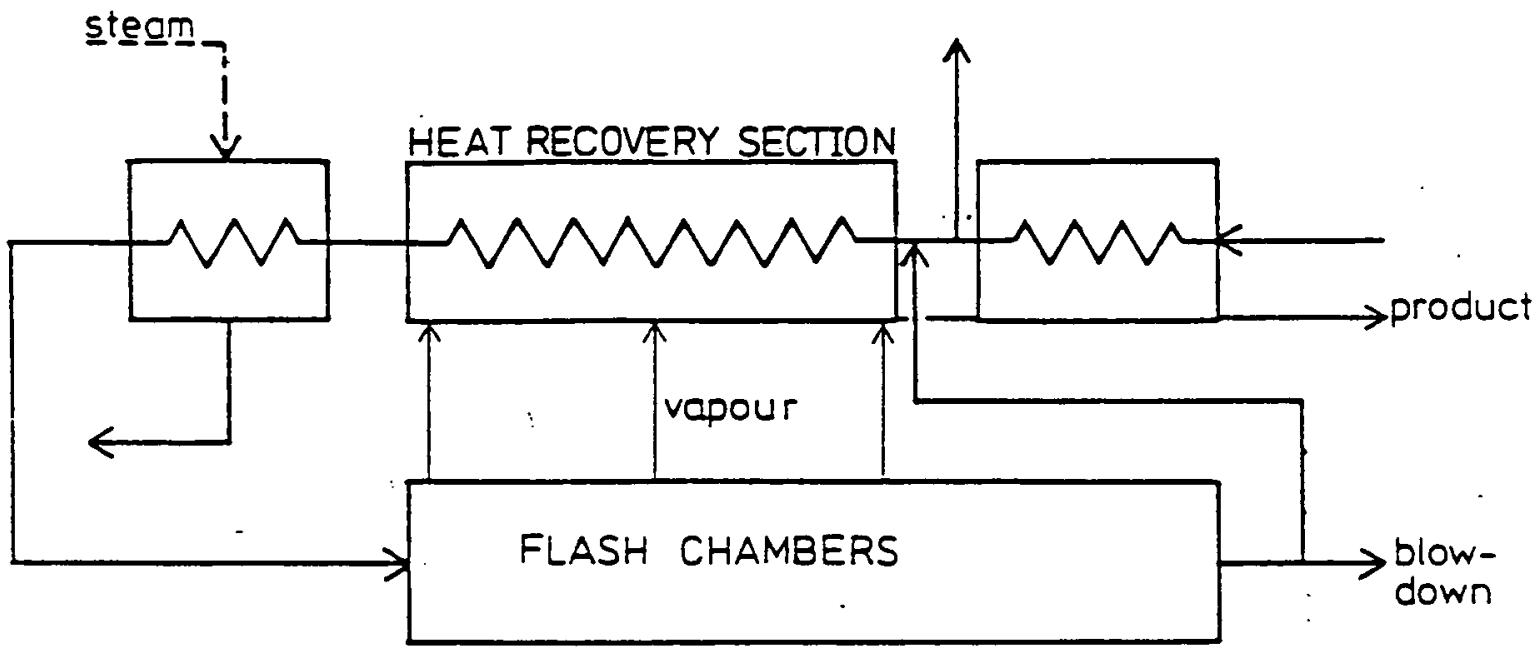

Figure (2.5) MSF Plant with Infinite Number of Stages

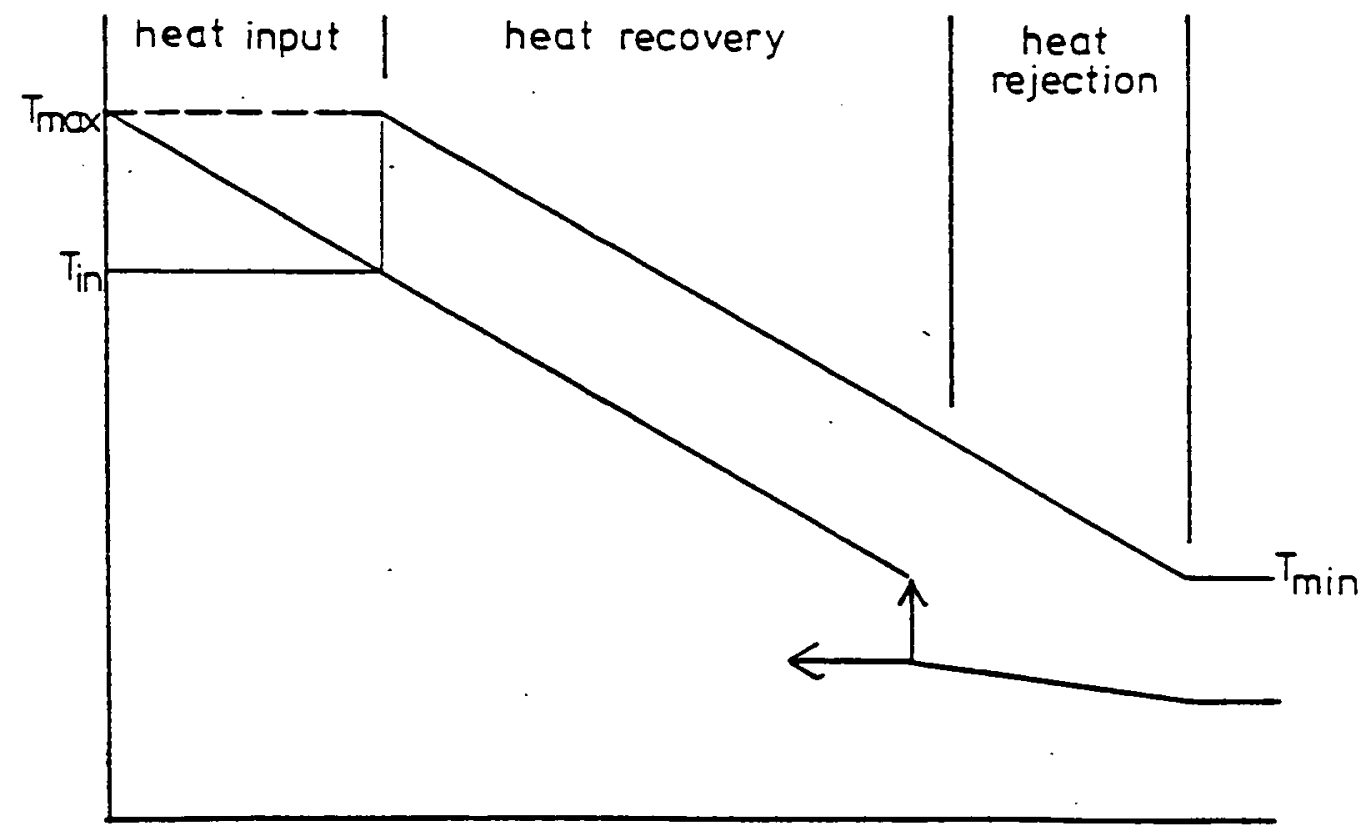

Figure (2.6) Temperature Profile Through Above Plant 


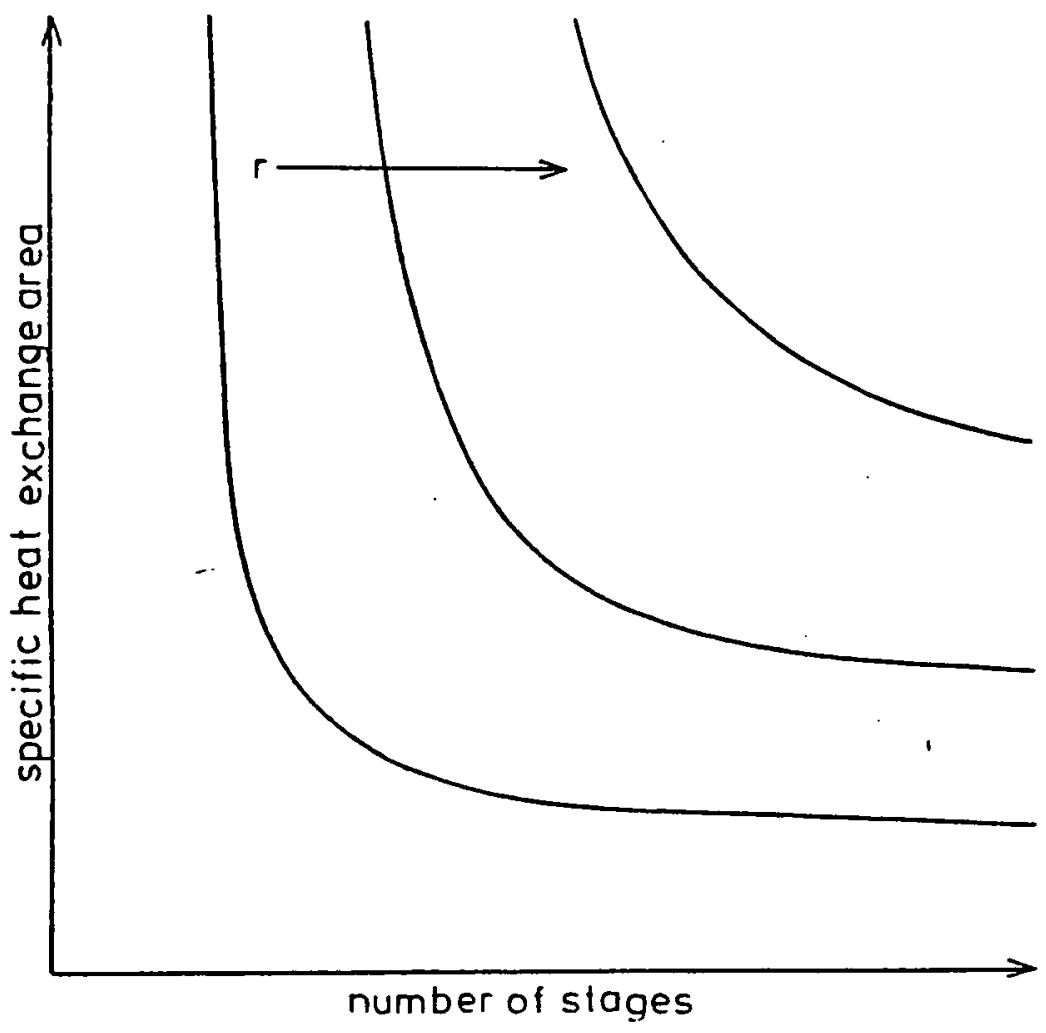

Figure (2.7) Specific Area vs. Performance Ratio for MSF Plant

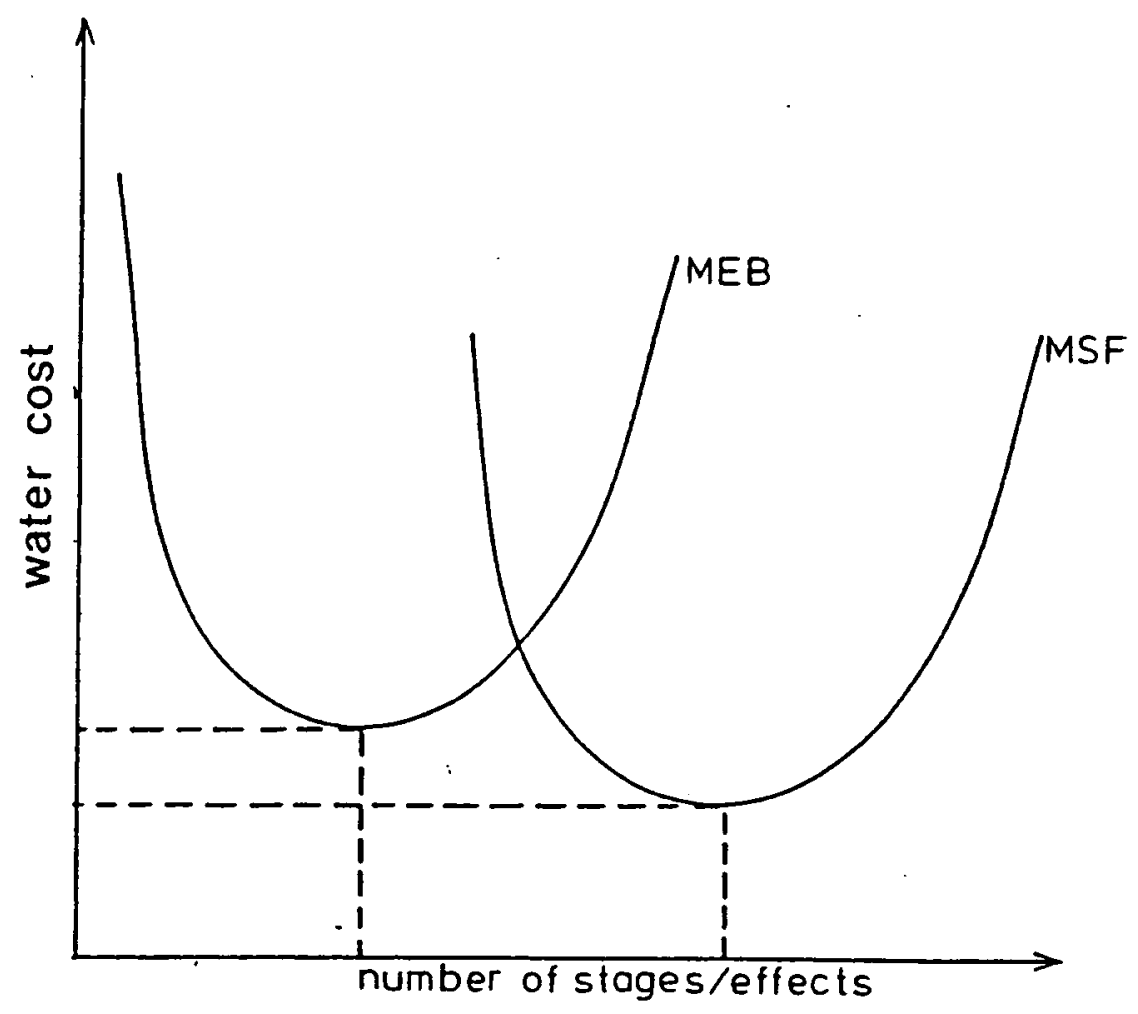

Figure (2.8) Water Cost vs. Number of Stages for MSF and MBE Plants 


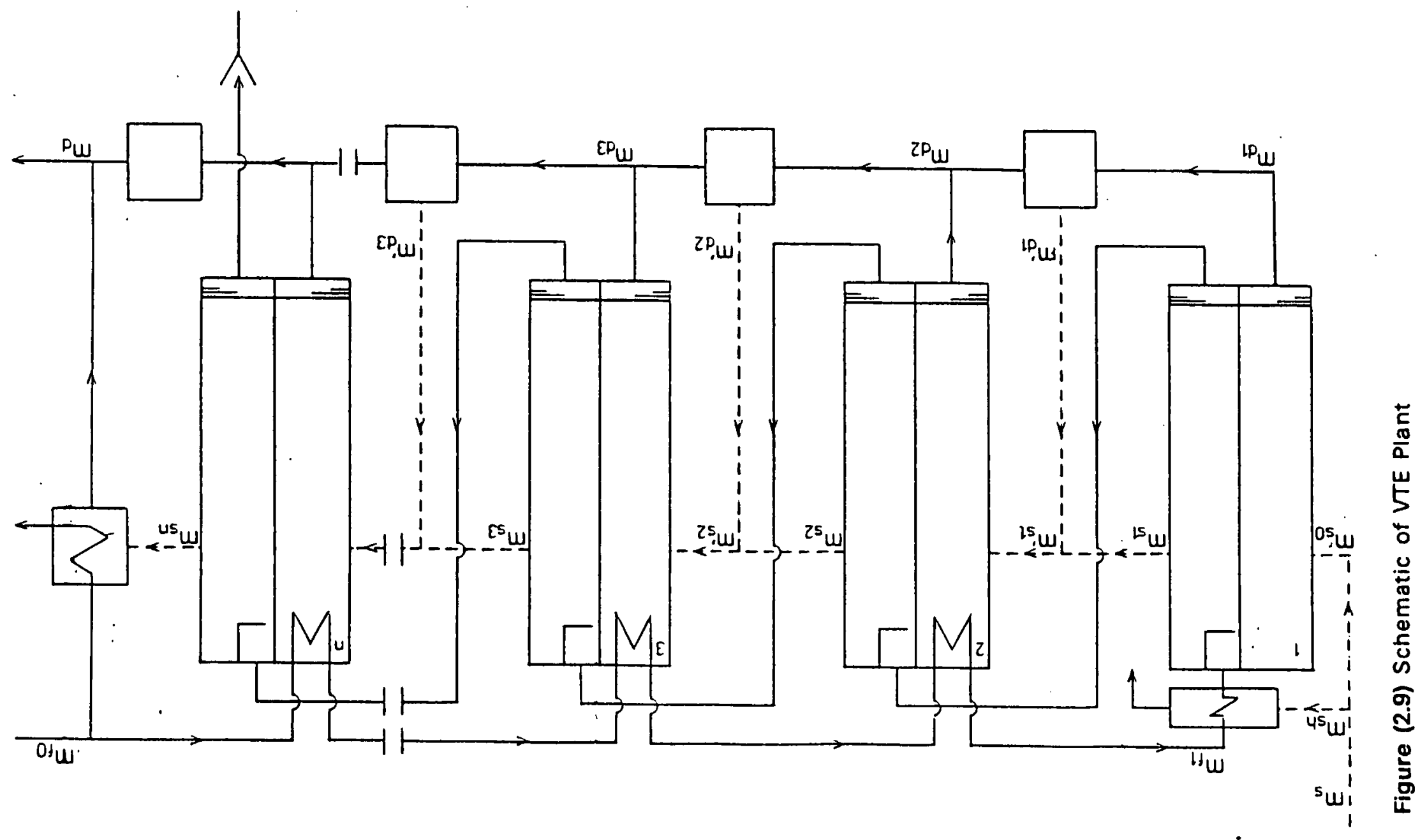




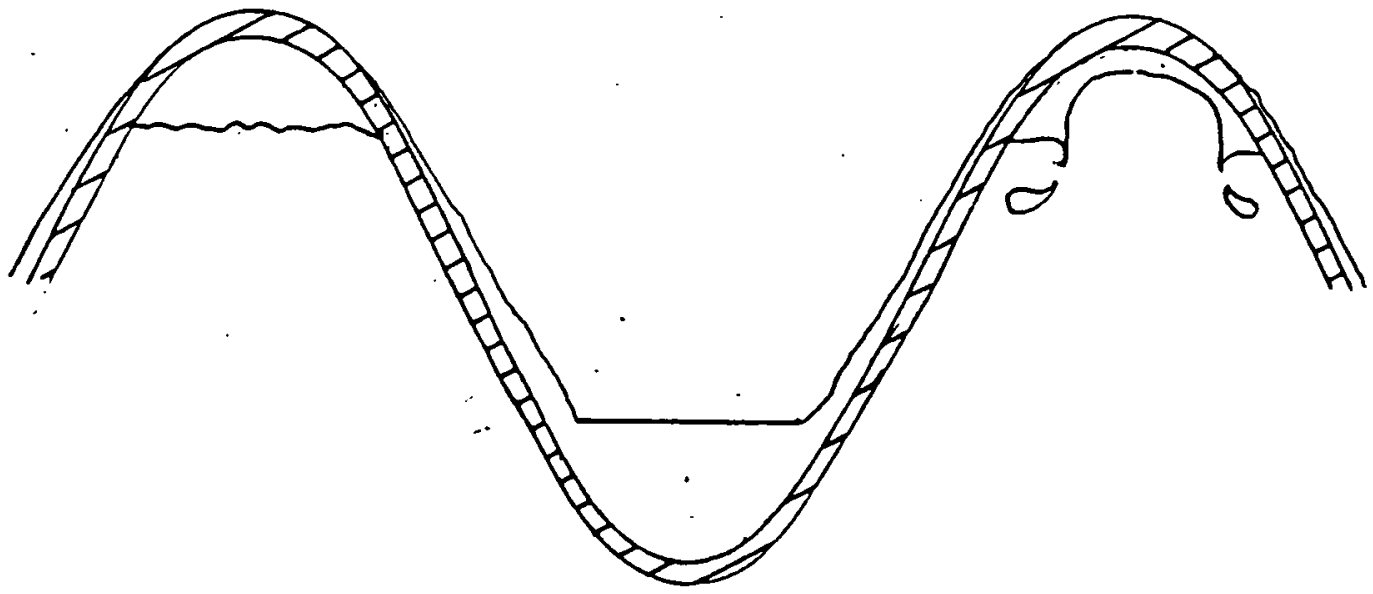

Figure (2.10) Cross Section Through Double-Fluted Tube Surface

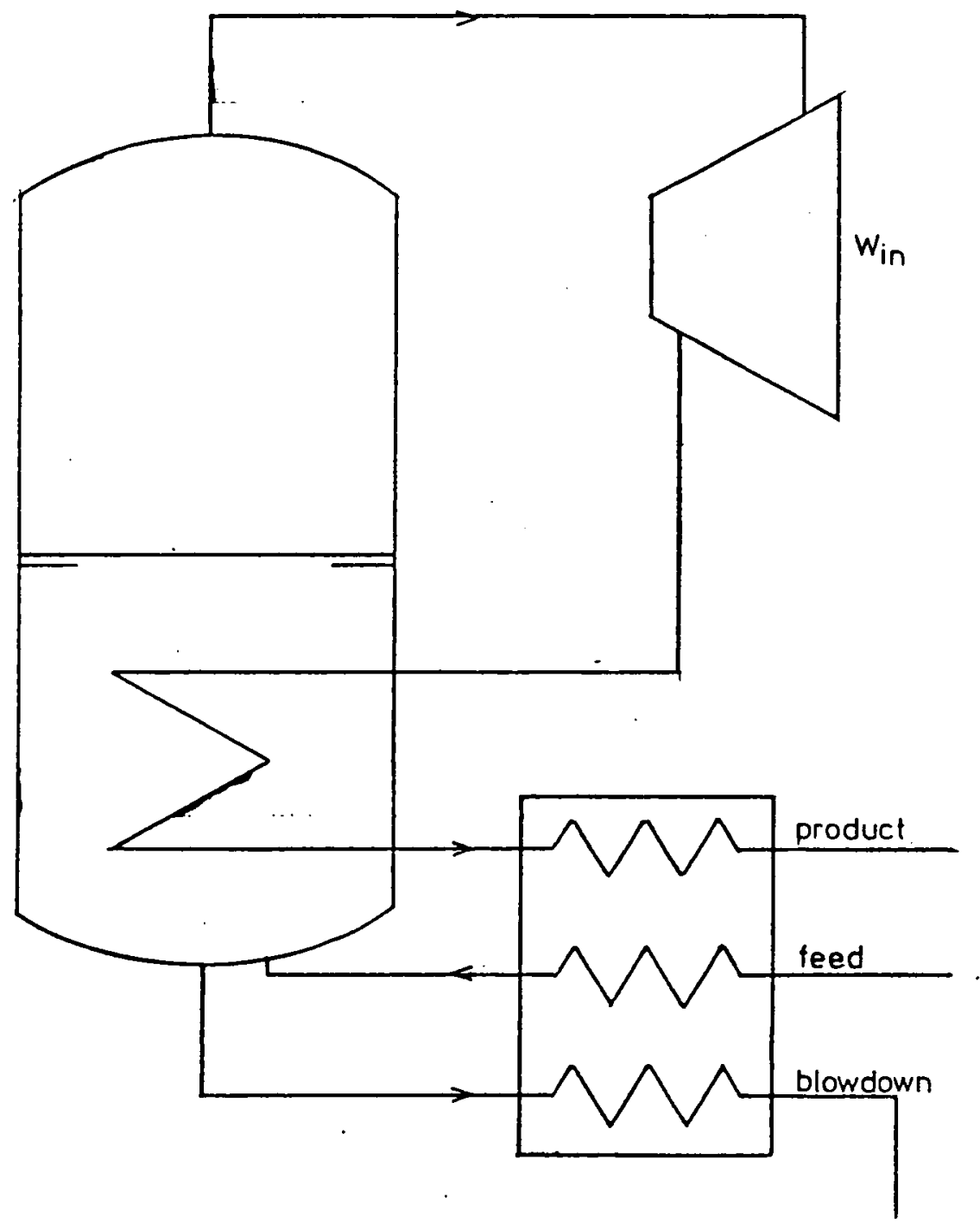

Figure (2.11) Schematic of Simple VC Unit 


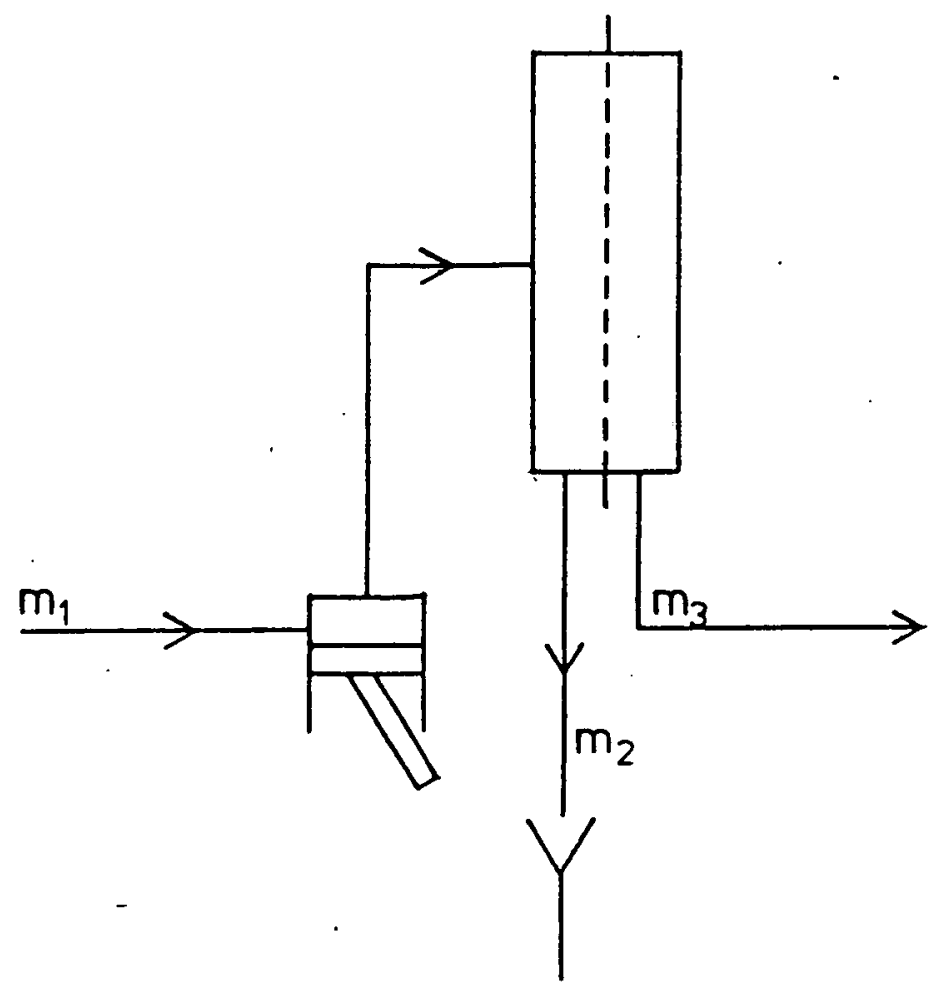

Figure (2.12) Simple RO Plant

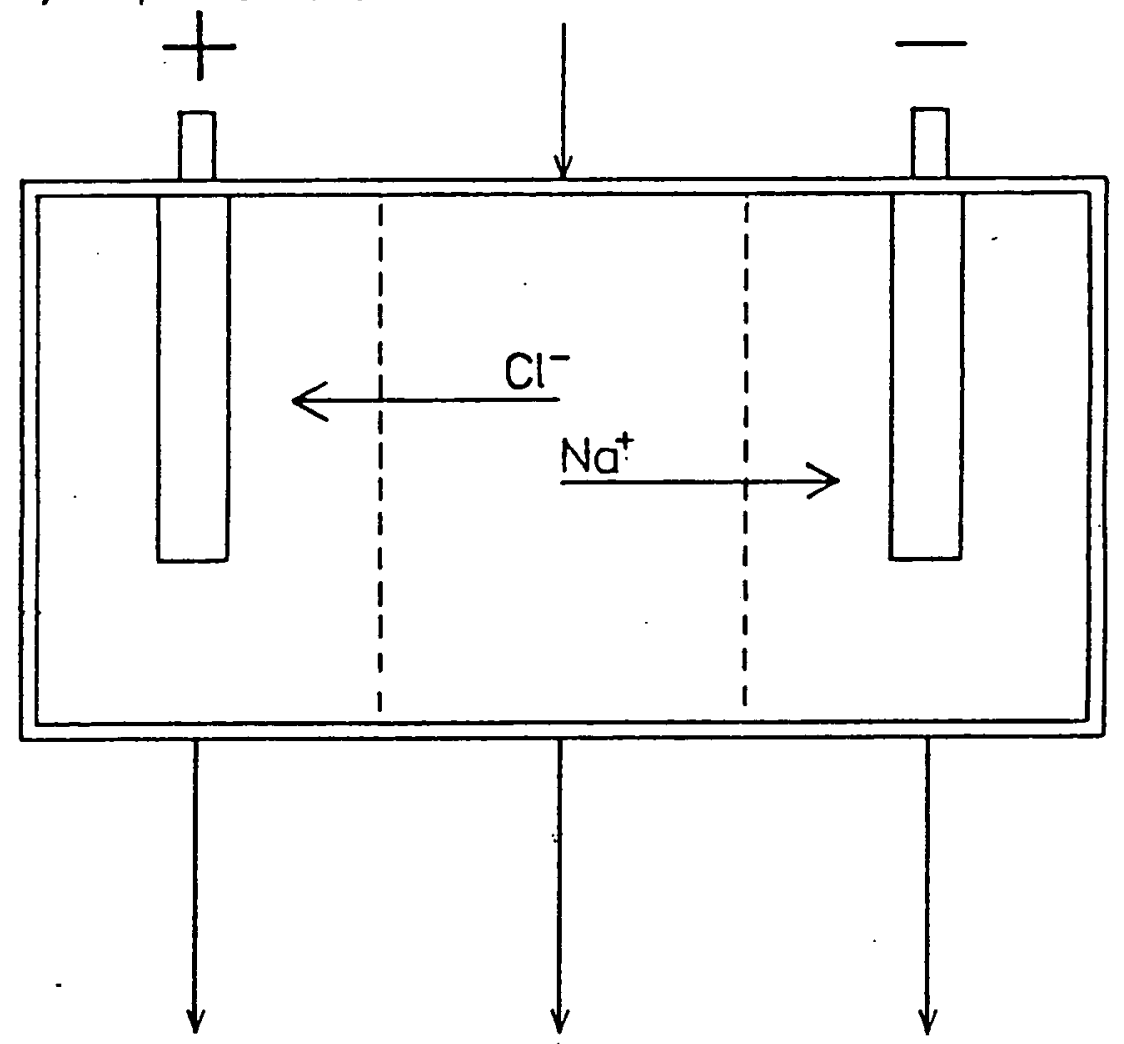

Figure (2.13) Principle of Electrodialysis 


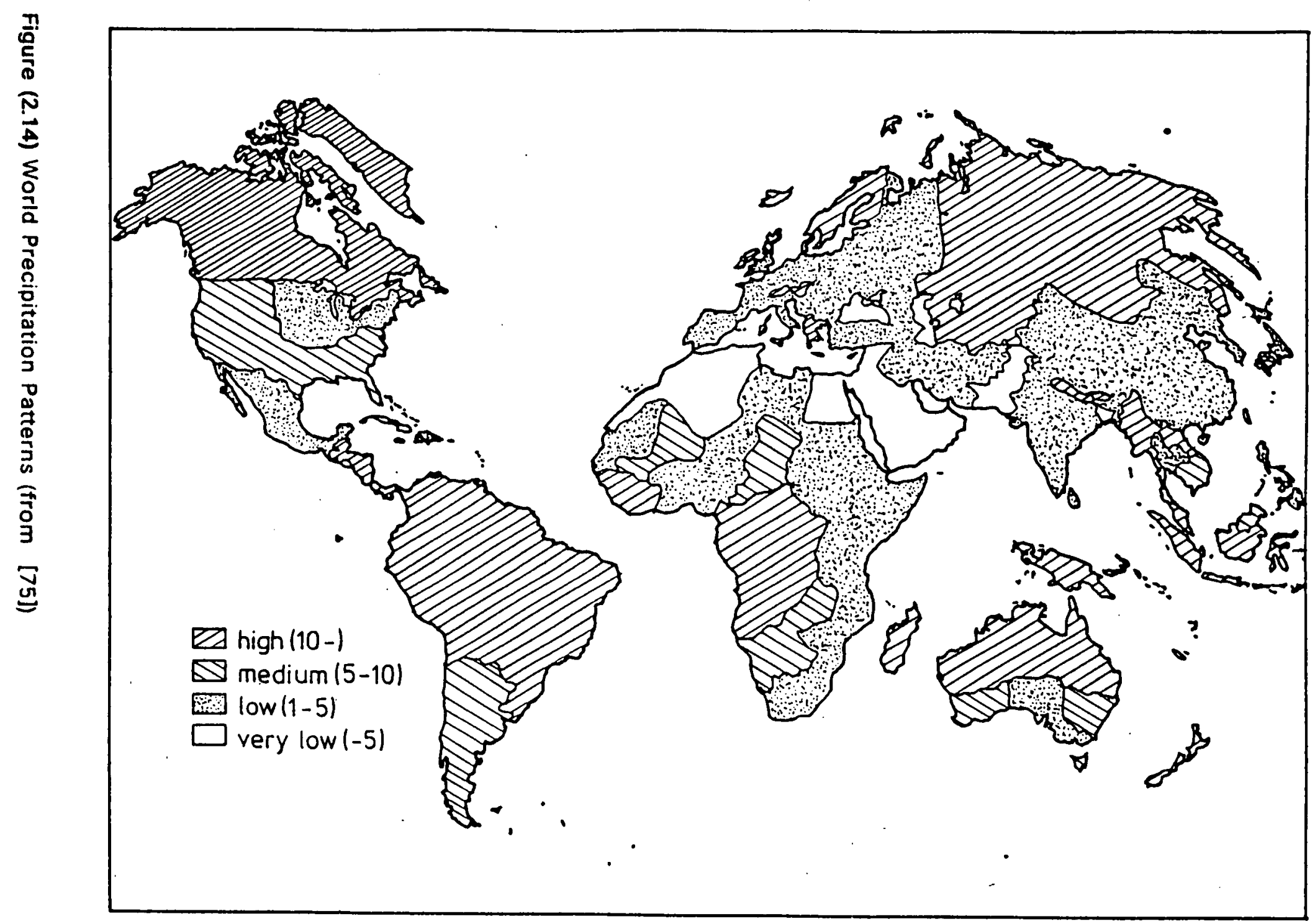




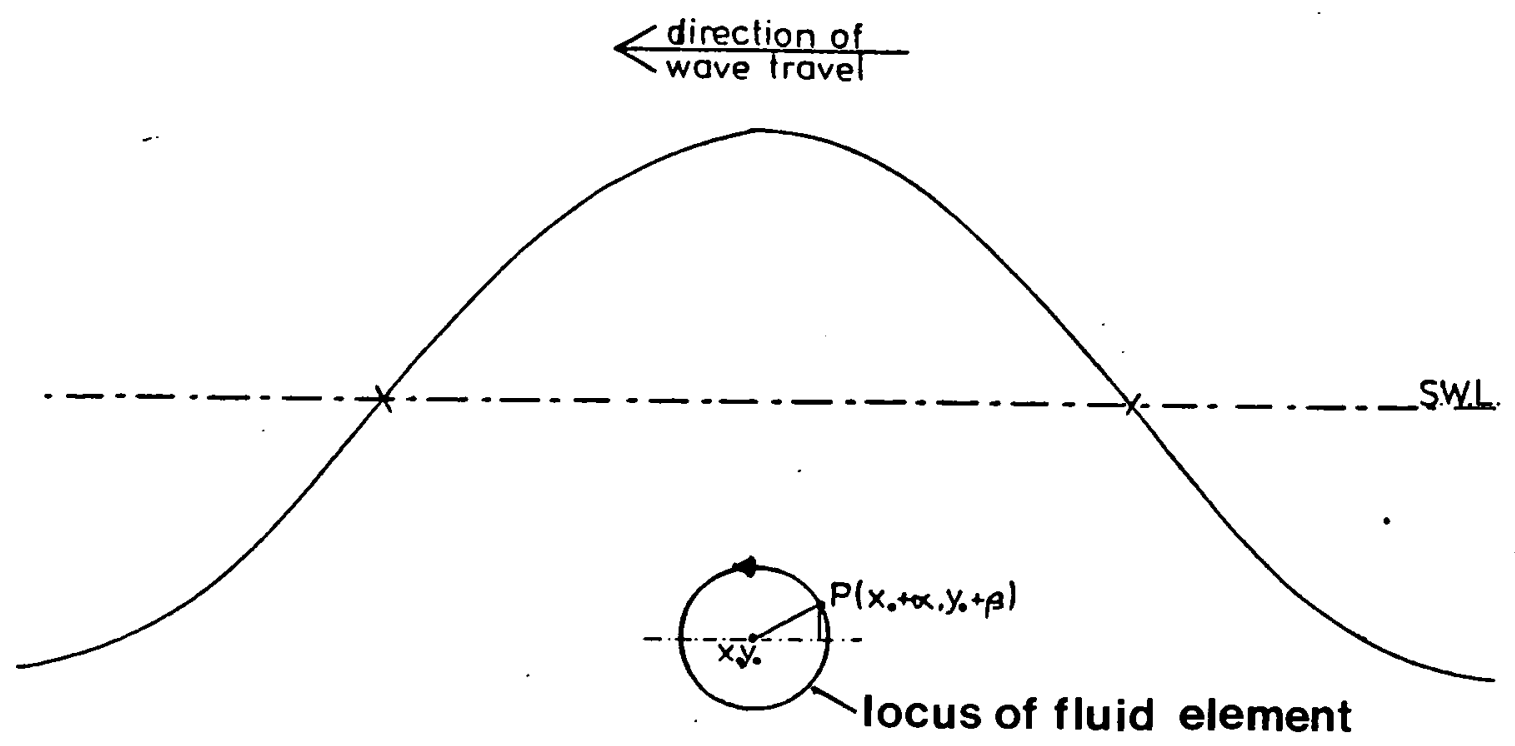

Figure (2.15) Progressive Water Wave

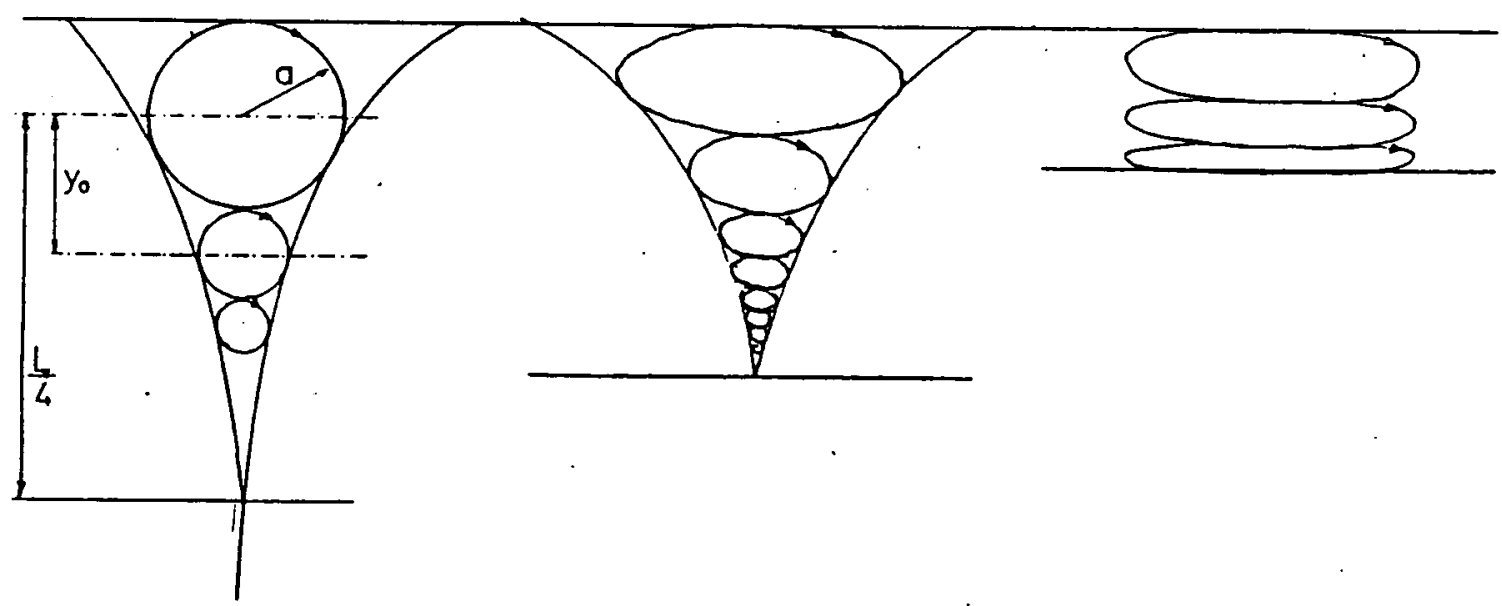

Figure (2.16) Circulation Beneath Water Waves 


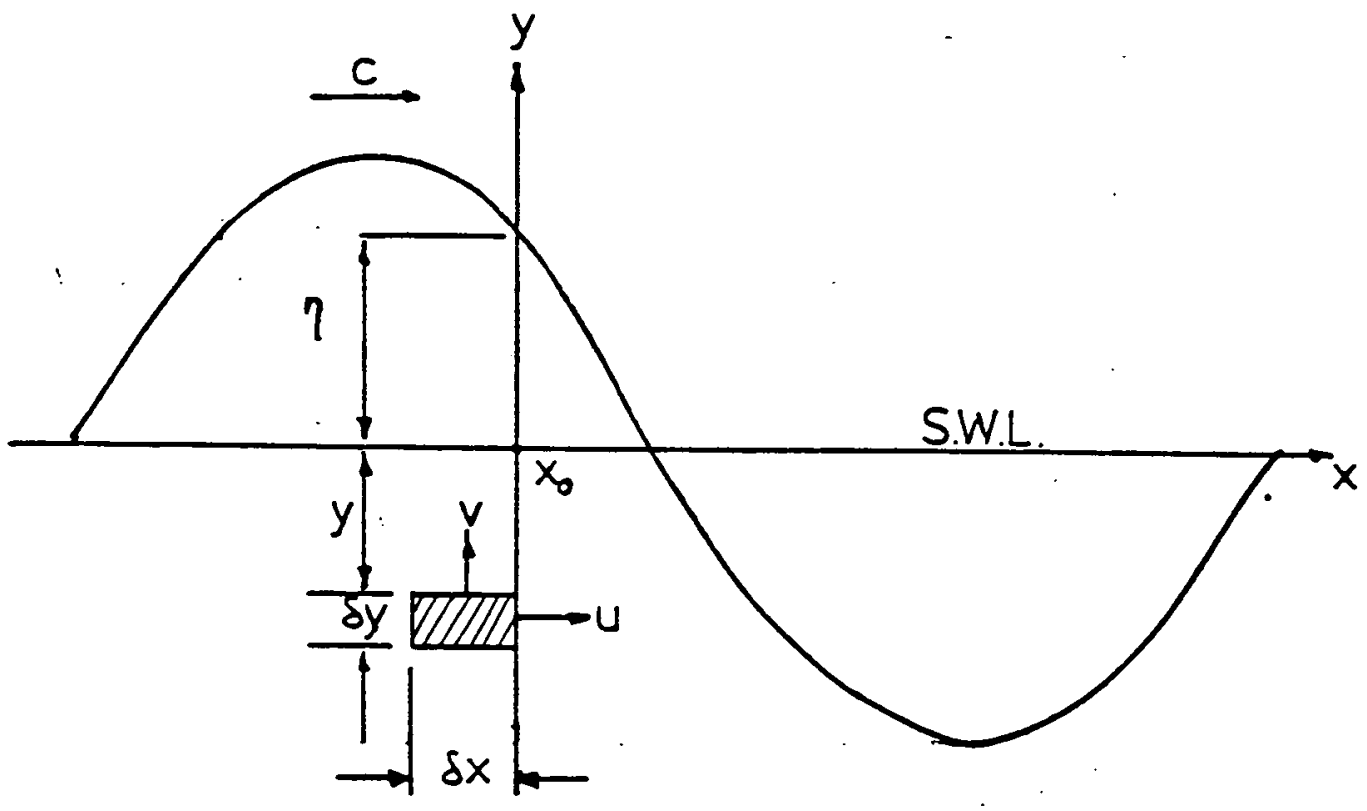

Figure (2.17) Momentum Transfer in Water Waves

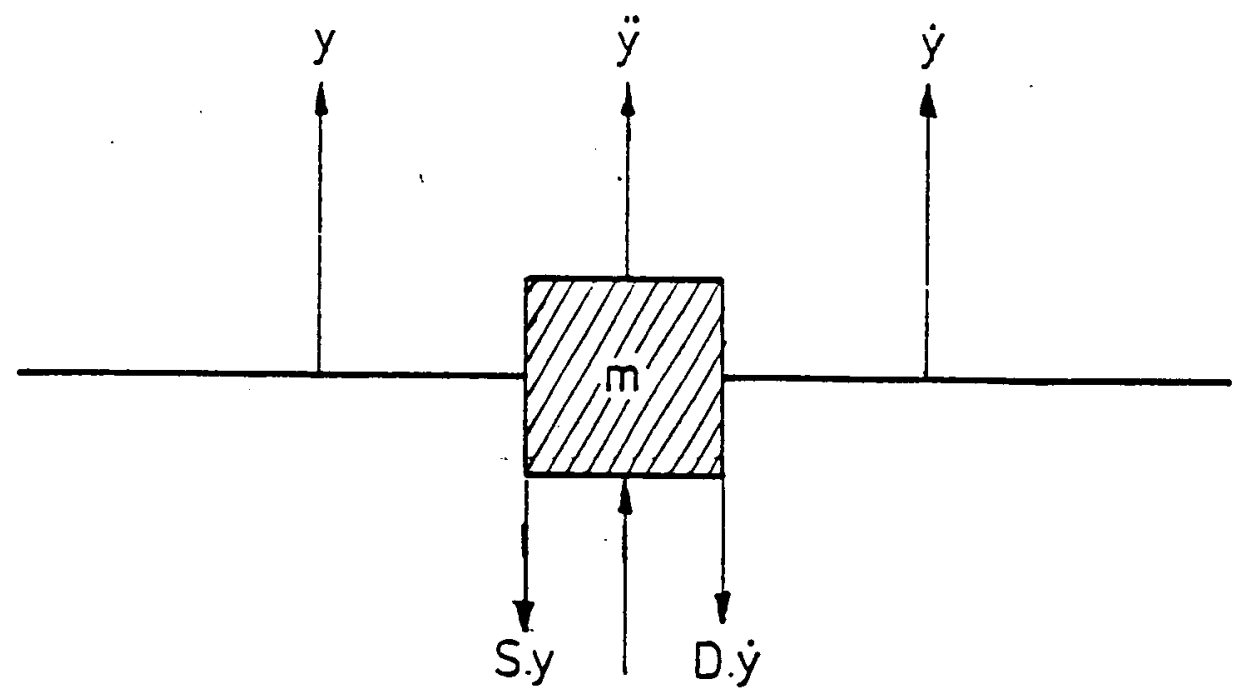

$F \cos (\omega t)$

Figure (218) Simply Shaped Body in a Wave Field 


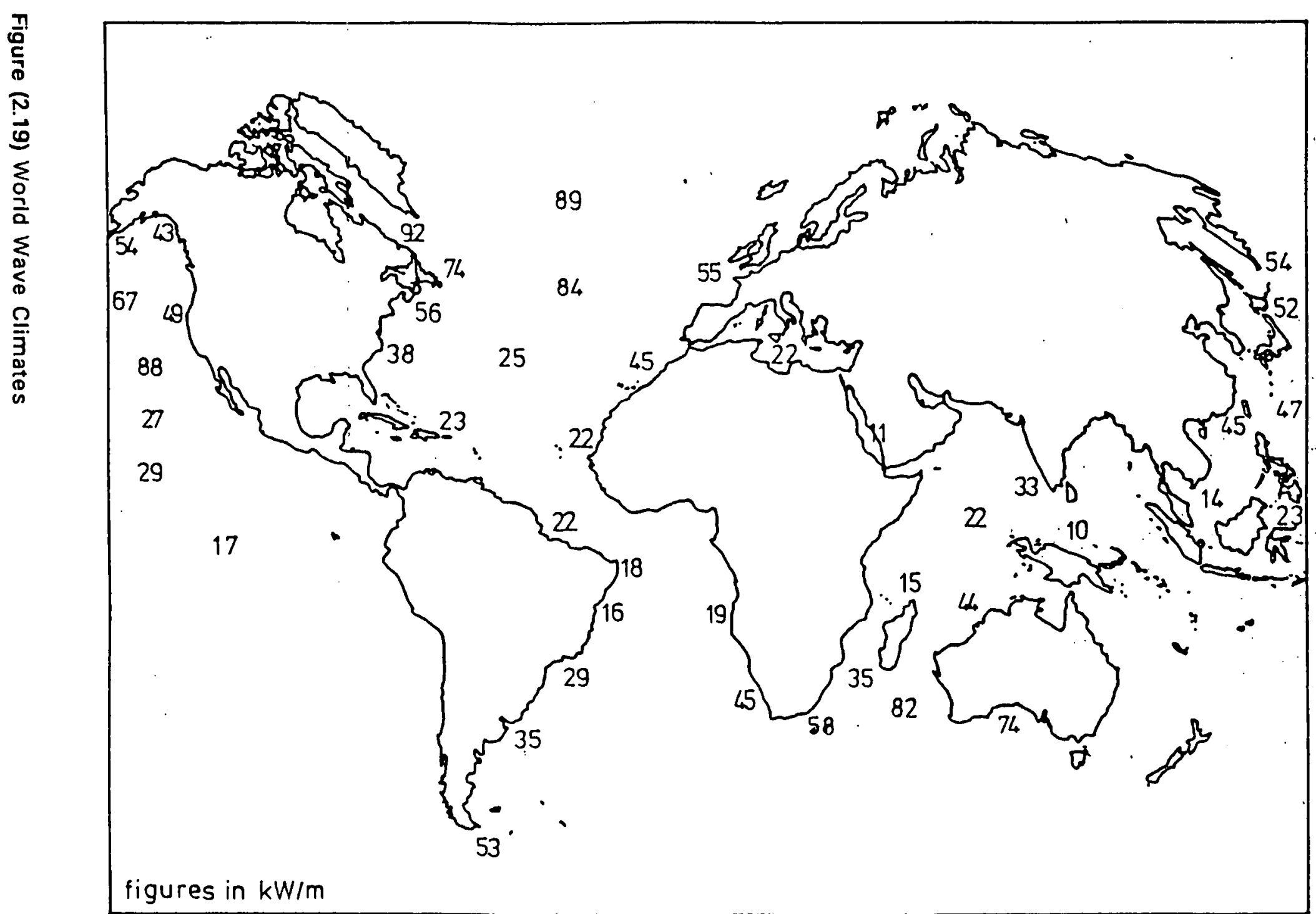




\section{CHAPTER 3}

MODELUNG AND SIMULATION OF THE DESALINATION PROCESS 


\subsection{INTRODUCTION}

Mathematical modelling has been of major importance in the study of this process. Indeed, without these techniques, it is difficult to see how this research could have progressed, given the impracticality of the construction of a demonstration plant (and the limited utility of such an exercise). During the course of the project, two mathematical models were developed and implemented; both were designed to describe the heat and mass transfer processes which would take place in a desalination duck under simulated sea conditions. The first model was developed at an early stage of the project and its purpose was to allow rapid "data" generation in order that a parametric analysis of the process could be carried out. It was hoped that, in this way, the ideas which were held about the project at that time could be clarified and a definite plan of research action constructed. As such, the first model was relatively crude in construction and unsophisticated in the solution methods used. Eventually, as the need for more reliable data became apparent, a second model was developed. The second model had many similarities with the first, as the insight and experience gained through the development of the first was of direct relevance to the formulation of the second. However, as shall be discussed in section 3.3.1, the differences between the two were important enough to justify the construction of a completely new computer program.

Both models were contained in programs written in the IMP programming language and mounted on the Edinburgh University EMAS-A mainframe computer system.

\subsection{THE FIRST MODEL}

\subsubsection{Objectives}

The basic objectives of this model were to investigate the influences of various process parameters (such as the heat exchanger areas, feed temperature etc.) on the performance of the ducks and to determine the effects of the unsteady-state nature of the process on the characteristics of the system. With particular regard to this second objective, it was important to determine whether or not the torque on the duck, generated by the pressure force across it, could be represented by a sum of linear functions of 
the angular displacement, velocity and acceleration of the duck: if this could be achieved, then the shape of the duck; its mooring design, and, in short, the questions pertaining to the performance of desalination ducks in real seas could be tackled through the study of small-scale models in wave tanks (see chapter 2, section 2.2.3).

\subsubsection{The Model Basis}

The model was essentially based on a two-state representation: that is, the structure of the model differed depending upon whether the non-return valves into and out of the main heat exchanger were chosen to be open or closed at any instant. With the valves closed, four chambers were modelled: a condensing space; an evaporating space; an adiabatic enclosure with increasing volume, and an adiabatic enclosure with decreasing volume (see figure (3.1)). With the valves open, only two chambers were modelled, with the condensing space being linked to the decreasing volume enclosure and the evaporating space being linked to the increasing volume enclosure. No pressure drop across the valves was modelled and it was assumed that the contents of the linked chambers mixed perfectly as soon as the valves opened to give a completely homogeneous vapour in the two chamber state. Furthermore, it was specified that both sets of non-return valves would operate simultaneously and that they would stay open (or closed) until the duck started to change its direction of rotation. This rigidly constrained mode of operation was obviously quite unrealistic but it had the advantages of being simple to analyse and easy to program.

Another major simplification which affected the entire model, was that the resultant motion of the duck and the fluid piston could be represented by a simple sine wave of known amplitude and frequency. This simplification, then, omitted any interaction between the duck and the waves (a necessary step because of the complex nature of wave/device interactions) and ignored the response of the fluid piston to the pressure force across it. It seemed likely that the motion of the fluid piston would resemble a sine wave with the same frequency as the duck, but there was no means of knowing what the phase angle between the two would be, nor the amplitude of the motion of the fluid piston. Once again, this simplification was entirely unrealistic, but was required in order to be able to write the first program as rapidly as possible. 
The ideal gas law:

$$
\text { p.V = n.R.T }
$$

was assumed to hold for the water vapour throughout. At typical process conditions (e.g. saturated steam at one bar), the compression factor, $z$, in the equation:

$$
\text { P.v = z.R.T }
$$

would be in the region of 0.98 , implying that the error resulting from this assumption would be of the order of $2 \%$ in predicted temperature, pressure or specific volume. It was felt that this error was not large enough to justify the use of a more complex form of gas equation than equation (3.1). The physical properties of the water vapour, condensed water and evaporating brine were assumed to be constant over the range of interest. Over a strictly limited range of temperature, pressure and salinity, this assumption was tenable; nevertheless, it was partially the desire to improve upon this situation that led to the development of the second model (see section 3.3.1). It was assumed that the equilibration of the vapour and liquid phases in the still would occur instantaneously; given the large wetted areas and low mass hold up of the liquid films, this was not unreasonable. This assumption, however, implied complete homogeneity of the vapour, which would in turn depend upon the design of the valves and of the heat exchanger.

Instantaneous equilibration of phases was not assumed, however, for the vapour spaces above the fluid piston; in fact it was assumed that no heat transfer could take place between the vapour in the "cylinders" and either the surface of the fluid piston and/or the structure of the duck. For a steady-state device with well insulated walls, heat loss from the duck to the surroundings would indeed be negligible, in terms of an overall energy balance over the system. For an unsteady-state process such as this, however, while the overall loss might be insignificant, of more importance could be the role of the structure of the duck as a thermal energy storage medium. A full understanding of this effect would require exact details of the geometry of the walls of the duck; the vapour flows around or over them, and their materials of construction. The unsteady-state heat conduction equation with variable boundary conditions (themselves dependent upon the operation of the duck) 
would then need to be solved. It was felt to be a justifiable simplification to assume that the thermal storage effects of the walls would be negligible.

The situation was similar in the case of the fluid piston. In a steady-state system, the fluid piston would be simply a large, U-shaped body of water, with a small temperature difference across it. Vapour could condense on one side of the piston and evaporate from the other side, at a rate determined by the rate of heat transfer by conduction through the fluid. This rate would be influenced by the degree of mixing in the water (for example, if the fluid piston were perfectly mixed, then no temperature difference across it could exist) and so it may be seen that, even for this comparitively simple situation, the effects of heat transfer to and from the fluid piston would be difficult to estimate without pilot plant data. For an unsteady-state system, the situation would be even more complex, as the piston could act both as a heat transfer medium and as a thermal energy storage device; and once again, the degree of mixing would be of crucial importance in either role. Indeed, one would also have to consider the heat transfer between the walls of the duck and the fluid piston; a complicated problem because of the relative motions of the two. It was the opinion of the author that the complete modelling of the thermal and kinetic states detailed above would be a project of immense complexity, requiring a great deal of experimental data input and verification.

In order to gain an order of magnitude estimate of the errors following from the above simplification, however, an approximate analysis of the heat transfer to a fluid piston was carried out as follows. Taking the case of a solid block of material with the same properties as the fluid piston, it was possible to study transient heat transfer in this system and therefore draw some tentative conclusions about the possible effects of heat transfer to the fluid piston. The block was taken to be a semi-infinite solid; qualitatively, it seemed likely that the temperature at the centre of the fluid piston (and hence of the postulated block) would be unaffected by short term variations in the temperatures of the surface regions. One may therefore apply the equation for unsteady-state heat conduction through a semi-infinite block: 


$$
Q_{1}=2 \cdot k \cdot A \cdot\left(T_{s}-T_{g}\right)^{t / \pi \cdot a}
$$

Taking $t$ as half of one wave period, say 5 seconds, and the typical temperature change over this period, $\left(T_{s}-T_{a}\right)$, to be of the order of $2{ }^{\circ} \mathrm{C}$ on either side of the duck, this gives $\mathrm{O}_{4}$ approximately equal to $300 \mathrm{~kJ}$. Thus the average rate of heat transfer to the piston would be of the order of $75 \mathrm{~kW}$, while the average rate of heat transfer through the main heat exchanger (for a $1000 \mathrm{~m}^{3} \cdot \mathrm{day}^{-1}$ duck) would be of the order of $25 \mathrm{MW}$. On the basis of this estimate, therefore, it would appear that the heat transferred between the fluid piston and the vapour above it would represent less than $0.5 \%$ of the total heat load of the duck.

The above analysis was, of course, very inaccurate: the real fluid piston would not be semi-infinite, as it would not be a simple shape; condensation and evaporation to and from the surfaces of the piston would alter the shape of the temperature profile within the fluid, as would bulk mixing and heat transfer with the structure of the duck. Ultimately, it is felt that only experimental work of some kind could determine the importance of these effects.

\subsubsection{Derivation of the Equations}

The principle underlying the equations for the heat and mass transfer in the duck was that the rate of change of pressure with time in any part of the system could be expressed as the sum of two parts: a term due to adiabatic volume change at constant mass and a term due to adiabatic change of mass at constant volume. Note that the second of these terms implies that mass transfer must take place isothermally. In order for the mass transfer to take place under conditions of adiabaticity, there can be no sensible heat transfer from the enclosure and therefore the contents must always be at their saturation conditions (the mass transfer under these circumstances is identical to the removal or addition of a portion of the vapour by mechanical means). The two terms were evaluated starting from the equation for the adiabatic compression/expansion of an ideal gas:

$$
\text { p.v } Y=\text { constant }
$$

where: 


$$
\gamma=C_{p} / C_{v}
$$

From equation (3.4), for adiabatic volume change at constant mass, it may be shown that:

$$
d p / d t=-\gamma \cdot(p / V) \cdot d V / d t
$$

This may be approximated by:

$$
\delta p=-\gamma \cdot\left(p_{i} N_{i}\right) \cdot \delta V
$$

so that:

$$
p_{i+1}=p_{i} \cdot\left(1-\gamma \cdot \delta V N_{i}\right)
$$

The adiabatic temperature change may be expressed in a similar manner:

$$
T_{i+1}=T_{i \cdot}\left(1+(1-\gamma) \cdot \delta V / N_{i}\right)
$$

Starting from equation (3.4), the expressions for the rates of change of temperature and pressure with respect to time with changing mass and constant volume, can also be found:

$$
d p / d t=\gamma \cdot(p / n) \cdot d n / d t
$$

or, approximately:

$$
p_{i+1}=p_{i} \cdot\left(1+\gamma \cdot \delta n / n_{i}\right)
$$

and

$$
T_{i+1}=T_{i \cdot} \cdot\left(1+(\gamma-1) \cdot \delta n / n_{i}\right)
$$

Thus, the two displacements $\delta n$ and $\delta V$ must be evaluated at a series of small time steps, $\delta t$. $\delta V$ can be found if the exact mode of motion of the duck is specified (eg. a sine wave of known radian amplitude and period) and its dimensions are known: 


$$
\delta V=0.5 \cdot r^{2} \cdot L \cdot \delta \theta
$$

where $\delta V$ is defined in figure (3.2), and:

$$
\theta=\alpha \cdot \sin (2 \cdot \pi \cdot t / T)
$$

Thus:

$$
\delta V=\left(\pi \cdot r^{2} \cdot L \cdot A / T\right) \cdot \cos (2 \cdot \pi \cdot t / T) \cdot \delta t
$$

Sn must be found from a heat balance around the main heat exchanger. Assuming the vapour is always saturated:

$$
d n / d t=q / \lambda
$$

where $q$ is the rate of heat transfer between the vapour and liquid films, given by:

$$
q=h \cdot A \cdot\left(T_{\text {sat }}-T_{w}\right)
$$

Heat balances over the liquid films also yield expressions for $q$; for the condensing side:

$$
q_{c}=A \cdot x \cdot \rho \cdot C_{p} \cdot d T_{s a t} / d t-A \cdot(k / w) \cdot\left(T_{w c}-T_{s a t}\right)
$$

and for the evaporating side:

$$
\begin{aligned}
q_{e}= & A \cdot x \cdot \rho \cdot C_{p} \cdot d T_{s a t} / d t+h \cdot e \cdot A \cdot\left(T_{w e}-T_{\text {sat }}\right)+ \\
& m_{f} \cdot C_{p} \cdot\left(T_{s a t}-T_{f}\right)-A \cdot(k / w) \cdot\left(T_{w e}-T_{\text {sat }}\right)
\end{aligned}
$$

Note that, in equations (3.18) and (3.19), terms taking account of the thermal inertia of the films and of the thermal conductivity of the heat transfer material were introduced. $T_{\text {sat }}$ the saturation temperature of the liquid or vapour, was found from a standard Antoine equation of the form:

$$
\log p_{\text {sat }}=A+\left(B /\left(C+T_{\text {sat }}\right)\right)
$$

The wall temperatures, $T_{\text {wc }}$ and $T_{\text {we }}$, were estimated from a knowledge of the liquid film temperatures and the relevant heat transfer coefficients: 


$$
\begin{aligned}
& T_{w c}=T_{f c}-\left(U / h_{c}\right) \cdot\left(T_{f c}-T_{f e}\right) \\
& T_{w e}=T_{f e}+\left(U / h_{e}\right) \cdot\left(T_{f c}-T_{f e}\right)
\end{aligned}
$$

The condensation heat transfer coefficient was estimated from the Nusselt correlation:

$$
h=0.725 \cdot\left\{k_{f}^{3} \cdot \rho_{f}^{2} \cdot g \cdot \lambda / \Delta T_{0} \cdot D_{0} \cdot \mu_{f}\right\}^{0.25}
$$

where:

$$
\Delta T_{0}=T_{\text {sat }}-T_{\text {we }}
$$

Equation (3.23) is a good fit to experimental data for laminar films of condensate at steady state, the conditions for which it was derived. It seems likely that the condensate film in the duck would always be in the laminar region (although the motion of the duck may have some effect), but the steady-state approximation, which cannot be said to properly apply in this case, is bound to lead to some inaccuracies. The condensate film thickness was found from the same analysis:

$$
\delta_{c}=\left\{3 \cdot \Gamma \cdot \mu_{f} / \rho_{f}^{2} \cdot g\right\}^{0.33}
$$

where:

$$
\Gamma=h_{c} \cdot L \cdot \Delta T_{0} / \lambda
$$

The evaporation side film coefficient was evaluated using a correlation developed by Struve (Struve, 1968, [69]) to represent the results of Dukler (Dukler, 1961, [16]) for turbulent evaporating films:

$$
\mathrm{Nu}=\{0.110-0.565 /(\mathrm{Pr}+5.47)\} \cdot \mathrm{Re}^{0.231}
$$

It was not necessary to calculate the brine film thickness from a correlation as this could be found from the user specified feed mass flowrate, $m_{f}$. It was assumed that this flowrate would always be significantly greater than the product flowrate, and therefore that $m_{f}$ would be approximately constant.

Equations (3.8) to (3.27) were algebraically manipulated to give a set of ten algebraic equations in ten unknowns. The method of solution employed and some details of the structure of the program containing the equations and the 
solution method, are given in the next section.

\subsection{Implementation of the First Model}

The equations were solved simultaneously using a multi-dimensional Newton-Raphson method with numerical derivative approximation, at a series of short time steps. The stability of the method was found to be highly sensitive to the step size and to the initial conditions chosen. It seems likely that such difficulities may have been due more to the form of the equations and the approximations inherent in the model, rather than fundemental problems with the solution method chosen (see section 3.3.1). For example, it seems likely that the equations containing logarithmic or fractional power terms could have led to instability problems. Stability problems most often occurred at the point of opening or closing of the valves, probably because this action resulted in a step change of volume. Attempts were made to limit the effects of this step change, mainly aimed at getting a better first estimate for the iterative procedure, and these proved to be a partial success. Away from these difficult points, the method typically converged to a solution in around 5-10 iterations with a step size of 0.05 seconds.

The structure of the program containing the model, written in the IMP programming language was basically simple (see appendix IV for detailed listings). The equations for adiabatic compression and/or expansion were held in a routine, ADIABAT, which was called at the appropriate points in the program to calculate the conditions of the chambers not involved in heat transfer, that is, the spaces outside the still with the valves closed. The equations describing conditions inside the still chambers with heat transfer occurring were held another routine, EVALUATE, in the form:

$$
f(x)=0
$$

This form was necessary, as EVALUATE was called by another routine, SOLVER, which held the Newton-Raphson algorithm and required the equations to be arranged in this way. With the definition of these three routines making up the bulk of the program, the rest of the code consisted of calling the routines at the correct points with the correct parameters. As there were two states in the model, open-valves/closed-valves, it was possible to divide the program into two sections divided by an if..then statement. With the valves closed 
ADIABAT and SOLVER(EVALUATE) were called at each time step, with the valves open, only SOLVER(EVALUATE) needed to be called.

At each time step an initial estimate was required for the iteration process, this was calculated from the rate of change of the variable over the last two steps, thus:

$$
x_{i+1}=x_{i}+\varepsilon \cdot\left(x_{i}-x(i-1)\right)
$$

Values of $\varepsilon$ between 0.5 and 0.75 were found to give reasonably reliable convergence. The results of the solution were sent to a file, "RESULT", other, more detailed, data could also be sent to other, named files (see appendix IV).

\subsection{THE SECOND MODEL}

\subsubsection{Objectives}

The objectives leading to the construction of the second model were largely the same as those leading to the construction of the first (see section 3.2.1). Added to this list, however, was the desire to improve upon the first model in terms of its perceived accuracy, numerical stability and the clarity of the resulting computer program. It had become clear, through the experience gained in the construction of the first model and in the analysis of its results, that some of the inherent assumptions were causing serious defects in the output produced. Notably, the basis of the first model, the two-state system, was felt to be unrealistic and unnecessarily constraining. Furthermore, a simple model of the fluid piston had been developed and it was felt to be important to be able to utilise this, but it would have been difficult to introduce this element into the first model. Finally, it had become apparent that the assumption that the liquid and vapour phases would always be at equilibrium, without the transfer of sensible heat, not only led to occasional anomalies in the results (such as liquid evaporating into a sub-cooled vapour), but also meant that any enthalpy of superheat of the vapour would be ignored, thus underestimating the efficiency of the system. To include such changes as were necessary in the existing model would have been complicated and generally unsatisfactory and so it was decided to start again on the construction of a completely new model. 


\subsubsection{The Model Basis}

In the second model, the duck was envisaged as a four chamber system with vapour flows between the chambers being controlled by "real" non-return valves. That is to say, the models for the valves operated on the basis of the pressure drop across the valve (to decide if it was to be open or not) and that a pressure drop across the valve, due to vapour flow through it, was also modelled; this was felt to be much closer to the operation of the real valves than the first model.

An attempt was made to model the motion of the fluid piston under the influence of the pressure difference across it, although the motion of the duck itself was still modelled by a simple sine wave of known amplitude and period.

As in the first model, no attempt was made to account for heat transfer between the structure of the duck, the fluid piston and the water vapour.

The behaviour of the vapour was assumed to be adequately modelled by the ideal gas law, but the physical properties of both liquid and vapour phases were estimated from a number of correlations, rather than being assumed to be constant over the range of interest.

\subsubsection{Derivation of the Equations}

\section{Motion of the Fluid Piston}

The motion of the fluid piston was modelled in two simple ways; as a U-tube of fluid and as a solid semi-cylindrical shell. Both methods gave some similarity in their results and the second method was chosen for inclusion in the final program (see below). It is, however, interesting to compare both methods:

(1) The fluid piston as a $U$-tube of water (see figure (3.3)).

A force balance on the fluid piston, neglecting drag at the walls of the duck gives: 


$$
M . d^{2} x / d t^{2}+2 \cdot x \cdot p \cdot g \cdot r=A \cdot \Delta p
$$

Where $x$ is this vertical displacement of the liquid surface from a datum level, and $M$ is the mass of the piston, given by:

$$
M=\rho . \pi \cdot\left(r^{2}{ }_{1}-r^{2}{ }_{2}\right)
$$

for unit length. For simple harmonic motion, with no pressure forcing term, equation (3.30) can be expressed as:

$$
\pi \cdot\left(r^{2}{ }_{1}-r^{2}\right) \cdot d^{2} x / d t^{2}+2 \cdot g \cdot x \cdot r_{1}=0
$$

Solution of this equation gives the harmonic frequency of the piston as:

$$
\omega=\left(2 \cdot g \cdot r_{1} / \pi \cdot\left(r^{2}{ }_{1}-r^{2}{ }_{2}\right)\right)^{0.5}
$$

Providing that $x$ is sma!l, so that $r$ is not a function of $x$, for typical duck dimensions, equation (3.33) gives $\omega$ to be equal to $1.61 \mathrm{rad}^{-\mathrm{s}^{-1}}$.

(2) The fluid piston as a solid semi-cylinder (see figure (3.4)).

For unit length, a torque balance about $X X$ gives:

$$
1 . d^{2} \theta / d t^{2}+M \cdot d \cdot \sin \theta=A \cdot \Delta p \cdot r / 2
$$

where:

$$
I=0.5 \cdot M \cdot r^{2}
$$

and:

$$
d=(4 / 3) \cdot r / \pi
$$

For simple harmonic motion:

$$
\omega=(8 . g / 3 . r \cdot \pi)^{0.5}
$$

If $\theta$ is small, so that $\sin \theta \simeq \theta$. For typical dimensions, equation (3.37) gives $\omega$ to be equal to $1.67 \mathrm{rad} . \mathrm{s}^{-1}$.

The difference in the natural frequencies of oscillation, $\omega$, as predicted by the two methods is only of the order of $4 \%$ of the lower value. Intuitively, it 
would seem likely that the real behaviour of the fluid piston could be somewhere between the two extreme cases studied; the fact the two approaches give such a small variance was therefore encouraging. The second method was implemented in the model, as this held for all $\omega$, whereas equation (3.32) was only true if $x$ was small.

\section{Physical Property Prediction}

1. Liquid density: the brine density prediction function used data from Isdale and Morris (Isdale and Morris, 1969, [30]) in the form of a look-up table with linear interpolation for intermediate values of temperature and/or salinity. For pure water, a simple second order polynomial in temperature was fitted to the data of Haywood (Haywood et al., 1972, [24]) and Perry (Perry et al., 1984, [49]). For brines, temperatures in the range $20-180^{\circ} \mathrm{C}$ and salinities in the range $10-150 \mathrm{~g} \cdot \mathrm{kg}^{-1}$ could be accommodated. The data for the pure water correlation was in the range $10-120^{\circ} \mathrm{C}$.

2. Liquid dynamic viscosity: the viscosity prediction routine used a correlation from Isdale et al. (Isdale et al., 1972, [31]) of the form:

$$
\begin{aligned}
& a=a_{1}+a_{2} \cdot s+a_{3} s^{2}+a_{4} \cdot s^{3} \\
& b=(T-20) /(T+109) \\
& c=b_{1} \cdot\left(1+b_{2} \cdot s+b_{3} \cdot s^{2}\right)+c_{1} \cdot(T-20) \cdot\left(1+c_{2} \cdot s+c_{3} \cdot s^{2}\right) \\
& d=b \cdot c \\
& \mu=0.001 \cdot a / 10^{d}
\end{aligned}
$$

The limits of applicability were given in [31] as: temperature $20-180^{\circ} \mathrm{C}$, salinity $0-150 \mathrm{~g} \cdot \mathrm{kg}^{-1}$.

3. Liquid specific heat capacity: the heat capacity function used a correlation from Isdale and Morris (ibid). The correlation was in the form of a cubic polynomial, with 
salinity dependent coefficients, that is:

$$
\begin{aligned}
& c_{p}=a+b \cdot T+c \cdot T^{2}+d \cdot T^{3} \\
& a=a_{1}+a_{2} \cdot s+a_{3} \cdot s^{2}+a_{4} \cdot s^{3} \\
& b=b_{1}+b_{2} \cdot s+b_{3} \cdot s^{2} \\
& c=c_{1}+c_{2} \cdot s+c_{3} \cdot s^{2} \\
& d=d_{1}+d_{2} \cdot s+d_{3} \cdot s^{2}
\end{aligned}
$$

The limits of applicability were given in [30] as: temperature $0-180^{\circ} \mathrm{C}$, salinity $0-180 \mathrm{~g} \cdot \mathrm{kg}^{-1}$.

4. Liquid thermal conductivity: the liquid thermal conductivity function used a correlation from Isdale and Morris (ibid), of a somewhat complex form;

$$
\begin{aligned}
& x=k_{1} \cdot s \\
& y=k_{2} \cdot s \\
& g=k_{3}+k_{4} \cdot s \\
& p=\log (240+x) \\
& q=(2 \cdot 3-g / T) \cdot\left(1-T_{a} /\left(T_{c}+y\right)\right)^{0.33} \\
& k=0.001 \cdot e^{p+q}
\end{aligned}
$$

The limits of applicability given in [30] were: temperature $20-180^{\circ} \mathrm{C}$, salinity $0-180 \mathrm{~g} \cdot \mathrm{kg}^{-1}$.

5. Boiling point elevation: the boiling point elevation (bpe) function used a correlation from Bromley et al. (Bromley et al., 1974, [5]) of the form: 


$$
\begin{aligned}
& x=s / 1000 \\
& a=k_{1} \cdot x \cdot T^{2} \\
& b=k_{2} \cdot T \\
& c=k_{3} \cdot T^{0 \cdot 5} \\
& d=k_{4} \cdot x \\
& \theta=k_{5} \cdot x \cdot T \cdot\{(T-225 \cdot 9) /(T-236)\} \\
& f=k_{6} \cdot x \cdot(x-1) / T \\
& b p e=a \cdot(1+b+c+d+e+f)
\end{aligned}
$$

The data to which this function was fitted lay in the range: temperature $0-200^{\circ} \mathrm{C}$, salinity $20-120 \mathrm{~g} \cdot \mathrm{kg}^{-1}$.

6. Vapour isobaric heat capacity: as the assumption was made that the vapour would behave as a perfect gas, then it followed that the isobaric heat capacity of the vapour would only be a function of temperature and not pressure. A four term polynomial was fitted to the saturated water vapour data given in Haywood et al. (ibid) and Perry et al. (ibid). The data to which the correlation was fitted, by a method of least squares, fell in the range $80-150^{\circ} \mathrm{C}$. The maximum error of the correlation over this range was of the order of $0.5 \%$.

7. Vapour isochoric heat capacity: as the vapour was assumed to behave as a perfect gas, the isochoric heat capacity could be calculated simply from;

$$
C_{v}=C_{p}-R
$$

where $C_{p}$ was found from the correlation above.

8. Adiabatic exponent: this function calculated the exponent, $\gamma$, in the equation for adiabatic compression of a perfect gas (equation (3.4)). As it was assumed that the 
compression processes were all adiabatic and the gas perfect, then;

$$
\gamma=C_{p} / C_{v}
$$

$C_{p}$ and $C_{v}$ were found using the methods described above.

9. Saturation temperature at a given pressure: for pure water this function used a standard Antoine relationship rearranged to give temperature as the dependent variable:

$$
T=C-(B /(A-\log p))
$$

The coefficients $A, B$ and $C$ were given by Ohe, (Ohe, 1972, [45]). For brines, the situation was rather more complex due to the presence of the boiling point elevation (bpe). As the bpe is a function of both temperature and salinity, it was necessary to solve two equations simultaneously, that is:

$$
\begin{aligned}
& T=C+b p e-(B /(A-\log p)) \\
& b p e=f(T, s)
\end{aligned}
$$

Equations (3.65) and (3.66) were solved simultaneously using a numerical Davidenko method. The limits of applicability for the Antoine coefficients were given in [45] as: $-17-139^{\circ} \mathrm{C}$.

10. Vapour pressure: the vapour pressure of a brine at a given temperature and salinity was calculated from a modified Antoine equation, thus:

$$
\log p=A-B /(C+T-b p e)
$$

The constants $A, B$ and $C$ and the limits of the function were the same as those given above.

11. Latent heat of vapourisation: the function to calculate the latent heat of vapourisation used the thermodynamically 
exect Clapeyron equation:

$$
d p / d T_{\text {sat }}=\lambda /(T \cdot \Delta v)
$$

where:

$$
\Delta v=v_{1}-v_{g}
$$

at saturation. From the Antoine equation, equation (3.67), it may be shown that:

$$
d p / d T_{\text {sat }}=-p \cdot B /(C-b p e+T)^{2}
$$

$v_{1}$ was found from the density correlation, above; $v_{g}$ was found from the ideal gas equation, equation (3.1); $p_{\text {sat }}$ was found using the vapour pressure function above.

12. Saturated vapour temperature at a given specific vapour volume: this function, which was essentially a restatement of (3.67), above, was for use in applications where it was easier to specify the specific vapour volume than the pressure. Rather than solving the gas equation and the relevant Antoine equation simultaneously, it was decided to fit a function to the saturated specific vapour vs. temperature data given by Perry et al. (ibid). The equation was of the form:

$$
T_{\text {sat }}=A-B \cdot \log v_{\text {sat }}
$$

for brines the function was corrected such that:

$$
T_{\text {sat }} \cdot=T_{\text {sat }}+\text { bpe }
$$

\section{Equations for the Heat and Mass Transfer Processes}

As with the first model, the rate of change of pressure with time was considered as being made up of two terms: change of volume at constant mass and change of mass at constant volume. Both of these changes represented, of course, changes in the specific volume of the vapour 
concerned. Also included in this model, however, was a third step in which the liquid and vapour phases were allowed to come to equilibrium instantaneously in a closed space. This meant that the vapour and liquid would always be at, or very close to, their equilibrium conditions during the mass transfer stage; which in turn meant that the adiabatic assumption would be more realistic. Each time step, could therefore, be divided into three, conceptual stages:

- Equilibration at constant mass and internal energy

- Adiabatic volume change at constant mass

- Adiabatic mass change at constant volume

The equations for the instantaneous equilibration step were derived as follows. The equilibration process was to take place inside an adiabatic enclosure of constant volume, with no mass transfer across it boundaries. Therefore:

$$
\Delta \mathbf{U}=\mathbf{0}
$$

and also;

$$
\delta n_{v}=-\delta n_{1}
$$

At the end of the equilibration process, both phases had to be be at the same temperature, the saturation temperature, $T_{s}$. Carrying out an energy balance on the enclosure gives, therefore:

$$
\begin{aligned}
& U_{1}=C_{v} \cdot n_{v 1} \cdot\left(T_{v 1}-T_{r}\right)+C_{1} \cdot n_{11} \cdot\left(T_{11}-T_{r}\right)+\lambda \cdot n_{v 1}+\Sigma \\
& U_{2}=C_{v} \cdot n_{v 2} \cdot\left(T_{s}-T_{s}\right)+C_{1} \cdot n_{12} \cdot\left(T_{s}-T_{r}\right)+\lambda \cdot n_{v 2}+\Sigma
\end{aligned}
$$

Where $\Sigma$ is the sum of the changes in internal energy associated with phase changes up to temperature $T_{s}$, excepting the vapour-liquid transformation. From equations (3.73) and (3.74), assuming $C_{v}, C_{1}$ and $\lambda$ are constant over the range of interest, and setting $T_{r}$ to be $O K$, it follows that: 


$$
\delta n_{v}=\left\{C_{v} \cdot n_{v 1} \cdot\left(T_{s}-T_{v 1}\right)+C_{1} \cdot n_{11} \cdot\left(T_{s}-T_{11}\right)\right\} /\left(\lambda-T_{s} \cdot\left(C_{1}-C_{v}\right)\right)
$$

Note that, since the differences between $T_{s}$ and the starting temperatures are always likely to be small (if the time step size is small), the errors in approximating $C_{v}, C_{l}$ and $\lambda$ as constants are also small. Equation (3.77) must be solved simultaneously with the saturation vapour temperature from equation (3.64). The equilibration process takes place only in the condensing and evaporation spaces of the main heat exchanger, which have a fixed, specified volume. This gives a system of four equations in four unknowns, for the condensation and the evaporation spaces, to be solved at each time step.

For the purposes of the derivation of the equations to describe the other two steps in the process (see above), the duck was divided into four chambers (see figure (3.5)); A, B, C and D from left to right. Unlike the first model, where the valves were assumed to operate with no pressure drops across them, or back leakage, an attempt was made in this version to simulate the behaviour of real valves. As there were four chambers, there were four possible vapour flows and, although the valves were conceptually uni-directional, some back flow could be reasonably expected. The four flows were thus:

$-m_{1}$ : flow between $A$ and $B$

$-m_{2}$ : flow between $D$ and $B$

$-m_{3}$ : flow between $C$ and $A$

$-m_{4}$ : flow between $C$ and $D$

Starting with the equation for adiabatic compression of an ideal gas, it was possible to derive the following general equation to describe the process taking place in any of the four chambers:

$$
d T / d t=(\gamma-1) \cdot T \cdot\{(1 / n) \cdot d n / d t-(1 N) \cdot d V / d t\}
$$

Added to this was a non-adiabatic mixing term, to take account of the fact that vapour flowing into a chamber may not be at the same temperature as that already there: 


$$
d T_{\text {mix }} / d t=\Sigma\left(m_{x y} / n_{y}\right) \cdot\left(T_{x}-T_{y}\right)
$$

So for the four chambers of the duck it may be shown that:

$$
\begin{aligned}
& d T_{A} / d t=\left(\gamma_{A}-1\right) \cdot T_{A} \cdot\left\{\left(1 / n_{A}\right) \cdot d n_{A} / d t-\left(1 / N_{A}\right) \cdot d V_{A} / d t\right\}+\left(m_{3} / n_{A}\right) \cdot\left(T_{C}-T_{A}\right) \\
& d T_{B} / d t=\left(\gamma_{B}-1\right) \cdot T_{B} \cdot\left\{\left(1 / n_{B}\right) \cdot d n_{B} / d t\right\}+\left(m_{1} / n_{B}\right) \cdot\left(T_{A}-T_{B}\right)+\left(m_{2} / n_{B}\right) \cdot\left(T_{D}-T_{B}\right) \\
& d T_{C} / d t=\left(\gamma_{C}-1\right) \cdot T_{C} \cdot\left\{\left(1 / n_{C}\right) \cdot d n_{C} / d t\right\}
\end{aligned}
$$

Note that the mixing term for chamber $\mathrm{C}$ was ignored; any flows into chamber $C$ would be small, so that the additional accuracy gained from the inclusion of this term was more than offset by the complication added to the model.

$$
d T_{D} / d t=\left(\gamma_{D}-1\right) \cdot T_{D} \cdot\left\{\left(1 / n_{D}\right) \cdot d n_{D} / d t-\left(1 N_{D}\right) \cdot d V_{D} / d t\right\}+\left(m_{4} / n_{D}\right) \cdot\left(T_{C}-T_{D}\right)
$$

For chambers $A$ and $D$, evaluation of the $\mathrm{dn} / \mathrm{dt}$ term was a simple task:

$$
\begin{aligned}
& d n_{A} / d t=m_{3}-m_{1} \\
& d n_{D} / d t=m_{4}-m_{2}
\end{aligned}
$$

In chambers $B$ and $C$ mass transfer could take place. Therefore:

$$
\begin{aligned}
& d n_{B} / d t=m_{1}+m_{2}-\psi \\
& d n_{C} / d t=\varepsilon-m_{3}-m_{4}
\end{aligned}
$$

where $\psi$ and $\varepsilon$ were the condensation and evaporation rates respectively.

$$
\begin{aligned}
& \psi=h_{B} \cdot A \cdot\left(T_{B}-T_{w B}\right) / \lambda_{B} \\
& \varepsilon=h_{C} \cdot A \cdot\left(T_{w C}-T_{C}\right) / \lambda_{C}
\end{aligned}
$$

$T_{w C}$ and $T_{w B}$ were estimated from:

$$
\begin{aligned}
& T_{W B}=T_{f B}-U \cdot\left(T_{f B}-T_{f C}\right) / h_{B} \\
& T_{W C}=T_{f C}+U \cdot\left(T_{f B}-T_{f C}\right) / h_{C}
\end{aligned}
$$

where: 


$$
U=\left\{\left(1 / h_{B}\right)+\left(1 / h_{C}\right)+(x / k)+\sum\left(1 / f_{i}\right)\right\}^{-1}
$$

Where $f_{i}$ were the fouling factors for the main heat exchanger, defined by the user, and $T_{f B}$ and $T_{f C}$ were the average film temperatures for the condensate and brine film temperatures respectively. Heat balances around these films gave rise to two more differential equations:

$$
\begin{aligned}
& d T_{f B} / d t=\left\{\Psi \cdot \lambda_{B}-k \cdot A \cdot\left(T_{w B}-T_{w C}\right) / x\right\} /\left(C_{p B} \cdot m_{B}\right) \\
& d T_{f C} / d t=\left\{-\varepsilon \cdot \lambda_{C}-C_{p C} \cdot m_{f} \cdot\left(T_{f C}-T_{f}\right)+k \cdot A \cdot\left(T_{w B}-T_{w C}\right) / x\right\} /\left(C_{p C} \cdot m_{C}\right)
\end{aligned}
$$

The vapour flows, $m_{1}$ to $m_{4}$ were estimated from the equation for incompressible flow through an orifice:

$$
m=\operatorname{Cd} .\left\{\left(p_{1}{ }^{2}-p_{2}{ }^{2}\right) / R \cdot T\right\}^{0.5}
$$

To account for the fact that the valves were nominally uni-directional, it was necessary to define $\mathrm{Cd}$, above, as a function of the pressure drop across the valve. This was achieved in the following manner; firstly by letting:

$$
C d=\text { Cv.AO. } \sigma
$$

where $\mathrm{Cv}$ and $\mathrm{AO}_{0}$ were user defined values of the valve coefficient and the valve opening area respectively and $\sigma$ was the fractional opening of the valve in the range 0-1. It was decided to model $\sigma$ as a sigmoid curve (see figure (3.6)) using an inverse tangent function. This form of characteristic meant that some backflow was allowed and had the additional advantage of being a continuous function, which helped to reduce some of the numerical difficulties at the solution stage. The slope of the steepest part of the curve and the position of the half-way point on the $\Delta p$ axis could be chosen by the user; that is, the user could choose how "sticky" and "leaky" the valves should be. In this model, all the valves were specified as having the same operating characteristics, although it would not have been difficult to incorporate variance should this have been required. The opening fraction $\sigma$ was fitted to:

$$
\sigma=0.5 \cdot\left(1+(2 / \pi) \cdot \tan ^{-1}(a \cdot \Delta p+b)\right)
$$

The constants, $a$ and $b$, were calculated from two user specified parameters; $a$ percentage valve opening and the pressure difference across the valve required to give that opening, for example, $50 \%$ open at 0.05 bar. It was than 
specified in the model that, at the reverse of the pressure difference, the valve should open $0.1 \%$ of the selected value, to allow for leakage. This gave enough information to be able to find $a$ and $b$ and hence the shape of the curve. The disadvantage of this method was that many different pairs of percentage opening and $\Delta p$ could give the same valve characteristics without the user necessarily being aware of this. The pressures involved, $p_{A}$ to $p_{D}$, were calculated from the ideal gas law.

The heat transfer coefficients, $h_{B}$ and $h_{C}$, were calculated using the Nusselt and the Struve-Dukler correlations respectively (equations (3.23) and (3.27)) as in the first model. In this model, however, both coefficients were evaluated at each time step, as the physical properties involved in the calculations were functions of temperature and/or salinity.

The terms in $V$ and $d V / d t$ were found from the included angle between the fluid piston and the main heat exchanger compartment as in the first model (equation (3.15)). In this case, however, the function $f(\theta)$ was modified to take account of the radial asymmetry of the heat exchanger compartment. From figure (3.7) it may be seen that, as the duck rotates anti-clockwise, a portion of the still compartment becomes submerged in the fluid piston. This phenomenon must cause a change in the level of the piston and hence affect the volume of the vapour spaces as a function of $\theta$. It may be shown that:

$$
\begin{aligned}
& V_{A}=L .\left\{r^{2} / 2 \cdot(\pi / 2-\theta)+d^{2} / 4 \cdot(\tan \theta-\theta)-d . r\right\} \\
& V_{D}=L .\left\{r^{2} / 2 \cdot(\pi / 2+\theta)-d^{2} / 4 \cdot(\tan \theta+\theta)-d . r\right\}
\end{aligned}
$$

It may be confirmed that, when $\theta=0$;

$$
V_{A}=V_{D}=L \cdot\left(r^{2} \cdot \pi-d \cdot r\right) / 4
$$

Thus, different volume calculations were carried out depending upon whether $\theta$ was greater than or less than zero. Instantaneous values of the rates of change of volume with time were also required. These were calculated from the analytical derivatives of equations (3.98) and (3.99): 


$$
\begin{aligned}
& d V_{A} / d t=L \cdot d \theta / d t .\left\{d^{2} \cdot\left(\left(1 / \cos ^{2} \theta\right)-1\right) / 4-r^{2} / 2\right\} \\
& d V_{D} / d t=L . d \theta / d t .\left\{r^{2} / 2-d^{2}\left(\left(1 / \cos ^{2} \theta\right)+1\right) / 4\right\}
\end{aligned}
$$

Equations (3.80) to (3.87) together with (3.93) and (3.94) and the equation of motion of the fluid piston, gave a system of differential equations which completely described the system within the given constraints.

\section{Heat Recovery Network Equations}

A simple model of the heat recovery network (see figure (3.8)) was set up to allow the entire system, as modelled, to come to a quasi steady-state solution. It was found that simply specifying a single brine feed temperature as a constant, as in the first model, tended to cause the results of the model to show gradual heating or cooling of the system. Whilst this did not necessarily suggest any difficulties in the design of the duck as interpreted in the model, it did tend to make the results of the simulations rather more difficult to analyse.

The model of the heat recovery network was based on a steady-state assumption. This implied that the heat exchangers involved had no thermal inertia and so, for example, the instantaneous feed temperature was only a function of the instantaneous blow down and condensate temperatures. This was obviously unrealistic, but the construction of a more complete, time-dependent model would at the very least require detailed specifications of the design of the heat exchangers to be used and, in the final analysis, the model for the heat recovery system could become just as complex as that for the rest of the system. As it was, the heat recovery model consisted of heat balances around exchangers 1. and 2. (see figure (3.8)) and a mass balance over the entire process:

$$
\begin{aligned}
& m_{2}=m_{1}+m_{3} \\
& m_{2}=s \cdot m_{3}
\end{aligned}
$$

(s is the salinity ratio, blowdown concentration/feed concentration) 


$$
\begin{aligned}
& q_{1}=C_{p} \cdot m_{1} \cdot\left(T_{1}-T_{2}\right)=C_{p} \cdot m_{2} \cdot\left(T_{6}-T_{5}\right) \\
& q_{1}=U_{1} \cdot A_{1} \cdot \Delta T_{I m 1} \\
& q_{2}=C_{p} \cdot m_{1} \cdot\left(T_{2}-T_{3}\right) \\
& q_{2}=1 / 2 \cdot U_{2} \cdot A_{2} \cdot \Delta T_{1 m 2} \\
& q_{3}=C_{p} \cdot m_{3} \cdot\left(T_{7}-T_{8}\right) \\
& q_{3}=1 / 2 \cdot U_{2} \cdot A_{2} \cdot \Delta T_{1 m 3} \\
& q_{2}+q_{3}=C_{p} \cdot m_{2} \cdot\left(T_{5}-T_{4}\right)
\end{aligned}
$$

$\Delta T_{1 m 1}, \Delta T_{1 m 2}$ and $\Delta T_{1 m 3}$ are the log mean temperature differences for the relevant streams. Note that, in the derivation of the log mean temperature difference, it is assumed that the $d T / d A$ for the streams are constant. For exchanger 2., in this case, this assumption would only be accurate if the condensate and blowdown streams entered the second exchanger with the same flowrate and at the same temperature. Otherwise, it is possible for heat to be transfered, indirectly, from one of these streams to the other, thus negating the above condition. All the specific heats were assumed to be constant and equal. It was necessary to specify: the condensate and brine flowrates and temperatures; the sea temperature; the heat transfer coefficients and areas of both exchangers, and the brine blowdown concentration. The equations were then solved simultaneously at each time step to find, in particular, the feed temperature $\mathrm{T}_{4}$, and also the product and blowdown outlet temperatures, $T_{6}$ and $T_{8}$. A numerical Newton-Raphson routine, written by the author, was used in the computer program to solve the equations.

\subsubsection{Implementation of the Second Model}

The purpose of this section is to describe in brief the structure of the program containing the code for the second model. Detailed listings may be found in appendix IV; the discussion in this section will, therefore, be limited to the general considerations leading to the structure adopted.

The code for the physical property functions and some other general purpose functions, such as those calculating heat transfer coefficients and so on, was held in a file, the compiled object file of which was inserted into the author's directory in order that all these routines and functions could be called by any subsequent program. Four routines were defined in the final versions of the 
program containing the model: MATHMOD, which contained the differential equations to be solved; ALGEBRAIC, which contained the set of non-linear algebraic equations to be solved for the instantaneous equilibration step; RECOVER, which contained the set of non-linear algebraic equations describing the heat recovery network, and PHYSPROP, which called the necessary physical property routines. A single global vector of variables, constants and parameters was used in the program in order to facilitate the passing of numerical values from one routine to another. This vector contained all the physical properties, dependent variables, user-set parameters, etc.

With the the four basic routines in place, the rest of the program was fairly straightforward. Once the necessary parameters had been supplied by the user (via an external routine inserted in the directory, SETUP) and allocated vector positions, the dependent variables (temperatures in the chambers, etc.) were set to their initial values, commensurate with the duck starting from rest at saturated state at 1 bar. The main time stepping cycle then began; at each step the routines were called in order: PHYSPROP, ALGEBRAIC, RECOVER and IMPDRUNGE. IMPDRUNGE was an external routine available from library software, which solved the differential equations in MATHMOD using a fourth order Runge-Kutta method. The equations in ALGEBRAIC were solved using the iterative Davidenko method, also available as an external routine from software. The linear equations in RECOVER were solved using a numerical Newton-Raphson technique, written by the author for the first model and held in the routine NDNEWTON. Results from the solution of the system were printed to a file at every fifth time step, immediately after ALGEBRAIC had been called, so that the results always represented equilibrium states. After the differential equations were solved, the results, stored in a vector, were passed to the appropriate slots in the global vector for use as the initial conditions for the next time step. When the time cycle was completed the program terminated with a "Finished" method to the user.

It may be clearly seen that this program had the capability to generate an immense amount of numerical data. For example, a typical wave cycle might be around 7 seconds long and it was usual to simulate 4 or 5 wave cycles in order to let the data "settle down" to a quasi steady-state. With a time step of 0.02 seconds (the largest possible to give reliable results), this implies $\mathbf{3 5 0}$ sets of numbers would be sent to the output file. There were fourteen 
dependent variables, so this meant that a total of 4,900 values would need to be stored. Obviously, this would be too much data to be manually analysed with ease and so a second suite of programs was developed with the purpose of manipulating the output data into a more comprehensible form. By having a number of different processing programs and a single simulation program producing "raw" data it was possible to maximise the amount of information available from each single run of the main program, thus cutting down on the amount of processor time used.

\subsection{SIMULATION OF EXPERIMENTAL APPARATUS}

The experimental equipment constructed for this project are described in detail in the next chapter; it was, however, necessary to use modified versions of the existing simulation programs, detailed in sections 3.2 and 3.3 , above, to aid in the design of the apparatus and in the analysis of the results produced. The precise uses to which the modified programs were put will be explained in chapters 4 and 6 ; the purpose of this section is to describe the construction of these programs with reference to the full-scale duck simulations developed.

\subsubsection{The First Model as a Design Tool}

Almost as soon as the detailed design of the apparatus had commenced, the need for a computer based design aid became apparent. The purpose of the equipment was, broadly, the same as that of the simulation models; ie. to simulate the performance of a full-scale duck as accurately as possible. Because of this and because of the complex, unsteady-state nature of the process, it was felt that the best means of proceeding with the design would be to modify the existing computer model in order that it could provide a description of the operation of the proposed design and then to change the relevant design parameters until the data generated by the duck model and the rig model matched each other as closely as possible. The advantages and disadvantages of this approach will be discussed in the following chapter.

The modifications made to the first model were quite simple: the values of the areas and volumes, that is, of the physical dimensions of the apparatus, were substituted in the model in place of the duck dimensions and the physical properties of water and brine were replaced with those of refrigerant R11, the proposed working fluid for the rig. An initial estimate of the scale of the rig 
was obtained from the following analysis. Starting from equation (3.1), and differentiating with respect to time, gives:

$$
d p / d t=R \cdot T N \cdot d n / d t+n \cdot V N \cdot d T / d t-n \cdot R \cdot T N^{2} \cdot d V / d t
$$

The most significant is the $\mathrm{dn} / \mathrm{dt}$ term, so:

$$
(d n / d t)_{\text {rig }}=(d n / d t)_{\text {duck }}
$$

was taken as the principle scaling guideline. Now, using mean values, $\mathrm{dn} / \mathrm{dt}$ can be estimated by:

$$
d n / d t=h \cdot A \cdot \Delta T / \lambda
$$

for both duck and rig. Thus, it may be shown that:

$$
V_{D} N_{R}=\left(T_{D} / T_{R}\right) \cdot\left(h_{D} / h_{R}\right) \cdot\left(A_{D} / A_{R}\right) \cdot\left(\lambda_{R} / \lambda_{D}\right)
$$

where $R$ and $D$ refer to rig and duck respectively. Taking mean values for the terms on the right hand side of equation (3.115), $V_{D} N_{R}$ was estimated to be $24 \times 10^{3}$. This factor was used, therefore, in initial etimates for the sizing of the rig. Other than these details, the structure of the program remained virtually unaltered.

\subsubsection{Modifications to the Second Model}

As described above, the apparatus was designed using a version of the first model. It was expected, at the time, that the results from the physical simulation (the apparatus) and from the mathematical simulation (the modified first model) could be compared and, therefore, provide a quantitative analysis of the accuracy of the approach of the first model. As the second model was developed, however, (while the apparatus was under construction) and began to produce data which differed significantly from that of the first, it became clear that it would be necessary to compare instead the data from the apparatus with data produced by a model of the equipment constructed on the same principles as the second model of the duck (which was felt to be more rigorously derived and thus, hopefully, more accurate than the first). The program containing the second model was, therefore, adapted in much the same manner as that containing the first: rig dimensions replaced those of the duck and the properties of seawater were replaced by those of R11. Other 
differences included: the model of the valve operation was changed from a continuous function, equation (3.97), to an on/off function incorporating variable hysteresis to mimic the action of the solenoid valves installed on the rig; most of the physical properties of R11 were expressed as constants (due to lack of time and the data to fit correlations), with the notable exception of the latent heat of evaporation which was calculated using the Clapeyron relationship as before, equation (3.68), and the fluid piston equations of motion and the heat recovery network equations were removed from the model (a constant feed temperature was specified instead). Apart from these changes and some changes to the data input specifications and the cylinder volume calculations, the program remained as above. 

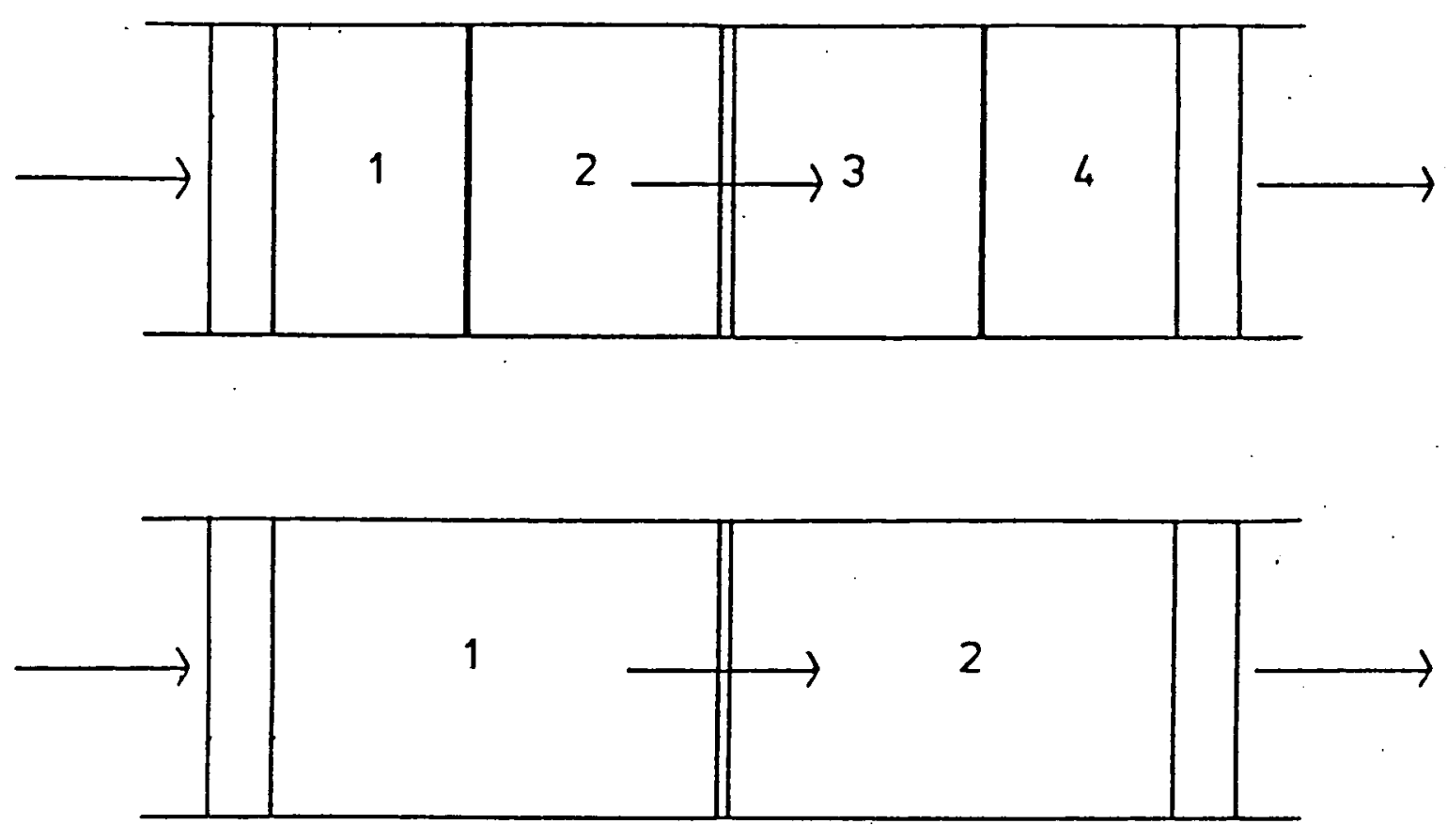

Figure (3.1) 2 Chamber/4 Chamber Basis for the First Model

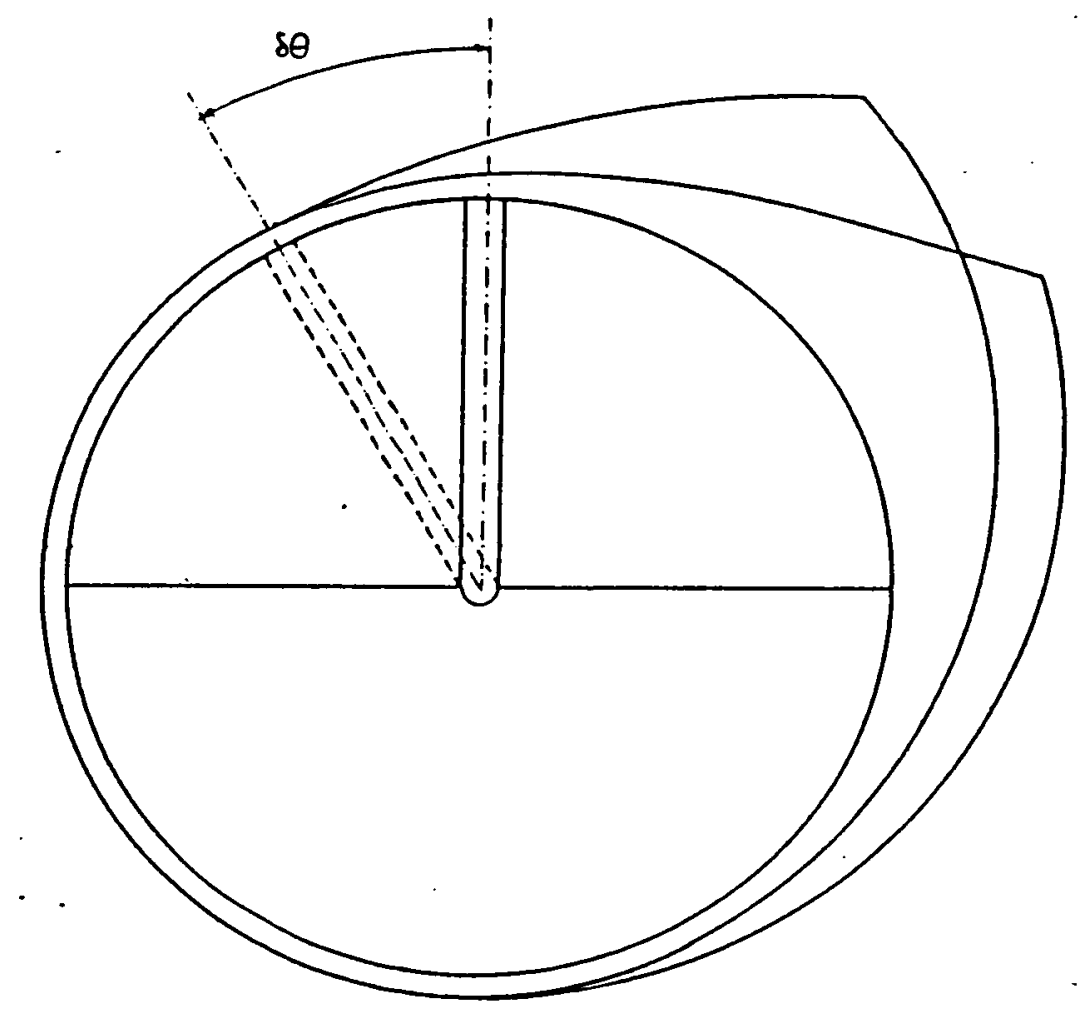

Figure (3.2) Definition of dV for the First Model 


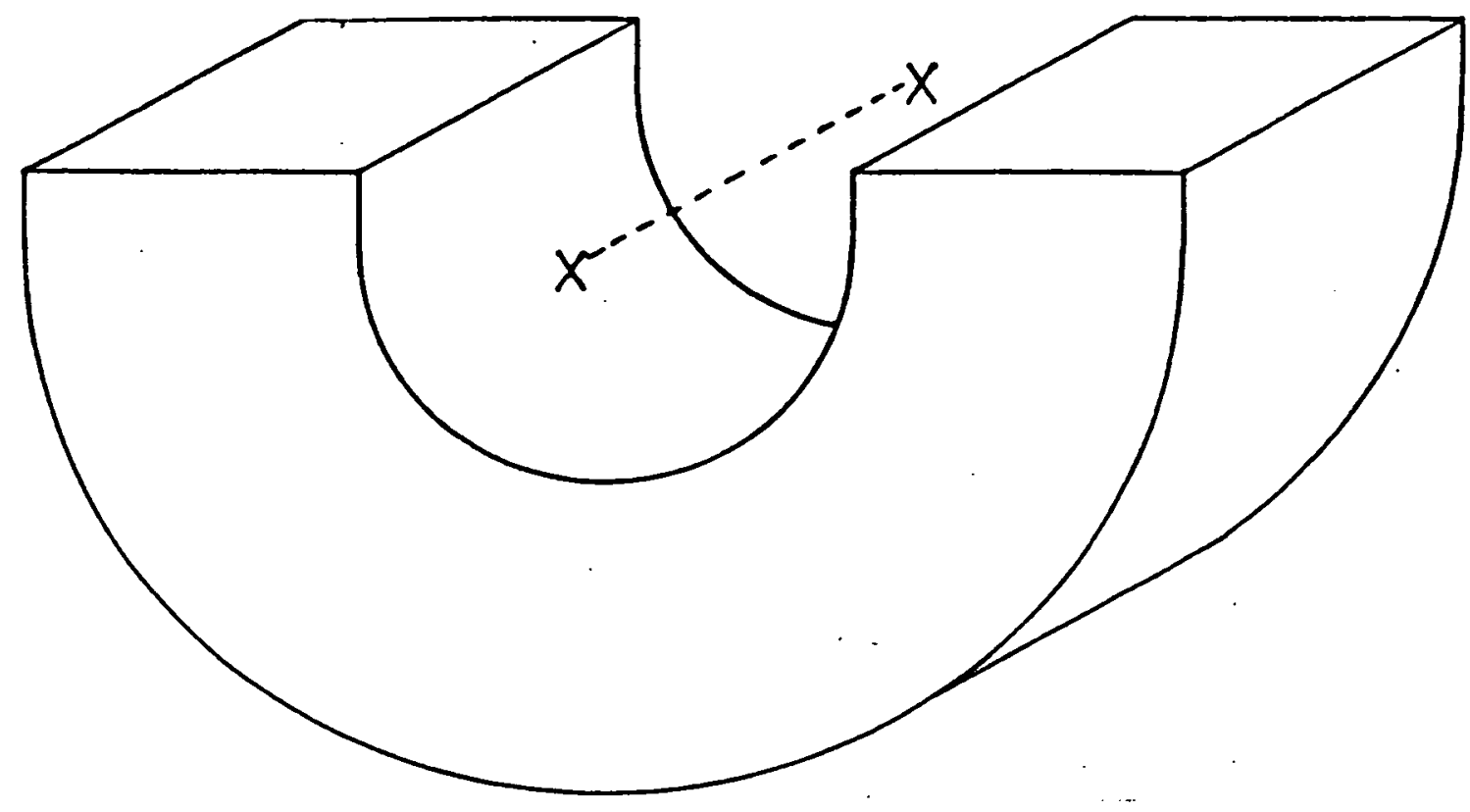

Figure (3.3) Fluid Piston as a U-Tube

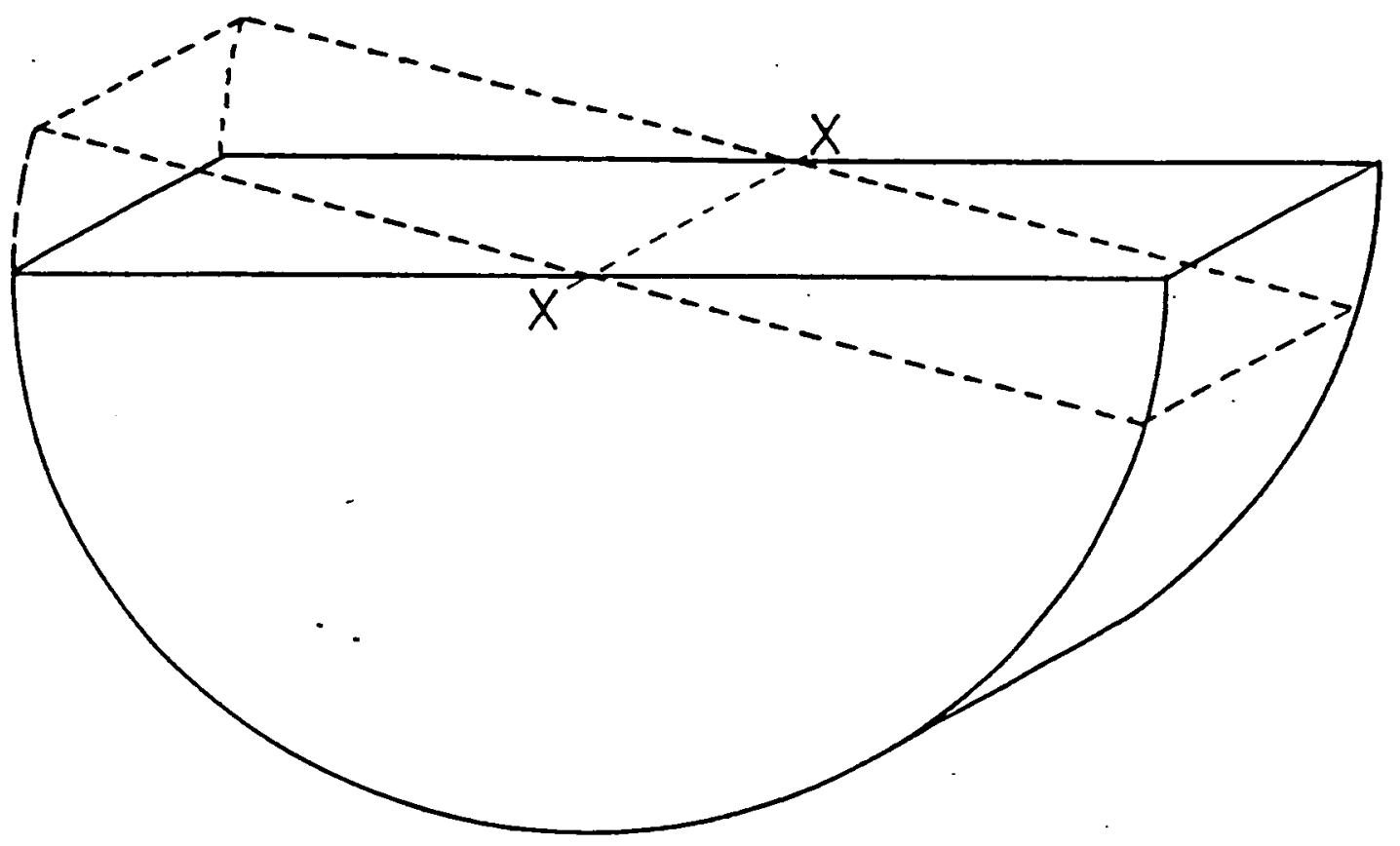

Figure (3.4) Fluid Piston as a Solid Semi-Cylinder 


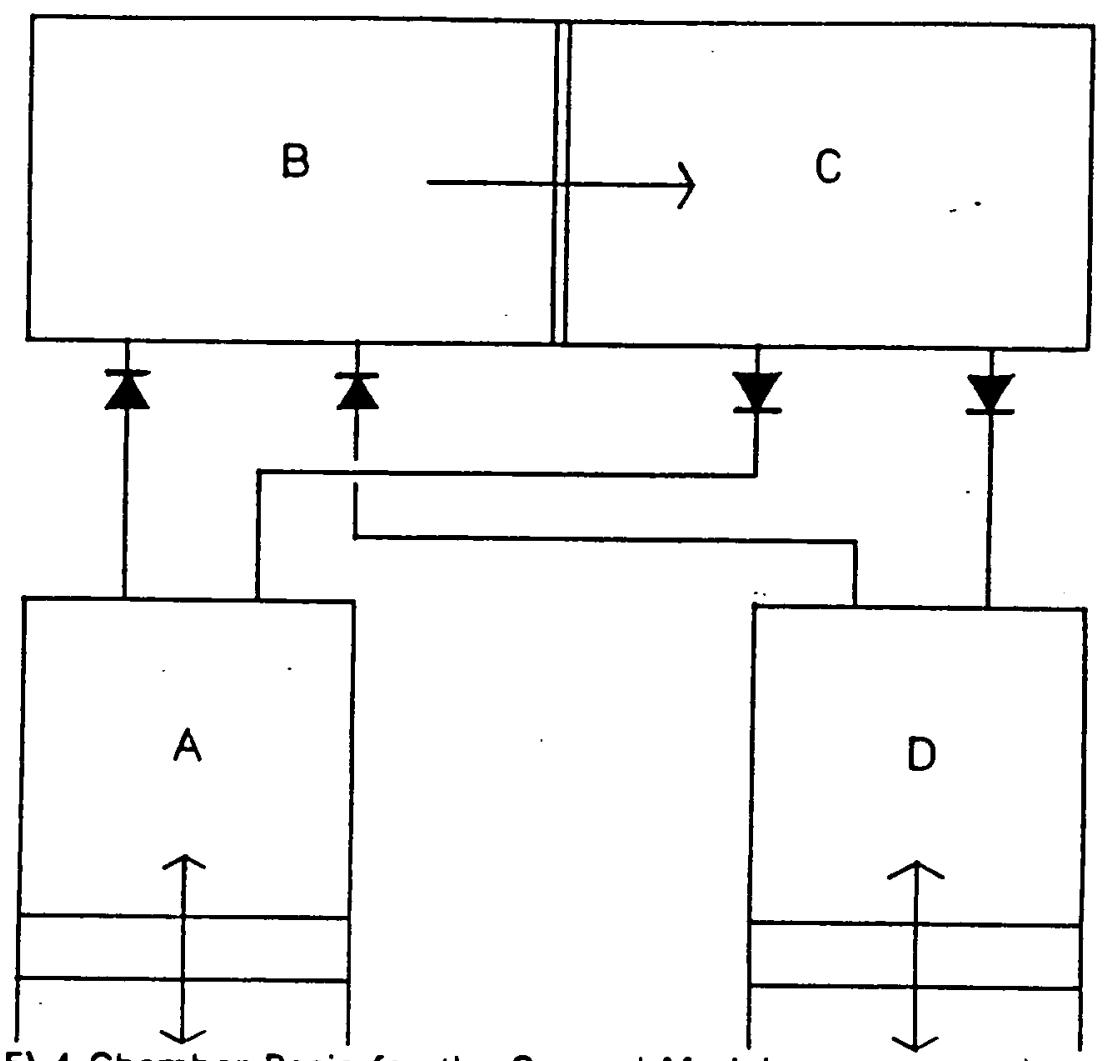

Figure (3.5) 4 Chamber Basis for the Second Model

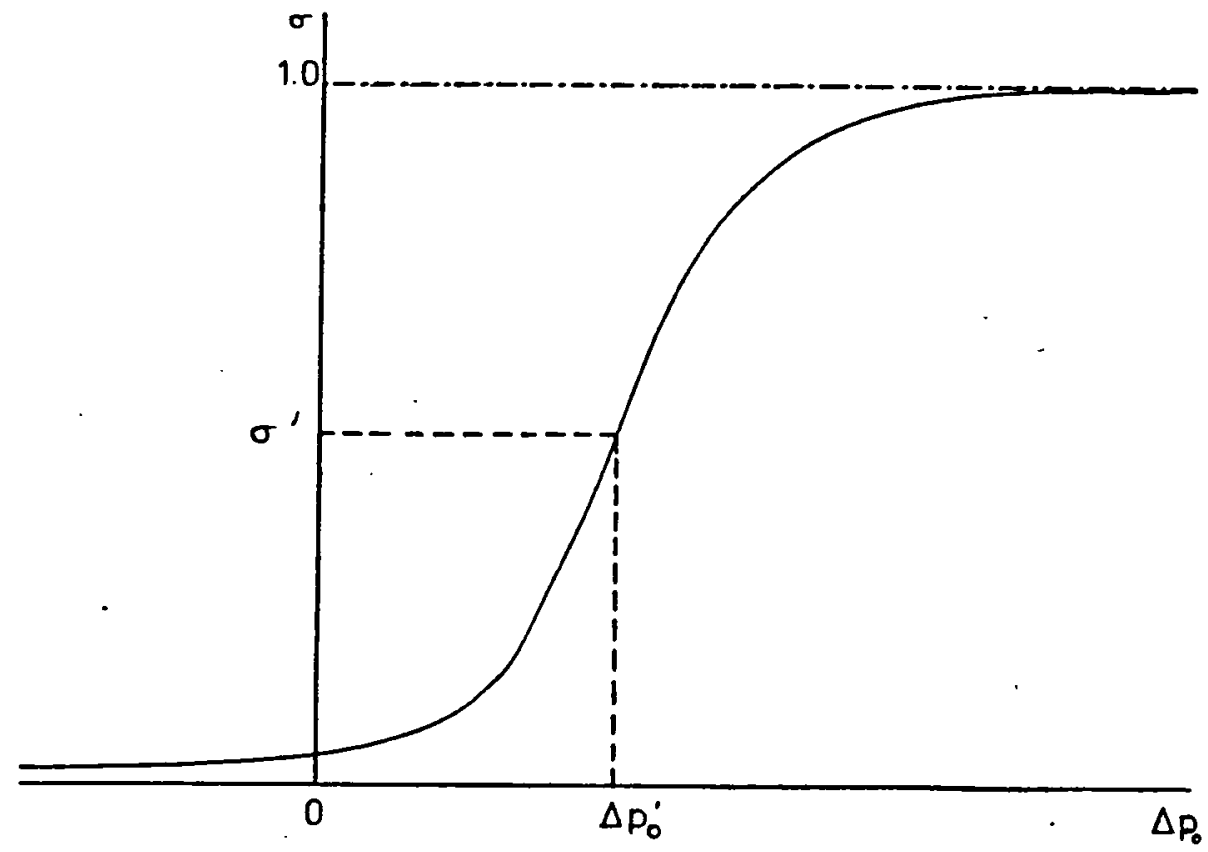

Figure (3.6) Fractional Opening vs. $\Delta p$ for Non-Return Valves 


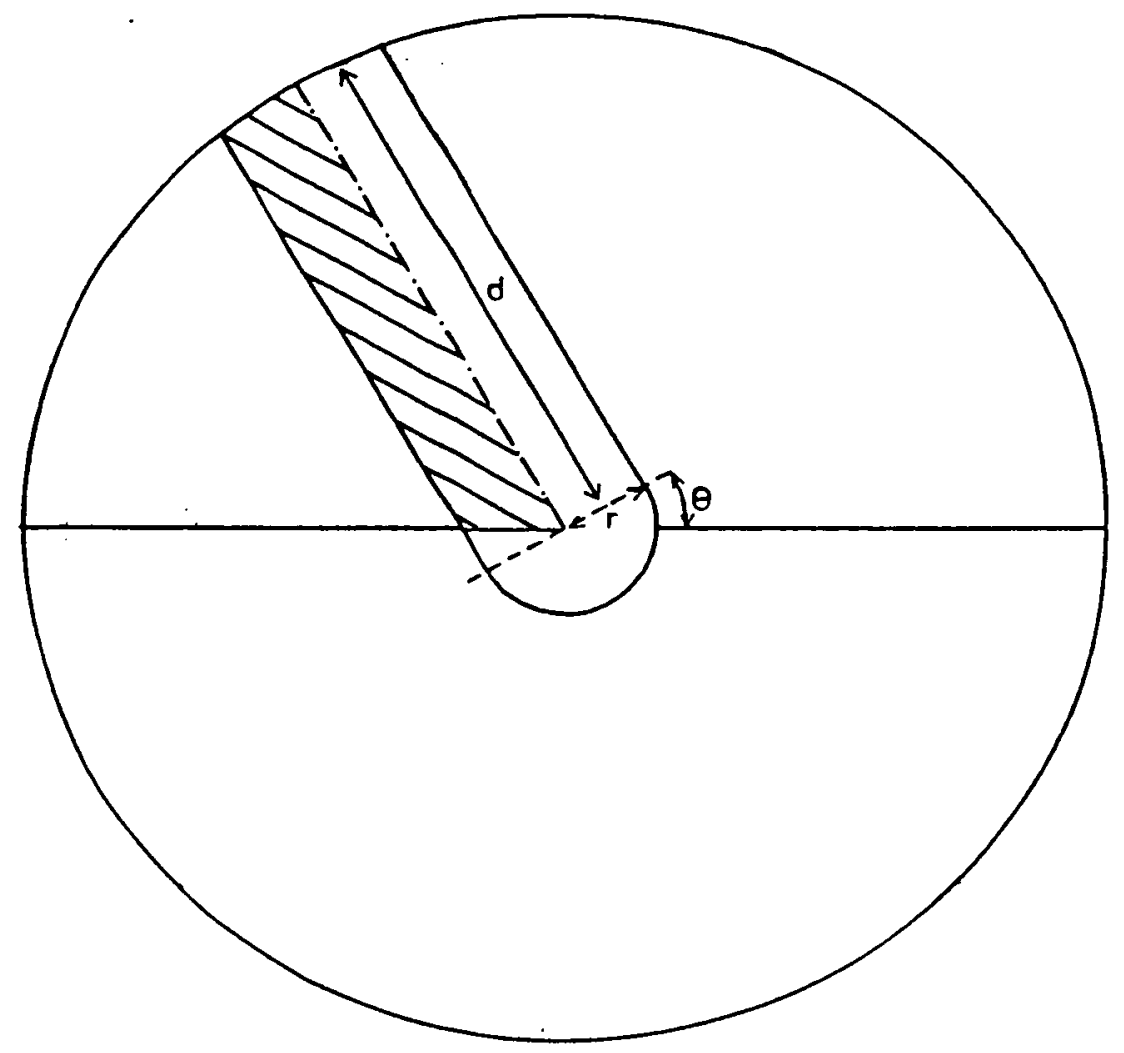

Figure (3.7) Definition of dV for the Second Model

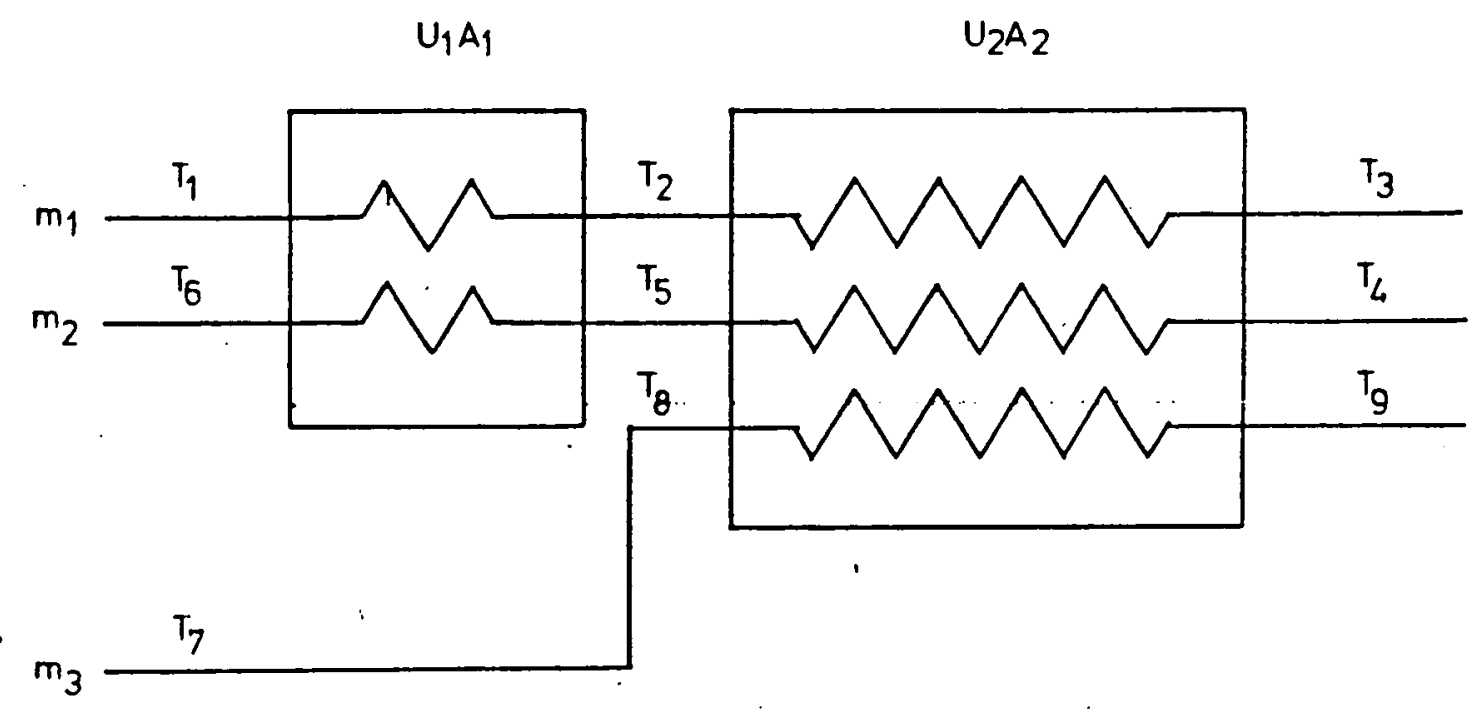

Figure (3.8) Heat Recovery Exchanger Model 
CHAPTER 4

DESIGN AND DEVELOPMENT OF THE EXPERIMENTAL FACILTY 


\subsection{INTRODUCTION}

The mathematical modelling and computer simulation work described in chapter 3 produced data which suggested that the heat and mass transfer processes taking place in the desalination cycle exhibited significant time dependencies. This conclusion had a profound effect on the possibilities for experimental work, for, as was discussed in chapter 2, the scaling laws which are applicable for such systems mean that small scale models of ducks can only yield useful results if the forces applied to them are linear functions of their angle of rotation, angular velocity and angular acceleration only (ie the spring, damping and inertial forces). The time dependency of the desalination cycle meant that the pressure force across the duck could not be modelled in such a manner and that, therefore, two different research strategies were available. It would have been possible either to study the parameters of duck design using small-scale models with a time-independent approximation to the pressure force; or to study the desalination process in detail using a specified wave shape in place of the real wave-duck interaction.

As the optimal design of ducks had already been studied extensively with reference to power generating devices, it was decided to concentrate on the latter option.

\subsubsection{The Experimental Approach}

Removing the "wave element" from any pilot plant apparatus immediately removed many of the design problems associated with the project. Essentially the design brief was reduced to producing a simple evaporator/condenser unit linked to a low frequency reciprocating compressor of suitable scale. The problem remained, however, of finding a means of taking into account the motion of the fluid piston in a physical piece of apparatus. The motion of the fluid piston is frequency dependent and so, for example, its resonant frequency would increase as the inverse square root of its mass, making a direct scaling-down procedure impossible. It was not until it was realised that the motion of the fluid piston, under the influence of gravity and the pressure force across it, could be modelled approximately by a simple, second-order differential equation of the form: 
that realistic designs could be studied.

The importance of equation (4.1) was that it implied that the fluid piston could be modelled using apparatus bearing no physical resemblance to the duck whatsoever, with the only constraint being that the ratios $1 / k, D / k$ and $S / k$ should remain the same for the rig and the real system. Equation (4.1) may be further simplified by neglecting the damping term, as this force would be generated by fluid friction at the walls of the duck and thus would be small in comparison to the inertia and spring forces.

Early conceptual designs for the compressor tended to be of the type shown in figure (4.1). A solid piston of known mass is anchored to a reference point by a leaf spring of known compliance, while the cylinders move around it driven in a sinusoidal motion. The cylinder assembly would be a direct analogue of the duck body and the piston of the fluid piston. While such designs were a significant step away from the use of a real fluid piston, they would, however, have been difficult or impossible to realise in practical terms. Instead of attempting to simulate the fluid piston by purely mechanical means, it was decided to use real-time feedback control to provide the necessary compliance of the pistons electronically. This approach utilised techniques, familiar to researchers in the Wave Power Unit, entailing the measurement of the acceleration, velocity and position of the pistons of the compressor, and of the pressure force across it. Equation (4.1) would then be solved electronically to yield a resultant drive signal. Equation (4.1) may be rearranged to give, with the damping force assumed to be negligible:

$$
\theta=\left(k \cdot F_{\Delta p}-1 . d^{2} \theta / d t^{2}\right) / S
$$

One may then define a variable, $y^{\prime}$, such that:

$$
y^{\prime}=\tau-\theta
$$

where: 


$$
\tau=A \cdot \sin (\alpha t)
$$

represents the motion of the waves. The acceleration term in equation (4.2) may be calculated from:

$$
d^{2} \theta / d t^{2}=d^{2}\{\tau-y\} / d t^{2}
$$

so that $d^{2} y / d t^{2}$ is the measured acceleration of the piston, and $d^{2} \tau / d t^{2}$ is the wave signal electronically differentiated twice with respect to time. $y^{\prime}$ represents a demand signal for a feedback control system producing an output of the form:

$$
E=G \cdot\left(y^{\prime}-y\right)
$$

where $y$ would be the measured position of the piston at any instant. The output signal, $E$, could then be used to drive the system as shown in figure (4.2). Such a design would be far more complicated, in terms of the necessary electronics, than that shown in figure (4.1), but would be much simpler mechanically.

\subsubsection{Use of an Analogue Working Fluid}

Seawater is a notoriously difficult substance to use in experimental equipment: it is aggressively corrosive, its properties change with time and with repeated usage (necessitating large storage volumes for closed cycle systems) and its comparatively high atmospheric boiling point can make heat losses a considerable problem for small-scale apparatus. The decision was taken, therefore, at an early stage in the design process to use trichlorofluoromethane, the refrigerant R11, as an analogue working fluid. R11 is essentially non-corrosive and non-toxic and has an atmospheric boiling point of $23.7^{\circ} \mathrm{C}$. However, R11, of course, differs completely from seawater in its thermodynamic and transport properties and so the main components of the apparatus, the still and the compressor, could not be directly scaled down from the real duck dimensions.

In order to be able to assess properly the effects of changing the working fluid on the main design parameters, the existing computer model (see chapter 3 section 3.4) was modified to simulate the performance of the experimental facility for different designs. The results of these simulations were than 
compared with the results of simulations for a full-scale duck, particularily with regard to the $\Delta p$ vs. $\theta$ characteristics, and the proposed design of the rig adjusted incrementally until a good match was found. The disadvantage immediately apparent in this method, was that all the errors in the computer model were then built into the experimental apparatus. If the rig should behave as predicted by the model, however, then this would mean that the model was accurate for one physical system, the rig, and by inference, another, the real duck.

\subsection{DETAILED DESIGN OF COMPONENTS}

\subsubsection{Design of Condenser/Evaporator Unit}

The specifications for the design of the condenser/evaporator unit were simple: the still was to operate in the falling film mode, so provision had to be made for the even distribution of a liquid film over the evaporation surface; the whole unit had to be capable of being sealed against the ingress of non condensable gases; there had to be some means of manifolding for the inlet and extraction of the high and low pressure vapour streams respectively. From this basis, and using the computer model (above), the following design was derived. The heat transfer surface consisted of a $2 \mathrm{~m}$ length of 2 " $(51 \mathrm{~mm})$ internal diameter stainless steel tubing with a wall thickness of $1 \mathrm{~mm}$. The tube was enclosed in a column made up of four sections of $3^{\prime \prime}$ (76mm) internal diameter QVF glassware, one of which had a $90^{\circ}$ branch of $1.5^{\prime \prime}(38 \mathrm{~mm})$ internal diameter for a vapour inlet/outlet port. It was decided to operate the unit with evaporation from the outside of the stainless steel tube in order to facilitate visual observation of the liquid film (to check for maldistribution for example) and as the design of the liquid film distributor was simplified by operation in this mode. The stainless steel tube projected through flanges at the top and bottom of the QVF column made of $3 / 8^{\prime \prime}(10 \mathrm{~mm})$ aluminium plate. The bottom of the tube was sealed with a uPVC flange (forming the condensate pot) and the top was connected to the outlet line from the compressor (see figure (4.3)).

The unit was designed as a closed system, ie condensed liquid and unevaporated feed liquid were withdrawn from the bottom flanges of the stainless steel tube and the QVF section respectively using two peristaltic 
pumps; mixed, and returned to the top of the column as the feed stream. Peristaltic pumps were chosen for the duty as they were the least expensive type of reciprocating pump available; centrifugal pumps would have been unsuitable as the liquids to be transported would almost always be at or near their saturation point. The pumps were powered by two DC motors chosen in order to be able to effect control of the liquid flow rate (see section 4.3.3).

Some concern was felt during the initial stages of the design process that the vapour volumes inside the steel tube, and between the tube and the QVF, were too large relative to the heat exchange area when compared to the volume/area ratios envisaged for the real duck. It would have been difficult to achieve the low ratios required for duck/rig correspondence in practice, and so the computer model (above) was used to evaluate the effects of the disproportion for practical designs. The result of this enquiry was that these ratios seemed to have little effect on the performance of the system and, therefore, that any practical design could be adopted.

\section{Design of the Liquid Distributor}

The design of the distributor was based on that used by Struve, 1965, [69]. The underlying principle of the design was to ensure an even distribution of the liquid film by forcing the feed through a concentric annular gap between the evaporation surface and a collar or ring fitted over it. In this case, it was decided that it would be advantageous to be able to adjust the size of this gap, in order to vary the pressure drop across the distributor in such a manner as to operate the unit with the space above the collar full of liquid at all times. It was felt that this would help to minimise maldistribution of the refrigerant film.

The distributor was, therefore, made in two parts, shown on figure (4.4)a. and b. Figure (4.4)a. shows the collar piece turned from a block of aluminium with eight $3 / 8^{\prime \prime}(16 \mathrm{~mm})$ holes drilled from the top to the bottom of the piece at an angle of $10^{\circ}$ to the vertical to allow liquid to reach the annulus. The vertical sections left between these passages come into contact with the parallel sided section of the cone piece, shown in figure (4.4)b., and ensure that the gap formed between the two is concentric. The cone piece was also turned from a single piece of aluminium and was designed to be a push fit into the top of the steel tube with a minimum of disturbance of the refrigerant flow at the 
join. The section was hollowed out to an internal diameter of 1 " $(25 \mathrm{~mm})$ to form the vapour inlet to the condensing space. By moving the collar piece up or down the cone section using the screwed rods as shown in figure (4.5), it was possible to vary the width of the gap from $0-1 \mathrm{~mm}$.

\subsubsection{Design of Liquid Cooler and Trim Heater}

As work was done on the system by the compressor, it was therefore necessary to remove an equivalent amount of heat at some point in the cycle. It was decided to remove the necessary heat by cooling down the evaporate return stream by passing it through some sort of heat exchanger against cold water. Control of the feed temperature was required and, as it is difficult to control the temperature of the liquid leaving a heat exchanger on such a small scale, an alternative approach was adopted whereby the heat exchanger was deliberately over-sized such that it would sub-cool the liquid more than was necessary for the heat balance. The liquid leaving the exchanger would then be mixed with the condensate stream and then re-heated in a controlled manner using an electrical heating element to the desired feed temperature.

\section{Design of the Cooler}

It was known from the computer simulation of the rig that the mean power rating of the compressor would be approximately 90-100W. This figure, then, gave a lower bound on the duty of the cooler unit. In order to over-size the unit for the purposes of control it was decided to base the design of the cooler on a mean duty of $150 \mathrm{~W}$. Several different heat exchanger types were studied (double pipe, U-tube etc) but it appeared that the simplest and cheapest to have made would be a copper coil held in a tank of iced water agitated by an impeller and calculations are presented on this basis.

The mean flowrate of refrigerant through the coil was taken to be $0.61 / \mathrm{min}$, that is, roughly equivalent to a film mass flowrate of $0.15 \mathrm{kgs}^{-1} \mathrm{~m}^{-1}$. It was assumed that the coil would be made of $1 / 8^{\prime \prime}(3.2 \mathrm{~mm})$ copper pipe as this was the piping material used throughout the rig. This gives:

$$
\operatorname{Re}=p . u \cdot d / \mu=23490
$$

The Seider-Tate relationship, with the coil correction factor for a $4^{\prime \prime}(102 \mathrm{~mm})$ coil, was used to find the heat transfer coefficient inside the coil: 


$$
N u=0.027 \cdot \operatorname{Re}^{0.8} \cdot \operatorname{Pr}^{0.33} \cdot\left(\mu / \mu_{w}\right)^{0.14} \cdot\left(1+3 \cdot 5 \cdot D_{i} / D_{c}\right)
$$

Whch gave $h_{c}=4,700 \mathrm{Wm}^{-2} \mathrm{~K}^{-1}$. The outer heat transfer film coefficient was calculated from a correlation for agitated helical coils from Perry et al., 1984, [49]:

$$
h_{0} \cdot d_{v} / k=0.87 \cdot \operatorname{Re}_{a}{ }^{0.62} \cdot \operatorname{Pr}^{0.33} \cdot\left(\mu / \mu_{w}\right)^{0.14}
$$

where the Reynolds' number $R_{\theta_{a}}$ is defined as:

$$
\operatorname{Re}_{\mathrm{a}}=L^{2} \cdot \mathbf{N} \cdot \rho / \mu
$$

For a typical $3^{\prime \prime}(76 \mathrm{~mm})$ paddle, operating at around 350rpm in a 12" (305 mm) vessel, this gives $h_{0}=1830 \mathrm{Wm}^{-2} K^{-1}$, giving an overall heat transfer coefficient of $1300 \mathrm{Wm}^{-2} \mathrm{~K}^{-1}$. For a mean temperature difference of $10^{\circ} \mathrm{C}$ and $150 \mathrm{~W}$ duty, the required area of the coil was then $11.54 \times 10^{-3} \mathrm{~m}^{2}$, or approximately 4 turns of a coil of the specified dimensions.

\section{Design of the Trim Heater}

The trim heater design was based on a commercial aquarium heater rated at 200W. It was envisaged that this heater could be switched on and off at a controlled rate in order to control the temperature of the feedstream (see section 4.3 .3 for details). The element was enclosed in an $18^{\prime \prime}(457 \mathrm{~mm})$ section of $2^{\prime \prime}(51 \mathrm{~mm})$ internal diameter QVF glassware. The resultant low fluid velocities around the element gave only rather poor heat transfer from the element to the refrigerant and so nucleate boiling was occasionally observed around the heater particularily at times of peak demand, such as around start-up. This boiling gave rise to fluctuations in the feedrate to the distributor and as such had some "nuisance value". It was not possible to use a QVF section of smaller diameter, as this would not have. left enough space on the flanges for the liquid inlet and outlet connections and for the seal around the element. The use of blanking pieces inside the QVF to reduce the cross-sectional area for flow around the heating element was considered, but this could not be implemented because of lack of time.

Also included in the heating element (a diagram of which is shown in figure (4.6)) was a small filter, consisting of two drilled aluminium plates with a plug of glass wool held between them. This was included to remove detritus from the liquid which might otherwise block the distributor. 


\subsubsection{Design of the Compressor}

The design of the compressor was based on the use of a section of $6^{\text {" }}$ $(152 \mathrm{~mm})$ internal diameter precision bored QVF glassware which was held in the departmental stores at the time. The $1 \mathrm{~m}$ long section was cut in half across its cross section to form the two cylinders of the compressor. Taking a mean pressure difference across the cylinders of 0.15 bar and with an estimate of the likely frictional forces involved (measured on a similarly constructed piece of equipment, a large gas syringe used in chemical kinetics experiments) it was calculated that a total force in the region of $500 \mathrm{~N}$ would act on each of the pistons. This meant that the construction of the compressor would need to be suitably robust, particularily at the mounting points for the cylinders and piston drive shafts, and this consideration influenced the specifications made throughout the design process.

Having produced an estimate of the forces involved, the next step was to size the motor for the duty. Taking an average piston velocity of $1 \mathrm{~ms}^{-1}$, corresponding to an amplitude of \pm 0.45 radians with a wave period of 10 seconds, it was found that a motor rated at $100 \mathrm{~W}$ continuous would be sufficient (this was later confirmed by the computer simulation of the rig with friction terms included). Fortunately, a DC servo-motor, eminently suited to the purpose, was made available by the Wave Power Unit, together with the relevant specification sheets. This motor had a continuous rating of $130 \mathrm{~W}$, but a maximum torque of only $0.4 \mathrm{Nm}$, giving an indication of the amount of geared reduction that was required in order for it to be able to drive the pistons. It was decided that an effective way of achieving this reduction would be to mount the pistons on the end of a screw shaft and drive the shaft via a nut held in the middle and turned by the motor, as shown in figure (4.7). In order to minimise gear train losses, a ball-nut and-screw assembly was specified instead of the more usual acme screw and nut arrangement. $A$ ball-screw assembly is similar to a normal acme-screw, except that, in ball-nuts, ball bearings run between the threads of the nut and the screw, describing a helical path before being "caught" at the end of their travel and put back to the beginning by an externally mounted return tube, as shown in figure (4.8). These assemblies, therefore, have very small frictional losses and hence exhibit low wear rates and high efficiences (typically $90 \%$ for the nut driving the screw). Ball nuts are extensively used in precision engineering 
automated machine tools, as their low wear rates give long life with minimal backlash and hence very small positional error. Double preloaded ball nuts were considered for use in this application, as these can reduce backlash to a negligible error. It was felt, however, that, on a cost basis, this could not have been justified.

\section{Design of the Drive System}

Initial designs for the compressor were based on the simple "in-line" arrangement, such as is shown in figure (4.7), with the ball nut in the middle of a single long screw shaft with the pistons mounted on either end. This design had three important disadvantages;

1. It would be extremely difficult to align both pistons with their cylinders and with the central nut-mounting assembly.

2. All the pressure and frictional forces would be concentrated on a single point at the nut-mounting.

3. The "long-and-thin" shape of the compressor would be difficult to accommodate in the laboratory space provided for . the equipment.

Finally, it was decided that such designs were not feasible and a better, though rather more expensive, design was produced. The pistons were to run parallel to each other, one mounted on a right-hand screw shaft and the other on a left-hand screw shaft. The nuts on the shafts would be driven from the motor mounted between the two shafts via toothed belts and pulleys. This design spread the load between two ball-nuts and two mounting assemblies and, although it did not completely remove the problem, significantly reduced the difficulties involved in the alignment of the pistons and the cylinders. The new design did not produce a smaller compressor, but one which would, however, be simpler to install, as all the non-return valves would be situated at one end of the unit, thus shortening the necessary vapour ducting.

A direct 1:1 drive from the motor to the ballnuts would require a screw with a lead length of approximately $2.5 \mathrm{~mm}$ (that is, $2.5 \mathrm{~mm}$ linear travel for every complete revolution of the nut). Upon consultation with suppliers' it was found 
that a lead length of $0.2^{\prime \prime}(5 \mathrm{~mm})$ was more common and could, therefore, be purchased at less cost. The final 2:1 reduction from the motor to the ball-nuts was achieved using toothed belts and pulleys. The belts and pulleys were specified eccording to the suppliers recommendations, taking into account the torques and speeds involved. With the design at this stage it was possible to include the equations for the motor performance, given in the specification sheets, in the simulation program. This confirmed that the motor would be capable of driving the system with the gearing chosen and gave an indication of the current-voltage characteristics of the motor under normal operation.

It was important that the ball-nuts should only experience axial and not radial loads. Therefore, any direct connection between the toothed belts and the ball-nuts could not be considered. Instead, the ball-nuts were attached to flanges which were in turn attached to two "drive cylinders" held between two ball bearing races sunk into $1 "(25 \mathrm{~mm})$ thick aluminium upstands as shown in figure (4.9)a. The ball bearing races were specified from estimates of the forces involved: it was not felt necessary to use thrust bearings. The driving pulleys were mounted on the drive cylinders and held with grub screws and the cylinders were hollowed out to allow the ball screw shaft to pass through them. The servo motor was mounted on the rearmost plate of a similar pair of upstands, with an extended drive shaft carrying the two small pulley wheels terminating in the front plate in a small ball bearing race, see figure (4.9)b. Each pair of upstands were made in such a way that the drive cylinder could be securely clamped between them with very little backlash.

\section{Design of Cylinders and Mountings}

As the cylinders were made from a single glass section cut in half, only one end of each cylinder had the belled shape necessary for the mounting of a standard QVF flange. It was obvious that these ends should form the cylinder heads, as a gas tight seal was required at this end. In order to secure the other ends of the cylinders, UPVC rings were turned to fit inside QVF flanges and over the outside of the cylinders; this required a trial-and-error fitting procedure as the outside surface of the glass was irregular. The cylinder heads were capped with $3 / 8$ " $(10 \mathrm{~mm})$ aluminium plates, drilled and tapped for the vapour inlet and outlet ports, a thermocouple probe and a pressure port, and sealed with PTFE gaskets. The QVF flanges at each end of the cylinders were then tied together with lengths of screwed rod to add rigidity to the 
structure.

\section{Design of the Pistons}

The pistons had to be constructed in such a way as to provide adequate sealing with reasonably low friction, without the possibility of using a lubricant, as this would have been rapidly dissolved by the refrigerant. It was also considered to be necessary to give the pistons a large enough aspect ratio to reduce the possibility of then jamming in, and thus damaging, the cylinders. The first pistons to be made were constructed from three uPVC sections as shown in figure (4.10)a. with two piston rings to each piston. Initially solid PTFE rings were used, PTFE having desirable low friction properties and high resistance to chemical attack. It was found, however, that the solid O-rings did not give adequate sealing, and it was speculated that this was because they were not compliant enough to conform to irregularities on the inner surface of the glass cylinders. The solution to this problem lay in finding a ring seal with the properties of PTFE but also with a degree of "give". These properties were exhibited by a commercially available composite seal consisting of a butyl-rubber backing ring (the energiser), square in cross-section, with a thin (approximately $2 \mathrm{~mm}$ thick) glass loaded PTFE sleeve fitting over it. The existing pistons were modified to accept the new seals as shown in figure $(4.10(b))$, and trials conducted which showed that the new seals gave good sealing without an unacceptably high frictional force.

These pistons worked well during testing of the compressor with air in the system, however, when R11 vapour was introduced it was found that the butyl-rubber energisers swelled to such an extent that the servo-motor could no longer drive the pistons. It was decided to replace the energisers with fluoro-rubber alternatives, as it was known that fluoro-rubbers (eg. Viton) would be more stable under the influence of R11 vapour. It transpired that fluoro-rubber replacement energisers would need to be custom manufactured to order, with a lead time of several months, and incurring a high cost, and, therefore, a different approach was adopted. Instead of using one square section rubber ring, it was decided to use two standard size Viton O-rings side by side to provide the backing for the PTFE sleeve, as shown in figure (4.10(c)). The pistons were modified a third time and tested in the compressor with R11 vapour and it was found that they operated in much the same way as the butyl-rubber backed pistons rings. After some weeks of commissioning 
and normal operation, however, it became clear that the friction had increased to such an extent that the servo-motor/power supply system could no longer drive the compressor without encountering overload conditions. Upon detailed inspection of the equipment it was found that, once again, the 0 -rings had swollen under the influence of the R11 vapour. It was decided that, rather than attempting to identify an alternative "exotic" elastomer immune to the effects of the refrigerant, an approach would be adopted whereby undersized fluororubber O-rings would be pre-swollen in R11 before being used on modified pistons in the usual way. As the fractional increase in the dimensions of the O-rings was difficult to measure accurately (because of the extreme flexibility and increased compliance of the swollen rubber) two pairs of O-rings of the standard sizes immediately below the correct, unswollen size were purchased and treated with R11, and the best fit obtained empirically as usual.

It should be noted that, as the pistons were fitted into the cylinders from the cut ends, where the inner surface was not bevelled in any way, it would have been impossible to obtain a really tight fit without damage to the PTFE sleeve. Thus the maximum piston outer diameter was determined by oversizing the piston and machining the diameter down incrementally until a good fit was obtained.

\section{Assembly of the Compressor}

The above units were mounted on a stable platform constructed from angle bracket and surfaced with a sheet of $3 / 8$ " $(10 \mathrm{~mm})$ thick aluminium measuring approximately $1.2 \mathrm{~m}$ by $1 \mathrm{~m}$ (see figure (4.11) and (4.12)). All the mounting holes for the components (the upstands and the pistons) were slotted to allow the relative orientations of the units to be adjusted for the correct tensioning of the toothed belts and for alignment of the pistons and cylinders.

It was then necessary to devise some means of ensuring that the pistons and screw shafts could not rotate on their long axis, as this would have caused an error in the measured position of the pistons, which could eventually cause an malfunctioning of the compressor. Early designs for this mechanism concentrated on passing one or more rods from the pistons through the drive unit upstands. This method would, however, have added to the frictional forces involved and, perhaps more crucially, made the task of aligning the pistons 
and the drive units unnecessarily complex. The method eventually adopted used small wheeled carriages attatched to the outer ends of the screw shafts, with their "wheels" (made from small ball bearing races) running on either side of a rail mounted perpendicular to the plane of the mounting table and to the rear plates of the upstands. Each carriage had two races mounted on stub axles fitted into a bracket that could be angled so that both races would come into contact with either side of the steady-rail thus preventing rotation of the screw shaft (see figures (4.13)a. and b.). The mounting of the steady rail did not require a high degree of precision, since any out of alignment error could not be cumulative and therefore could not cause the compressor to fail. It was fortunate that the length of the screw shafts had been rather over-specified, as this meant that the steady carriages could be attatched with no danger of them obstructing the motion of the pistons.

The compressor was completed by fitting solenoid valves on the vapour ports and connecting up the unit to the still using 1 " $(25 \mathrm{~mm})$ diameter uPVC piping.

\subsection{INSTRUMENTATION AND CONTROL}

\subsubsection{Introduction}

The instrumentation and control requirements of the experimental facility can be divided into two main areas:

1. real time control of the piston motion

2. data logging and control of process variables

The first of these functions was carried out using dedicated hardware, built for the rig, and described in detail in section 4.3.2. The second area was covered using equipment organised in a three-tier structure. Most of the actual data logging and process control functions were carried out by a stand-alone processor unit, the MFI 1010 (see section 4.3.3), which then relayed the information gathered to a BBC-B microcomputer for visual display, floppy disc storage and user interaction. Another stand alone unit, the MFI 1000, was used to control the action of the solenoid valves (see below). The BBC-B micro was itself used to log transient process variables, effectively in real-time, via its analogue input port (see figure (4.14) for details). 


\subsubsection{Piston Position Control}

The original aim of the piston control scheme, as stated in section 4.2.2, was to use electronic feedback control to model the resultant motion of a sinusoidally acting duck and a free fluid piston, ie. to add pressure compliance to the motion of the pistons. In the event, due to unforseen technical problems, this aim was not wholly achieved and the pistons were only ever driven in a simple sine wave. Having completed this part of the control system, however, it would now be quite possible to "add-on" the necessary equipment to complete the simulation and some details of the apparatus required to do this are given in appendix III.

The control loop implemented ensured that the pistons were driven according to the demand signal, in this case a sine wave, irrespective of the load on the motor (within the mechanical limits of the equipment). For the purposes of analysis, the piston control system may be divided into four subsections: measurement, control, amplification and actuation.

\section{Actuation of the Pistons}

The actuation section of the control loop consisted of the servo-motor and drive system described in section 4.2.3. The servo-motor was a printed armature permanent magnet type, manufactured by Printed Motors Ltd., type G12 M4. These motors have low rotational inertia and linear current/voltage vs. speed/torque curves making them ideally suited to position control operations. In order to ensure that the particular motor chosen was capable of driving the compressor, the motor performance equations were included in the computer model of the rig (see section 4.2.3) and an operating map generated. This model was slightly inaccurate as it did not include the acceleration torques required to overcome inertias in the system. It had been calculated, however, that the magnitude of these moments would be small in comparison to the pressure and friction generated moments.

\section{Control of the Pistons}

The basic form of the piston control scheme was that of a position control system with loop stabilisation by output derivative damping. This means that the feedback loop was first closed on the position of the pistons and then some velocity feedback was added in order to reduce the transient time period (see Morris N.M., 1983, [44]). The circuit diagram for the control electronics is 
shown in figure (4.15). The fundamental element of the circuit was amplifier A4; this was a summing amplifier with an output voltage of the form:

$$
V_{\text {out }}=G \cdot\left(k_{1} \cdot V_{1}+k_{2} \cdot V_{2}+k_{3} \cdot V_{3}\right)
$$

In this case $V_{1}$ was the sine wave demand signal (from a function generator) and $v_{2}$ and $v_{3}$ were the inverted position and velocity signals respectively, and so equation (4.11) represents the familiar proportional plus derivative control loop equation. The values of $G, k_{1}, k_{2}$ and $k_{3}$ and thus of the components in the circuitry, were determined from closed loop trequency tests conducted on the compressor. (The author is indebted to D. Rogers of the Wave Power Unit, now in the Department of Computer Science, Edinburgh University, for his assistance with these tests). Amplifiers $A 1$ to $A 3$ were voltage followers used to impart the correct sense to the incoming signals and to provide current buffering and improve the common mode rejection ratio. The frequency response testing showed that the compressor exhibited a resonance at around $40 \mathrm{~Hz}$, probably the resonant frequency of the drive belts: amplifier A5 was therefore included as part of an active Butterworth filter to cut out frequencies around $40 \mathrm{~Hz}$ and so improve the stability of the system. The final amplifier, A6, reinverted the output drive signal to the correct sense for negative feedback. Capacitive-resitive low pass filters were installed on all the input lines in order to cut out $50 \mathrm{~Hz}$ "mains hum" noise, and the entire circuit enclosed in an earthed metal case, as summing amplifiers are particularily sensitive to electrical noise. Component values and some specifications are given in figure (4.15).

\section{Amplification of the Drive Signal}

Using the computer model of the rig it was possible to determine the voltage/current requirements of the motor under normal operation. This information was used as a basis for the specification of the amplification and motor power supply network. It was found that the motor would require an amplifier capable of producing up to $\pm 10 \mathrm{~A}$ at up to $\pm 30 \mathrm{~V}$. Upon making enquiries with suppliers of suitable equipment, it became clear that commercially available units were too expensive to be considered and that, therefore, it would be necessary to build an amplifier specifically for this application. As the author was unfamiliar with the design of such devices, it was fortunate that, around that time, an addition was made to the existing 
range of easily available semi-conductor components, namely the LM12CLK power amplifier i.c. manufactured by National Semi-Conductor Ltd. The availability of this device simplified the design work required to produce an amplifier to a considerable extent, as included in the i.c. package was almost all of the transistor and protection circuitry necessary for the construction of an operable "push-pull" amplifier (see Carlson A.B. and Gisser D.G., 1981, [7]). Furthermore, the data sheets for the LM12 contained much useful information and technical advice regarding the implementation of the device in high-power applications.

The amplifier was designed to be a current amplifier, this means that it would produce a given output current for a given input voltage, irrespective (within specified limits) of the nature of the load on the output. This mode of operation was chosen because the results of the rig simulation suggested that the motor current draw would more often be limiting than the voltage requirement; essentially the motor characteristic was that low speed plus high torque required high current and high speed plus low torque required high voltage, and the former was dominant in this case. The resulting circuit, without external protection circuitry, is shown in figure (4.16). This design, suggested in the manufacturers data sheet, gave 20A output for every IV input (note that this is an inverting design, so $+1 \mathrm{~V}$ in would give $-20 \mathrm{~A}$ out).

One of the primary design considerations was the problem of heat dissipation from the LM12 package. The heat generated for a DC output can be easily calculated from equation (4.12):

$$
Q=\left(V_{s}-V_{0}\right) \cdot 1
$$

where $V_{s}$ and $V_{0}$ are the supply rail and output voltages for the output transistor (PNP or NPN) of the LM12 respectively and $\mathrm{I}$ is the output current. The maximum power rating is determined by the maximum junction temperature allowed in the package (in this case $150^{\circ} \mathrm{C}$ ). The junction temperature can be calculated from equation (4.13):

$$
T_{j}=T_{c}+Q \cdot \theta_{j c}
$$

where $T_{c}$ and $T_{j}$ are the case and junction temperatures respectively, and $\theta_{i c}$ is the thermal resistance of the package at the temperature of the output 
transistor. Equations (4.12) and (4.13) were added to the computer model for the rig and it was found that one LM12 package on its own would tend to over-heat, but that two could operate in parallel with a reasonable margin for error (note that over-heating would not damage the LM12, the internal protection circuitry included a thermal cut-off sensor). It was decided to mount the LM12's on $1.1^{\circ} \mathrm{CW}^{-1}$ heat sinks and to use forced convection cooling: it was perhaps unlikely that this would have much effect on the heat dissipation from the packages, as the rate of heat transfer was limited by $\theta_{j c}$ however both the heat sinks and the fan were available at the time, and the heat sinks provided a convenient means of mounting the LM12's and their circuit boards.

The power supply for the amplifier consisted of two centre tapped, 120VA continuous rating toroidal transformers stepping down from $240 \mathrm{~V}$ to $25 \mathrm{~V}$ rms; a solid state bridge rectifier package rated at $25 \mathrm{~A}$ continuous (if mounted on a suitable heat sink, see below), and $39,800 \mu \mathrm{F}$ of smoothing capacitance on each rail. The two transformers were used in parallel as this provided the correct supply voltage $( \pm 35.4 \mathrm{~V}$ after rectification) and a reasonable continuous current rating $( \pm 6.4 \mathrm{~A})$ fairly inexpensively and in a compact form. The amount of smoothing capacitance on the supply rails gave a ripple voltage variation of approximately $2.5 \mathrm{~V}$, or $7.4 \%$, at the maximum design current of $10 \mathrm{~A}$; this variation could not affect the operation of the LM12's under normal circumstances.

The external protection circuitry for the power amplifiers consisted of two $47 \mu \mathrm{F}$ electrolytic bypass capacitors; output clamp diodes to the positive and from the negative supply rails, and inductor-resistor networks on the output lines from both the LM12's to increase the stability of the amplifiers when driving series resonant loads. A single $L-R$ circuit was first installed, but during tests of the compressor it was found that the amplifiers exhibited a high frequency oscillation instability when driving large negative currents: the addition of a second $L-R$ network effectively removed this problem. The amplifier circuit, including the power supply section and all the protection circuitry is shown in figure (4.17). All the earth points in the circuit were connected directly to a single star earthing point, in order to avoid ground looping problems, and the external circuitry (particularly the bypass capacitors) were mounted as close as possible to LM12 packages in order to avoid 
The LM12's were mounted on the heat sinks with their terminals fitting through holes drilled in the bodies of the heat sinks for that purpose. As the negative supply terminal for the LM12 was its outer case, and therefore the heat sink would be live, the other terminals were insulated using double layers of heat shrink material. The two heat sinks were joined together by two aluminium plates to form on open-ended box construction, with the LM12 packages facing inwards (see figure (4.18)). The cooling fan was mounted at one end of the "box" so that it blew air through and over the LM12's. The bridge rectifier was bolted to the uppermost aluminium plate in order that it too could benefit from forced convection cooling. The circuit boards for the LM12's were attatched to the heat sinks on the outside of the box and connected to the LM12 terminals. Finally a UPVC box was constructed to contain the heat sink unit, the fan, the transformers etc. A $\pm 30 \mathrm{~V}$ full-range voltmeter was installed across the output terminals of the amplifier and a 0-10A ammeter in the positive supply line; although this meant that only positive output currents could be monitored, some indication of the state of the output could generally be gained from the voltmeter. The amplifier was tested using a dummy load of $1 \Omega$, made up of $10 \times 0.1 \Omega, 25 \mathrm{~W}$ resistors mounted on a heat sink, first with only the "master" LM12 connected and then with the "slave" LM12 in circuit. In both cases the amplifier was found to give a stable and linear output for all loads.

\section{Measurement of Position and Velocity of Pistons}

The simplest, most accurate and most reliable means of measuring the position of the pistons would have been to use a linear potentiometer attatched to the outer end of one of the ball screw shafts. With the stroke lengths and velocities envisaged, however, this option had to be discounted on economic grounds. The second means considered was the use of an optical encoder mounted on the motor drive shaft. Reasonably inexpensive optical encoders were available; or it would have been possible to manufacture one "in house": but their use would have entailed the construction of a number of digital and digital-analogue circuits in order to condition the pulsed output of the encoders for use in the purely analogue control circuitry and therefore this method was "held in reserve" while a simpler approach was sought. 
The simplest means of generating an analogue position signal seemed to be to use some sort of potentiometer; the use of linear potentiometers had been ruled out (see above) and so it was decided to look for a means of using a standard, cheap, ten-turn potentiometer. The problem with this sort of device was finding a method of converting the 160rev/stroke of the motor shaft to $10 \mathrm{rev} / \mathrm{stroke}$ of the potentiometer. The use of commercial gearboxes was considered, but found to be impractical due the combination of the large reduction required and the high shaft velocities of the motor. The solution to the problem is shown in figure (4.19). The potentiometer was mounted on a bracket attatched to one of the steady carriages (see section 4.2.3). A small wheel, approximately $12 \mathrm{~mm}$ in diameter, with two O-ring "tyres" located in grooves turned in its surface, was attatched to the shaft of the potentiometer and made to bear on one side of the steady-rail as shown. This arrangement gave a gearing ratio of approximately seven turns per full stroke. The potentiometer bracket was made of light aluminium sheet, so the wheel could be sprung against the steady-rail to reduce slippage; a strip of glass paper was glued along the steady-rail face in order to further improve the adhesion.

In practice it was found that, due to the light construction of the bracket, the potentiometer tended to shift in its vertical alignment depending upon the direction of travel of the piston shaft, resulting in a drift in the position of the null point of the sensor. This problem was eventually overcome by tensioning the potentiometer against the steady carriage using a cable tie (see figure (4.19)). In this way the tension in the tie could be minutely adjusted until very little cumulative drift of the null position was observed. Although the resulting apparatus does have a rather gimcrack appearance, it was found to function quite satisfactorily.

For the velocity feedback signal it would have been possible to differentiate the position signal electronically using a simple differentiating amplifier. This method, however, is very susceptible to noise on the input line: a single sharp spike on the input would have an quasi-infinite gradient and hence would produce a very large differentiated output. For this reason it was preferable to use an electro-mechanical tachometer to measure the motor shaft speed directly. It was necessary to mount the tachometer securely in order to reduce backlash errors to a minimum, the resulting arrangement is shown in figure (4.20). (The author is indebted to the Wave Power Unit for the use of the 


\subsubsection{Data Logging and Process Control}

The data logging and process control system was based on a C.I.L. Ltd MFI (multi-functional instrument) 1010 and a BBC-B microcomputer. As the capabilities of the MFI 1010 effectively constrained the design of the monitoring apparatus, its specifications will be discussed first, before moving on to the individual elements of the system.

The MFI 1010 was a data logging instrument with a high degree of local intelligence, designed for stand-alone operation. This means that it had its own programmable memory and could compile and run fairly complex programs written in a simple dialect of BASIC. Programs up to 999 lines could be accommodated and were automatically compiled before being run, for maximum speed of execution. The MFI communicates with the outside world via an RS 232/432 link and was in this case connected to a BBC-B microcomputer; although, once programed, it could easily be connected, for example, to a printer, if hard-copy output was required. The unit had twelve analogue input ports (with cold-junction compensation performed in software so that thermocouples could be read directly); eight logic input/output ports; two analogue voltage outputs; an analogue current output; a constant current source, and a reed relay switch. Each analogue input had a programmable gain, set in software, for either $\pm 100 \mathrm{mV}$ or $\pm 10 \mathrm{~V}$ input. The logic ports could be configured as either input or output, again in software, and, if set up for input, could also be used to read the frequency of a pulsed input signal in the range 1-10 000 Hz. The three analogue outputs were: $01, \pm 10 \mathrm{~V} ; 02,0-10 \mathrm{~V}$; and $03,0-20 \mathrm{~mA}$ at $10 \mathrm{~V}$ maximum.

As thermocouples are fairly inexpensive and reliable, it was decided to fully instrument the rig for temperature measurement, as shown in figure (4.21), thus using eight of the analogue input ports for thermocouples and leaving four free. Four pressure transducers, one on each of the cylinders and two for the condensing and evaporating spaces, were necessary both for measurement and for control of the solenoid valves. Two flowmeters and two liquid level meters were required on the liquid circuit in order to carry out a mass balance over the system and to be able to control the feed flowrate. This gave a total of sixteen analogue inputs, four more than the MFI could accommodate 
directly. It was decided, therefore, to configure the logic 10 ports for output and use three of them to control the status of a multiplexer i.c., giving eight inputs switched in turn into a single MFI channel (see figure (4.31)).

The low pressure side pump DC motor was used to control the liquid feed flowrate. MFI analogue output 01 was used for this purpose and details of the motor speed control circuitry and of the control scheme operated are given below. The high pressure side (condensate) return pump was operated on an on/off basis depending on the liquid level in the condensate pot. The reed relay switch was used to switch a small voltage to a mains switching solid state relay.

The feed temperature was controlled by switching the feed heater in a pulsed manner at a mark-space ratio determined by output $\mathrm{O} 2$ (from software). An astable 555 timer circuit was used to produce the pulsed output from the analogue input; details of the circuitry and control scheme are given below.

\section{MFI 1010 Operation}

A suite of programs was developed to be used by the BBC-B microcomputer in order to be able to operate the MFI effectively. The first requirement was to produce a terminal emulation program to enable direct communication with the MFI via the BBC. A simple version of a program to fulfill this function was supplied by C.I.L. Ltd in the instruction manual for the MFI (the flowsheet for this program is reproduced in figure (4.22)). The disadvantage of this program was that it could not recognise the delete character and thus typing errors could only be corrected by re-entering the entire statement; a tedious process when creating long programs. A modified version of this program was developed, therefore, in which each character entered at the keyboard of the BBC was stored in a line vector and only when the carriage return key was pressed would the entire line be downloaded to the MFI (the flowsheet for this program is shown in figure (4.23)). If the delete character was pressed the last character in the line array would be deleted by decrementing the array counter by the appropriate amount and in this way the program dealt successfully with corrections.

The above terminal emulation program was most suitable for direct communication with the MFI but it was limiting in some respects: for example, there were occasions when it was required to download two or more fairly 
lengthy programs to the MFI sequentially, perhaps a testing or calibration program followed by the normal operational program. Using the terminal emulation program alone, this would have involved typing in each program line by line; a time-consuming method and one prone to error. A third program was created therefore, specifically suited to the task of loading programs into the memory of the MFI and not for general, direct communication. This program could operate in two ways: either the MFI program lines could be typed in by the user (for alteration of an existing program for example), in which the characters would be stored on disc and then downloaded, or a suitable disc file, created using the first option, could be accessed and the characters in it read directly into the MFI. This program not only cut down on the time spent loading programs into the MFI, it also produced useful back-up copies of the programs on disc which could be used, for example, in the event of accidental erasure of the MFI program.

Finally, it was sometimes necessary to get hard copies of the programs in the memory of the MFI. This could have been easily achieved by attaching a printer to the $B B C$ and using a terminal emulation program to print the characters from the MFI to the printer as well as the VDU screen. However, no printer was available and so an alternative method had to be devised. Two programs were developed; one to read the characters from the MFI and store their numerical ASCII codes in a disc file and the other to read the codes from the disc and print out the corresponding characters to a printer. The flowsheets for the two programs are given in figures (4.24) and (4.25) and it may be seen that the second, the output program, was trivially simple. The other program was rather more complex than would at first appear to be necessary for the following reason. Due to the time taken for the disc drive to be operated, data from the MFI could not be stored immediately as it was received: early versions of the program operated in this way, but it was found that the RS 232 buffer space in the BBC soon overflowed and characters were lost. In the program as implemented, the ASCII codes were stored in an array as they were received and then written on to the disc at the appropriate time. The limitations on the size of arrays available on the BBC meant that not all of a long MFI program could be stored at one time and so the storage program had to make a number of passes in order to get the complete program on to the disc; getting characters 1-1000 on the first pass, 1001-2000 on the next, and so on. This method was found to work satisfactorily although for long 
programs it was not a rapid process.

The operational program run by the MFI was quite straightforward, as the dialect of BASIC available and the requirement of speed of operation dictated that it should be. The MFI was provided with an inbuilt array, $D(x)$, containing up to 382 variables. It was decided to use this array to store input values, control parameters and intermediate calculation variables, as, since the array would always exist whether it was used or not, this seemed to make the best use of the available memory space of the MFI. A table showing the array location allocations for the $D(x)$ is given in figure (4.26). Another useful facility of the MFI was an inbuilt "filtering" routine used to smooth the values of the input variables by arithmetic averaging of a user-specified number of channel readings. For example, if FILTER was set to five, then each channel would be read five times when called and the average of the five values returned. For the majority of the input streams this variable was set to twenty-five, as this was found to produce reliable readings without reducing the speed of the program execution noticeably. However, for the two flowmeters, FILTER had to be set to fifty in order to get steady values.

The program consisted of a seven subroutines called by a short length of code at the start, see figure (4.27). The function of each of the subroutines was as follows (with reference to figure (4.27)): GOSUB 1 read the eight thermocouple inputs and performed the cold-junction compensation and linearisation to give direct temperature readings; GOSUB 2 operated the multiplexer circuitry and read input $I(10)$ at each of the eight binary switched steps; GOSUB 3 printed the values of the sixteen inputs to the BBC; GOSUB 4 controlled the liquid feed flowrate using output 01 on a proportional plus reset basis; GOSUB 5 printed the value of a single, chosen variable to the $B B C$ when pompted; GOSUB 6 controlled the liquid level in the condensate pot using the reed relay to switch the condensate return pump; GOSUB 7 controlled the feed temperature using output $\mathrm{O} 2$ on a proportional plus integral basis.

The control parameters (array locations $D(50)-D(89)$ ) were set by the user, stored on a disc and downloaded to the MFI using a program for which the flowsheet is shown in figure (4.28). The variables were stored in a disc file so that the chosen default values could be conserved from one run to another. 
The program was basically simple, although it contained a lot of code because of the amount of user-prompting and screen display instructions necessary.

The final program used was the normal running program for the BBC. The function of this program was to get data from the MFI during the course of an experimental run and display it in a graphical and tabular form. The program also had the facility to cause a shut-down if requested by the user (all MFI outputs set to zero), to put the gathered data onto a disc file, and to call the control parameter changing program (above). Again, this program was not fundamentally complex, but it was sizeable mainly because of the routines for graphical and tabular display. The ability to produce a real time graph of any chosen variable on any timescale desired proved most useful in, for example, leak testing the rig, where $\mathrm{dP} / \mathrm{dt}$ and thus $\mathrm{dn} / \mathrm{dt}$ could be easily illustrated. The routine was less useful in normal operation, however, because the BBC could not get data rapidly enough from the MFI to allow transient states to be studied.

\section{BBC Microcomputer Operation}

The BBC-B micro had the capability, aside from being able to communicate with the MFI 1010 and $\log$ data in this manner, to log up to four analogue input channels at very high sampling rates. It was decided that this facility could be used to gather transient data from the rig, effectively in real-time. The analogue input ports required input voltages in the range $0-1.8 \mathrm{~V}$ : the interface circuit shown in figure (4.29) was used to condition the transducer output voltages as necessary.

Three inputs were used; logging piston position (from the piston position potentiometer, see above) and the two cylinder pressures. Coupled with the accurate internal BBC clock (measuring elapsed time to $1 / 100^{\text {th }}$ of a second), this allowed five transient logging and display programs to be written to provide graphical display and floppy disc storage of: cylinder pressure vs. time; pressure difference vs. time; piston position vs. time; cylinder pressure vs. cylinder volume and pressure difference vs. cylinder volume.

These five programs, those described above associated with the operation of the MFI 1010, a program to create a reference disc file for each run and a simple program to calculate display and store mean process variables from the raw data collected, could all be called from a single "menu" program as 
requred by the user. The user was also able to choose precisely when the disc storage of gathered data should commence (see figure (4.30) for details).

\section{Multiplexer Operation and Circuitry}

The multiplexer circuit was based on the DG508 CMOS multiplexer i.c., a standard, general purpose device. Three logic outputs of the MFI, L1, L2 and L3, were used to switch any one of eight transducer signals into a single channel of the MFI, 1(10). A truth table and circuit diagram are given figure (4.31). As the multiplexer i.c. required a $\pm 15 \mathrm{~V}$ supply voltage and as, at the time of construction, only a $+10 \mathrm{~V}$ supply was available, a $+5 \mathrm{~V}$ to $\pm 15 \mathrm{~V} D C-D C$ convertor package and a voltage divider network were included in the circuit.

\section{Solenoid Valve Operation}

The four solenoid valves installed at the inlet and outlet ports of the compressor cylinders were mains-actuated, one-way valves with $3 / 4$ " couplings. The mains supply was switched to the valves by four solid state relays. These relays were originally controlled by four of the logic outputs from the $\mathrm{MFI} 1010$, via a quad operational amplifier wired for voltage following to provide current gain. It was found, however, that the MFI could not operate the valves frequently enough to be able to mimic the actions of direct-acting mechanical non-return valves and carry out all the other data processing and control functions required of it. One solution to this problem would have been to operate the valves from a hardware control circuit, based on the use of voltage comparators. However, as a short-term step, another $\mathrm{MFI}$, which happened to be available at the time (an MFI 1000, similar in operational terms to the MFI 1010, but with slightly different input/output specifications) was employed to control the solenoid valves only. In this way, with the MFI 1000 running only a very short program, the valve control routine could be entered frequently enough to provide an effective alternative to hardware based control.

The advantage of using a programmable controller was that the characteristics of the valves could be manipulated, to a certain extent, simply by changing the relevant control parameters in software: for example, the routine used included provision for a variable degree of hysteresis in the valve operation. A wiring diagram for the solenoid valve control network is given in figure (4.32). 


\section{Pressure Measurement}

The pressure was measured at four points on the rig (at the inlet and outlet to the still and at each cylinder of the compressor) using Honeywell 143PC differential pressure transducers (pressure range \pm 15 psi ( \pm 1.03 bar) gauge). The transducers were calibrated off the rig against a mercury manometer and were found to give stable and linear outputs over the entire range.

\section{Flow Measurement and Control}

Two RS standard size flowmeters $(0.05$ to $1.71 / \mathrm{min})$ were installed in the liquid return line, one before the condensate return liquid junction and one after it. This allowed the condensate flowrate to be determined from a mass balance rather than by direct measurement, this method being preferred as the condensate flowrate tended to be in the lower, and least accurate, range of flows measurable using these flowmeters. The transducers produced a pulsed output the frequency of which varied with the flowrate, which was converted to an analogue voltage using a tachometer i.c. and associated circuitry (see figure (4.33)). The flowmeters and their interfaces were calibrated together and linear functions of flowrate vs. voltage fitted to the results. Some drift of the calibrations was observed over a period of time and so the calibration process had to be occasionally repeated.

Control of the liquid feed flowrate was achieved through the variation of the speed of the evaporation side pump motor. The motor, a Parvalux DC permanent magnet type, was purchased along with a speed control unit designed to be operated manually using a potentiometer. In order to effect control of the motor using an analogue voltage from the MFI, the potentiometer was taken out of circuit and replaced with the opto-isolator circuit shown in figure (4.34). The opto-isolator i.c. incorporated a photo-diode and a light sensitive BIFET transistor. A voltage applied across the diode terminals could be used to control the base current of the transistor and hence its output voltage at the emitter terminal; the use of the photo-diode gave protection to the controlled voltage source (the MFI 1010). Although the opto-isolator used was primarily designed for use as a switch, by choosing the component values carefully, it was possible to operate the transistor in a narrow active range. Unfortunately, the operable range of voltages was very small, about 3 to $3.5 \mathrm{~V}$, and within this range the output of the circuit was very non-linear. It was possible, however, by manipulating the control parameters 
effectively, to produce an operable control system. For as long as the pump was operating under steady-state conditions, the control was satisfactory the fact that peristaltic pumps exhibit virtually linear relationships between pump speed and liquid flowrate helped to stabilise the control scheme).

A proportional plus integral control scheme was implemented in software via the analogue output 01 . This control scheme was of the form:

$$
\begin{aligned}
& E_{i}=\text { flow }- \text { setpoint } \\
& 01=G \cdot\left(E_{i}+G_{2} \cdot \Sigma^{n}{ }_{i=0} E \cdot d t\right) \\
& d t=\text { time }- \text { time of last control function }
\end{aligned}
$$

the constant $G_{1}$ and $G_{2}$ were determined empirically. The summation term was only evaluated when 01 was within certain limits, to prevent controller "wind-up".

\section{Temperature Measurement and Feed Temperature Control}

Eight $K$-type thermocouples were installed in the apparatus at the locations shown in figure (4.21). All the thermocouples were made from a single roll of wire and spot welded at the tips to form a junction, before being fitted into one or other of the probe assemblies shown in figure (4.35a. and b.). The feed temperature (measured directly above the distributor collar) was controlled through pulsed switching of the feed trimmer heater using a pulse position modulation circuit based on a 555 time i.c. running in the astable mode. This circuit produced a pulsed voltage where the mark-space ratio of the output could be varied by application of an external voltage, in this case output 02 from the MFI. This signal was used to switch a solid-state relay which in turn switched the mains supply to the heating element. It was found to be possible to vary the length of the "on" pulse from around $0.5 \mathrm{~s}$ to $2 \mathrm{~s}$, thus giving a turndown ratio of approximately $60 \%$. The fundamental problem with this means of control, however, was the large thermal inertia of the heating element itself, leading to a poor transient response. One way of reducing this problem would have been to increase the fluid velocities around the element (see section 4.2.2).

Proportional plus integral control via output 02 was implemented in software (see equations (4.14), (4.15) and (4.16)), the control parameters were again 
determined empirically.

\section{Liquid Level Measurement and Control}

The liquid levels of both the condensate and evaporate return pots were measured at the bottom of the still (see figure (4.21)). The level of the evaporate return liquid was measured so that the evaporation rate could be determined from a mass balance thus:

$$
\text { Evaporation rate }=m_{f}-m_{\theta}-k \cdot d L_{e} / d t
$$

The rate of change of the liquid level was approximated by:

$$
d L / d t=\left(L_{i}-L_{i-1}\right) / \delta t
$$

The condensate level was measured for two reasons: firstly, in order to find the rate of condensation from:

$$
\text { Condensation rate }=k \cdot d L_{c} / d t-m_{c}
$$

and, secondly, to be able to control the condensate pump on an on/off basis via the $\mathrm{MFI}$ reed relay.

Both levels were measured using the apparatus shown in figure (4.36), a simple float actuating a potentiometer. The only problem experienced with the operation of the "level boxes" was some initial difficulty in sealing them effectively, otherwise they proved reliable and accurate. 


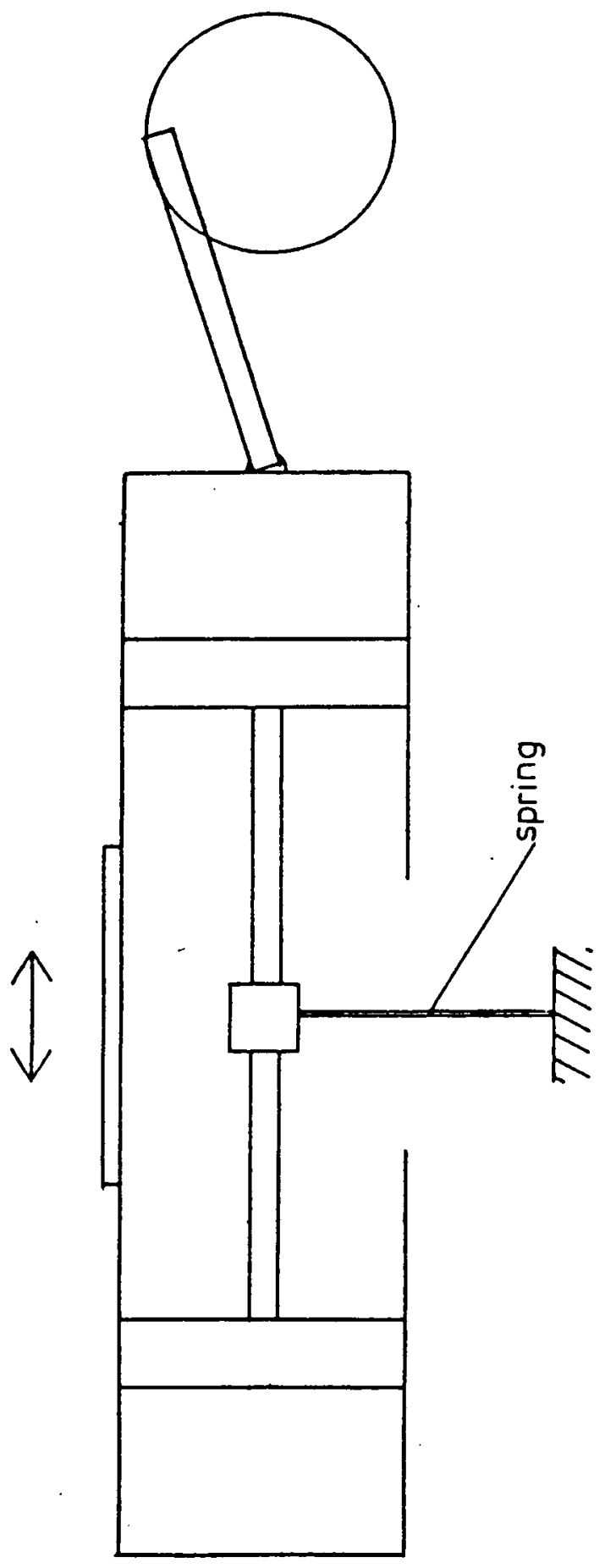

Figure (4.1) Early Design of Compressor 


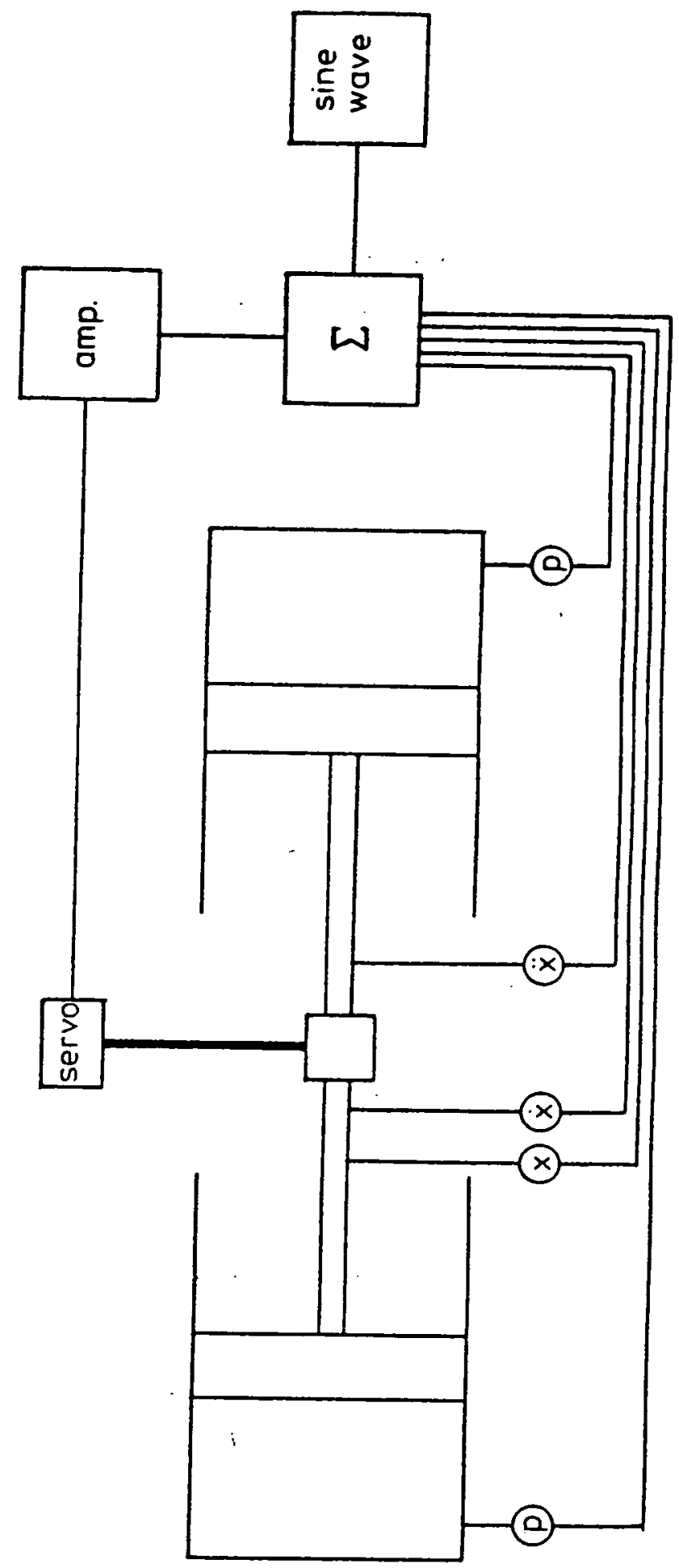

Figure (4.2) Compressor with Compliance Feedback Loop 


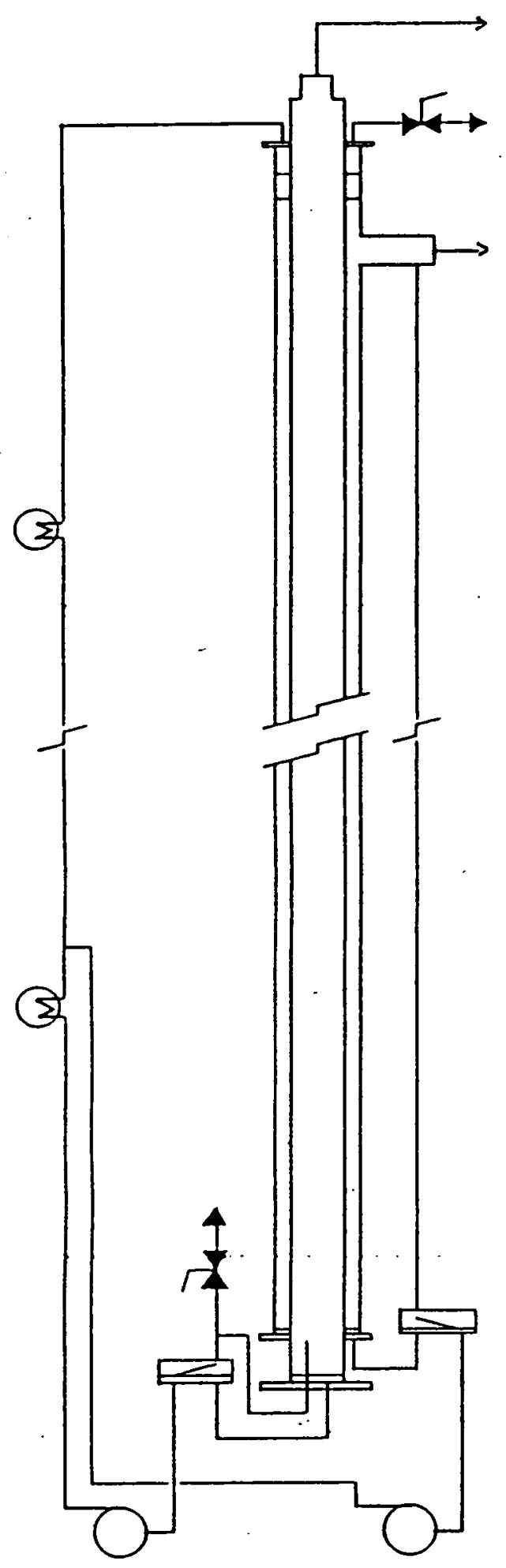

Figure (4.3) Sketch of Still with Liquid and Vapour Lines 

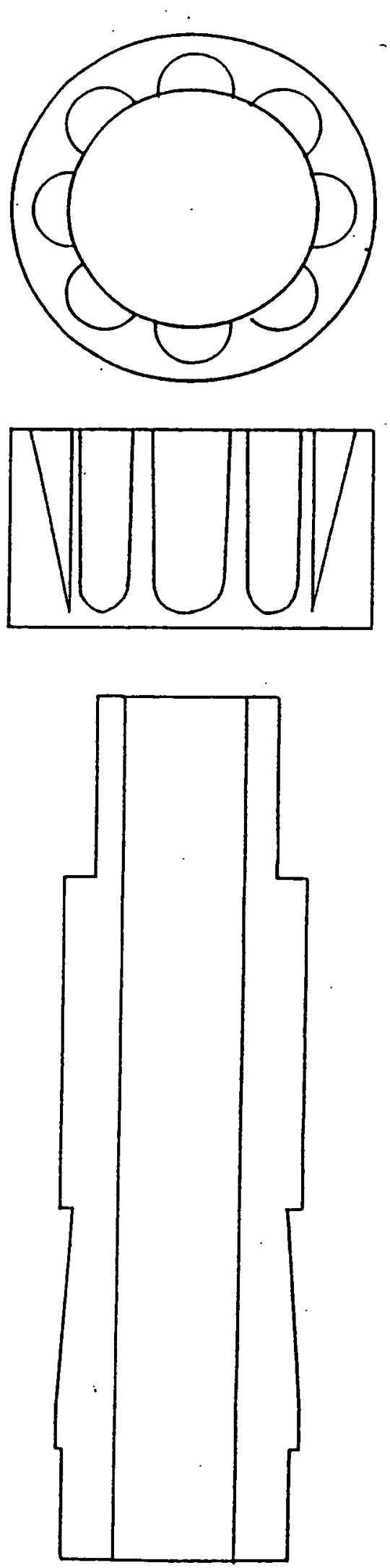

Figure (4.4) Distributor Components 


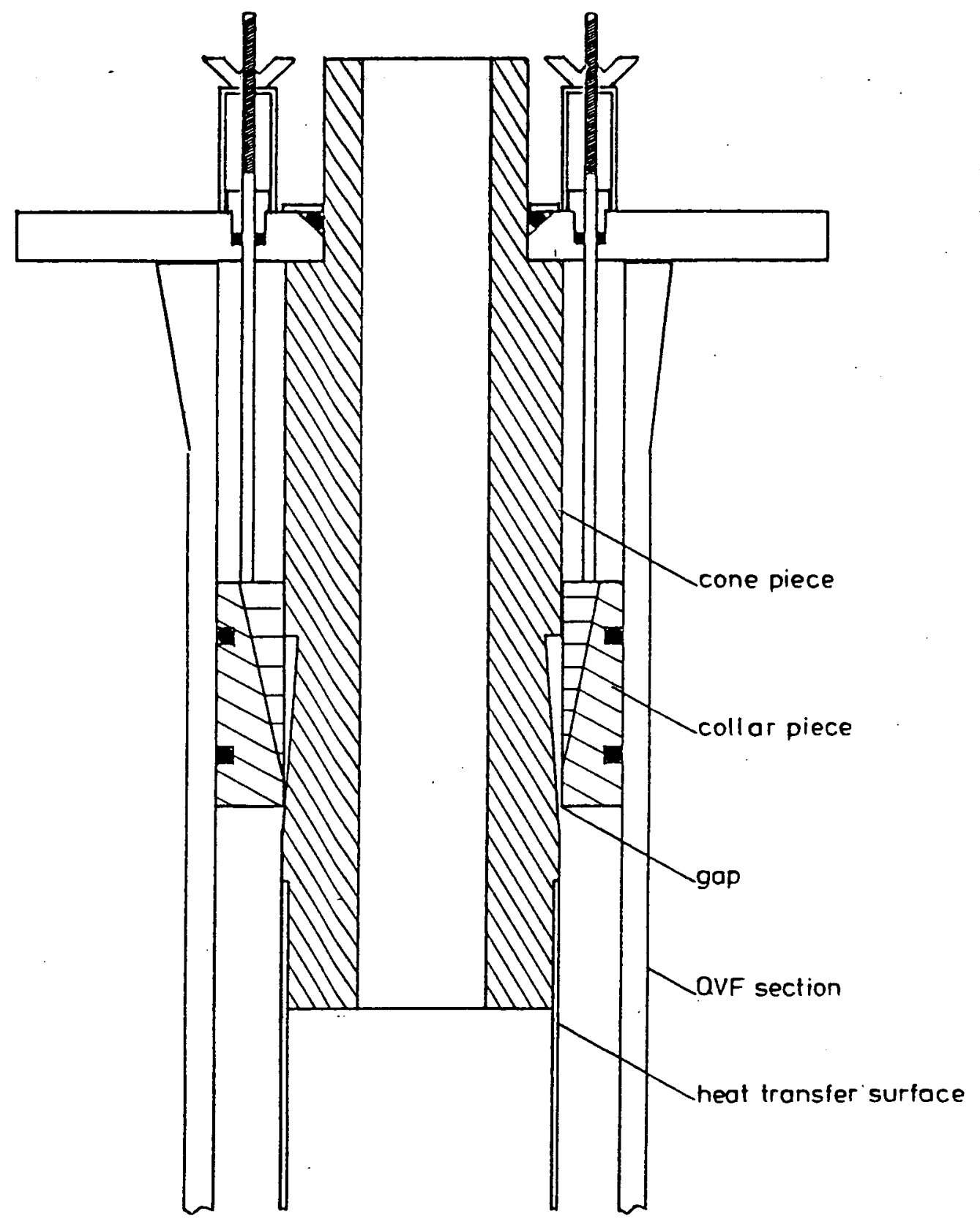

Figure (4.5) Assembled Distributor 


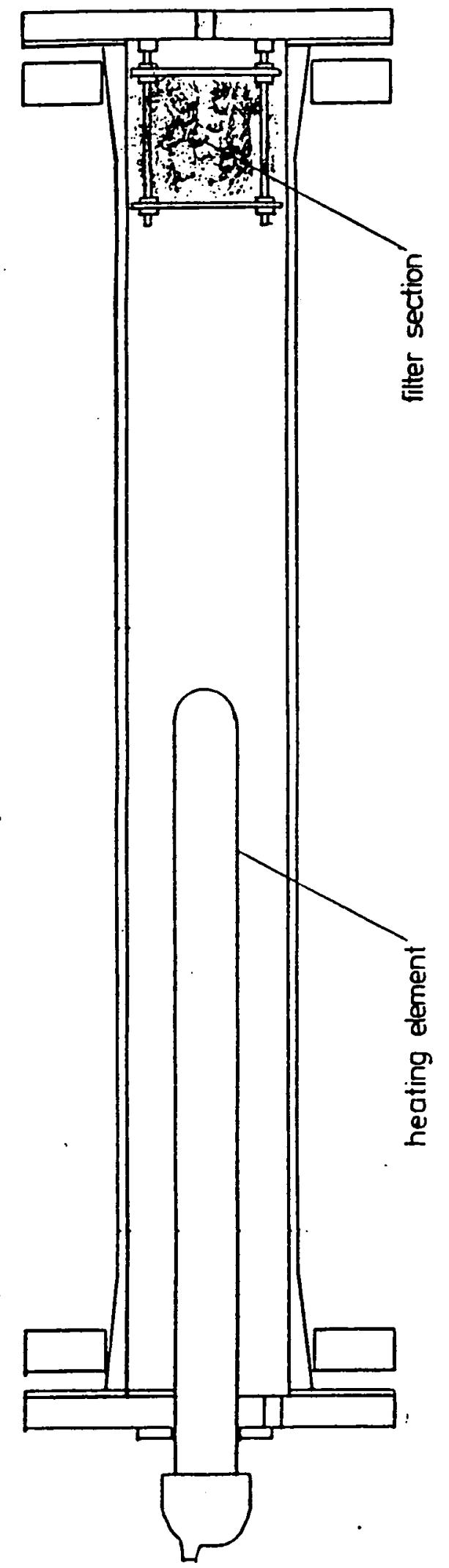

Figure (4.6) Feed Preheater 


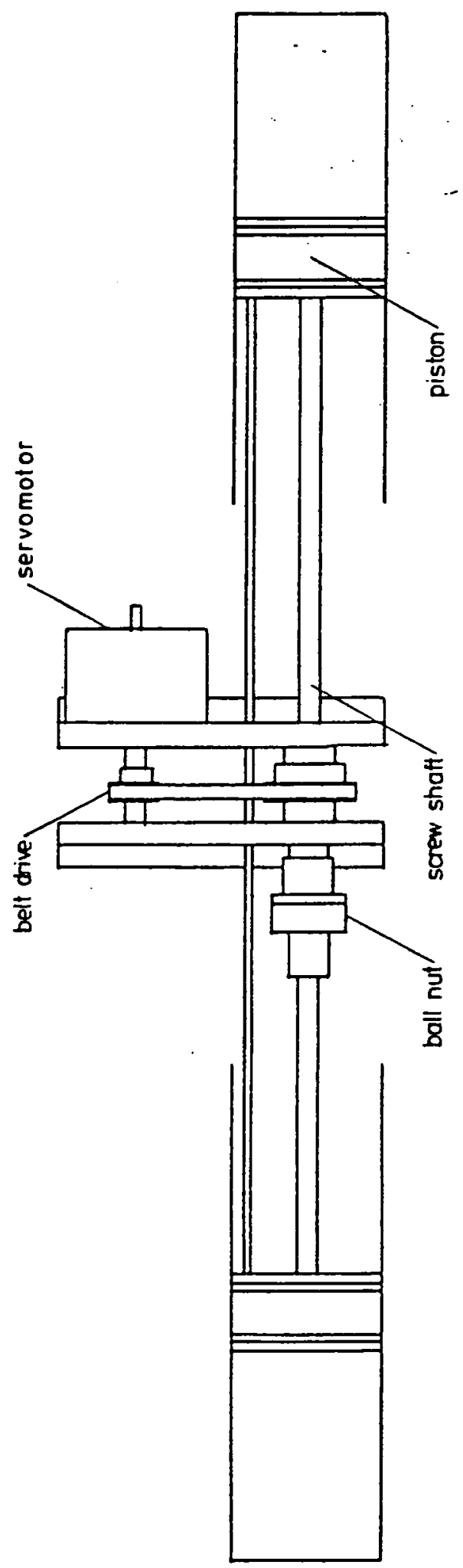

Figure (4.7) Sketch of Single Screw Compressor 


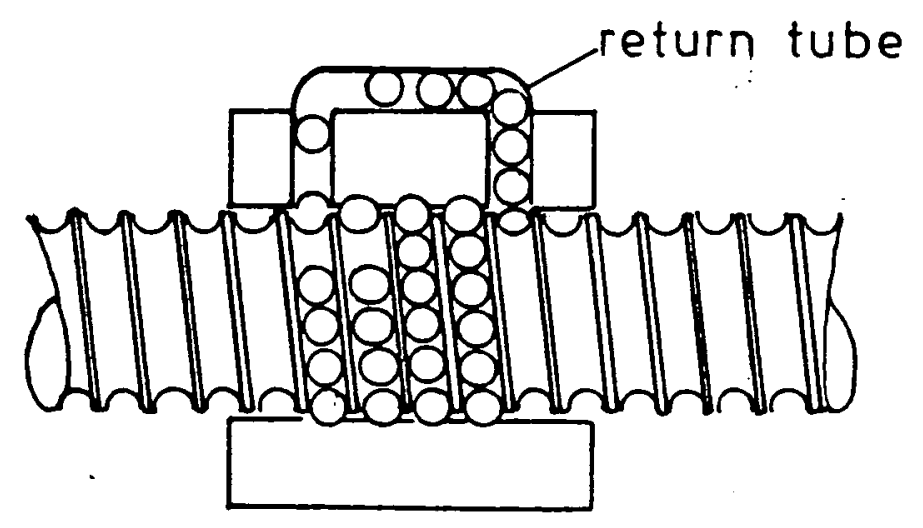

Figure (4.8) Section Through a Ball-Nut 

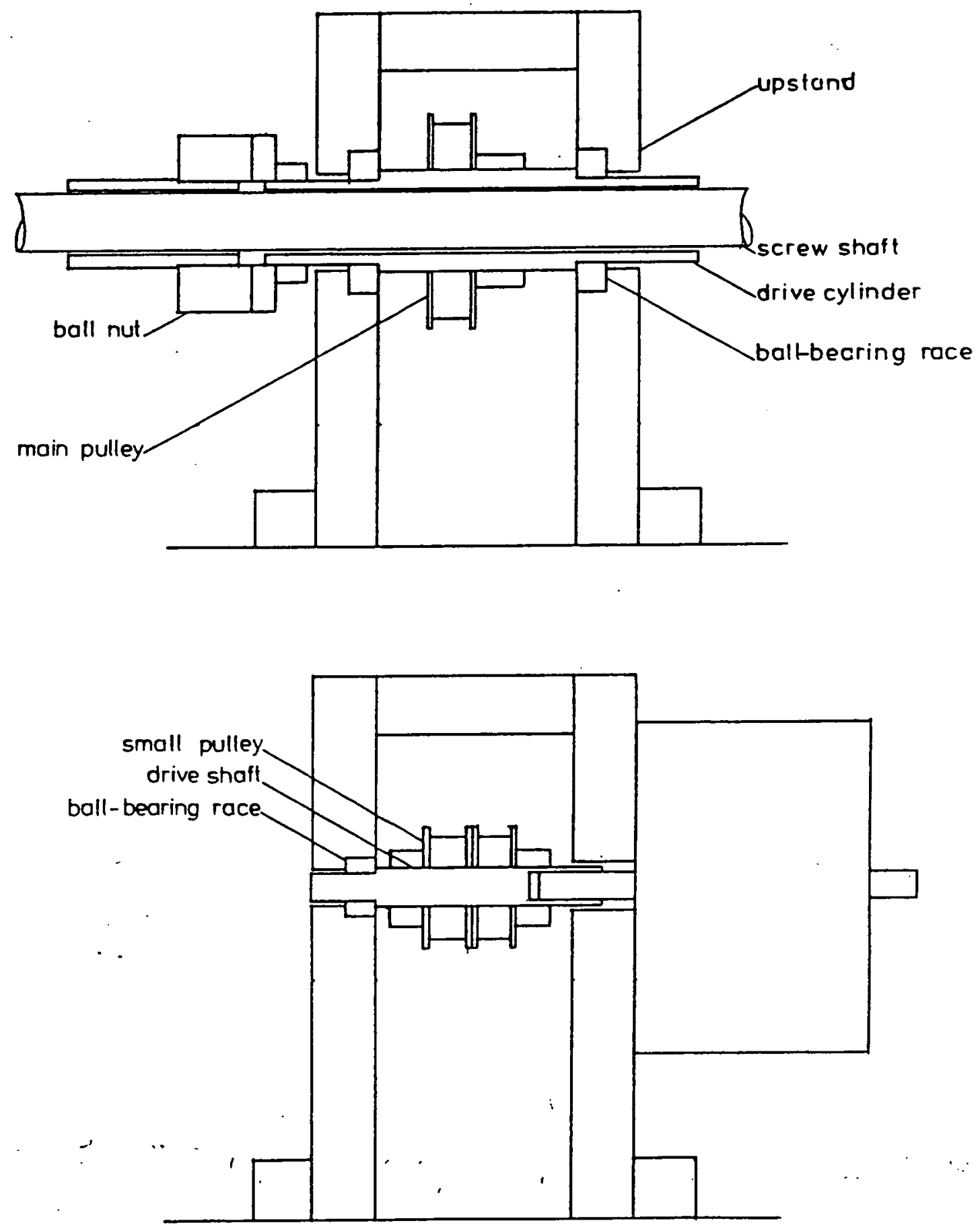

Figure (4.9) Compressor Components 


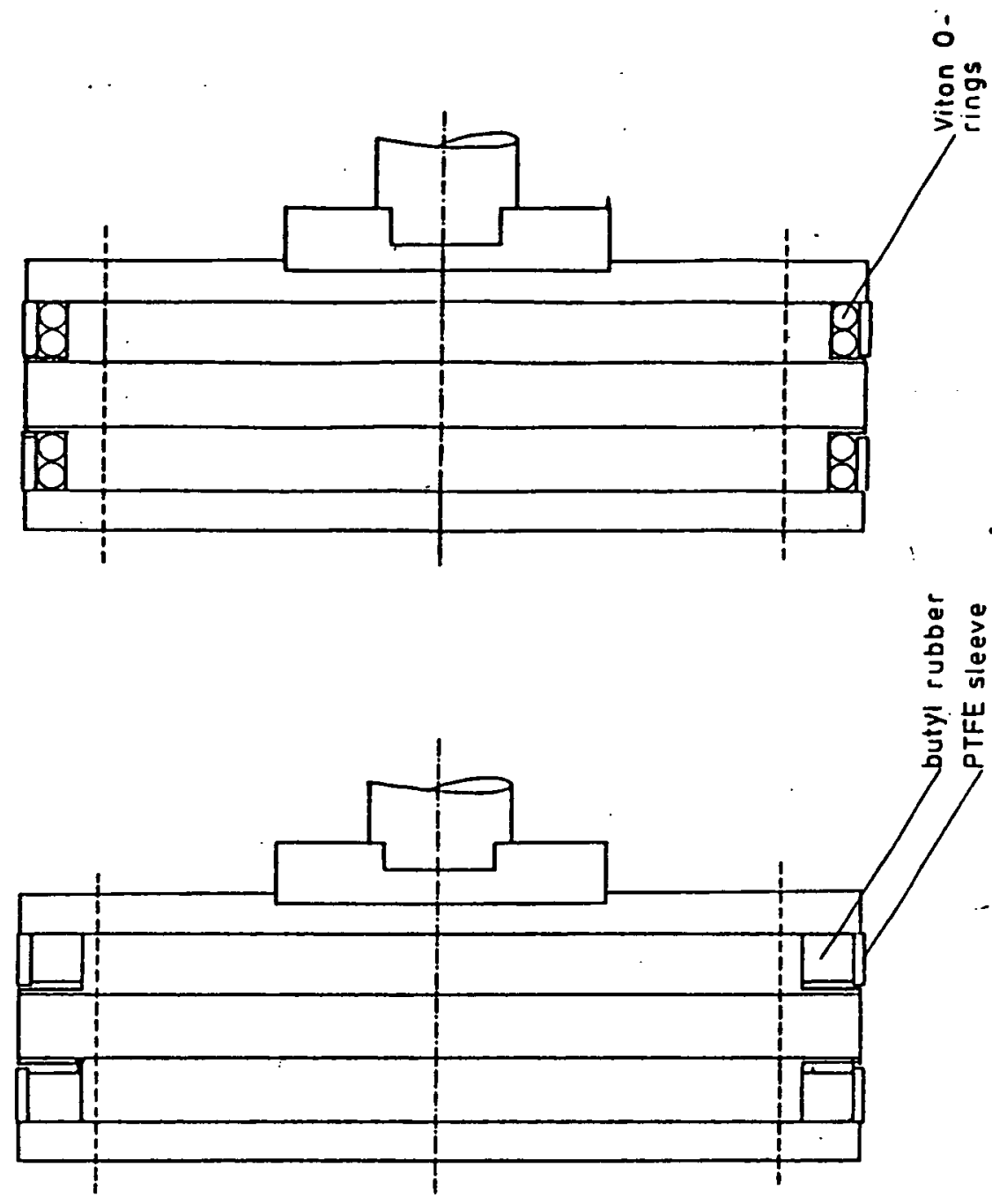

c.

b.

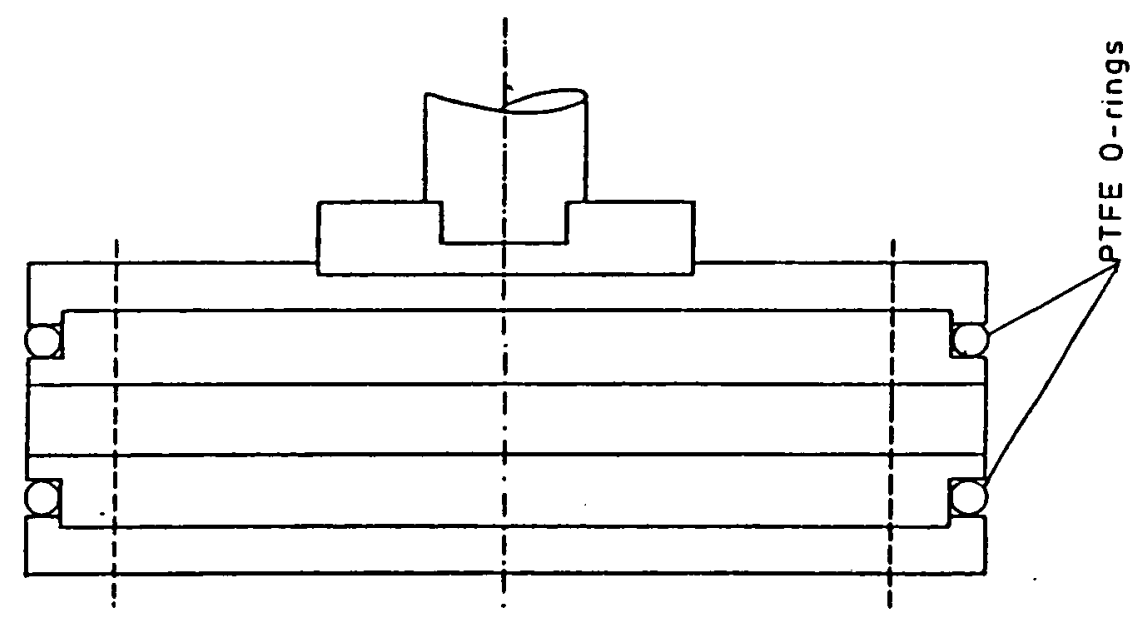

a.

Figure (4.10) Development of Piston Design 


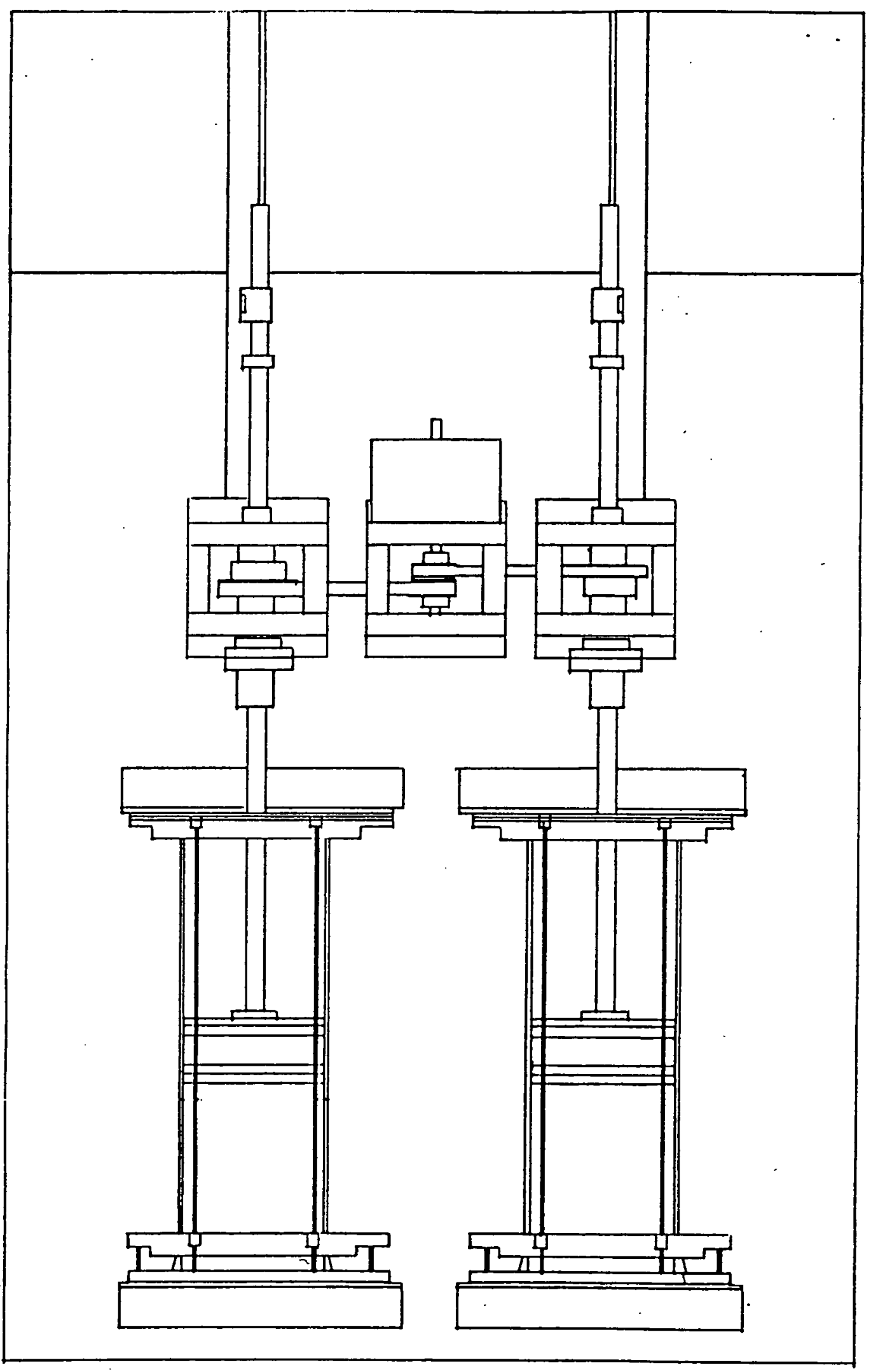

Figure (4.11) Compressor - Plan View 
$\vec{\Xi}$

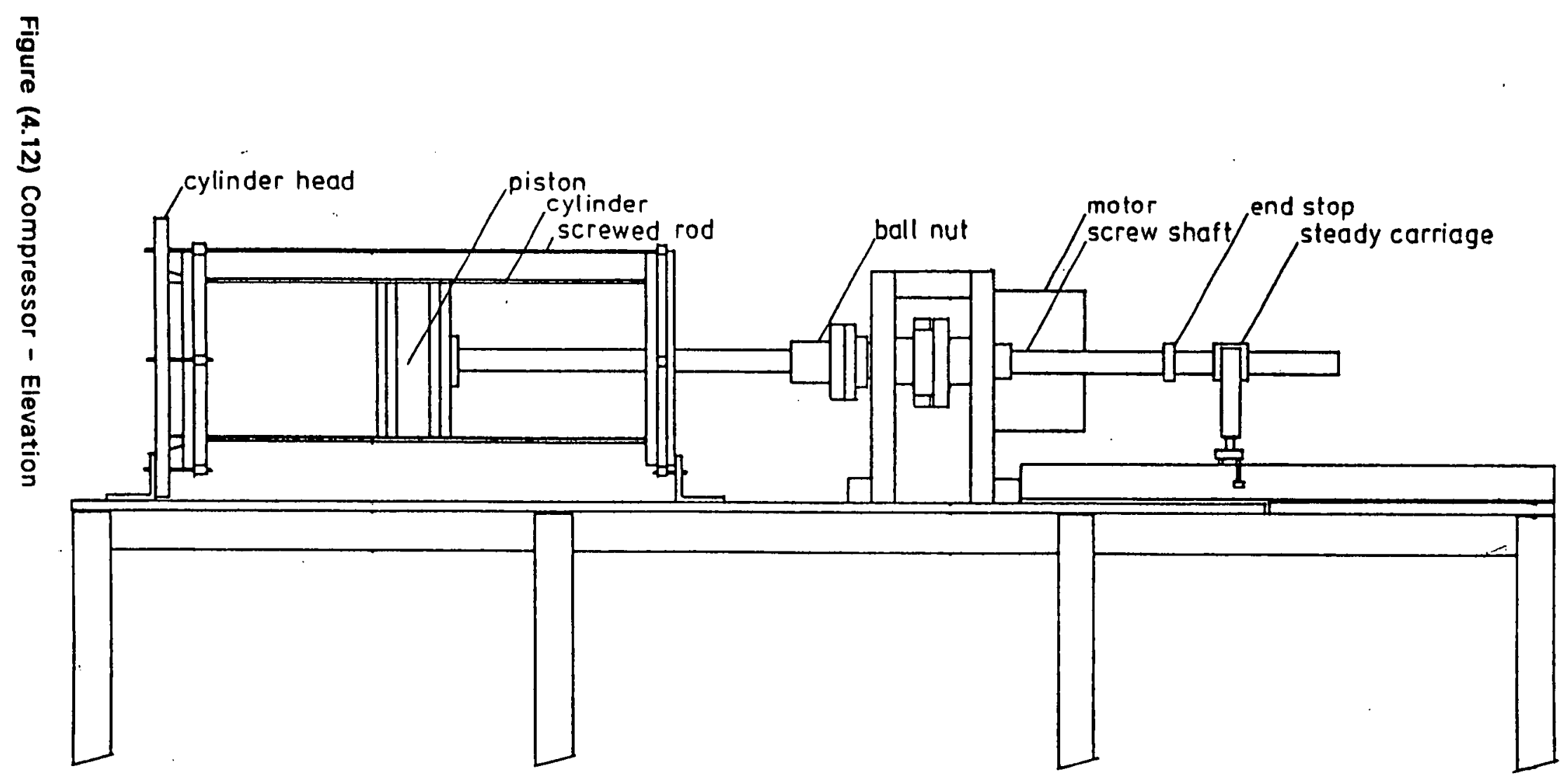




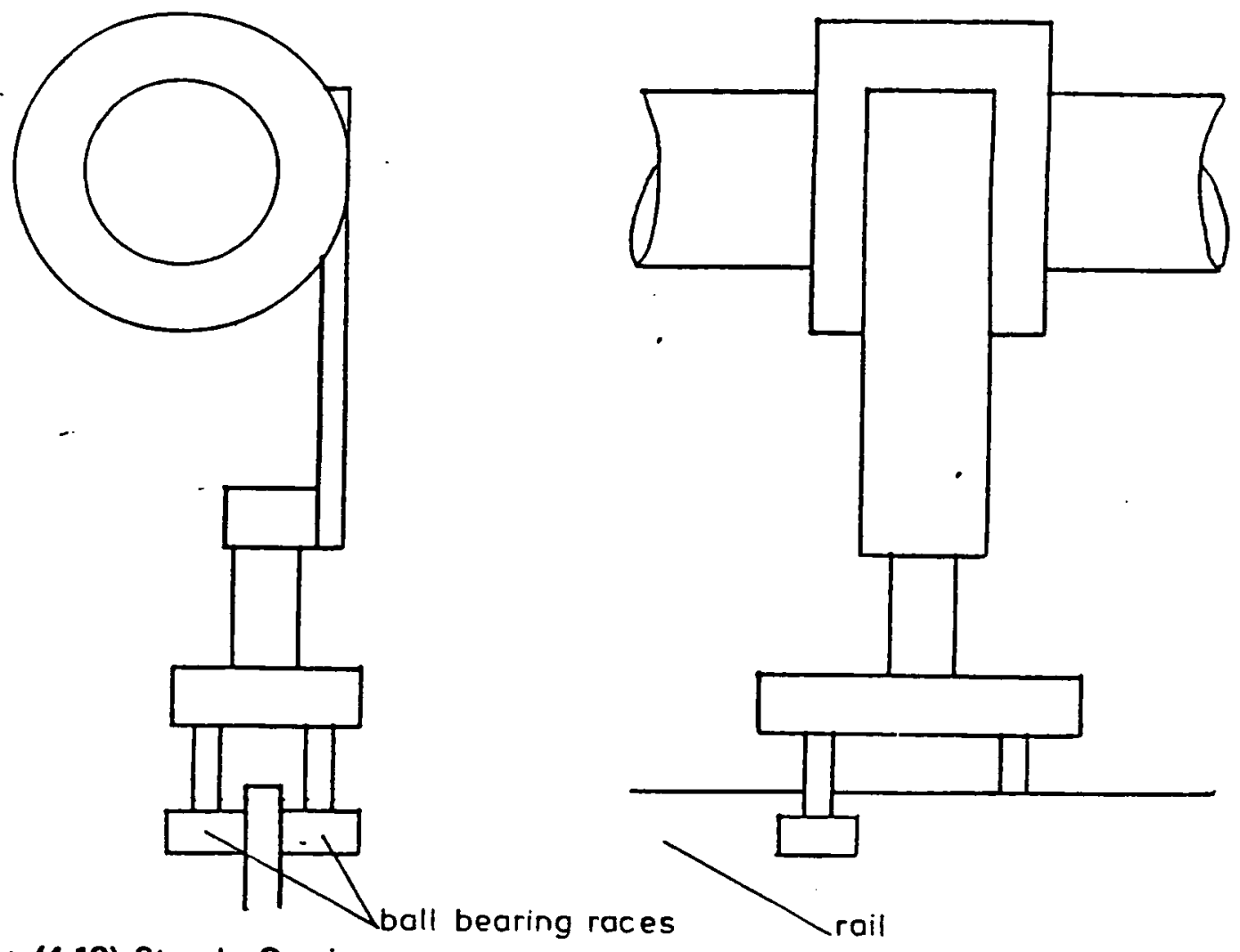

Figure (4.13) Steady Carriage

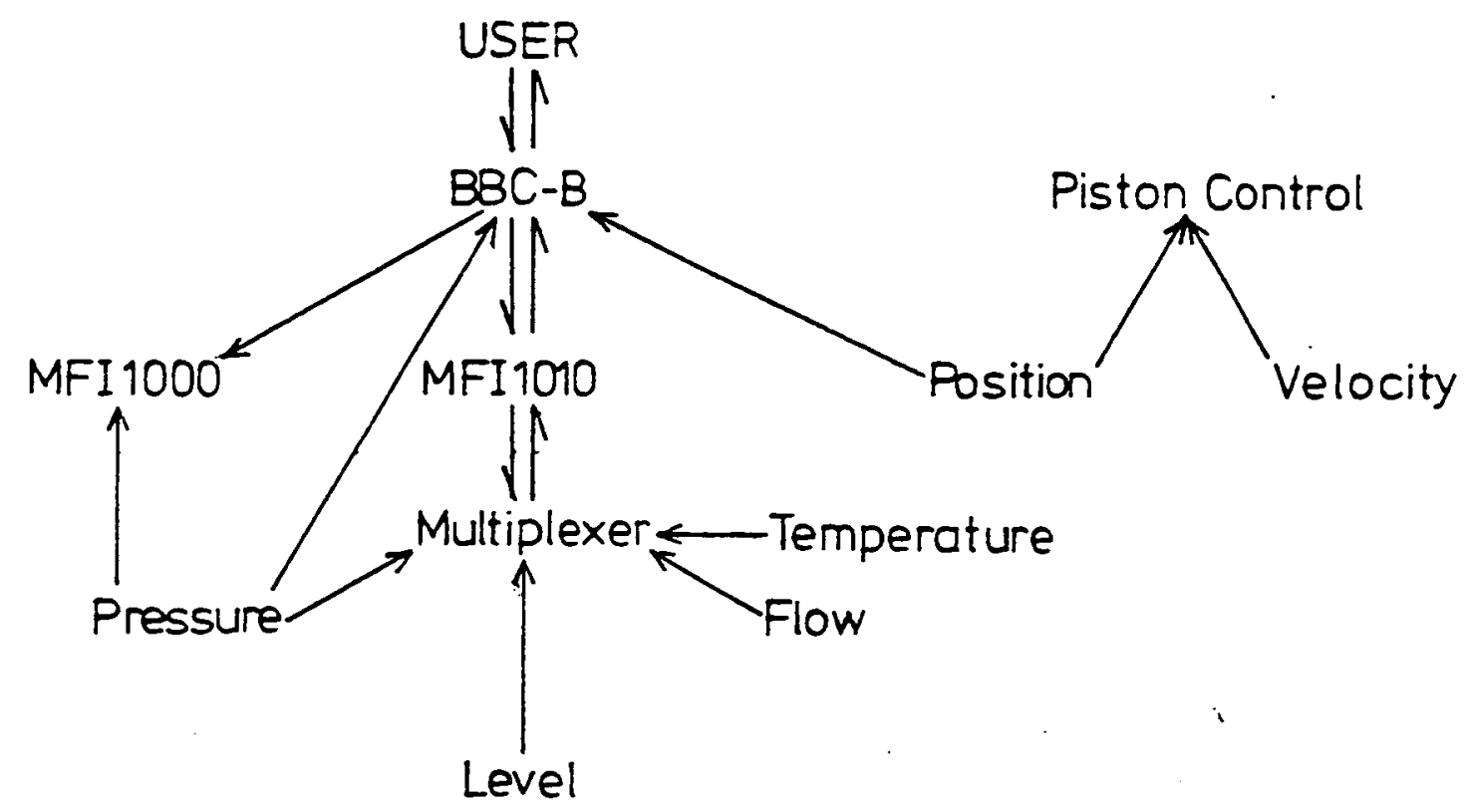

Figure (4.14) Break-Down of Control Functions 


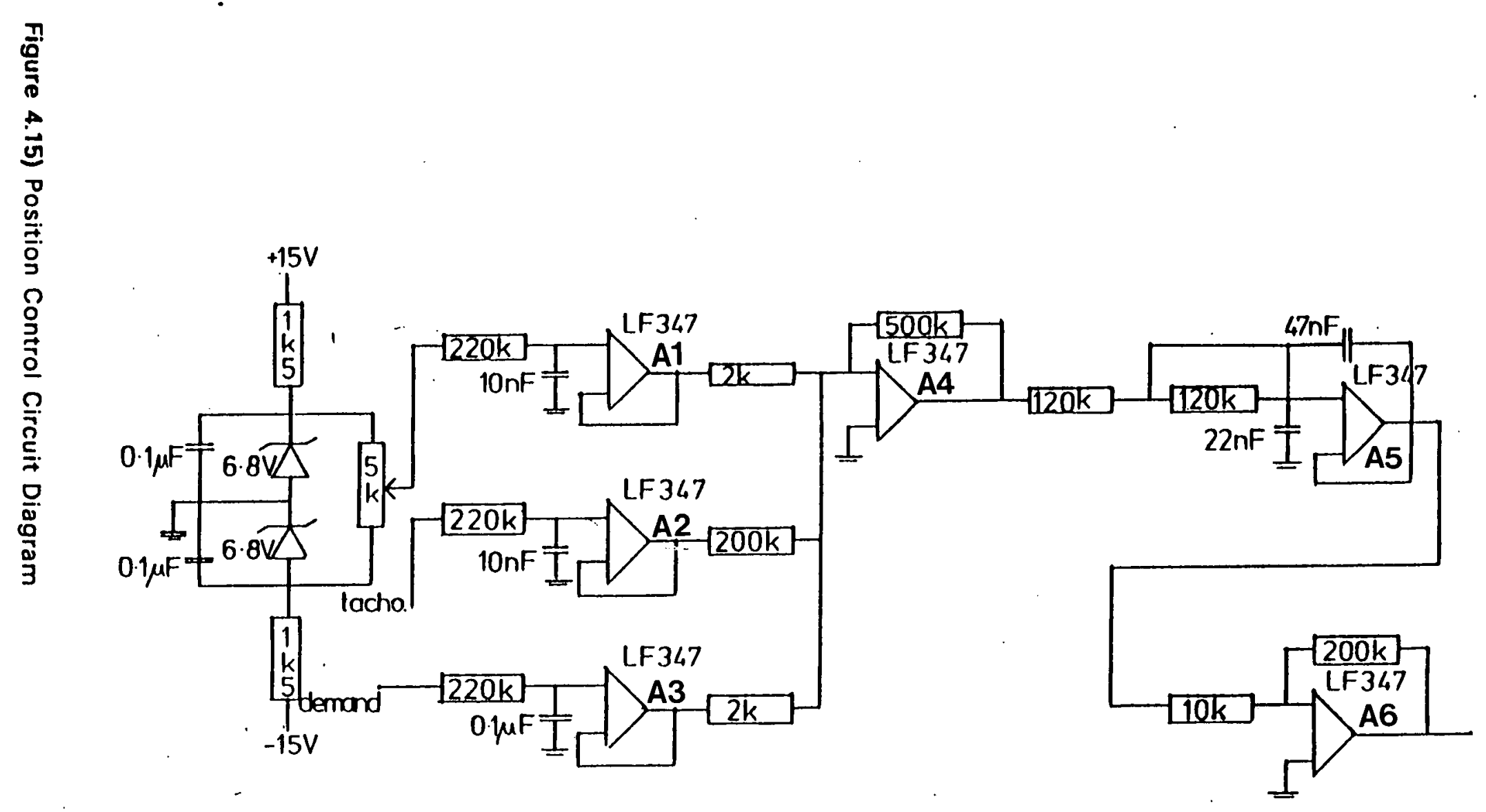




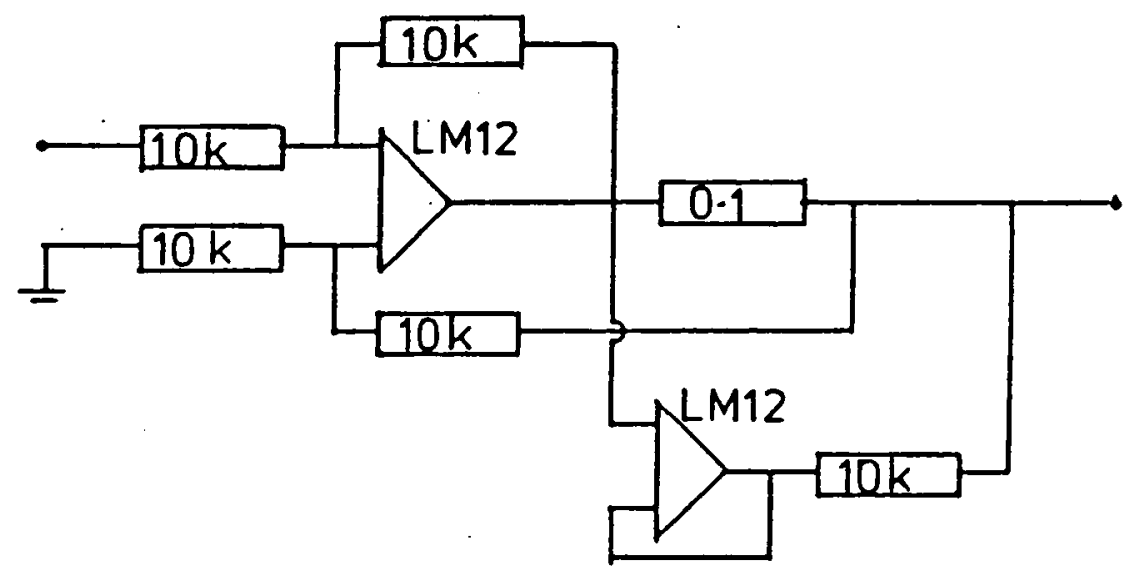

Figure (4.16) Simple Amplifier Circuit 


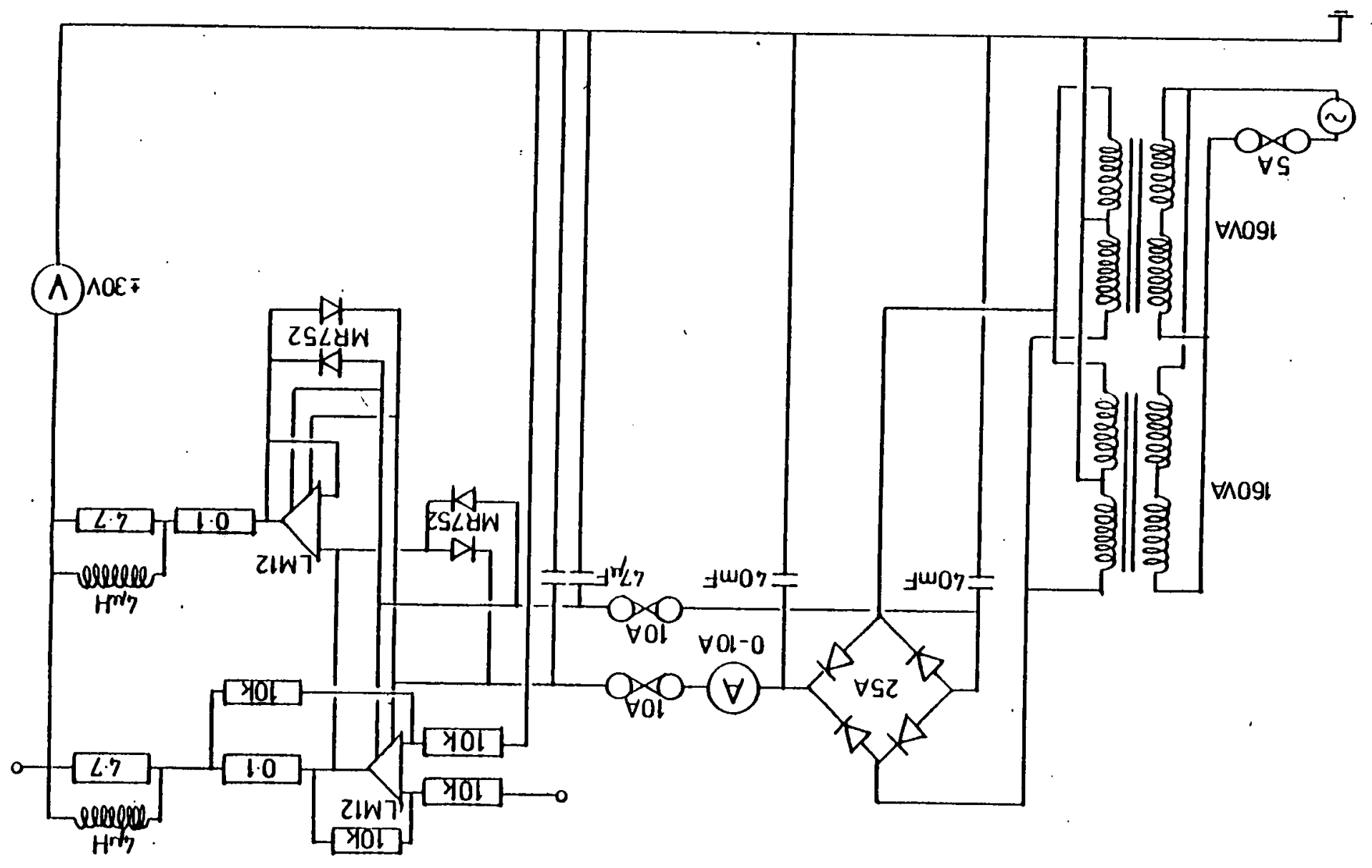

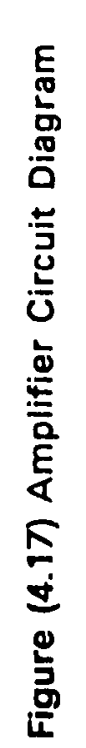



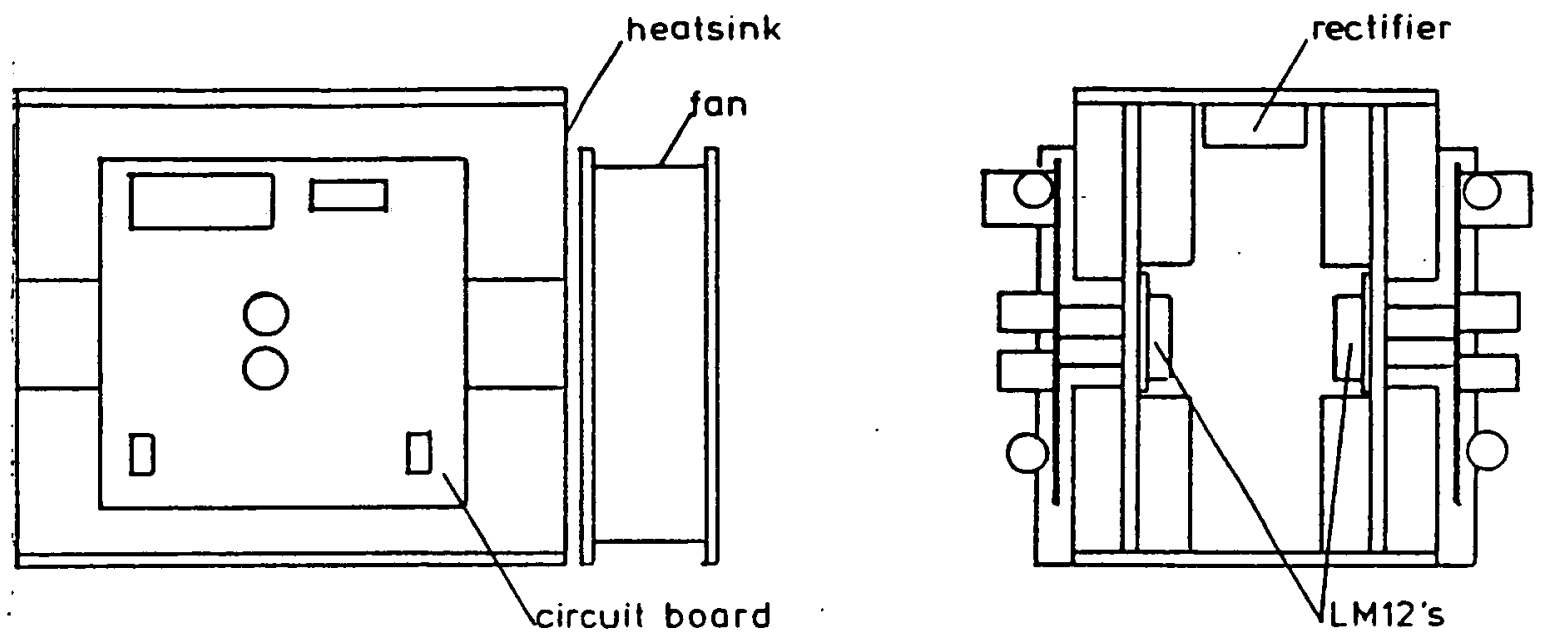

Figure (4.18) Mounting of LM12's
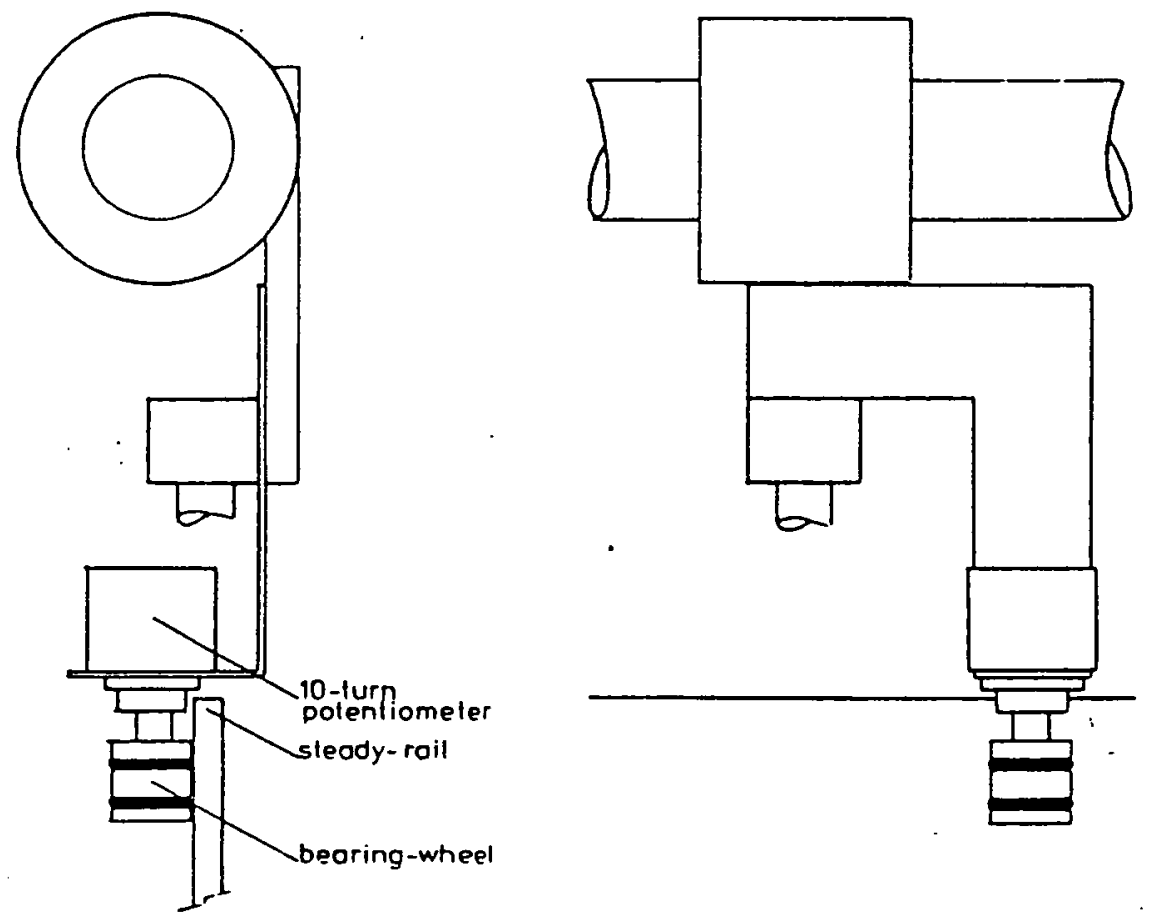

Figure (4.19) Mounting of Position Sensor 


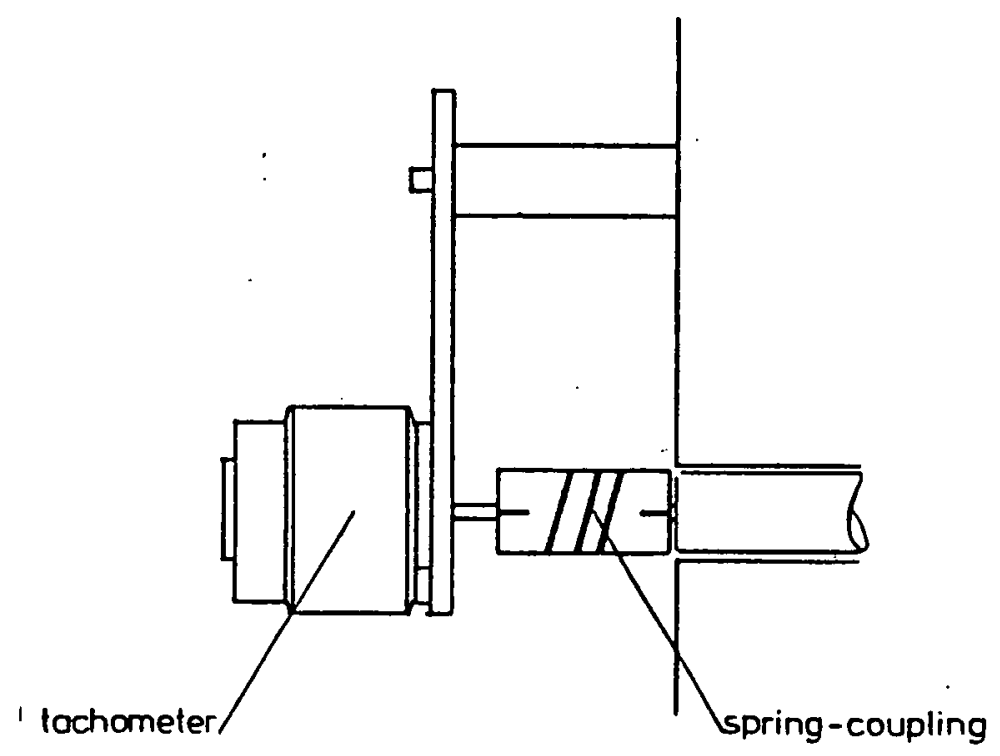

Figure (4.20) Mounting of Tachometer 


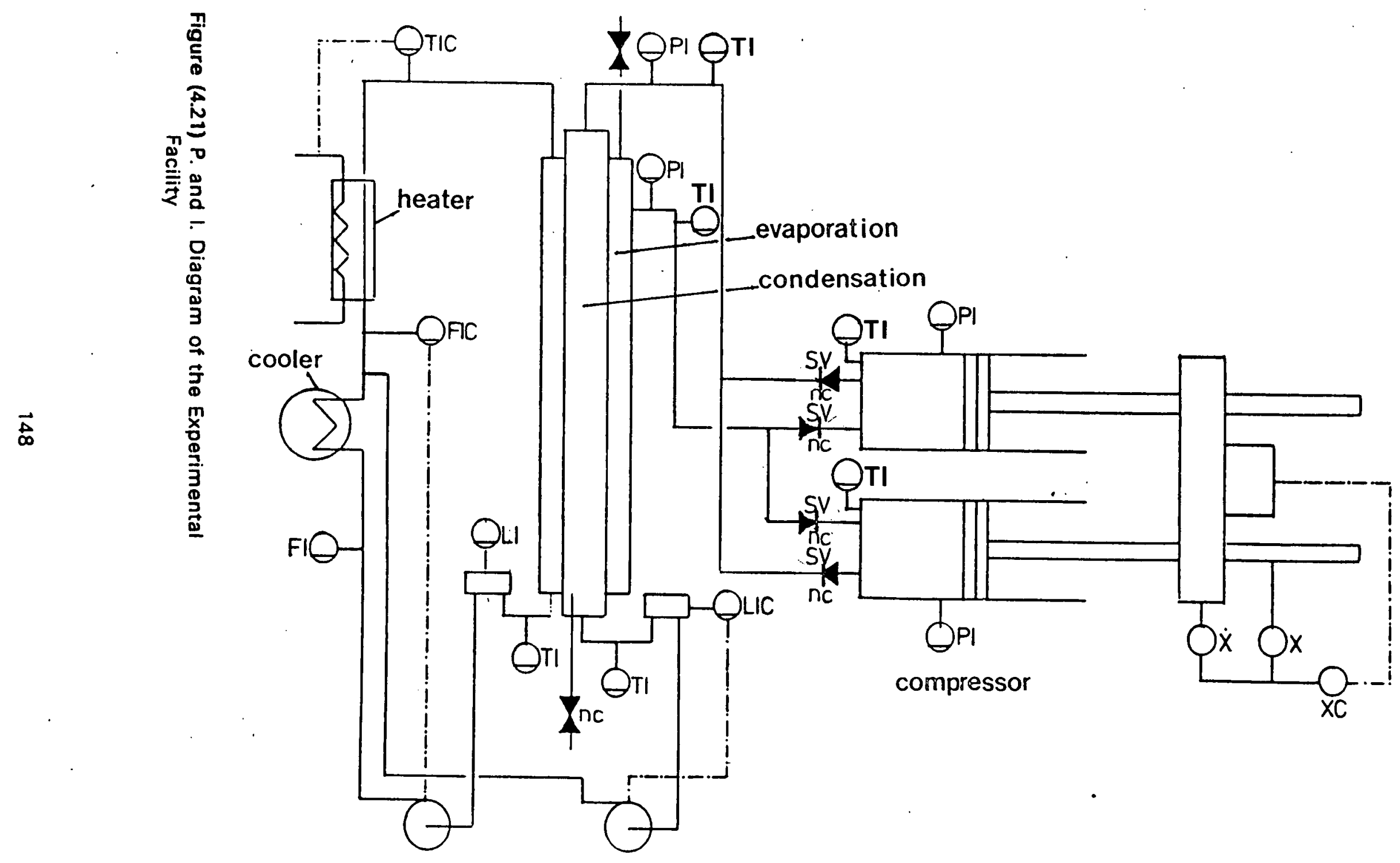




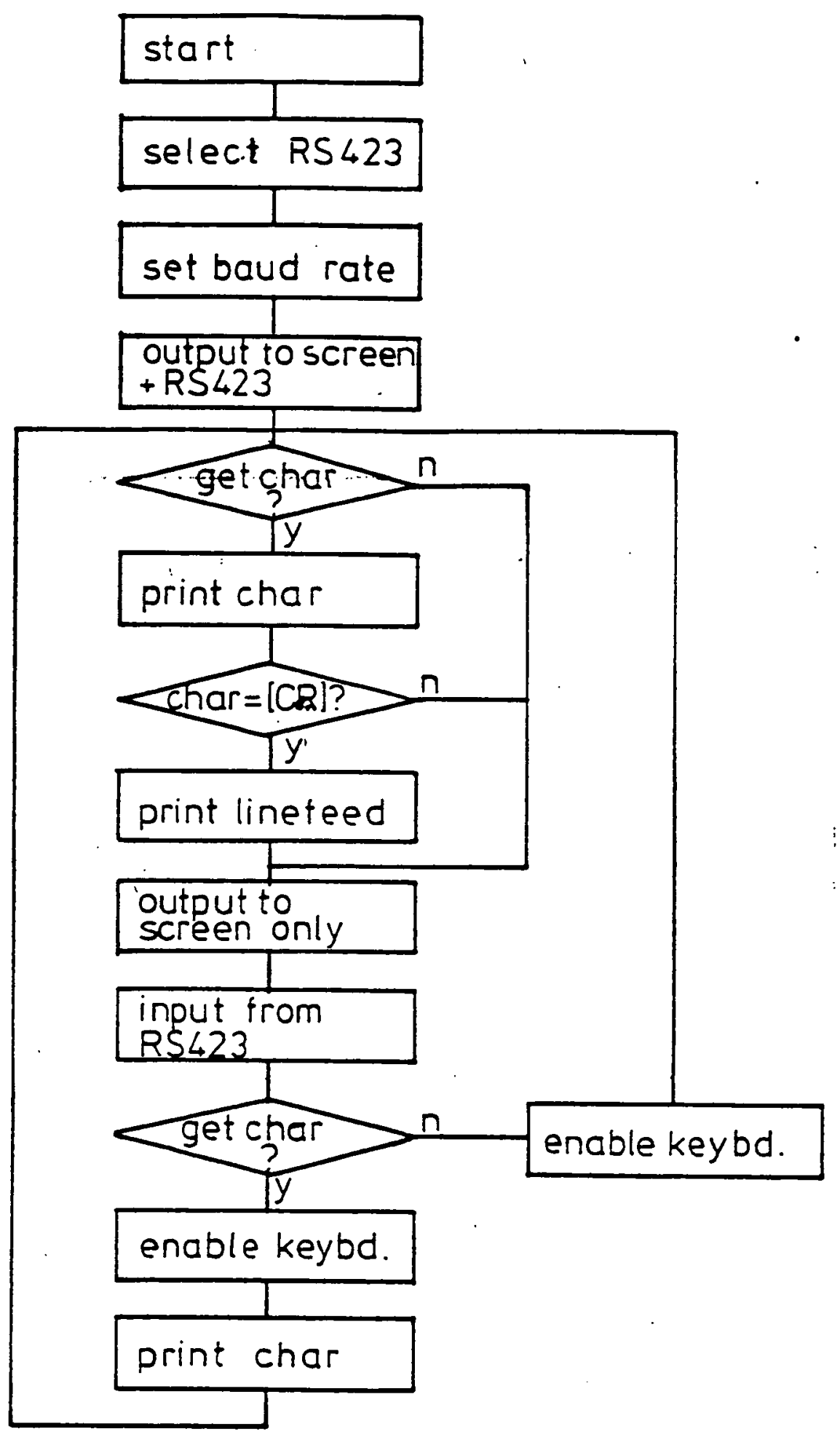

Figure (4.22) Simple Flowsheet for MFI Communication 


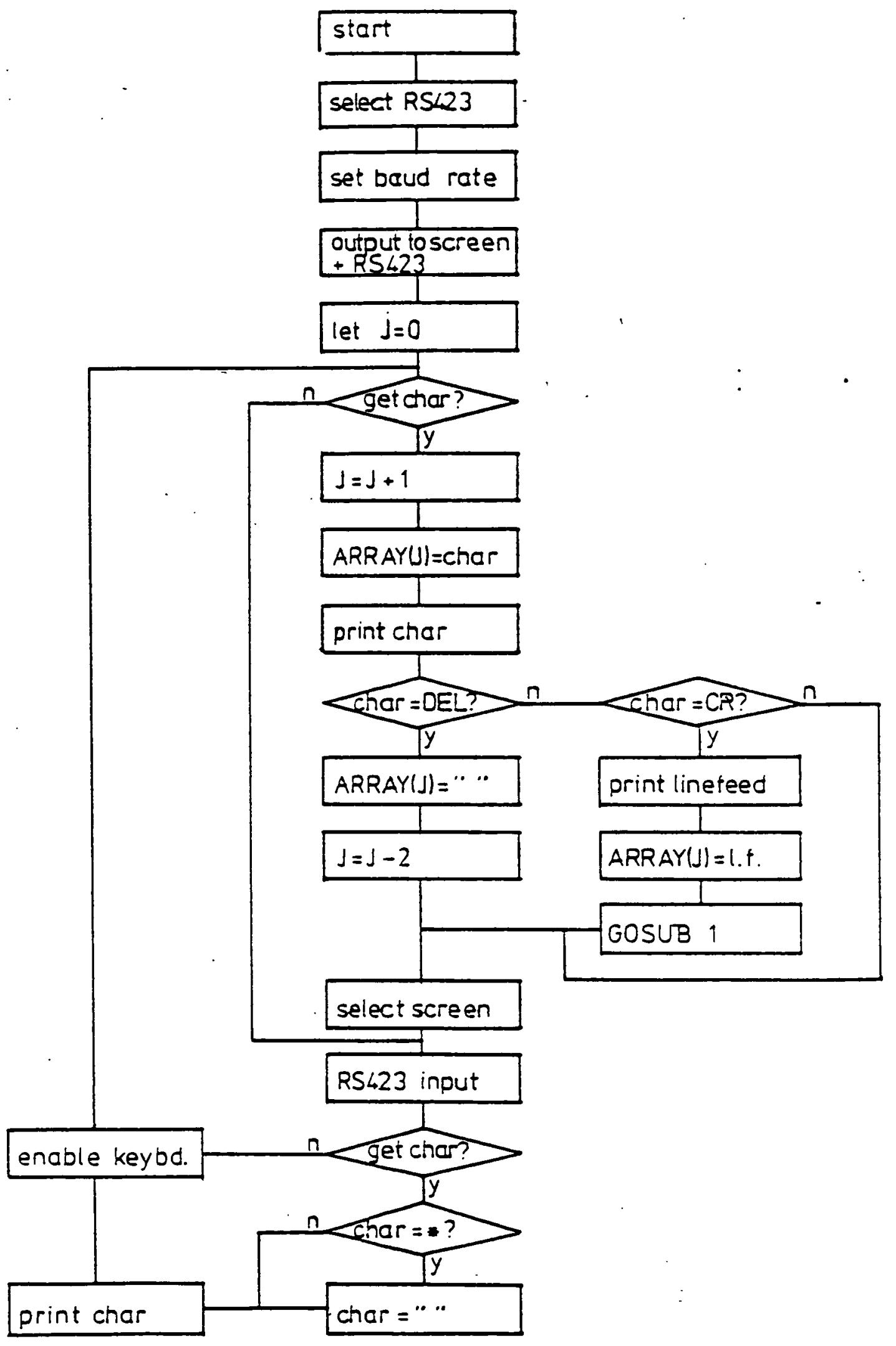

Figure (4.23) Improved FLowsheet for MFI Communication 


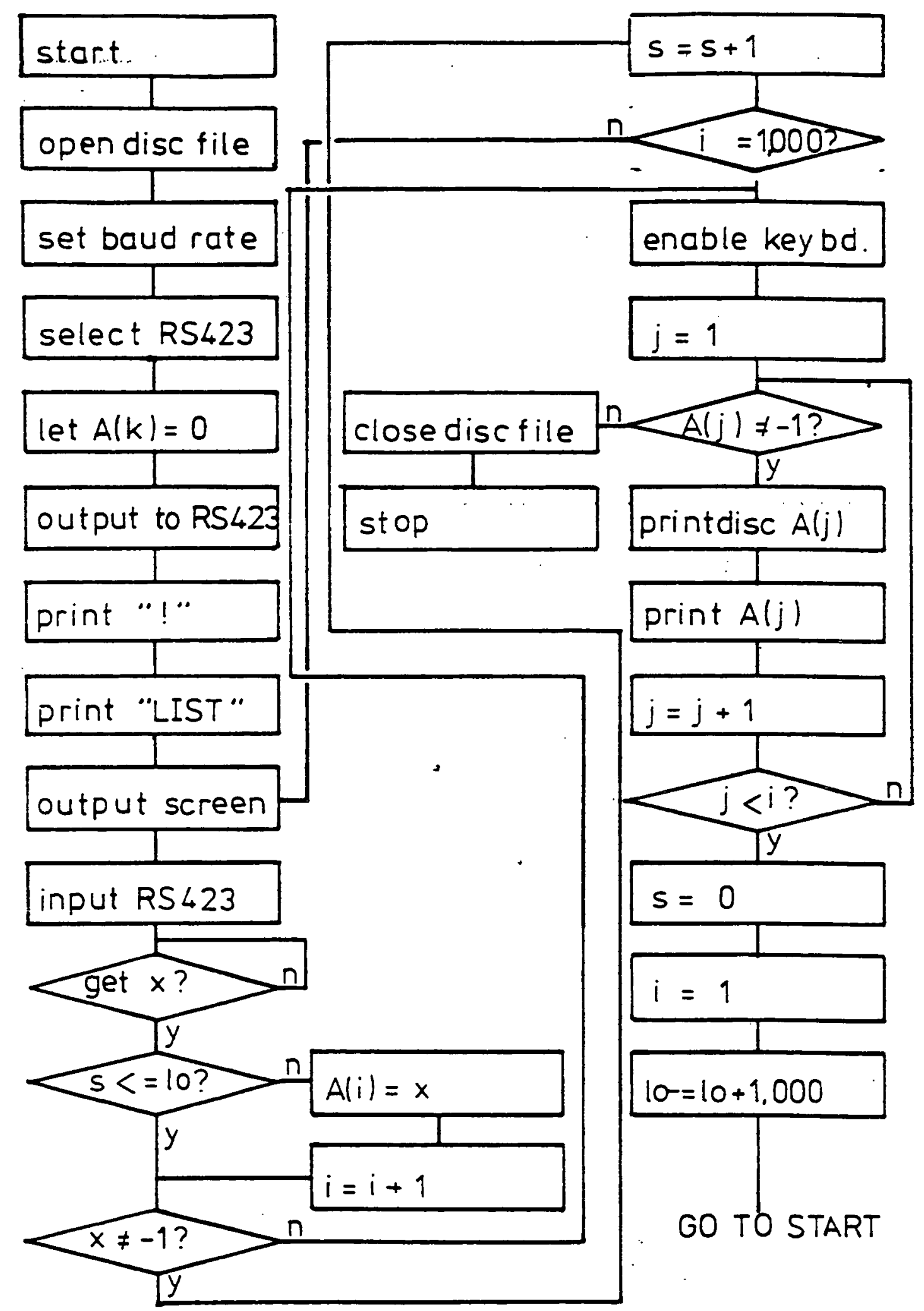

Figure (4.24) Flowsheet for Disc Storage of MFI Program 


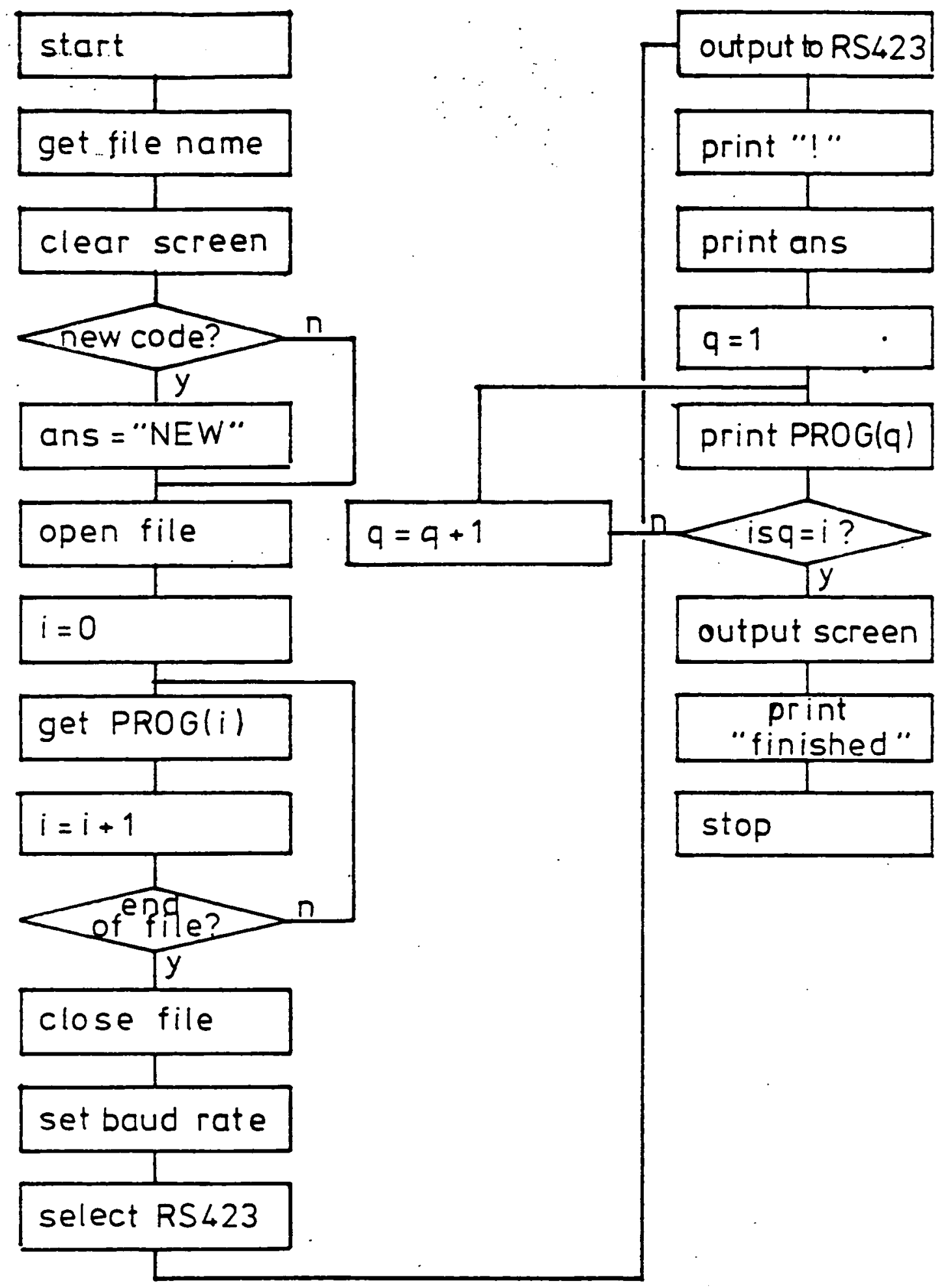

Figure (4.25) Flowsheet for Retrieval of Stored Program 
$D(1)$ : temp. cylinder $1 \quad D(20)-D(24)$ : feed flow control $D(2)$ : temp. cylinder 2 variables $D(3)$ : temp. evap. liquid $D(4)$ : temp. condensate $D(25)-D(29)$ : feed temp.control $D(5)$ : temp cond vapour variables $D(6)$ : temp. evap. vapour $D(7)$ : temp. feed liquid $D(31)$ : cold junction compen$D(B)$ : ambient temp. sation

$D(9)$ : pres. cond. vapour $D(51)$ : feed temp. setpoint $D(10)$ : pres. evap. vapour $D(11)$ : pres.cylinder $2 \quad D(61)$ : feed flow setpoint D(12): pres. cylinder 1

$D(71)$ : condensate level set $D(13)$ : condensate level point . $D(14)$ : evap. liquid level $D(72)$ : condensate level reset

$D(16)$ : evap. liquid flow $D(17)$ : feed liquid flow

Figure (4.26) Parameters Stored in MFI Array 


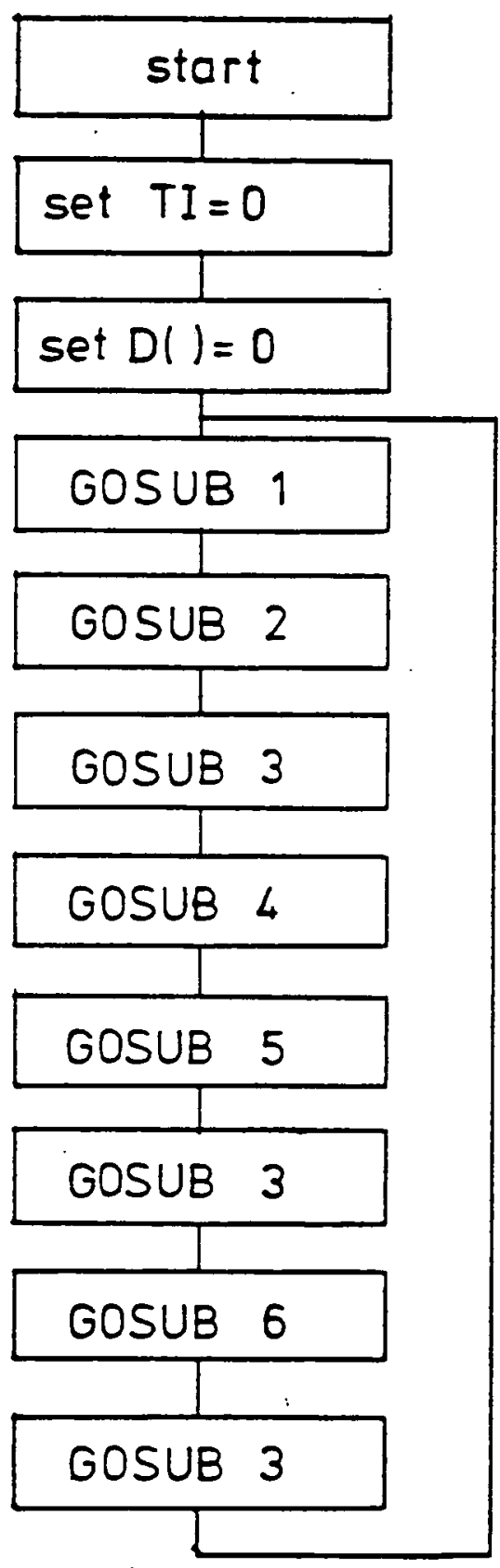

GOSUB 1: reads I (1-8); convert to $C$; store as $D(1-8)$

GOSUB2: switch multiplexer; read I(10): convert; store as $D(9-16)$

GOSUB 3: print dato to BBC on demand

GOSUB L: switch condensate pump on level

GOSUB 5: control feed pump on flow vio 01

GOSUB 6:control heater on feed temperature vio 02

Figure (4.27) Flowsheet for MFI Control and Monitoring Program 


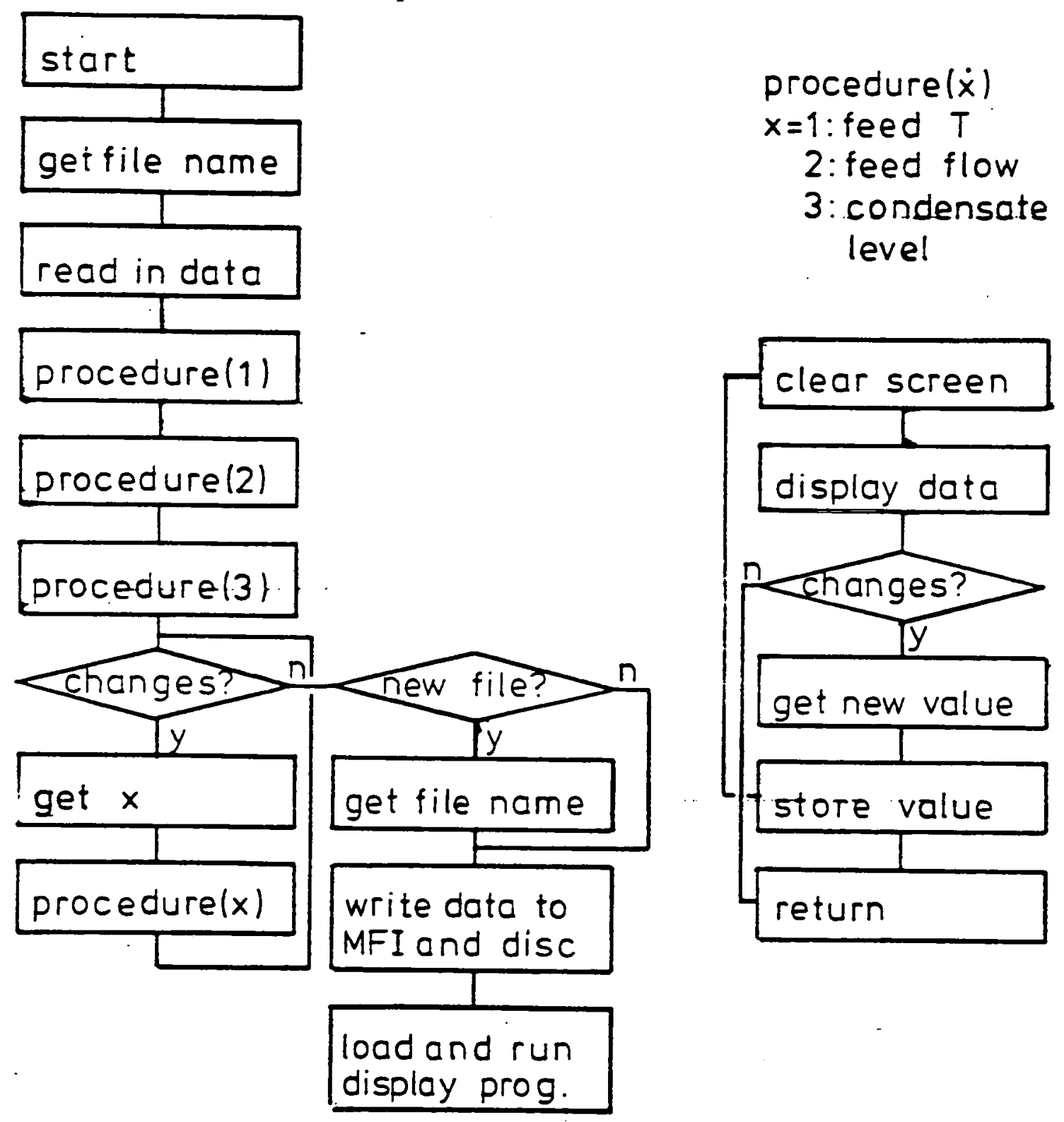

Figure (4.28)Flowsheet for Control Parameter Setting Program 


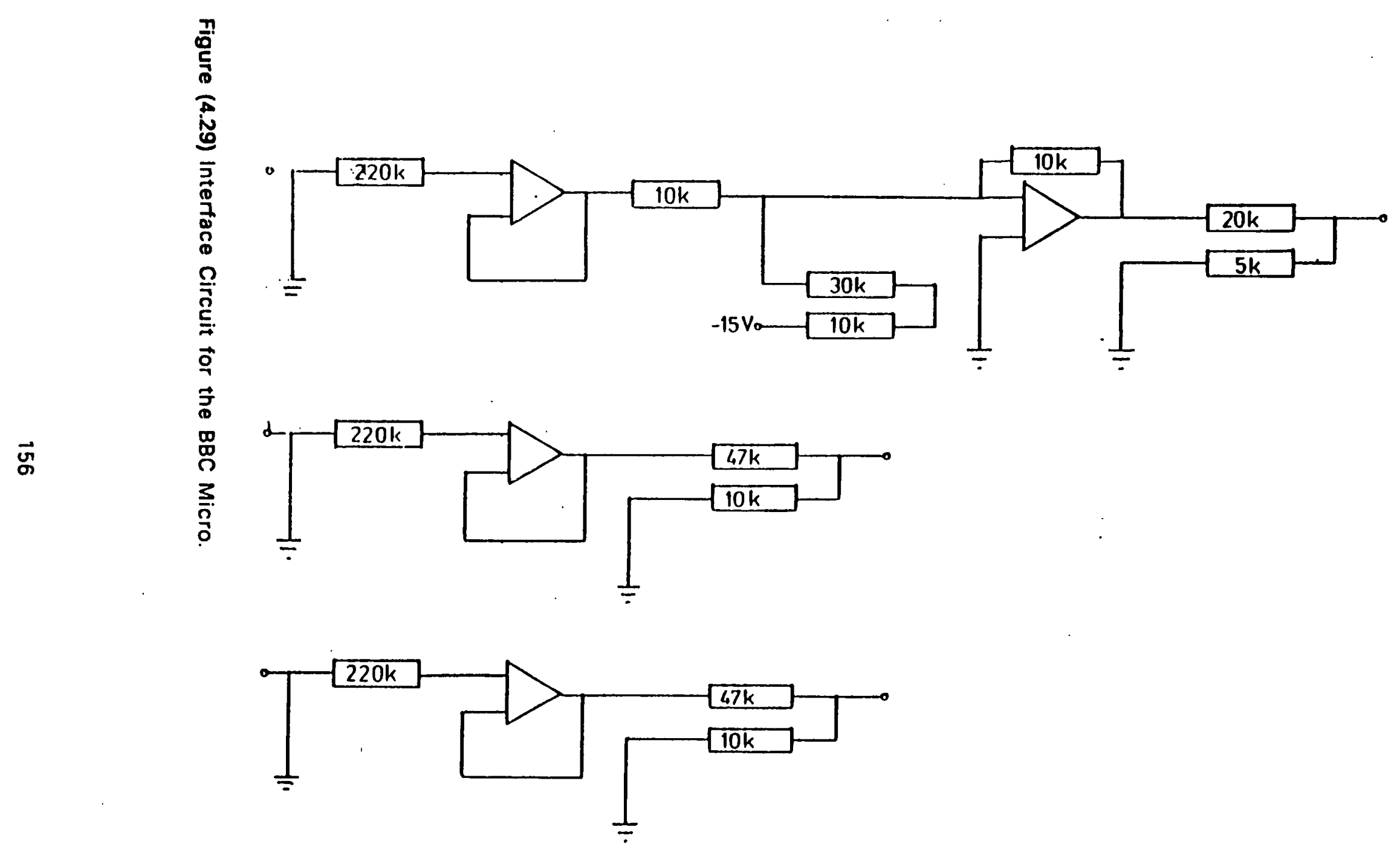




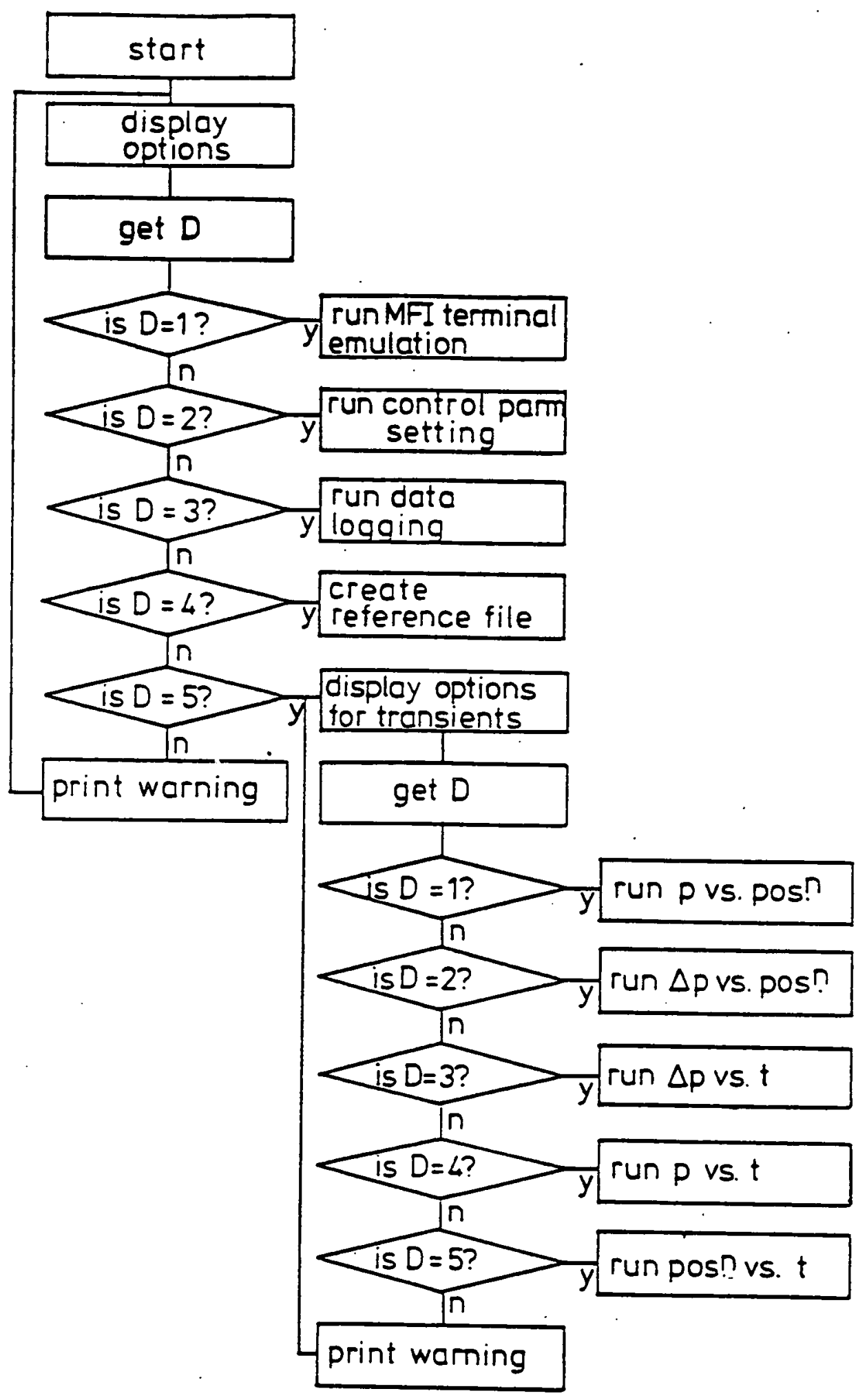

Figure (4.30) Flowsheet for Menu System 


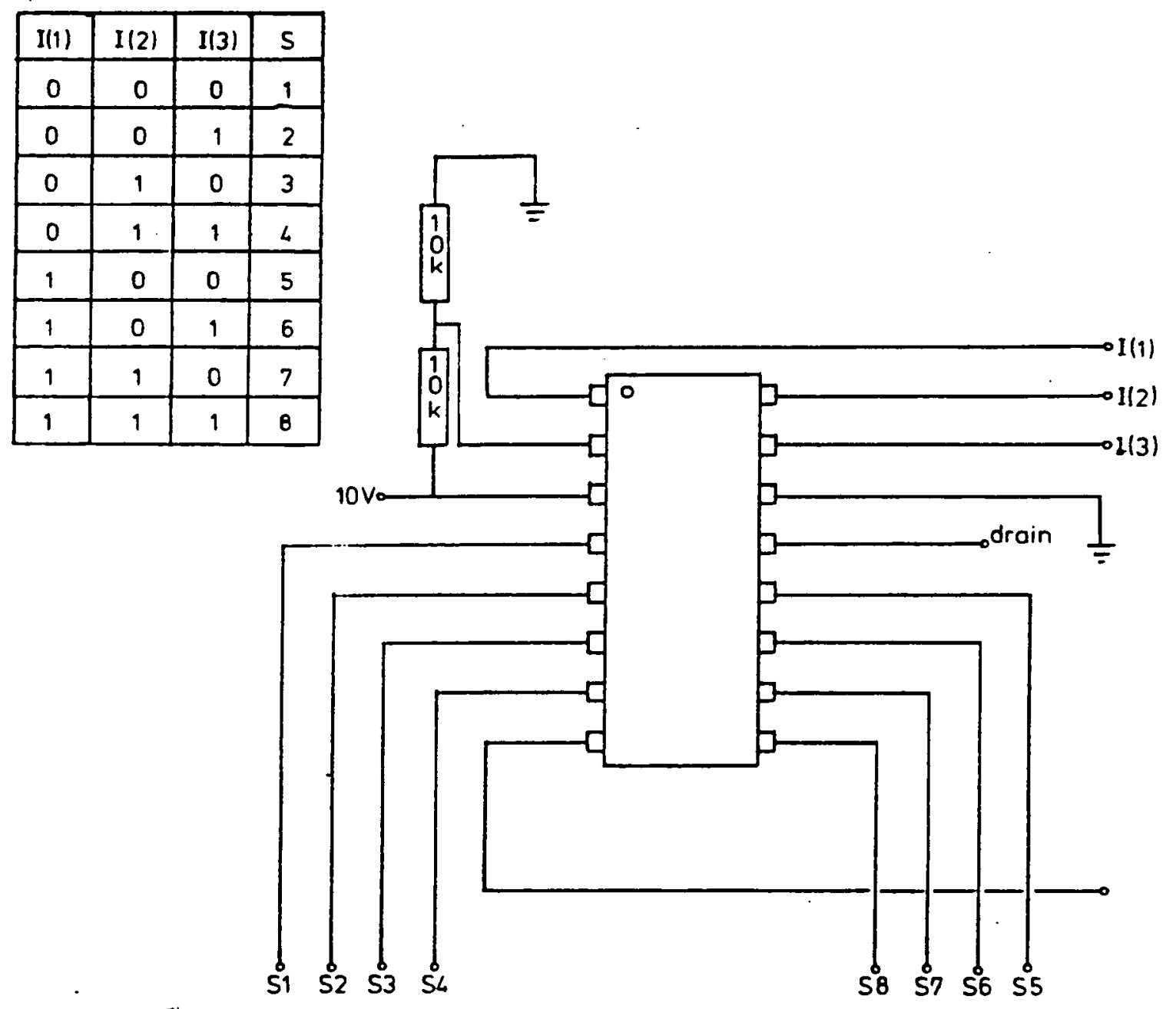

Figure (4.31) Multiplexer Circuit Diagram 


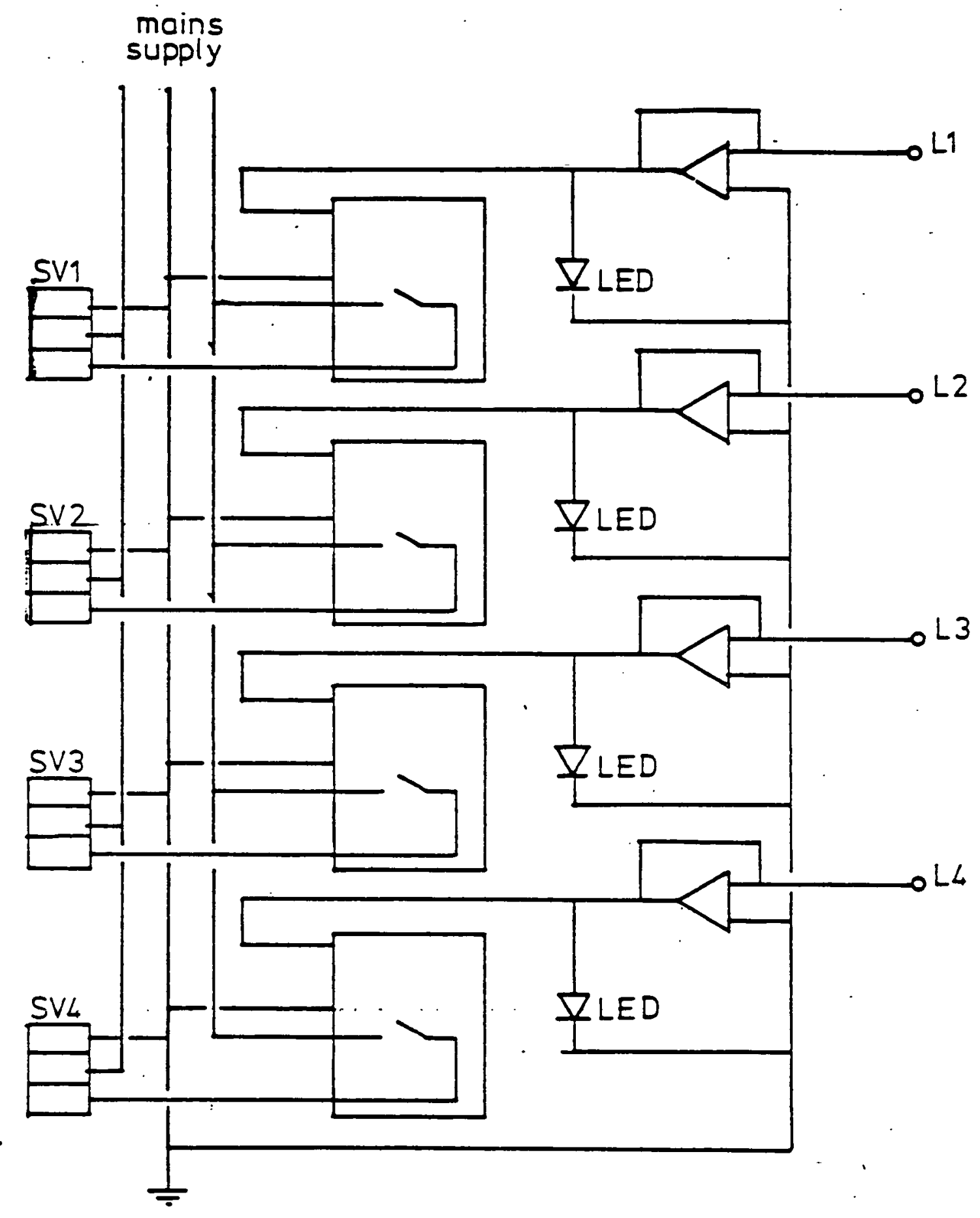

Figure (4.32) Solenoid Valve Relay Wiring Diagram 


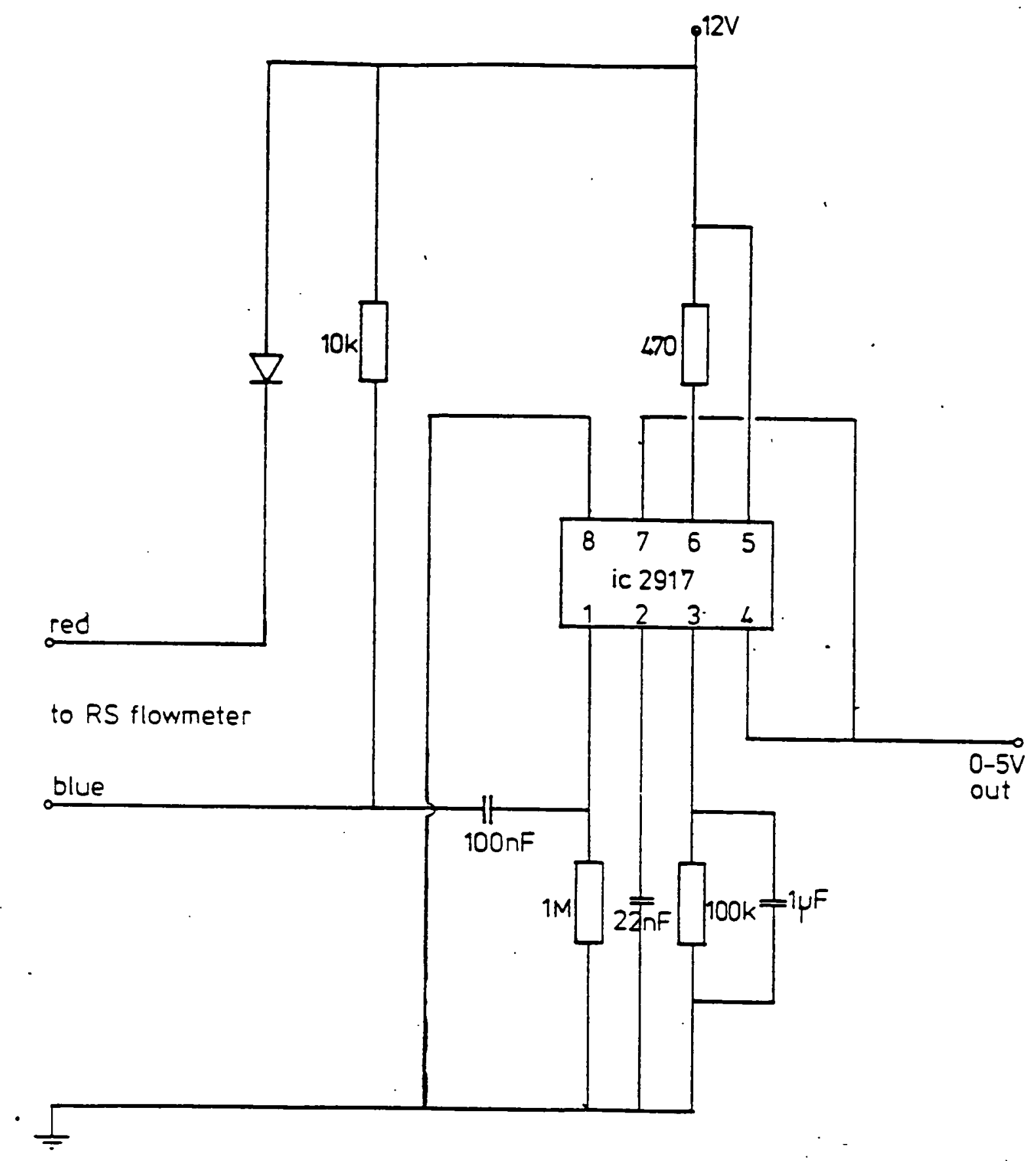

Figure (4.33) Tachometer i.c. Circuit Diagram 


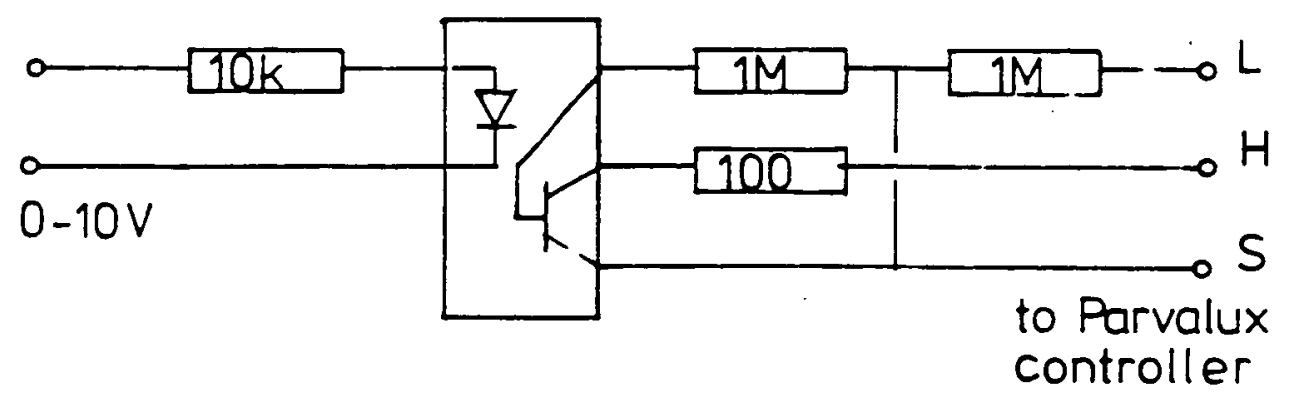

Figure (4.34) Opto-Isolator i.c. Circuit Diagram 

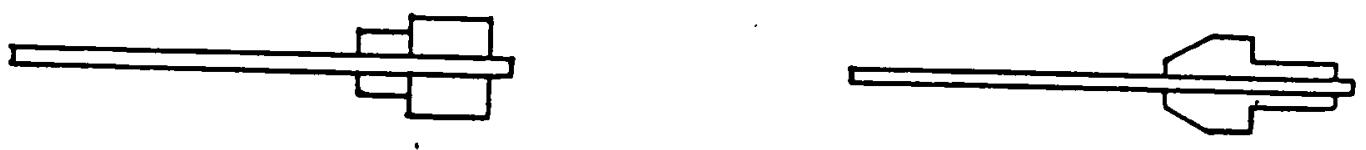

Figure (4.35) Thermocouple Probe Assemblies
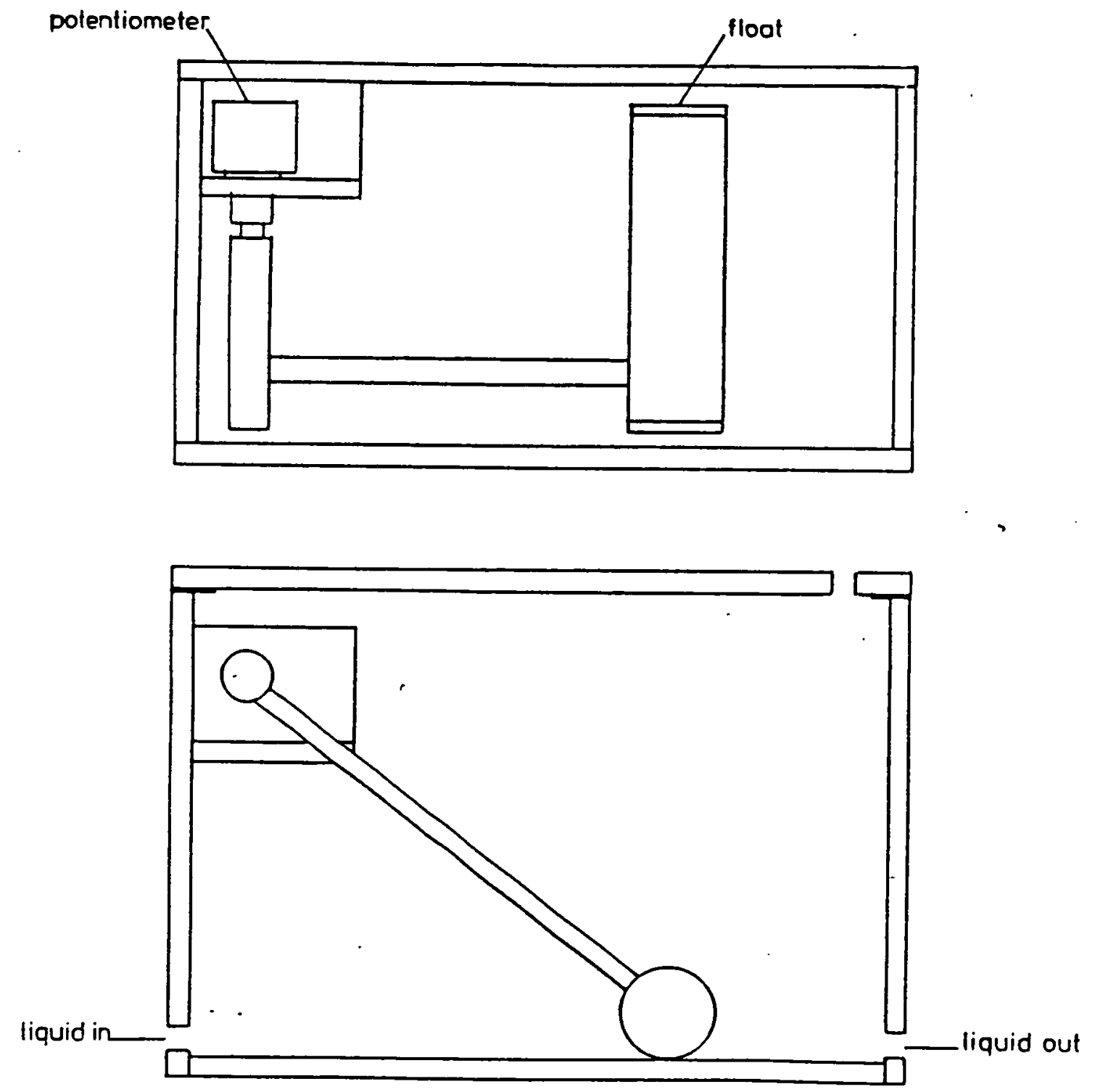

Figure (4.36) Level Measurment Boxes 


\section{CHAPTER 5}

RESULTS AND ANALYSIS 


\subsection{RESULTS FROM THE FIRST MODEL}

\subsubsection{Transient Performance Predictions}

The first model was formulated at an early stage in the project. At that point, only approximate, steady-state calculations had been carried out, with the purpose of estimating the likely performance of the duck in terms of fresh water production and specific energy consumption. Such calculations could not, however, reveal the nature of the transient behaviour of the duck and therefore could not begin to provide any evidence of the feasibility of a desalination duck as a wave power absorber. It was important to be able to study transient performance predictions ( $\Delta p$ vs. $\theta$ ); this was therefore one of the principal motives behind the construction and use of the first model.

As discussed in chapter 2, section 2.2.3, much of the research work carried out in the study of the Edinburgh duck as a wave energy absorber had been concerned with the optimisation of its performance through the choice of the inertial, spring and damping coefficients in the equation of motion:

$$
1 . d^{2} \theta / d t^{2}+D . d \theta / d t+S . \theta=F_{\text {wave }}
$$

Thus, the force produced by the duck in reaction to the wave induced force was always a linear function of $\theta$ and its first and second time derivatives. It was generally assumed that such a force characteristic as was required for optimum absorption efficiency could be achieved through sophisticated manipulation of the power take-off mechanisms in a full-scale device (as well as through the careful design of the shape of the duck; of its ballasting, and its mooring arrangements). It was recognised that for a desalination duck no such manipulation would be possible, as there would be no suitable mechanism available to carry it out. The "global" parameters of duck shape, mooring design and ballast distribution, however, were still available to the designer and so it was hoped that a programme of small-scale tank tests could be used to study the absorption characteristics of ducks in much the same way as for power generating ducks. In order for this to have been possible it would have been necessary to impose a reactive force on the model duck to simulate the scaled pressure difference force that would be experienced by a full-scale device. A primary aim for the first model was, 
therefore, to determine whether the $\Delta p$ across the duck could be represented by an equation such as equation (5.1)

Plotting $\Delta p$ vs. $\theta$ from an expression such as equation (5.1) produces a "tilted" ellipsoid, as in figure (5.1). The ratio of the lengths of the axes of the ellipse and the angle of the major axis from the horizontal are related to the coefficients in the equation. Plots of $\Delta p$ vs. $\theta$ as predicted by the first model produced similar shapes, although they were less regular than the linear model (see figure (5.2)). Qualitatively, therefore, it seemed possible that $\Delta p$ could be adequately represented as a linear function of $\theta$ and its time derivatives. The problem, then, was to find a means of determining the motion coefficients from the predicted data; to this end, a number of program runs were carried out using a series of sinusoids with variable amplitudes and periods (as opposed to the more usual monochromatic "seas" used). It was noted that, in these cases, the pressure difference seemed to depend not only upon $\theta$ and its derivatives, but also upon the shape of the preceding wave. This observation implied that the pressure force characteristic was not only non-linear but also time dependent. In order to check more rigorously for the existence of time dependency, a simple test was devised. Two sets of four waves were used for two program runs; the first three waves in either set were different, but the final waves were the same in both cases. If time dependency was not present, then the pressure force vs. time plots for both sets would be the same. Results of such a test are shown in figure (5.3); it was clear from such plots that time dependency did exist. As previously discussed (see chapter 2, section 2.2.3), the existence of time dependency effectively removed the possibility of small-scale tank testing for desalination ducks. It was immediately clear, therefore, that it would be necessary to adopt a different approach to the study of this subject.

\subsubsection{Performance Predictions}

The first model was also used to analyse the performance of the duck as a desalination process. This study consisted of running the program with various values of amplitude and period of motion; primary heat exchange area; feed temperature, and blowdown salt concentration and noting the effect on mean fresh water production and specific energy consumption (SEC). 


\section{Amplitude and Period}

For a conventional VC process (see section 2.1.3) with a reciprocating compressor, one would expect the output of the plant to be proportional to the product of stroke length and frequency. Quite simply, this product represents the amount of vapour handled by the compressor and, therefore, the amount of condensate produced (for constant suction and exit conditions). The results from the first model (see figure (5.4)) suggested that the duck would exhibit just such a characteristic. Amplitudes in the range 0.3-0.6 radians (in 0.1 radian steps) and periods in the range 4-12 seconds (in 1 second steps) were simulated. Plotting the mean rate of production against the ratio of amplitude to period gave a straight line passing through the origin.

The SEC was calculated from equation (5.2):

$$
S E C=\Delta p \cdot(d \theta / d t) /\left.m_{p}\right|_{\text {mean }}
$$

It has already been shown that the mass flowrate is proportional to the ratio of swept angle to period (the mean angular velocity):

$$
m_{p} \propto \theta / T \propto d \theta /\left.d t\right|_{\text {mean }}
$$

then equation (5.2) may be rearranged to give:

$$
\left.S E C \propto \Delta p\right|_{\text {mean }}
$$

Now, over a small pressure range:

$$
\Delta T \propto \Delta p \propto m_{p}
$$

Therefore:

$$
S E C \propto m_{p}
$$

The same result may be derived from a consideration of the overall mass and energy balances over the system:

$$
\text { SEC }=C_{p} \cdot\left(m_{p} \cdot\left(T_{D}-T_{f}\right)+m_{b} \cdot\left(T_{b}-T_{f}\right)\right) / m_{p}
$$

To a first approximation, the blowdown temperature will be similar to that of the feed, and so: 


$$
S E C=C_{p} \cdot\left(T_{p}-T_{b}\right)
$$

therefore:

$$
S E C \propto m_{p}
$$

as in equation (5.6). A plot of the SEC vs. $m_{p}$ for a primary heat exchange area of $1500 \mathrm{~m}^{2}$ is shown in figure (5.5).

The model was also used to investigate the effect of primary heat exchange area, feed temperature and blowdown/feed ratio upon the performance of the duck. As one would expect for a conventional vC system, output was found to rise linearly with area (see figure (5.6)); to increase slowly with feed temperature (see figure (5.7)) and to be virtually unaffected by blowdown salt concentration. Thus, the analysis of the results from this model showed that the predicted performance of the duck was very similar to that of a conventional VC plant.

\subsection{EXPERIMENTAL PROGRAMME}

As described in chapter 1 , the experimental work of the project was designed to produce results which could be used to estimate the validity of the mathematical models constructed. Accordingly, the second part of this section will deal with the comparison between experimental and simulated results; the first part will deal with the experimental programme in detail.

\subsubsection{Procedure}

A schedule of experimental runs was planned in which transient data ( $p$ s. $v$ and $\Delta p$ vs. $v$ ) were to be logged at a series of compressor frequencies and amplitudes. Amplitudes in the range $6-11 \mathrm{~cm}$ and periods in the range 4-12 seconds were to be used; not all possible combinations of these parameters were available because of the limits of operation of the servo motor and the amplifier in the compressor drive system. Low piston velocities could lead to too high currents at too low voltages (leading to over-heating of the LM12's, see section 4.3.2) and high velocities could lead to amplifier voltage limiting. Between these extremes, however, there was found to be a good range of sinusoids which could be employed. It was originally planned to go on to study the effects of other process parameters on both transient and long term 
mean performance; time constraints, however, meant that only the transient data described could be gathered.

Whatever run was planned, each experiment started in the same manner, as follows. During the course of the experimental programme, a thermostatically controlled fan heater was left switched on in the equipment room; maintaining the temperature in the room, and thus of the equipment, at around $24-25^{\circ} \mathrm{C}$, close to the normal operating temperature of the rig. This not only reduced heat losses from the rig during operation, it also allowed the equipment to come to a steady-state more rapidly. Using the appropriate programs on the $B B C$ microcomputer, the feed temperature setpoint; feed flowrate setpoint, and condensate level switching band would be set and downloaded to the MFI1010 (see chapter 4, section 4.3.3). The control of the feed heater and the two peristaltic pumps would then be pessed to the MFI1010 and that of the solenoid valves to the MFI1000. With the still section of the rig thus in operation, the equipment would be left for some time to allow the feed temperature to come to its setpoint temperature (usually $24.5^{\circ} \mathrm{C}$ ) and for the equipment in general to warm up close to its operating temperature: a period of 20-30 minutes. After a suitable time had elapsed, the compressor could be started.

It was important to start the compressor very carefully so as to avoid sudden step changes in the demand signal to the position control hardware, as this could have lead to damage of the drive train and/or the amplifier. Firstly, with the amplifier switched off, the output from the control circuitry would be checked with a cathode ray oscilloscope (CRO). At this juncture any null point offset could be removed through adjustment of the position sensor (after checking that the pistons were indeed at the correct rest position). Still with the amplifier switched off, the waveform generator would be switched on, with the amplitude setting as low as possible and with the attenuation switch set to $-20 \mathrm{~dB}$. The output from the control circuitry would again be observed on the CRO and its period adjusted to an intermediate value (usually approximately 8 seconds). At such low periods it was necessary-to check the period using a stopwatch; the frequency dial of the wave form generator was only useful as a rough guide. As the CRO trace from the control circuitry passed through the null position, the amplifier itself would be switched on and at this point the pistons would start to move with a very low amplitude, 
generally about $2-3 \mathrm{~cm}$. After checking for normal operation (noise, vibration, current and voltage levels, etc.), the attenuation switch would be set to $0 \mathrm{~dB}$, again as the CRO trace passed through the null point. Finally the stroke length would be gradually increased to around $7 \mathrm{~cm}$ and the compressor allowed to run in this manner for, perhaps, 20 minutes.

During this period, the operation of the compressor would be carefully monitored. The drive system would be checked for normal operation (es above) and also for null point drift, which could be corrected through adjustment of the tension of the tie-wrap around the position sensor support. The apparatus would be purged of air using the shut-off valve on the high pressure side of the still. After some time it would become clear that the equipment had reached a steady state of operation. This state was characterised by regular or continuous operation of the condensate pump; a reasonably steady feed temperature, and by a constant low pressure level: all of these variables would be monitored using the MF11010/BBC microcomputer data acquisition system. The amplitude and period of the compressor stroke would then be set to the required values and the rig left for another period, perhaps 10-15 minutes, before data logging would commence. The operator would be prompted for number of the run, the date and the process parameters set; this information was stored on the data disc and also used to name the file in which the logged data would be held. Cylinder pressure and pressure difference against piston position were logged for each run: 200 data points, i.e. approximately 40 seconds. At a later date, the data would be transferred to the EMAS mainframe system via the XTALK program and stored in a group as: BASIC.<runfilename>, eg. BASIC.DPPOS23. The form in which the data was transferred from the BBC to EMAS was not suitable for use in any of the programs available; consequently it had to be converted or "detokenised" to a useful format. A command was set up to detokenise the data and to edit out unnecessary characters: DET. This command acted on a given file and stored the reformatted data in the same file, thus no "raw" data was actually stored, save for that on the disc used by the BBC microcomputer. Details of each run were also manually recorded for future reference along with any pertinent observations. 


\subsection{Analysis of Experimental Data}

The purpose of the experimental programme was to attempt to quantify the accuracy of the mathematical models; not to provide data which could be directly applied to a full-scale process. The value of the experimental results lay, therefore, in their comparison with the results from the mathematical model of the rig (see chapter 3, section 3.4.2). A necessary step towards the realization of this end was the manipulation of the experimentally obtained date into a useful format. The ordinate of the results, piston position, was recorded in units of centimetres measured from the cylinder head. The values of these displacements did not necessarily correspond to a sine wave between the maximum and minimum points set by the nominal amplitude. A combination of sensor inaccuracy and errors in the null point setting usually meant that some of the values lay outwith the specified range. In order that experimental and simulated results could be directly compared it was required that the ordinates of both sets of data should be in a dimensionless form. This was a trivial task for the simulated data and was easily obtainable for the experimental data. An IMP program was written which scanned through the experimental values to find the maximum and minimum positions of the cylinder, $x_{\max }$ and $x_{\min }$. The data was then transformed into a dimensionless form using the equation:

$$
x^{*}=\left(x-x_{\min }\right) /\left(x_{\max }-x_{\min }\right)
$$

With the data in this form, experimental and simulated results could be plotted on the same axes. Plots of $\Delta p$ vs. $x^{*}$ for all the experimental runs are reproduced in figures (5.8) to (5.24), together with the simulated data for the same amplitude, frequency and process conditions. It may be seen from these plots that the two sets match, in general, well. There were some particular points in the cycle where significant discrepancies could be observed, notably around the points where the valves were likely to have been opening and/or closing. The overall impression, however, was that the model seemed to be capable of describing the physical system with reasonable accuracy. It was difficult to assign a numerical index to this perceived accuracy; with results of this nature it is the correspondence of the shape of the plots which is as important as the numerical correspondence, if not more so. While it was easy visually to note the coincidence of the two plots, a suitable quantitative method proved elusive. 
One method used was to plot $\Delta p$ for the same ordinate from each data set against one another: for perfect correspondence this would, of course, produce a straight line of unity gradient passing through the origin. Another program was written (in IMP) to carry out this task. As the ordinates and the cycle starting points of the two data sets did not match, a simple scanning and interpolation method was used. Plots of the data produced with values of the best fit gradient (by linear regression) and intercept are shown in figures (5.25) to (5.41). These plots confirmed the initial impressions formed from a study of the whole cycle plots. Furthermore, it appeared that the accuracy of the simulation was unaffected by amplitude or period over the range tested. as illustrated in figures (5.42) and (5.43) (gradient, intercept and standard deviation vs. amplitude and period respectively).

Attention was next turned to the cylinder pressure vs. $x$ " diagrams (figures $(5.44)$ to $(5.60)$ ). Once again, it was noted that the loci of the simulated and experimental data were of a similar shape; also obvious was the fact that the experimental data was displaced some 0.15-0.2 bar above the simulated data. The physical property routines in the model and the pressure transducers on the rig were re-checked, but no errors were found that could account for the observed difference. The possibility that the presence of non-condensables in the rig could have increased the total pressure was considered but rejected on the grounds that the mole fraction of air needed to cause such a difference would have had to be of the order of 0.1-0.15. The rig was always run with the lowest pressure in excess of atmospheric pressure and was regularly purged on start-up; it seemed very unlikely, therefore, that such a concentration of air could have built up. One explanation of this behaviour which seemed feasible, however, was related to the assumption of adiabatic compression and expansion inherent in the model. Frictional heating of the walls of the cylinders was routinely noted during normal operation of the compressor. It was thus obvious that the compressor was not only putting work into the system, but heat as well; thus, the compression/expansion processes cannot have been adiabatic. In order to find out if this explanation was really tenable it would have been necessary to carry out a heat and work balance over the entire rig; in particular, to carry out a heat balance around the heat rejection cooling coil and to measure the V.I product at the motor. It was unfortunate that neither the time nor the additional equipment was available to carry out the further experiments necessary for the testing of this 
theory.

In addition to frictional heating, the formation and recession of thin films of liquid on the cylinder walls were observed during the compression and expansion strokes respectively. As the film grew thick enough to be seen flowing down the walls of the cylinders and forming a pool at the bottom, an interesting phenomenon could be observed: the frictional heating of the cylinders was most noticeable on their upper surfaces (which became warm to the touch) whereas the lower surface felt appreciably cooler than its surroundings. The lower surface was, in effect, being cooled by evaporation of the liquid above it. The films were always formed first on the cylinder head plate and the piston face, before forming a front which moved through the cylinder towards the piston. This suggested that the temperature distribution in the cylinder walls was both radial and axial (it should be noted that the presence of phase change in the cylinders is evidence in itself of non-adiabaticity). The inclusion of the above effects in the rig simulation would have been a task of immense complexity. In addition to the solution of the existing equations, the equation for unsteady state heat transfer (with change of phase) in three dimensional cylindrical coordinates would have to be included and an estimate of the rate of frictional heating taking place would be required. It is the opinion of the author that the work involved in producing such a detailed model could not have been justified by the extra information gained.

The conclusion that was drawn from the above analysis was that, although the physical situation was a good deal more complex than that modelled (evinced by the $p$ vs. $x^{*}$ data), nevertheless the model did provide predictions of behaviour with reasonable accuracy (evinced by the $\Delta p$ vs. $x^{*}$ data). By implication, therefore, the model of the duck could be used with a similar degree of confidence. In fact, since the least tenable assumption in the model proved to be that of adiabatic compression due to the effects of friction and because friction is unlikely to be significant in the real duck, then it may be that the real duck model is a better description of that system than the rig 
model was of the rig'.

\subsection{RESULTS FROM THE SECOND MODEL}

With the second model validated, as far as possible, through the use of the experimental data, the next task was to use the model in much the same way as the first. Once again, the model was used to investigate both transient behaviour and long-term process performance.

\subsubsection{Transient Performance Predictions}

The results from the first model suggested that time dependencies would exist in the heat and mass transfer process in a full-scale system (see section 5.1.1). One of the major differences between the first and second models was the inclusion in the latter of the equations of motion for the fluid piston. The fluid piston was, essentially, a mechanism for energy transfer and/or storage: set in motion by the pressure difference across it at one point in the cycle, it could transfer that energy to the vapour at another point in the cycle. The behaviour of the fluid piston was not, in itself, time dependent; at any instant its motion would depend only upon the pressure forces across it; its angular acceleration, and its angular displacement. Its inclusion in the global system of equations, however, and its storage capacity (or "memory") tended to exacerbate the time dependencies already noted in the $\Delta p$ vs. $\theta$ characteristic.

In the program containing the code for the second model, no provision was made for the simulation of anything other than monochromatic "seas". It was not possible, therefore, to carry out tests for time dependency in the same manner as those carried out using the first model (see section 5.1.1). It was possible, however, to obtain some information about the effect of the fluid piston from diagrams such as figure (5.61), $\Delta p$ vs. time. During the first two wavelengths the trace is very irregular, as the fluid piston starts moving from rest (initial conditions were zero displacement and acceleration). Even after four wavelengths the trace does not become completely regular, although one might assume that the fluid piston must eventually arrive at a steady-state

\footnotetext{
'Note: the duck would, of course, exhibit condensation and evaporation inside its "cylinders": there is no way at present of judging the importance of that effect.
} 
motion. In a random sea, it is clear that the fluid piston would always be in a transient state: it is difficult, therefore, to envisage that $\Delta p$ could ever be expressed as a simple function of $\theta$ and its derivatives.

\subsubsection{Performance Predictions}

The second model was also used to investigate the effects of the following parameters on the predicted performance of the duck as a desalination plant: wave (sinusoid) amplitude and period; primary heat exchanger area; blowdown salt concentration, and the non-return valve specifications.

\section{Amplitude and Period}

Forty standard sinusoids were used in these runs: amplitudes in the range 0.3-0.7 radians (in 0.1 radian steps) and periods in the range 5-12 seconds (in 1 second steps). The heat exchanger area was set at $4000 \mathrm{~m}^{2}$; the blowdown concentration at $70 \mathrm{~g} / \mathrm{I}$ (i.e. approximately twice normal seawater concentration); valve area specified as $4 \mathrm{~m}^{2}$; valve orifice coefficient as 0.5 , and the valves were set to be $50 \%$ open at 0.01 bar pressure difference. The data generated by the main program were held in a partitioned file, MK3.RUNS (eg. MK3.RUNS_RUN12) for later use by any one of a number of short programs written for that purpose (see chapter 3, section 3.3.4, and appendix IV). In this way it was possible to reduce the number of times the main program needed to be used and consequently to reduce the total amount of processor time required. The data were analysed to provide values for the predicted mean rate of production of fresh water and SEC for each sinusoid, as plotted in figures (5.62) and (5.63).

The rate of production exhibited dependencies that radically differed from those predicted by the first model. In figure (5.62)a., output is plotted against amplitude for various periods. For a simple system, an approximately linear relationship between output and amplitude would be expected (see section 5.1.2, above). The plot in (5.62)a. shows an upward trend, but the relationship is definitely non-linear. Figure (5.62)b. is a plot of the output against period for various amplitudes. Once again, for a simple system, one would expect that the output would increase with the inverse of the period, the frequency of operation. The opposite trend is illustrated in figure (5.62)b.: for periods under about 6.5 seconds, the output increased sharply with period, before levelling off to a more gradual upwards slope towards 12 seconds. As a major 
difference between the first and second models was the inclusion of the model of the fluid piston, it wes decided to determine whether or not this change could have caused the differences in the predictions.

To this end, the date processing program was used to calculate the ratio $\alpha$ : the ratio of the actual vapour volume "swept out" by the duck to the ideal maximum swept volume for a given amplitude. Plots of $a$ vs. period and amplitude are reproduced in figures (5.64) and (5.65) respectively. It may be clearly seen that $\alpha$ was a very strong function of the period; increasing from practically zero at around 4 seconds, to around 0.5 at 12 seconds. The calculations carried out to determine the approximate resonant period of the fluid piston (see chapter 3, section 3.3.3) predicted that it would resonate at around 3.5 to 3.9 seconds. At the higher end of the frequency scale, therefore, one would expect the fluid piston to be resonating in phase with the $\Delta p$ force and, therefore, for a to become vanishingly small in this region. Moving down the frequency scale should produce larger values of $a$, as observed, for the same reasons. The trend of a with the duck amplitude was found to be much less marked, see figure (5.65). The increase in $\alpha$ with amplitude can, however, account for the non-linear output/amplitude relationship noted in figure (5.67)a.

These simulations gave not only an indication of the performance of the duck in numerical terms, they also provided an insight into the importance of the fluid piston as a system component. In an attempt to quantify the effects of the fluid piston, a new version of the simulation program was created (called VERIFY), identical to the first in every respect save for the removal of the fluid piston motion equations. Plots of output vs. period for a single 0.5 radian wave from both programs are shown in figure (5.66). Without the fluid piston, the system exhibits the inverse period relationship as expected; it also produces much more fresh water for obvious reasons. As the equations of motion for the fluid piston were part of the whole system of equations describing the behaviour of the vapour compression process, it seemed reasonable to expect that its effects would be observed throughout this study.

\section{Primary Heat Exchange Area}

Plots of output and SEC against heat exchanger area are shown in figures (5.67) and (5.68); the data points were generated for an 8 second, 0.5 radian 
wave. As anticipated, output increased and SEC decreased with area; the output vs. area curve was, however, non-linear. It was decided to investigate the behaviour of the fluid piston in order to determine whether or not this could account for the anomalous result. Figure (5.69) is a plot of a vs. heat exchange area; it may be seen that $\alpha$ increases with area, suggesting a relationship of the form:

$$
m \propto \alpha^{1 / n}
$$

Now, since output is proportional to the amplitude and hence $\alpha$, then it follows that:

$$
m \propto A^{1+1 / n}
$$

which could describe the curve in figure (5.67). Confirmation of this result came from the data produced by VERIFY; the production rate was found to rise approximately linearly with a very slight tailing off at the greatest areas, possibly caused by flow limitations imposed by the non-return valves.

\section{Blowdown Salt Concentration}

According to the laws of conservation of mass and energy, all the mechanical work absorbed by the duck must be accounted for in the difference in the enthalpies between product and blowdown, and the feed streams. Thus:

$$
S E C=C_{p} \cdot\left(m_{p} \cdot\left(T_{p}-T_{f}\right)+m_{b} \cdot\left(T_{b}-T_{f}\right)\right) / m_{p}
$$

as before. Now, if the heat recovery exchanger is large enough, then the exit temperatures of blowdown and product must be similar; so, approximately:

$$
S E C \simeq C_{p} \cdot \Delta T_{0} \cdot m_{f} / m_{p}
$$

where $\Delta T_{0}$ is the terminal temperature difference of the recovery exchanger. From the mass balances around the process, equation (5.7) may be rearranged to give:

$$
S E C=C_{p} \cdot \Delta T_{0} \cdot s /(s-1)
$$

For a given recovery heat exchanger area and heat transfer coefficient: 


$$
\Delta T_{0} \propto m_{f}=m_{p} \cdot s /(s-1)
$$

Combining equations (5.15) and (5.16) gives:

$$
\operatorname{SEC} \propto m_{p} \cdot s^{2} /(s-1)^{2}
$$

Thus SEC decreases with salinity, as one might expect; hence the drive towards improved methods of scale control in conventional desalination plant, see appendix I. The rate of production is, of course, expected to decrease with salinity due to the increase in the bpe causing a reduction in the effective temperature difference in the primary exchanger.

For the simulation runs carried out, the salinity of the blowdown was varied from 50 to $90 \mathrm{~g} / \mathrm{l}$, using the standard 0.5 radien, 8 second test sinusoid. Figures (5.70) and (5.71) show the results of these simulations as output and SEC, respectively, plotted against salinity. Over the given range, output fell by approximately $25 \%$ and the SEC rose by around $4 \%$. It is possible to see that the output would drop because of the increase in the boiling point elevation with salinity reducing the effective heat transfer difference available for heat (and therefore mess) transfer. The increase in bpe would introduce further irreversibilities into the system and so one could envisage that this could cause an increase in the SEC. The process modelled was a closed system, however, and therefore the arguments previously advanced to explain the relationship between production rate, SEC and salinity must equally apply in this situation. The reduction in the SEC caused by the reduction in the output and increase in the salinity ratio would to some extent be offset by the increase in the mean working temperature of the primary exchanger; it seemed unlikely, however, that this could account for the increase in the SEC observed. It was at this point decided to more closely examine the performance of the heat recovery exchanger model, as this could have a great influence on the SEC. When this part of the model was studied in isolation from the rest of the system of equations, it was found that, if the temperature of the working fluid was too great, or if the flowrates of the streams too large, then the model no longer produced satisfactory heat balances over the exchanger. It was surmised that this was because the $d T / d A$ for the streams involved were no longer approximately constant and therefore that the log mean temperature difference calculations used in the model were no longer valid. This effect became most serious, of course, when the specified duty of 
the exchanger was at its greatest level. An outline of a more rigorous scheme for the description of this exchanger is given in appendix III, but, unfortunately, lack of time prevented the implementation of such a scheme in the existing model. Given that the recovery model was flawed in this way, it was obvious that there was little value in the continued consideration of the trends in the SEC in terms of the overall energy balance around the duck. Nevertheless, the observed dependencies (on amplitude, period, area, etc) did make sense qualitatively when thought of in terms of the irreversible losses involved in the process, or in terms of the pressure difference force/angular velocity product.

The decrease in a with salinity of the blowdown was found to be quite marked. One could qualitatively envisage that the higher salinities would lead to larger bpe's and so to a reduction in output and $\alpha$. For a conventional process, the only mechanism for the reduction in output would be the increase in bpe; for this process the "knock on" effect of the bpe on the motion of the fluid piston appears to be much more important. This was reflected in the results from VERIFY (figure 5.72), where output dropped from $9.47 \mathrm{kgs}^{-1}$ to $9.28 \mathrm{kgs}^{-1}$ (for a $0.2 \mathrm{rad}$ wave) over the same salinity range; a decrease of only $2 \%$.

\section{Valve Parameters}

Four values needed to be supplied by the user in order to completely model the operation of the non-return valves. These were: cross sectional area for flow through the valves; the orifice coefficient for flow through the valves; a fractional opening, and the pressure drop across the valve at which that fractional opening would occur. The last two parameters were used to fit a sigmoid (inverse tangent) curve to the fractional opening $(\sigma)$ vs. $\Delta p_{0}$ curve, as described in chapter 3 , section 3.3.3. It soon became clear that this method of specification of the valve characteristic was far from ideal: the relationship between the given values of $\sigma$ and $\Delta p_{0}$ and the shape of the curve was too complex. Figures (5.72) and (5.74) are plots of $\sigma$ and $\Delta p_{0}$ for various specified pairs (say, $\sigma^{\prime}$ and $\Delta p_{0}$ ). It may be seen that the main effect of $\Delta p_{o}^{\prime}$ was to shift the position of the entire curve along the $\Delta p_{0}$ axis, with a slight, secondary effect on the slope of the steepest part of the curve. The main effect of $\sigma^{\prime}$ (from figure (5.74)) was to alter the maximum gradient of the curve; although it was also found that extreme values of $\sigma^{\prime}$ could also affect the position of the curve along the $\Delta p_{0}$ axis (i.e. values of $\sigma^{\prime}$ greater than $90 \%$ 
or less than $10 \%$ ). Of the two parameters, then, it was clear that it was $\Delta p_{0}^{\circ}$ which could be used to effect the greater changes in the behaviour of the valves. It was decided to use only one value of $\sigma^{\prime}$ (taken as $50 \%$ ) in all the simulations and to vary $\Delta p_{0}{ }^{2}$.

A series of simulation runs were carried out, using the standard 0.5 radian, 8 second wave, in which $\Delta p_{0}$ was varied from 0.005 to 0.05 bar in 0.005 bar steps. Once again, mean production rate and SEC were calculated for each run. The condensate mass flowrate was found to decrease with $\Delta p_{0}$ from around $8.4 \mathrm{kgs}^{-1}$ at 0.005 bar to around $5.1 \mathrm{kgs}^{-1}$ at 0.05 bar (see figure (5.75)). Over the same range, the SEC was found to increase from $9.0 \mathrm{kJkg}^{-1}$ to $18.2 \mathrm{kJkg}^{-1}$ (see figure (5.76)). One might intuitively expect the rate of production to decrease (a larger pressure drop across the valves must lead to a reduction in $\Delta T$ for heat transfer) and SEC to increase (the valves introduce more irreversibilities into the system and therefore the thermodynamic efficiency must decrease, hence the SEC must increase).

A slight change in $\alpha$ was noted with $\Delta p_{0}$, see figure (5.77). Further investigation showed that $\Delta p_{0}$ had an effect on the phase angle between the motion of the duck and the fluid piston. The phase angle is related to the shape of the $\Delta p$ vs. $\theta$ curve and so these plots were generated for each value of $\Delta p_{0}$, see figure (5.78). A marked change in the shape of the plots over the range was noted: at the lowest values of $\Delta p_{0}$, the plots were appreciably parallelogram-shaped, as one would expect for an ideal compressor; as the $\Delta p_{0}$ was increased, so the plots became more rounded. The area inside the plots remained approximately the same; indicating that the compression work per cycle was roughly constant. The shape of the plots suggested that at low values of $\Delta p_{0}$ the valves were either fully open or fully closed; higher values produce valves which were nearly always only partially open. This would seem to tally fairly well with what might be expected from real valves and with the evidence of the production rate and SEC data. Although these results seem to agree with a qualitative description of the process, it is unfortunate that the method of specification of the valve behaviour makes it difficult to use the

\footnotetext{
${ }^{2}$ Note: it is proposed that a new method of specifying the valve behoviour should be found for future use, see chapter 6 and appendix III
} 
model as a design aid in a more exact manner.

The valve orifice coefficient $\left(C_{d}\right)$ and orifice area $\left(A_{0}\right)$ for the valves were also specified by the user. For convenience, the product of the two parameters was used, rather than testing their effects separately, in a number of runs, varying from $3 \mathrm{~m}^{2}$ to $0.5 \mathrm{~m}^{2}$ using the standard sinusoid. The primary heat exchanger area was also varied for each value of $C_{d} \cdot A_{0}$ from $4000 \mathrm{~m}^{2}$ to $9000 \mathrm{~m}^{2}$ : it was felt that, especially at the larger values of area and smaller values of $C_{d} \cdot A_{o}$, the condensate flux might be limited by mass transfer through the valves and not by heat transfer. In fact, $C_{d} . A_{0}$ was found to have very little effect, if any, on either the SEC or the rate of production; with or without the fluid piston. In retrospect, this result could have been anticipated: taking a mean mass flowrate of $10 \mathrm{kgs}^{-1}$ and $C_{d} \cdot A_{o}$ es $1 \mathrm{~m}^{2}$, then even with the valve only $50 \%$ open the pressure drop across it would only be of the order of 0.001 bar. Thus, it would appear that flow restriction by the valve only becomes important when it occurs as a result of the valve not being able to open enough, as above.

\subsection{SUMMARY}

There were three important points emergent from the work described above:

1. The heat and mass transfer processes taking place in the duck, as predicted by both models, are time dependent and therefore small-scale tank tests would be inappropriate.

2. The results from the second model agreed reasonably well with experimental results.

3. The inclusion of the equations of motion of the fluid piston radically change the predicted behaviour of the duck from the initial estimates.

The second model was used to investigate the performance of the duck as a desalination process. It was found that, aside from the effects attributable to the fluid piston, the process was much like any other simple VC desalination plant in its characteristics. It would appear to be able to produce flowrates close to $1000 \mathrm{~m}^{3}$ of fresh water per day as originally proposed and to do so in 
an highly energy efficient manner. The results suggested that it may be important to be able to design the duck shape so as to produce the largest of angles of rotation possible, as output was found to be directly related to the amplitude of motion. The utility of the results is limited in this respect, however, because the model could not take account of the motion of the duck; it was thus impossible to produce any estimates of the wave climates that might be required.

Other than these rather general considerations, it is difficult to provide any more substantive evidence with which to judge the viability of the proposed process. Perhaps the best interpretation of the work carried out so far, is that its results suggest that there is still good deal of research to be done and that such research would be justifiable. What form the continuing work might take is considered in detail in appendix III but the key areas might be:

1. The performance of the duck as a wave energy absorber

2. Gathering of specific site information

3. Design and evaluation of auxiliary machinery

4. Study of the fluid piston kinetic and thermal behaviour

5. Use of data from the above to improve the existing model

The difficulties in studying these topics, particularly 1. and 2., may be compounded by the difficulty in decoupling one part of the system from the rest, so that it may be studied in isolation. For example, the design of the auxiliary equipment; duck/shore pumps, deaeration equipment and so on; may prove as complex an issue as the design of the duck itself: all the proposed systems are novel and all rely on the direct conversion of wave energy, in the same way as the duck. Without additional information on the above subjects it has been possible to improve upon the confidence in the basis of the initial cost estimates, but not to the point where these estimates could be considered definitive. 


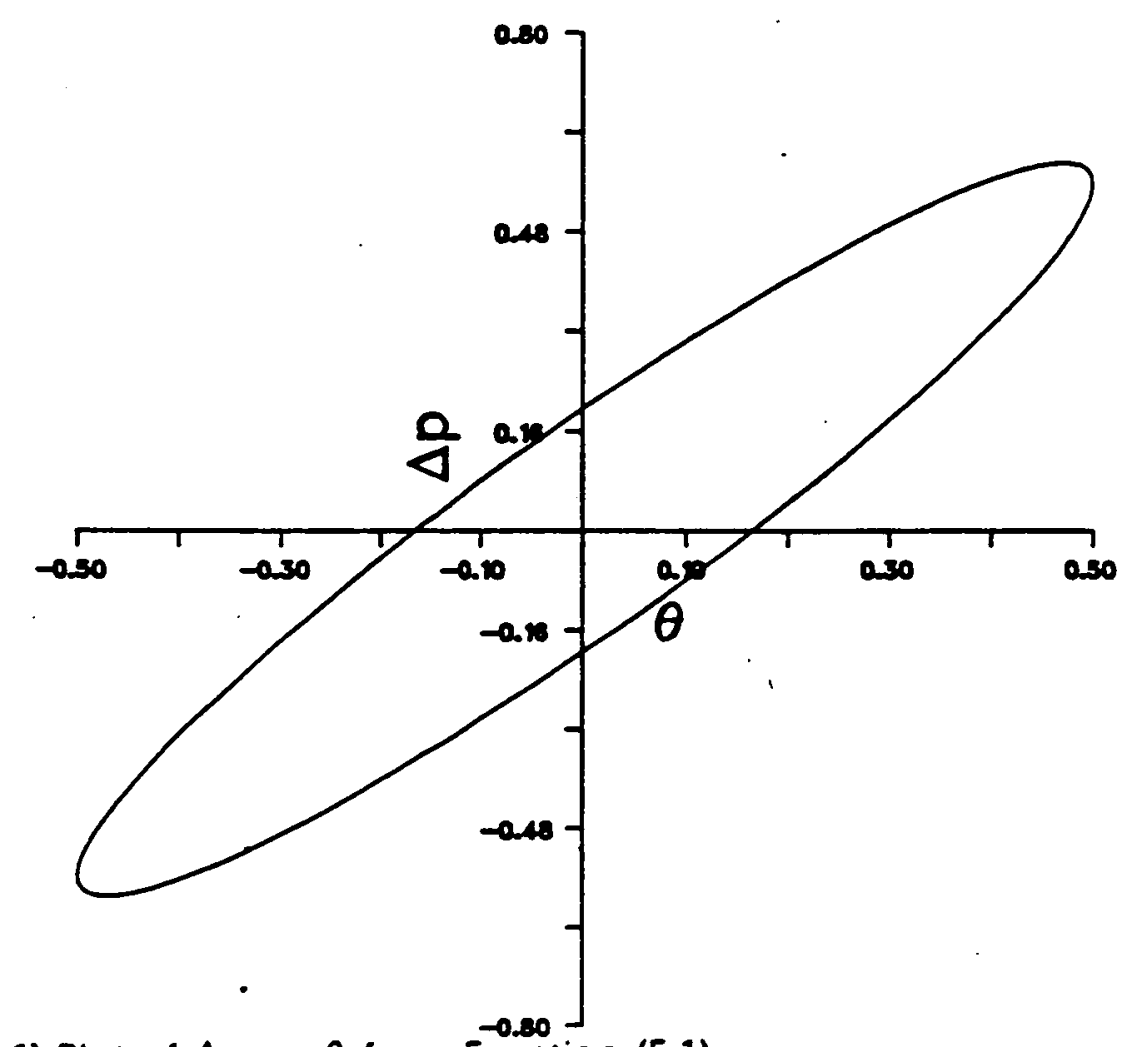

Figure (5.1) Plot of $\Delta p$ vs. $\theta$ from Equation (5.1)

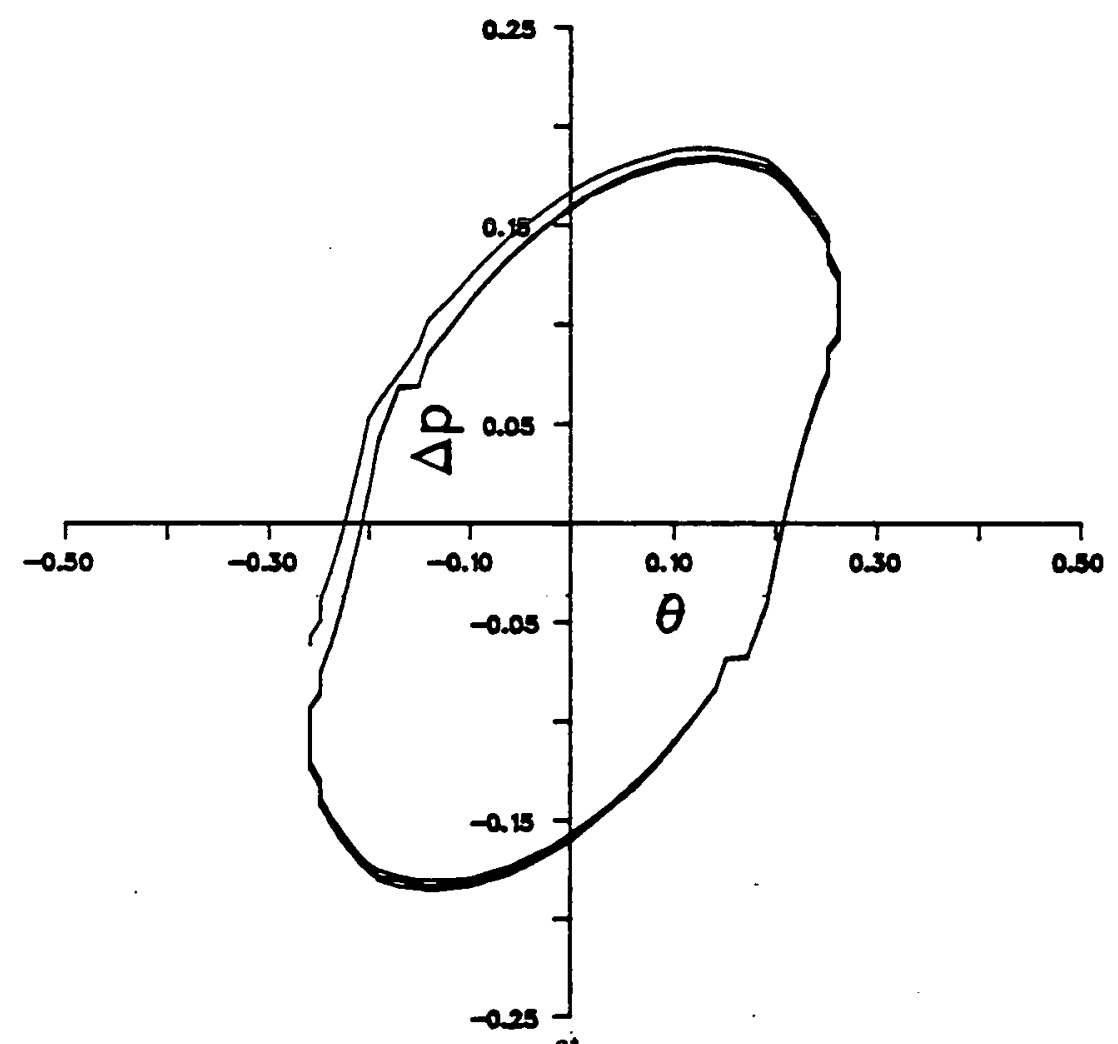

Figure (5.2) Plot of $\Delta p$ vs. $\theta$ from the $1^{\text {st }}$ Model 


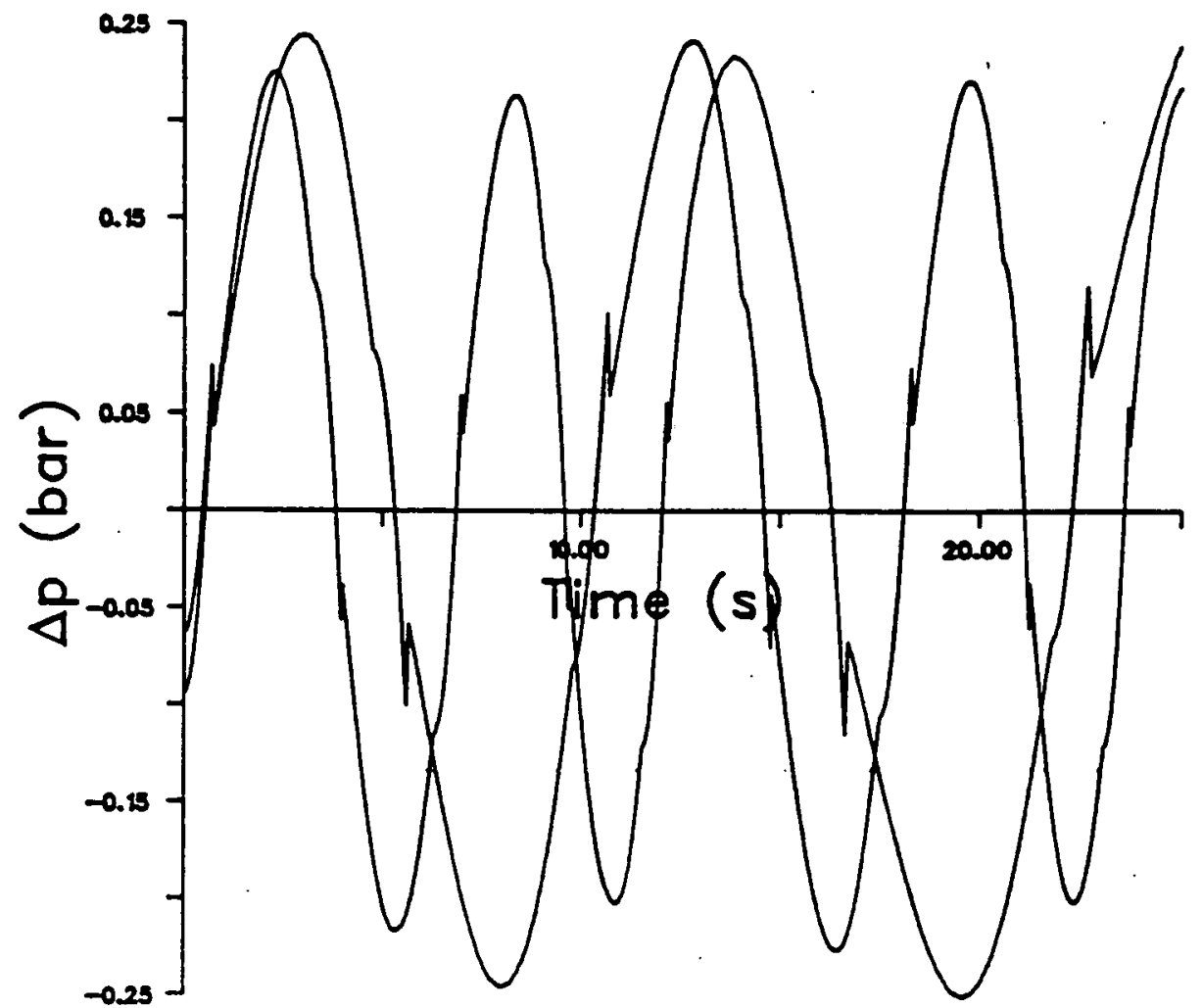

Figure (5.3) Plot of $\Delta p$ vs. time for time dependency testing, $1^{\text {st }}$ Model 


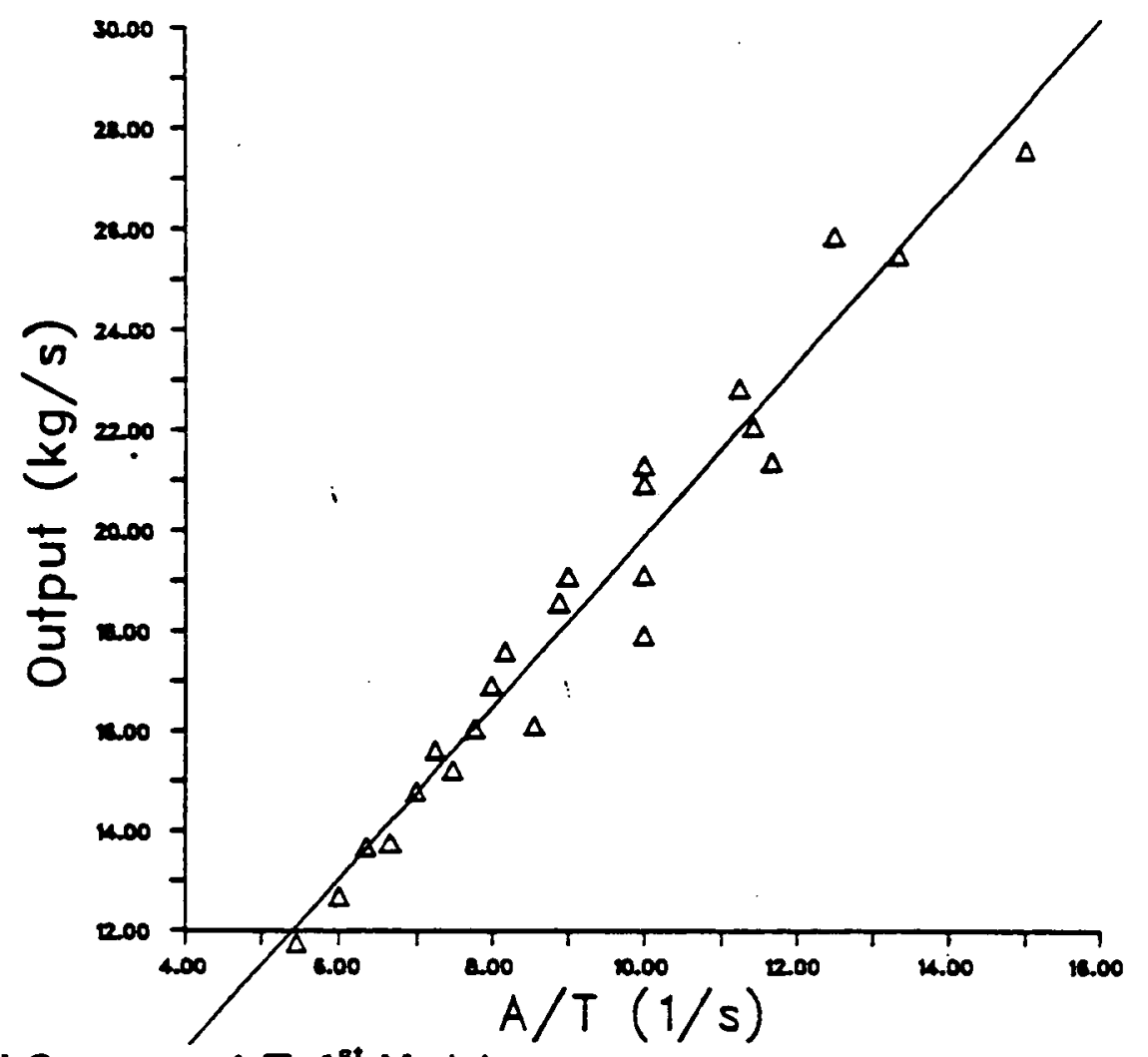

Figure (5.4) Output vs. $A \sqrt{T}, 1^{\text {st }}$ Model

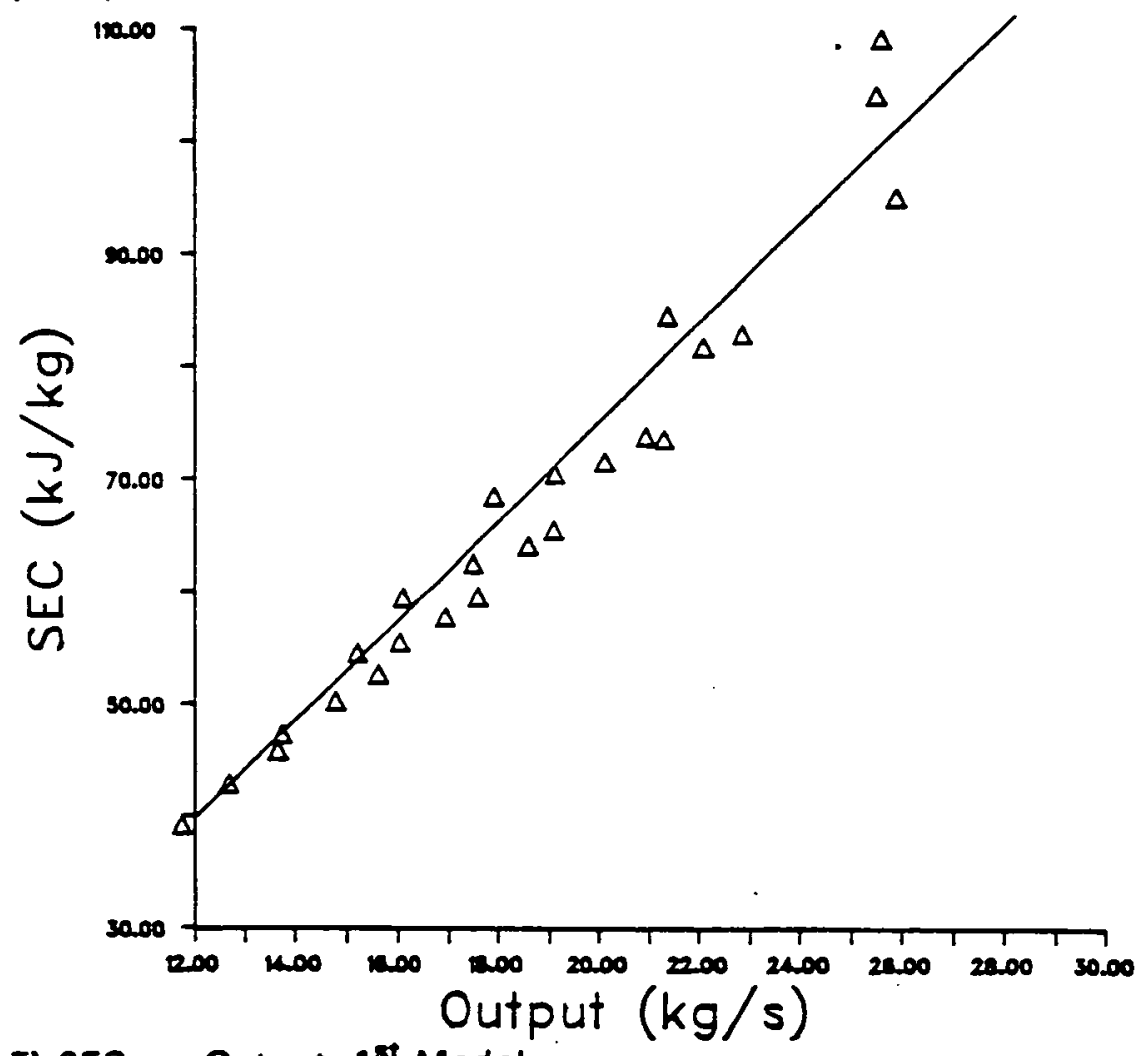

Figure (5.5) SEC vs. Output, $1^{\text {st }}$ Model 


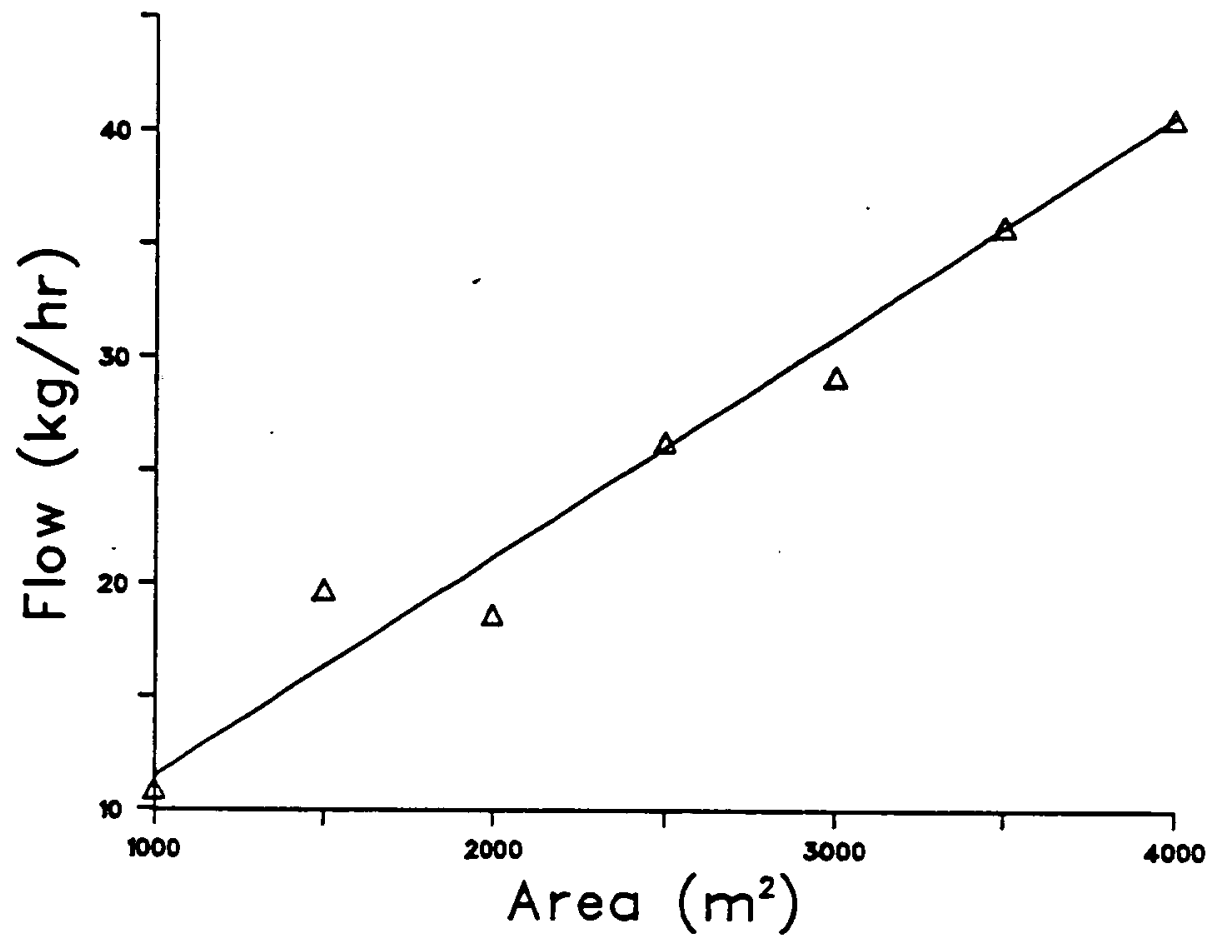

Figure (5.6) Output vs. Area, $1^{\text {st }}$ Model

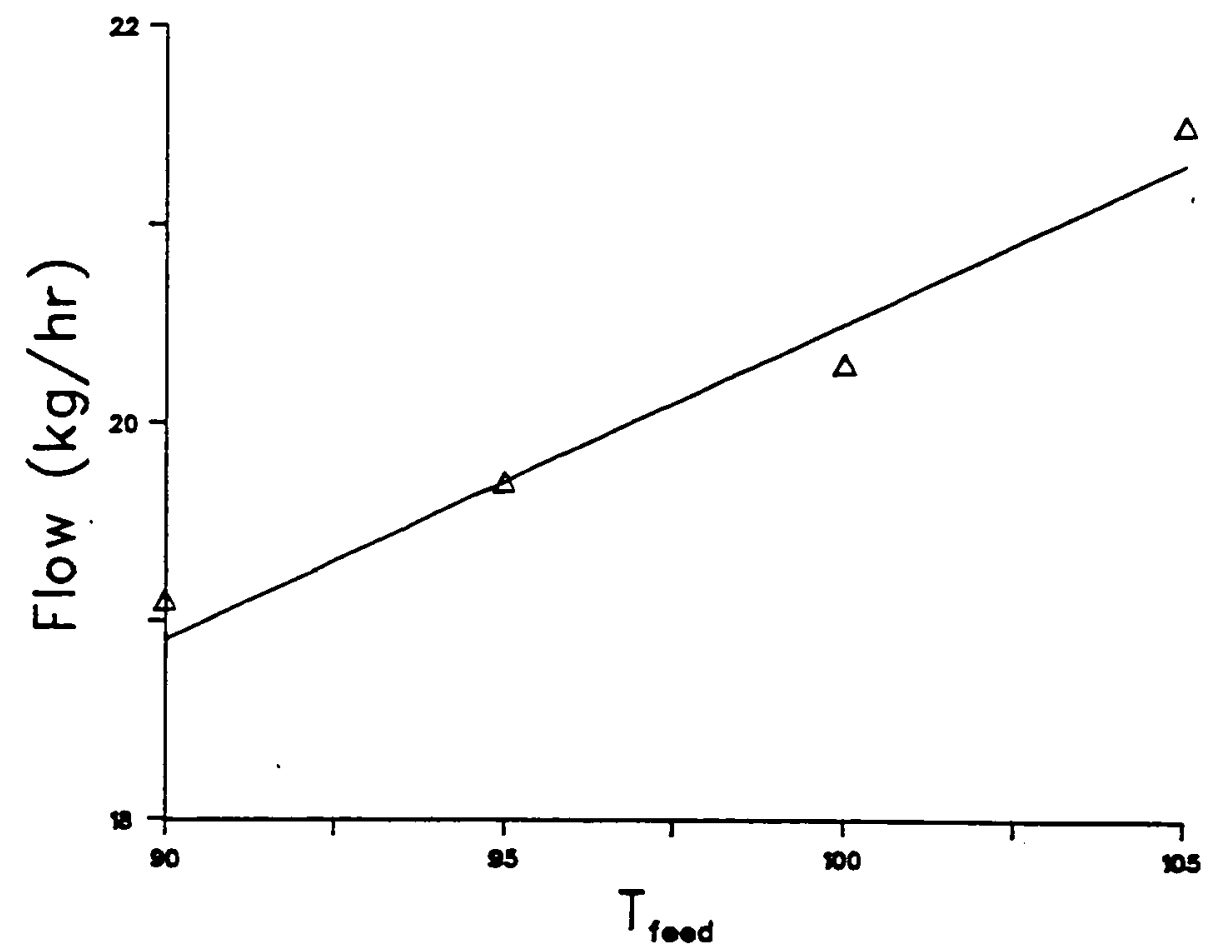

Figure (5.7) Output vs. $T_{\text {feed }} 1^{\text {st }}$ Model 


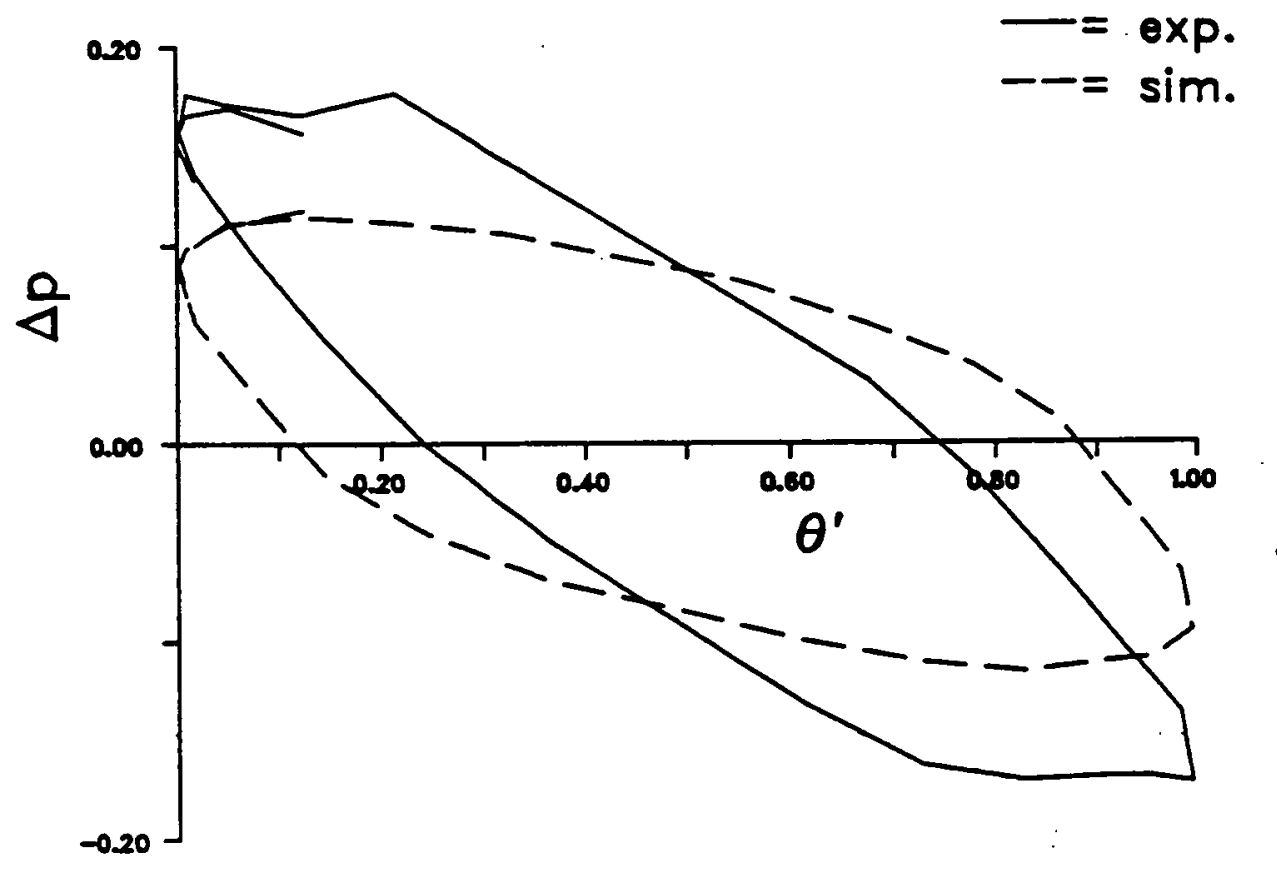

Figure (5.8) $\Delta p$ vs. $\theta$; Experimental and Predicted Period $=6 \mathrm{~s} ;$ Amplitude $=4 \mathrm{~cm}$

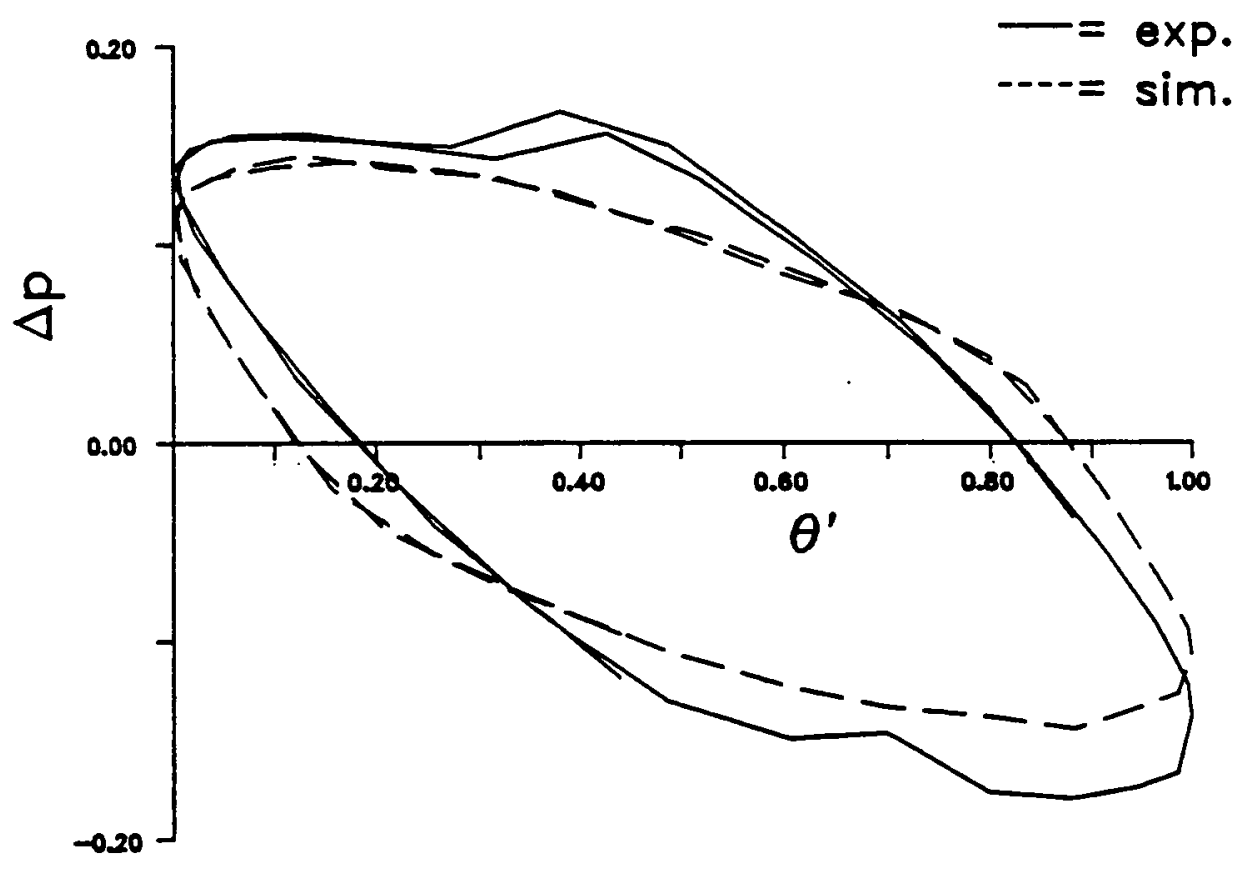

Figure (5.9) $\Delta p$ vs. $\theta$; Experimental and Predicted Period $=6 \mathrm{~s} ;$ Amplitude $=5 \mathrm{~cm}$ 


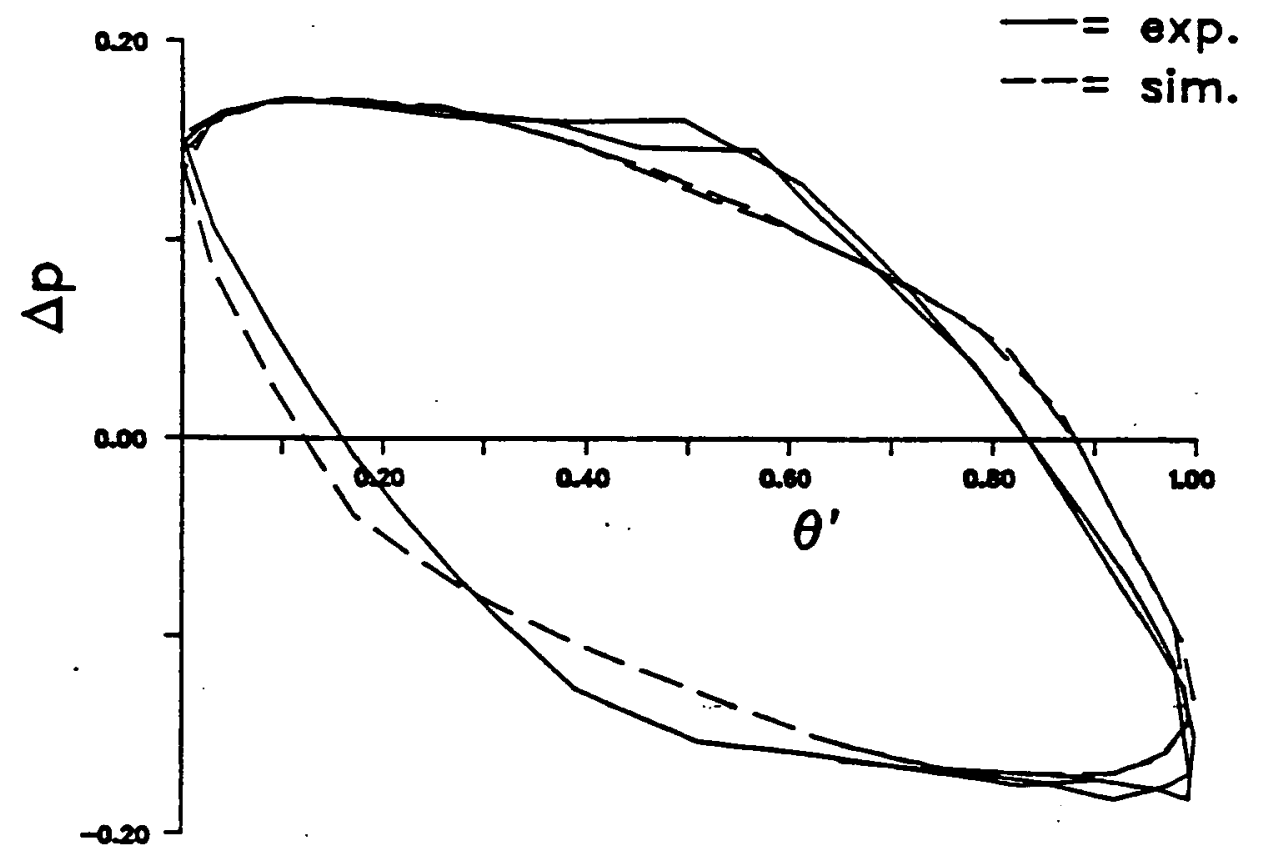

Figure (5.10) $\Delta p$ vs. $\theta$; Experimental and Predicted Period $=6 \mathrm{~s} ;$ Amplitude $=6 \mathrm{~cm}$

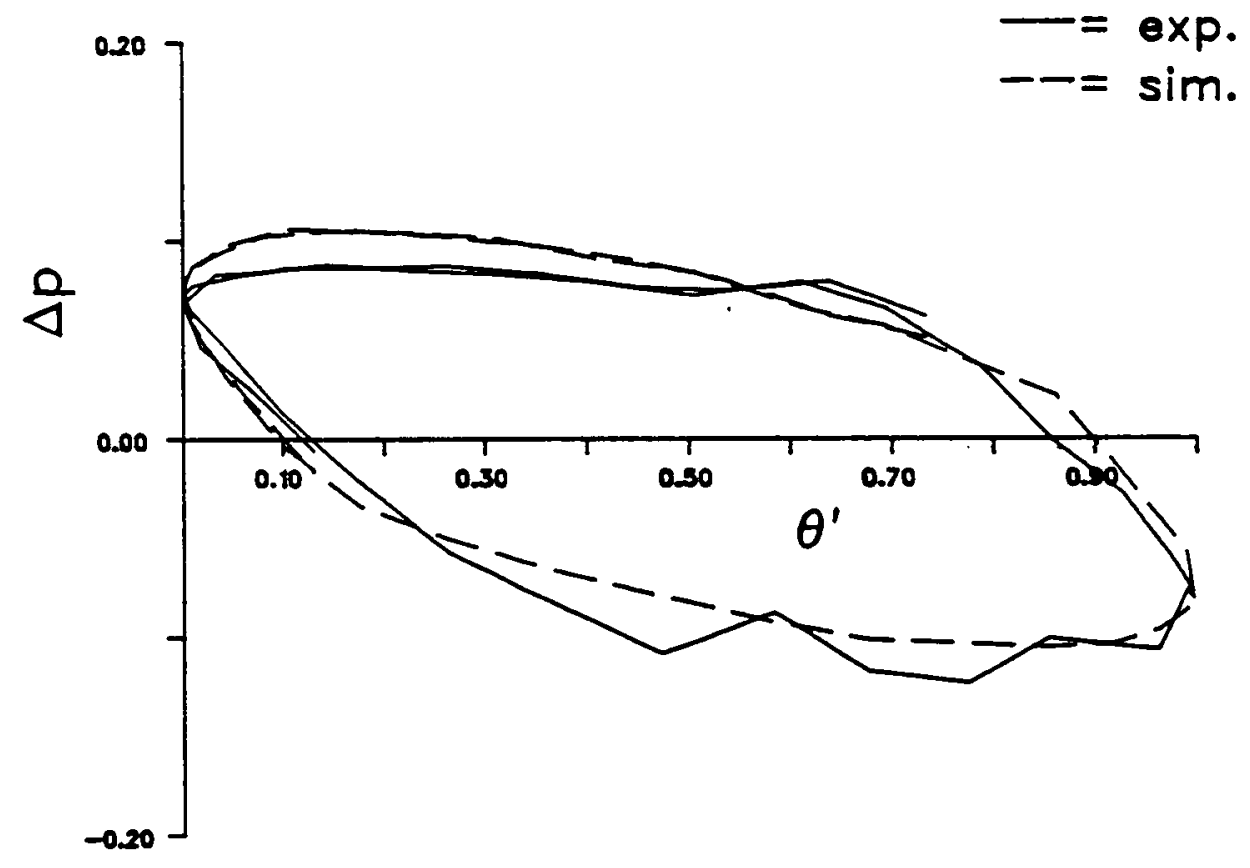

Figure (5.11) $\Delta p$ vs. $\theta$; Experimental and Predicted Period $=7 \mathrm{~s} ;$ Amplitude $=4 \mathrm{~cm}$ 


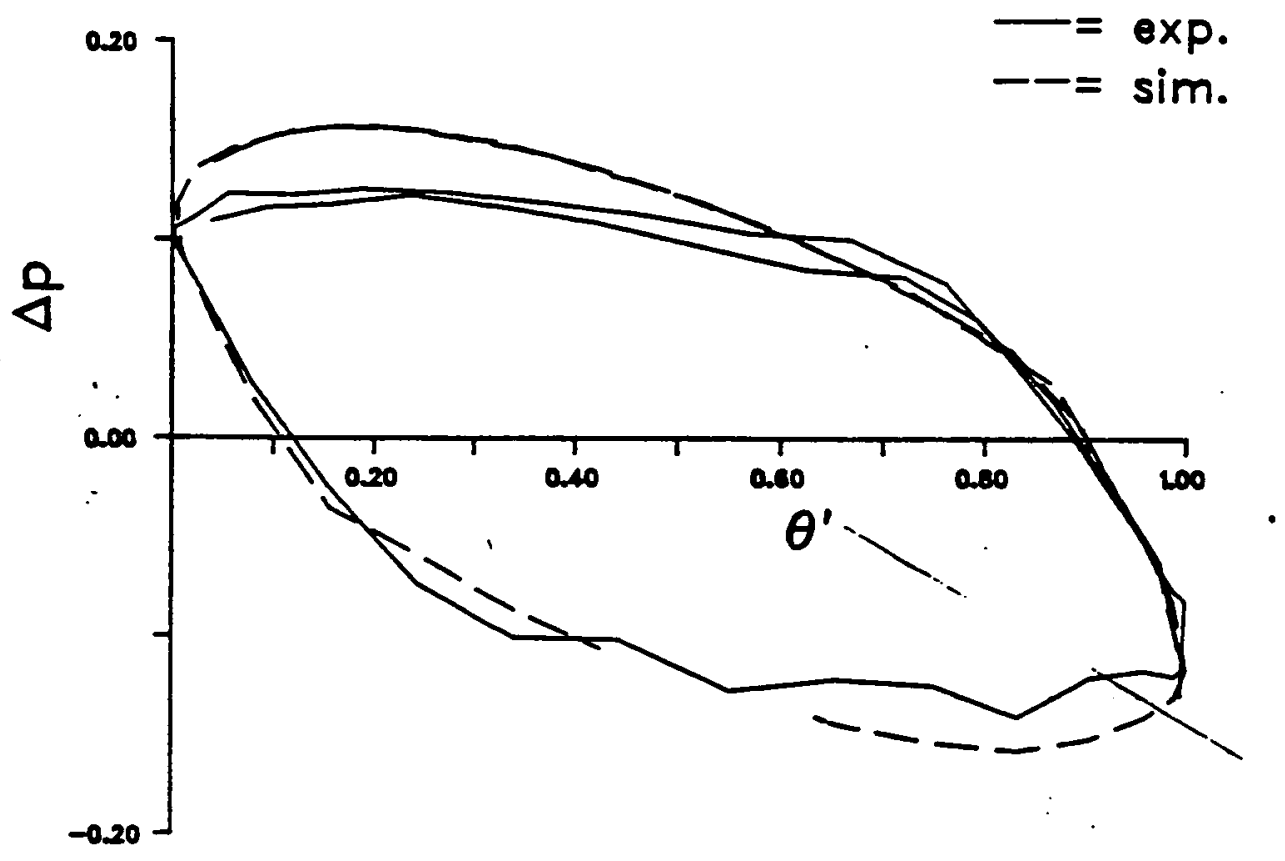

Figure (5.12) $\Delta p$ vs. $\theta$; Experimental and Predicted Period $=7 \mathrm{~s} ;$ Amplitude $=6 \mathrm{~cm}$

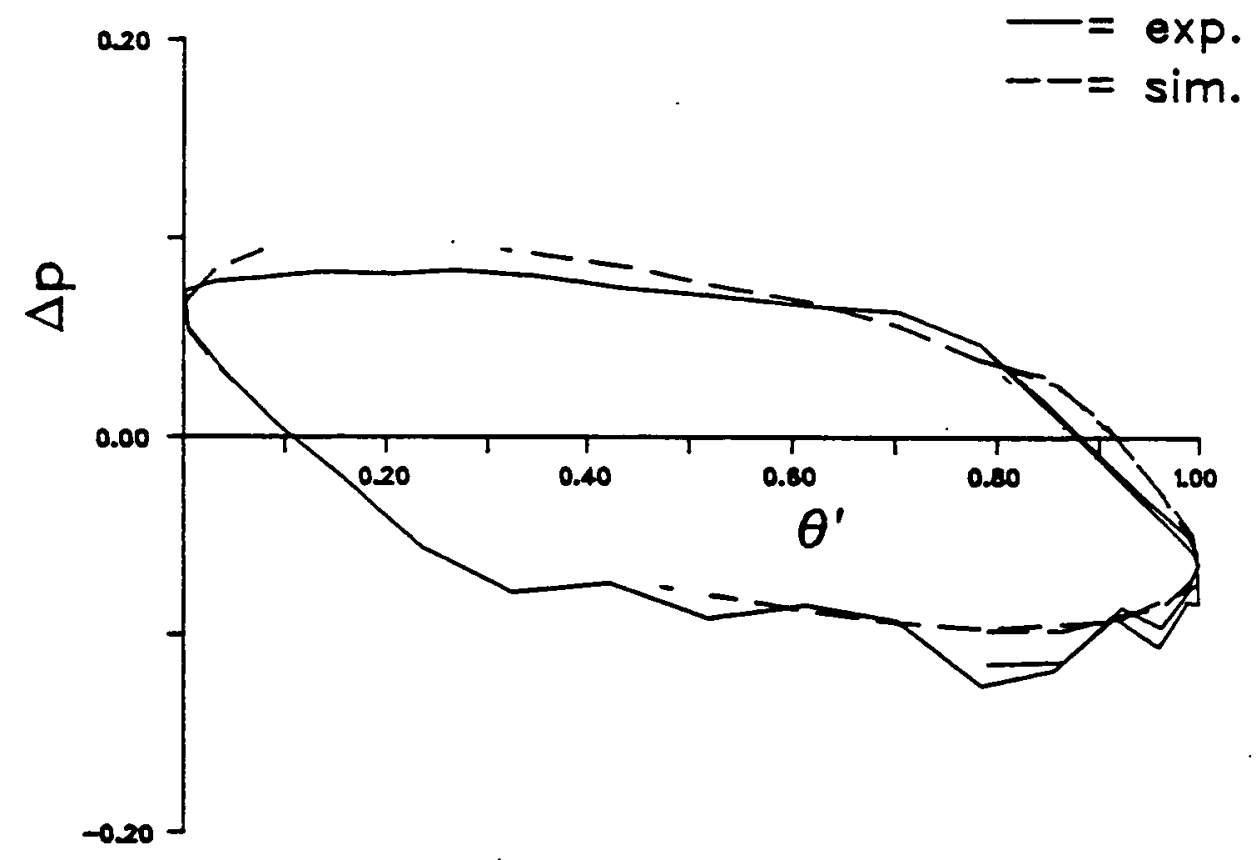

Figure (5.13) $\Delta p$ vs. $\theta$; Experimental and Predicted Period $=8 \mathrm{~s} ;$ Amplitude $=4 \mathrm{~cm}$ 


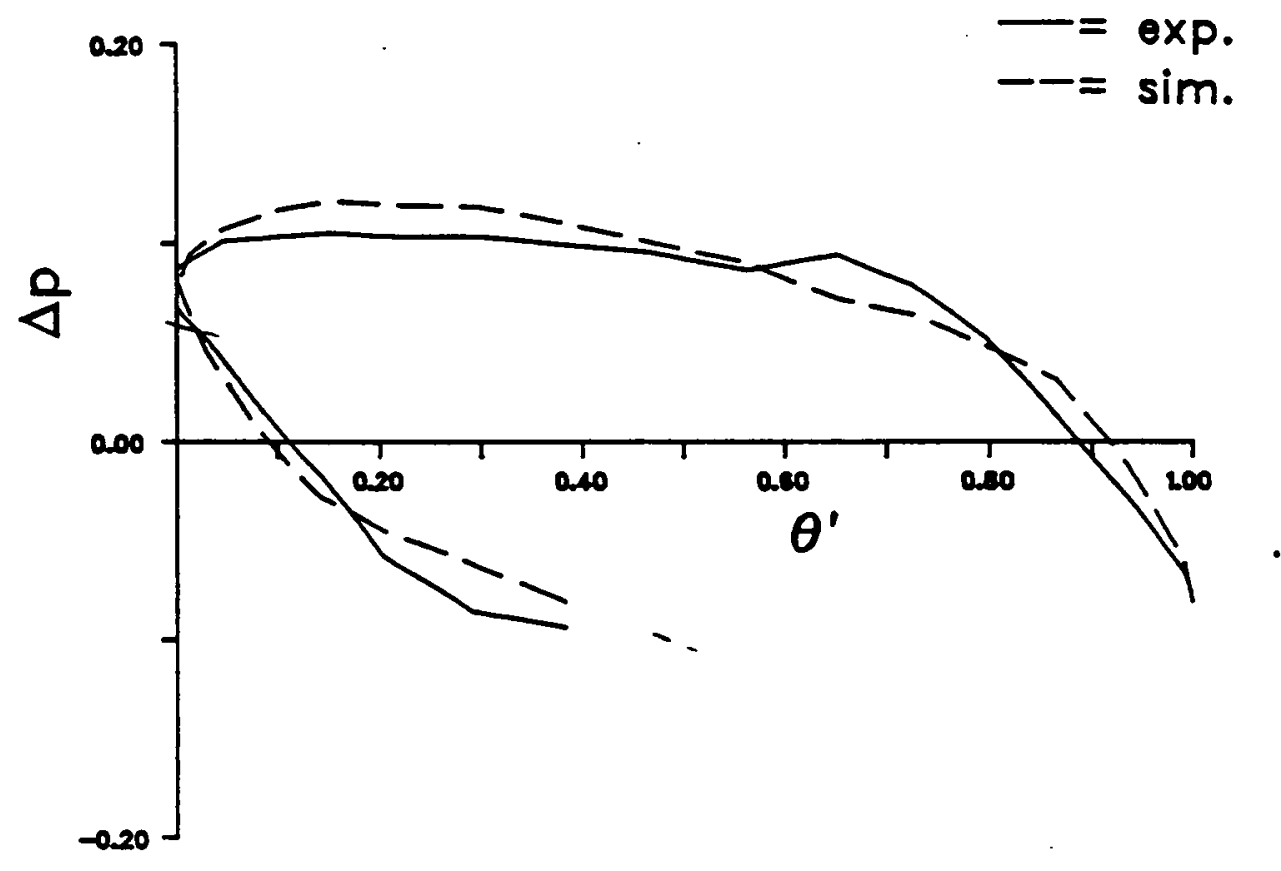

Figure (5.14) $\Delta p$ vs. $\theta$; Experimental and Predicted

Period $=8 \mathrm{~s} ;$ Amplitude $=5 \mathrm{~cm}$

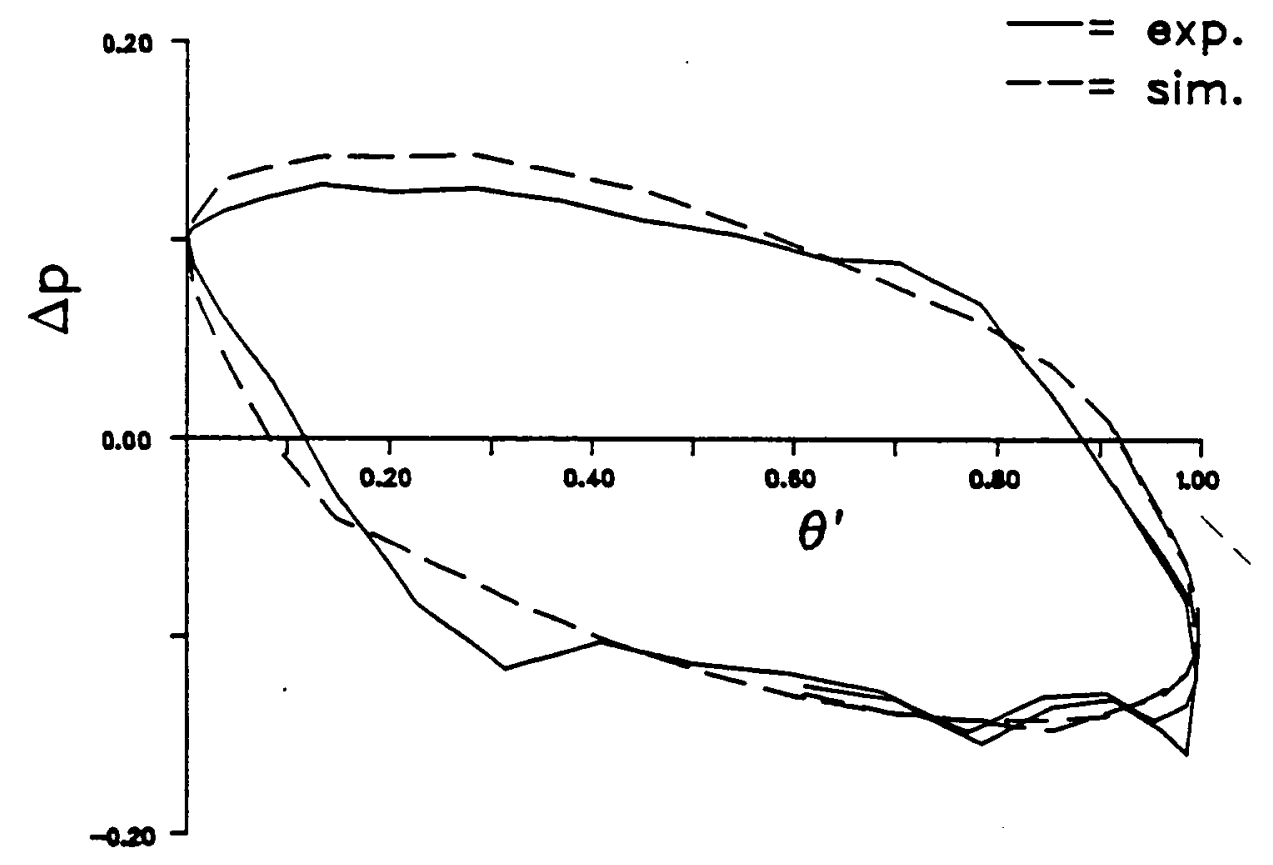

Figure (5.15) $\Delta p$ vs. $\theta$; Experimental and Predicted

Period $=8 \mathrm{~s} ;$ Amplitude $=6 \mathrm{~cm}$ 


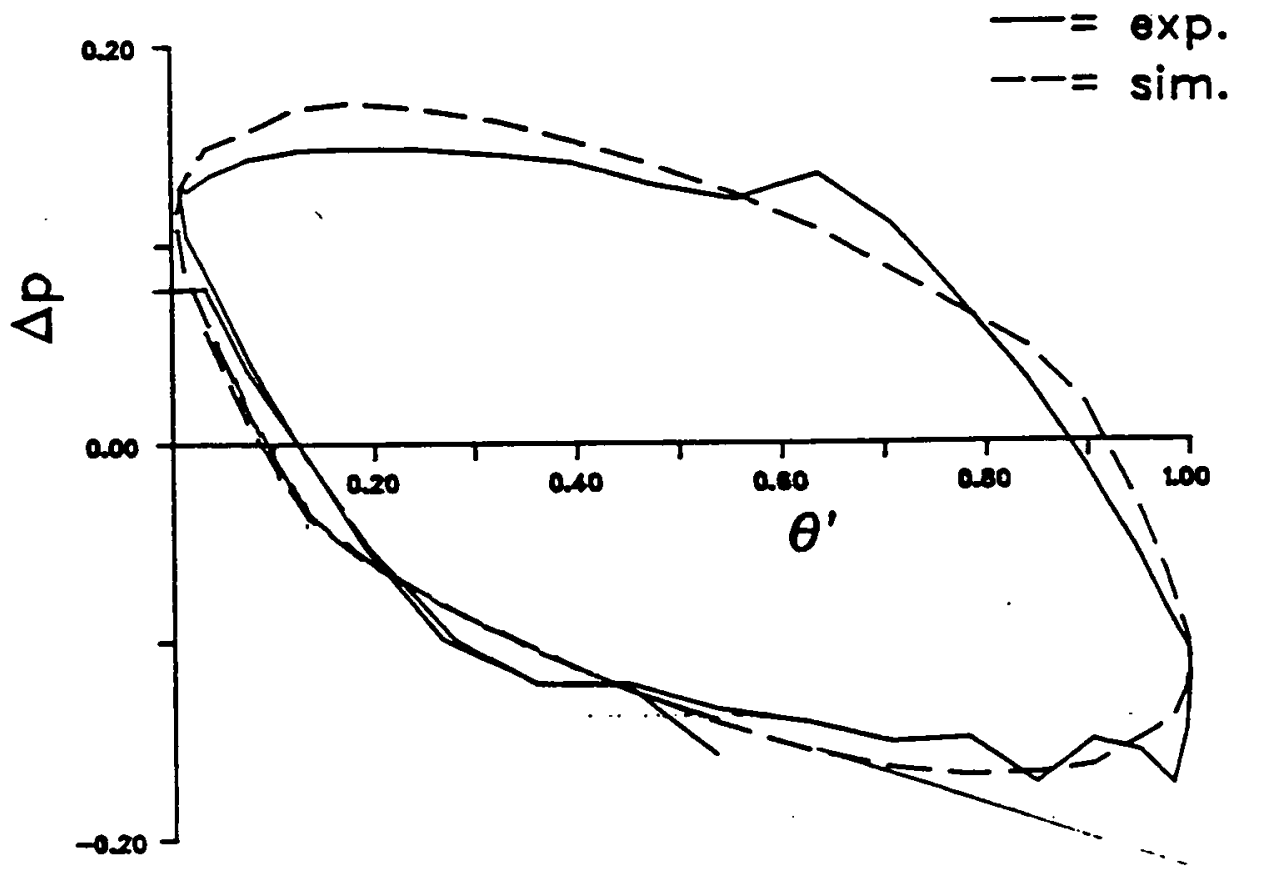

Figure (5.16) $\Delta p$ vs. $\theta$; Experimental and Predicted

Period $=8 \mathrm{~s} ;$ Amplitude $=7 \mathrm{~cm}$

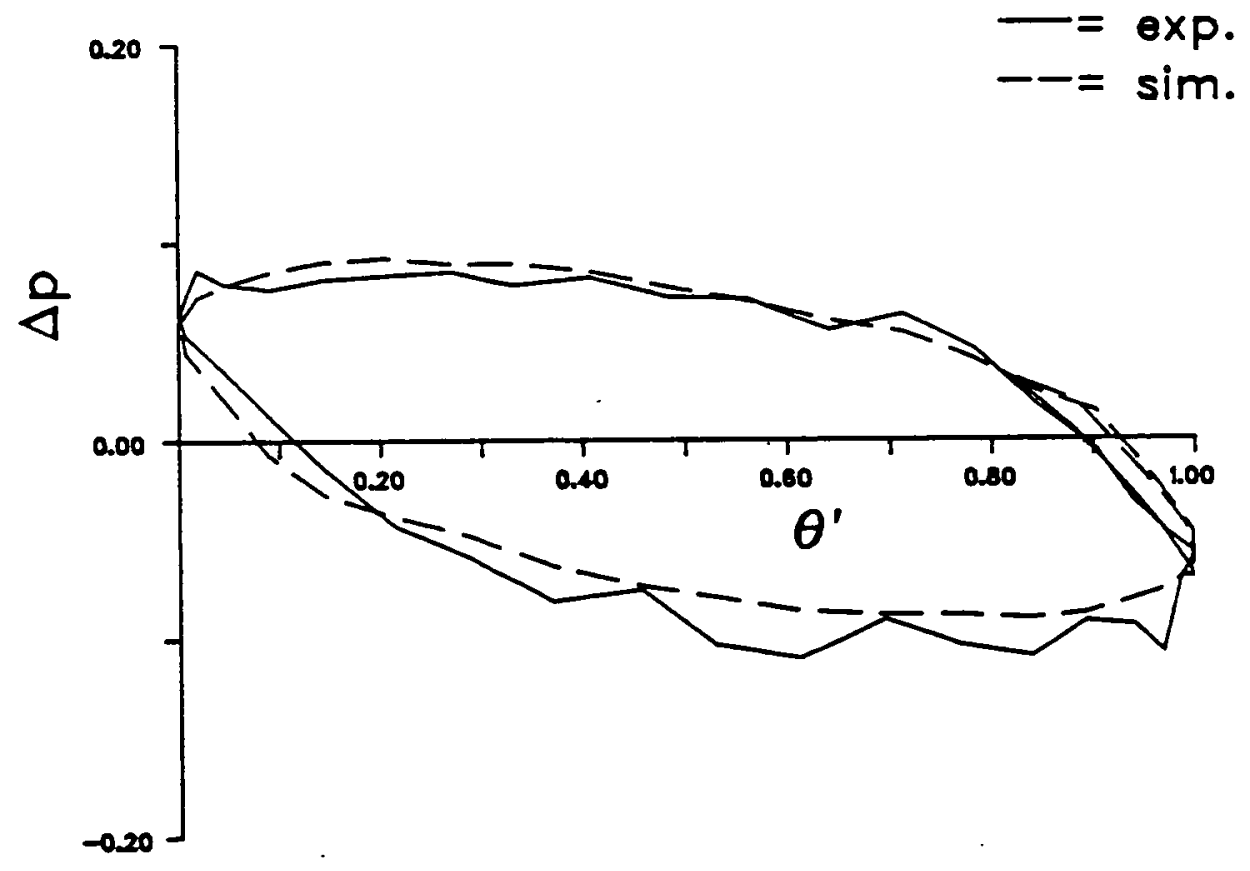

Figure (5.17) $\Delta p$ vs. $\theta$; Experimental and Predicted Period $=9 \mathrm{~s} ;$ Amplitude $=4 \mathrm{~cm}$ 


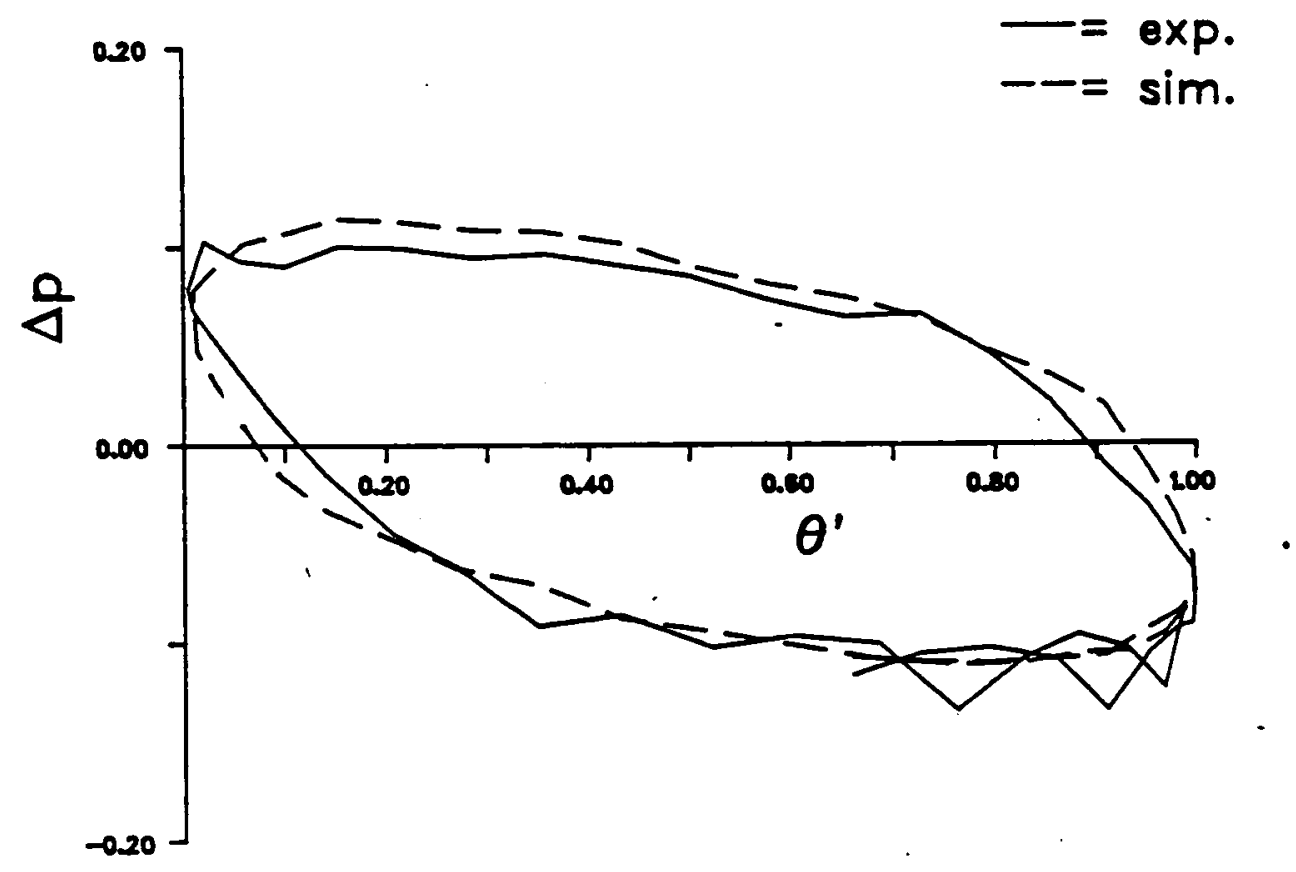

Figure (5.18) $\Delta p$ vs. $\theta$; Experimental and Predicted Period $=9 \mathrm{~s} ;$ Amplitude $=5 \mathrm{~cm}$

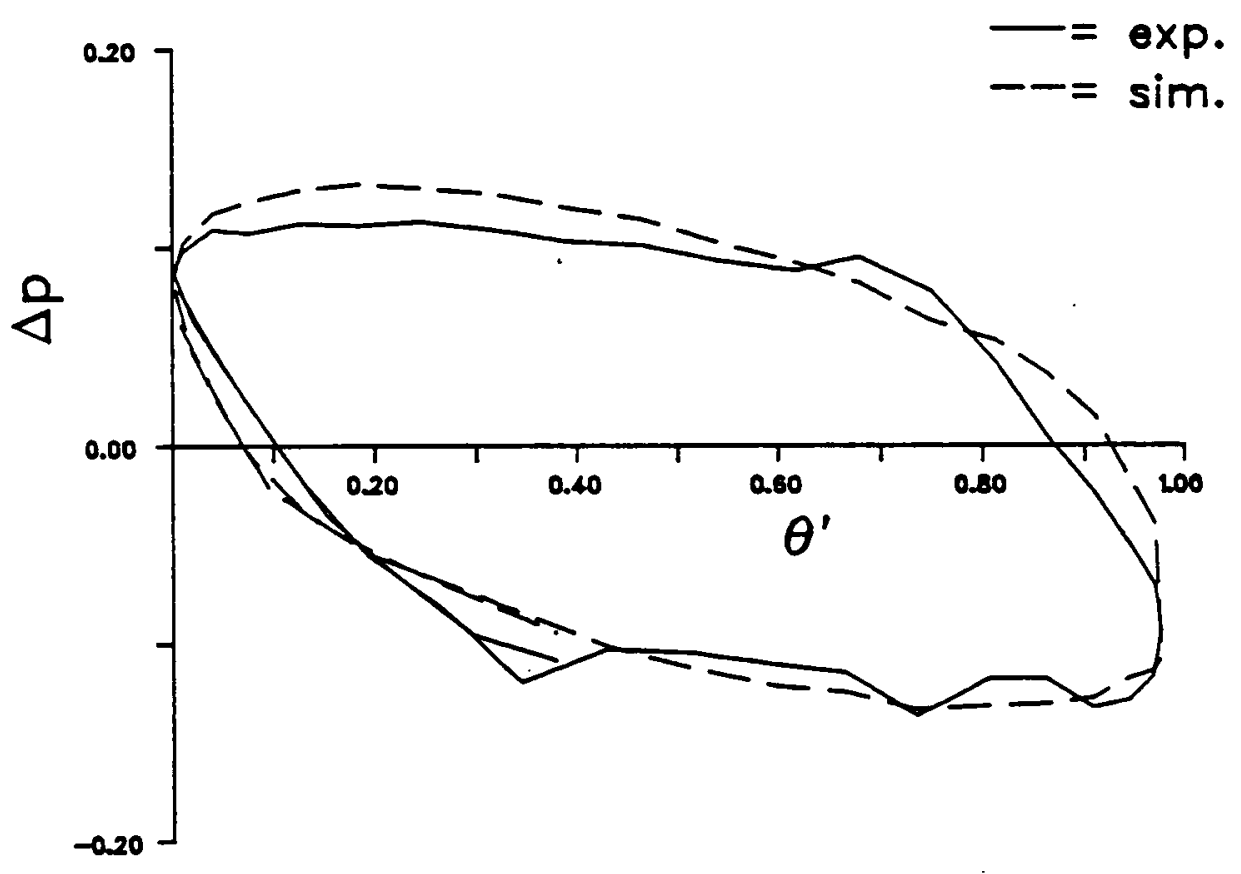

Figure (5.19) $\Delta p$ vs. $\theta$; Experimental and Predicted Period = 9s; Amplitude $=6 \mathrm{~cm}$ 


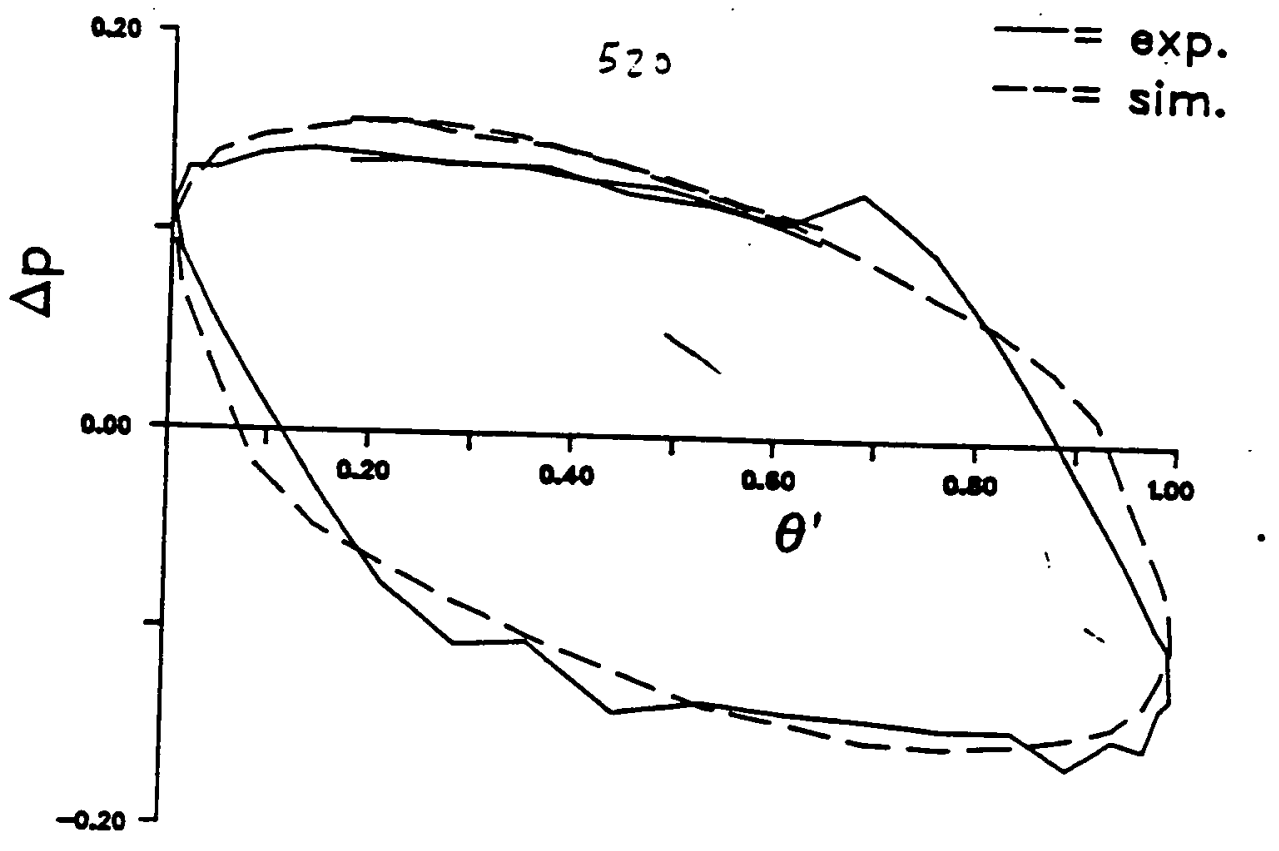

Figure (5.20) $\Delta p$ vs. $\theta$; Experimental and Predicted

Period $=9 s ;$ Amplitude $=7 \mathrm{~cm}$

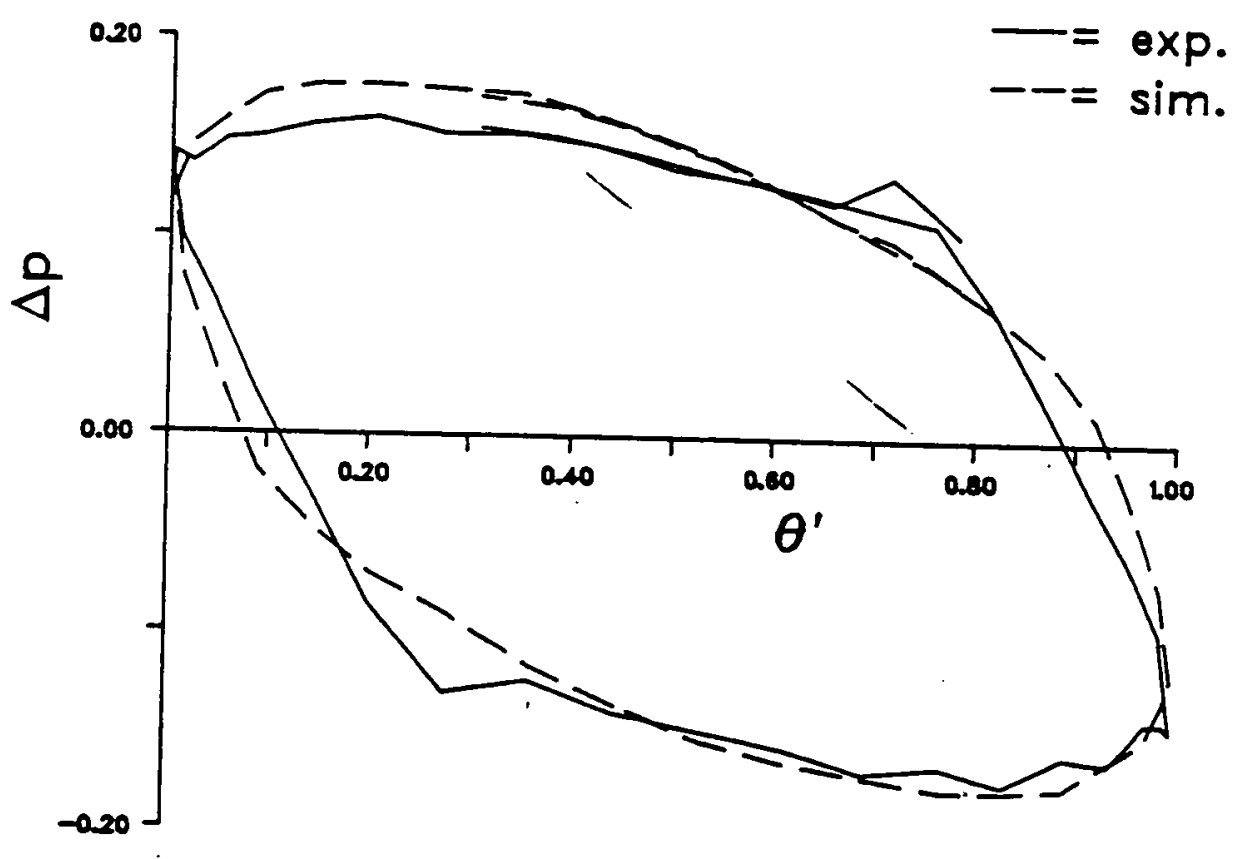

Figure (5.21) $\Delta p$ vs. $\theta$; Experimental and Predicted Period $=9 s ;$ Amplitude $=8 \mathrm{~cm}$ 


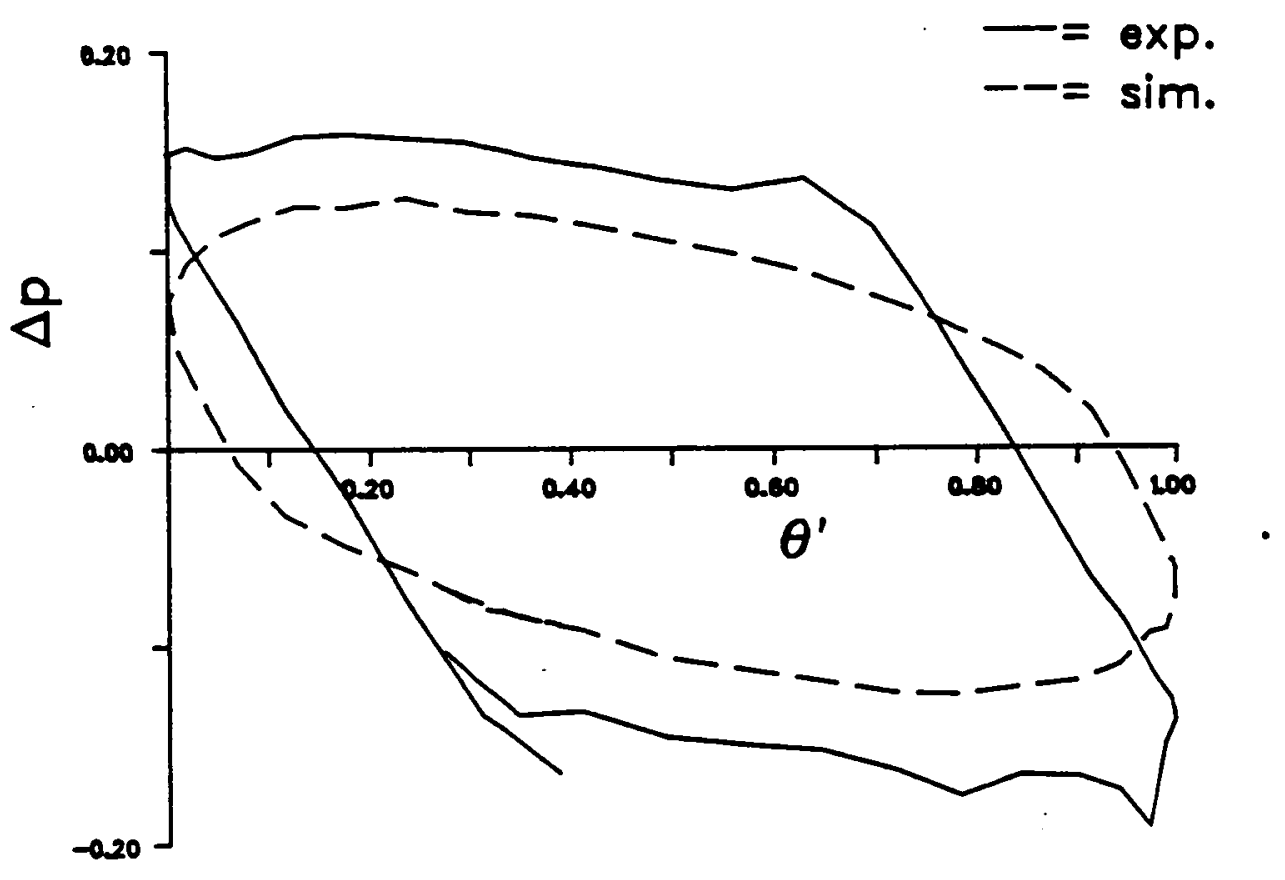

Figure (5.22) $\Delta p$ vs. $\theta$; Experimental and Predicted Period $=10 \mathrm{~s} ;$ Amplitude $=6 \mathrm{~cm}$

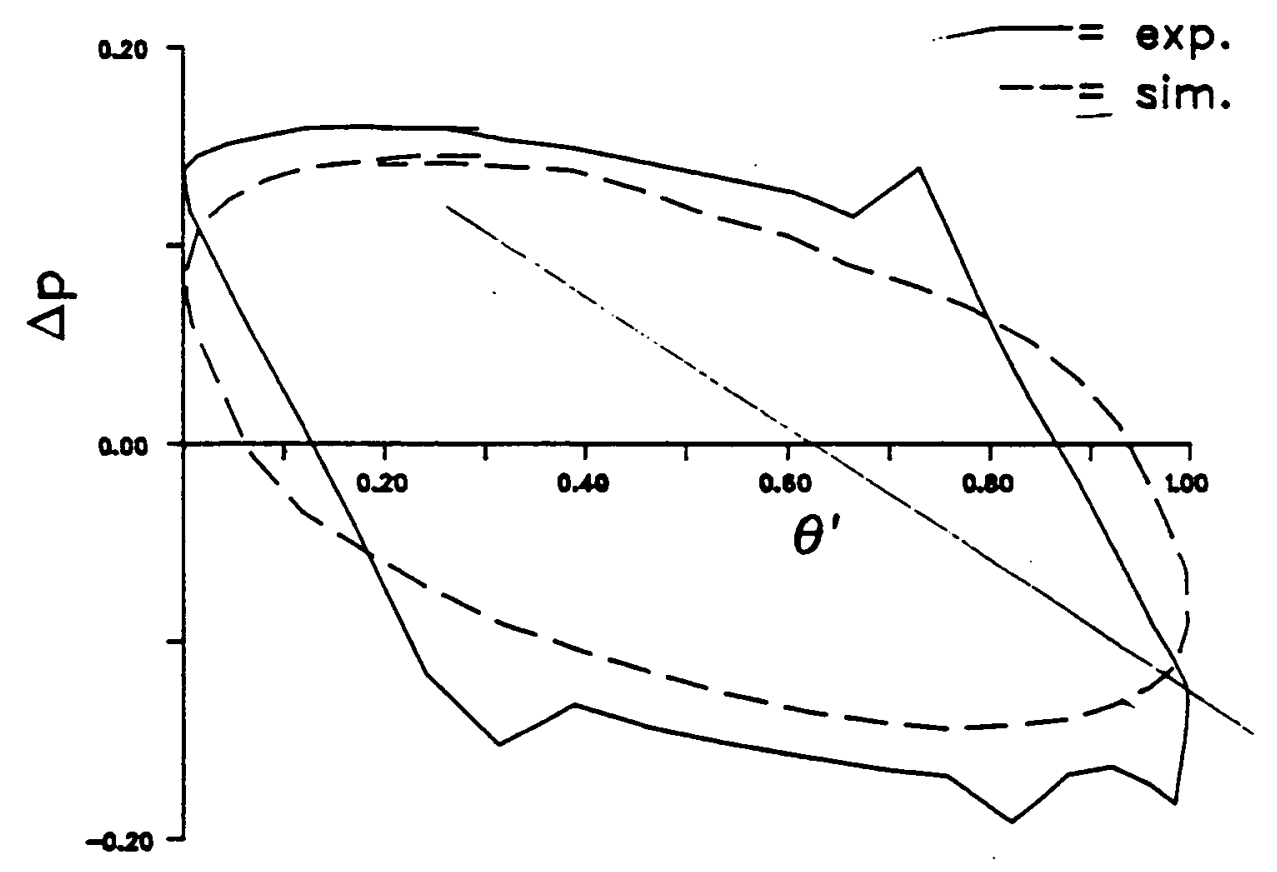

Figure (5.23) $\Delta p$ vs. $\theta$; Experimental and Predicted Period $=10 \mathrm{~s} ;$ Amplitude $=7 \mathrm{~cm}$ 


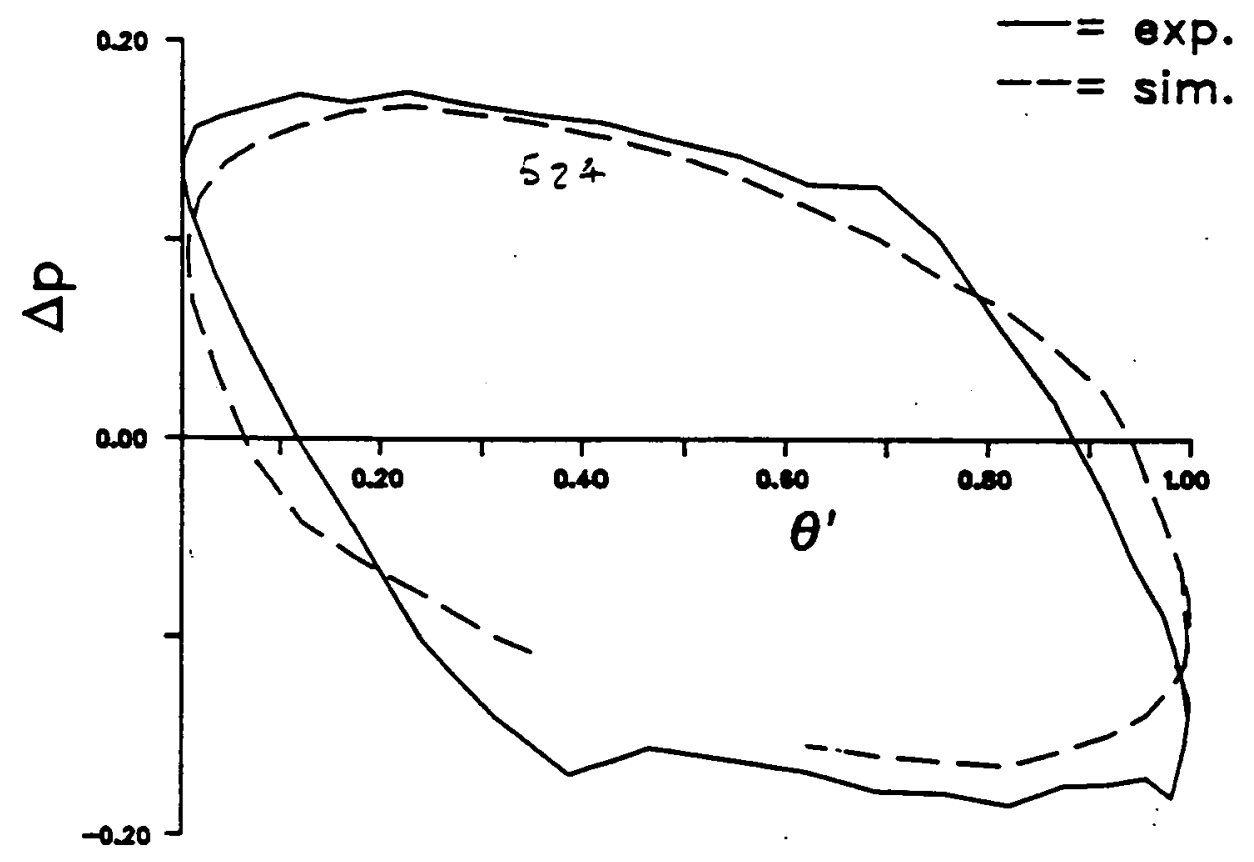

Figure (5.24) $\Delta p$ vs. $\theta$; Experimental and Predicted Period = 10s; Amplitude $=8 \mathrm{~cm}$ 


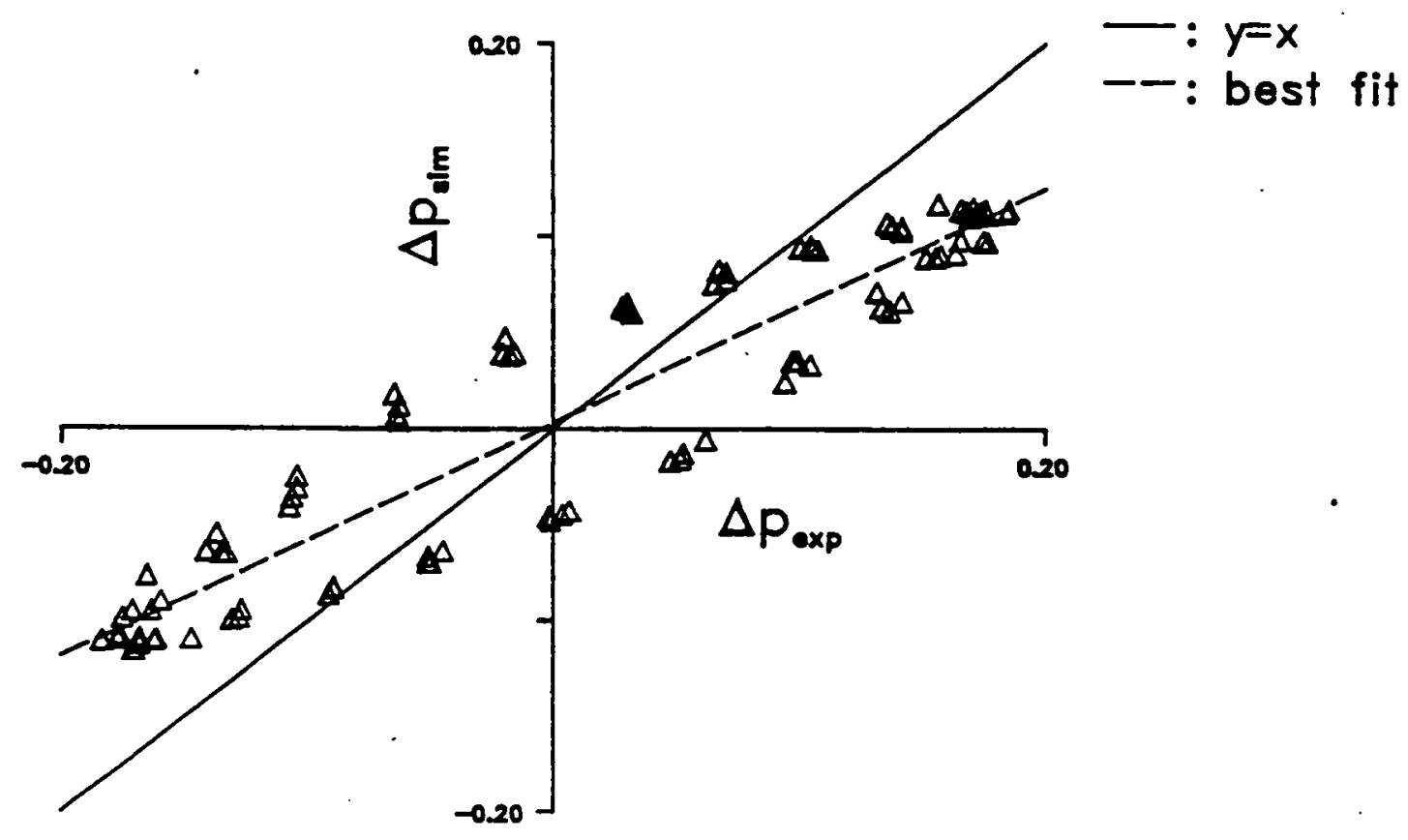

Figure (5.25) $\Delta p_{\text {sim }} v s . \Delta p_{\text {exp }}$

Period $=6 \mathrm{~s} ;$ Amplitude $=4 \mathrm{~cm}$

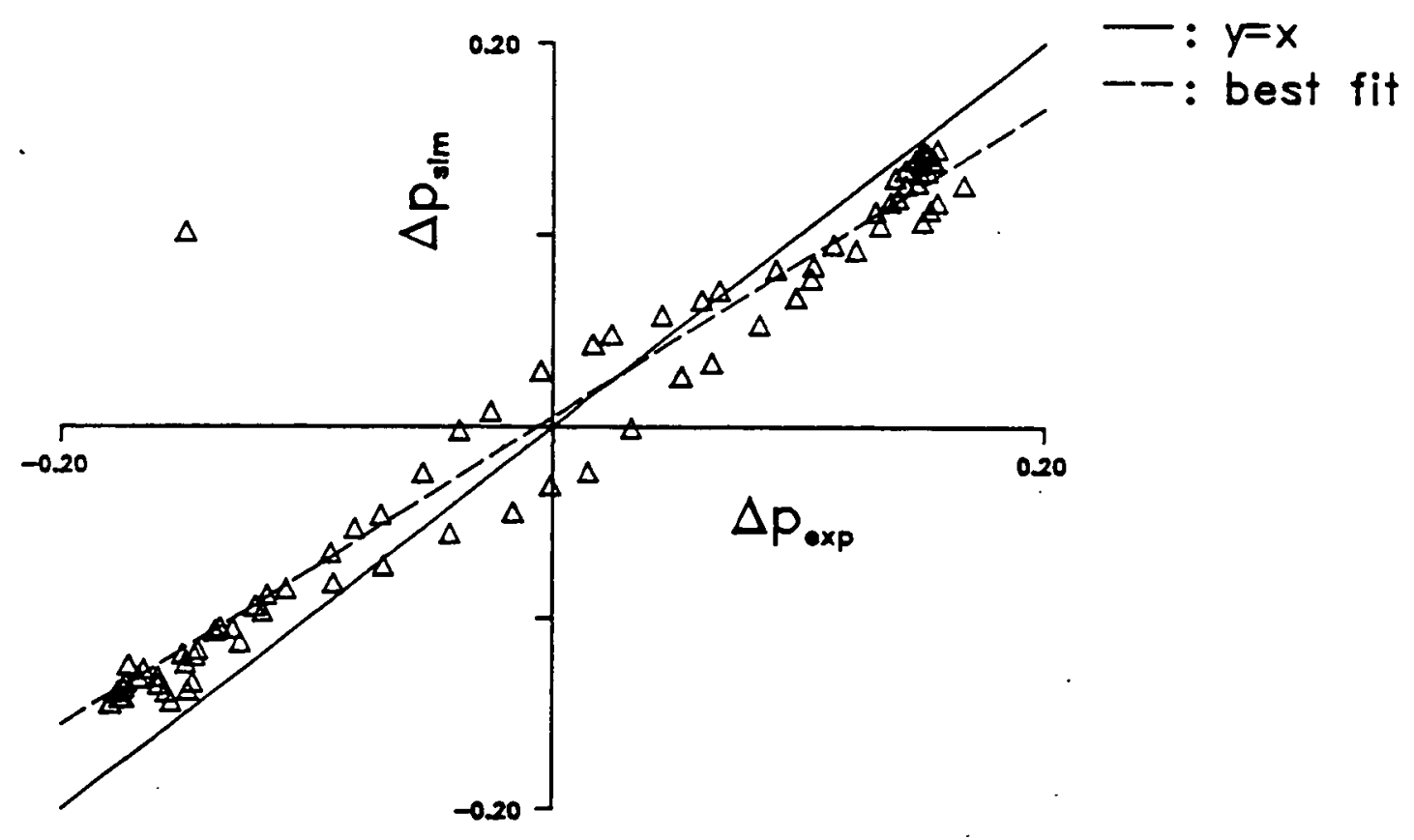

Figure (5.26) $\Delta p_{\text {sim }}$ vs. $\Delta p_{\text {exp }}$

Period $=6 \mathrm{~s} ;$ Amplitude $=5 \mathrm{~cm}$ 


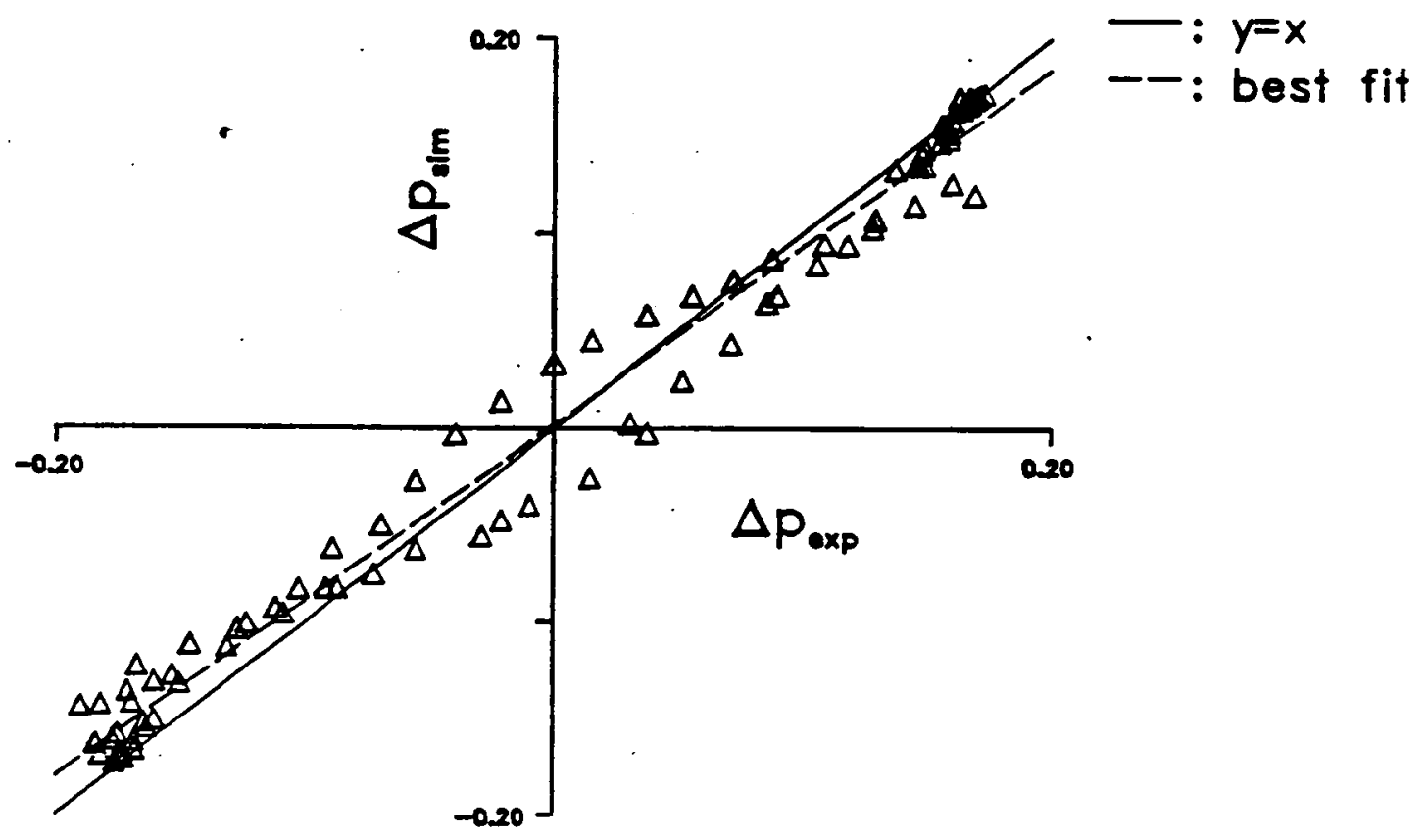

Figure (5.27) $\Delta p_{\text {sim }}$ vs. $\Delta p_{\text {exp }}$

Period $=6 \mathrm{~s} ;$ Amplitude $=6 \mathrm{~cm}$

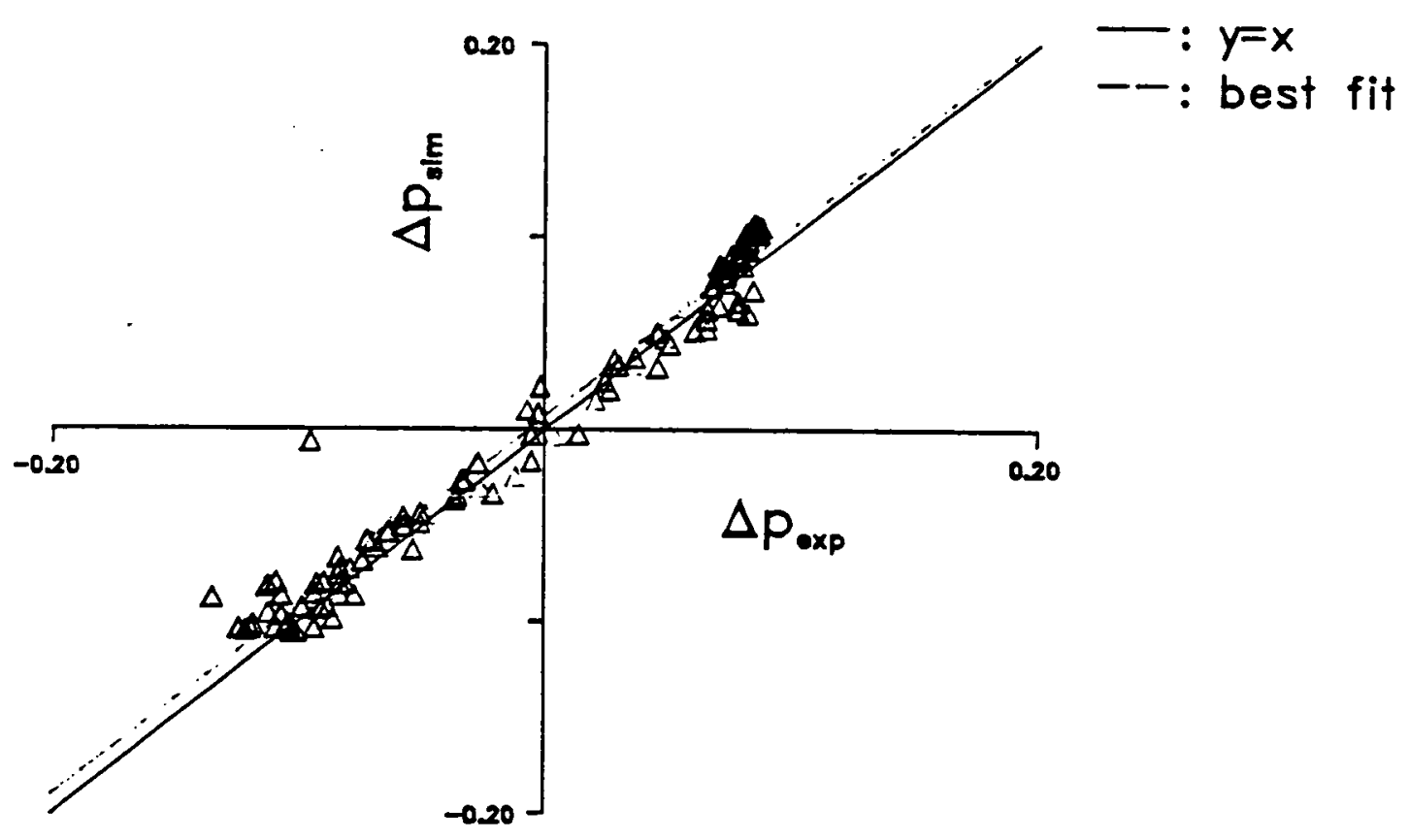

Figure (5.28) $\Delta p_{\text {sim }} v s . \Delta p_{\text {exp }}$

Period $=7 \mathrm{~s}$; Amplitude $=4 \mathrm{~cm}$ 


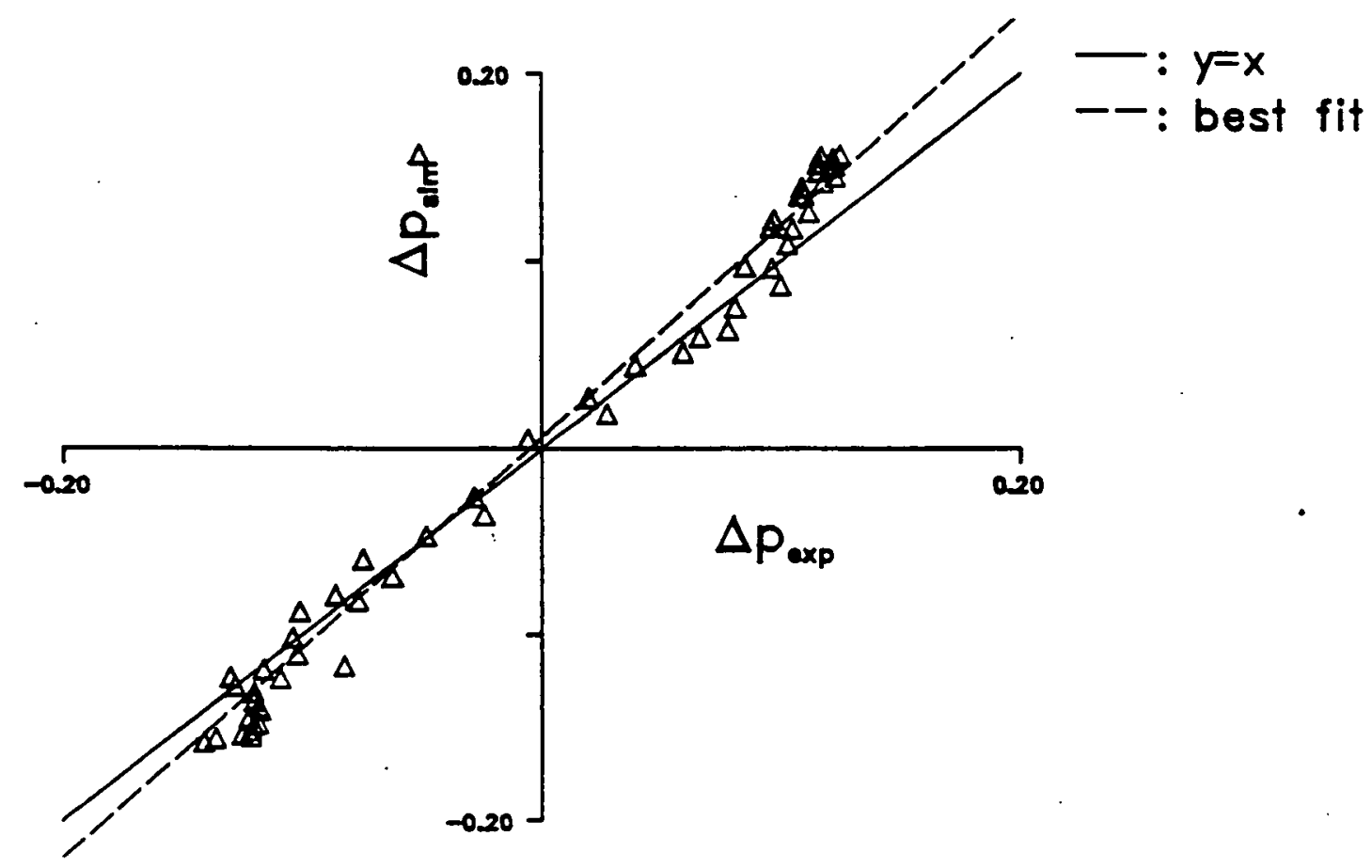

Figure (5.29) $\Delta p_{\text {sim }}$ vs. $\Delta p_{\text {exp }}$

Period $=7 \mathrm{~s} ;$ Amplitude $=6 \mathrm{~cm}$

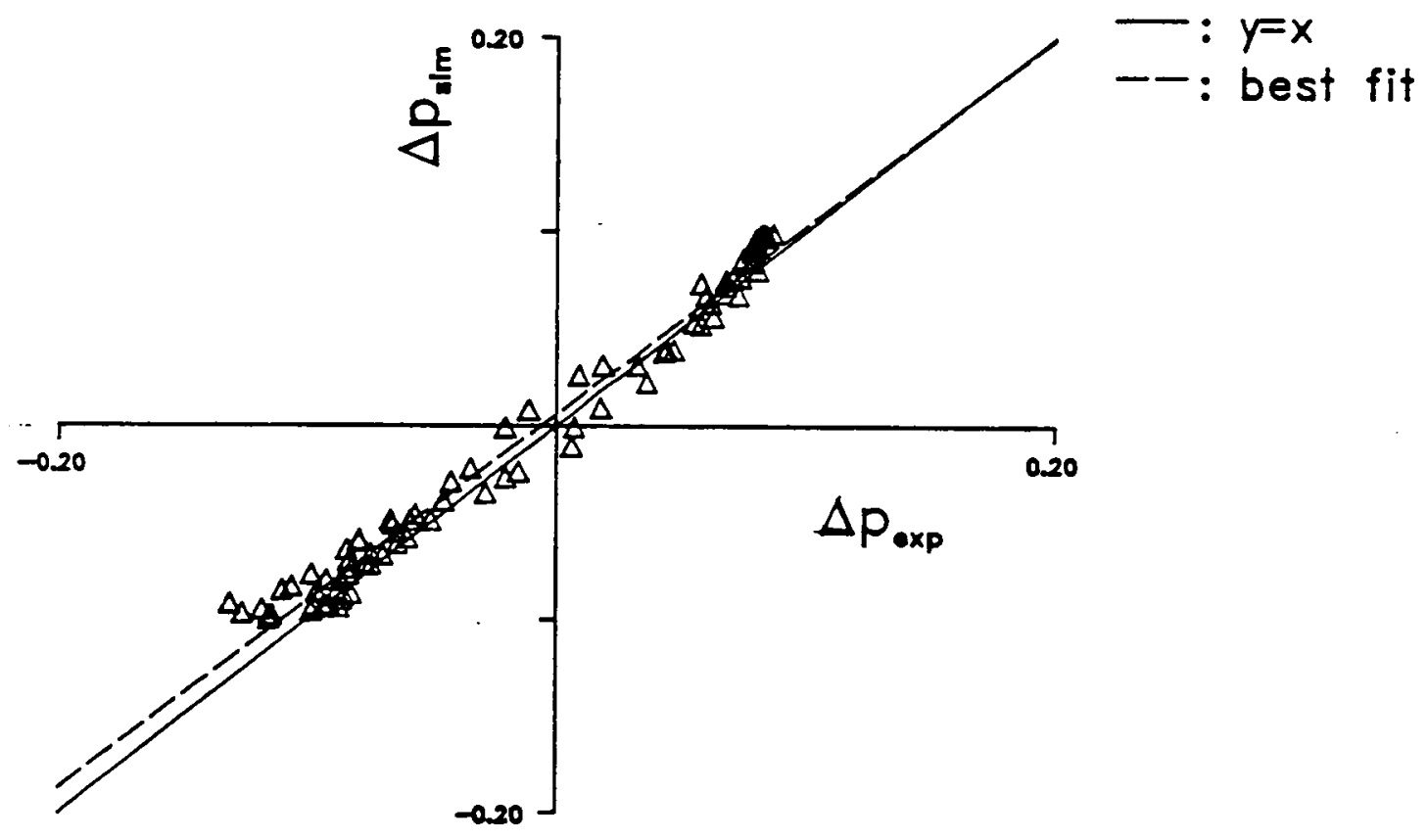

Figure (5.30) $\Delta p_{\text {sim }}$ vs. $\Delta p_{\text {exp }}$

Period $=8 \mathrm{~s} ;$ Amplitude $=4 \mathrm{~cm}$ 


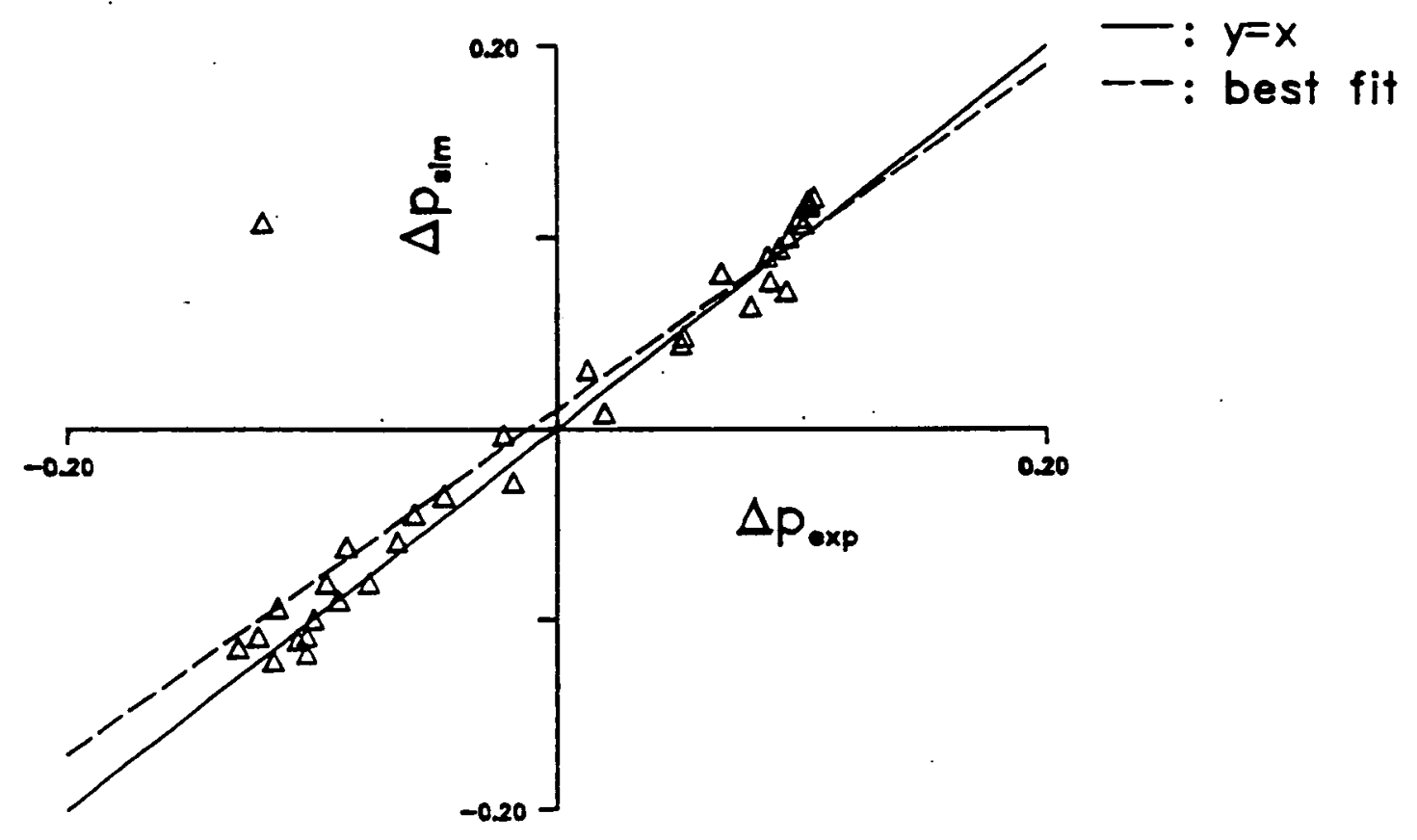

Figure (5.31) $\Delta p_{\text {sim }}$ vs. $\Delta p_{\text {exp }}$

Period $=8 \mathrm{~s} ;$ Amplitude $=5 \mathrm{~cm}$

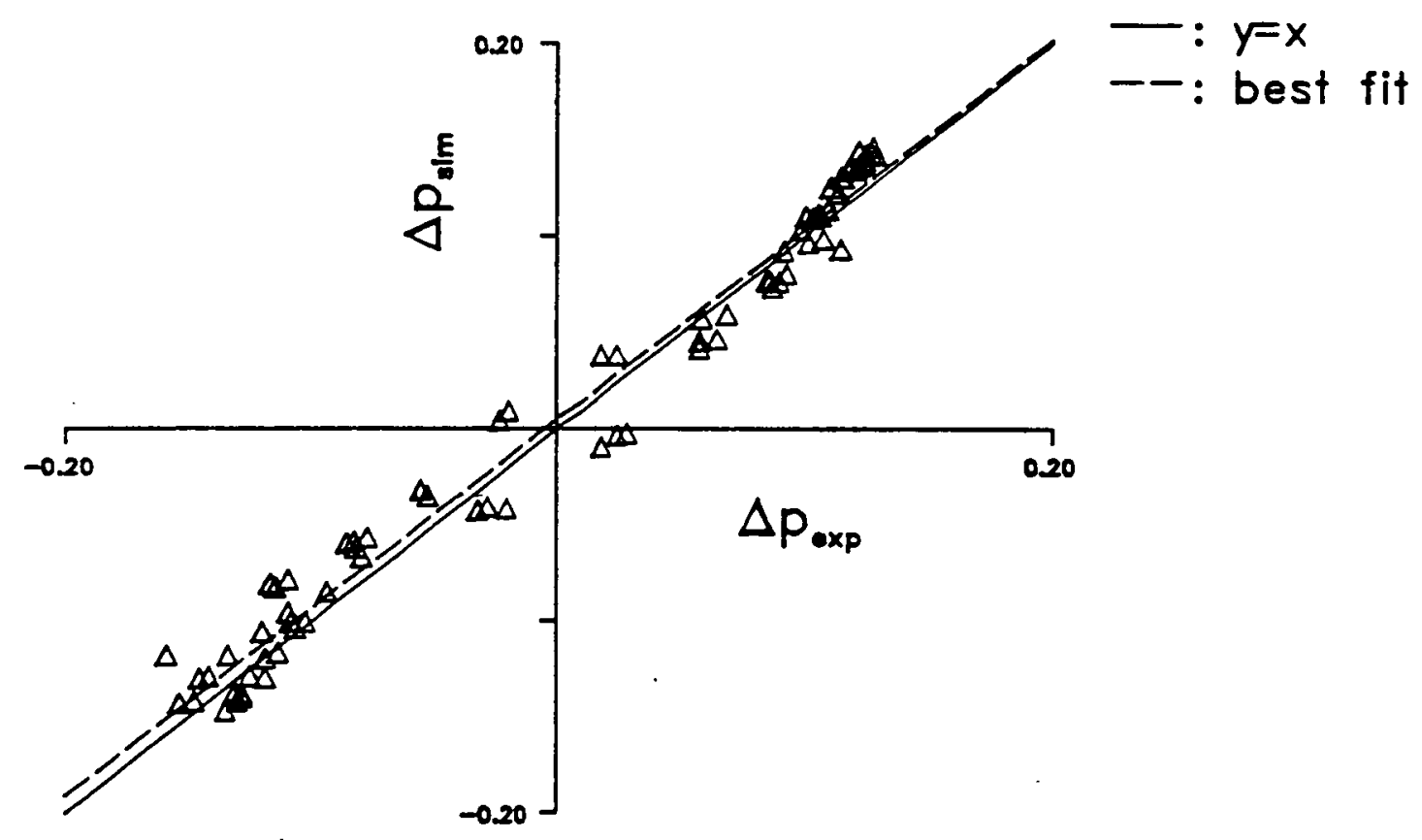

Figure (5.32) $\Delta p_{\text {sim }} v s . \Delta p_{\text {exp }}$

Period $=8 \mathrm{~s}$ : Amplitude $=6 \mathrm{~cm}$ 


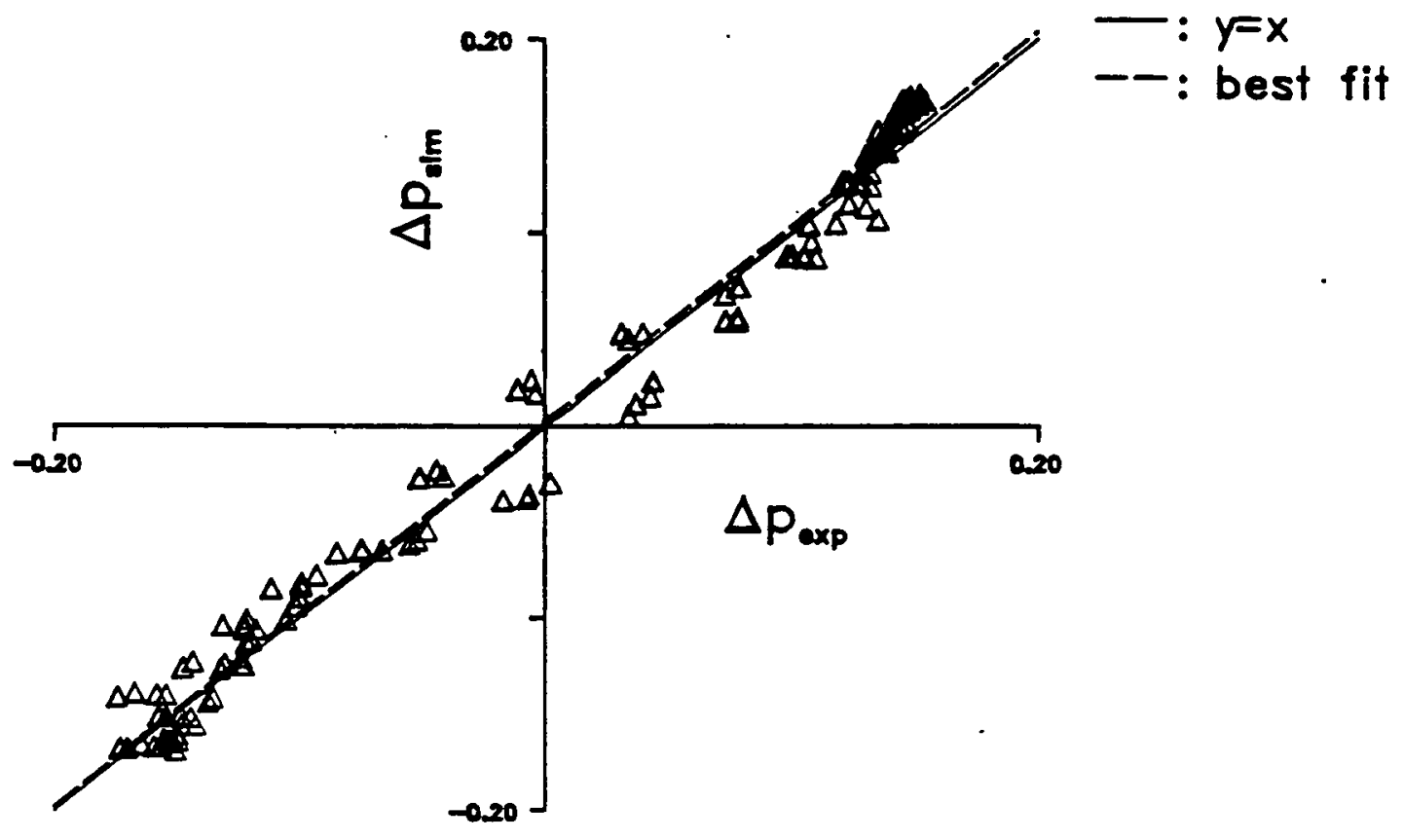

Figure (5.33) $\Delta p_{\text {sim }}$ vs. $\Delta p_{\text {exp }}$

Period $=8 \mathrm{~s} ;$ Amplitude $=7 \mathrm{~cm}$

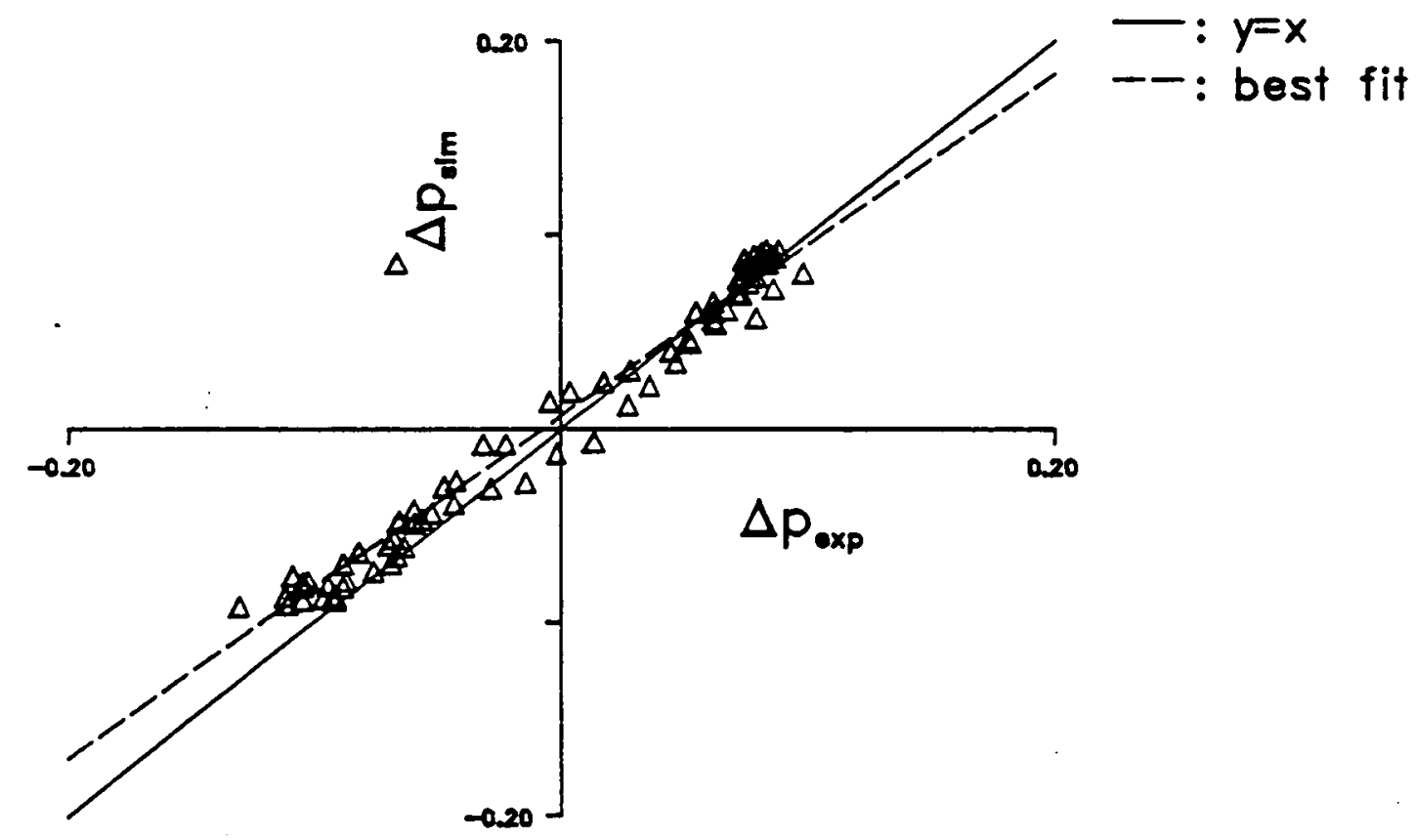

Figure (5.34) $\Delta p_{\text {sim }}$ vs. $\Delta p_{\text {exp }}$

Period $=9 s ;$ Amplitude $=4 \mathrm{~cm}$ 


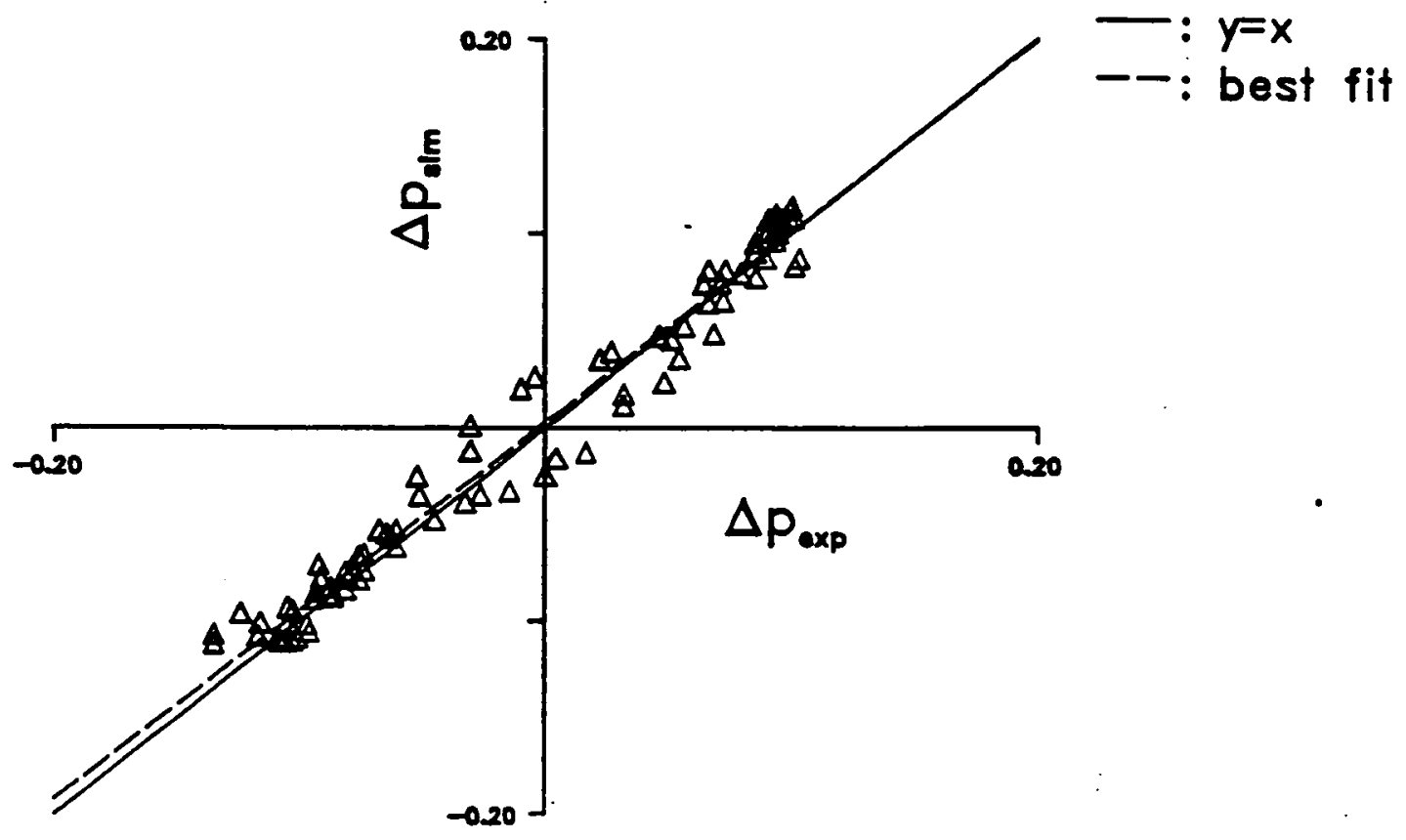

Figure (5.35) $\Delta p_{\text {sim }}$ vs. $\Delta p_{\text {exp }}$

Period $=9 \mathrm{~s} ;$ Amplitude $=5 \mathrm{~cm}$

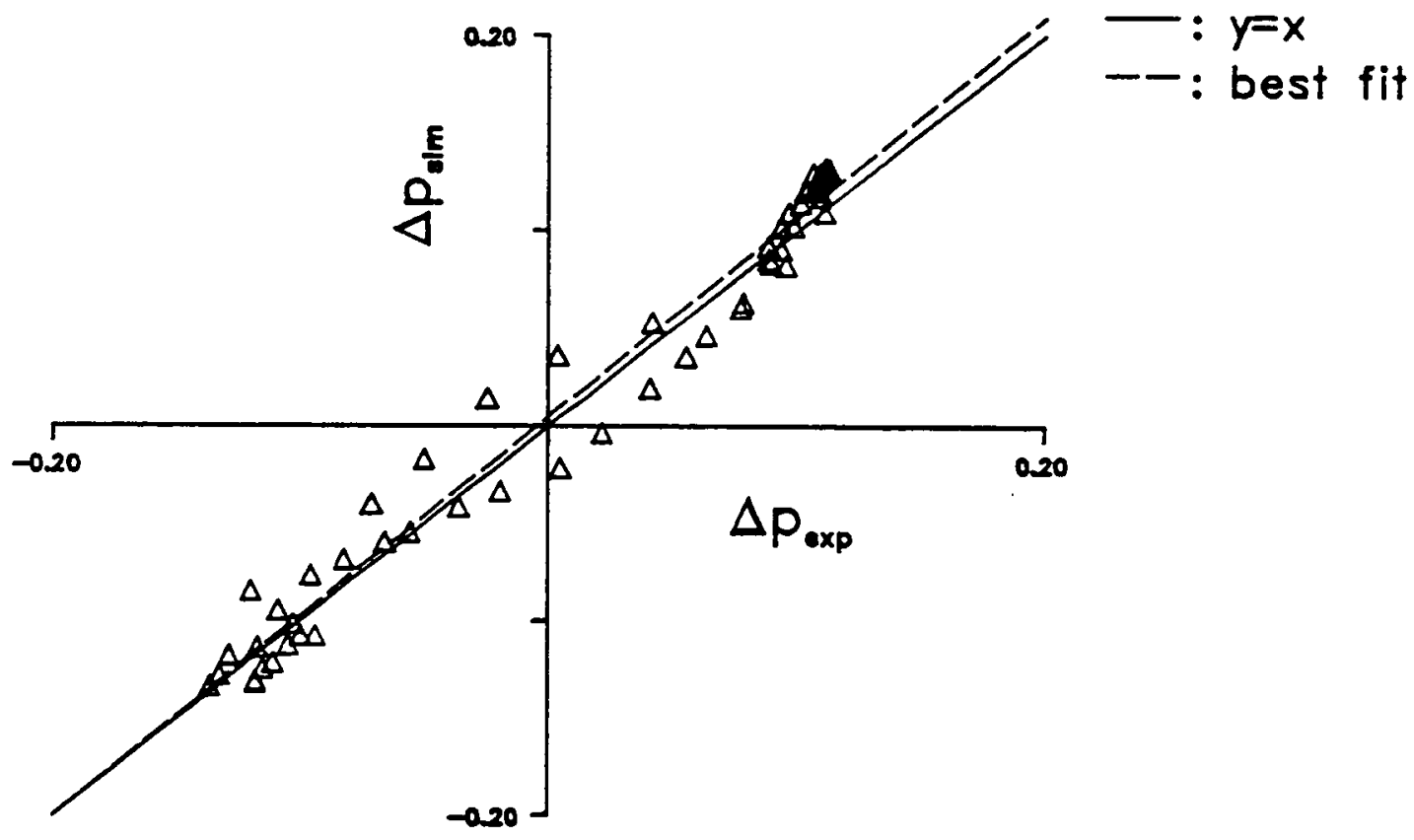

Figure (5.36) $\Delta p_{\text {sim }}$ vs. $\Delta p_{\text {exp }}$

Period = 9s; Amplitude $=6 \mathrm{~cm}$ 


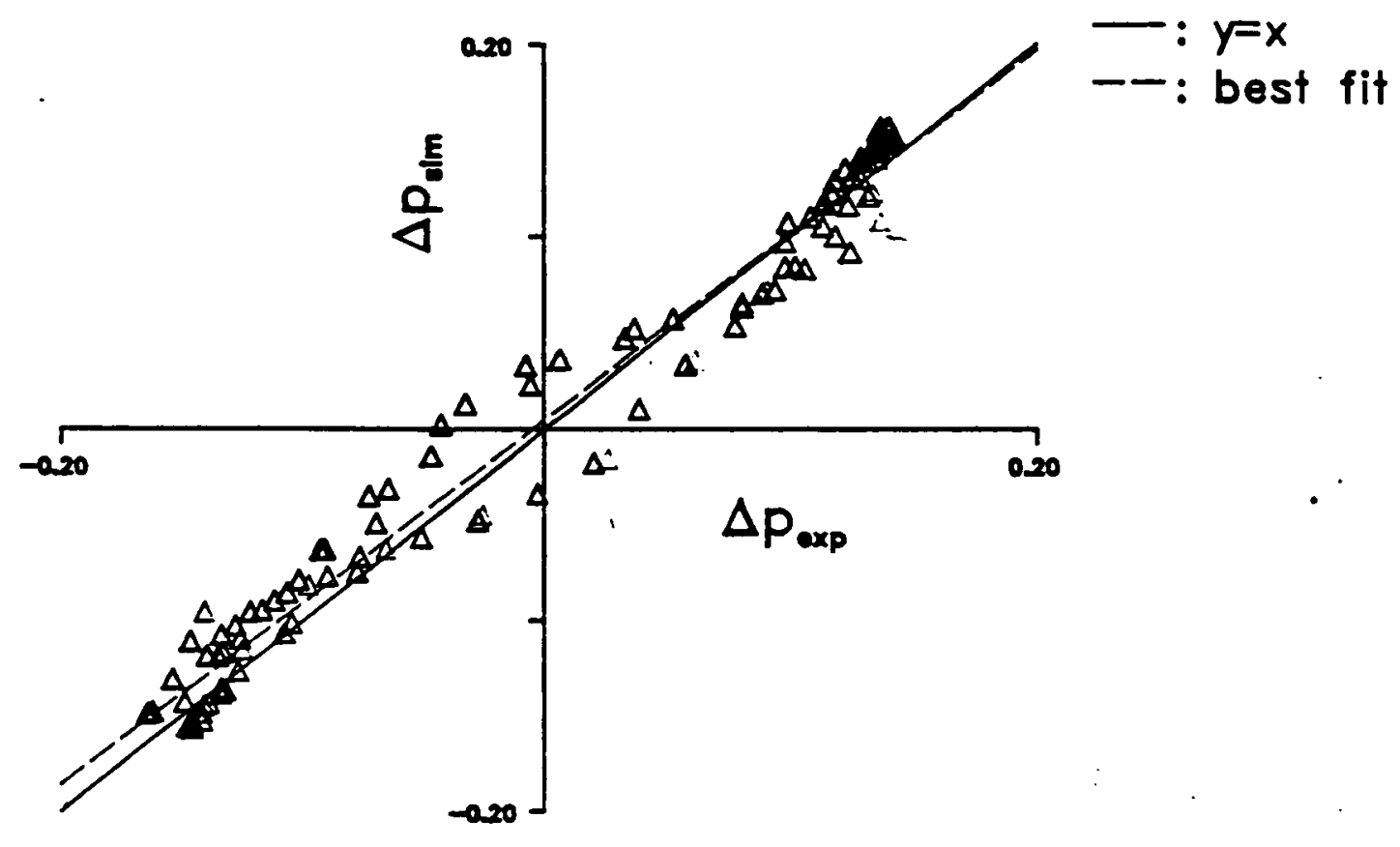

Figure (5.37) $\Delta p_{\text {sim }}$ vs. $\Delta p_{\text {exp }}$

Period $=9 \mathrm{~s} ;$ Amplitude $=7 \mathrm{~cm}$

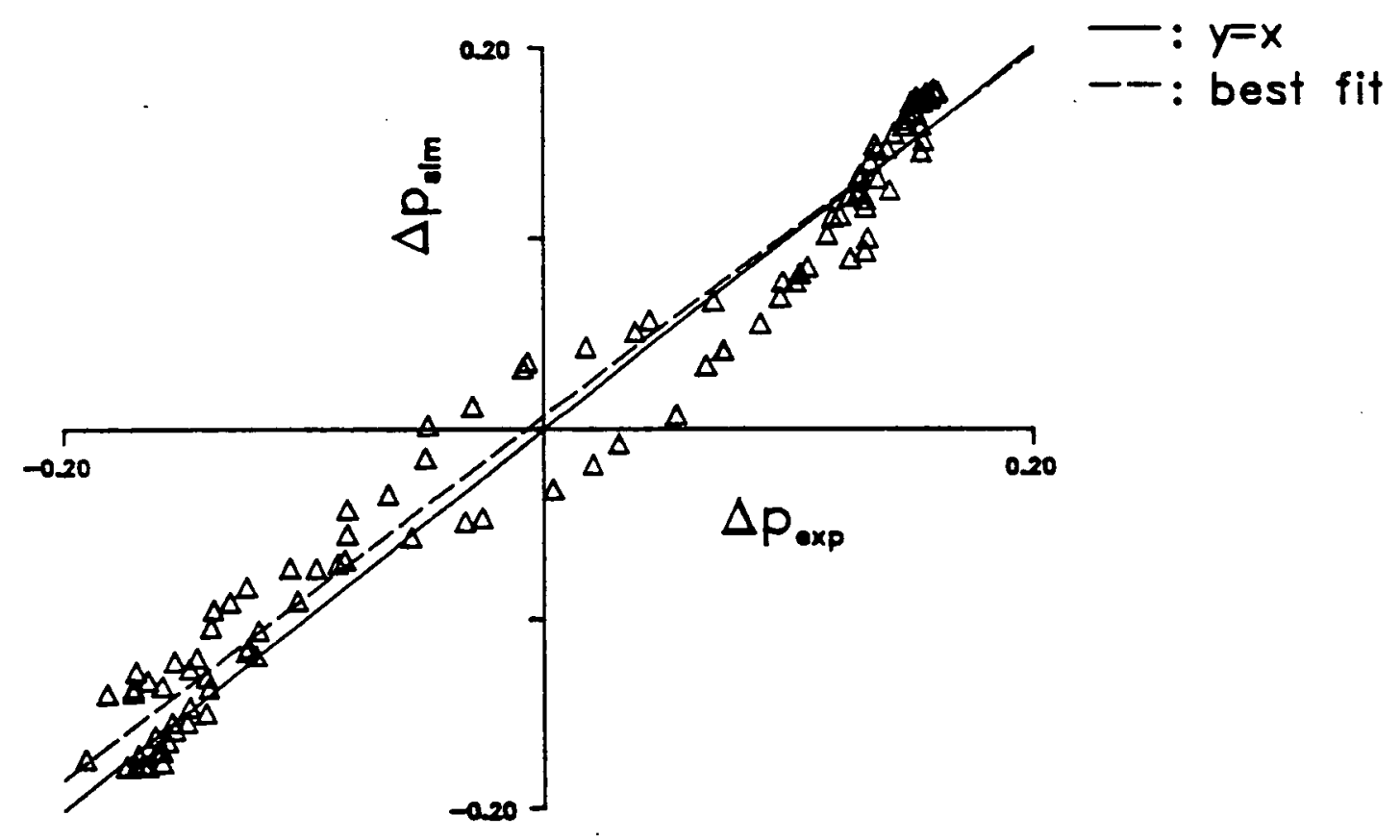

Figure (5.38) $\Delta p_{\text {sim }}$ vs. $\Delta p_{\text {exp }}$

Period $=9 \mathrm{~s} ;$ Amplitude $=8 \mathrm{~cm}$ 


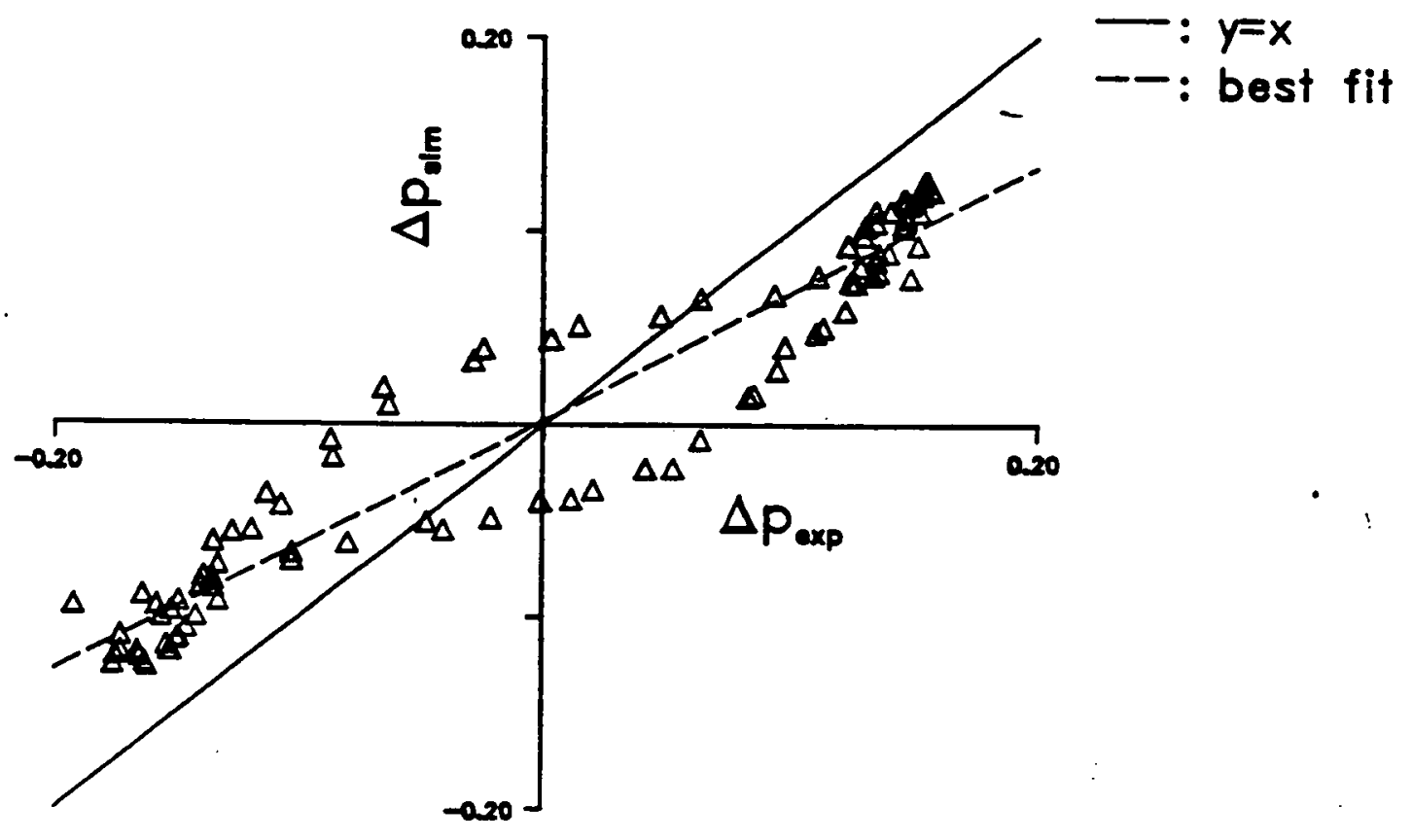

Figure (5.39) $\Delta p_{\text {sim }}$ vs. $\Delta p_{\text {exp }}$

Period $=10 \mathrm{~s} ;$ Amplitude $=6 \mathrm{~cm}$

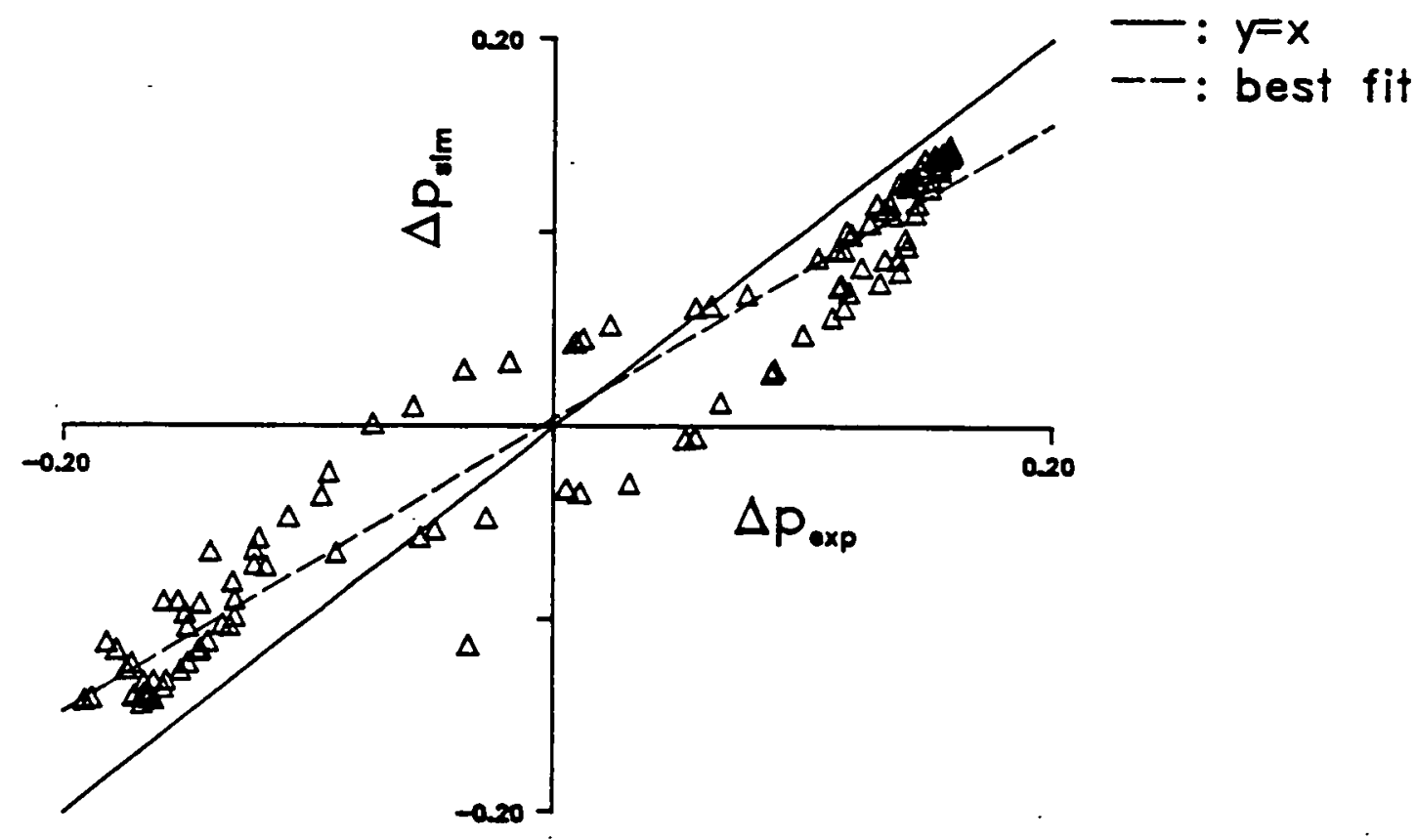

Figure (5.40) $\Delta p_{\text {sim }}$ vs. $\Delta p_{\text {exp }}$

Period $=10 \mathrm{~s} ;$ Amplitude $=7 \mathrm{~cm}$ 


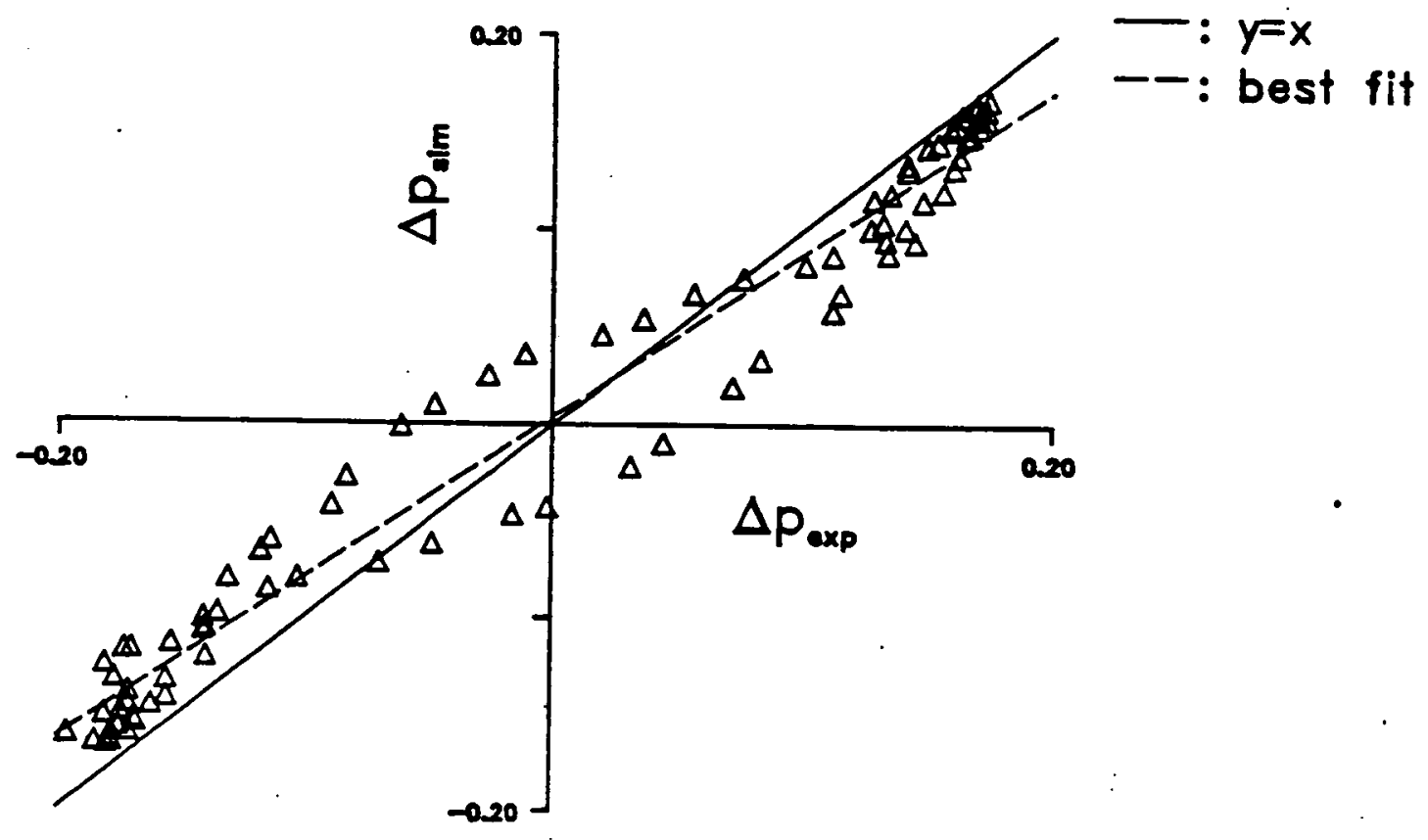

Figure (5.41) $\Delta p_{\text {sim }}$ vs. $\Delta p_{\text {exp }}$

Period = 10s; Amplitude $=8 \mathrm{~cm}$ 

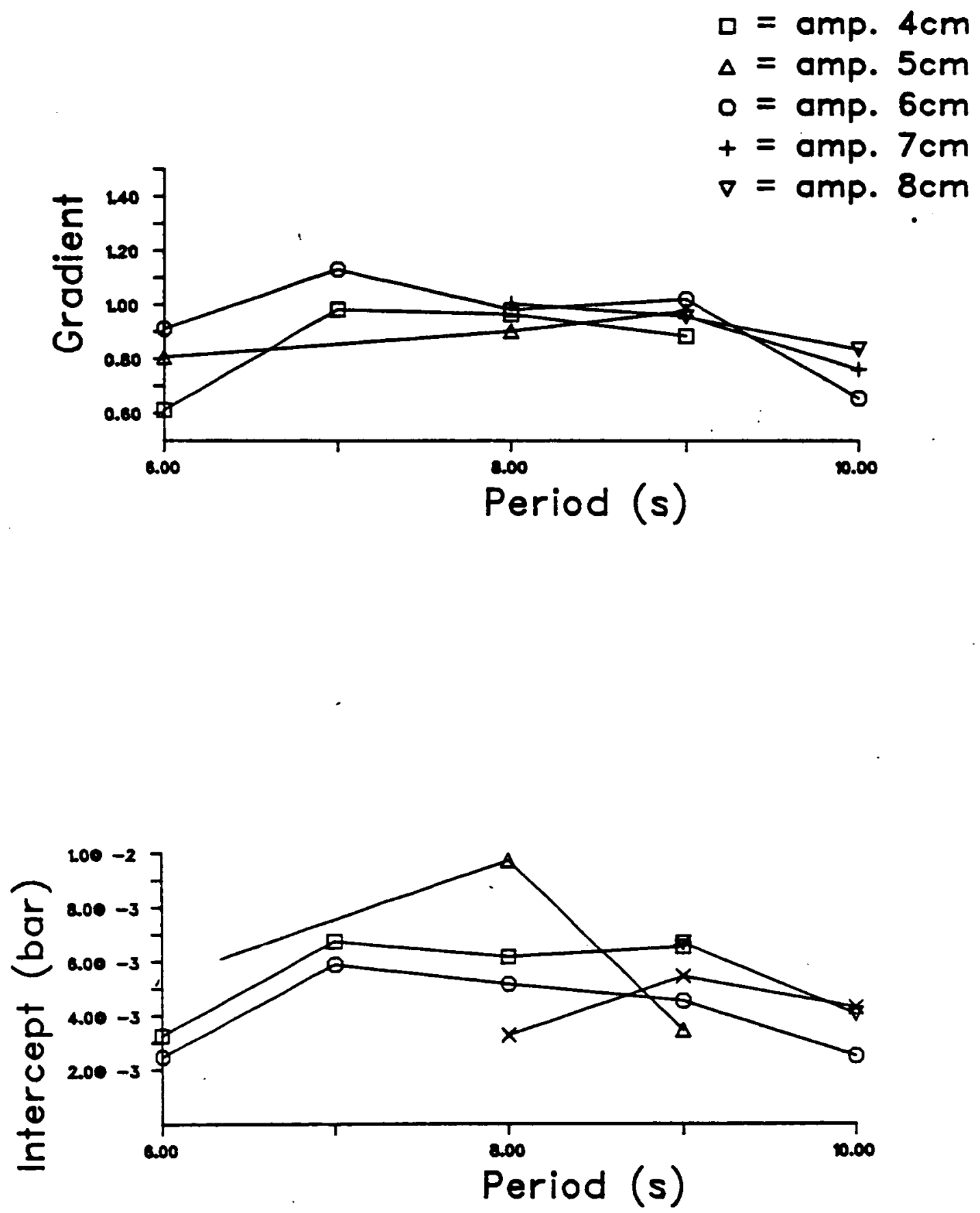

Figure (5.42) Gradient, and Intercept vs. Period 

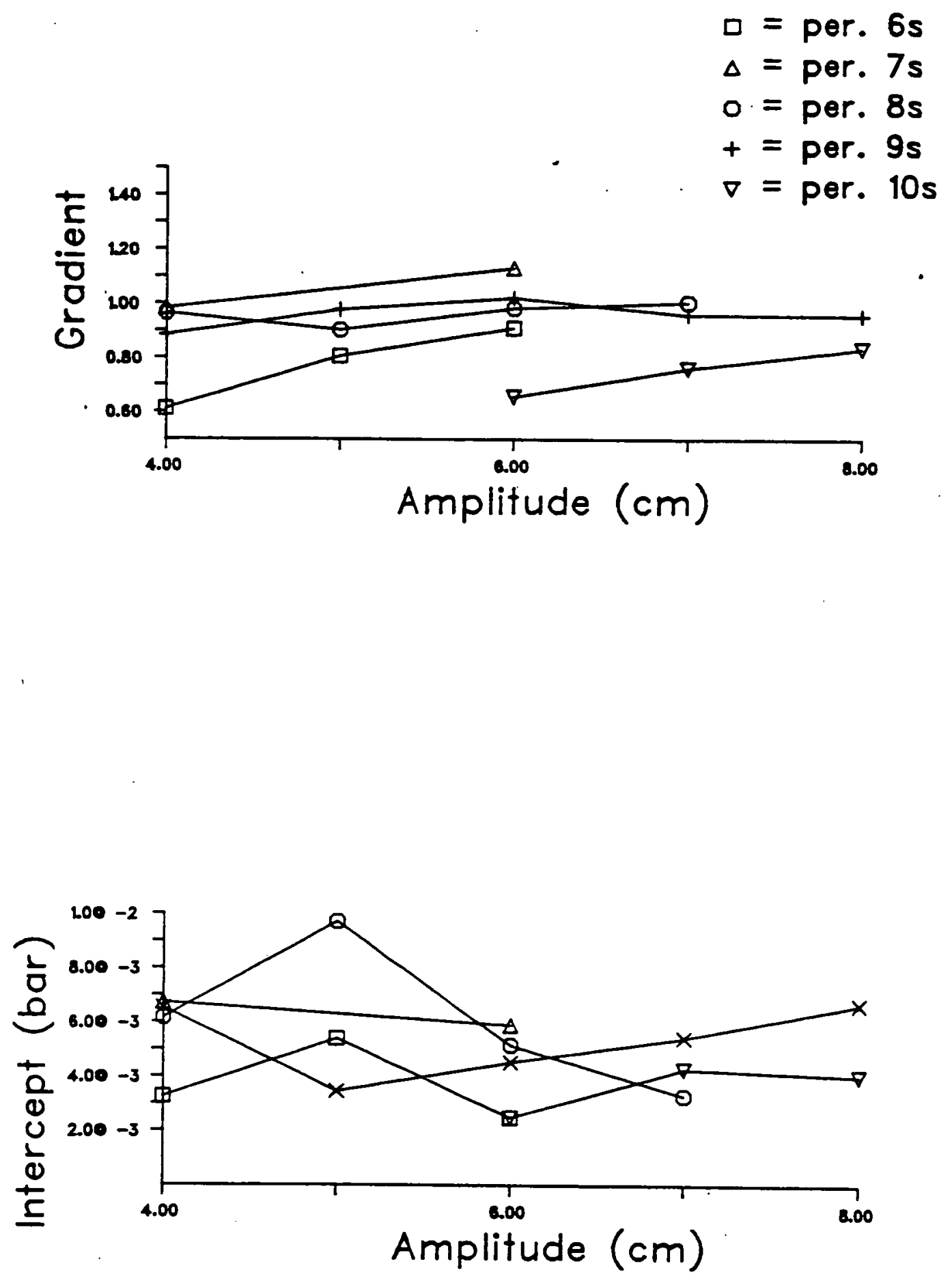

Figure (5.43) Gradient and Intercept vs.Amplitude 


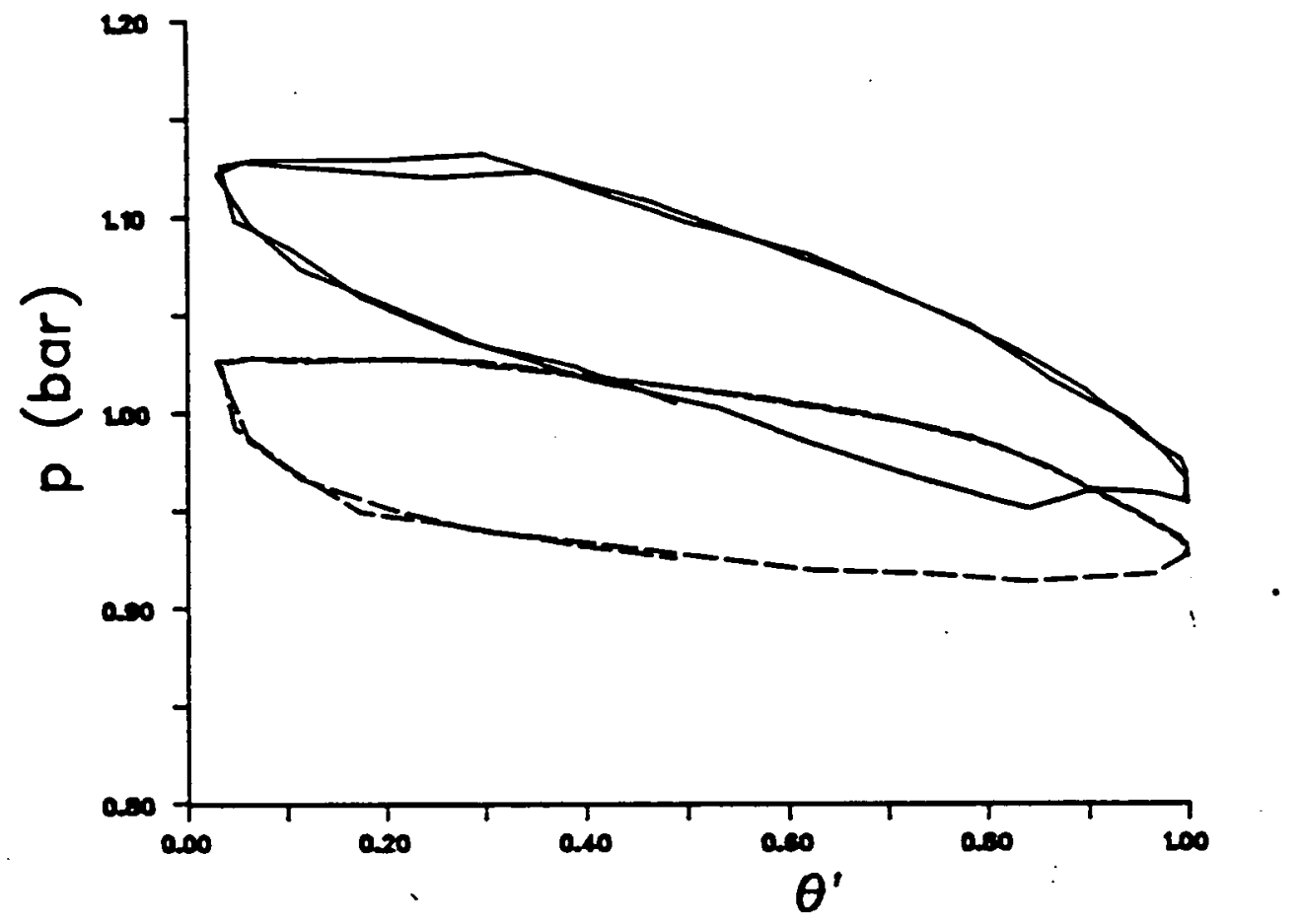

Figure (5.44) D us. $\theta$ : Experimental and Predicted Data Period $=6 \mathrm{~s} ;$ Amplitude $=4 \mathrm{~cm}$

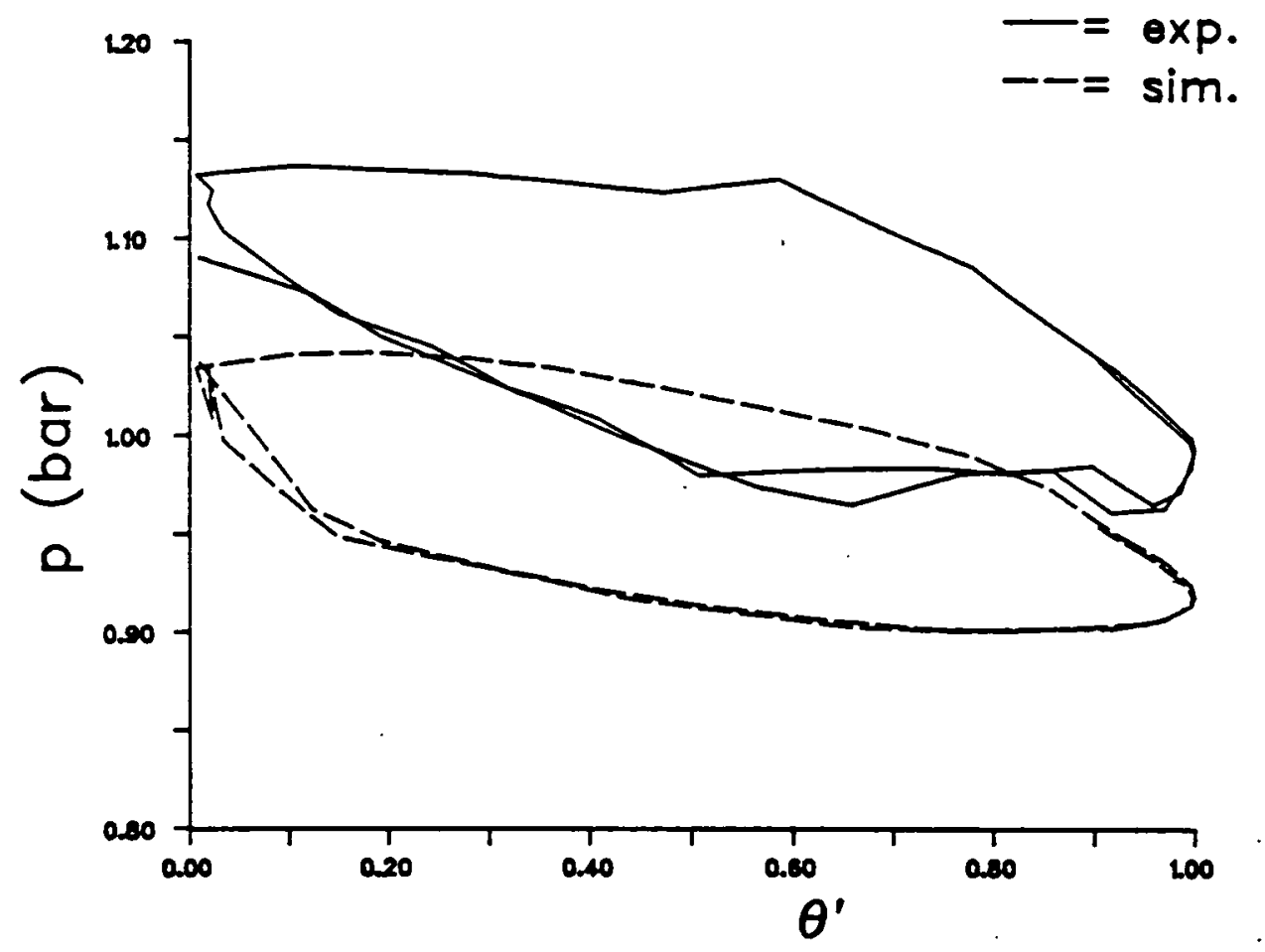

Figure (5.45) p vs. $\theta$; Experimental and Predicted Data Period = 6s; Amplitude $=5 \mathrm{~cm}$ 


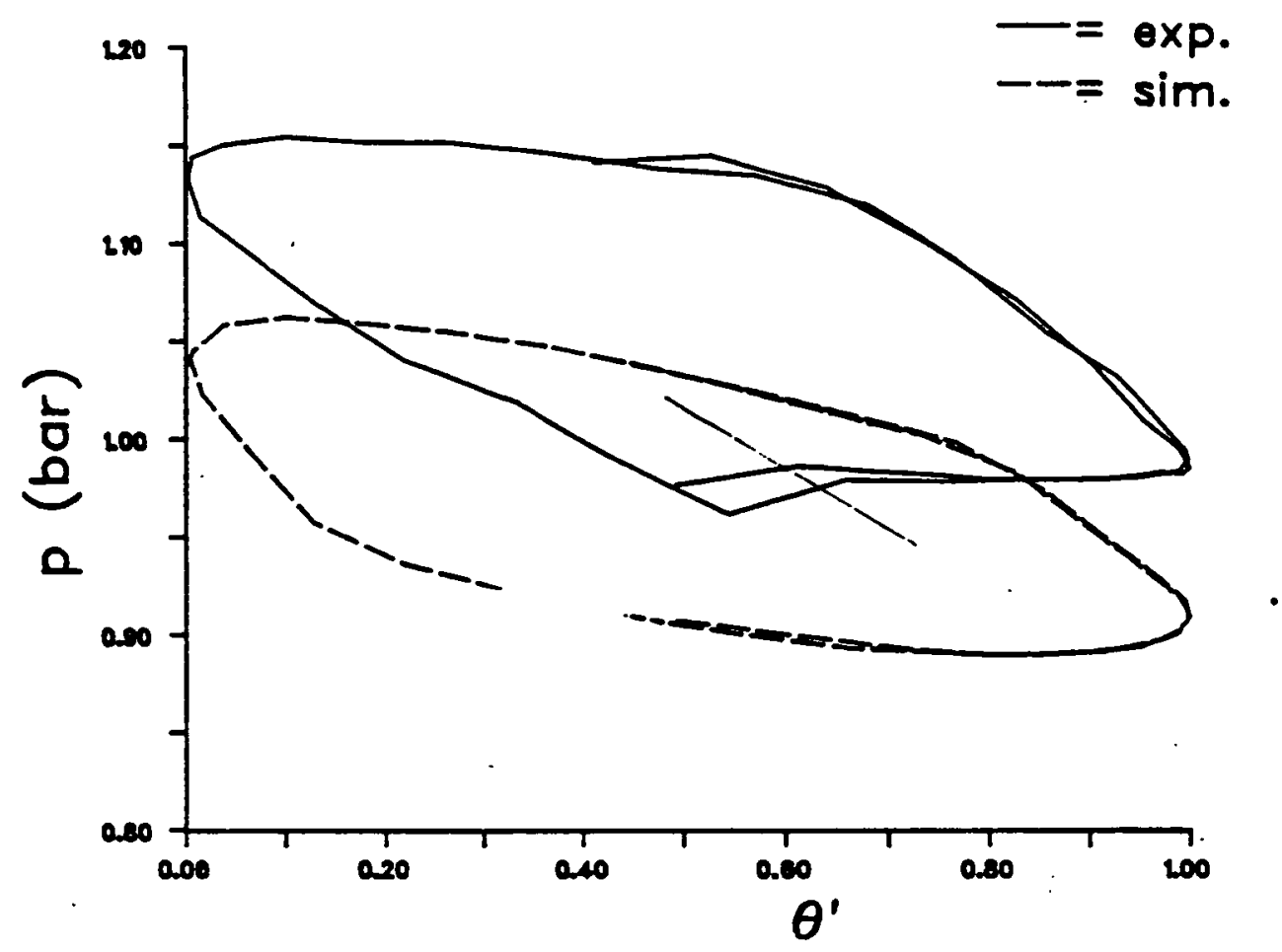

Figure (5.46) p vs. $\theta$; Experimental and Predicted Data Period = 6s; Amplitude $=6 \mathrm{~cm}$

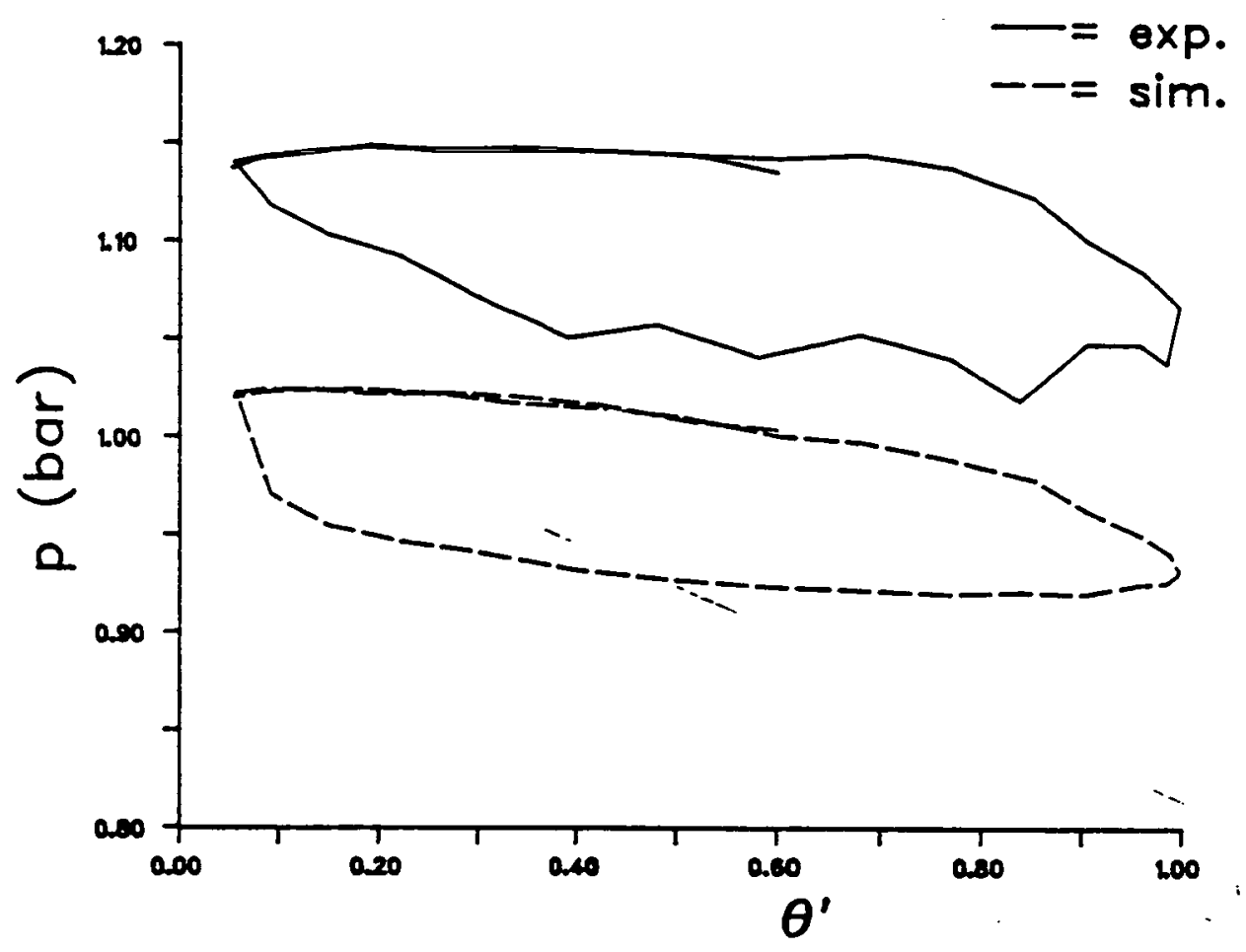

Figure (5.47) p vs. $\theta$; Experimental and Predicted Data Period $=7 \mathrm{~s} ;$ Amplitude $=4 \mathrm{~cm}$ 


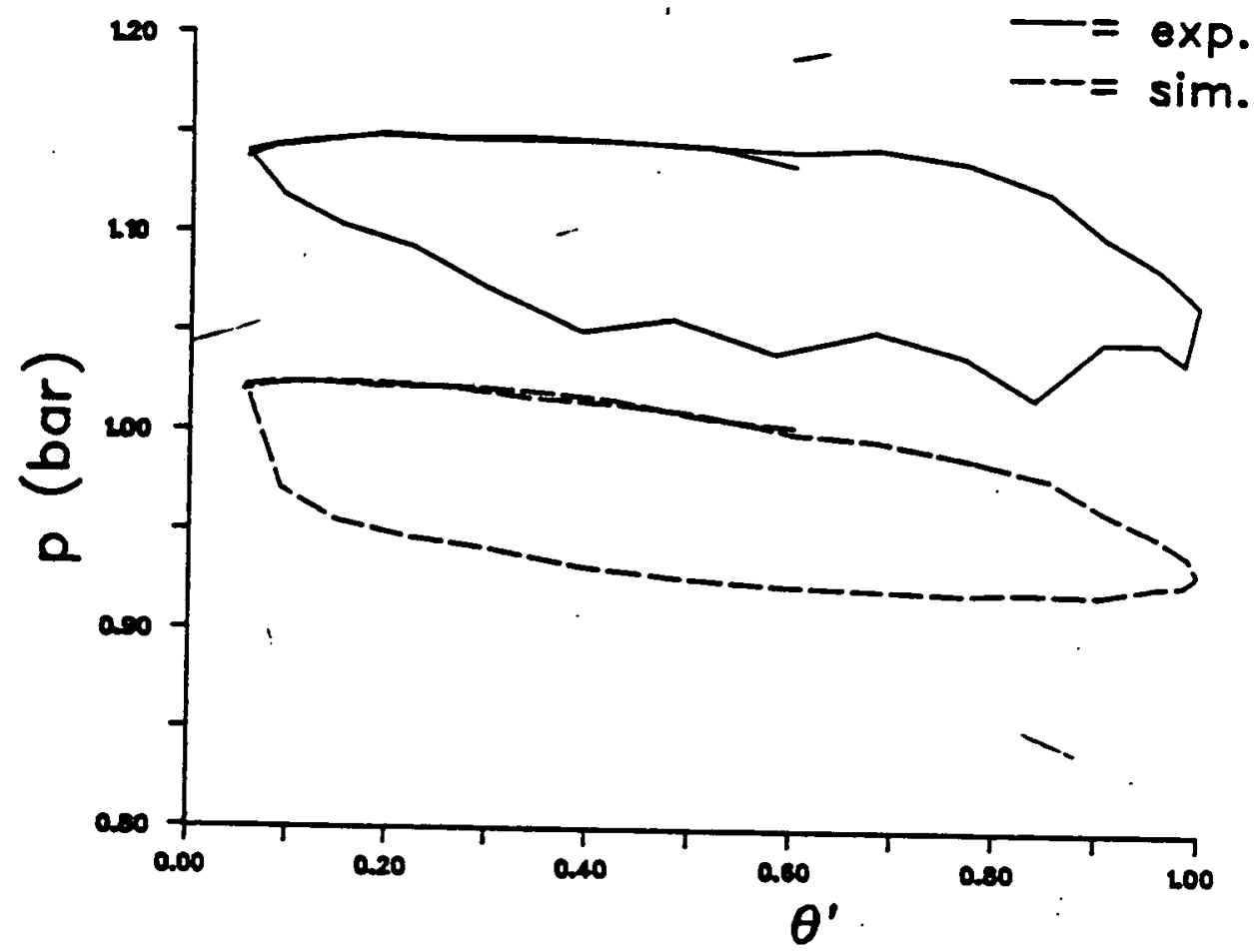

Figure (5.48) p vs. $\theta$; Experimental and Predicted Data Period $=7 \mathrm{~s}$; Amplitude $=6 \mathrm{~cm}$

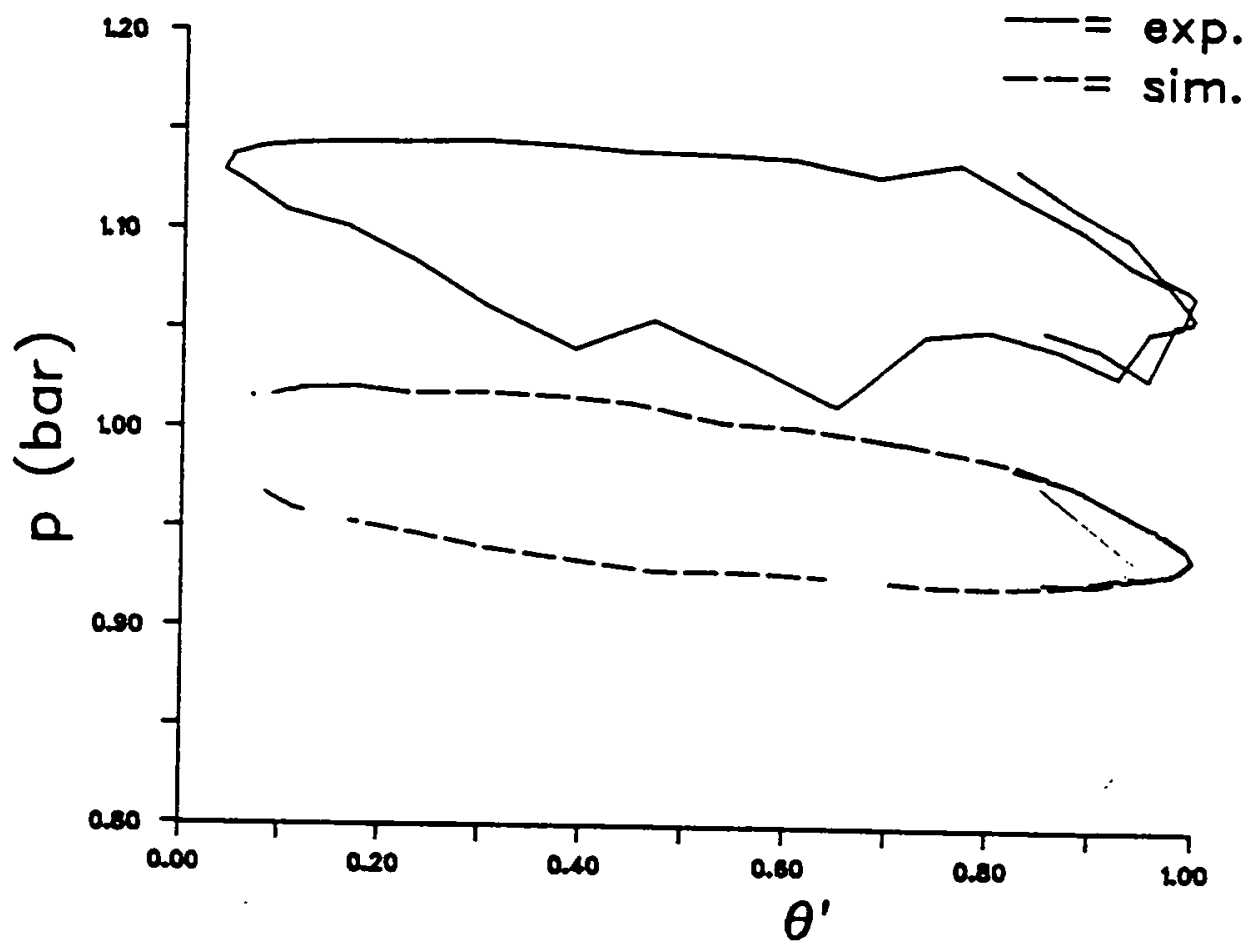

Figure (5.49) p vs. $\theta$; Experimental and Predicted Data Period $=8 s ;$ Amplitude $=4 \mathrm{~cm}$ 


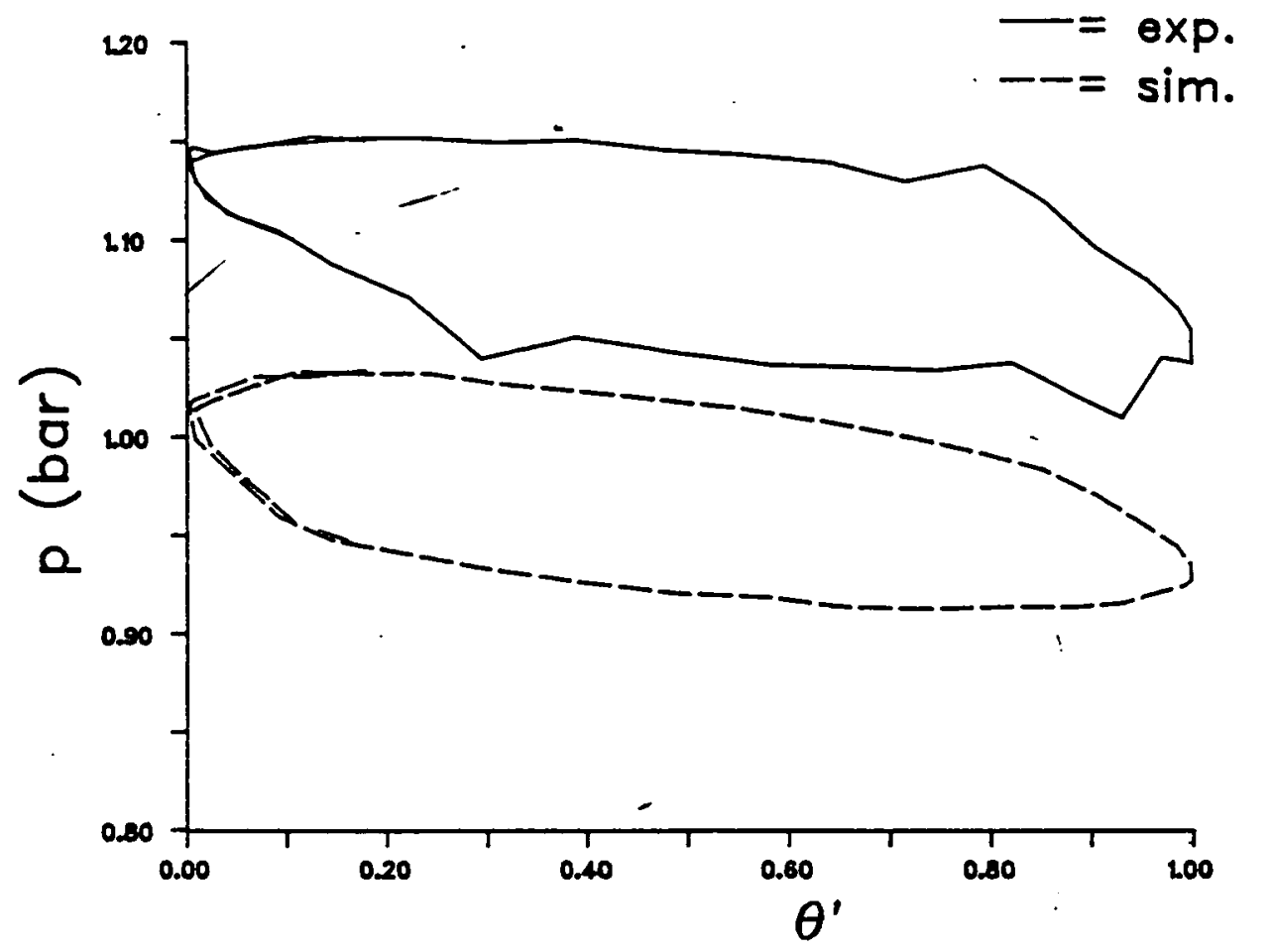

Figure (5.50) p vs. $\theta$; Experimental and Predicted Data

Period $=8 \mathrm{~s} ;$ Amplitude $=5 \mathrm{~cm}$

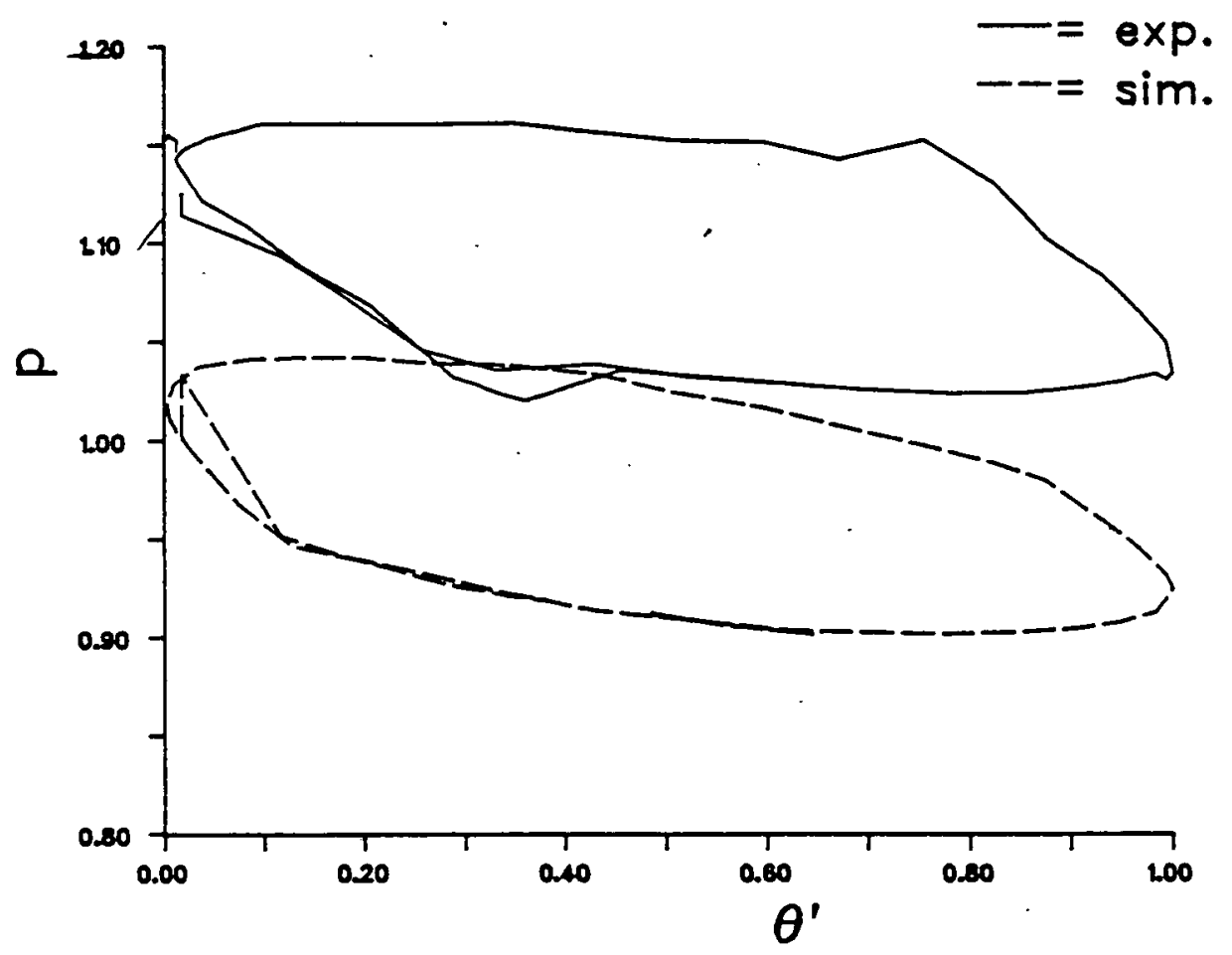

Figure (5.51) p vs. $\theta$; Experimental and Predicted Data

Period $=8 \mathrm{~s} ;$ Amplitude $=6 \mathrm{~cm}$ 


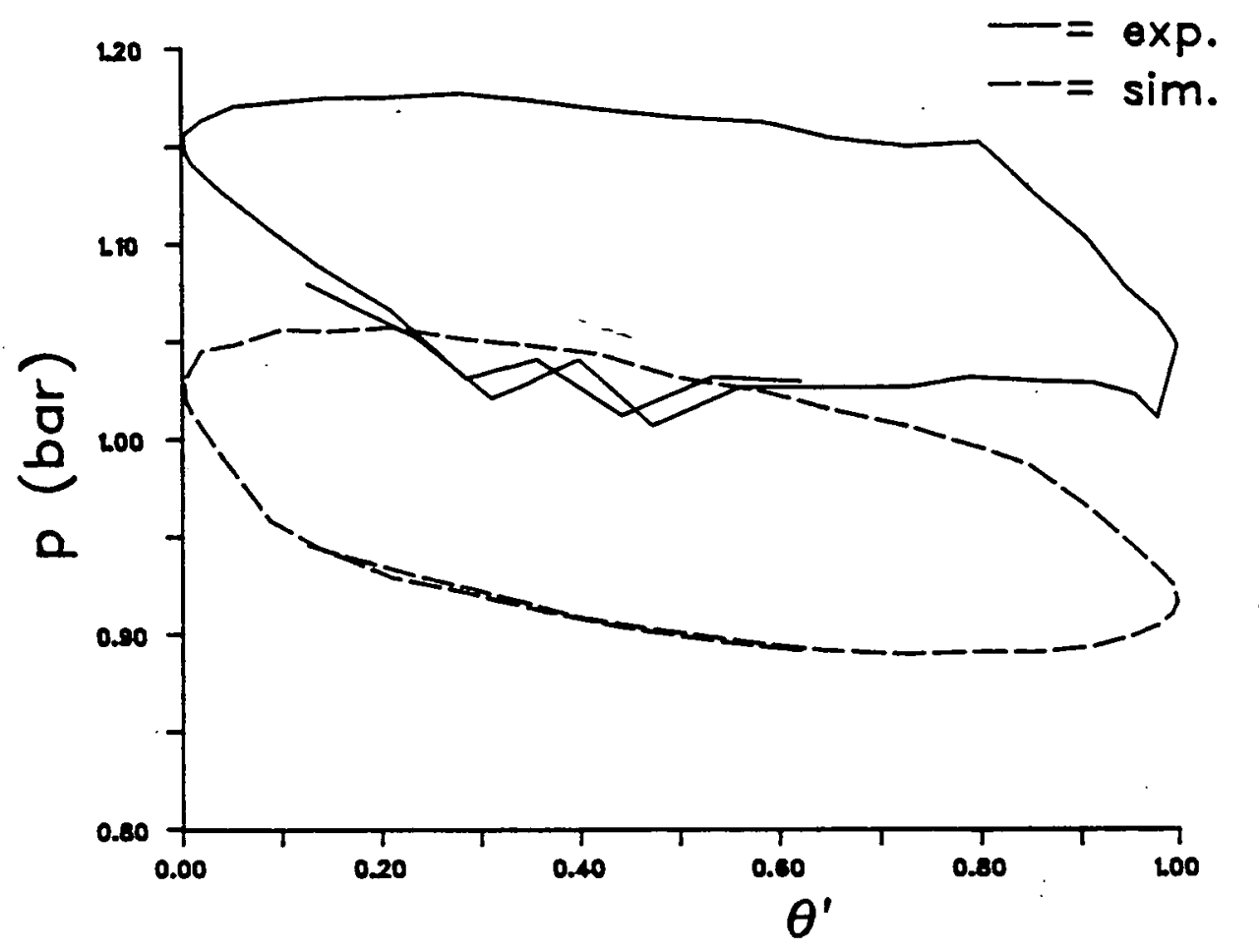

Figure (5.52) p vs. $\theta$; Experimental and Predicted Data Period $=8 \mathrm{~s} ;$ Amplitude $=7 \mathrm{~cm}$

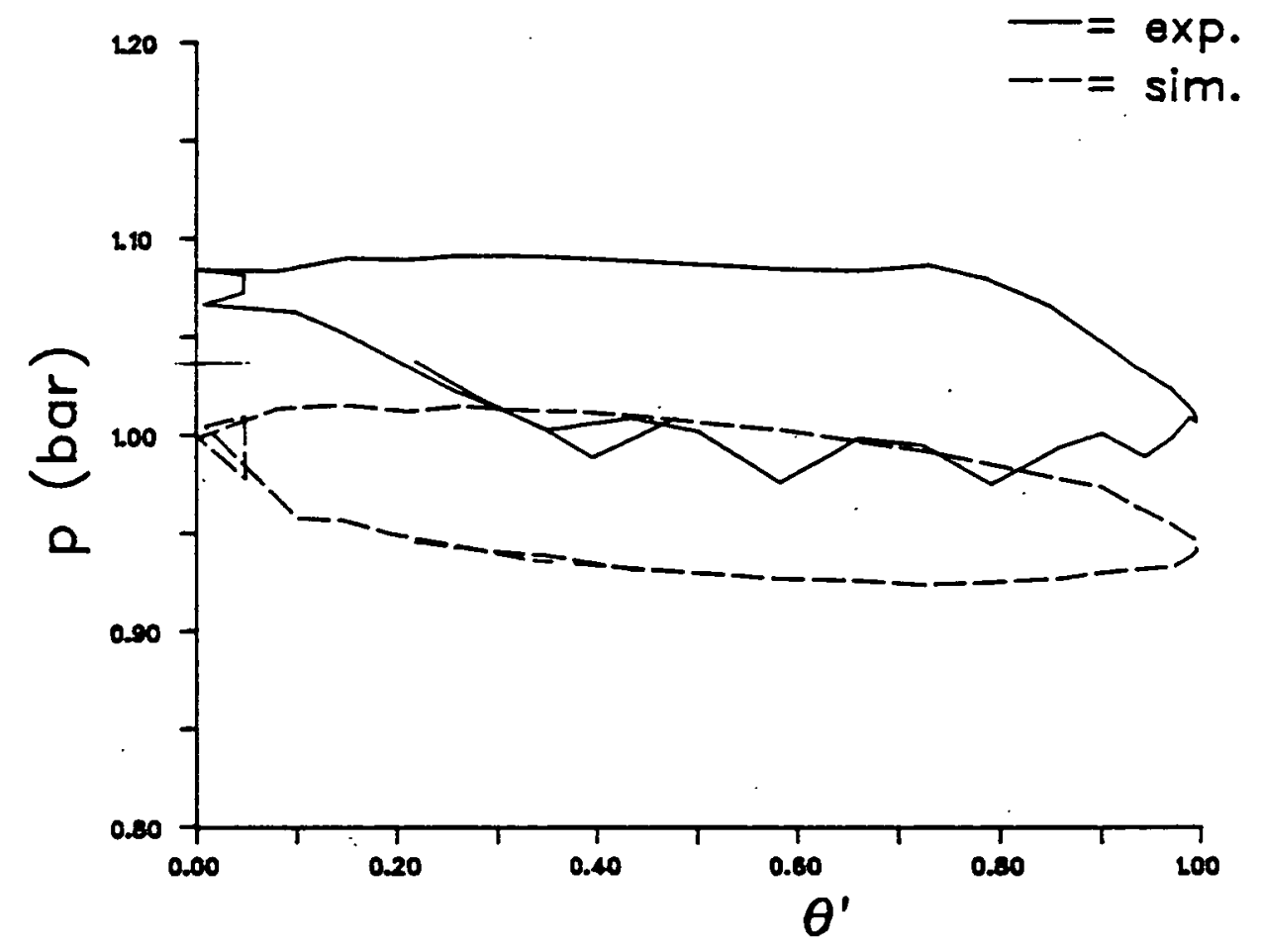

Figure (5.53) p vs. $\theta$; Experimental and Predicted Data

Period = 9s; Amplitude $=4 \mathrm{~cm}$ 


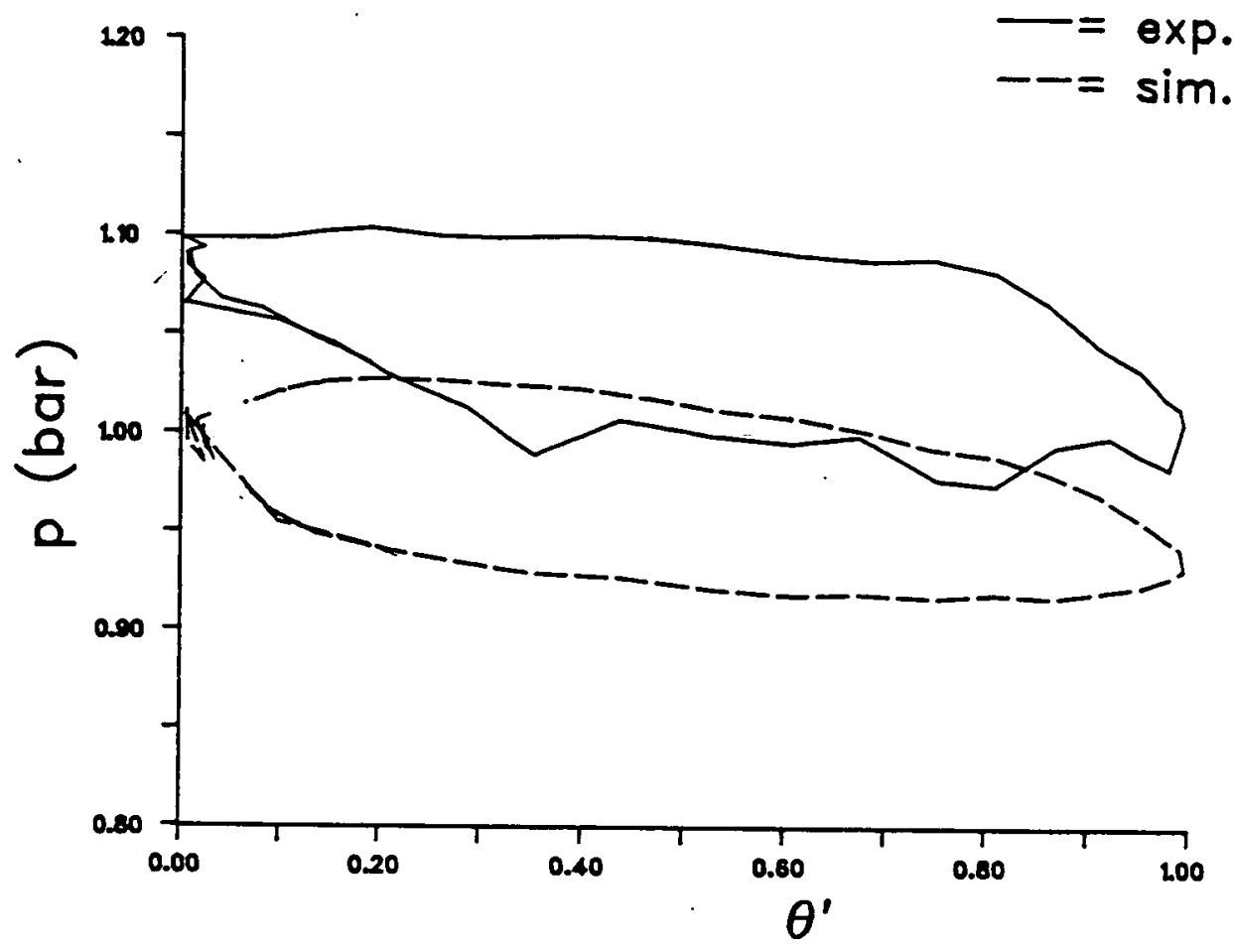

Figure (5.54) p vs. $\theta$; Experimental and Predicted Data Period $=9 \mathrm{~s} ;$ Amplitude $=5 \mathrm{~cm}$

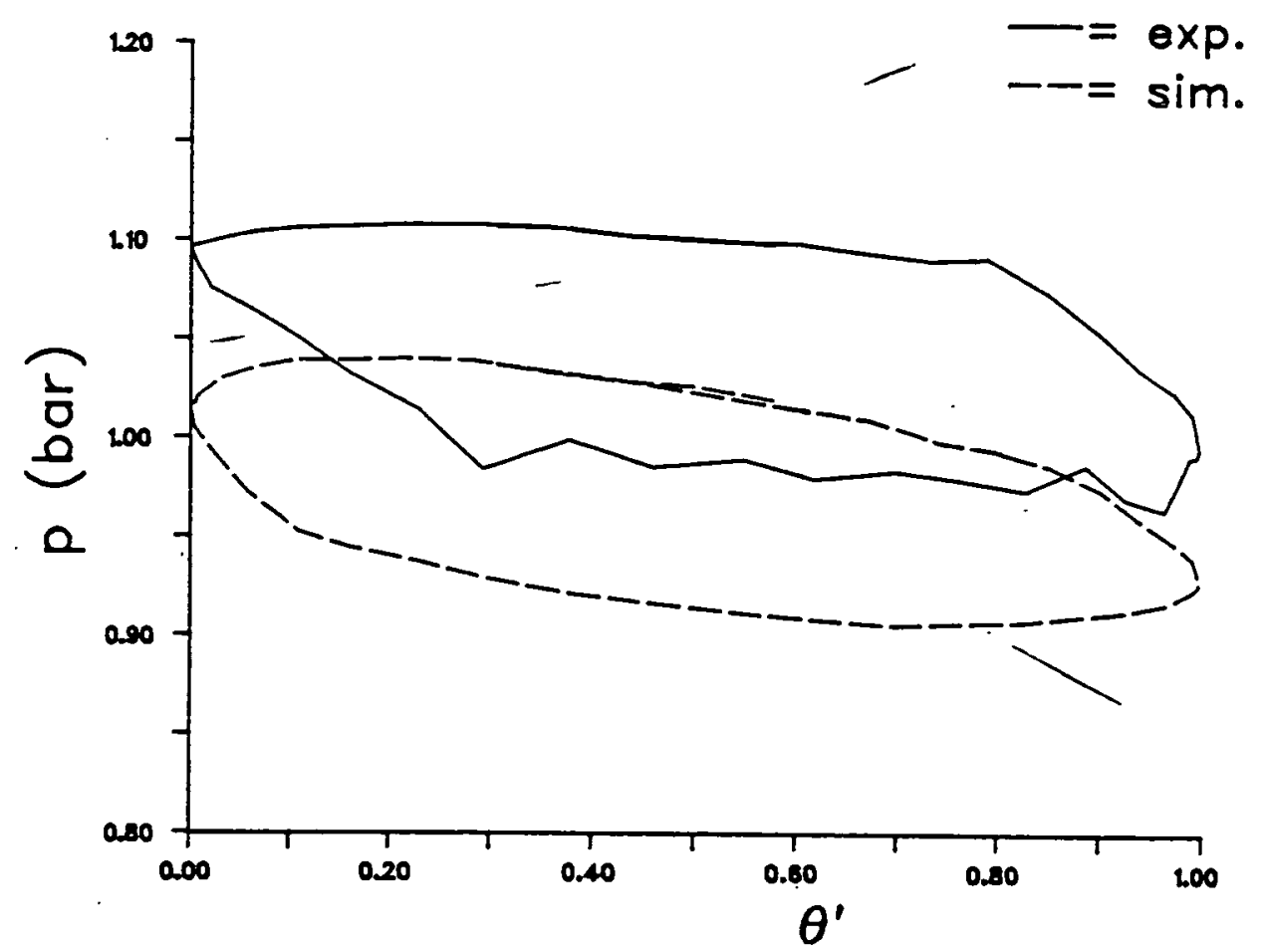

Figure (5.55) p vs. $\theta$; Experimental and Predicted Data Period $=9 \mathrm{~s} ;$ Amplitude $=6 \mathrm{~cm}$ 


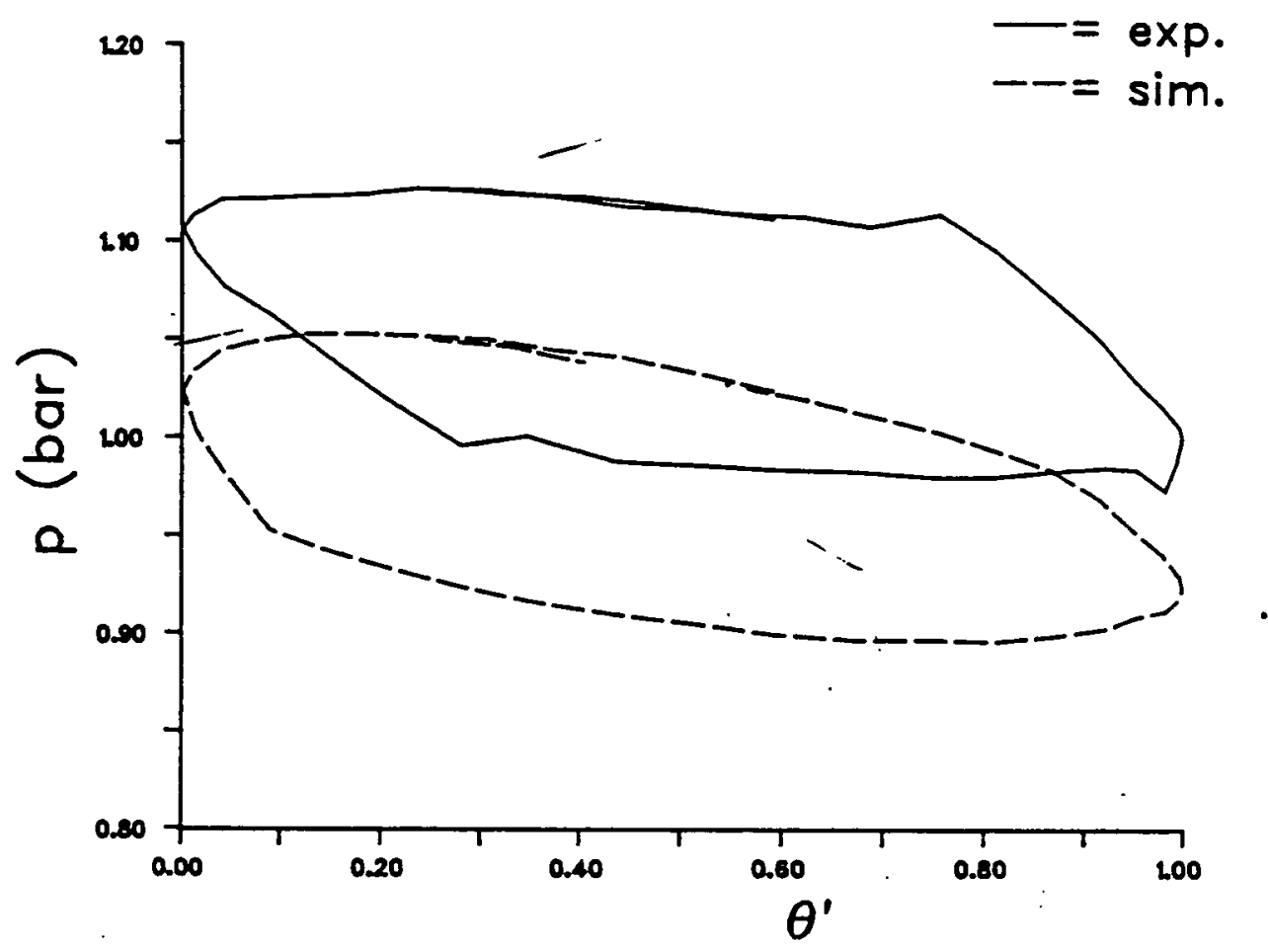

Figure (5.56) $p$ vs. $\theta$; Experimental and Predicted Data Period $=9 \mathrm{~s} ;$ Amplitude $=7 \mathrm{~cm}$

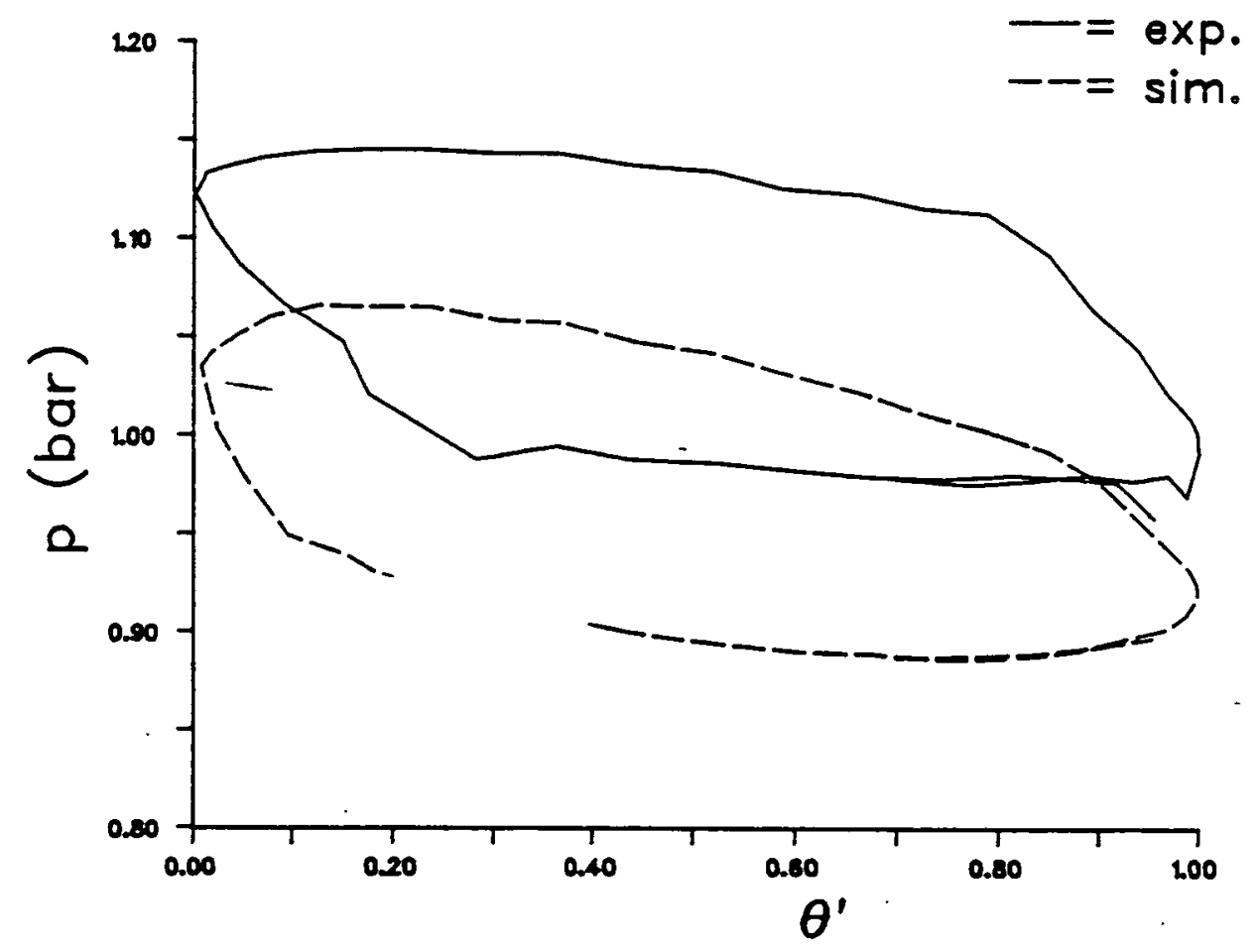

Figure (5.57) p vs. $\theta$; Experimental and Predicted Data Period $=9 \mathrm{~s} ;$ Amplitude $=8 \mathrm{~cm}$ 


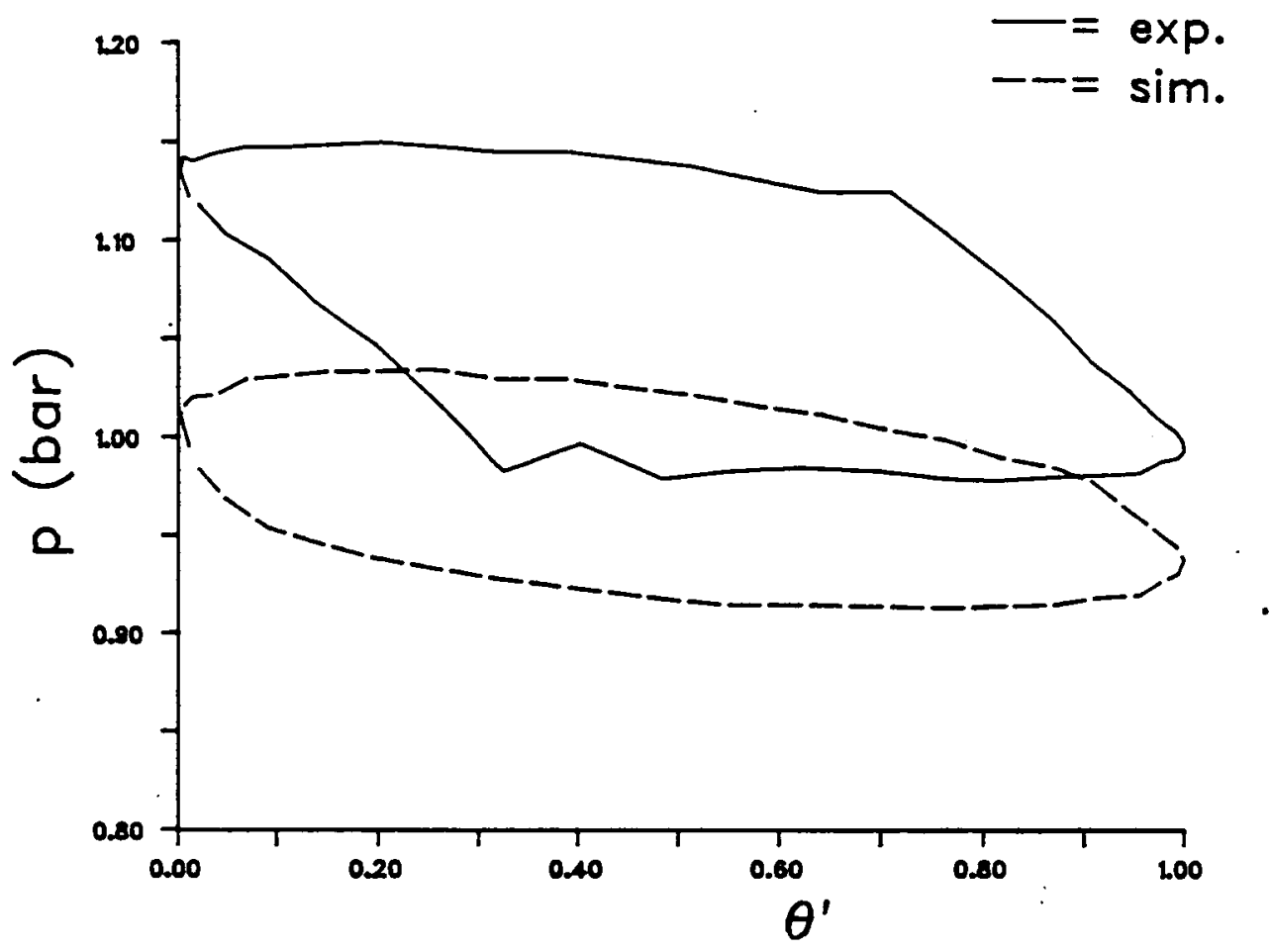

Figure (5.58) $\mathrm{p}$ vs. $\theta$; Experimental and Predicted Data Period $=10 \mathrm{~s} ;$ Amplitude $=6 \mathrm{~cm}$

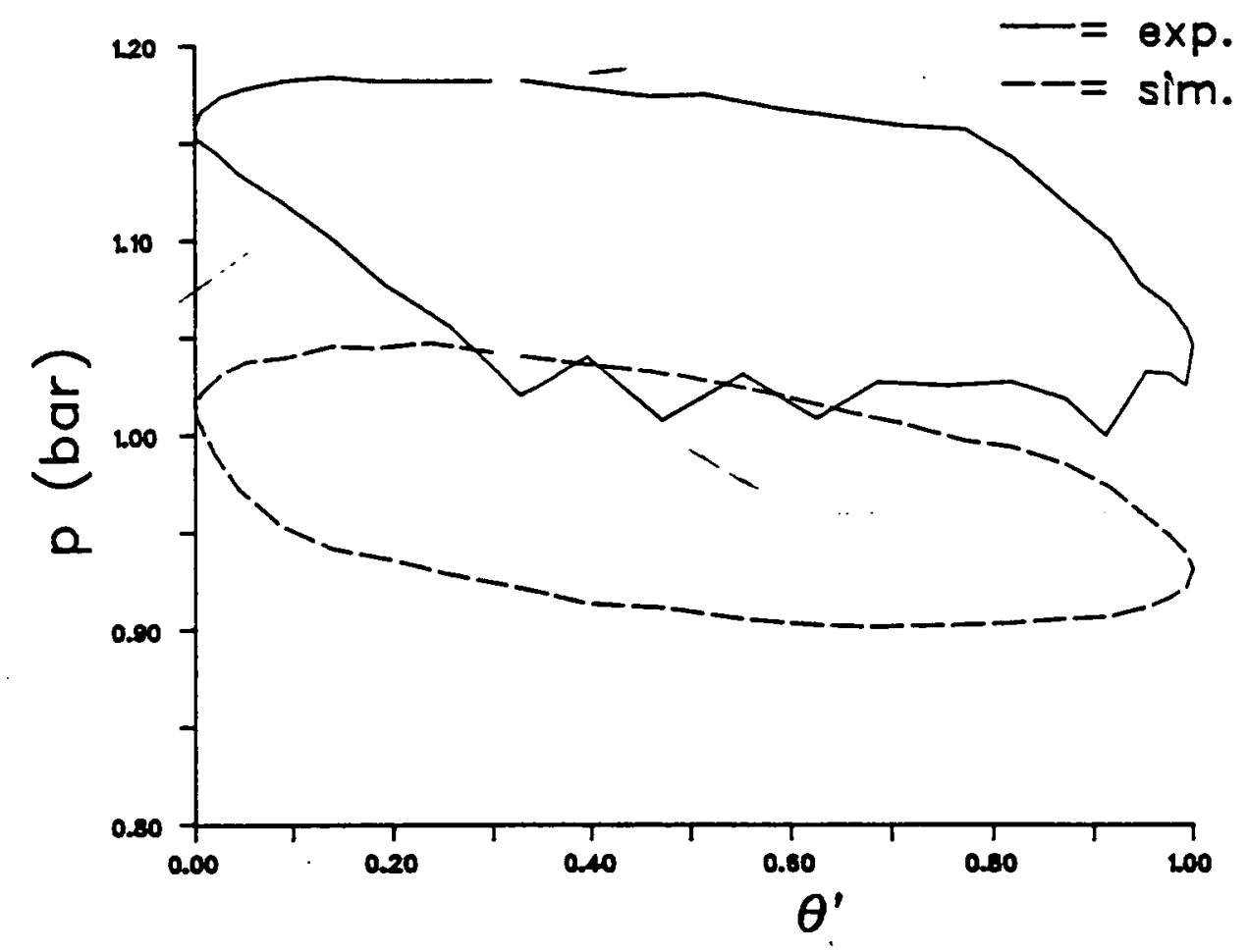

Figure (5.59) p vs. $\theta$; Experimental and Predicted Data Period $=10 \mathrm{~s} ;$ Amplitude $=7 \mathrm{~cm}$ 


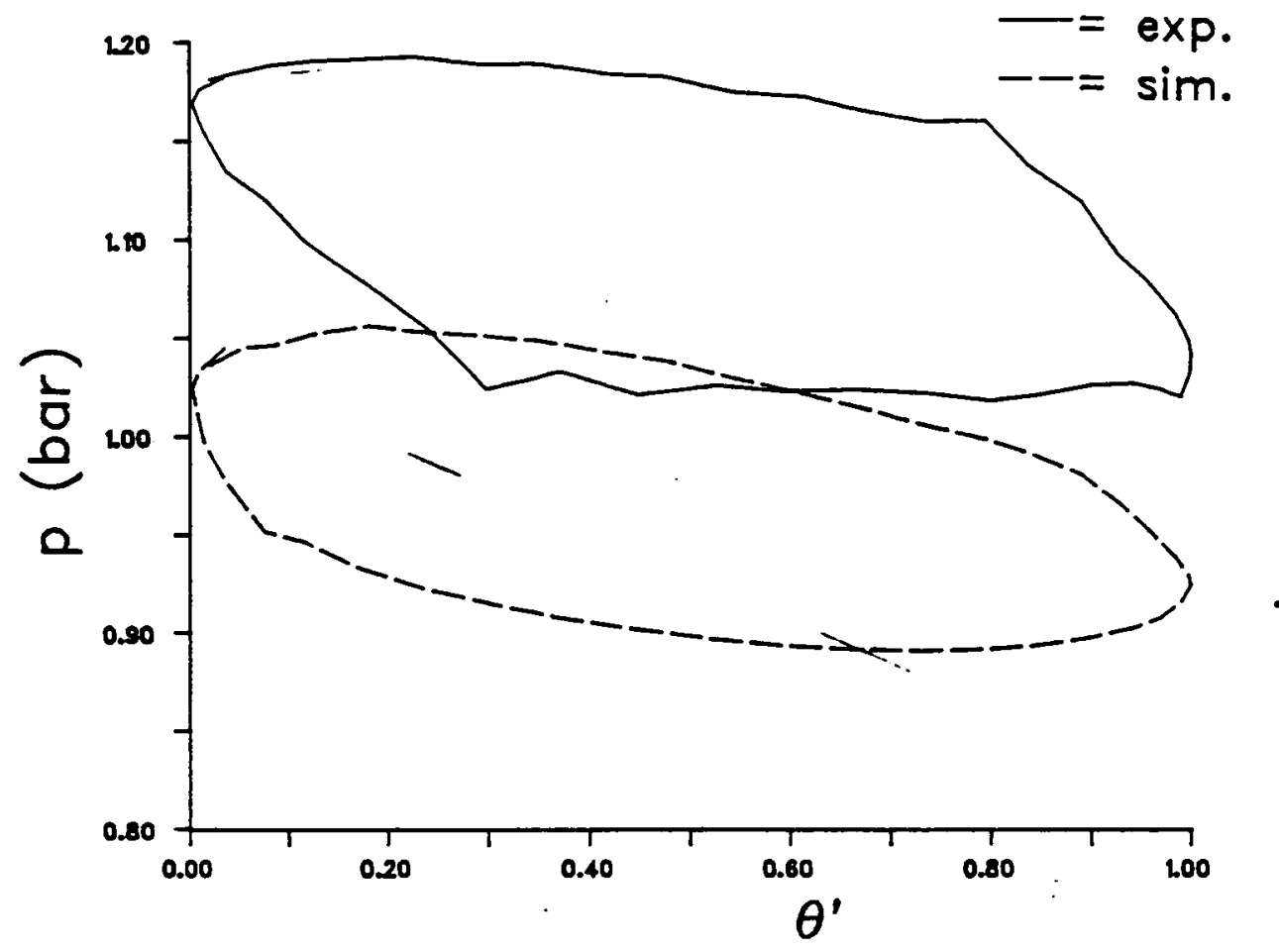

Figure (5.60) $p$ vs. $\theta$; Experimental and Predicted Data

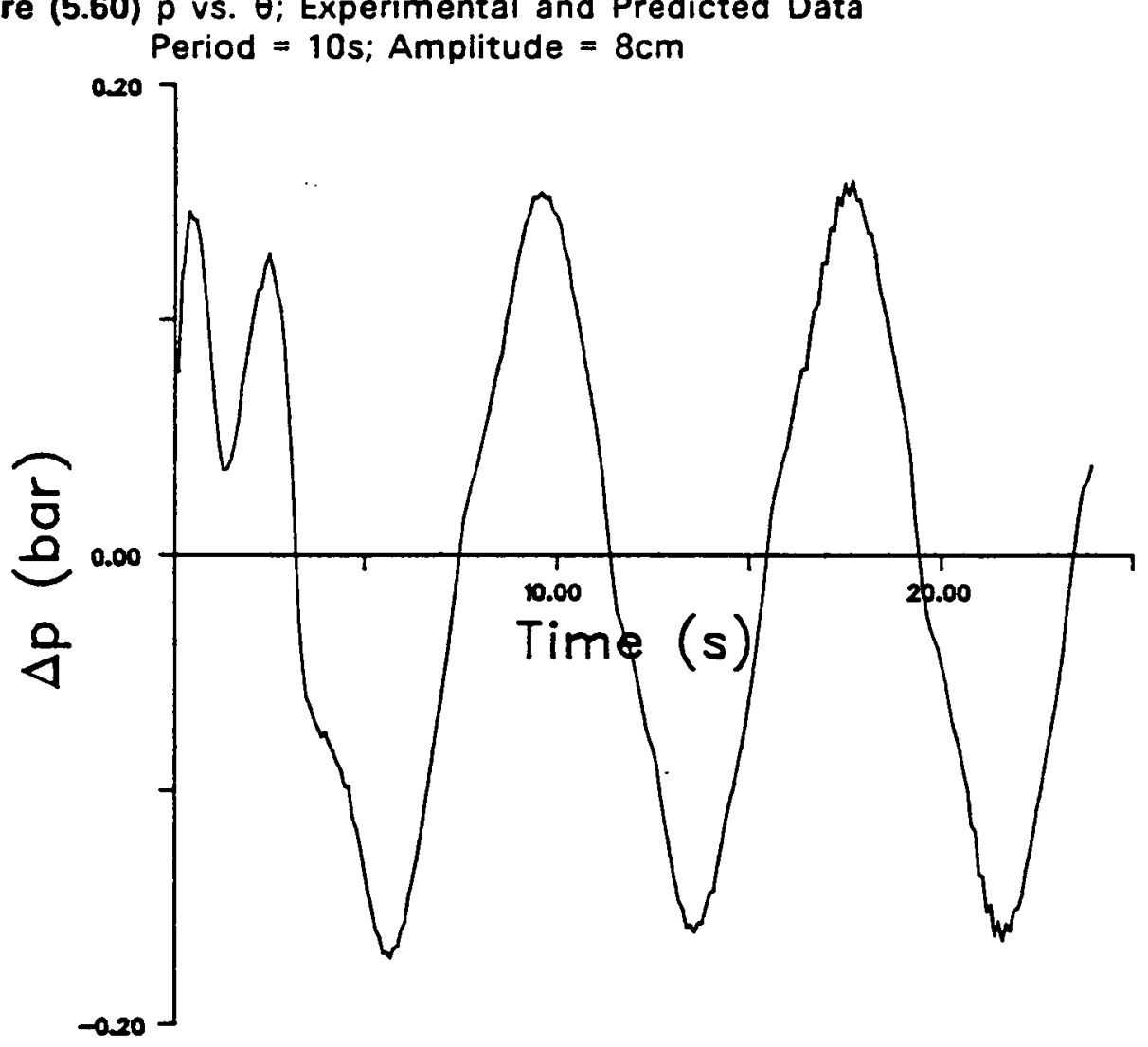

Figure (5.61) Transient Behaviour Prediction from the $2^{\text {nd }}$ Model 

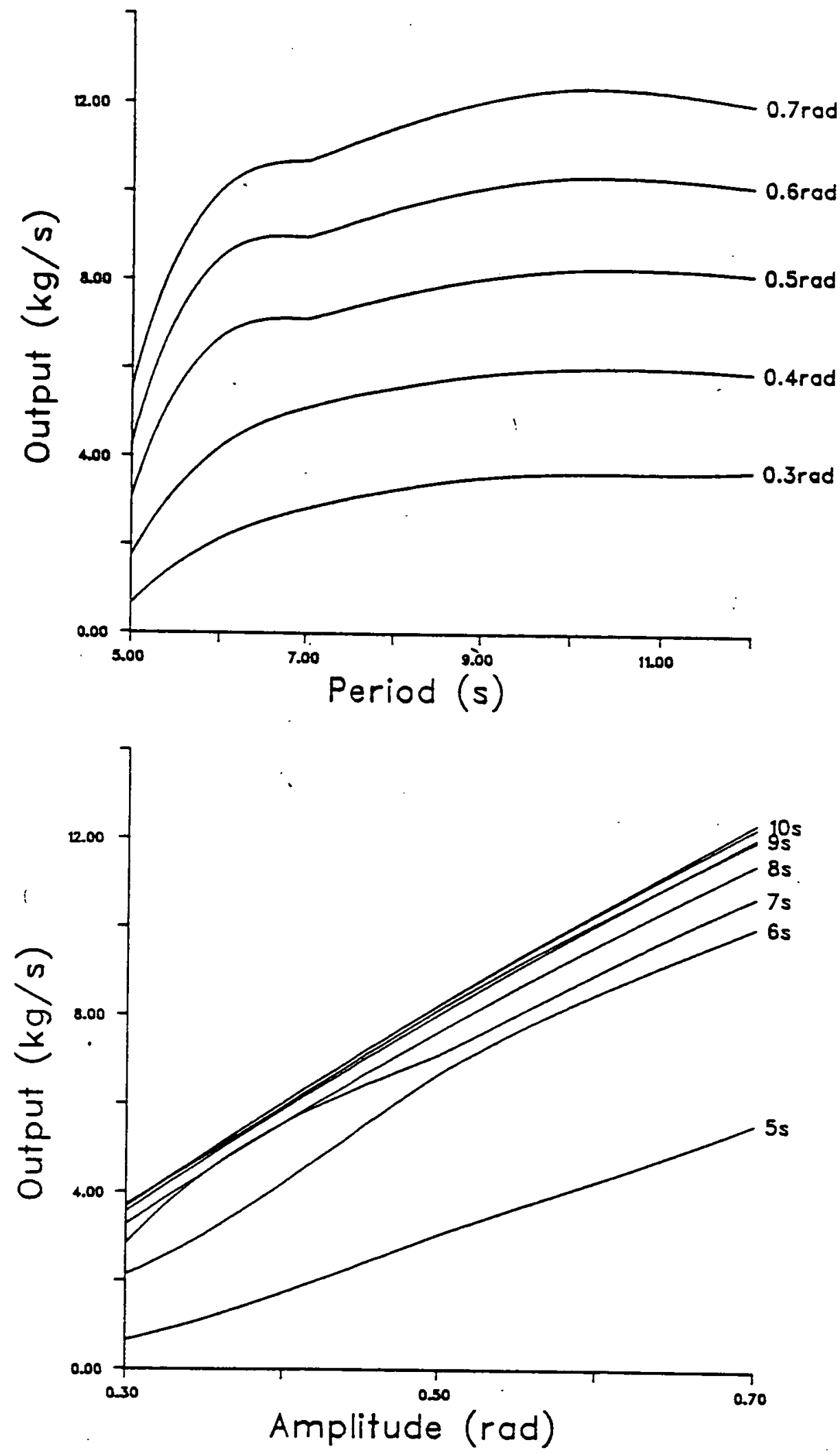

Figure (5.62) Output vs. Period and Amplitude from the $2^{\text {nd }}$ Model 

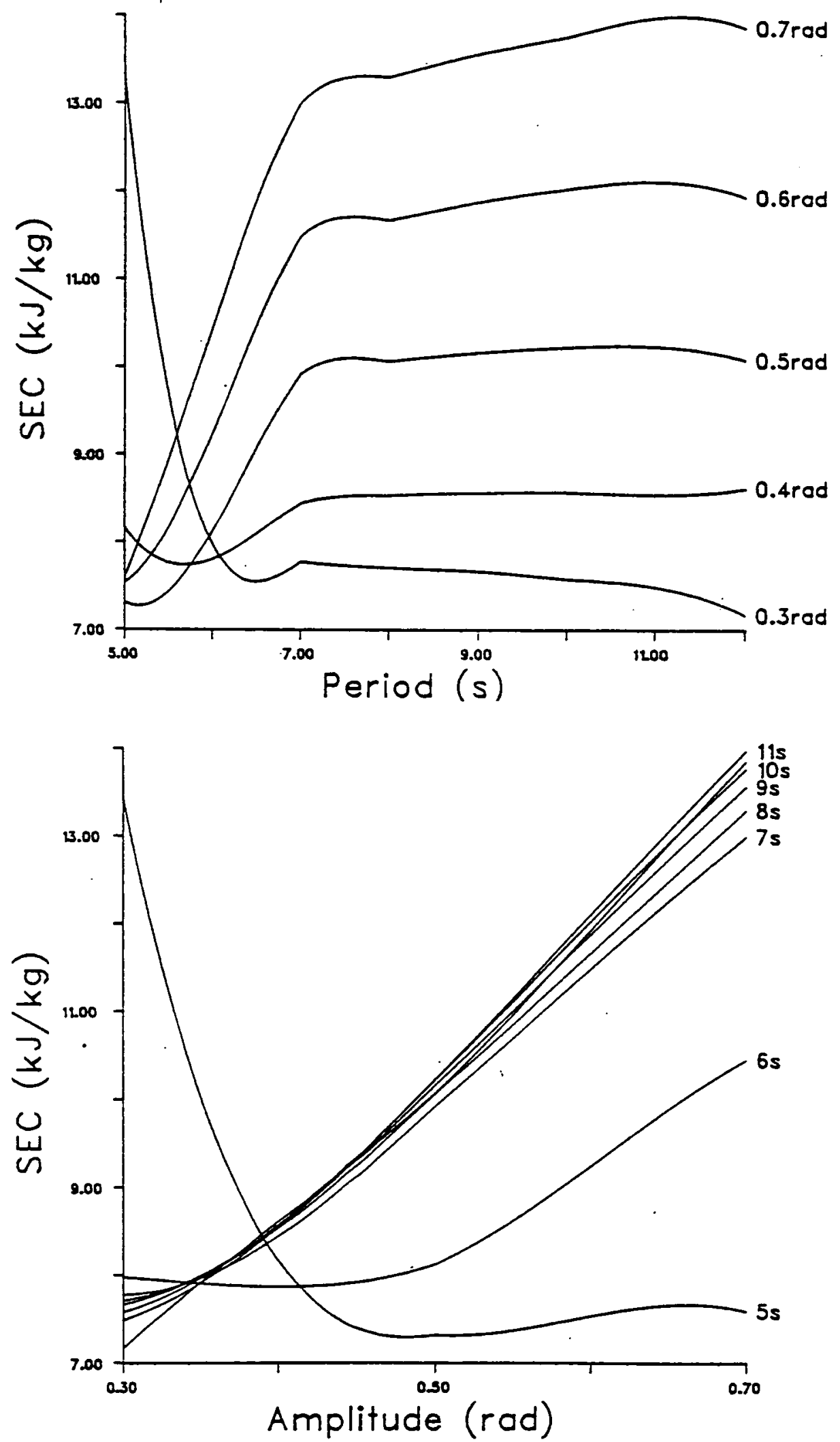

Figure (5.63) SEC vs. Period and Amplitude from the $2^{\text {nd }}$ Model 


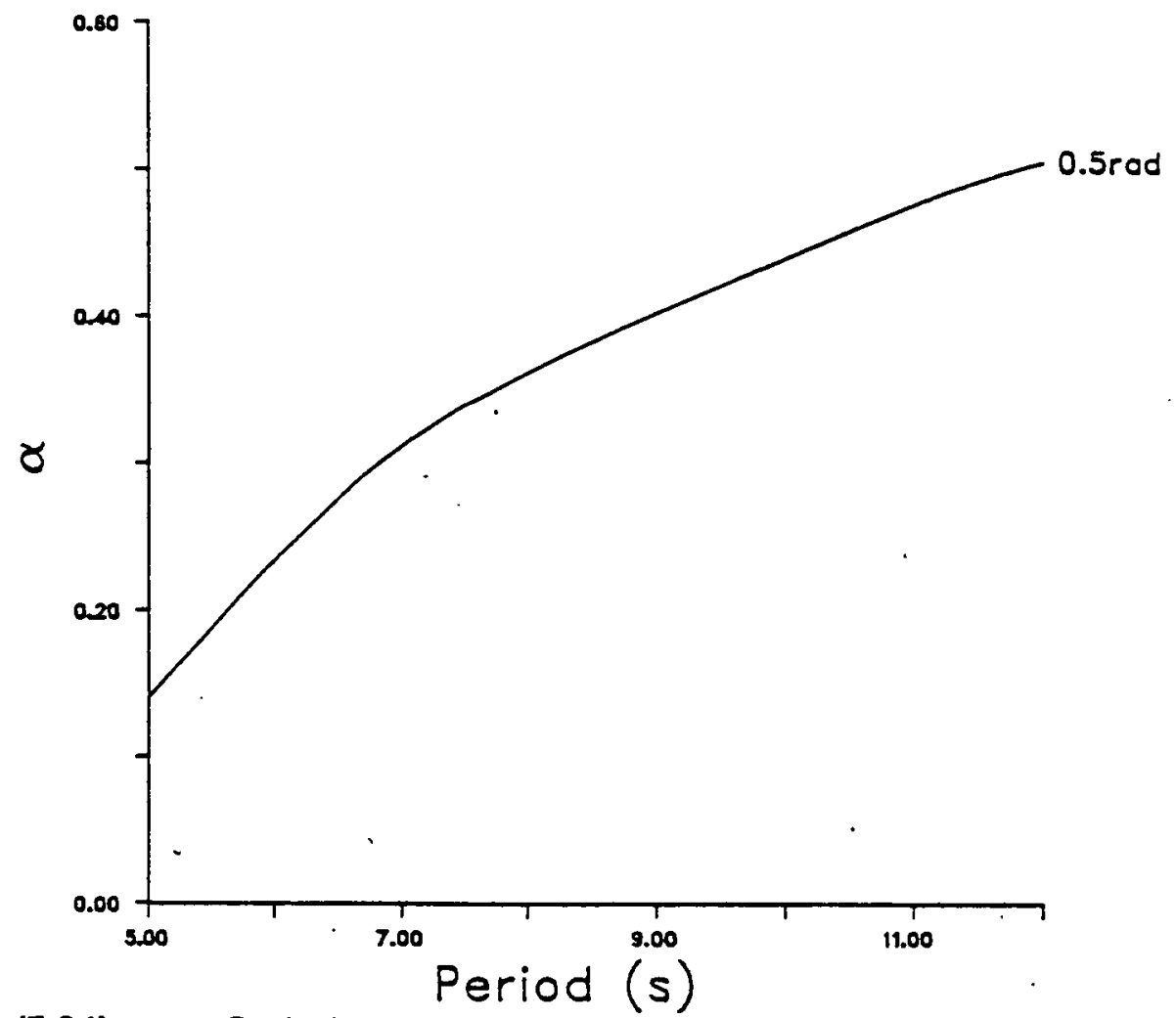

Figure (5.64) $\propto$ vs. Period

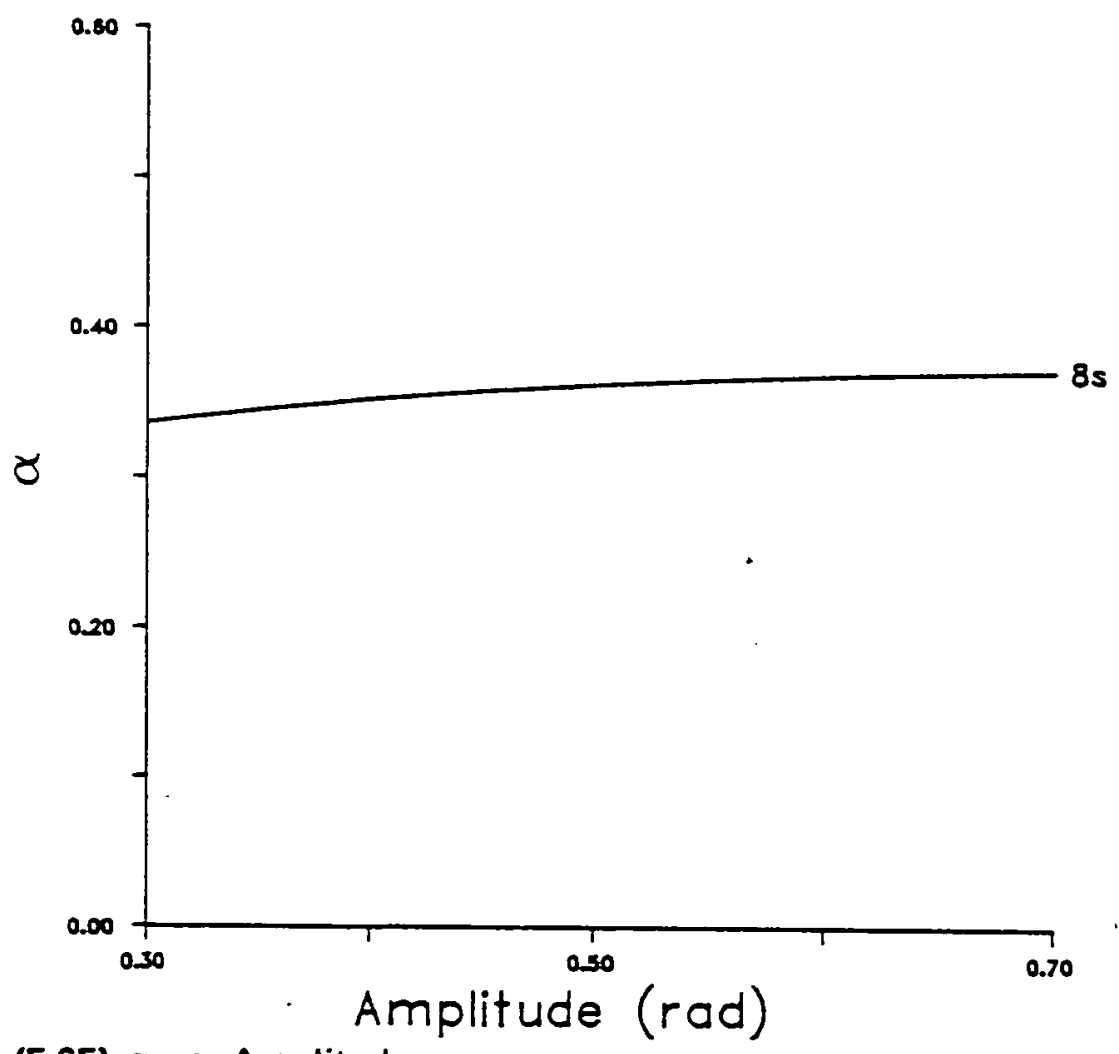

Figure (5.65) $\propto$ vs. Amplitude 


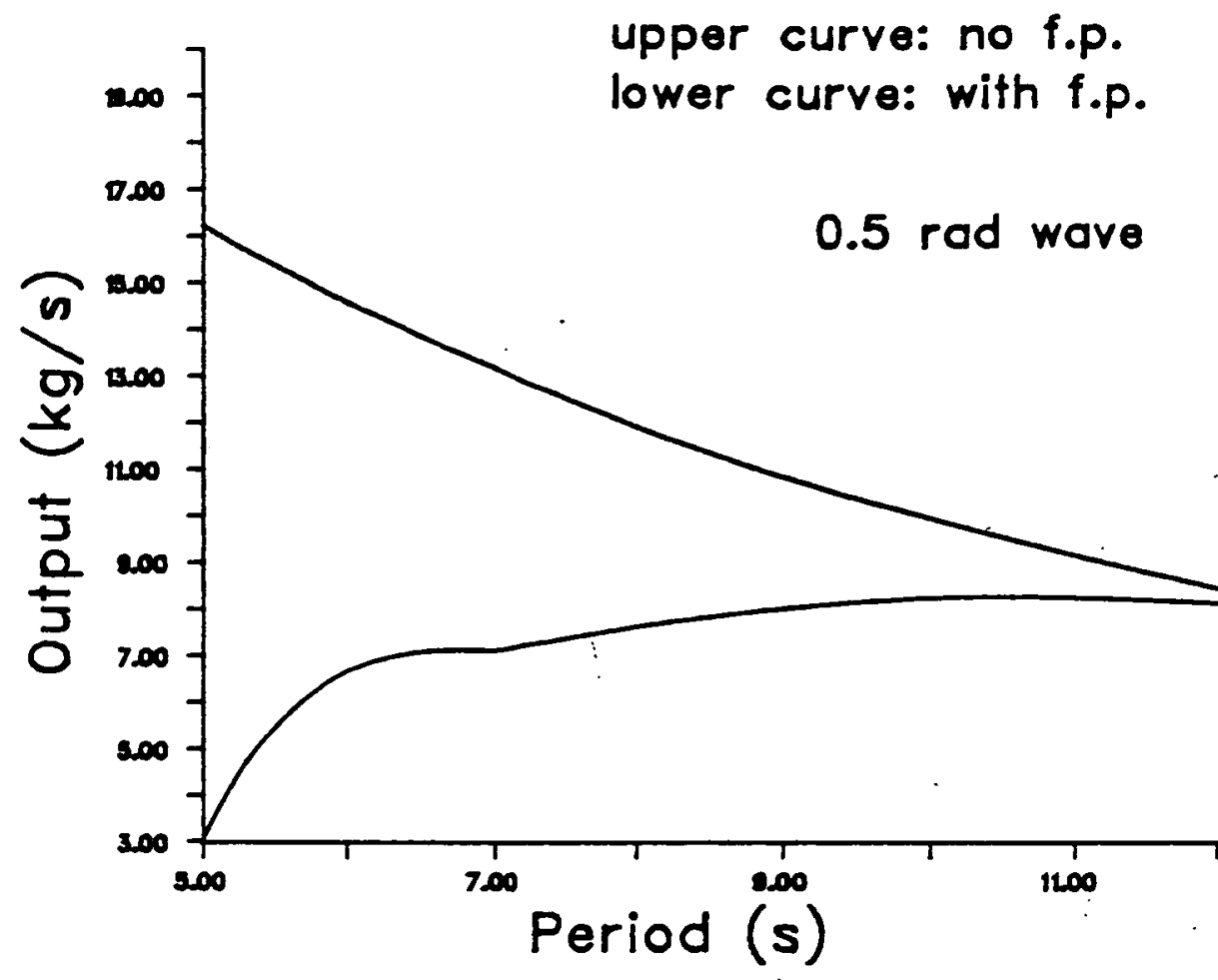

Figure (5.66) Output vs. Period without the fluid piston

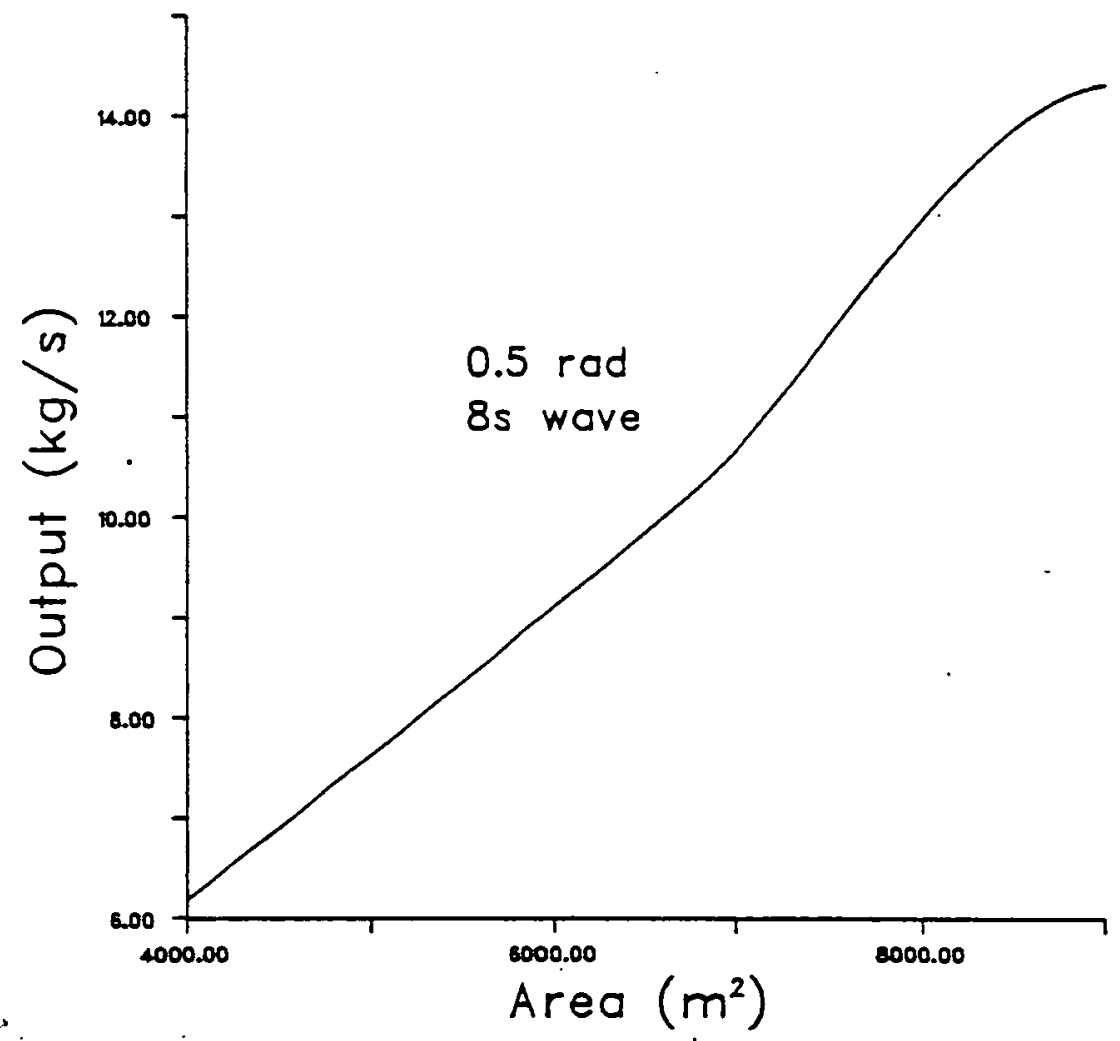

Figure (5.67) Output vs. Area from the $2^{\text {nd }}$ Model 


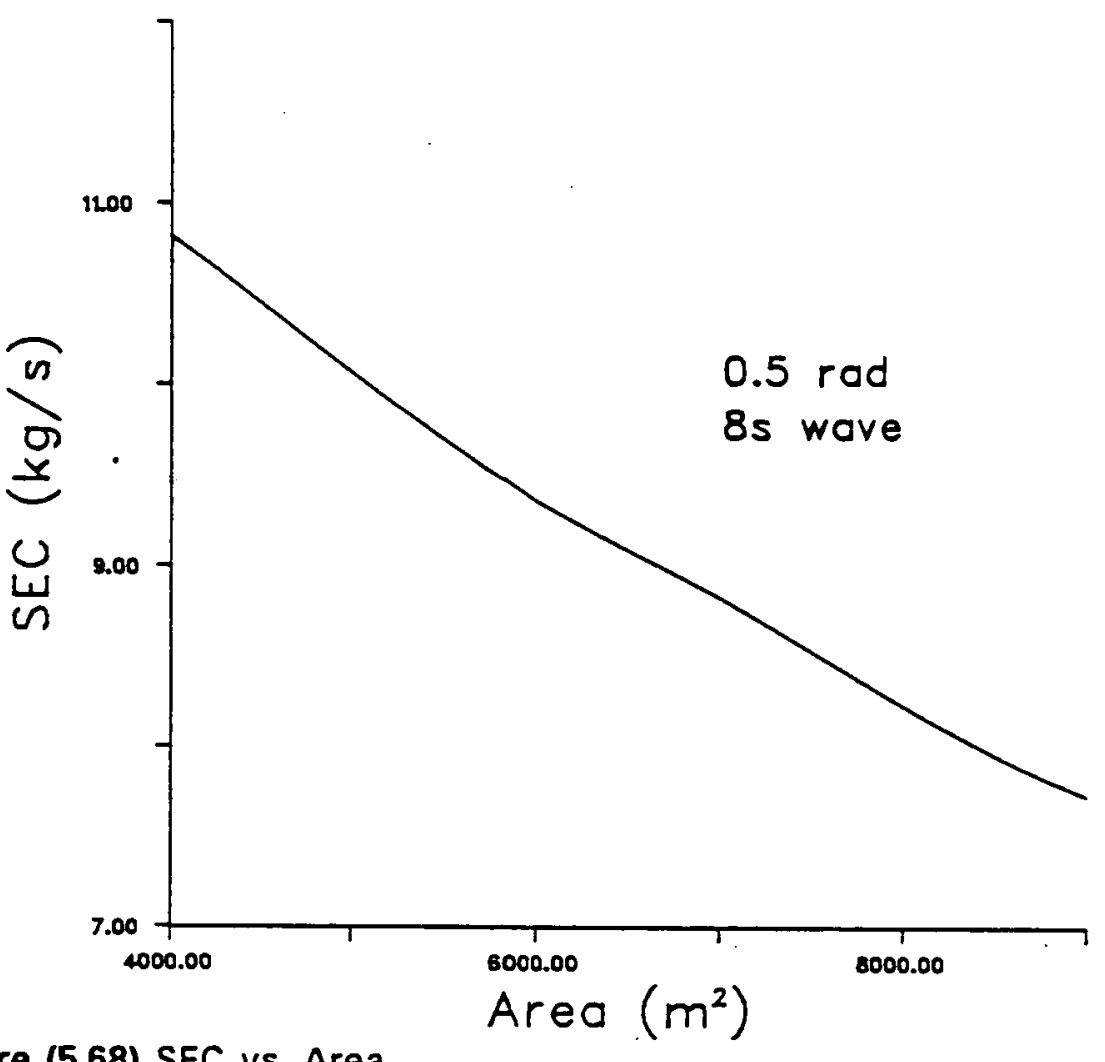

Figure (5.68) SEC vs. Area

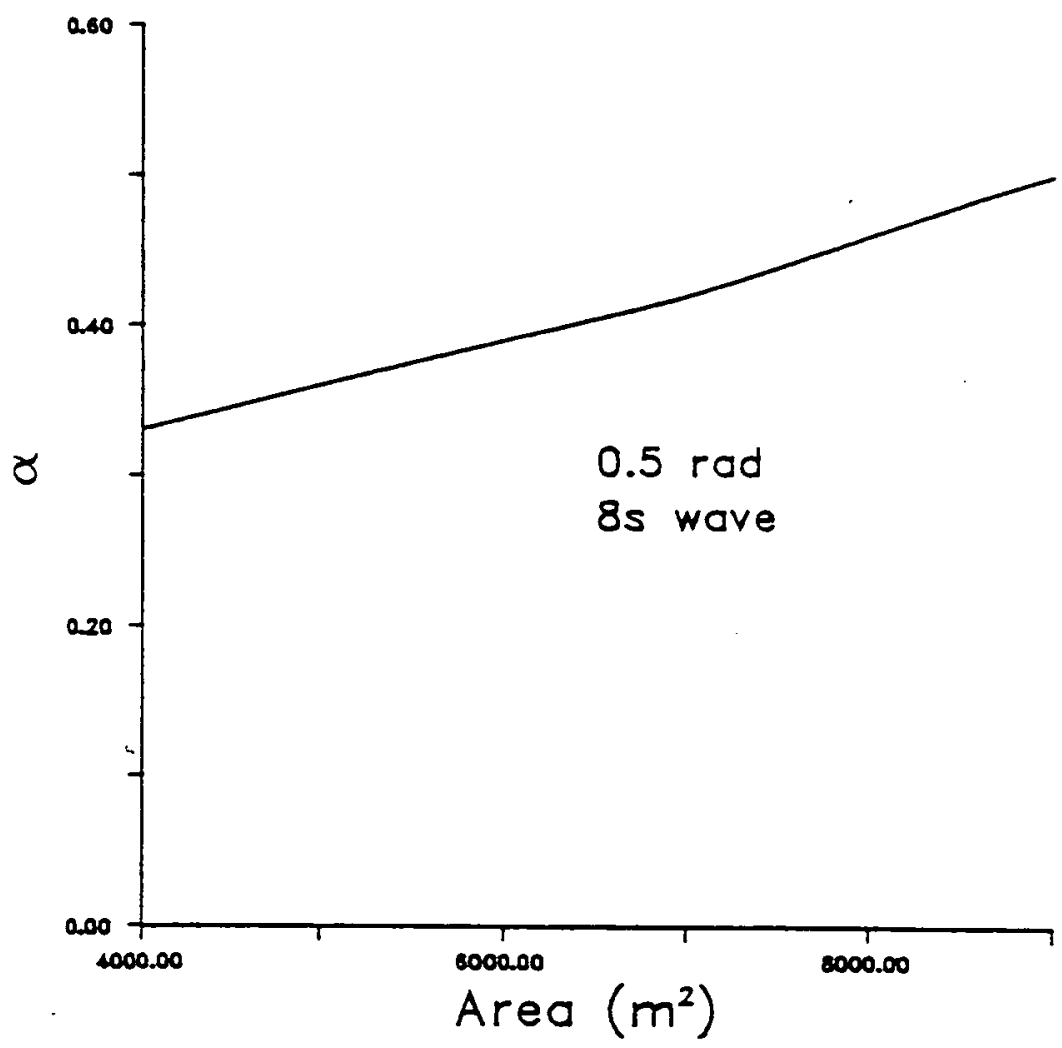

Figure (5.69) $\propto$ vs. Area 


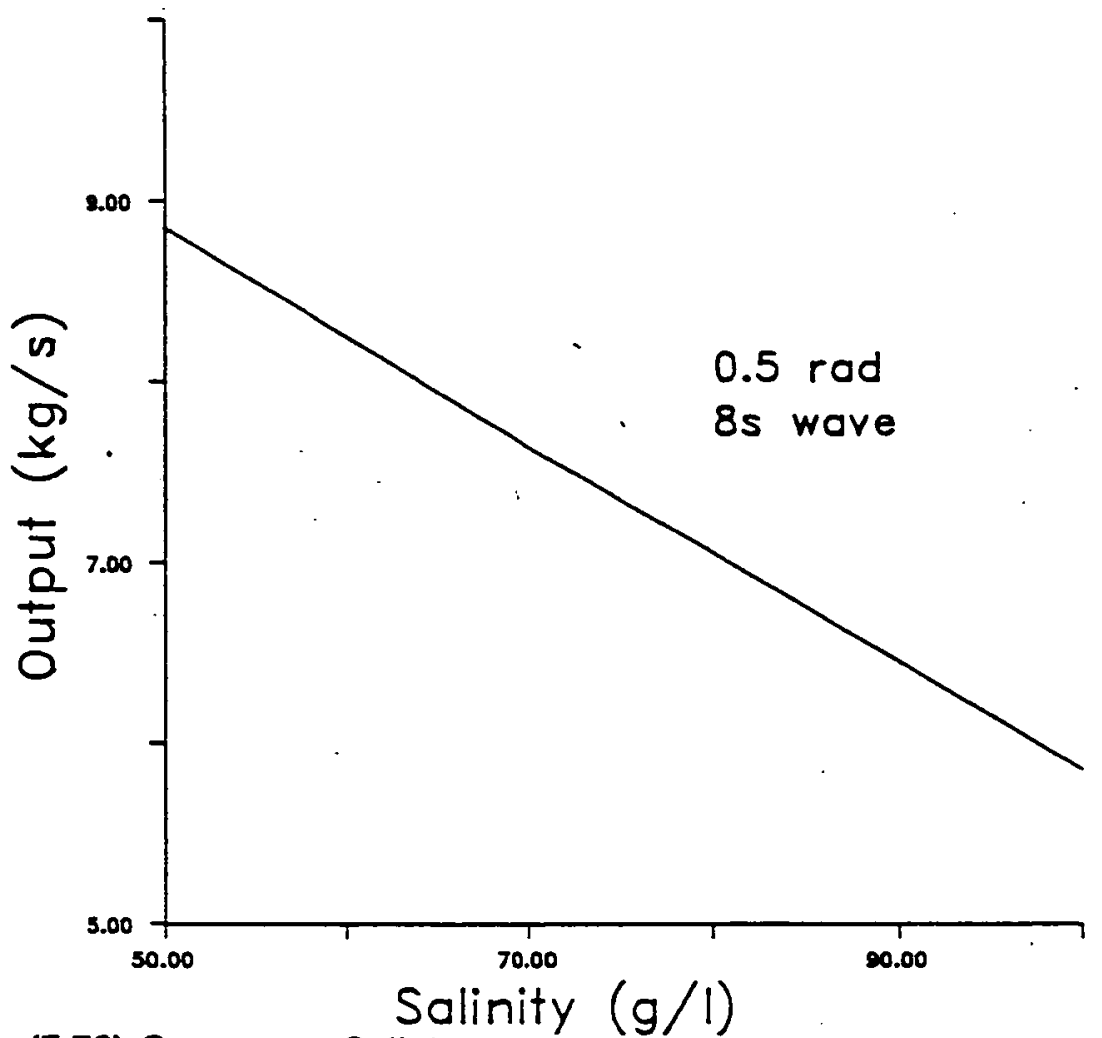

Figure (5.70) Output vs. Salinity

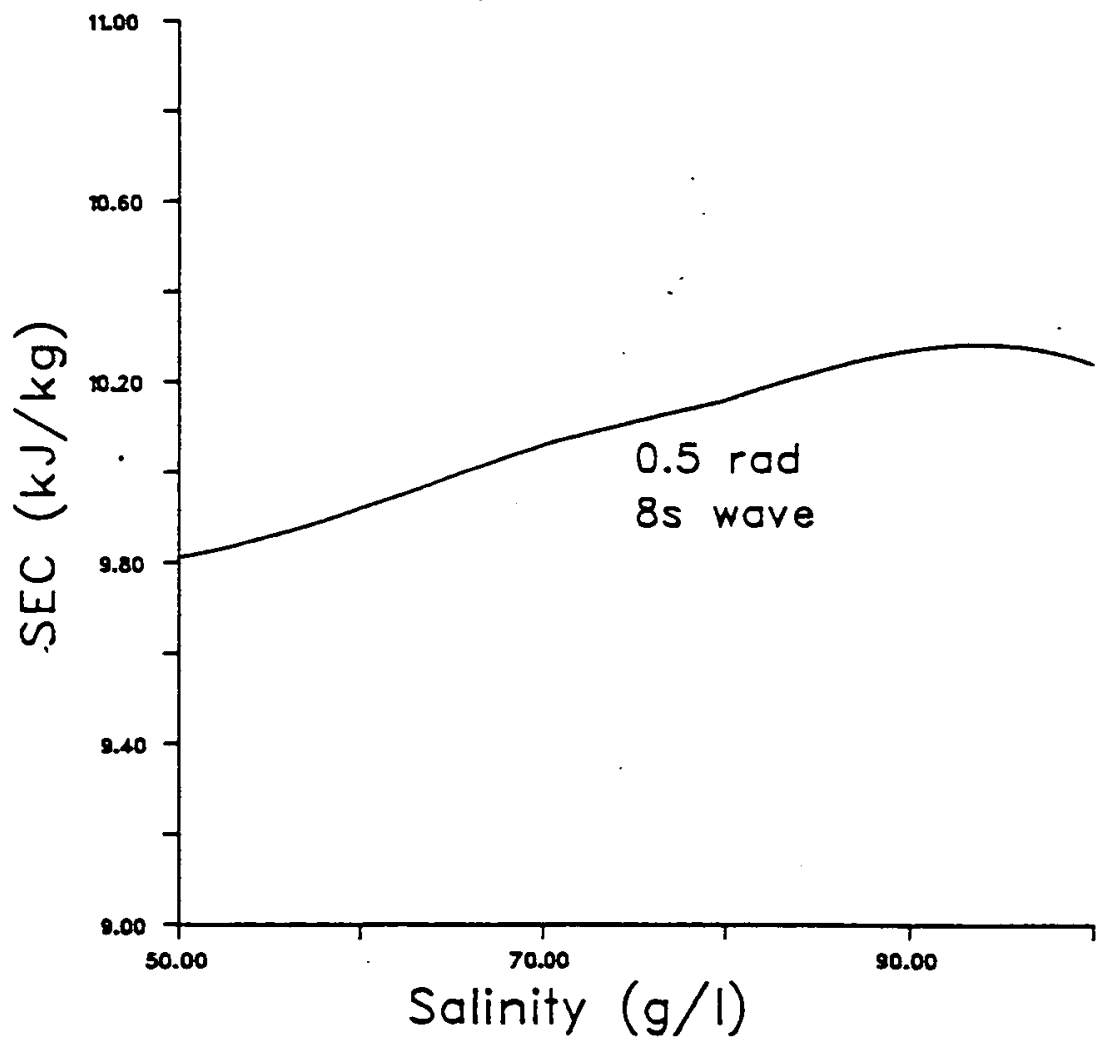

figure (5.71) SEC vs. Salinity 


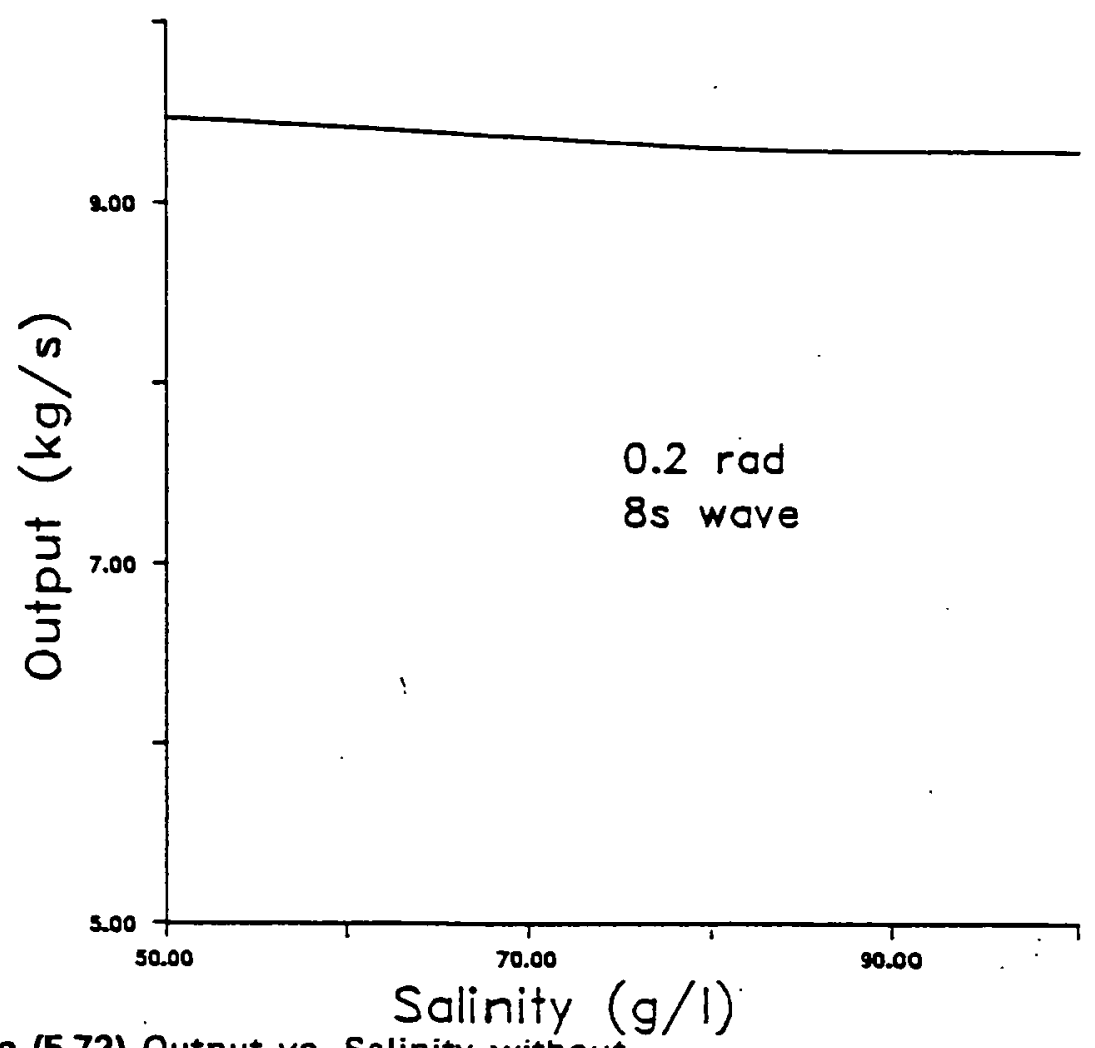

Figure (5.72) Output vs. Salinity without the fluid piston

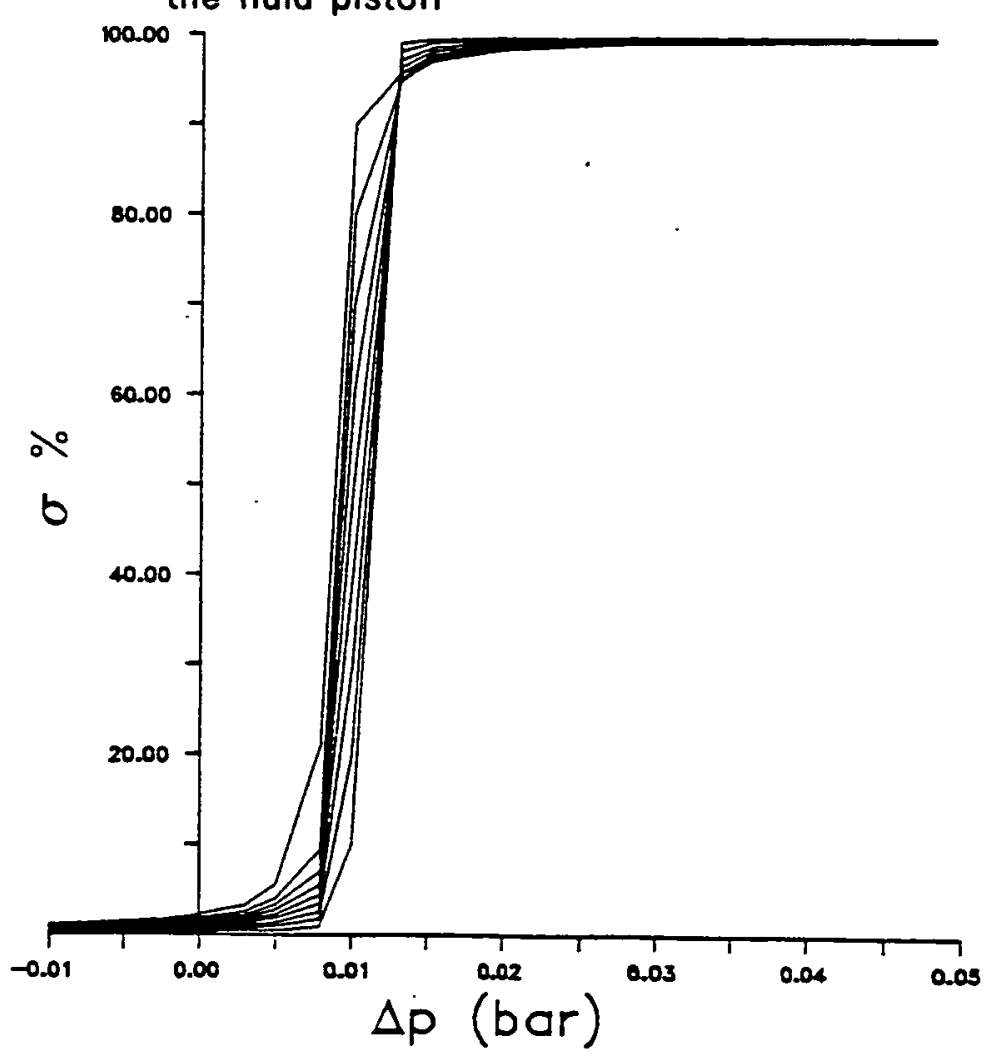

Figure (5.73) $\sigma$ vs. $\Delta p_{\circ}{ }^{\prime}$ for various values of $\sigma^{\prime}$ 


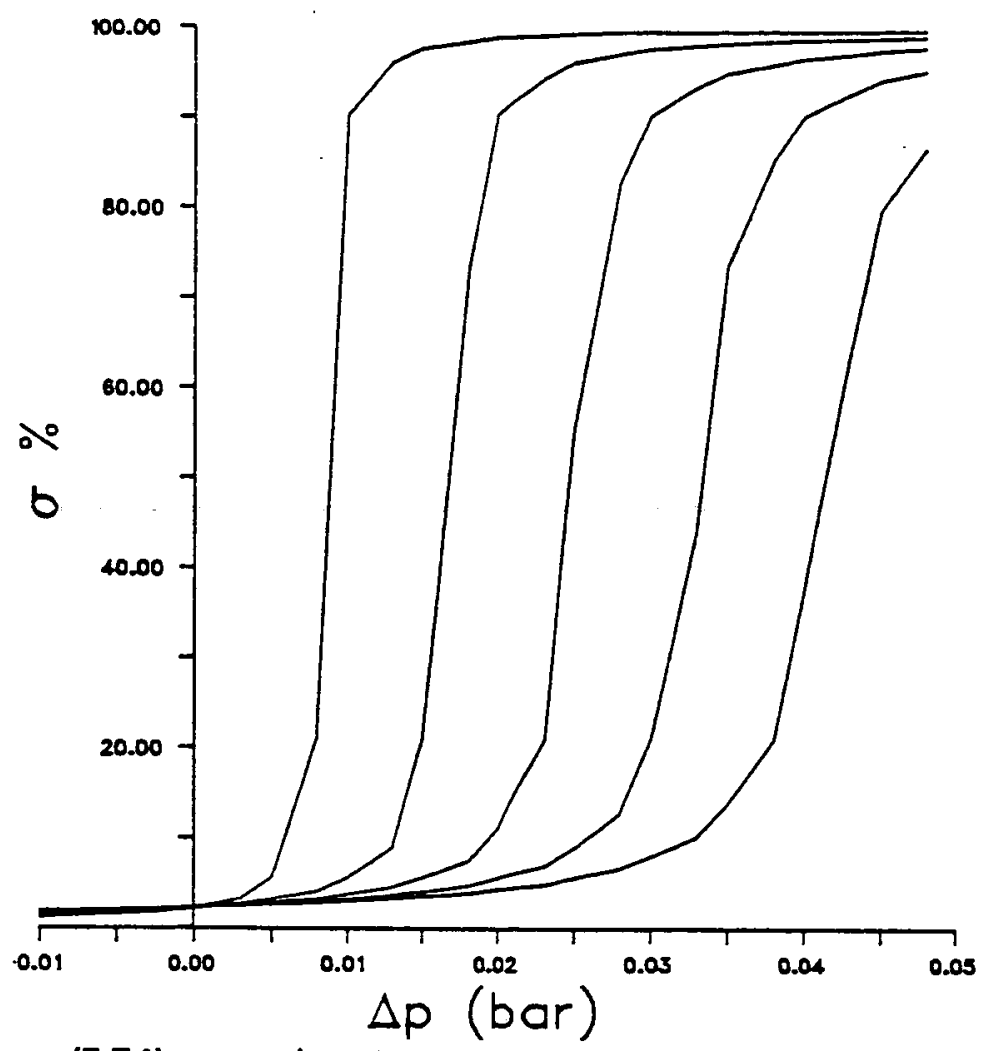

Figure (5.74) $\sigma$ vs. $\Delta p_{\circ}$ for various values of $\Delta p_{o}^{\prime}$

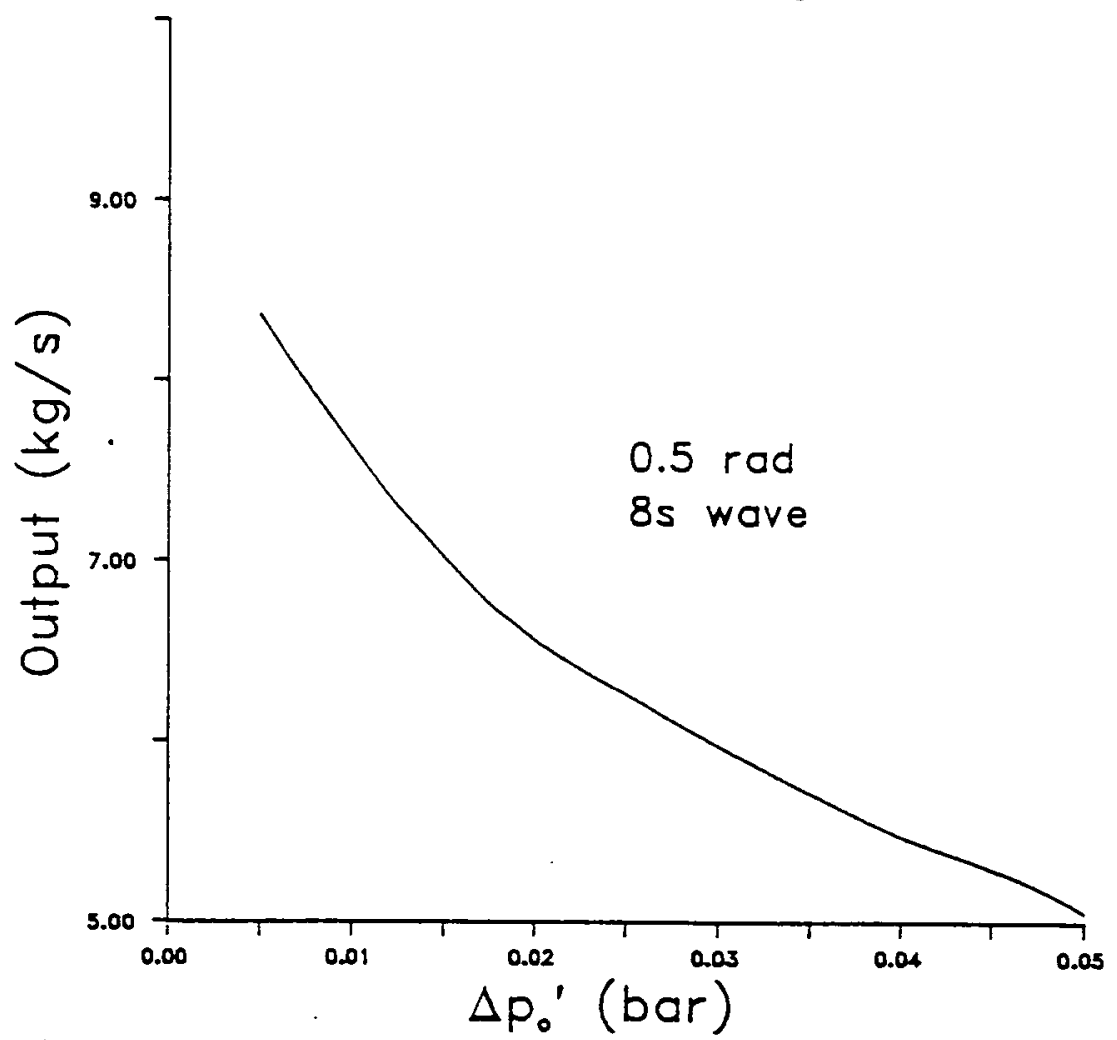

Figure (5.75) Output vs. $\Delta p_{0}{ }^{\prime}$ 


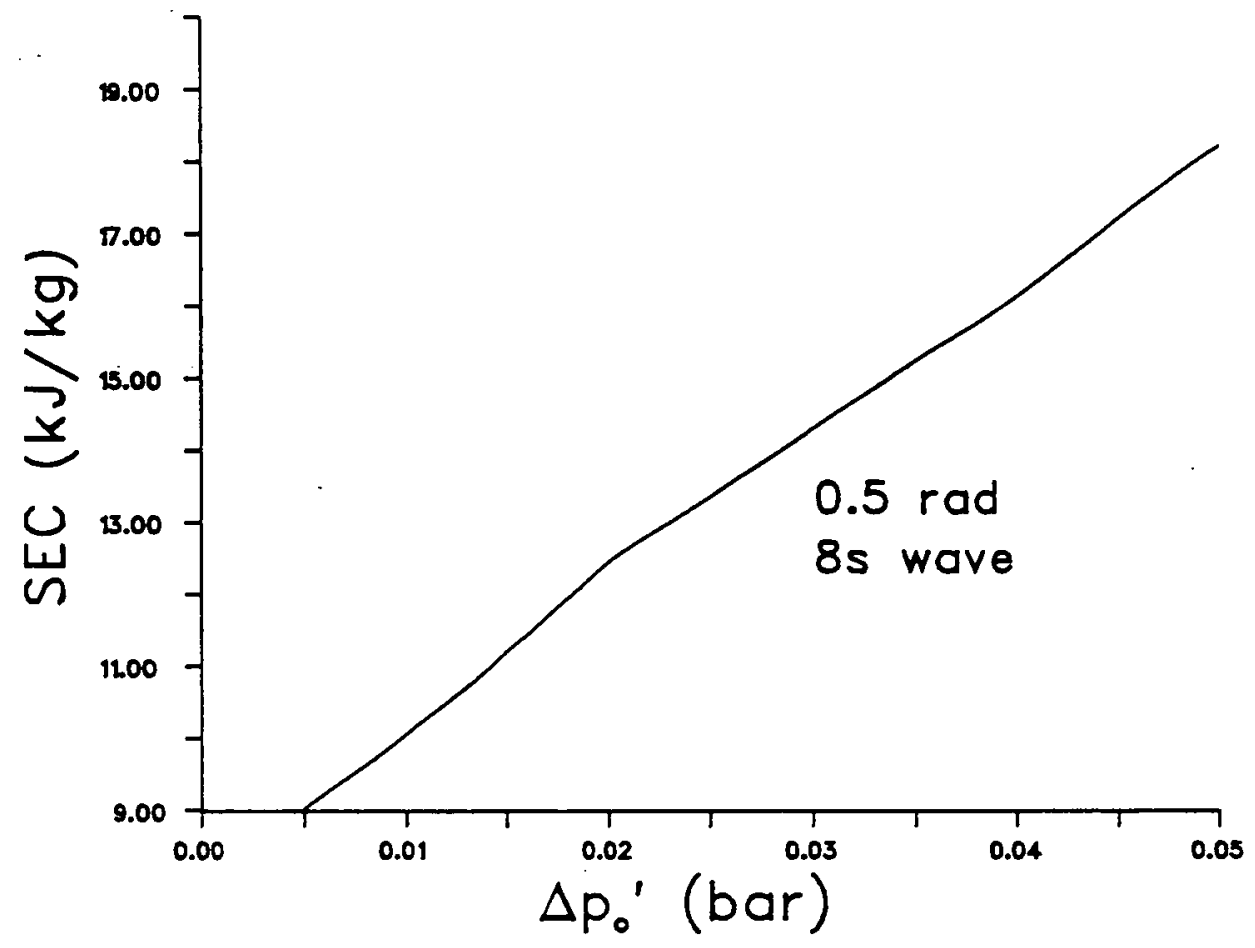

Figure (5.76) SEC vs. $\Delta p_{\circ}^{\prime}$

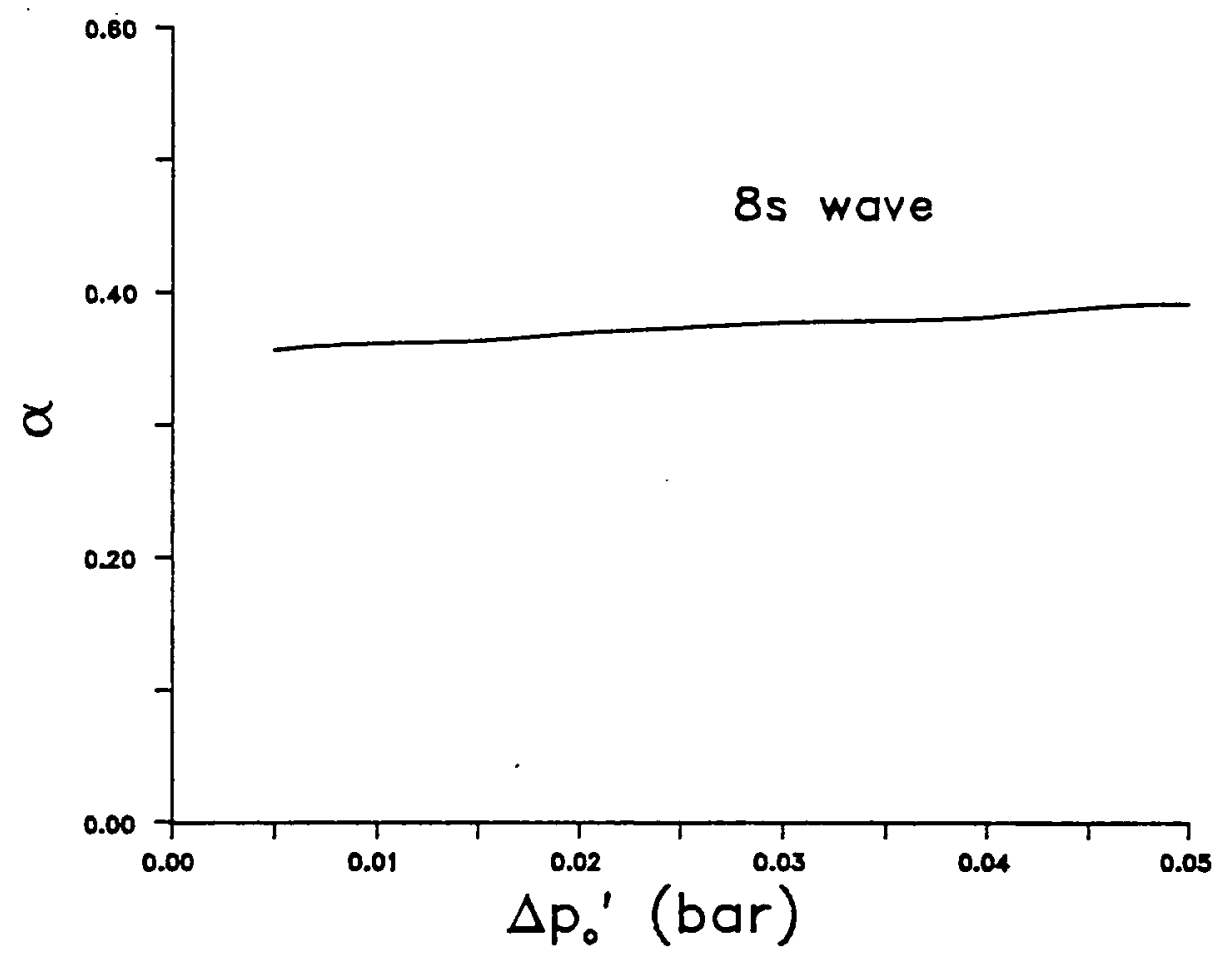

Figure (5.77) $\alpha$ vs. $\Delta p_{\circ}^{\prime}$ 


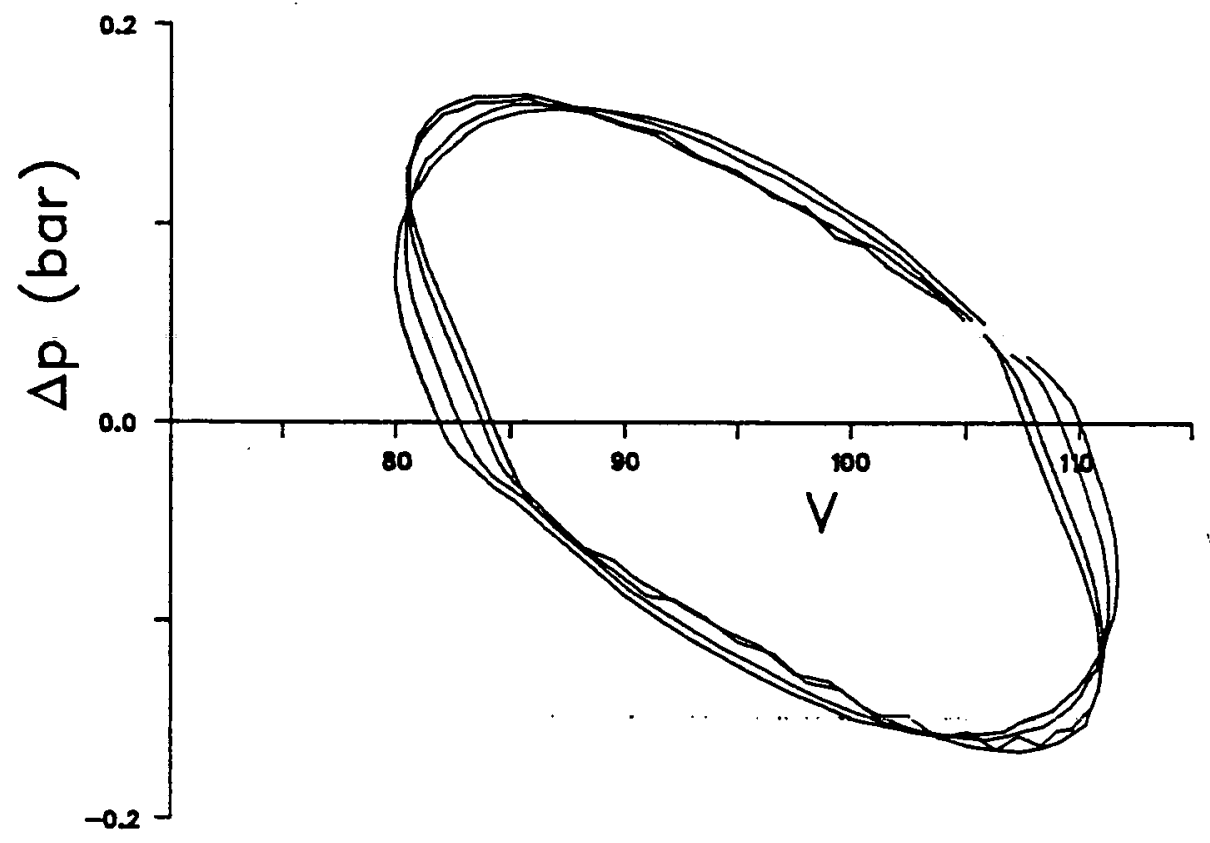

Figure (5.78) $\Delta p$ vs. $\theta$ for various values of $\Delta p_{0}$ ' 
CHAPTER 6

CONCLUSIONS AND RECOMMENDATIONS 
The function of this chapter is to precis the conclusions that may be drawn from the work presented and the recommendations for future work which are stated elsewhere in the text. More detailed analysis of the points made may be found in the preceding chapters; particularly chapters 3 and 5 .

Two mathematical models and associated computer programs were developed to describe the heat and mass transfer processes that would take place in a VC desalination duck as proposed. The second model was much more rigorously derived and was found to be more reliable and accurate than the first. An experimental facility was constructed which took the form of a small-scale analogue of the VC distillation system using the refrigerant $R 11$ $\left(\mathrm{CCl}_{3} \mathrm{~F}\right)$ as the working fluid. A mathematical model of the apparatus was derived from the mathematical model of the duck with appropriate changes of dimensions, fluid properties, etc. Transient data from the rig were compared with those predicted by the model in order that the validity of the principles upon which the model was based might be tested. It was found that the model described the system well, within the limitations imposed by the approximations in its derivation.

Having thus validated, as far as possible, the use of the mathematical model, the computer program was used to provide performance predictions for a full-scale device. These predictions suggested that the duck would operate in a manner similar to a simple, steady-flow VC desalination process except for the effects of the motion of the fluid piston. The action of the fluid piston was characterised using the ratio, " $\alpha$ "; the ratio of the actual vapour volume swept out by the duck to the ideal volume were the fluid piston to remain steady. This ratio was found to be highly dependent upon the frequency of the oscillation; tending towards zero at the resonant period of the piston as modelled (around 4.5 seconds). Correlations between $\alpha$ and the duck amplitude of motion, the heat transfer area, the brine salinity and the specification of the non-return valves were also noted. From these predictions it would appear that a vC duck, $20 \mathrm{~m}$ long by $6 \mathrm{~m}$ in diameter, with a heat transfer surface area of around $4500-5000 \mathrm{~m}^{2}$, could produce the initial design target figure of $1000 \mathrm{~m}^{3} \cdot \mathrm{day}^{-1}$ of fresh water in a wave climate of around $10-20 \mathrm{~kW} \cdot \mathrm{m}^{-1}$. It was also found, however, that the output was critically dependent upon the swept angle, as well as the frequency, and therefore that it would be important to find a duck shape that could produce large amplitudes of motion in quite small 
There would now appear to be several routes along which future research work could develop. A number of these could arise from the relaxation of the various constraints and approximations made in the derivation of the mathematical model. The most fundamental of these was that the duck would always move as a sine wave of specified amplitude and period, rather than according to the forces acting upon the duck It is difficult to see how this approximation could be removed, but it is the opinion of the author that, without any progress in this direction, there would be little point in further refining the existing model. Allied to this is the question of the design of the duck shape for optimal absorption of wave energy, alluded to above. Normally, this would have been studied through the use of small scale replica ducks in a wave tank; in this case this option was not open because of the complexity of the force characteristic generated by the compression process. In the future, however, it might be possible to derive an approximate model which could be used in this manner. If the project is to proceed, then some means of tackling this question must be devised.

It would also be important to study the fluid piston; firstly to test the existing models (and perhaps to improve upon them) and secondly, to analyse the effects of the fluid piston as a modifier of the thermal processes of distillation (ie. heat storage effects etc). It seems likely that some sort of experimental work would be necessary in both of these areas, ideally coupled to further modelling work. (A suggestion for the experimental investigation of some of the kinetic aspects of the behaviour of the fluid piston is made in appendix III.) Less significant improvements, and ones which might be easily incorporated into the model as it stands, include changes in the manner of the specification of the non-return valve behaviour and in the model of the heat recovery system (see III for details).

As the idea behind this project was entirely novel, only the most fundamental aspects of the process have been studied. The reasoning was that, if these investigations should prove the process to be feasible, then it would be reasonable to proceed with more detailed design calculations. If these calculations should uncover any difficulties, then it should, in principle, be possible to circumvent them; as long as the basis of the process is "secure". 
This approach has, naturally, left many aspects of the process out of the investigation. For example; device-to-shore pumping; heat recovery exchanger design; seawater deaeration, etc., are all important but have yet to be studied. Perhaps most important of all, however, may be the requirement to find out more about the potential market for the device. The supply side of the economic equation may now be evaluated with some confidence; it is possible to determine (within limits) how much water a given duck could produce; how much it might cost to construct and install, and the wave climate it would require. The demand side, on the other hand, remains completely unknown; indices of water cost and projected demand cannot be applied in this case because of the novelty of the process and its fundamentally different operating philosophy. A study of desalination processes which use renewable energy may provide some useful economic indicators. Information from wind powered plant studies, in particular, may be relevant as these installations are based on mechanical systems, as opposed to solar desalination units which are often purely thermal. 
CHAPTER 7

APPENDICES 


\section{SEAWATER SCALE FORMATION AND CONTROL}

The formation of scale on heat transfer surfaces during the distillation of seawater and the subsequent reduction in system performance, is one of the most important limiting factors in the design of desalination distillation equipment. The topic has, therefore, been thoroughly investigated with the aim of developing effective means of scale prevention and/or removal: there are several general texts which may be recommended for their treatments of the subject (Porteous, 1974, [53]; Porteous, 1983, [54], and Speigler and Laird, 1982, [67]).

Mineral scale is caused by mineral salts crystallizing from solution directly onto solid surfaces. Most scaling compounds have inverse solubility curves; that is, they become less soluble at higher temperatures and will thus tend preferentially to form on heating surfaces. Scale is sometimes confused with sludge, another commonly occurring phenomena in distillation equipment, but the two are not the same. Sludges are deposits consisting of, for example, corrosion products, detached scale particles, silt and so on, which are held in suspension in the process fluid and tend to settle out in regions where the fluid velocity is low enough. They are, generally, non-adherent and so present few problems in operational terms. Scale, by comparison, results from direct crystal nucleation and growth on heat exchange surfaces (or wherever the conditions are favourable). Depending upon the type of scale formed, they can be very hard; of low thermal conductivity, and extremely difficult to remove. Scale growth does not only result in a decrease in the heat transfer coefficient; scale thicknesses in the brine pre-heaters of MSF plants have been found to be so great that flow restriction was taking place to a significant extent. Scales can also form on RO and ED membranes (because of locally high concentrations) blocking the pores and reducing mass transport (see Gilron and Hasson, 1987, [22]; Okazaki and Kimura, 1984, [46]). Less than 10\% by weight of the minerals dissolved in seawater are capable of producing scale; the other minerals present (chiefly $\mathrm{NaCl}, \mathrm{MgCl}_{2}$ and $\mathrm{KCl}$ ) pose no problems at the concentrations normally encountered. The principal scale forming constituents are: $\mathrm{CaCO}_{3}, \mathrm{Mg}(\mathrm{OH})_{2}$ and $\mathrm{CaSO}_{4}$; small quantities of alumina, silica and ferric and other metal chlorides may also be found in the scales, but these are not the main constituents. The types of scale formed may be first divided into two groups: alkaline scales and sulphate scales. 


\section{I.I. Alkaline Scales}

A typical alkaline scale consists of a mixture of $\mathrm{CaCO}_{3}$ and $\mathrm{Mg}(\mathrm{OH})_{2}$ : the predominant type depends upon the conditions under which the scale is formed. Alkaline scales are so called because they result from reactions involving the total alkalinity of the brine. The alkaline component in the water, the bicarbonate ion, $\mathrm{HCO}_{3}{ }^{-}$, breaks down on heating to produce $\mathrm{CO}_{3}{ }^{-}$ions and $\mathrm{OH}^{-}$ions. As the brine reaches the solubility limits for a particular scale forming constituent, so that scale will tend to appear. These solubility limits are a function of the temperature and of the composition of the brine, see Porteous, 1983, [53], for details. Of rather more fundamental importance, however, is the actual chemistry of scale formation. A comprehensive theory on the formation of alkaline scales has yet to emerge because of the myriad factors affecting the reaction $(\mathrm{pH}$, temperature, concentration, residence time, etc). The most generally accepted treatment, however, is that of Langelier et al, 1950, [35]. This theory proposes the thermal decomposition of the bicarbonate ion as a first step:

$$
2 \mathrm{HCO}_{3}{ }^{-} \rightleftharpoons \mathrm{CO}_{3}{ }^{2-}+\mathrm{CO}_{2}+\mathrm{H}_{2} \mathrm{O}
$$

(note that this reaction produces gaseous $\mathrm{CO}_{2}$ which has some nuisance value in thermal processes: as a condensation inhibitor and as a corrosive gas). The carbonate ion evolved in this reaction can then take part in two competing equilibria:

$$
\begin{aligned}
& \mathrm{Ca}^{2+}+\mathrm{CO}_{3}^{2-} \rightleftharpoons \mathrm{CaCO}_{3} \\
& \mathrm{CO}_{3}{ }^{2-}+\mathrm{H}_{2} \mathrm{O} \rightleftharpoons \mathrm{HCO}_{3}^{-}+\mathrm{OH}^{-}
\end{aligned}
$$

Reaction (7.2) can only occur if the ion product of the $\mathrm{CaCO}_{3}$ exceeds its solubility at the given temperature and total salinity. If sufficient magnesium ions exist in the solution, such that the ion product of the $\mathrm{Mg}(\mathrm{OH})_{2}$ exceeds its solubility product, then a third reaction can take place:

$$
\left.\mathrm{Mg}^{2+}+2 \mathrm{OH}^{1} \rightleftharpoons \mathrm{Mg}(\mathrm{OH})_{2(\mathrm{~s}}\right)
$$

The key factor in the formation of alkaline scales is the ratio of the concentration of carbonate and hydroxyl ions. This ratio is dependent on $\mathrm{pH}$, $\mathrm{HCO}_{3}{ }^{-}$concentration, the dissociation constant of water and the secondary dissociation constant of carbonic acid, thus: 


$$
\left[\mathrm{CO}_{3}{ }^{2-}\right] /\left[\mathrm{OH}^{-}\right]^{2}=\mathrm{K}_{2} \cdot\left[\mathrm{HCO}_{3}{ }^{-}\right] \cdot\left[\mathrm{H}^{+}\right] / \mathrm{K}_{\mathrm{w}}{ }^{2}
$$

According to Langelier, $K_{w}$, in expression (7.5), known to be a function of temperature, is the critical component. For example, $K_{w}{ }^{2}$ is estimated to change by a factor of $10^{4}$ between 35 and $100^{\circ} \mathrm{C}$; which could account for the variation in the composition of the different scaling compounds formed in different parts of operational plants.

The above reaction scheme was supported by the work of Hillier, 1952, [26], which was based on studies of a pilot plant scale submerged tube evaporator. Hillier reported $\mathrm{CaCO}_{3}$ as the dominant scale at low temperatures and $\mathrm{Mg}(\mathrm{OH})_{2}$ at higher temperatures. Hillier's paper also included other, controversial, findings: that scale growth was not related to brine concentration; scale growth was inversely related to temperature, and that minimum scaling was observed at around $80^{\circ} \mathrm{C}$, the "transition" point between $\mathrm{CaCO}_{3}$ and $\mathrm{Mg}(\mathrm{OH})_{2}$. Later researchers (Dooly and Glater, 1972, [14]) have found it difficult to reproduce these findings and so have proposed a different alkaline scaling mechanism. The first step, again, is the breakdown of the bicarbonate ion, but to differing products:

$$
\mathrm{HCO}_{3}^{-} \rightleftharpoons \mathrm{CO}_{2}+\mathrm{OH}^{-}
$$

The hydroxide ion formed can be buffered by fresh bicarbonate:

$$
\mathrm{OH}^{-}+\mathrm{HCO}_{3}{ }^{-} \rightleftharpoons \mathrm{CO}_{3}{ }^{2-}+\mathrm{H}_{2} \mathrm{O}
$$

or it may form $\mathrm{Mg}(\mathrm{OH})_{2}$ as in equation (7.4). The $\mathrm{CO}_{3}^{-}$ion produced by reaction (7.7) can react as in (7.2). The balance between the two processes is related to temperature, brine concentration and residence time. It is now felt that the classical Langelier mechanism cannot be valid at lower temperatures, but that the actual mechanism may be a complex combination of the two proposed above.

\section{I.II. Calcium Sulphate Scaling}

Alkaline scales form as a result of a system of $\mathrm{pH}-d e p e n d e n t$ ionic reactions (see above); $\mathrm{CaSO}_{4}$ scaling occurs as a result of a purely physical precipitation process, when the solubility limits for $\mathrm{CaSO}_{4}$ are reached (through increasing temperature and/or salinity). Once formed, $\mathrm{CaSO}_{4}$ scales severely restrict the 
operation of distillation plants as the scale is extremely hard, adherent and resistant to chemical treatment. Reliable methods of $\mathrm{CaSO}_{4}$ scale control have yet to be developed and so distillation plants operate in the non-saturated region if this form of scaling is to avoided; an understanding of the limits of operability is, therefore, vital for practical plant design.

Three forms of $\mathrm{CaSO}_{4}$ can be produced, depending upon the conditions: gypsum $\left(\mathrm{CaSO}_{4} \cdot 2 \mathrm{H}_{2} \mathrm{O}\right)$ and anhydrite $\left(\mathrm{CaSO}_{4}\right)$ which are formed by natural processes and a metastable form, hemihydrate $\left(\mathrm{CaSO}_{4} \cdot 1 / 2 \mathrm{H}_{2} \mathrm{O}\right)$ which is formed when natural seawater is heated above $100^{\circ} \mathrm{C}$ (commercially known as "plaster of Paris", manufactured by heating gypsum at $125^{\circ} \mathrm{C}$ ). Recently, other modifications to these crystal structures were discovered, giving a total of six forms; it has since been confirmed, however, that only the three above are formed from equilibria with aqueous solutions. The papers published on $\mathrm{CaSO}_{4}$ solubility limits are too numerous to mention (the reader is referred to Spiegler and Laird, 1982, [67], for more detail); the data most widely accepted is from one of the first papers, that of Partridge and White, 1929, [48], reproduced in figure (7.1). This data was determined for $\mathrm{CaSO}_{4}$ precipitation from pure water; the extrapolation of this data to describe precipitation from natural seawater is fraught with difficulties and it is at this point that experimental, pilot-plant and full-scale operational data must be included. When these sorts of data are studied, it becomes clear that solubility thresholds and scaling limits can be quite different. This is because, for example, as the brine is concentrated not only does the $\mathrm{CaSO}_{4}$ become more saturated, but also other salts in the solution. Under realistic conditions the equilibrium between the various forms of $\mathrm{CaSO}_{4}$, (see figure (7.1)) must also be taken into consideration. To illustrate why this should be so, the reader is referred to figure (7.2) which is a representation of the mean results from many researchers (see Porteous, 1974, [53]). According to this diagram, it should be impossible to operate above atmospheric boiling point conditions without encountering anhydrite formation. Although anhydrite is the stable high temperature form, however, hemihydrate (which is produced first) is stable enough to remain for time periods longer than the normal residence time in most evaporators, thus allowing higher temperatures (around $120^{\circ} \mathrm{C}$ ) and higher concentration factors (around 2 to 2.5) to be used. Thus, most plants routinely operate above the anhydrite line and below the hemihydrate line and can do so in a scale-free manner (effectively). The kinetics of the 
transition between the phases, especially between hemihydrate and anhydrite, has attracted a great deal of attention, as it is of considerable importance. A complete description of $\mathrm{CaSO}_{4}$ scaling has, however, proved elusive; not only temperature and concentration are important, residence times (local and bulk), existence of nucleation sites, local superheating, local supersaturation, all play important roles in the determination of the rate of $\mathrm{CaSO}_{4}$ growth and all are a function of the design and operation of the plant.

\section{I.III. Methods of Scale Control}

On early plants it was often the case that the cost of the water was unimportant compared to its availability, such as on shipboard evaporators. In such applications, intermittent and expensive mechanical means of scale removal were often employed: thermally shocking the tubes or physical removal of the scale by chipping or hammering were two common methods. In larger plants, the economics of operation becomes critical and on-line continuous feed treatment becomes a necessity.

\section{Akaline Scale Control}

The most common method of alkaline scale control is acid pre-treatment. An acid, normally $\mathrm{H}_{2} \mathrm{SO}_{4}$, is added to the feed make-up water to lower the $\mathrm{pH}$ to around 4.0. The bicarbonate ion in the water is converted to $\mathrm{CO}_{2}$ at this $\mathrm{pH}$, which is removed ("decarbonation" or "acid-degassification") to avoid corrosion and/or heat transfer problems downstream. The acid feed water is then neutralised by the addition of sodium hydroxide to a pH of 7-8.0. Obviously, the design of the acid addition and caustic neutralisation plant is largely determined by the need to avoid corrosion problems.

Another method of scale formation inhibition is known as "threshold treatment" (see, for example, Austin et al, 1975, [2]). In this technique, small amounts (hence the title) of complex chemical agents, normally polyelectrolytes such as polyacrylic acids, methacrylate polymers and so on, are added to the feed water. The normal dosage is less than 10ppm, which is why the technique has attracted commercial interest. The mechanism of scale inhibition is not well understood; recent theories suggest that similarities in the crystal structures of the scale and of polyphosphates (a popular additive) could result in the additive effectively "blanking off" nucleation sites to crystal growth. It has been suggested that there is, then, the possibility of designing 
inhibitors for particular types of crystal formation. The principal disadvantage of the threshold treatment methods is the thermal instability of the additives used, limiting the maximum temperature of operation. Threshold agents are, however, routinely used as scale inhibitors in RO operations, usually in the form of dosing with sodium hexametaphosphate $\left(\mathrm{NaPO}_{3}\right)_{6}$

\section{Calcium Sulphate Scale Control}

There are no reliable means of $\mathrm{CaSO}_{4}$ scale control at the time of writing. The only means available for the prevention of the formation of $\mathrm{CaSO}_{4}$ precipitate is operation of the plant outside the scaling thresholds. Active methods of scale prevention have been studied, including removal of sulphate ions by resin exchange (Hunter et al, 1968, [29]) and $\mathrm{CaSO}_{4}$ crystal seeding (Gainey et al, 1963, [21]). In seeding methods, small crystals of $\mathrm{CaSO}_{4}$ are deliberately kept in suspension in the evaporating brine. These crystals tend to provide preferential nucleation sites for the formation of further precipitate, thus avoiding its formation on heat exchange surfaces. This method has been proposed by many different workers, but, unfortunately, the results of these tests have proved at best inconclusive. 


\section{ENHANCED HEAT TRANSFER IN FALUNG FILMS}

The fluid mechanics of liquid flow in thin films are such that high heat transfer coefficients between the film and the surface over which it flows can be obtained without any form of enhancement. This is related to the fact that almost all of the thickness of the film is made up of the laminar boundary layer, the rate of conduction through which determines the rate of heat transfer. In turbulent flow in a pipe, for example, the resistance of the boundary layer is only part of the overall resistance which includes bulk eddy transport and transport through the turbulent boundary layer. The mechanics of flow and heat transfer have been extensively studied; the classical work on the topic, that of Nusselt, has been continuously revised (particularly for turbulent and/or evaporating films). It would not be appropriate to include here a review of all of this work, as the subject area is so large; instead the reader is referred to the works of Struve, 1965, [69]; Wilke, 1962, [78]; Dukler, 1960, [15], and Schnabel, 1983, [61], all of which contain reviews of the field as well as important contributions from the authors.

\section{II.I. Condensation}

The very high heat transfer coefficients offered by the maintenance of dropwise condensation (see, for example, Perry et al., 1984, [49]) have provided encouragement to the development of techniques aimed at increasing the range of condensate mass fluxes over which this regime could occur. Such techniques have generally involved the injection of promoters into the condensing vapour (eg. mercaptans) and/or treatment of the heat transfer surface. These measures may be counted, at best, as a partial success; dropwise condensation can be promoted beyond its normal operating range, but the effects of the additives have tended to wear off with time. With the development of passive techniques, dropwise enhancement has all virtually been abandoned.

If operating in the continuous film regime, the obvious way to increase the heat transfer coefficient would be to somehow thin the liquid film so as to reduce its resistance to heat transfer. A number of methods have been considered: vapour shear thinning (ie. blowing the liquid film to spread it more thinly); wiped films using a mechanical apparatus (more common for evaporation duties), and surface tension methods. The effects of vapour shear 
on liquid films has been studied in great detail from a fundamental point of view (Dukler, 1961, [16], [17]), but it seems unlikely that the method could have a great deal of practical application. Wiped film systems are already used in the evaporation of viscous liquors. The use of a mechanical wiper can introduce sealing problems as well as an increased maintenance requirement. Surface tension methods have proved more popular and effective to the point where coefficients achieved through their use can approach those obtained in dropwise condensation.

Surface tension heat transfer enhancement methods rely on the use of surface profiles locally to thin the condensing film. The most common type is the fluted surface (see figure (7.3)) originally studied by Gregorig, 1954, [23]. Gregorig showed, experimentally and theoretically, that machining flutes into the surface of a condenser tube could significantly augment the condensation heat transfer film coefficient; increases of up to $800 \%$ of the performance of plain tubes was observed. The enhancement is caused by surface tension in the film setting up a pressure gradient "along" the flute, causing liquid to flow from the crests into the troughs. The effect may be described analytically, starting from Laplace's equation:

$$
p=\sigma / r_{i}
$$

where $p$ is the overpressure inside the convex film due to surface tension; $\sigma$ is the surface tension, and $r_{i}$ is the radius of curvature of the liquid/vapour interface. Over a flute, equation (7.8) may be differentiated with respect to I, the distance along the flute, to give the pressure gradient:

$$
d p / d l=\left(-\sigma / r_{i}^{2}\right) \cdot d r_{i} / d l
$$

Under the influence of $d p / d$ the liquid flows from the crests to the troughs (at a rate determined by the viscous friction). Thus, most of the condensation takes place on the crests of the flutes, with the condensate being drawn off in turbulent flow in the troughs. Enhancements of 3-4 times the coefficients of plain tubes are commonly achievable in practice. From a practical viewpoint, the immediate difficulty with equation (7.9) is the definition of the shape of the vapour/liquid interface. The shape of the boundary will be affected by the shape of the flute but also by the local condensate mass flux and how full the troughs are (which will depend upon the amount of condensate flowing down 
the tube from above). Much of the work carried out in this field has, therefore, been concerned with the optimisation of fluted surfaces, both experimentally and theoretically.

Through this research, it has become clear that the original tube profile used by Gregorig need not be the best choice and, therefore, many different flute profiles have been tried. One interesting development from this work arose from the work of Thomas et al., 1968, [70]. The initial investigation studied the use of simple fins, rectangular in cross section, attached to the outside of a condenser tube. As the fins were only lightly fixed, their role as an extension of the heat transfer surface would have been limited; nevertheless, heat transfer enhancement was observed and it was surmised that this was caused by surface tension drawing the film into a rivulet at the join of the fin and the tube (see figure (7.4)). In an extension of this idea, a plain tube was wrapped with wire to form a helix with a long lead length. Enhancement was once again observed as the condensate was drawn into the angle where the wire came into contact with the tube (see figure (7.5)). For applications with a high mass flux requirement, however, it would appear that fluted (or roped, ie. helical fluted) tubes were to be preferred because of the greater liquid capacity of the troughs. Horizontally mounted tubes may be treated in a similar manner with the addition of closely spaced radial fins along their length. Studies have been directed at finding the optimum values for the fin spacing and fin height. Fluted tubes and their variants are now routinely used in multi-effect evaporation plants for desalination.

\section{II.II. Evaporation}

The search for methods of improving evaporation film coefficients has not proved to be as successful as that above. One common method is wiped film evaporation where the film is mechanically thinned using a set of rotating wipers to physically spread the film over the inside of the tube. This technique, however, has not proved suitable for desalination duties because of the maintenance requirement (a problem for large desalination plants with many tubes) and because of the vulnerability of the wiper mechanism to corrosive attack by the boiling brine. Another mechanical method, rotating disc evaporation in which the evaporation surface consists of a series of rapidly spinning discs over which the liquid is thinned by centrifugal force, has failed 
to be adopted for similar reasons. (see Wang et al., 1980).

If the evaporation duty is likely to include extensive nucleate boiling, then there are a number of extended or porous boiling surfaces which may be suitable (see Webb, 1980, [76], for details). The principle behind these surfaces is the provision of a large number of nucleation sites so that nucleate boiling can be extended to regions where film boiling would normally have started to take place. In falling film desalination, however, nucleate boiling tends to be limited because of the danger of creating locally high concentrations of scale-forming and corrosive solutions.

It has been found that fluted tubing can increase evaporation coefficients as well as condensation coefficients for which they were originally designed. The enhancements are not as good as those for condensation; the mechanism seems to be based on the local thinning of the film on the crests in combination with rapid, turbulent flow in the troughs. It has also been suggested that the crests may be wetted by nucleation in the troughs or by the propagation of waves down the surface of the tubes (see Rothfus and Lavi, 1976, [58]). Other work in this area has involved the addition of small amounts of surfactant to the evaporator feed which can, under the right conditions, cause the formation of a very thin, foamy film over the crests. Surfactant addition has also been studied with reference to rising film evaporation systems (see, for example, Sephton, 1976, [64]). 


\section{I. Addition of Compliance to the Compressor}

The addition of pressure force compliance to the compressor drive control circuitry was considered in order that the pistons of the compressor could mimic the resultant motion of the duck and the fluid piston. It quickly became clear, however, that there was little point in such an exercise as all that was required of the experimental facility was that its performance could be compared with a model of it. This section has been included, therefore, primarily to illustrate the techniques by which compliance may be electronically introduced into mechanical systems; techniques extensively used by the Edinburgh Wave Power Group in the course of their research.

For the fluid piston, the following approximate equation was derived:

$$
1 . d^{2} x / d t^{2}+D \cdot d x / d t+S \cdot x=k \cdot \Delta p
$$

In this case, $D$, the damping coefficient, could be neglected, so:

$$
I . d^{2} x / d t^{2}+S . x=k . \Delta p
$$

where $x$ is the position of the fluid piston (measured from a datum). Now if $y$ is the resultant displacement between the duck and the fluid piston (or the compressor piston and the cylinder head) and $z$ is the imposed duck position (from a sinusoid) then:

$$
\begin{aligned}
& y=z-x \\
& I . d^{2} x / d t^{2}+S \cdot(z-y)=k \cdot \Delta p
\end{aligned}
$$

Rearranging equation (7.13) gives:

$$
y=k_{1} \cdot d^{2} x / d t^{2}+k_{2} \cdot 2-k_{3} \cdot \Delta p
$$

In this form, y can be the demand signal to the control circuitry; $z$ the output from a sine wave generator, which can be electronically differentiated twice to give $d^{2} z / d t^{2} ; \Delta p$ can be measured in the usual way, and $d^{2} x / d t^{2}$ may be measured using an accelerometer (or by a position sensor or tachometer with appropriate differentiation of the resulting output signal). A block diagram for the implementation of such a feedback scheme is shown in figure (7.6) with a 
The practical implication of the implementation of such a system would be that the pistons would act in a manner similar to a fluid piston. For example, with a null demand signal from the sine wave generator, the pistons could nevertheless move if there existed a pressure difference across them. A null signal plus a step change in the $\Delta p$ (perhaps caused by the sudden opening of a solenoid valve) would cause the pistons to oscillate exactly like a pendulum. Although such a device would be most interesting to behold, it would not be without its operational problems. Principal amongst these would be the possibility of a sudden change in the control signal to the power amplifier and thus to the drive system, with the attendant risk of overload. With the compressor set up as it was, an overload could only occur if the pistons or drive system were to fail mechanically or if a sinusoid outside the operating range were specified. In either case, the fault would lie, ultimately, with the operator. With a compliant system, it might be possible for a set of conditions to exist which would cause an overload entirely outwith the control of the operator (eg. resonant behaviour). The implementation of a compliant system, therefore, would not only require the addition of a circuit such as that shown, but also such interlocks and cut-outs as would be required for reliable operation?.

\section{III.II. Verification of the Fluid Piston Equation}

Two models of the fluid piston were derived: one based on a U-tube; the other on a semi-cylindrical body (see section 3.3.3). The difference in the approximate resonant periods predicted by the two methods was small enough to suggest that the behaviour of the fluid piston could be adequately approximated by either model. It would be useful, however, to be able to test this hypothesis and so the following outline for a suitable experiment has been devised.

The principle of the experiment would be to displace a replica fluid piston of

\footnotetext{
'An accelerometer and a more reliable and accurate method of position sensing would be a worthwhile improvement
} 
known dimensions from rest, then to release it and measure its resonant period as it returned to its null position. The apparatus could consist of a length of pipe, half-filled with water and with a barrier bisecting the air space above the liquid; in essence, a small scale replica of the core of the VC duck. On one side of the barrier several large holes would be made in the upper surface of the pipe section so that, if the fluid piston were to move, the air so displaced could flow back and forth with the minimum of disturbance. In the pipe surface on the other side of the barrier a similar set of passages would be made, but these would require some means of being shut off and opened suddenly (or instantaneously). This could be achieved through the the use of fast acting solenoid valves, or perhaps the rupture of a stretched membrane. $A$ further, smaller, sealable nozzle would also be required on this side so that the piston could be displaced from rest using a pressure difference (see figure (7.8)).

Once the fluid piston had been displaced by a given amount by the pressure difference across it, the valves would be opened, or the membrane perforated, and the water would then oscillate at its resonant period until its motion became damped out by fluid friction. It might be possible visually to determine the period of this oscillation, but it would not be difficult to install some kind of interface sensor; the water could be used to make and break a pair of electrical contacts, for instance. The values thus obtained for the resonant period could then be compared with those from a model of the system (as described) and so a quantitative measure of the accuracy of the approach arrived at.

\section{III.III. New Model for the Non-Return Valves}

As described in chapter 3, section 3.3.3, the simulation of the non-return valves in the second model was based upon the use of an inverse tangent function for the opening vs. $\Delta p$ curve. In general, it was found that this was a suitable function to use as it was of the correct sigmoid shape and could not return a completely shut or fully open valve (which seems reasonable for any practical value). The disadvantage with the method lay, however, with the means used to specify the exact shape of the curve; two parameters were used but their influence on the shape of the curve was difficult to predict (see chapter 5, section 5.3.2). What was required, therefore, was a new means of 
specifying the exact shape of the curve which would be more "user friendly".

The form of the function was:

$$
\sigma=(2 / \pi) \cdot \tan ^{-1}(a \cdot \Delta p+b)
$$

Thus, it may be seen that the two constants, a and b, determine the shape of the plot and its position along the $\Delta p$ axis. This means that the user must specify two points on the curve in order for $a$ and $b$ to be evaluated. Perhaps the simplest and most straightforward way to do this would be to specify the $\Delta p$ 's at which the valves would open (say) $10 \%$ and $90 \%$. It is easy to see how this would automatically fix the position of the curve and the gradient of its near-linear middle section. Two linear equations would result:

$$
\begin{aligned}
& \text { a. } \Delta p_{1}+b=\tan (0.1 . \pi / 2) \\
& \text { a. } \Delta p_{2}+b=\tan (0.9 . \pi / 2)
\end{aligned}
$$

which could be simply solved to find $a$ and $b$. The chosen openings, $10 \%$ and $\mathbf{9 0 \%}$, may not necessarily be optimal; this would need to be tested by trial and error.

\section{III.IV. New R:odel of the Heat Recovery Exchanger}

The simulation of the three-way heat recovery exchanger included in the second model of the duck is described in chapter 3 , section 3.3.3. One of the assumptions implicit in the derivation of these equations was that the $\mathrm{dT} / \mathrm{dA}$ for all of the streams in the exchanger would be approximately constant and that, therefore, the usual definition of the log mean temperature difference $(\Delta T)$ would hold. As discussed in chapter 5, section 5.3.2, however, it was found that, under certain circumstances, this condition was not valid and so that for future use a more complex (and accurate) model would need to be derived.

The problem with the existing model was that the assumption that the dT/dA for any stream would be nearly constant along the entire length of the exchanger. As this manifestly was not the case, so some means of modifying this assumption had to be found. The most obvious way to do this was to divide the exchanger into a number of conceptual stages; if the stage $\delta A$ was small enough, then $d T / d A$ for each stream in any stage would be constant and 
so the log mean $\Delta T$ could be calculated (see figure (7.9)). Heat and mass balances around each stage would be carried out giving rise to $6 x n$ equations (where $n$ is the number of stages) and $6 x n+6$ unknowns, so that 6 parameters (eg. 3 temperatures and 3 flows) would need to be specified as before. Such a scheme would be uncomplicated to implement in a computer based model but would require additional processing time. With a large enough number of stages, however, it might be possible to use an arithmetic mean $\delta T$ and so to require only the solution of linear equations. 


\section{N. PROGRAM FLOWSHEETS AND USTINGS}

N.I. First Model

\section{Listing of First Model Program}

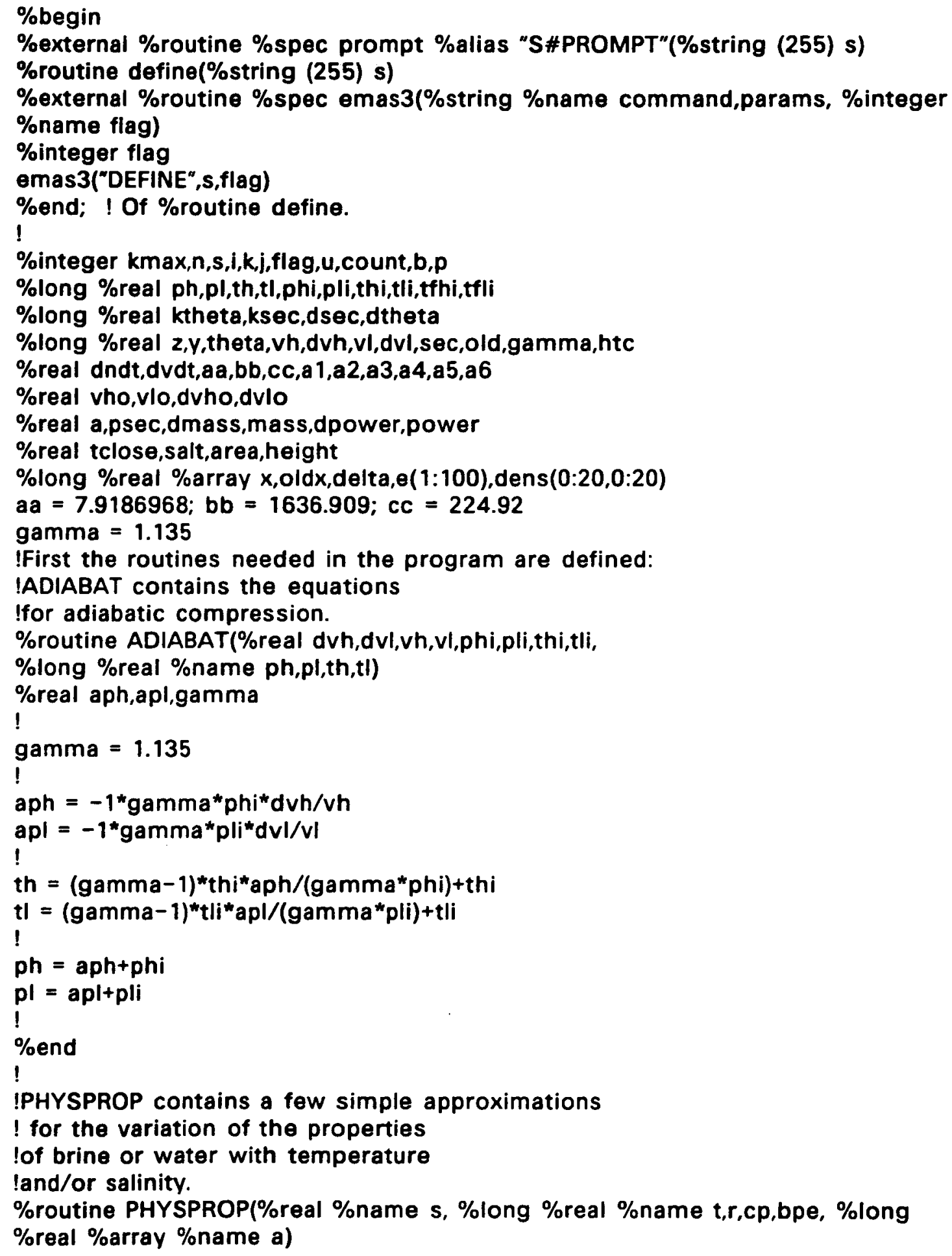




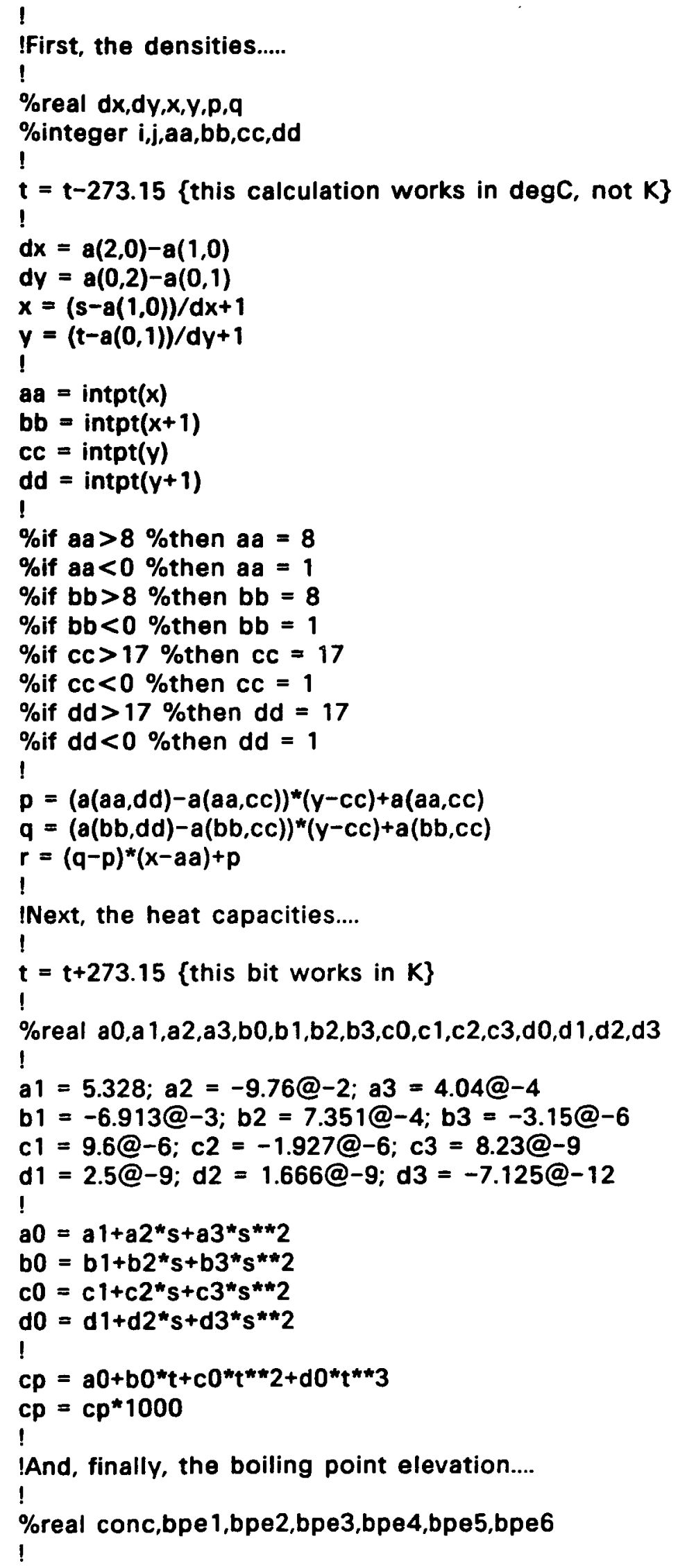




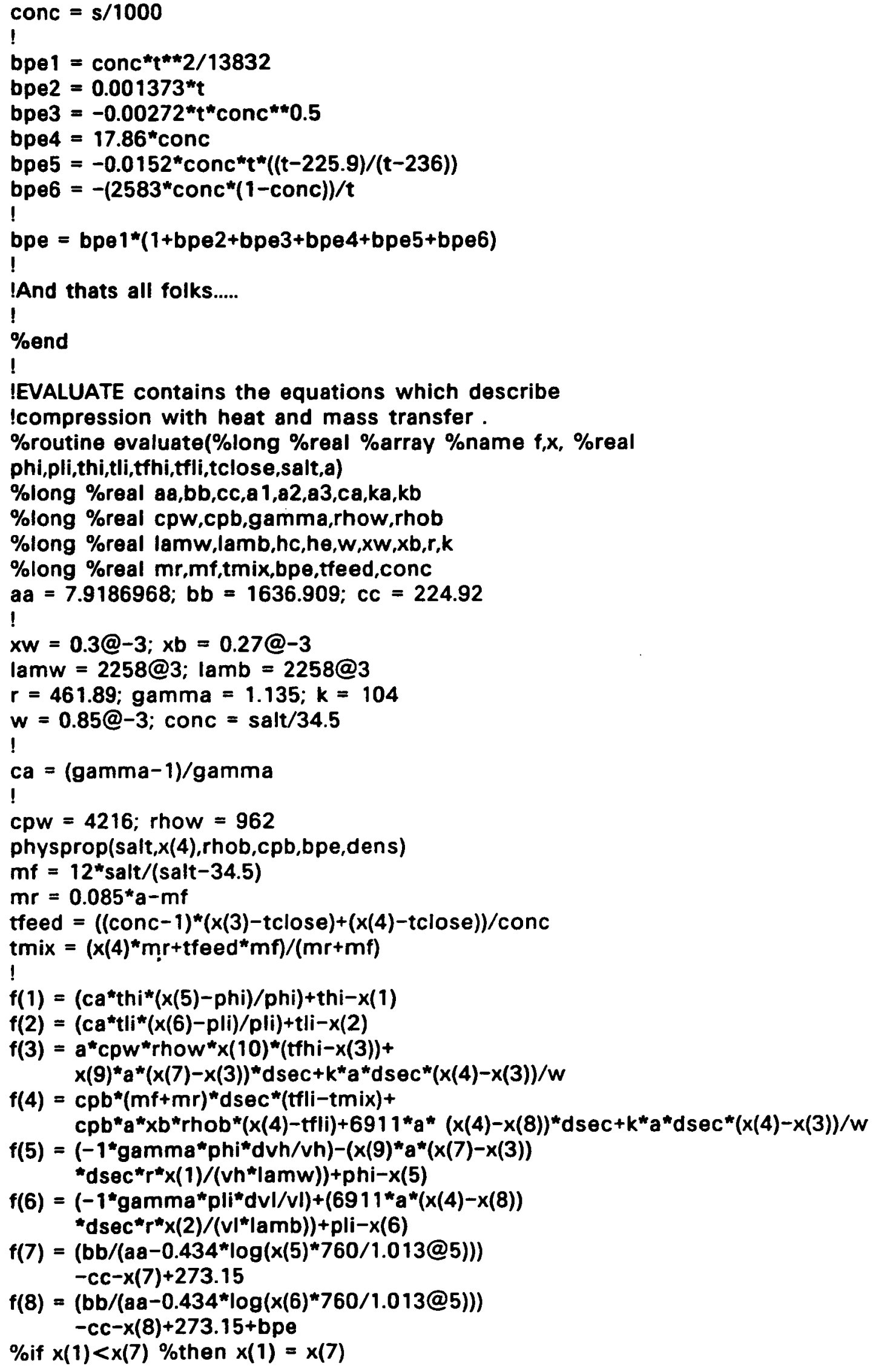




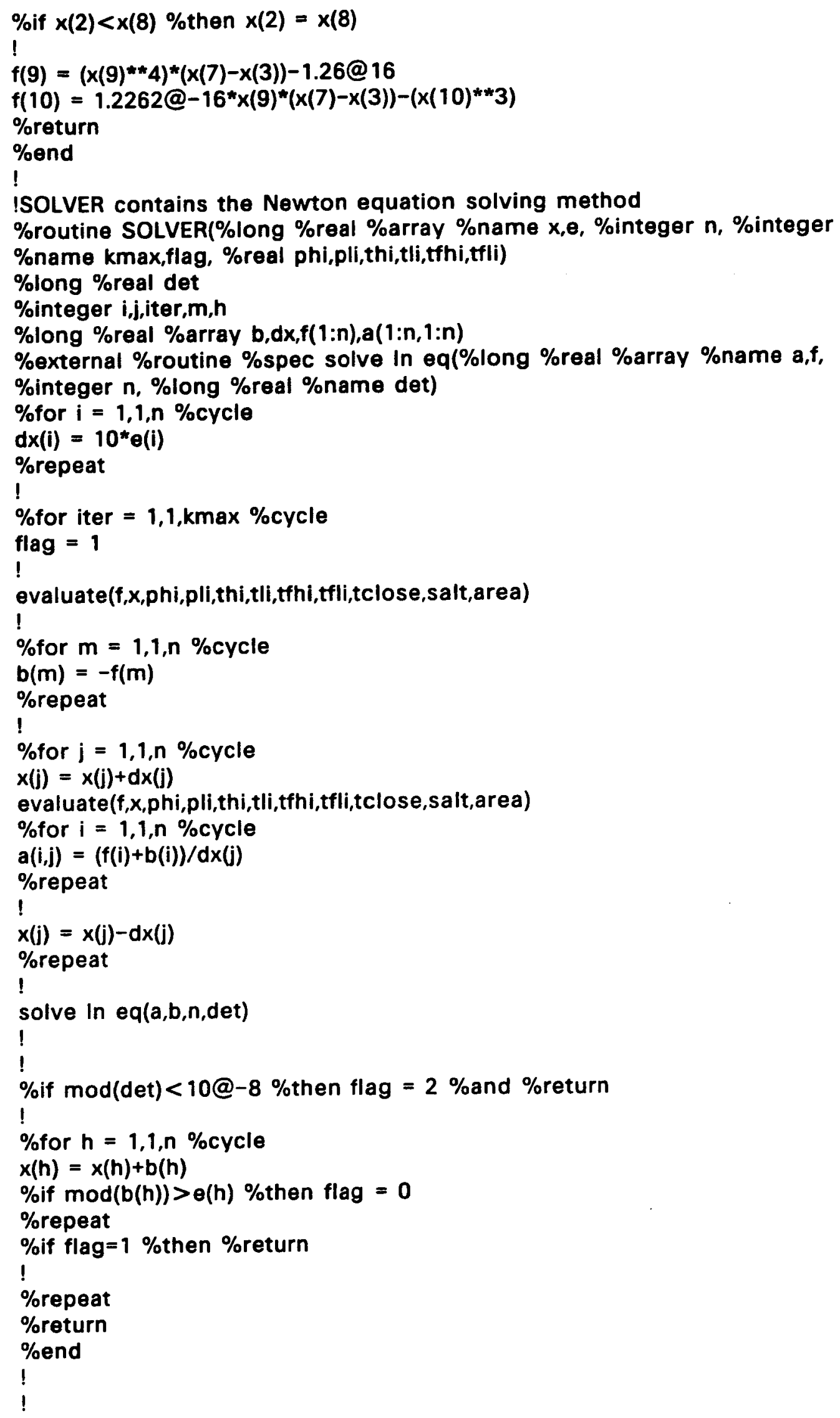


!nput of initial data from the terminalprompt("Initial pressure on h.p. side "); read(phi) prompt("Initial pressure on I.p. side "); read(pli) prompt("Initial temperature on h.p. side "); read(thi) prompt("Initial temperature on I.p. side "); read(tli) prompt("Initial water film temperature "); read(tthi) prompt("Initial brine film temperature "); read(tfli) prompt("Temp. approach in H.Recov. "); read(tclose) prompt("Area for heat exchange "); read(area) prompt("Brine concentration factor "); read(salt) salt $=$ salt $\star 34.5$ prompt("Swept angle "); read(ktheta) ktheta = ktheta*pi/180 prompt("Period of oscillation "); read(ksec) prompt("Number of timesteps "); read(s)

!Output files are defineddefine("1,theta")

define("2,temp")

define("3,delp")

define("4,time")

define("5,mass")

define("6,data")

define("10,emct 12:general.densities")

!

selectinput(10)

$\%$ for $\mathrm{i}=0,1,17 \%$ cycle

\%for $j=0,1,8 \%$ cycle

$\operatorname{read}(\operatorname{dens}(\mathrm{j}, \mathrm{i}))$

$\%$ repeat

\%repeat

closestream(10)

!

The timestep size is calculated

$\mathrm{dsec}=\mathrm{ksec} /(\mathrm{s})$

$\mathrm{n}=10$

!Initial guess for $x()$ variables

! $x(1)=t h ; x(2)=t \mid ; x(3)=t f h ; x(4)=t f l ; x(5)=p h$

! $x(6)=$ pl; $x(7)=$ tsat; $x(8)=$ tsat; $x(9)=h c ; x(10)=x w$

$x(1)=$ tli; $x(2)=$ thi; $x(3)=$ tfhi; $x(4)=$ tfli

$x(5)=$ pli; $x(6)=$ phi

$x(9)=10000 ; x(10)=0.2 @-3$

!lteration tolerances are defined

$e(1)=0.05 ; e(2)=0.05 ; \theta(3)=0.05 ; e(4)=0.05$

$\theta(5)=1000 ; e(6)=1000 ; e(7)=0.05 ; \theta(8)=0.05$;

$\theta(9)=100 ; \theta(10)=0.01 @-3$

!

count $=0$

old $=-k$ theta $/ 2$

$\mathrm{kmax}=\mathbf{5 0 0}$

!

!

$u=1$

$b=1$

dmass $=0$ 


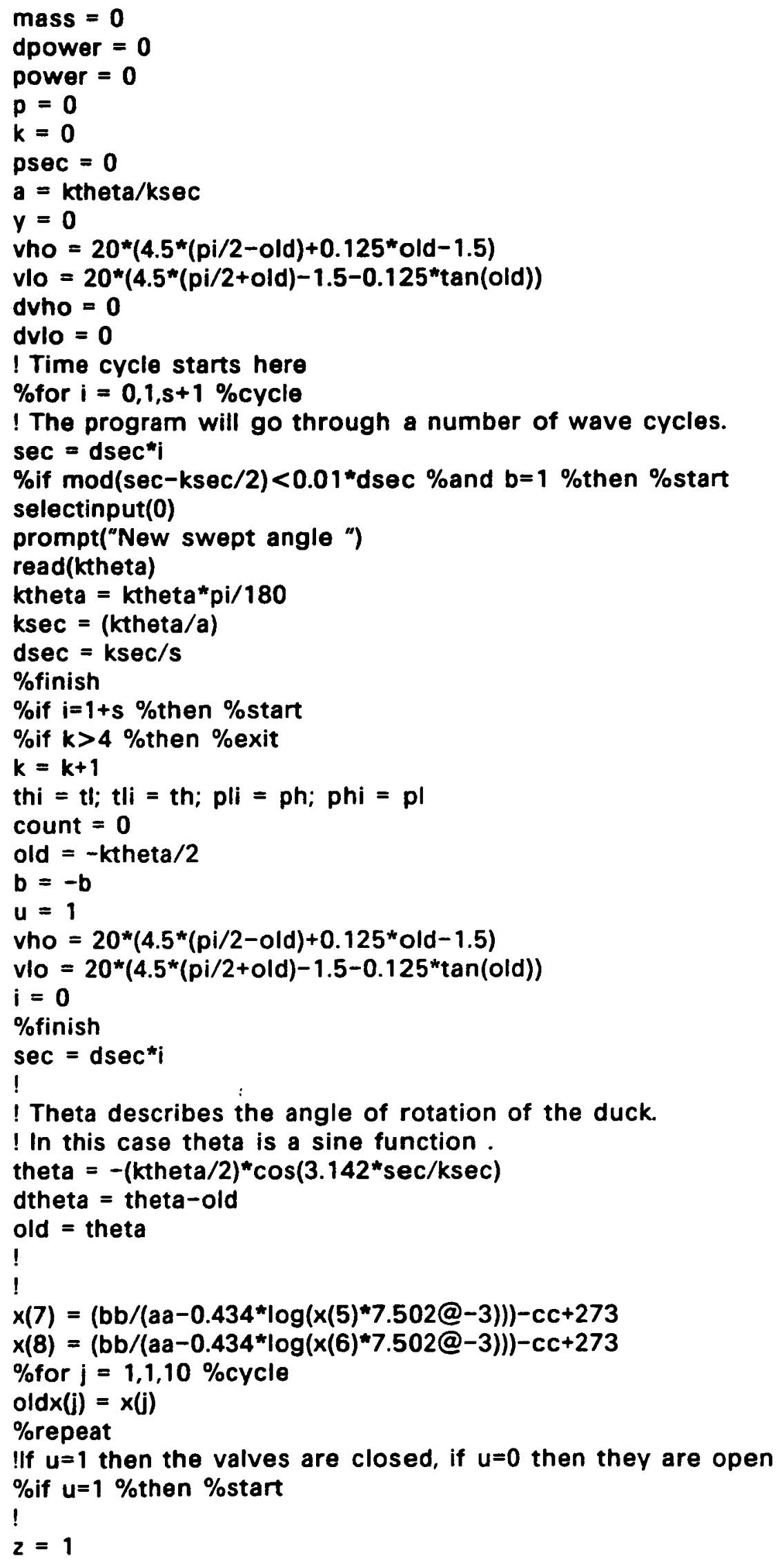




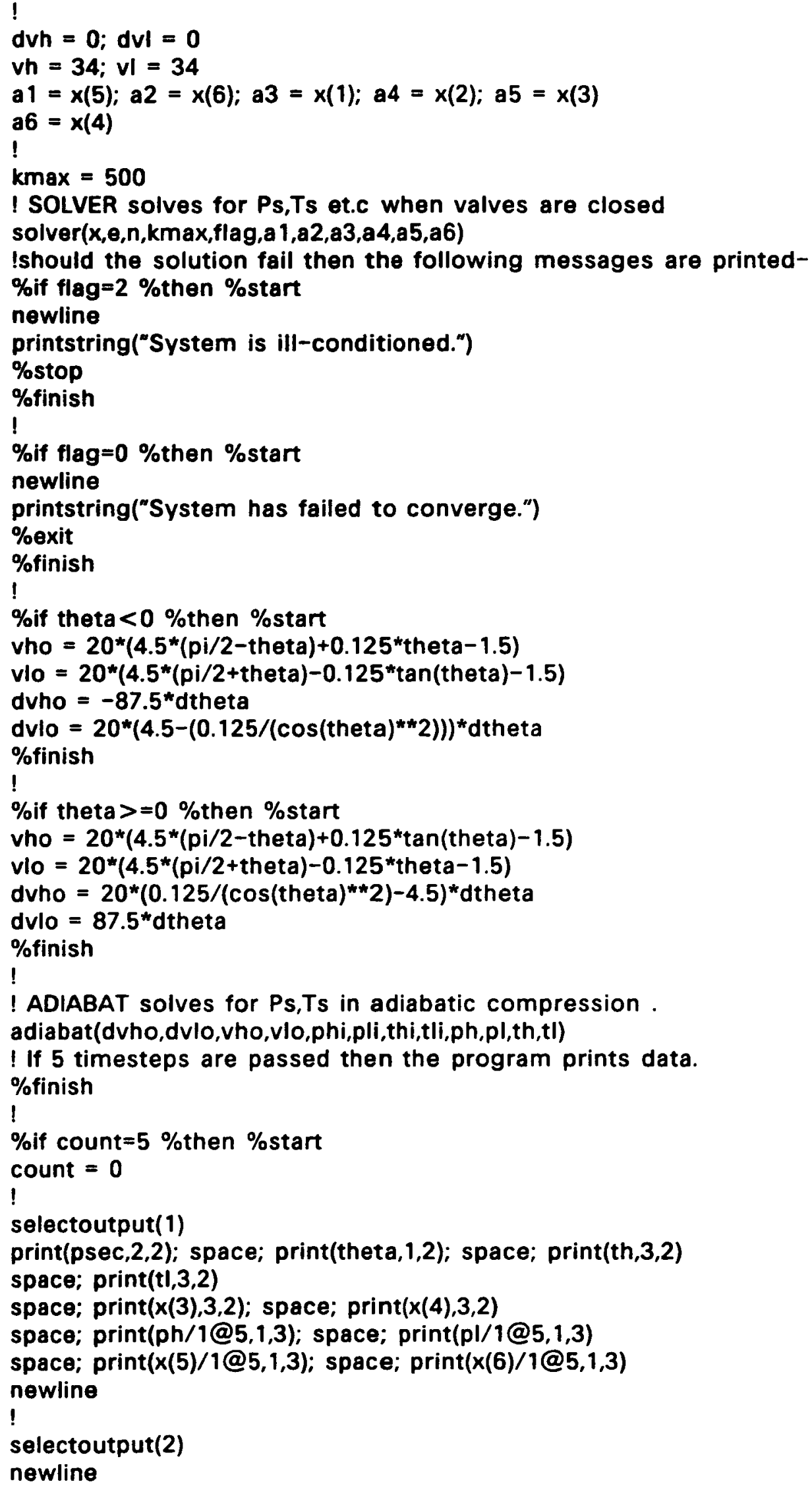


$\operatorname{print}(x(9), 4,2)$; space; print $(x(10) * 1000,1,3)$

!

selectoutput(3)

newline

print(psec, 1,2); space; print(b*(ph-pl)/1@5,1,3)

!

selectoutput(4)

newline

\%finish

! Test to see if the valves are open

I (with a 0.01 bar pressure drop).

\%if $x(5)+0.01 @ 5<p h \%$ and $x(6)>p \mid+0.01 @ 5 \%$ then $u=0$

! Update the $x$ values according to previous changes .

\%for $j=1,1,10 \%$ cycle

delta(j) $=x(j)-$ old $x(j)$

$x(j)=x(j)+0.75^{\star}$ delta $(j)$

$\%$ repeat

! Reset initial values

phi $=$ ph; pli $=$ pl; thi $=$ th; $t$ li $=t l$

tfhi $=x(3)$; tfli $=x(4)$

I Check if the valves are open.

$\%$ if $u=0 \%$ then \%start

!

$!$

\%if theta $<0 \%$ then \%start

$d v h=-87.5^{*}$ dtheta

$d v l=20^{*}\left(4.5-\left(0.125 /\left(\cos (\text { theta })^{* \star} 2\right)\right)\right)^{*}$ dtheta

\%finish

!

\%if theta $>=0 \%$ then \%start

$d v h=20 *(0.125 /(\cos (\text { theta }) * * 2)-4.5)^{*}$ dtheta

$d v I=87.5^{*}$ dtheta

\%finish

$!$

$\mathrm{kmax}=200$

! If the valves are open for the first time, then a

! different type of guess is needed.

\%if $z=1 \%$ then \%start

vh $=$ vho +34

$v l=v l o+34$

$\mathrm{kmax}=300$

thi $=0.5^{\star}$ thi $+0.5^{*} \times(1)$

tli $=0.5^{*} t\left(i+0.5^{*} \times(2)\right.$

phi $=0.5^{*}$ phi $+0.5^{*} \times(5)$

pli $=0.5^{\star}$ pli $+0.5^{\star} \times(6)$

$x(1)=$ thi

$x(2)=$ tli

$x(5)=p h i$

$x(6)=$ pli

$x(7)=\left(b b /\left(a a-0.434^{*} \log \left(p h i^{*} 7.502 @-3\right)\right)\right)-c c+273$

$x(8)=(b b /(a a-0.434 * \log (p / i * 7.502 @-3)))-c c+273$

$x(9)=9000$

$x(10)=0.2 @-3$

\%finish 
$z=0$

! Solve for HE behaviour with open valves.

solver(x,e,n,kmax,flag,phi,pli,thi,tli,tfhi,tfli)

! If equation fails the following messages will be printed !

\%if flag=2 \%then \%start

newline

printstring("System is ill conditioned.")

$\%$ stop

\%finish

$\%$ if flag=0 \%then \%start

newline

printstring("System has failed to converge.")

\%exit

$\%$ finish

! Update initial values

$p h=x(5) ; p l=x(6) ;$ th $=x(1) ; t I=x(2)$

tfhi $=x(3) ; t f l i=x(4)$

\%finish

$\%$ if theta $<0 \%$ then \%start

vh $=20 *(4.5 *($ pi $/ 2-$ theta $)+0.125 *$ theta -1.5$)+34$

$v I=20^{*}\left(4.5^{*}(\mathrm{pi} / 2+\right.$ theta $)-0.125^{\star} \tan ($ theta $\left.)-1.5\right)+34$

\%finish

!

$\%$ if theta $>=0 \%$ then \%start

$v h=20^{*}\left(4.5^{*}(\mathrm{pi} / 2-\right.$ theta $)+0.125^{*} \tan ($ theta $\left.)-1.5\right)+34$

$v I=20^{*}\left(4.5^{*}(\mathrm{pi} / 2+\right.$ theta $)-0.125^{*}$ theta -1.5$)+34$

$\%$ finish

$!$

count $=$ count +1

psec $=$ psec $+d s e c$

$p=p+1$

dmass $=x(9)^{*} \operatorname{area}^{*}(x(7)-x(3)) / 2258 @ 3$

dmass $=\mathrm{dmass}^{\star \star 2} 2$

mass $=$ mass $+d$ mass

!

dpower $=b *(p h-p l) * 90 * d$ theta/dsec

dpower $=$ dpower $* 2$

power $=$ power+dpower

!

\%repeat

! Take next timestep

!

mass $=\operatorname{mass} / p$

mass $=$ mass ${ }^{\star \star} 0.5$

!

power $=$ power $/ p$

power $=$ power ${ }^{\star \star} 0.5$

!

selectoutput(6)

printstring("Area for heat exchange ="); print(area,4,1)

newline

printstring("Temp. approach $=")$; print(tclose, 3,2) 
newline

printstring("RMS Fresh Water Flowrate $=")$; print $($ mass,3,3)

printstring( $\mathrm{kg} / \mathrm{s}$ "); newline

printstring("RMS Power Consumption ="); print(power $/ 1000,3,3$ )

printstring("kW"); newline

printstring("RMS Specific Energy Usage ="); print(power/(mass"1000),3,3)

printstring(" $\mathrm{kJ} / \mathrm{kg}$ "); newline

printstring("Mean Wave Climate ="); print(power/20@3,2,2)

printstring("kW/m"); newline

height $=\left(\text { power } /\left(22 @ 3^{* k s e c}\right)\right)^{\star *} 0.5$

printstring("RMS Wave Height $=$ "); print(height,2,2)

printstring("m"); newline

closestream(6)

1

\%end \%of \%program

\section{N.Il. Second Model}

Listing of the Second Model Program

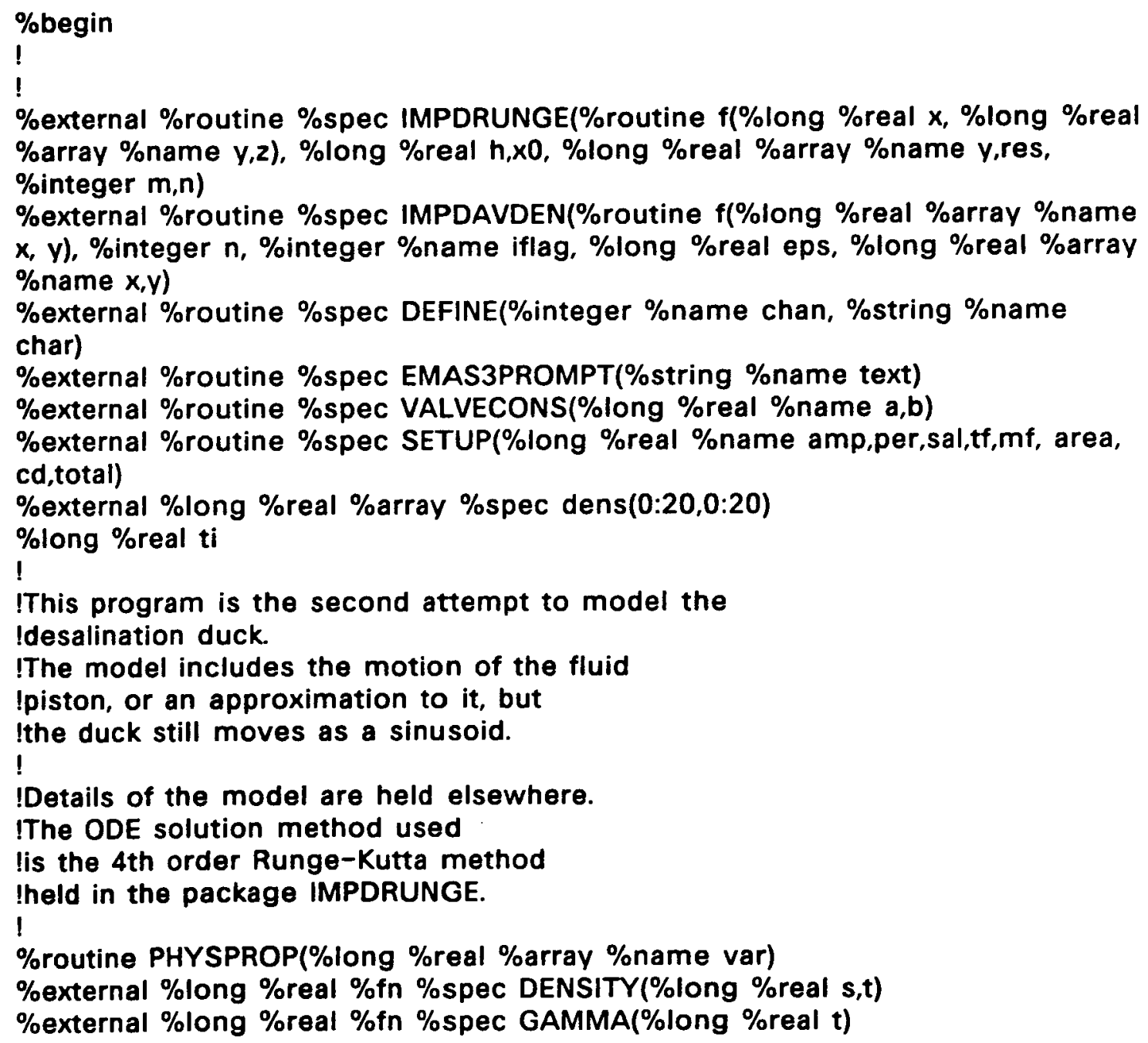


\%external \%long \%real \%fn \%spec HEATCAP(\%long \%real s,t)

\%external \%long \%real \%fn \%spec LATHEAT(\%long \%real s,t)

\%external \%long \%real \%fn \%spec TCOND(\%long \%real s,t)

\%external \%long \%real \%fn \%spec VOLUCV(\%long \%real t)

\%external \%long \%real \%fn \%spec VISC(\%long \%real s,t)

\%external \%long \%real \%fn \%spec SATV(\%long \%real s,vg)

!

\%long \%real vg1,vg2,tsat1,tsat2

!

$\operatorname{vg} 1=\operatorname{var}(39) / \operatorname{var}(8)$

$\operatorname{vg} 2=\operatorname{var}(40) / \operatorname{var}(9)$

tsat $1=\operatorname{SATV}(0, \mathrm{vg} 1)$

tsat2 = SATV(var(20),vg2)

IVAR() is a global array that holds all

Ithe process variables that need

Ito be passed from one routine to another.

IPHYSPROP assigns those that are to be

!physical properties.

$\operatorname{var}(25)=$ LATHEAT $(0$, tsat 1$)$

$\operatorname{var}(26)=$ LATHEAT(var(20),tsat2)

$\operatorname{var}(27)=\operatorname{VOLUCV}($ tsat 1$)$

$\operatorname{var}(28)=\operatorname{VOLUCV}($ tsat2)

$\operatorname{var}(29)=\operatorname{HEATCAP}(0$, tsat 1$)$

$\operatorname{var}(30)=$ HEATCAP(var(20),tsat2)

$\operatorname{var}(31)=\operatorname{DENSITY}(0$, tsat 1$)$

$\operatorname{var}(32)=$ DENSITY $(\operatorname{var}(20)$, tsat2)

$\operatorname{var}(33)=$ GAMMA $(\operatorname{var}(3))$

$\operatorname{var}(34)=$ GAMMA(var(4))

$\operatorname{var}(35)=$ GAMMA $(\operatorname{var}(5))$

$\operatorname{var}(36)=$ GAMMA(var(6))

$\operatorname{var}(37)=\operatorname{VISC}(0$, tsat 1$)$

$\operatorname{var}(38)=\operatorname{VISC}(\operatorname{var}(20)$, tsat2)

$\operatorname{var}(43)=\operatorname{TCOND}(0$, tsat 1$)$

$\operatorname{var}(44)=T C O N D(\operatorname{var}(20)$, tsat 2$)$

!

$\%$ return

\%end

!

$!$

\%routine RECOVER(\%long \%real \%array \%name var)

\%external \%routine \%spec NDNEWTON(\%routine f(\%long \%real \%array \%name $x, y), \%$ long \%real \%array \%name x,e, \%integer $n$, \%integer \%name kmax, flag)

$\%$ long \%real \%array $x, e r r(1: 17)$

\%long \%real s

\%integer kmax,flag,i

!

\%routine BAL(\%long \%real \%array \%name $x, y$ )

$\%$ long \%real \%array del(1:5)

\%long \%real cp,u1,u2,a1,a2, Im 1,Im2, Im3,s

!

$s=\operatorname{var}(20) / 35$

$\operatorname{del}(1)=x(1)-x(6) ; \operatorname{del}(2)=x(2)-x(5)$

$\operatorname{del}(3)=x(3)-x(4)$

$\operatorname{del}(4)=x(7)-x(5) ; \operatorname{del}(5)=x(8)-x(4)$ 


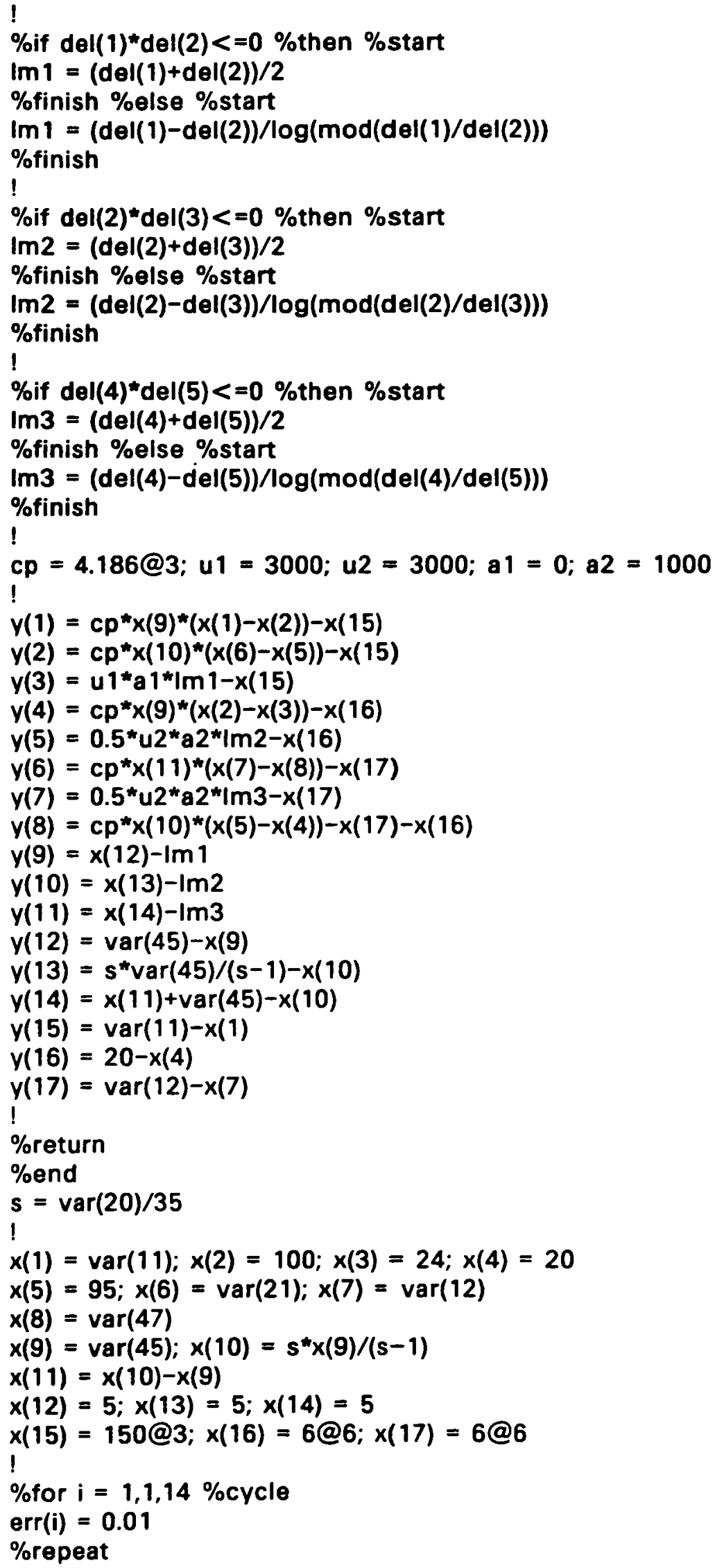




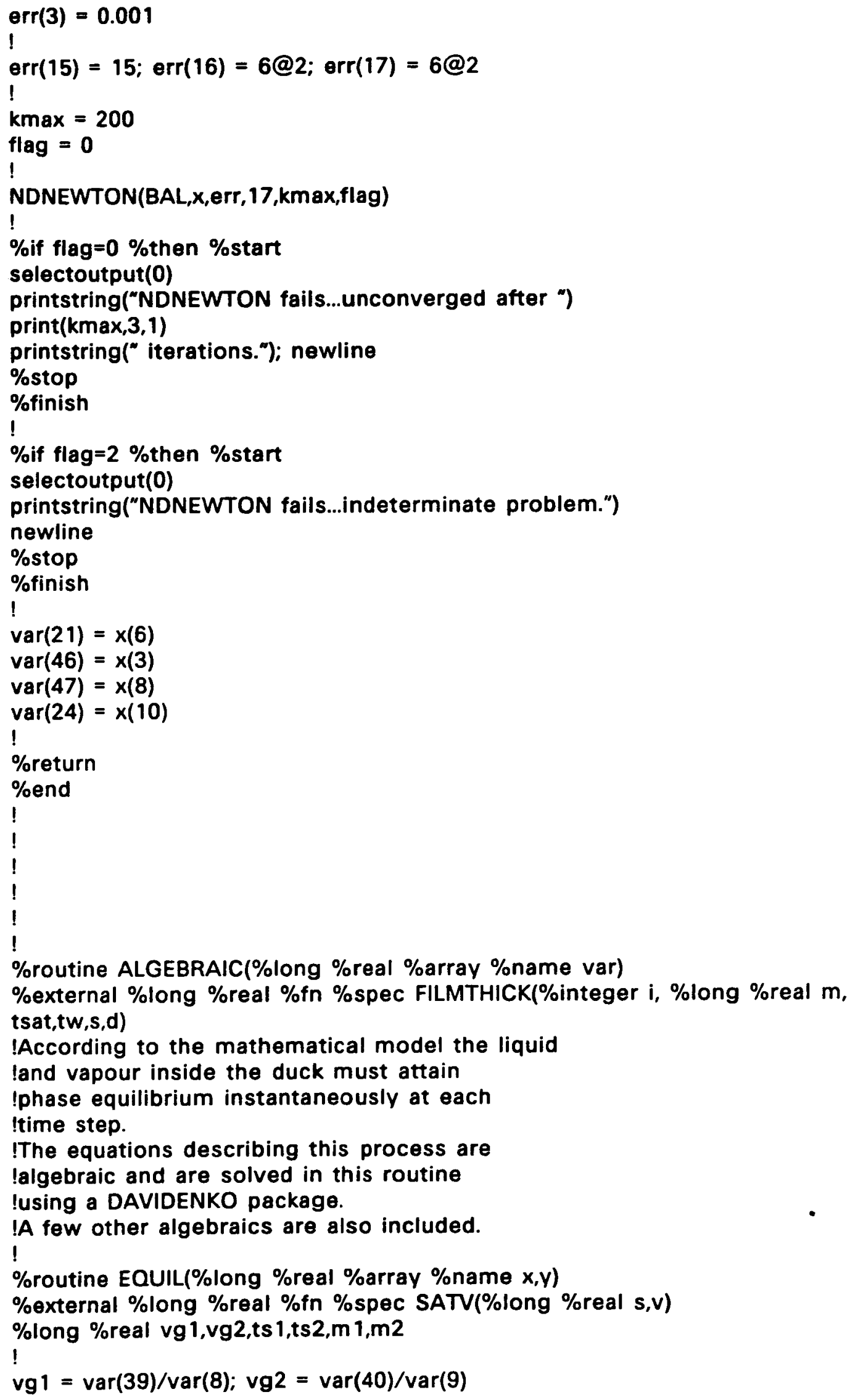


ts 1 = SATV $(0, v g 1) ;$ ts2 = SATV(var(20),vg2)

$m 1=\operatorname{var}(8)+\operatorname{var}(13) ; m 2=\operatorname{var}(9)+\operatorname{var}(14)$

$!$

IThe equations below describe equilibration at constant volume !mass and enthalpy

$y(1)=\operatorname{var}(27)^{*} \operatorname{var}(8)^{*}(\operatorname{var}(4)-t s 1)+$ $\operatorname{var}(29)^{*} \operatorname{var}(13)^{*}(\operatorname{var}(11)-\mathrm{ts} 1)$

$y(1)=((y(1)+\operatorname{var}(29) * m 1 *(\operatorname{ts} 1-x(2))) / \operatorname{var}(25))$ $+\operatorname{var}(8)-x(1)$

!

$y(2)=\operatorname{SATV}(0, \operatorname{var}(39) / x(1))-x(2)$

!

$y(3)=\operatorname{var}(28)^{*} \operatorname{var}(9)^{*}(\operatorname{var}(5)-t s 2)+$

$\operatorname{var}(30)^{*} \operatorname{var}(14)^{*}(\operatorname{var}(12)-t s 2)$

$y(3)=((y(3)+\operatorname{var}(30) * m 2 *(t s 2-x(4))) / \operatorname{var}(26))$ $+\operatorname{var}(9)-x(3)$

$!$

$y(4)=\operatorname{SATV}(\operatorname{var}(20), \operatorname{var}(40) / x(3))-x(4)$

!

!

\%end

!

$!$

$!$

\%long \%real \%array $x(1: 12)$,res(1:12)

\%long \%real tw,alpha

\%integer $\mathrm{i}, \mathrm{j}$

!

1

$x(1)=\operatorname{var}(8) ; x(2)=\operatorname{var}(4)$

$x(3)=\operatorname{var}(9) ; x(4)=\operatorname{var}(5)$

!The above equations are solved in IMPDAVDEN.

IMPDAVDEN(EQUIL, $4, i, 0.05, x$, res)

!Error trapping....

\%if $i=1 \%$ then $\%$ start

selectoutput $(0)$

printstring("DAVDEN fails"); newline

\%for $j=1,1,12 \%$ cycle

$\operatorname{print}(\operatorname{res}(j), 1,3)$

\%repeat

newline

closestream(0)

\%finish

!Reset....

$\operatorname{var}(4)=x(2) ; \operatorname{var}(11)=x(2) ; \operatorname{var}(8)=x(1)$

$\operatorname{var}(5)=x(4) ; \operatorname{var}(12)=x(4) ; \operatorname{var}(9)=x(3)$

!

$\mathrm{tw}=0.5^{*}(\operatorname{var}(11)+\operatorname{var}(12))$

!Liquid film thicknesses are

!calculated from correlations.

!This may be inaccurate due to the

!steady-state assumptions made

lin the derivation of such correlations.

$\operatorname{var}(13)=\operatorname{FILMTHICK}(1,0, \operatorname{var}(4), \mathrm{tw}, 0,2.5)^{*} \operatorname{var}(22)^{*} \operatorname{var}(31)$ 


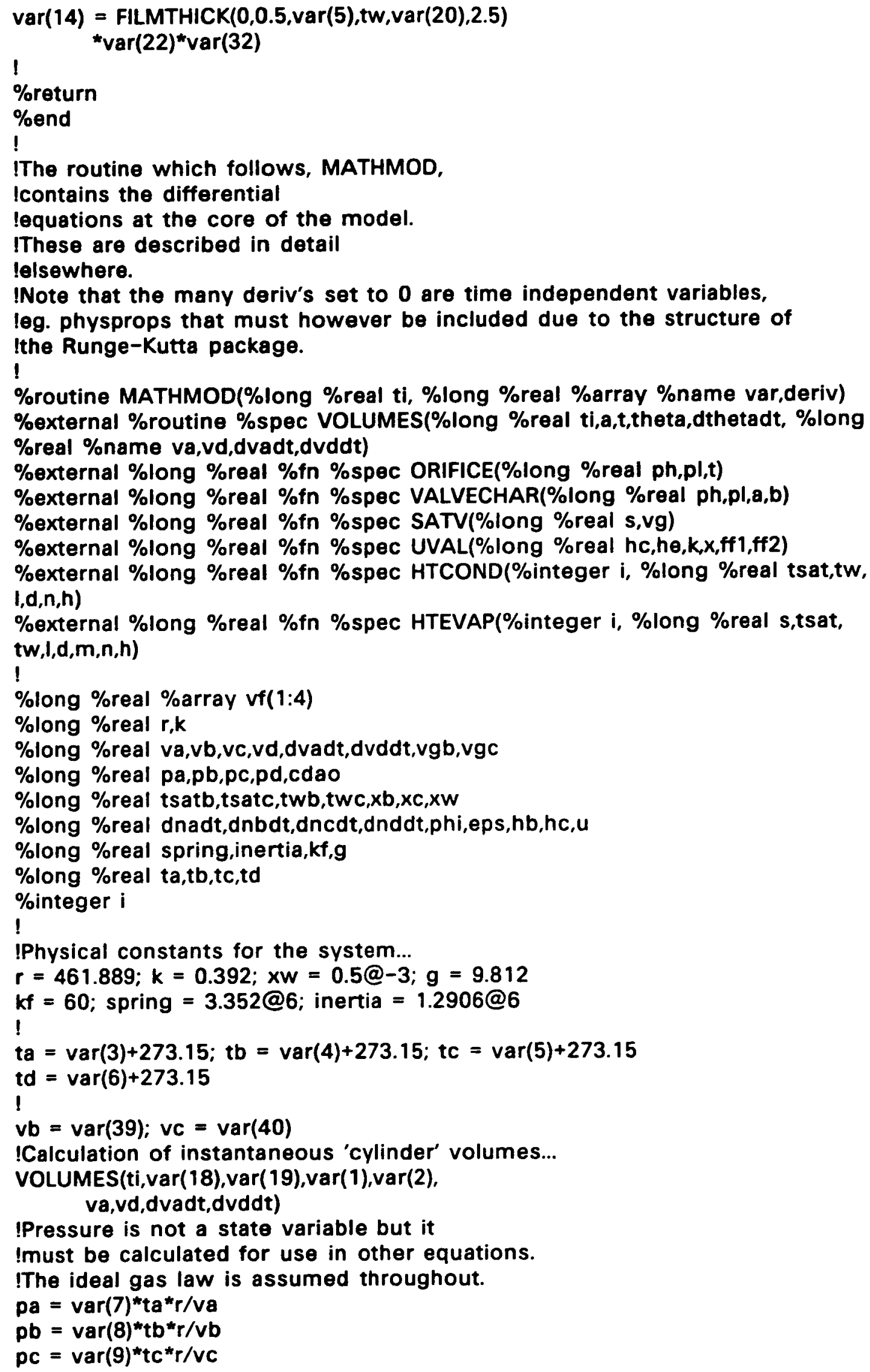




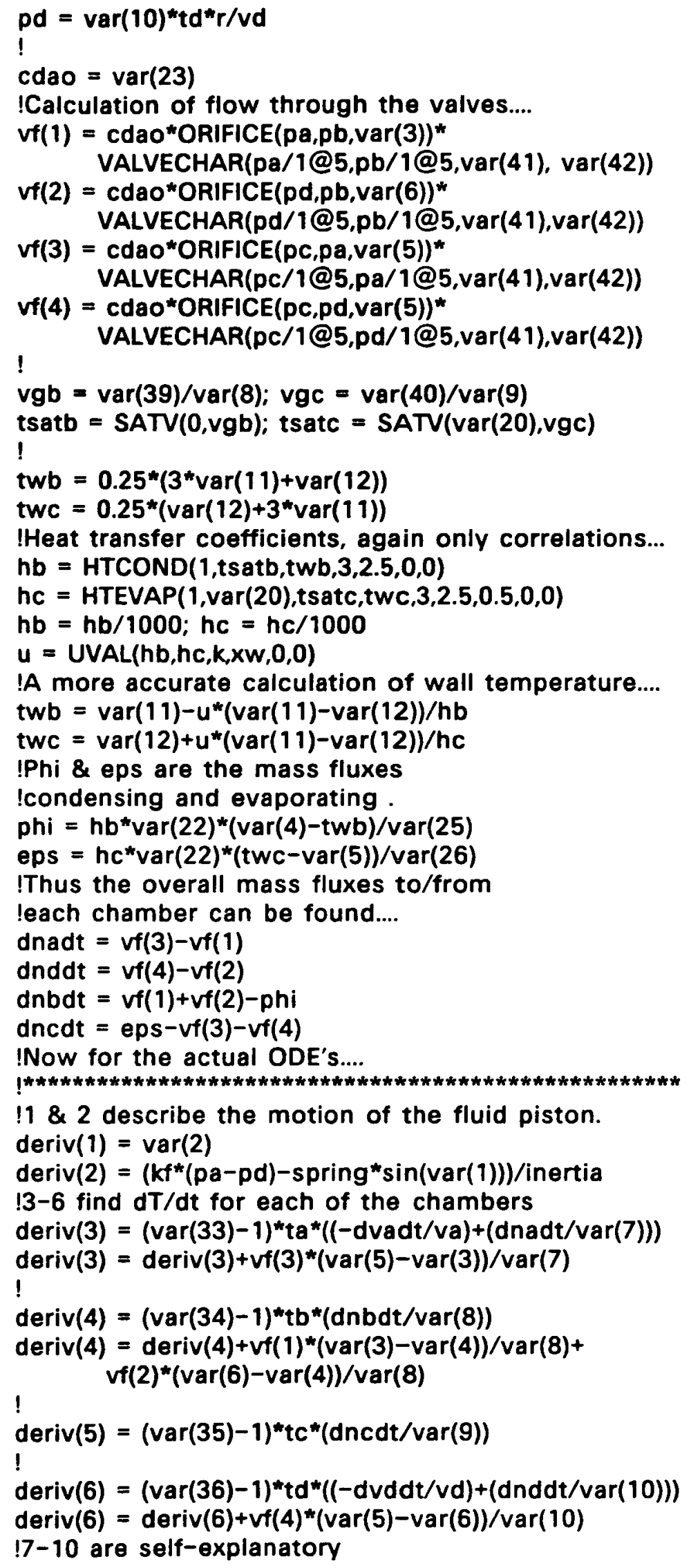




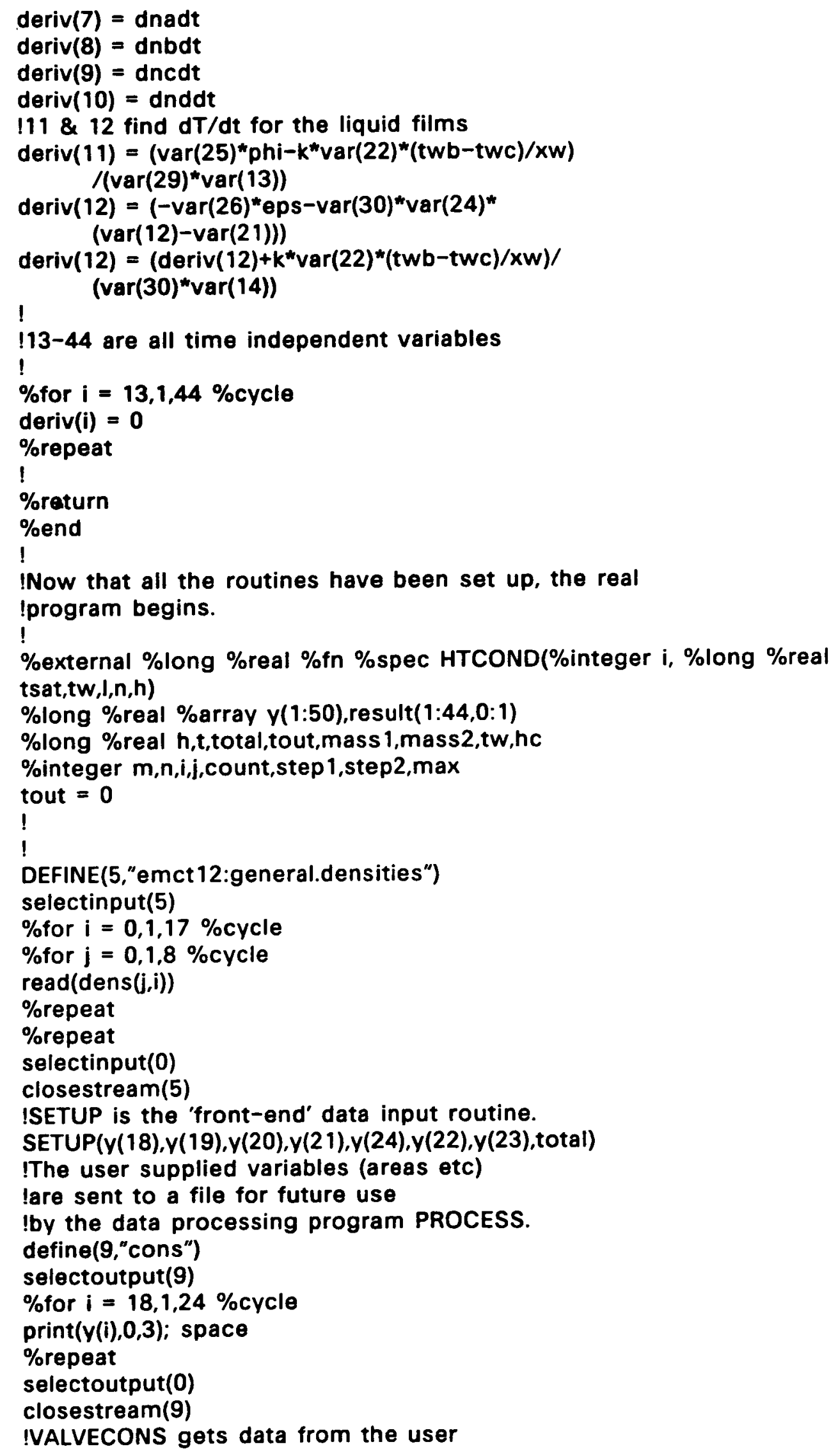




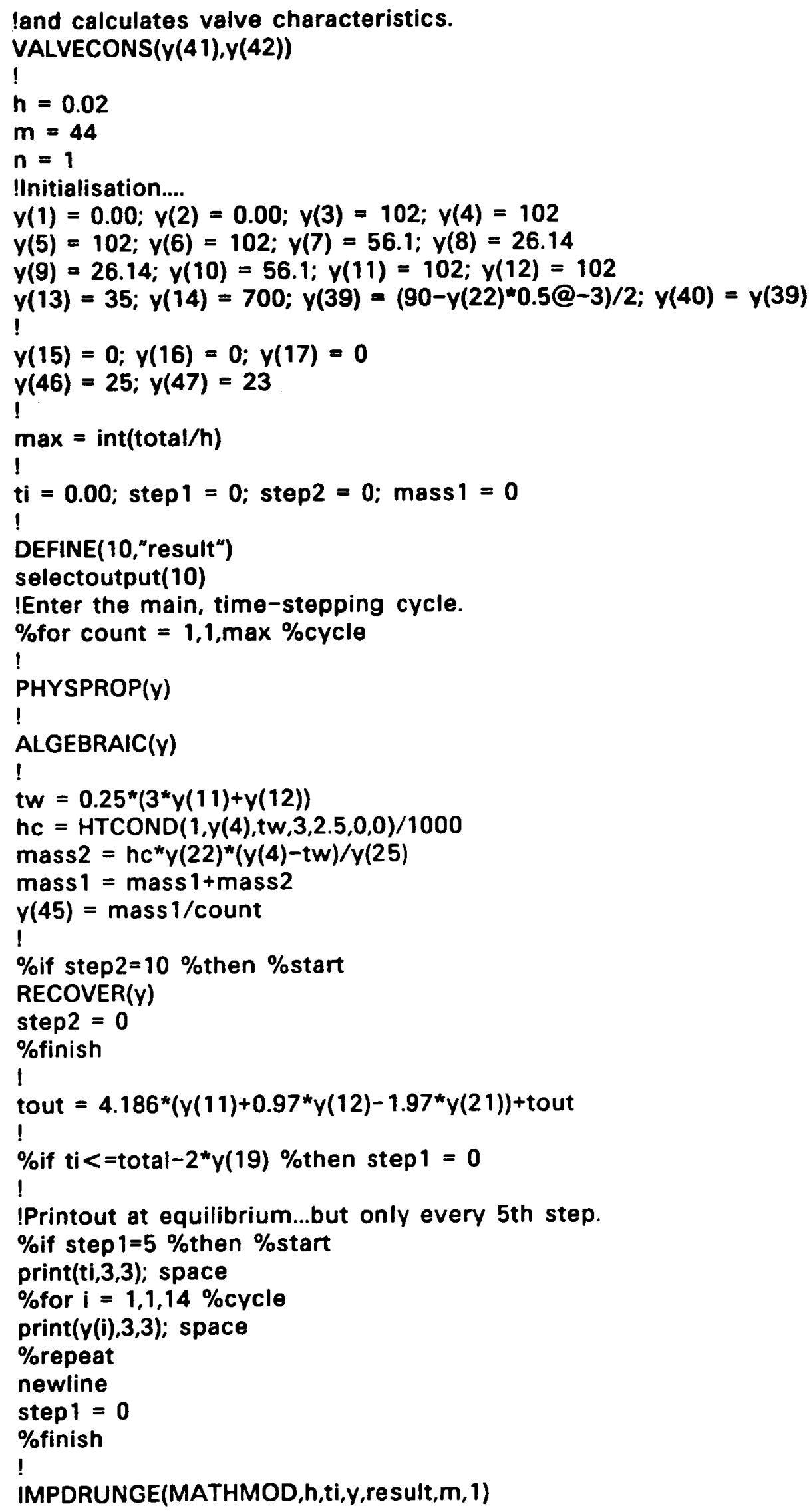


$!$

\%for $j=1,1,44 \%$ cycle

$y(j)=\operatorname{result}(j, 1)$

\%repeat

IUpdate....

step $1=$ step $1+1$

step2 $=$ step $2+1$

$t i=\mathbf{t i}+\boldsymbol{h}$

$!$

\%repeat

$!$

print $(101010.10,6,2)$

newline

selectoutput $(0)$

closestream(10)

newlines(3)

printstring("Finished")

!That's all Folks!

\%end \%of \%program 
!All these routines/functions may called as lexternals in any program.

\%external \%routine \%spec emas3(\%string \%name command,params, integer $\%$ name flag)

\%external \%routine \%spec emas3itos(\%integer \%name i, \%string \%name s) $\%$ externallongrealarray dens(0:20,0:20)

!

\%external \%routine define(\%integer \%name chan, \%string \%name file)

$\%$ string (8) number

\%integer flag

emas3itos(chan,number)

emas3("define","sto".number.",.file,flag)

\%return

!

\%end

!

\%external \%long \%real \%function density(\%long \%real s,t)

!Liquid density calculation for brines

land pure water.

\%externalroutinespec emas 3 (\%stringname comm,parms \%integer flag)

\%integer aa,b,c,d,i,j,flag

\%long \%real $x, y, p, q, d x, d y, r$

!

\%if $t>180 \%$ then \%result $=1000$

$\%$ if $t<20 \%$ then \%result $=1000$

\%if $s<=0 \%$ then \%start

$r=1.00386 @ 3-\left(2.23115 @-1^{*} t\right)-\left(2.40169 @-3^{*} t^{* *} 2\right)$

$\%$ result $=r$

\%finish \%else \%start

!

$!$

$d x=\operatorname{dens}(2,0)$-dens $(1,0)$

$d y=\operatorname{dens}(0,2)$-dens $(0,1)$

$x=(s-\operatorname{dens}(1,0)) / d x+1$

$y=(t-\operatorname{dens}(0,1)) / d y+1$

!

$a a=\operatorname{intpt}(x)$

$b=\operatorname{intpt}(x+1)$

$c=\operatorname{intpt}(y)$

$d=\operatorname{intpt}(y+1)$

!

$p=(\text { dens }(a a, d)-\text { dens }(a a, c))^{\star}(y-c)+$ dens $(a a, c)$

$q=(\operatorname{dens}(b, d)-\operatorname{dens}(b, c))^{*}(y-c)+\operatorname{dens}(b, c)$

$r=(q-p)^{*}(x-a a)+p$

\%finish

\%result $=r$

\%end

$!$

\%external \%long \%real \%function VISC(\%long \%real $s, t)$

IViscosity correlation for brines and 


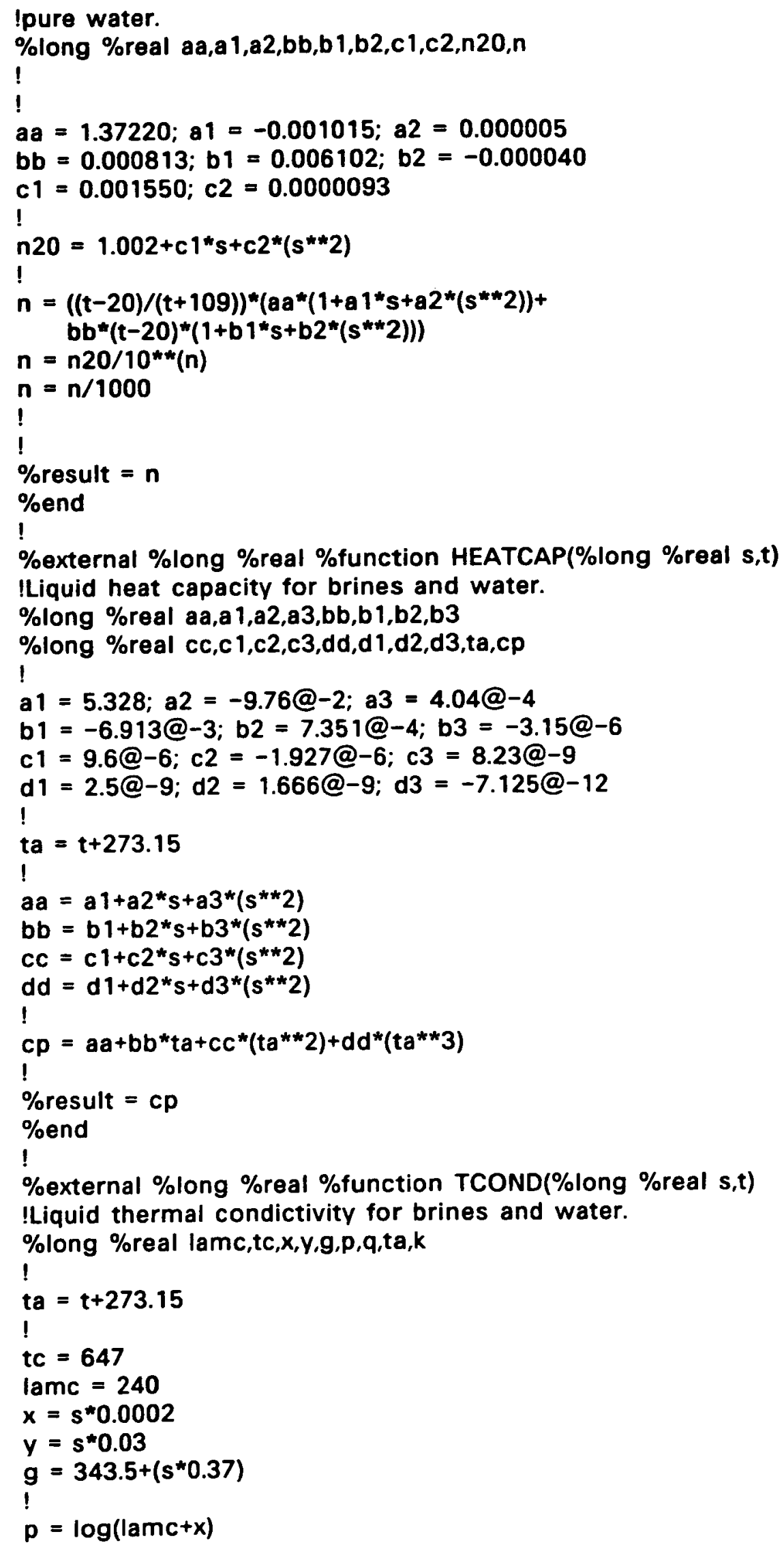




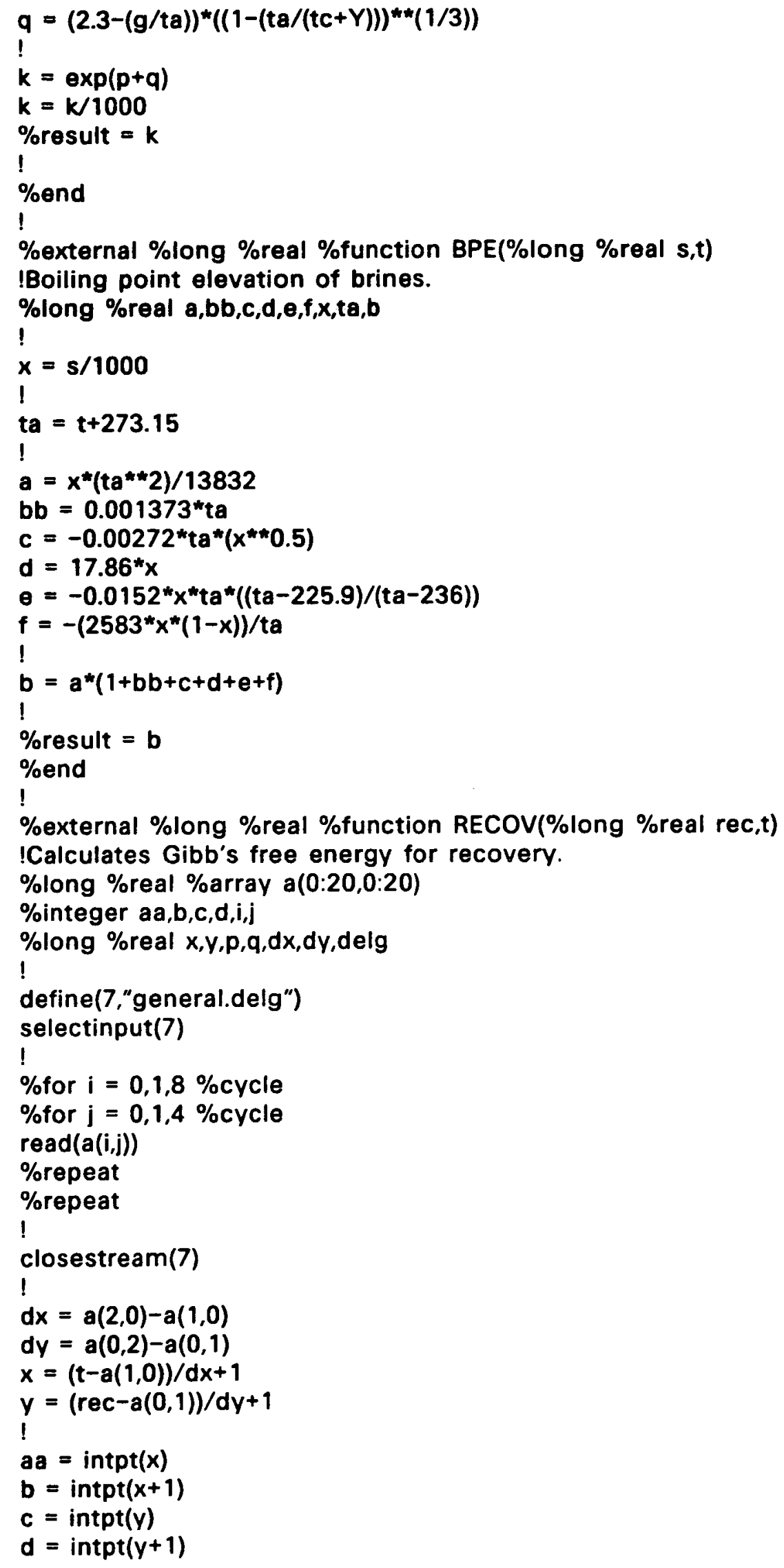


!

$p=(a(a a, d)-a(a a, c))^{*}(y-c)+a(a a, c)$

$q=(a(b, d)-a(b, c))^{\star}(y-c)+a(b, c)$

delg $=(q-p)^{*}(x-a a)+p$

I

\%result $=$ delg

\%end

!

\%external \%long \%real \%function BARCP(\%long \%real t)

IHeat capacity of vapour at constant pressure.

\%long \%real a,b,c,d

$a=-1.28115 ; b=9.76656 @-2 ; c=-9.90726 @-4 ; d=3.44861 @-6$

\%long \%real cp

!

$c p=a+b * t+c^{*} t * 2+d^{*} t * 3$

!

\%result $=\mathbf{c p}$

\%end

!

\%external \%long \%real \%function VOLUCV(\%long \%real t)

!Heat capacity of vapour at constant volume.

\%external \%long \%real \%fn \%spec barcp(\%long \%real t)

\%long \%real $r$

$r=0.462$

\%long \%real cv,cp

$!$

$c p=\operatorname{barcp}(t)$

$c v=c p-r$

$!$

$\%$ result $=\mathrm{cv}$

$\%$ end

!

\%external \%long \%real \%function GAMMA(\%long \%real t)

IAdiabatic compression index.

\%long \%real $c p, c v, g a m m a$

!

$c p=\operatorname{barcp}(t)$

$c v=\operatorname{volucv}(t)$

gamma $=\mathrm{cp} / \mathrm{cv}$

!

$\%$ result $=$ gamma

\%end

$!$

\%external \%routine SATEQ(\%long \%real \%array \%name $x, y)$

\%external \%long \%real \%fn \%spec BPE(\%long \%real s,t)

!

\%long \%real a,b,c

$a=7.9186968 ; b=1636.909 ; c=224.92$

1

$y(1)=\left(b /\left(a-0.434294^{*} \log \left(x(3)^{*} 750.06\right)\right)\right)-c+x(2)-x(1)$

$y(2)=\operatorname{BPE}(x(4), x(1))-x(2)$

!

$\%$ return

\%end 


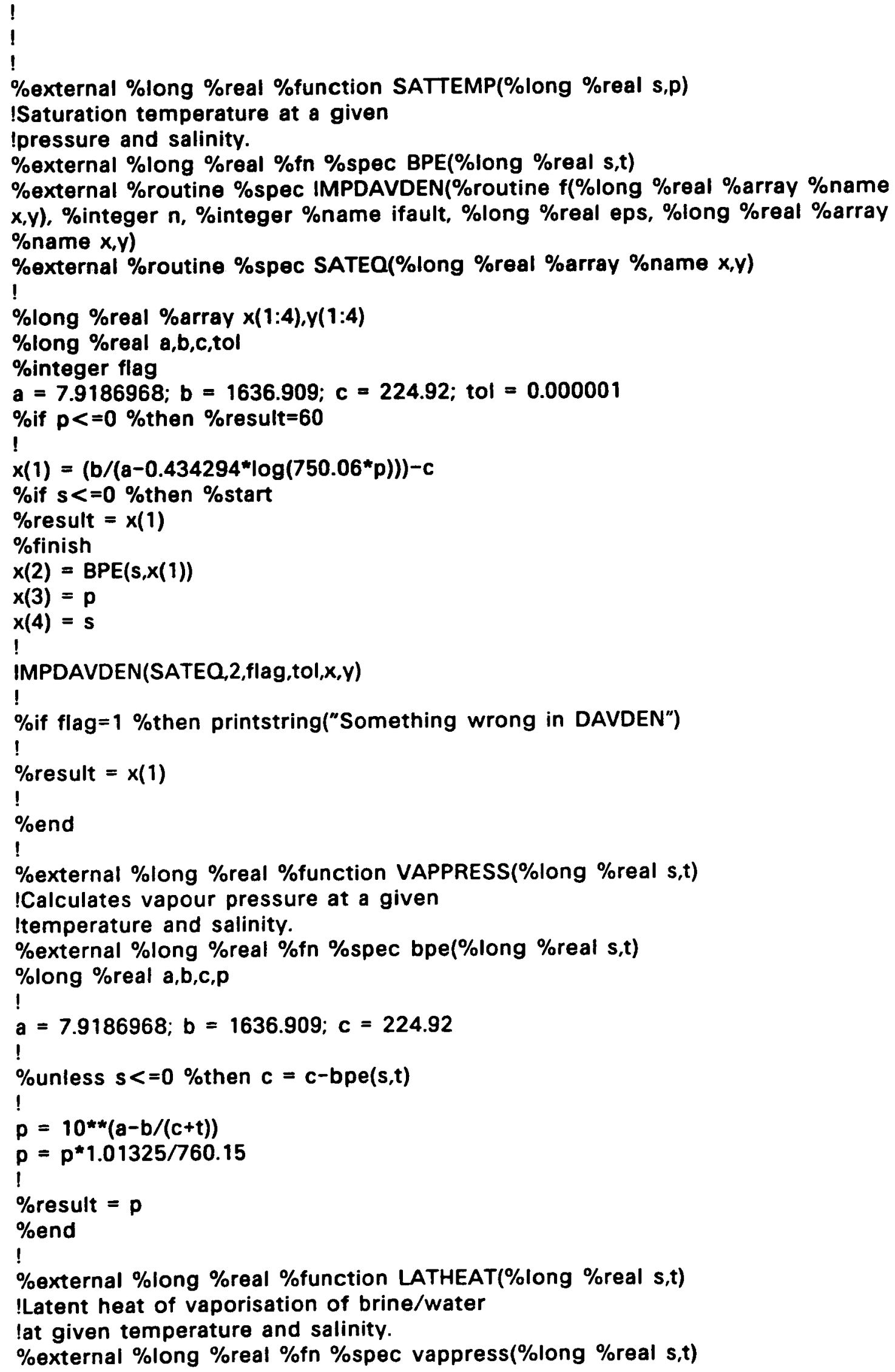




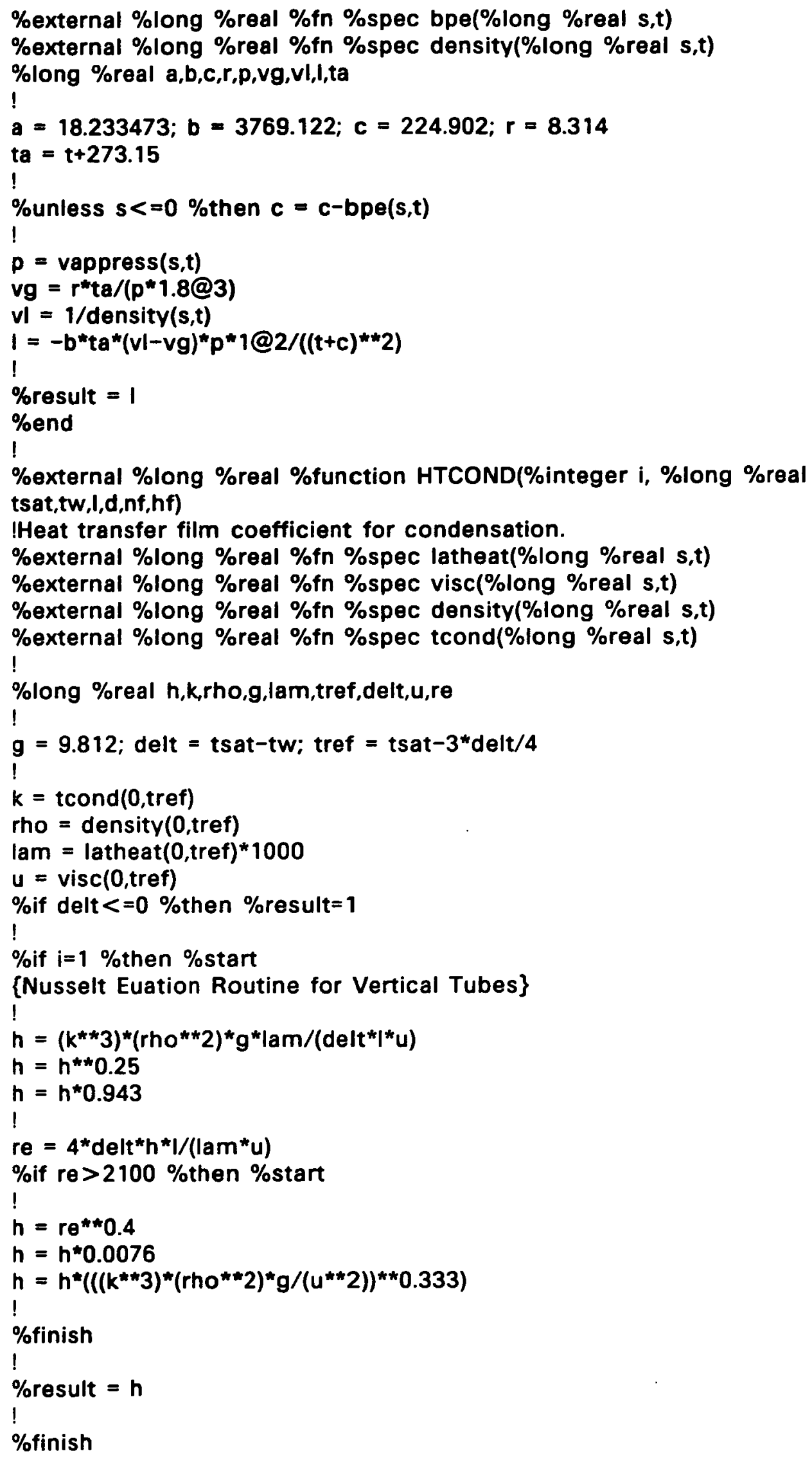




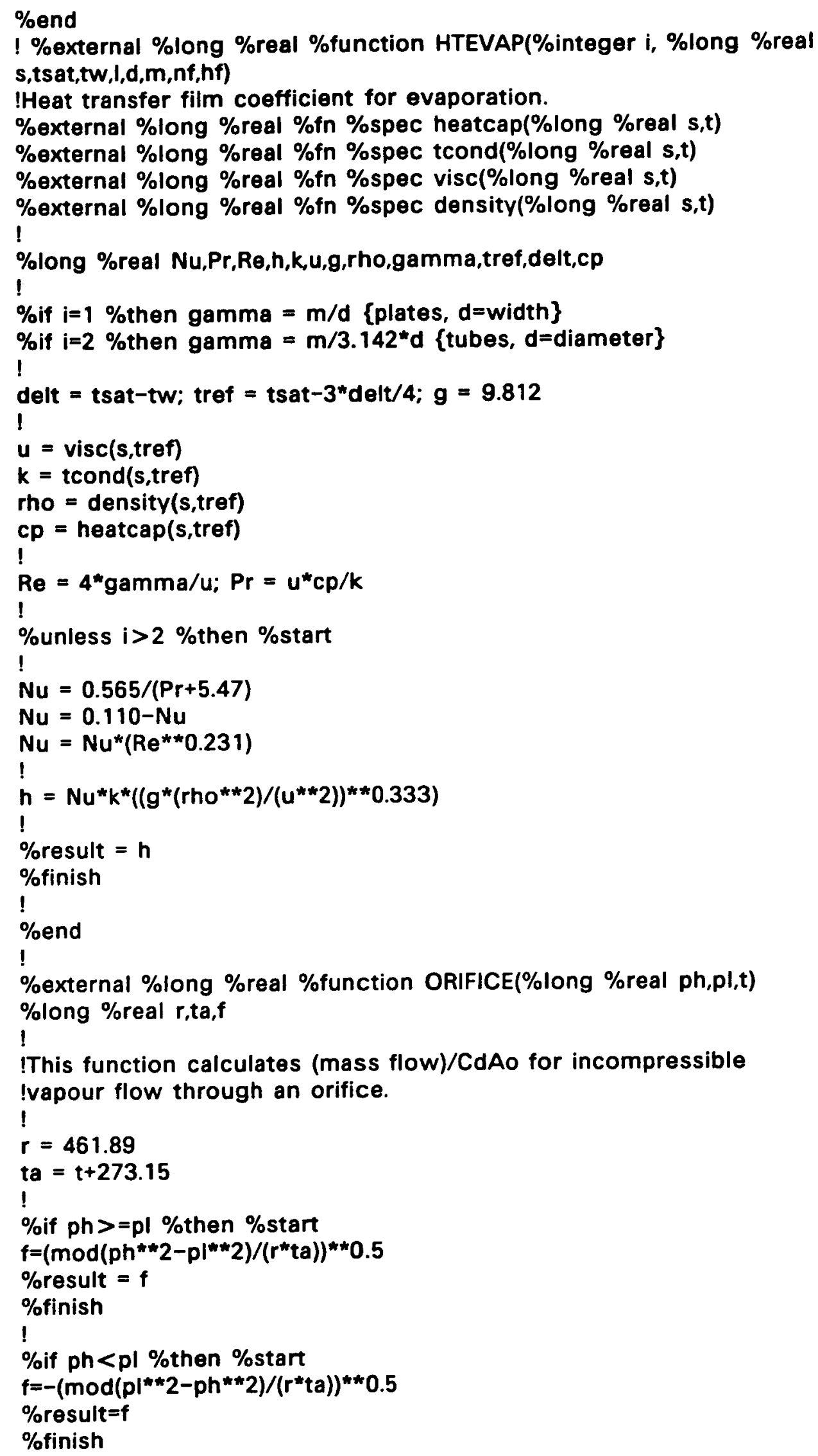




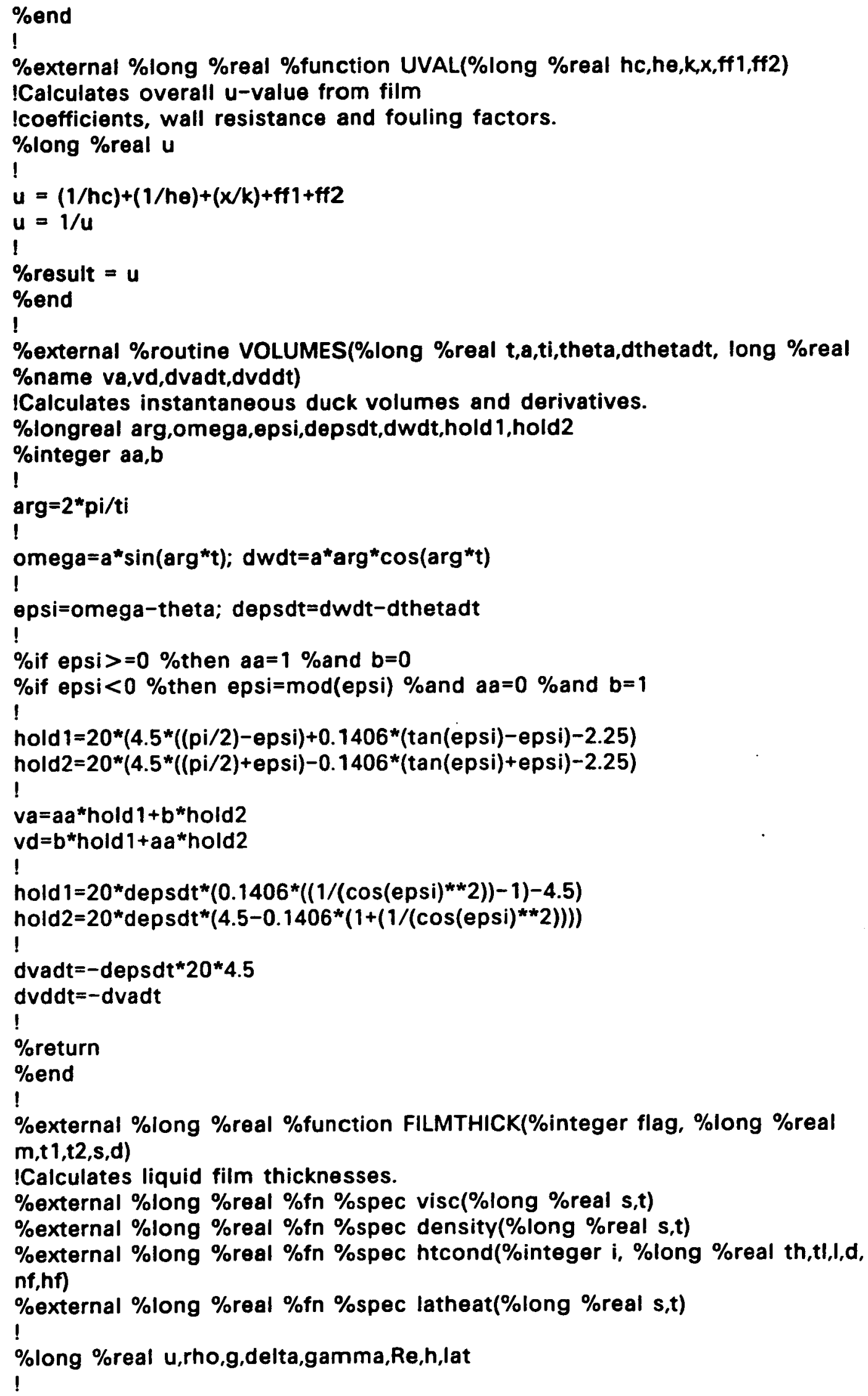




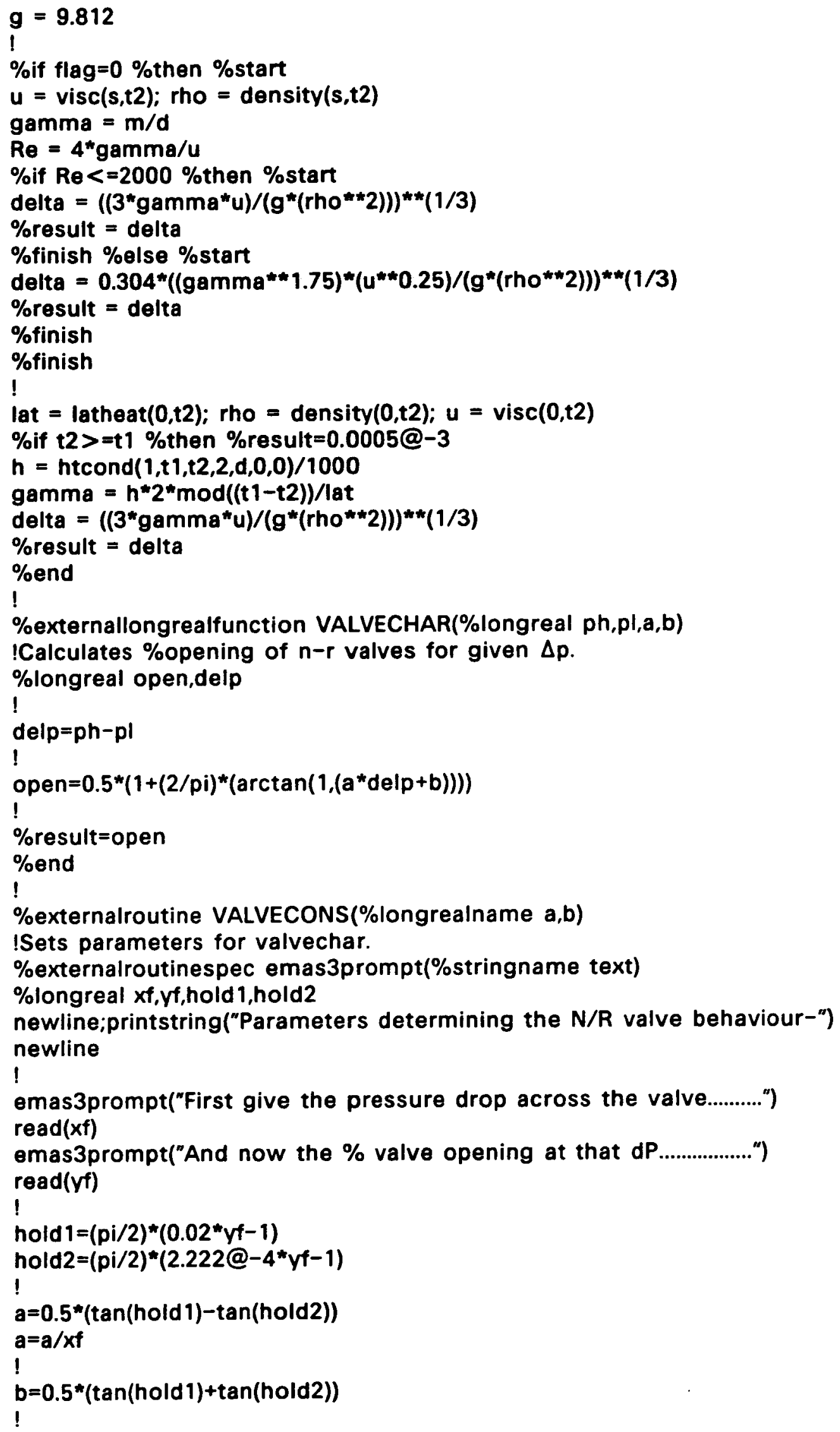




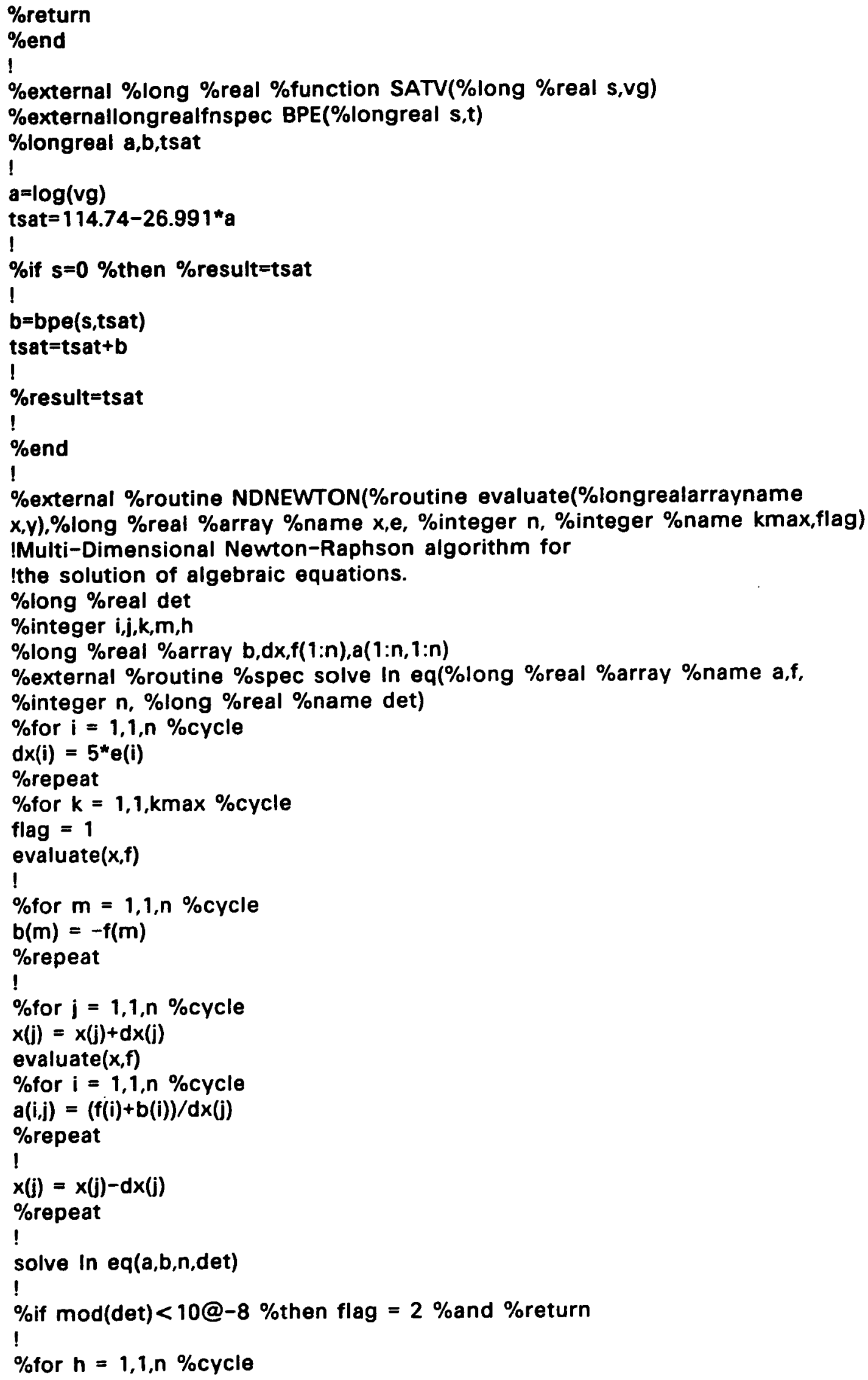


$x(h)=x(h)+b(h)$

$\%$ if $\bmod (b(h))>\theta(h) \%$ then flag $=0$

\%repeat

!

\%if flag=1 \%then $k \max =k$ \%and \%return

\%repeat

\%return

\%end

\%end \%of \%file 


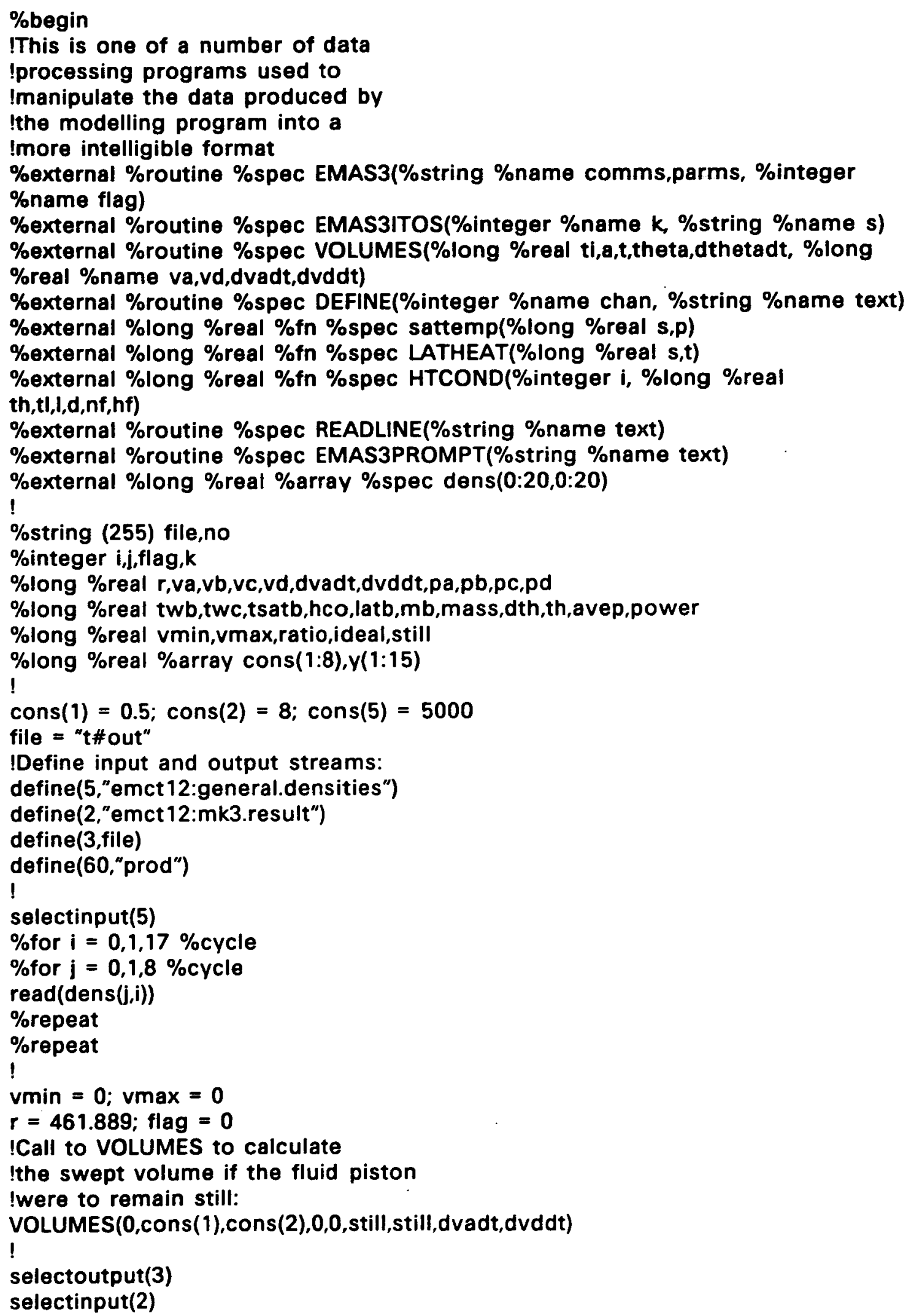




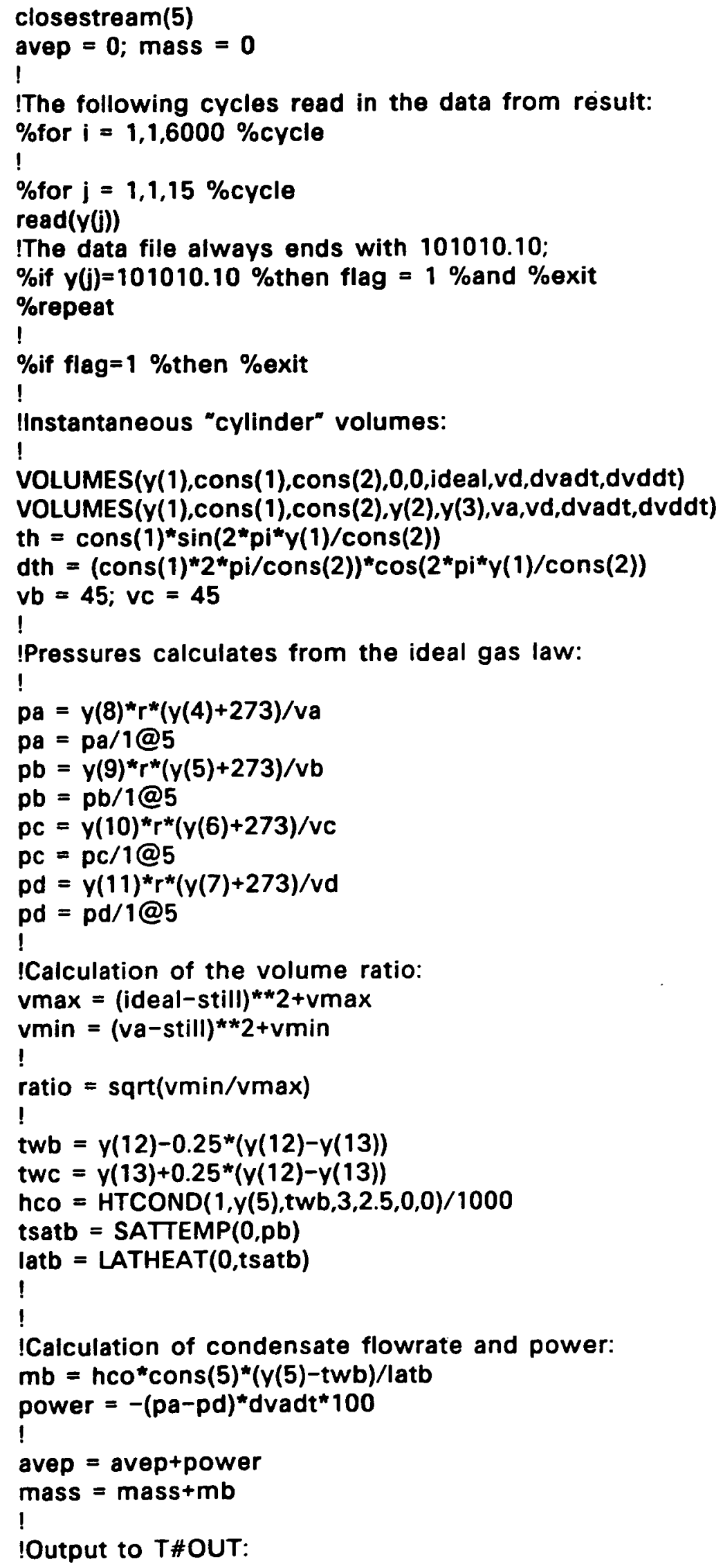


!

$\operatorname{print}(y(1), 2,2) ; \operatorname{print}(p a, 3,3) ; \operatorname{print}(p b, 3,3) ; \operatorname{print}(p c, 3,3)$

print(pa-pd,3,3); print(va,3,3)

newline

!

$!$

\%repeat

mass $=$ mass $/ \mathbf{i}$

avep $=$ avep $/ \mathrm{i}$

selectinput(0)

selectoutput(0)

closestream(3)

closestream(2)

selectoutput(60)

!Output to PROD:

printstring("The average mass flowrate of condensate is ")

print(mass,2,2)

printstring(" kg/s.")

!

newline

printstring("Mean Power input ="); print(avep,3,3); printstring("kW")

newline

printstring("Mean SEC ="); print(avep/mass,3,3); printstring \%c

("kJ/kg")

newline

printstring("Ratio of swept volume to ideal volume = "); print(ratio,3,3)

selectoutput(0)

closestream(60)

\%end \%of \%program 


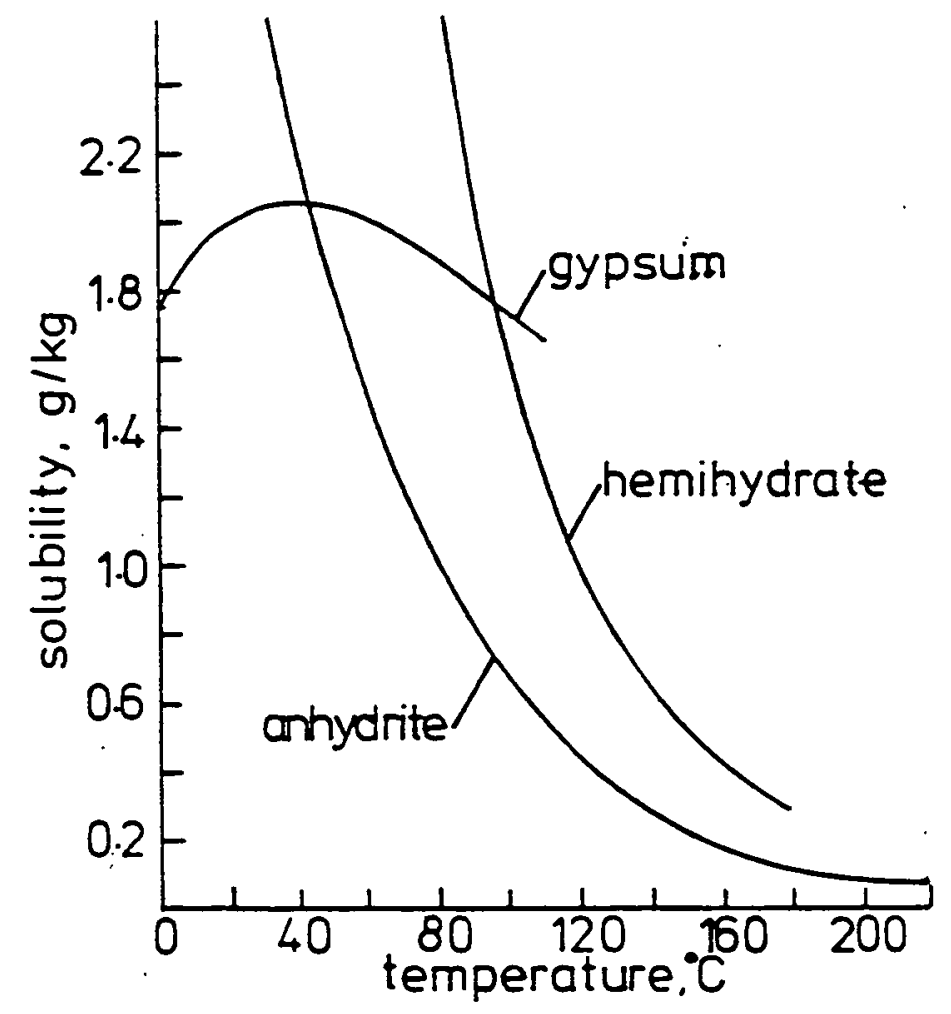

Figure (7.1) $\mathrm{CaSO}_{4}$ Solubility Limits after [48]

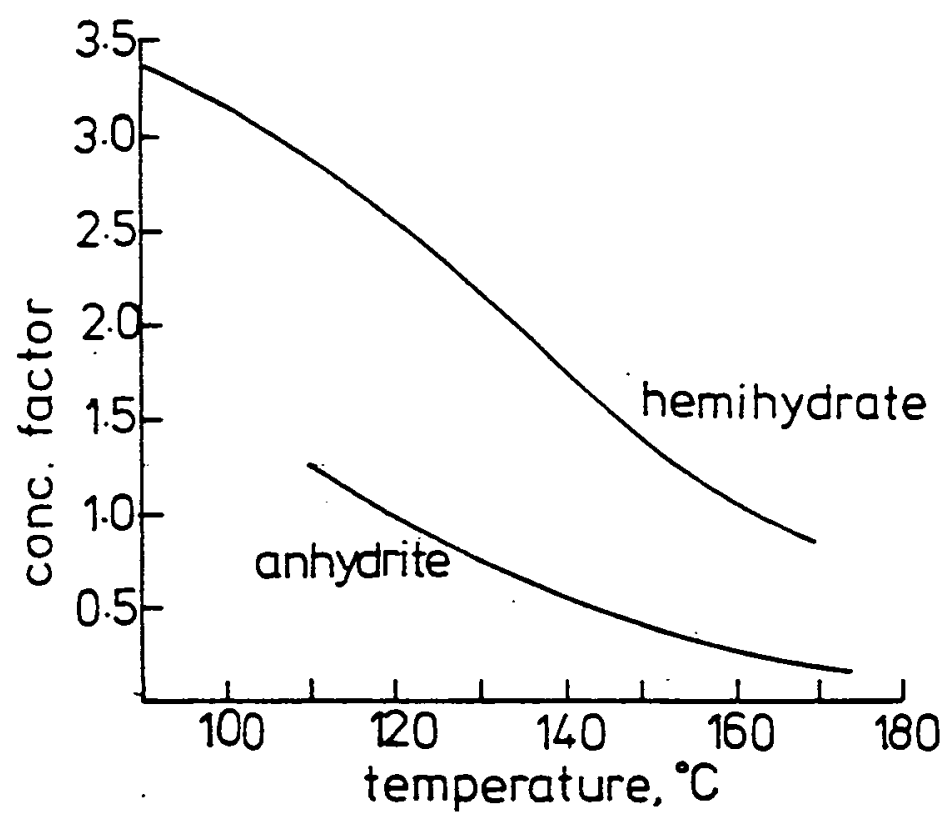

Figure (72) Equilibrium Diagram for Crystalline Forms of $\mathrm{CaSO}_{4}$ after [53] 


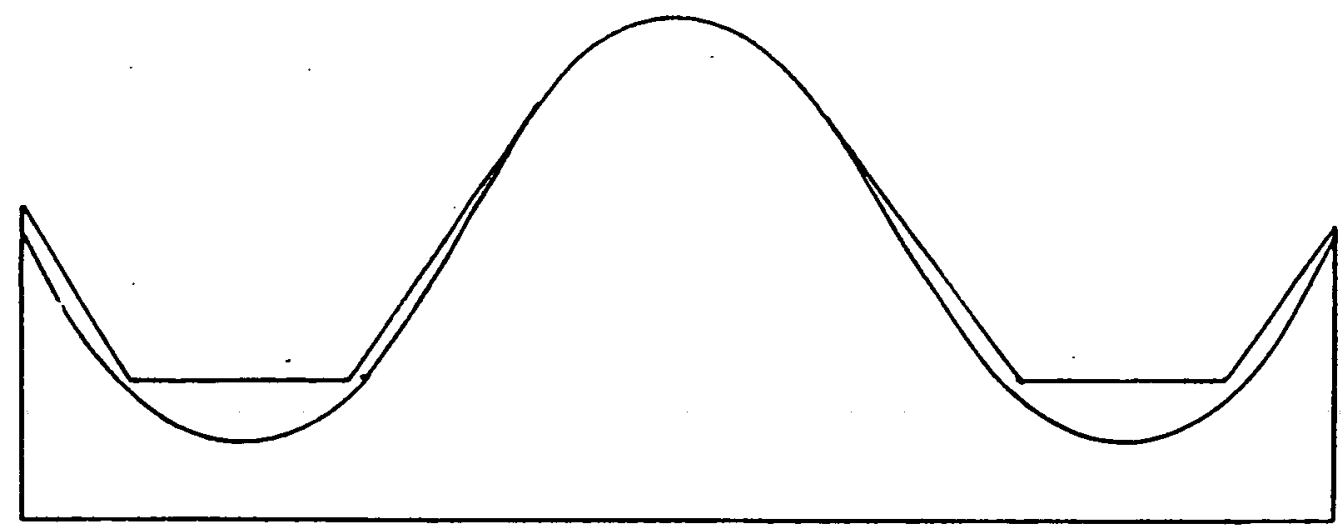

Figure (7.3) Gregorig Condensation Surface

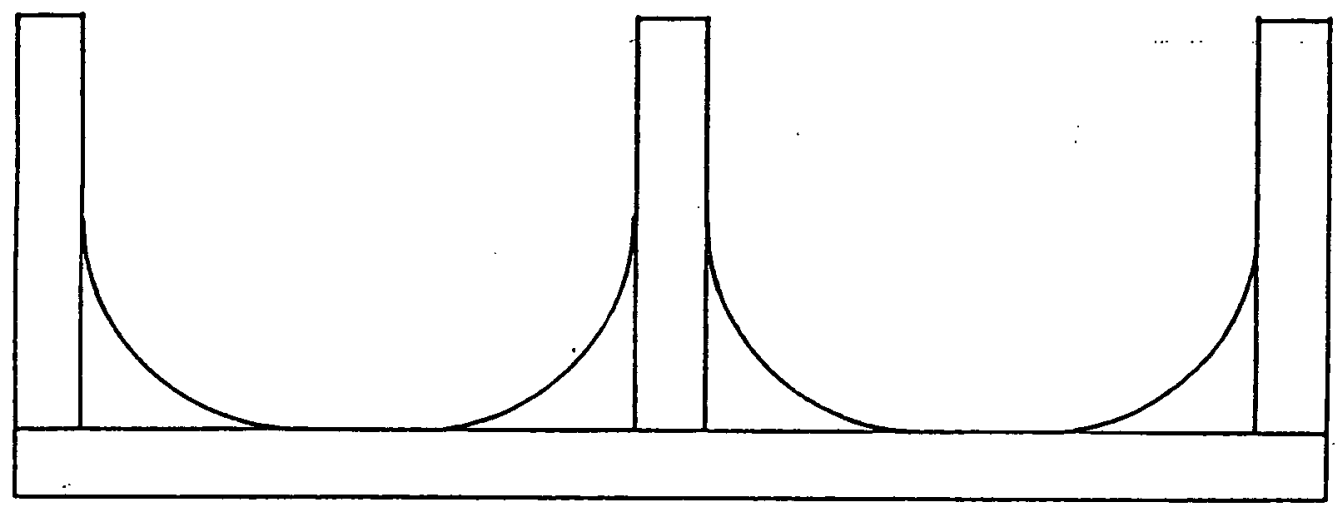

Figure (7.4) Finned Condensing Surface after [70]

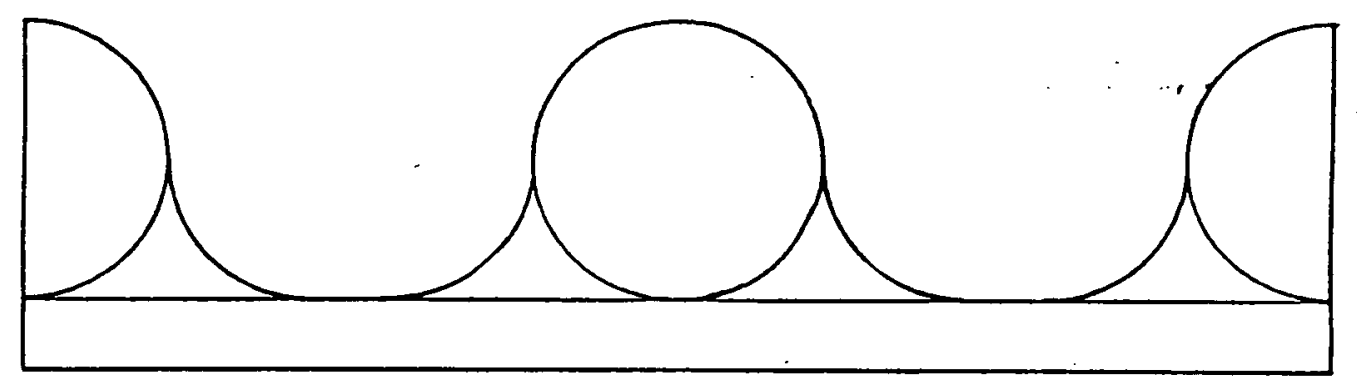

Figure (7.5) Wire-Wrapped Tube after [70] 


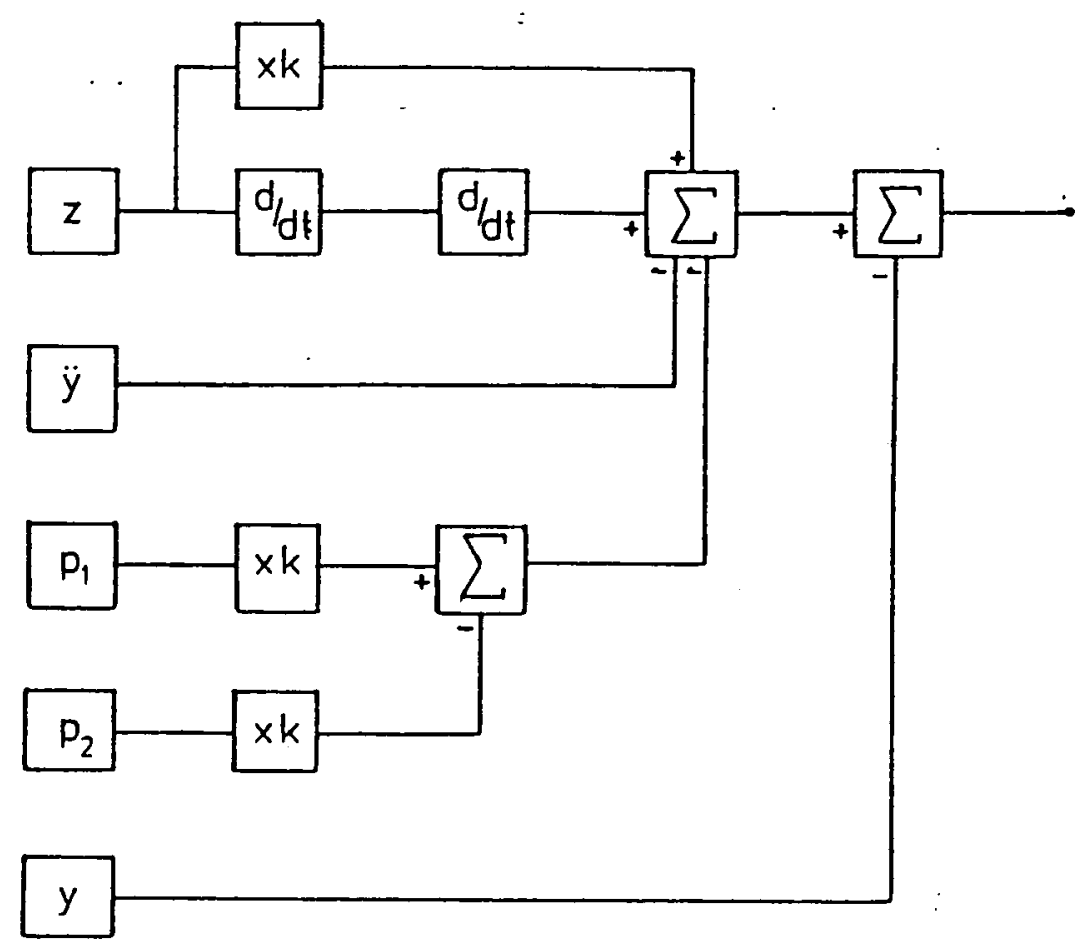

Figure (7.6) Block Diagram of Pressure Compliance Control System

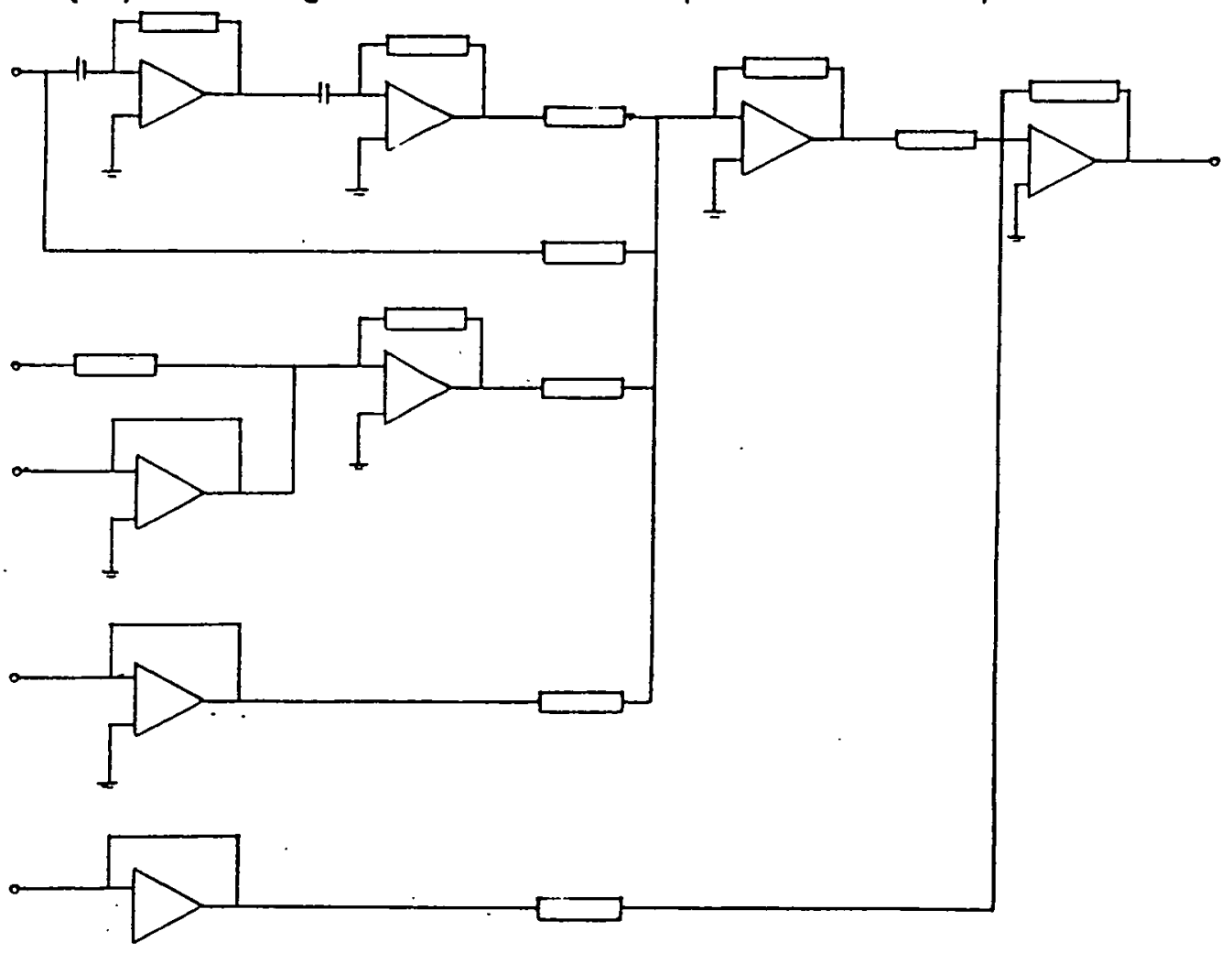

Figure (7.7) Simplified Circuit Diagram for Compliance System 


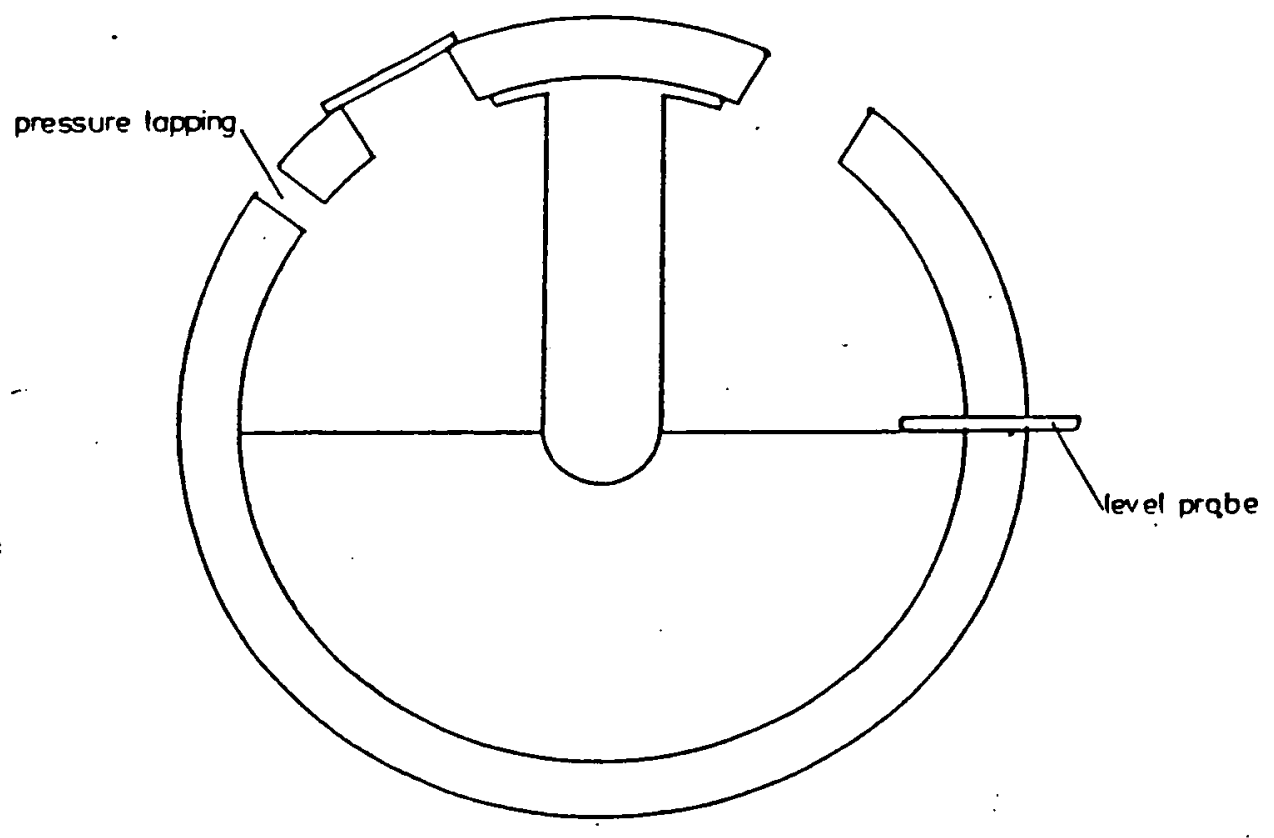

Figure (7.8) Apparatus for Study of Fluid Piston Motion

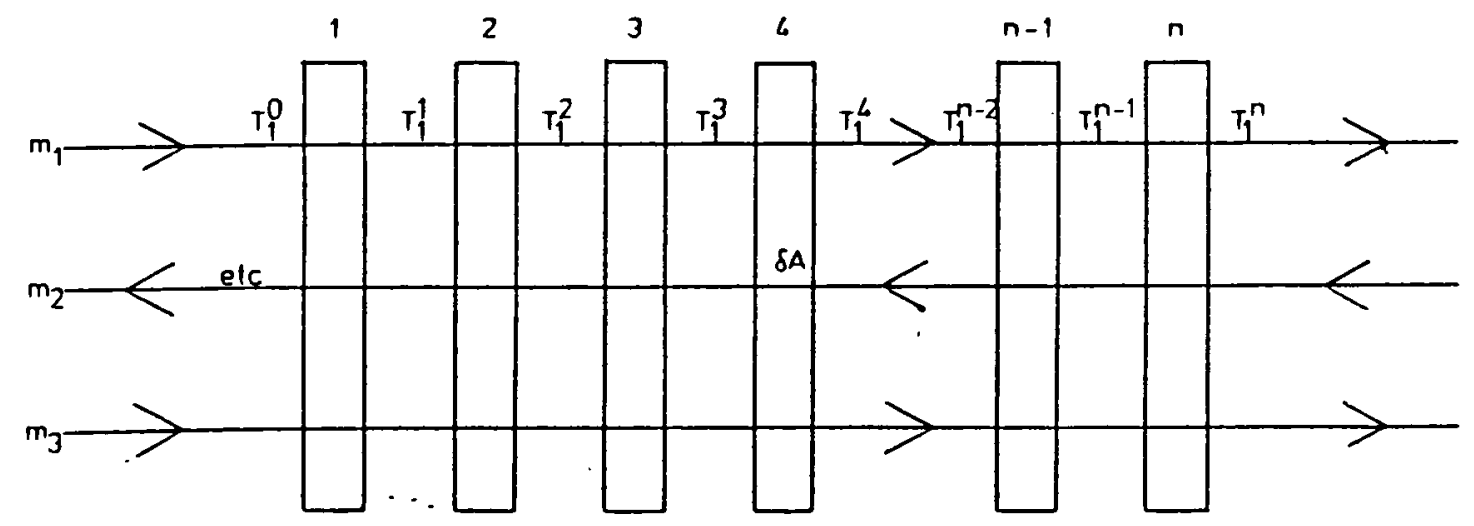

Figure (7.9) Stage-Wise Heat Recovery Exchanger Model Basis 


\section{NOMENCLATURE}

A Antoine coefficient

area

$m^{2}$

Ao area of vena contracta

$m^{2}$

a activity

amplitude

rad

B Antoine coefficient

bpe boiling point elevation

${ }^{\circ} \mathrm{C}$

C Antoine coefficient

concentration

$\mathrm{kg} \cdot \mathrm{m}^{-3}$

Cd orifice coefficient

C specific heat capacity

$J . \mathrm{kg}^{-1} \cdot \mathrm{K}^{-1}$

c

celerity

m.s ${ }^{-1}$

D damping coefficient

N.s.rad ${ }^{-1}$

diameter

m

diffusion coefficient

$\mathrm{m}^{2} \cdot \mathrm{s}^{-1}$

d radius of centre of mass of

fluid piston (equation (3.34))

m

F force

$\mathbf{N}$

f fouling factor

$W \cdot m^{-2} \cdot k^{-1}$

frequency

$\operatorname{rad.s}{ }^{-1}$

G controller gain

g gravitational acceleration

m.s s $^{-2}$

h depth of sea below

still water line

m

heat transfer film

coefficient

$W \cdot m^{-2} \cdot k^{-1}$

I current

A 
inertial coefficient

N.s $\mathbf{s}^{2} \cdot \mathrm{rad}^{-1}$

K

dissociation constant

k

general constant

thermal conductivity

$W \cdot m^{-1} \cdot K^{-1}$

L

length

m

level

m

wavelength

m

membrane thickness

m

M

mass of fluid piston

kg

m

mass flow

kg. $\mathrm{s}^{-1}$

mass

$\mathbf{k g}$

number of stages

P mechanical power

w

membrane permeability

$$
\text { p }
$$

Q

pressure

N. $\mathrm{m}^{-2}$

thermal power

w

q heat flux

resultant velocity

R gas constant

W. $\mathrm{m}^{-2}$

$\mathrm{m} . \mathrm{s}^{-1}$

$\mathrm{J} . \mathrm{kg}^{-1} \cdot \mathrm{K}^{-1}$

performance ratio

radius

m

spring coefficient

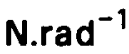

salinity

$\mathrm{kg} \cdot \mathrm{m}^{-3}$

scaling factor

$T$

period

temperature

s

${ }^{\circ} \mathrm{C}$

time

s

internal energy

J.kg ${ }^{-1}$

overall heat transfer

coefficient

$W \cdot m^{-2} \cdot k^{-1}$ 
$\mathbf{u}$

velocity component

voltage

volume

windspeed

$\mathbf{v}$

w

$x$

Y

$\mathbf{z}$

Greek Letters

$\alpha$

amplitude of duck motion

constant in equation (2.18)

displacment of fluid element

volume ratio

B

$\Gamma$

$\gamma$

$\gamma_{t}$

$\Delta$

$\delta$

displacement of fluid element

condensate loading

polytropic compression index

minimum temperature approach

prefix, denoting difference

boiling point elevation

film thickness

prefix, denoting increment

E

$\varepsilon$ $\mathrm{m} \cdot \mathrm{s}^{-1}$

v

$\mathrm{m}^{3}$

$\mathrm{m} \cdot \mathrm{s}^{-1}$

$\mathrm{m}^{3} \cdot \mathrm{kg}^{-1}$

$\mathrm{m} \cdot \mathrm{s}^{-1}$

m

$\mathrm{rad}$

m

m

$\mathrm{kg} \cdot \mathrm{s}^{-1} \cdot \mathrm{m}^{-1}$

${ }^{\circ} \mathrm{C}$

${ }^{\circ} \mathrm{C}$

m 


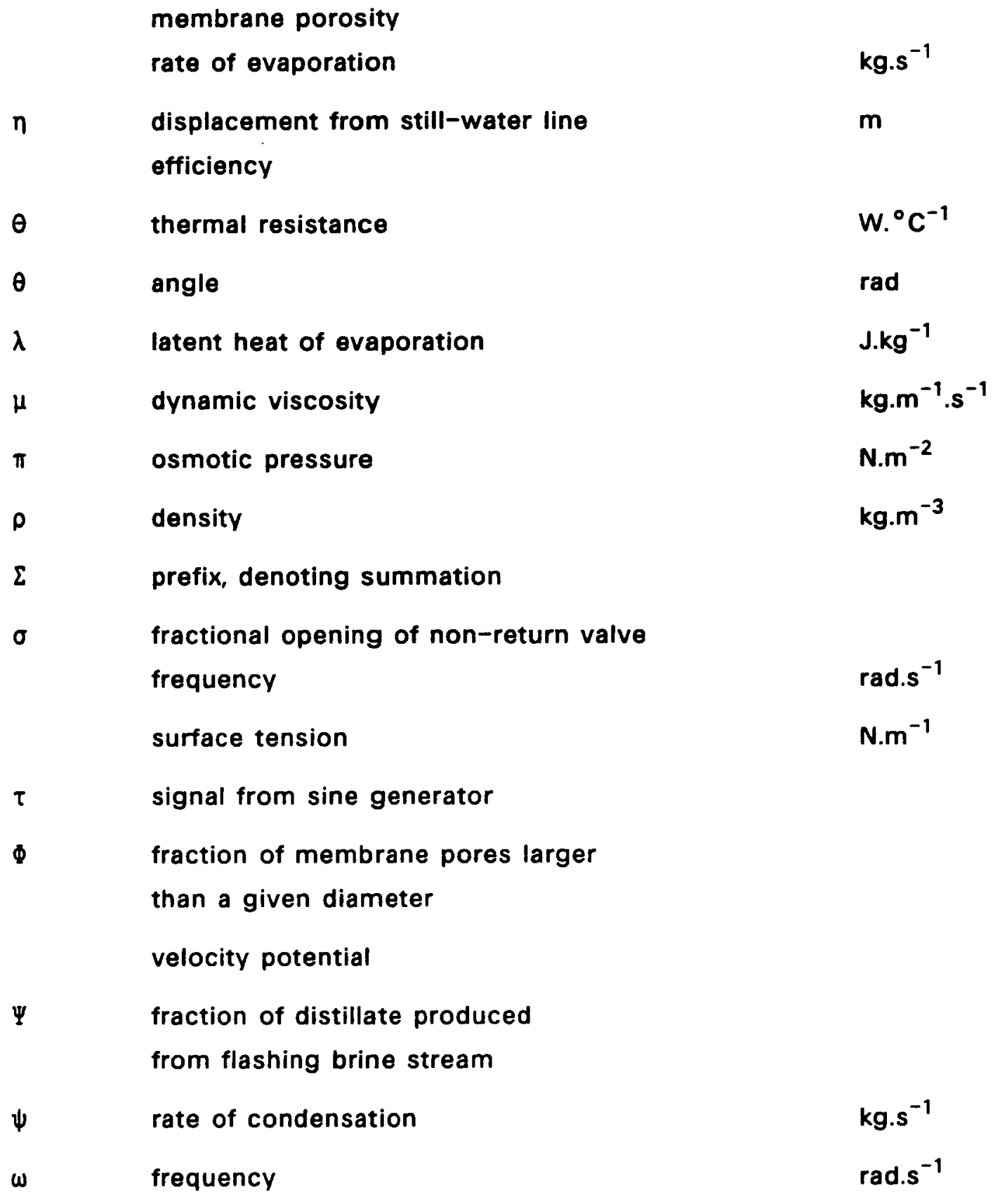

Non-Dimensional Groups

$=u /(g . L)^{0.5}$

Nu Nusselt no.

$=h . L / k$

Pr

Prandt no.

$=\mu . C_{p} / \lambda$ 


$\begin{array}{llll}\text { Re } & \text { Reynolds no. } & = & \rho . U . L / \mu \\ \mathrm{Re}_{\mathrm{a}} & \begin{array}{l}\text { Modified Reynolds no. } \\ \text { for agitated vessels }\end{array} & =\mathrm{L}^{2} \cdot \mathrm{N} \cdot \rho / \mu \\ & \text { Strouhal no. } & = & \omega . \mathrm{L} / \mathrm{u}\end{array}$

Subscripts

A refers to chamber A of duck; second model

a agitated

B refers to chamber B of duck; second model

C refers to chamber C of duck; second model

c case of LM12 package

coil

condensation

D refers to chamber D of duck; second model

d discharge

distillate

e evaporation

f feed

film

h heater

i at step "i"

inlet

interfacial

internal

j junction

1 - liquid

Im log-mean

m mechanical 


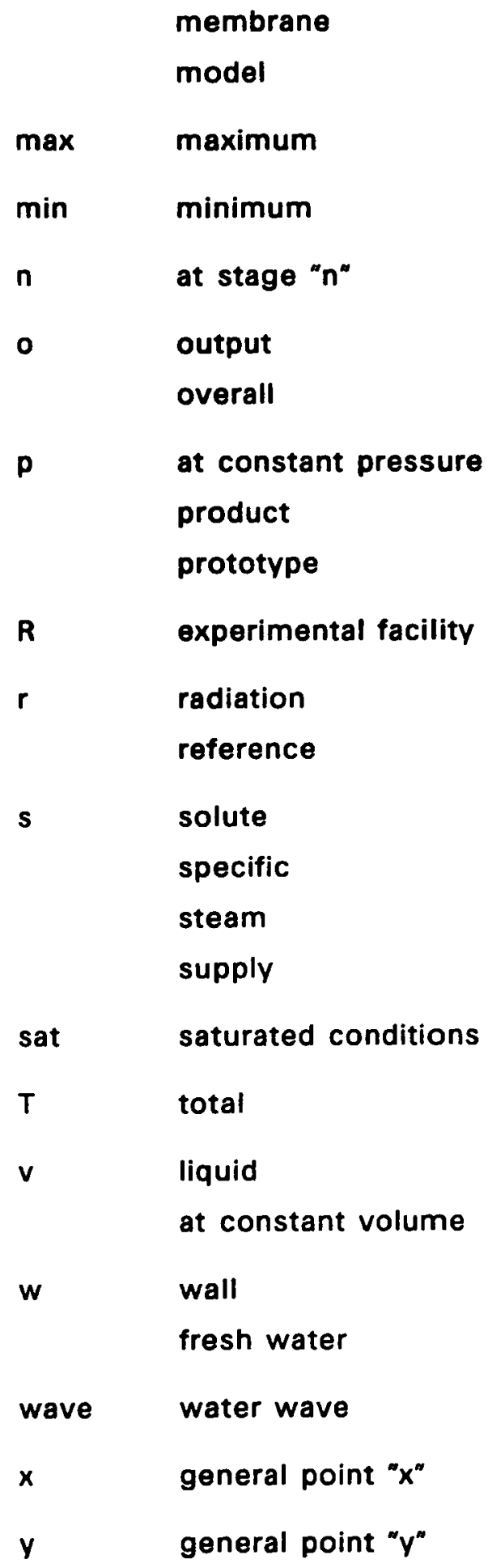

Numerical subscripts refer to streams; points, etc. as identified in the relevant diagrams. 
Superscripts

* dimensionless 
BIBLOGRAPHY 
[1] Alward R.; Lising E.R, and Laward T.A, The Effect of a Variable Power Input on the Performance of a Reverse Osmosis Desalination Unit In: Proceedings of the $4^{\text {th }}$ International Symposium on Fresh Water from the Sea: Volume 4, Elsevier, pp 25-34, 1974

[2] Austin A.E.; Miller J.F.; Vaughan D.A., and Kircher J.F., Chemical Additives for Calcium Sulphate Scale Control Desalination, no.16, pp 345-357, January 1975

[3] Awerbuch $L$ and Fernelius W.A., Geothermal Desalination In: Proceedings of the $5^{\text {th }}$ International Symposium on Fresh Water from the Sea: Volume 2, Elsevier, pp 415-433, 1976

[4] Brian P.L.T, Potential Advantages and Development Problems in Water Desalination by Freezing The Chemical Engineer, pp 191-197, May 1971

[5] Bromley L.A.; Singh D.; Parthasarathi R.; Sribhar S. and Read S.M., Thermodynamic Properties of Sea Salt Solutions, A.I.Chem.E. Journal, 20, pp 326-334, March 1974

[6] Budal K. and Lillebeken P.M., Wave Forces on a Horizontal, Submerged, Spinning Cylinder, In: I.U.T.A.M. Symposium on the Hydrodynamics of Ocean Wave Energy Utilization, I.U.T.A.M., September 1985

[7] Carison A.B. and Gisser D.G., Electrical Engineering-Concepts and Applications, Addison-Wesley, 1981.

[8] Count B.M., (ed.), Power from Sea Waves, Academic Press, 1980.

[9] Crapper G.D., Introduction to Water Waves, Ellis Horwood, 1935.

[10] Crerar A.J.; Pritchard C.L., and Salter S.H., Wave Power for Desalination by Direct Vapour Compression In: Proceedings of the $5^{\text {th }}$ International Conference on Energy Options, Institution of Electrical Engineers, pp 201-203, 1986

[11] Crerar A.J.; Low R.E., and Pritchard C.L., Wave Powered Desalination In: Proceedings of the $3^{\text {rd }}$ World Congress on Desalination and Water Reuse: Volume 4, Elsevier, pp 127-138, September 1987 
[12] Davies P.G.; Cloke M.S.; Major K.A.; Page D.I., and Taylor R.J., Wave Energy - The Department of Energy's R\&D Programme 1974-1983, E.T.S.U. Report R26, U.K.A.E., Harwell,

[13] Delyannis E.E., Status of Solar Assisted Desalination - a Review, In: Proceedings of the $3^{\text {rd }}$ World Congress on Desalination and Water Reuse: Volume 4, Elsevier, pp 3-19, September 1987

[14] Dooly R. and Glater J., Alkaline Scale Formation in Boiling Sea Water Brines Desalination, no.11, pp 1-16, 1972

[15] Dukler A.E., Fluid Mechanics and Heat Transfer in Vertical Falling Film Systems Chem. Eng. Progress Symposium Series, 56, no.30, pp 1-10, 1960

[16] Dukler A.E., Predicting Heat Transfer Coefficients in Film Flow - Part 1: Theory, Petro/Chem. Engineering, pp 52-54, September 1961

[17] Dukler A.E., Predicting Heat Transfer Coefficients in Film Flow - Part2: Design Charts and Equations Petro/Chem. Engineering, pp 46-53, October 1961

[18] Feron P., The Use of Windpower in Autonomous Reverse Osmosis Seawater Desalination Wind Engineering, 9, no.3, pp 180-199, 1985

[19] Fisher U., Compressor and System Matching in Vapour Compression Distillation Units, Israeli Journal of Technology, 15, pp 102-111, 1977

[20] Frankel A., Flash Evaporators for the Distillation of Seawater, Proceedings of the I.Mech.E., 174, no.7, pp 312-324, 1960

[21] Gainey R.J.; Thorp C.A., and Cadwallader E.A., $\mathrm{CaSO}_{4}$ Seeding Prevents $\mathrm{CaSO}_{4}$ Scale Ind. Eng. Chem., 55, no.3, pp 39-43, 1963

[22] Gilron J. and Hasson D., Calcium Sulphate Fouling of Reverse Omosis Membranes, Chem. Engng. Sci., 42 (2), pp 2351-2360, 1987

[23] Gregorig R., Hautkondensation an feingewellten Ober-flachen bei Beruksichtigung der Oberflachenspannungen Zeitchrift fur augewandte Mathematik und Physik, 5, pp 36-49, 1954 
[24] Haywood R.W., Thermodynamic Tables in S.I. (metric) Units, Cambridge University Press, 1972.

[25] Hicks D.C. and Pleass C.M., Physical and Mathematical Modelling of a Point Absorber Wave Energy Conversion System with Nonlinear Damping I.U.T.A.M., pp 113-124, September 1985

[26] Hillier H., Scale Formation in Seawater Distilling PLants and its Prevention, Transactions of the I.Mech.E., January 1952

[27] Hoffman D., Second Generation Low Temperature Vapour Compression Plants. Desalination, no23, pp 449-454, 1977

[28] Howarth J.R.; Havers R.I., and Coombes S., Operation of a 300,000 G.P.D. V.T.E. Plant In: Proceedings of the $5^{\text {th }}$ International Symposium on Fresh Water from the Sea: Volume 2, Elsevier, May 1976

[29] Hunter R.F.; Simmons P.J.; Tan K.S., and Spinner I.H., Reciprocating Flow Ion Exchange: Part Application to Water Softening Can. J. Chem. Engng., 46, pp 57-61, 1968

[30] Isdale J.D. and Morris R., Physical Properties of Seawater Solutions, Desalination, no.7, pp 23-30, 1969

[31] Isdale J.D.; Spence C.M., and Tudhope J.S., Pysical Properties of Seawater Solutions: Viscosity, Desalination, no.10, pp 219-328, 1972

[32] Kammal 1.; Emmerman D.K., and Tusel G.F., RO, MSF and VTENC Desalination: a Comparitive Evaluation Desalination, no.33, pp 229-239, 1980

[33] Kirk-Othmer, Encyclopedia of Chemical Technology, 9 see Alberger Flash Process 1980

[34] Kremmen S.S., Means By Which Reverse Osmosis Desalting Costs Can Be Substantially Reduced Well Before the Year 2000, Desalination, no.30, pp 56-68, 1980

[35] Langelier W.F.; Caldwell D.W.; Lawrence W.B., and Spaulding C.H., Scale Control in Sea Water Distillation Equipment Industrial and Engineering 
Chemistry, 42, pp 126-130, 1950

[36] Le Martret J. Ph., Desalination Through Vapour Compression Process in Large Plants, Desalination, no.19, pp 337-348, 1976

[37] Liberti L.; Passino R.; Santori M., and Boari G., Technical and Economic Trends of Seawater Desalting in the '90's In: Proceedings of the $2^{\text {nd }}$ World Congress on Desalination and Water Reuse: Volume 2, Elsevier, pp 503-514, 1985

[38] Lighthill M.J., Sir, Waves in Fluids, Cambridge University Press, 1978.

[39] Loebel F.A., Vapour Compression for Brakish and Sea Water Desalting In: Proceedings of the $2^{\text {nd }}$ European Symposium on Fresh Water from the Sea: Volume 2, Elsevier, pp 28.1-28.8, May 1967

[40] Lucas M. and Murat F., Desalination by Vapour Compression: Operational Results After One Year's Operation of Flammanville Unit In: Proceedings of the $3^{\text {rd }}$ International Congress on Desalination and Water Reuse: Volume 2, Elsevier, pp 33-42, 1985

[41] Margolis G.; Sherwood T.K.; Brian P.L.T., and Sarofim A.F., The Performance of a Continuous Well Stirred lce Crystallizer, Ind. Eng. Chem. Fundam., 10, no.3, pp 439-452, 1971

[42] Matz R. and Fisher U., A Comparison of the Relative Economics of Seawater Desalination by Vapour Compression and Reverse Osmosis for Small to Medium Capacity Plants. Desalination, no.36, pp 137-151, 1981

[43] Mollison D., Wave Climate and the Wave Power Resource In: I.U.T.A.M. Symposium on the Hydrodynamics of Ocean Wave Energy Utilization, I.U.T.A.M., September 1985

[44] Morris N.M., Control Engineering M $^{\mathrm{C}} \mathrm{Graw}-\mathrm{Hill}, 1983$.

[45] Ohe S., Computer Aided Data Book of Vapour Pressure Data Book Publishing Co., Tokyo, 1972.

[46] Okazaki M. and Kimura S., Scale Formation on Reverse Osmosis Membranes J. Chem. Engng. Japan, 17 (2), pp 145-151, 1985 
[47] Ouahes C.; Ouahes R.; Le Goff J., and Le Goff P., A Hardy, High-Yield Solar Distiller of Brakish Water, In: Proceedings of the $3^{\text {rd }}$ World Congress on Desalination and Water Reuse: Volume 4, Elsevier, pp 43-42, September 1987

[48] Partridge E.P. and White A.H., The Solubility of Calcium Sulfate from 0 to $200^{\circ}$, Journal of the American Chemical Society, 51, pp 360-370, 1929

[49] Perry R.H. and Green D. (ed.s), Perry's Chemical Engineer's Handbook $M^{c}$ Graw-Hill, 1985.

[50] Petersen G.; Fries S.; Mohn J., and Mueller A., Wind-and Solar-Powered Reverse Osmosis Desalination Units, Desalination, no.31, pp 501-509, 1971

[51] Petersen G.; Fries S.; Mohn J., and Mueller A., Wind-and Solar-Powered Reverse Osmosis Desalination - Design Start-Up, Operating Experience, Desalination, no.39, pp 125-135, 1981

[52] Pleass C.M., The Use of Wave Powered Systems for Desalination In: Proceedings of the International Symposium on Wave and Tidal Energy, B.H.R.A. Fluid Engineering, pp D1.1-D1.10, $27^{\text {th }}-29^{\text {th }}$ September, 1978

[53] Porteous A., Sea Water Distillation Processes, $M^{\mathrm{C}} \mathrm{Graw}$-Hill, 1975.

[54] Porteous A. (ed.), Desalination Technology - Developments and Practice, Applied Science Publishers, 1983.

[55] Posnansky M., Technical and Economic Aspects of Solar Desalination with Particular Emphasis on Solar Pond Powered Distillation Plants In: Proceedings of the $3^{\text {rd }}$ World Congress on Desalination and Water Reuse: Volume 4, Elsevier, pp 81-95, 1987

[56] Rautenbach R. and Arzt B., Large Scale Diesel Driven Vapor Compression Units, Desalination, no.38, pp 85-97, 1981

[57] Roach P.D. and Holtz R.E., Plastic Heat Exchangers for Heat Recovery, Final Report ANL-83-81, Argonne National Laboratory, Illinois, 
[58] Rothfus R.R. and Lavi G.H., Vertical Falling Film Heat Transfer - A Literature Survey, Proceedings of the $5^{\text {th }}$ International O.T.E.C. Conference,

[59] Salter S.H., Progress on Edinburgh Ducks, In: I.U.T.A.M. Symposium on the Hydrodynamics of Ocean Wave Energy Utilization, I.U.T.A.M., September 1985

[60] Salter S.H., Wave Powered Desalination In: Proceedings of the $4^{\text {th }}$ Conference on Energy for Rural and Isolated Communities, Institution of Electrical Engineers, 1985

[61] Schnabel G., Analysis of Local and Overall Heat Transfer Enhancement on Double-Fluted Condenser/Evaporator Tubes Verfahrenstechnik, 17, pp 616-632, October 1983

[62] Senatore S.J., Vapour Compression Distillation In: Proceedings of the $7^{\text {th }}$ International Conference on Fresh Water from the Sea: Volume 1, Elsevier, pp $351-358,1980$

[63] Senatore S.J., Vapour Compression Distillation Desalination, no.42, pp $151-161,1982$

[64] Sephton S.H., New Developments in Vertical Tube Evaporation of Sea Water, In: Proceedings of the $5^{\text {th }}$ International Conference on Fresh Water from the Sea: Volume 2, Elsevier, pp 279-287, May 1976

[65] Shaw R., Wave Energy - a Design Challenge, Ellis Horwood, 1980.

[66] Souririjan S., The Mechanism of Demineralisation of Aqueous Sodium Chloride Solutions by Flow, Under Pressure, Through Porous Membranes, Ind. Engng. Chem., 2, pp 51-55, February 1976

[67] Speigler K.S. and Laird A.D.K (ed.s), Principles of Desalination; Parts A and $B$ Academic Press, 1980.

[68] Stone F.E. and Rogers A.N., A Projected World Market for Seawater Desalination Equipment In: Proceedings of the $3^{\text {rd }}$ International Symposium on Desalination and Water Reuse; Volume 2, Elsevier, pp 529-556, 1985 
[69] Struve H., Heat Transfer to an Evaporating Liquid Film V.D.I. Forschungsheft, no.534, 1965

[70] Thomas D.G. and Young G., Thin-Film Enhancement by Finned Surfaces Ind. Eng. Chem. Process Design and Development, 9, no.2, pp 317-323, 1970

[71] Tliemat B.W. and Howe E.D., Comparative Productivity of Distillation and Reverse Osmosis Using Solar Energy from Solar Ponds Trans. A.S.M.E. Journal of Solar Engineering, 104, pp 299-304, November 1982

[72] Unione A.; $M^{c}$ Clymont A.; Dix T., and Husseiny A., Wind Powered Reverse Osmosis for Self-Contained Village Applications, a Design Study, In: Proceedings of the $7^{\text {th }}$ International Symposium on Fresh Water from the Sea: Volume 2, Elsevier, pp 319-330, 1980

[73] The United Nations Organisation, Water Desalination in Developing Countries, United Nations Publications, Report no.ST/ECA/82, 1964

[74] United Wire Group Ltd., Edinburgh, MPC Development Product Internal evaluation report for U.W.G. Ltd. on mesh plastic composite,

[75] The United Nations Organisation, $2^{\text {nd }}$ United Nations Desalination Plant Operation Survey, United Nations Publications, Report no.GP/UN/702, 1973

[76] Webb R.L., Special Surface Geometries for Heat Transfer Augmentation Developments in Heat Exchanger Technology, 101, pp 179-215, 1980

[77] Whittaker, Recent Developments in Wave Energy Systems, In: Proceedings of the $5^{\text {th }}$ International Conference on Energy Options, The Institution of Electrical Engineers, 1987

[78] Wilke W., Warmeubergang an Reiselfilme (Heat Transfer in Falling Films), V.D.I. Forschungsheft, no.490, 1962

[79] Wood F.C, The Changing Face of Desalination - A Consulting Engineer's Vewpoint Desalination no.42, pp 17-25, March 1982

[80] Wang G.E., Greig R. and Laird A.D.K., Study of Heat Transfer in a Rotating Disc Evaporator, Desalination, no.33, pp 259-276, March 1980 